University of Tennessee Health Science Center

UTHSC Digital Commons

\title{
Synthesis of Novel Cannabinoid Ligands and Their Use as Anti- Glioma and Anti-Inflammatory Agents
}

\author{
Steven Neal Gurley \\ University of Tennessee Health Science Center
}

Follow this and additional works at: https://dc.uthsc.edu/dissertations

Part of the Pharmacy and Pharmaceutical Sciences Commons

\section{Recommended Citation}

Gurley, Steven Neal , "Synthesis of Novel Cannabinoid Ligands and Their Use as Anti-Glioma and AntiInflammatory Agents" (2010). Theses and Dissertations (ETD). Paper 111. http://dx.doi.org/10.21007/ etd.cghs.2010.0122.

This Dissertation is brought to you for free and open access by the College of Graduate Health Sciences at UTHSC Digital Commons. It has been accepted for inclusion in Theses and Dissertations (ETD) by an authorized administrator of UTHSC Digital Commons. For more information, please contact jwelch30@uthsc.edu. 


\title{
Synthesis of Novel Cannabinoid Ligands and Their Use as Anti-Glioma and Anti- Inflammatory Agents
}

\begin{abstract}
Following the discovery of the cannabinoid receptors, research in the field of cannabinoids has grown exponentially over the last two decades. Cannabinoids have been shown to have tremendous therapeutic potential in the treatment of several pathological conditions ranging from inflammation to asthma, multiple sclerosis, Parkinson's disease, epilepsy, glaucoma, septic shock, hemorrhagic shock, and cancer. Our research has focused on two major conditions for which cannabinoids hold great promise for drug development, namely, cancer and inflammation.

Our focus in the field of cancer has been on the devastatingly lethal brain tumor glioblastoma multiforme. Due to the high expression of the CB2 receptor in human gliomas, we chose to focus on two CB2 selective lead compounds for these studies: KM-233 (CB2 antagonist) and HB-I-172 (CB2 inverse agonist). Both compounds showed in vitro efficacy against four human glioma cell lines ( $\mathrm{EC}_{50 \mathrm{~S}}=2.27 \pm$ $0.18 \mu \mathrm{M}$ to $6.34 \pm 4.10 \mu \mathrm{M}$ ) while HB-I-172 also showed efficacy against prostate, lung, and colorectal tumor lines $(2.38 \pm 0.75 \mu \mathrm{M}$ to $5.22 \pm 1.2 \mu \mathrm{M})$. Significant reductions in tumor load were also observed in vivo in both side flank and intracranial models of glioblastoma. The anti-glioma efficacies of the two lead compounds were not altered by pharmacologic blockade of the CB1 and CB2 receptors which points to a non-CB1/CB2 mediated signaling pathway. This pathway was examined in the human glioma line U-87 MG and determined to involve reductions in pMEK1/2, pERK1/2, pAkt, and p-p70S6K with increases in pGSK3B, p53, and cleaved caspases 3 and 9, and PARP. Immunohistochemical studies revealed loss of mitochondrial membrane polarization with release of cytochrome-C, DNA fragmentation and condensation, and alterations in cytoskeletal structure.

The current focus in the field of cannabinoids as anti-inflammatory agents is on CB2 selective compounds which are devoid of the CNS side-effects which could limit their therapeutic usefulness. We screened a variety of novel cannabinoids and the CB2 inverse agonist HB-I-172 emerged as a lead. In vitrotreatment with HB-I-172 caused a significant reduction in a number of proinflammatory cytokines and chemokines in both neoplastic lung epithelial cell and macrophage models and in primary human lung epithelial and endothelial cells, macrophages, and microglia. We believe that HB-I-172 and other CB2 selective cannabinoids are promising treatments for a variety of inflammatory conditions from neuroinflammatory disorders such as multiple sclerosis and Huntington's disease to systemic conditions such as atherosclerosis and inflammatory pain.
\end{abstract}

Finally, three new series of heterocyclic cannabinoid ligands were synthesized and screened. The hexahydro pyrimidine classical, pyrimidine nonclassical, and furanopyrimidine cannabinoids were designed with CB2 selectivity in mind. After many failed synthetic routes were abandoned, the three new classes were successfully generated. Many of these compounds showed good CB2 selectivity (77-fold) and showed efficacy in both anti-glioblastoma and anti-inflammatory studies.

Document Type

Dissertation

Degree Name

Doctor of Philosophy (PhD)

Program

Pharmaceutical Sciences 


\section{Research Advisor}

Bob M. Moore II, Ph.D.

\section{Keywords}

Cannabinoids, CB2, Glioblastoma, Heterocyclic, Inflammation

\section{Subject Categories}

Medicine and Health Sciences | Pharmacy and Pharmaceutical Sciences 


\title{
Synthesis of Novel Cannabinoid Ligands and Their Use as Anti-Glioma and Anti-Inflammatory Agents
}

\author{
A Dissertation \\ Presented for \\ The Graduate Studies Council \\ The University of Tennessee \\ Health Science Center
}

\author{
In Partial Fulfillment \\ Of the Requirements for the Degree \\ Doctor of Philosophy \\ From The University of Tennessee
}

\author{
By \\ Steven Neal Gurley \\ May 2010
}


Copyright (C) 2010 by Steven Neal Gurley

All rights reserved 


\section{Dedication}

This dissertation is dedicated to my wife Amanda who has also endured the many years of research that this document represents. 


\section{Acknowledgements}

I would like to thank my major professor, Dr. Bob M. Moore II for his support, guidance, and patience. I would also like to thank my committee members, Dr. Duane D. Miller, Dr. Isaac O. Donkor, Dr. Wei Li and Dr. Andrea Elberger for their useful suggestions and guidance.

I would like to thank my colleagues Dr. Himanshu Bhattacharjee, Dr. Suni Mustafa, and Dr. Bret Koertge for all of their support and useful discussions. I would also like to thank other members of our laboratory, both past and present, for their support and cooperation: Dr. Mathangi Krishnamurthy, Patrick Allison, Peihong Guan, Dr. Xiofeng Zhang, Erin Oliver, Dr. Supriya Bavadekar, Dr. Christopher Surdock, Dr. Galina Dulatov, Negin Nouraei, and Ammaar Abidi.

Finally I would like to thank the College of Pharmacy at The University of Tennessee Health Science Center for giving me the opportunity to pursue my research. 


\begin{abstract}
Following the discovery of the cannabinoid receptors, research in the field of cannabinoids has grown exponentially over the last two decades. Cannabinoids have been shown to have tremendous therapeutic potential in the treatment of several pathological conditions ranging from inflammation to asthma, multiple sclerosis, Parkinson's disease, epilepsy, glaucoma, septic shock, hemorrhagic shock, and cancer. Our research has focused on two major conditions for which cannabinoids hold great promise for drug development, namely, cancer and inflammation.

Our focus in the field of cancer has been on the devastatingly lethal brain tumor glioblastoma multiforme. Due to the high expression of the CB2 receptor in human gliomas, we chose to focus on two CB2 selective lead compounds for these studies: KM233 (CB2 antagonist) and HB-I-172 (CB2 inverse agonist). Both compounds showed in vitro efficacy against four human glioma cell lines $\left(\mathrm{EC}_{50} \mathrm{~S}=2.27 \pm 0.18 \mu \mathrm{M}\right.$ to $6.34 \pm$ $4.10 \mu \mathrm{M}$ ) while HB-I-172 also showed efficacy against prostate, lung, and colorectal tumor lines $(2.38 \pm 0.75 \mu \mathrm{M}$ to $5.22 \pm 1.2 \mu \mathrm{M})$. Significant reductions in tumor load were also observed in vivo in both side flank and intracranial models of glioblastoma. The anti-glioma efficacies of the two lead compounds were not altered by pharmacologic blockade of the $\mathrm{CB} 1$ and $\mathrm{CB} 2$ receptors which points to a non-CB1/CB2 mediated signaling pathway. This pathway was examined in the human glioma line U-87 MG and determined to involve reductions in pMEK1/2, pERK1/2, pAkt, and p-p70S6K with increases in pGSK3B, p53, and cleaved caspases 3 and 9, and PARP.

Immunohistochemical studies revealed loss of mitochondrial membrane polarization with release of cytochrome-C, DNA fragmentation and condensation, and alterations in cytoskeletal structure.
\end{abstract}

The current focus in the field of cannabinoids as anti-inflammatory agents is on CB2 selective compounds which are devoid of the CNS side-effects which could limit their therapeutic usefulness. We screened a variety of novel cannabinoids and the CB2 inverse agonist HB-I-172 emerged as a lead. In vitro treatment with HB-I-172 caused a significant reduction in a number of proinflammatory cytokines and chemokines in both neoplastic lung epithelial cell and macrophage models and in primary human lung epithelial and endothelial cells, macrophages, and microglia. We believe that HB-I-172 and other CB2 selective cannabinoids are promising treatments for a variety of inflammatory conditions from neuro-inflammatory disorders such as multiple sclerosis and Huntington's disease to systemic conditions such as atherosclerosis and inflammatory pain.

Finally, three new series of heterocyclic cannabinoid ligands were synthesized and screened. The hexahydro pyrimidine classical, pyrimidine nonclassical, and furanopyrimidine cannabinoids were designed with CB2 selectivity in mind. After many failed synthetic routes were abandoned, the three new classes were successfully generated. Many of these compounds showed good CB2 selectivity (77-fold) and showed efficacy in both anti-glioblastoma and anti-inflammatory studies. 


\section{Table of Contents}

Chapter 1: Introduction ....................................................................................................1

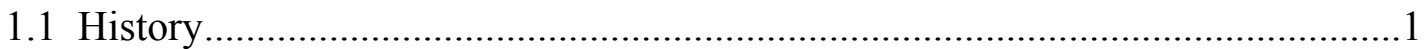

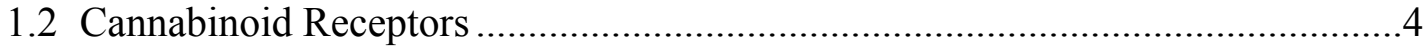

1.3 Cannabinoids as Anti-Glioma Agents ......................................................

1.3.1 Current Standard of Care for Glioblastoma .............................................

1.3.1.1 Surgical Intervention ........................................................ 7

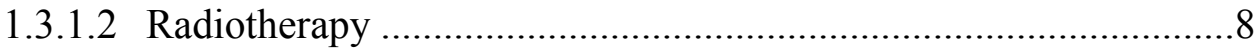

1.3.1.3 Chemotherapy .......................................................................

1.3.2 Cannabinoids as Chemotherapeutic Agents for the Treatment of High-Grade Gliomas ................................................................9

1.4 Cannabinoids as Anti-Inflammatory Agents .............................................. 10

1.4.1 Cannabinoid Receptors in the Immune System ...............................10

1.4.2 Cannabinoid Effects on Immunity ....................................................11

Chapter 2: Anti-Glioma Activity of KM-233 and Analogs .....................................14

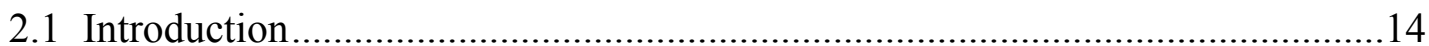

2.2 Murine Flank Model ............................................................................... 19

2.3 Efficacy of KM-233 and Analogs against Multiple Glioma Lines ....................23

2.4 Receptors Involved in the Anti-Glioma Activity of KM-233 .........................26

2.5 Mechanistic Studies to Determine the Anti-Glioma Activity of KM-233 ..........31

2.6 Experimental Methods .............................................................................4

2.6.1 Cytotoxicity Assays .......................................................................4

2.6.2 Cytotoxicity Assays with Pertussis Toxin .......................................4 41

2.6.3 CB1/CB2 Functional Assays ............................................................43

2.6.4 MSD Protein Quantification .............................................................43

2.6.5 Mitochondrial Membrane Potential Assay ...........................................44

2.6.6 Microscopic Analysis of Cytoskeleton and Cytochrome C Release ......44

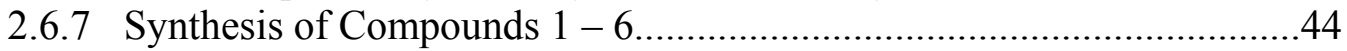

Chapter 3: Anti-Glioma Activity of HB-I-172 and Analogs .....................................45

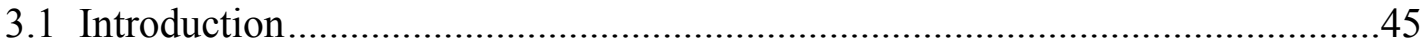

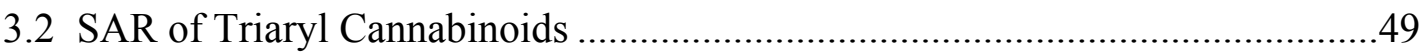

3.3 Antineoplastic Activity of Triaryl Cannabinoids and Selection of HB-I-172 ....52

3.4 Receptors Involved in the Anti-Glioma Activity of HB-I-172 .......................55

3.5 Comparison of HB-I-172 to Standard GBM Treatments .................................58

3.6 Mechanistic Studies to Determine the Anti-Glioma Activity of HB-I-172 ........62

3.6.1 Methods and Results .....................................................................62

3.6.2 Proposed Mechanism of the Anti-Glioma Activity of HB-I-172 „..........80

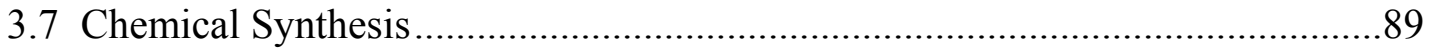

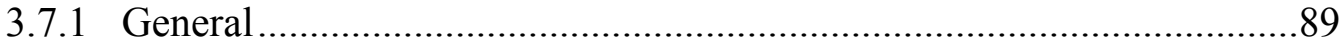

3.7.2 Synthesis of 4-formyl-2,6-dimethoxyphenyl trifluoromethanesulfonate (18) 
3.7.3 Synthesis of 3',5'-dichloro-2,6-dimethoxybiphenyl-4-carbaldehyde (20)

3.7.4 Synthesis of (3',5'-dichloro-2,6-dimethoxybiphenyl-4yl)(phenyl)methanol (8).

3.7.5 Synthesis of (3',5'-dichloro-2,6-dimethoxybiphenyl-4yl)(phenyl)methanone (14)

3.7.6 Synthesis of 3',5'-dichloro-2,6-dimethoxy-4-(2-phenylpropan-2yl)biphenyl (15)

3.7.7 Synthesis of (3',5'-dichloro-2-hydroxy-6-methoxybiphenyl-4yl)(phenyl)methanone (9) and (3',5'-dichloro-2,6-dihydroxybiphenyl-

4-yl)(phenyl)methanone (10)

3.7.8 Synthesis of 3',5'-dichloro-6-methoxy-4-(2-phenylpropan-2-yl) biphenyl-2-ol (16) and 3',5'-dichloro-4-(2-phenylpropan-2-yl) biphenyl-2,6-diol (11).... .96

3.7.9 Synthesis of 3',5'-dichloro-4-(hydroxy(phenyl)methyl)-6methoxybiphenyl-2-ol (12)

3.7.10 Synthesis of 3',5'-dichloro-4-(hydroxy(phenyl)methyl)biphenyl2,6-diol (13) .97

3.8 Experimental Methods

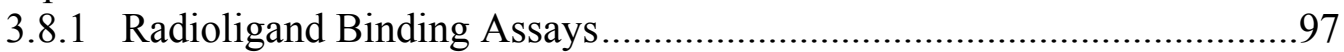

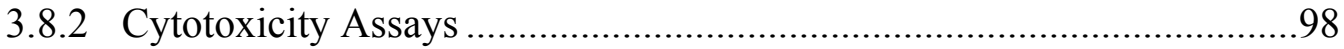

3.8.3 Cytotoxicity Assays with Pertussis Toxin ............................................98

3.8.4 CB1/CB2 Functional Assays ..........................................................98

3.8.5 MSD Protein Quantification ............................................................99

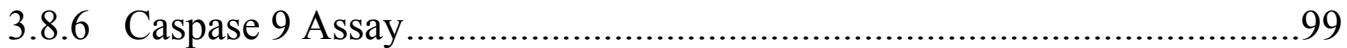

3.8.7 Mitochondrial Membrane Potential Assay ...........................................100

3.8.8 Microscopic Analysis Using Immunohistochemical Techniques..........100

Chapter 4: Design, Synthesis, and Testing of Novel Cannabinoid Ligands ...............101

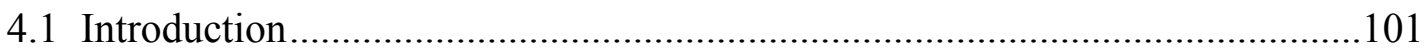

4.2 Design and Synthesis of Novel Compounds...................................................107

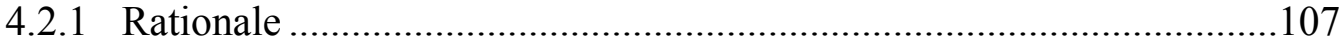

4.2.2 Synthesis of Hexahydro Pyrimidine Classical Cannabinoids ................109

4.2.2.1 Synthesis of 2-methyl-2-phenylpropanenitrile (22) ....................124

4.2.2.2 Synthesis of 2-cyclohexyl-2-methylpropanenitrile (23).............124

4.2.2.3 Synthesis of 2-methyl-2-(thiophen-2-yl)propanenitrile (24)......124

4.2.2.4 Synthesis of 2,2-dimethyloctanenitrile (25) ..............................125

4.2.2.5 Synthesis of 2-methyl-2-phenylpropanimidamide hydrochloride (26)

4.2.2.6 Synthesis of 2-methyl-2-(thiophen-2-yl)propanimidamide hydrochloride (27)

4.2.2.7 Synthesis of 2-cyclohexyl-2-methylpropanimidamide hydrochloride (28)

4.2.2.8 Synthesis of 2,2-dimethyloctanimidamide hydrochloride (29)..126

4.2.2.9 Synthesis of 2-(2-phenylpropan-2-yl)pyrimidine-4,6-diol (30) 126 
4.2.2.10 Synthesis of 2-(2-(thiophen-2-yl)propan-2-yl)pyrimidine-

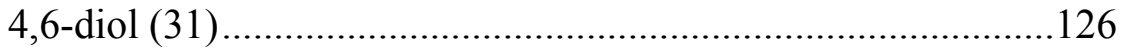

4.2.2.11 Synthesis of 2-(2-cyclohexylpropan-2-yl)pyrimidine4,6-diol (32).....

4.2.2.12 Synthesis of 2-(2-methyloctan-2-yl)pyrimidine-4,6-diol (33) ...127

4.2.2.13 Synthesis of (6aS,9R,10aR)-6,6,9-trimethyl-3(2-phenylpropan-2-yl)-6a, 7,8,9,10,10a-hexahydro-6Hisochromeno[3,4-d]pyrimidin-1-ol (34) .....

4.2.2.14 Synthesis of (6aS,9R,10aR)-6,6,9-trimethyl-3-(2-(thiophen2-yl)propan-2-yl)-6a, 7,8,9,10,10a-hexahydro-6Hisochromeno[3,4-d]pyrimidin-1-ol (35)

4.2.2.15 Synthesis of (6aS,9R,10aR)-3-(2-cyclohexylpropan-2-yl)6,6,9-trimethyl-6a, 7,8,9,10,10a-hexahydro-6H-isochromeno [3,4-d]pyrimidin-1-ol (36).

4.2.2.16 Synthesis of (6aS,9R,10aR)-6,6,9-trimethyl-3-(2methyloctan-2-yl)-6a, 7,8,9,10,10a-hexahydro-6Hisochromeno[3,4-d]pyrimidin-1-ol (37) ....................................128

4.2.3 Synthesis of Pyrimidine Non-Classical Cannabinoids ..........................129

4.2.3.1 Synthesis of diethyl 2-m-tolylmalonate (38) .............................129

4.2.3.2 Synthesis of diethyl 2-(3,5-dichlorophenyl)malonate (39) ........135

4.2.3.3 Synthesis of diethyl 2-cyclohexylmalonate (40) .......................135

4.2.3.4 Synthesis of diethyl 2-(pyridin-2-yl)malonate (41)....................135

4.2.3.5 Synthesis of diethyl 2-benzylmalonate (42) ...............................135

4.2.3.6 Synthesis of diethyl 2-hexylmalonate (43)................................136

4.2.3.7 Synthesis of 2-(2-phenylpropan-2-yl)-5-m-tolylpyrimidine4,6-diol (44) .......................................................................136

4.2.3.8 Synthesis of 2-(2-(thiophen-2-yl)propan-2-yl)-5-mtolylpyrimidine-4,6-diol (45).

4.2.3.9 Synthesis of 2-(2-cyclohexylpropan-2-yl)-5-mtolylpyrimidine-4,6-diol (46).

4.2.3.10 Synthesis of 2-(2-methyloctan-2-yl)-5-m-tolylpyrimidine4,6-diol (47).

4.2.3.11 Synthesis of 5-(3,5-dichlorophenyl)-2-(2-phenylpropan-2yl)pyrimidine-4,6-diol (48)

4.2.3.12 Synthesis of 5-(3,5-dichlorophenyl)-2-(2-(thiophen-2-yl) propan-2-yl)pyrimidine-4,6-diol (49).

4.2.3.13 Synthesis of 2-(2-cyclohexylpropan-2-yl)-5-(3,5dichlorophenyl)pyrimidine-4,6-diol (50) 138

4.2.3.14 Synthesis of 5-(3,5-dichlorophenyl)-2-(2-methyloctan-2yl)pyrimidine-4,6-diol (51)

4.2.3.15 Synthesis of 5-cyclohexyl-2-(2-phenylpropan-2-yl) pyrimidine-4,6-diol (52).

4.2.3.16 Synthesis of 5-cyclohexyl-2-(2-(thiophen-2-yl)propan-2yl)pyrimidine-4,6-diol (53)

4.2.3.17 Synthesis of 5-cyclohexyl-2-(2-cyclohexylpropan-2yl)pyrimidine-4,6-diol (54) 
4.2.3.18 Synthesis of 5-cyclohexyl-2-(2-methyloctan-2-yl)pyrimidine4,6-diol (55).

4.2.3.19 Synthesis of 5-(pyridin-2-yl)-2-(2-(thiophen-2-yl)propan-2yl)pyrimidine-4,6-diol (56)

4.2.3.20 Synthesis of 5-benzyl-2-(2-methyloctan-2-yl)pyrimidine-4,6diol (57)

4.2.3.21 Synthesis of 5-hexyl-2-(2-methyloctan-2-yl)pyrimidine-4,6diol (58)

4.2.4 Synthesis of Furanopyrimidine Cannabinoids .....

4.2.4.1 Synthesis of (2-chloro-2-nitrovinyl)benzene (59)

4.2.4.2 Synthesis of 1-(2-chloro-2-nitrovinyl)-3-methylbenzene (60)...147

4.2.4.3 Synthesis of 1,3-dichloro-5-(2-chloro-2-nitrovinyl)benzene (61)

4.2.4.4 Synthesis of 2-(2-chloro-2-nitrovinyl)thiophene (62)

4.2.4.5 Synthesis of 5-phenyl-2-(2-phenylpropan-2-yl)furo[2,3d]pyrimidin-4-ol (63)

4.2.4.6 Synthesis of 5-phenyl-2-(2-(thiophen-2-yl)propan-2-yl)furo [2,3-d]pyrimidin-4-ol (64).

4.2.4.7 Synthesis of 2-(2-cyclohexylpropan-2-yl)-5-phenylfuro[2,3d]pyrimidin-4-ol (65)

4.2.4.8 Synthesis of 2-(2-methyloctan-2-yl)-5-phenylfuro[2,3-d] pyrimidin-4-ol (66).

4.2.4.9 Synthesis of 2-(2-phenylpropan-2-yl)-5-m-tolylfuro[2,3d]pyrimidin-4-ol (67)

4.2.4.10 Synthesis of 2-(2-(thiophen-2-yl)propan-2-yl)-5-m-tolylfuro [2,3-d]pyrimidin-4-ol (68)

4.2.4.11 Synthesis of 2-(2-cyclohexylpropan-2-yl)-5-m-tolylfuro[2,3d]pyrimidin-4-ol (69)

4.2.4.12 Synthesis of 2-(2-methyloctan-2-yl)-5-m-tolylfuro[2,3d]pyrimidin-4-ol (70)

4.2.4.13 Synthesis of 5-(3,5-dichlorophenyl)-2-(2-phenylpropan-2yl)furo[2,3-d]pyrimidin-4-ol (71).....

4.2.4.14 Synthesis of 5-(3,5-dichlorophenyl)-2-(2-(thiophen-2-yl) propan-2-yl)furo[2,3-d]pyrimidin-4-ol (72).

4.2.4.15 Synthesis of 2-(2-cyclohexylpropan-2-yl)-5-(3,5dichlorophenyl)furo[2,3-d]pyrimidin-4-ol (73).....

4.2.4.16 Synthesis of 5-(3,5-dichlorophenyl)-2-(2-methyloctan-2yl)furo[2,3-d]pyrimidin-4-ol (74)

4.2.4.17 Synthesis of 2-(2-phenylpropan-2-yl)-5-(thiophen-2-yl)furo [2,3-d]pyrimidin-4-ol (75)

4.2.4.18 Synthesis of 5-(thiophen-2-yl)-2-(2-(thiophen-2-yl)propan-2yl)furo[2,3-d]pyrimidin-4-ol (76).

4.2.4.19 Synthesis of 2-(2-cyclohexylpropan-2-yl)-5-(thiophen-2yl)furo[2,3-d]pyrimidin-4-ol (77)

4.2.4.20 Synthesis of 2-(2-methyloctan-2-yl)-5-(thiophen-2-yl)furo [2,3-d]pyrimidin-4-ol (78) 


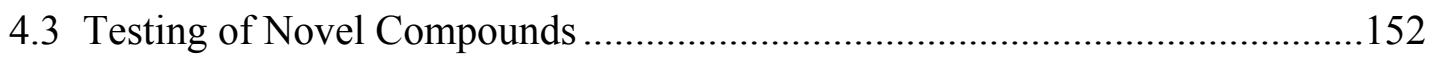

4.3.1 Pyrimidine Hexahydro Classical Cannabinoids.....................................153

4.3.2 Pyrimidine Non-Classical Cannabinoids …………...............................153

4.3.3 Furanopyrimidine Cannabinoids.........................................................153

4.4 Experimental Procedures ……………………….................................158

4.4.1 Radioligand Binding Assays............................................................... 158

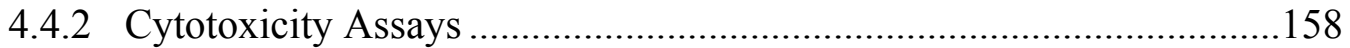

4.4.3 CB1/CB2 Functional Assays ………………………………….........160

Chapter 5: Cannabinoids as Anti-Inflammatory Agents ...............................................161

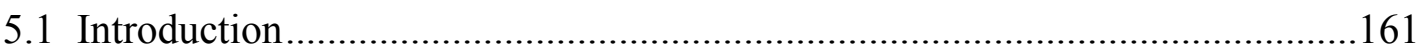

5.2 Evaluation of CB2 Agonists in Preclinical Models .........................................162

5.2.1 Pain ........................................................................................162

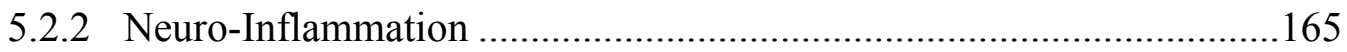

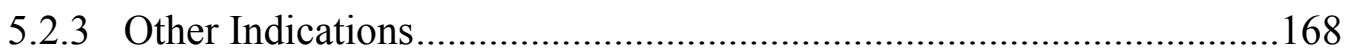

5.3 Evaluation of CB2 Agonists in Clinical Trials ..................................................168

5.4 Evaluation of Novel Cannabinoids as Anti-Inflammatory Agents ....................170

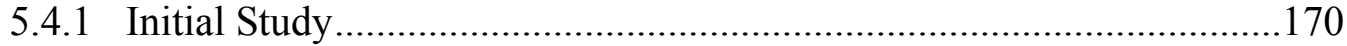

5.4.2 Selection of Compounds to Screen ........................................................172

5.4.3 Cytokine and Chemokine Release Profiles.........................................175

5.4.4 Screening of Initial Eight Compounds..................................................177

5.4.5 Dose Response of Four Lead Compounds ..............................................191

5.4.6 Further Evaluation of HB-I-172 ..........................................................191

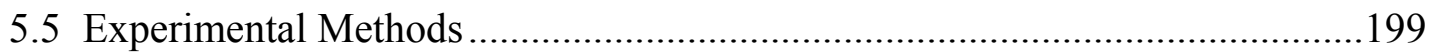

5.5.1 Transformation of THP-1 and KG-1 _.............................................199

5.5.2 Cytokine and Chemokine Assays ......................................................199

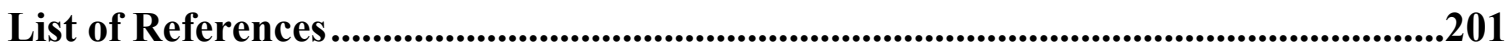

Appendix A: Cytokine and Chemokine Release Profiles of THP-1, KG-1, A549,

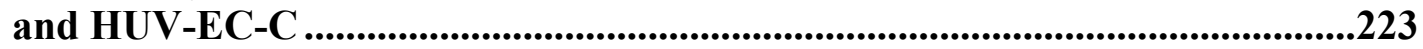

Appendix B: Effects of Eight Cannabinoids on Cytokine and Chemokine

Release of THP-1, A549, and HUV-EC-C .............................................................232

Appendix C: Dose Response of Four Cannabinoids on Cytokine and

Chemokine Release of THP-1 and HUV-EC-C ....................................................244

Appendix D: Cytokine and Chemokine Release Profiles of HMVEC-LBI, Monocyte Derived Macrophages, and SAEC.........................................................263

Appendix E: Effects of HB-I-172 and SMM-I-229 on the Cytokine and Chemokine Release of HMVEC-LBI, Monocyte Derived Macrophages, and SAEC

Appendix F: Cytokine and Chemokine Release Profiles of Primary Human Microglia ..................................................................................................................227

Appendix G: Effects of HB-I-172 and KM-233 on the Cytokine and Chemokine Release of Primary Human Microglia 
Appendix H: Comparison of the Effects of HB-I-172 to Dexamethasone on the Cytokine and Chemokine Release of THP-1, A549, and HMVEC-LBI ..........283

Vita

.290 


\section{List of Tables}

Table 2.1: $\quad \mathrm{EC}_{50}$ values for $\mathrm{KM}-233$ and BCNU against human GBM cell lines........25

Table 3.1: $\quad$ Binding affinity $\left(K_{\mathrm{i}}\right)$ of compounds 8-11 for the CB1 and CB2 receptors compared to KM-233 1 and $\Delta^{8}$-THC ....................................48

Table 3.2: $\quad$ Binding affinity $\left(K_{\mathrm{i}}\right)$ of compounds 8-16 for the $\mathrm{CB} 1$ and $\mathrm{CB} 2$ receptors compared to KM-233 1 and $\Delta^{8}$-THC

Table 3.3: Efficacy of three triaryl cannabinoids against a variety of human cancer cell lines.

Table 3.4: $\quad$ Efficacy of four triaryl cannabinoids against a variety of human cancer cell lines.

Table 3.5: $\quad$ Efficacy of HB-I-172 against U-87 MG and LN-229............................61

Table 4.1: $\quad$ Binding affinity constants $\left(K_{\mathrm{i}}\right)$ for compounds 34-37 compared to $\Delta^{8}$-THC, KM-233, and HB-I-172

Table 4.2: $\quad$ Efficacy of pyrimidine hexahydro classical cannabinoids compared to HB-I-172, KM-233, and BCNU against LN-229 155

Table 4.3: $\quad$ Binding affinity constants $\left(K_{\mathrm{i}}\right)$ for select pyrimidine non-classical cannabinoids compared to $\Delta^{8}$-THC, KM-233, and HB-I-172 .

Table 4.4: Comparison of selected pyrimidine non-classical cannabinoids to HB-I-172, KM-233, and BCNU against LN-229 


\section{List of Figures}

Figure 1.1: $\quad$ Structures of cannabinol and $\Delta^{6 \mathrm{a}, 10 \mathrm{a}}$-tetrahydrocannabinol .......................2

Figure 1.2: $\quad$ Structures of $\Delta^{9}-$ THC, cannabidiol, cannabigerol, cannabichromene,

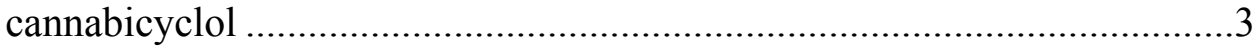

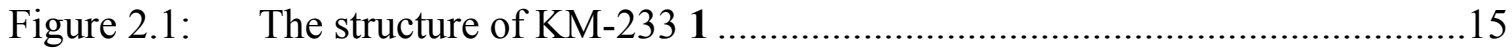

Figure 2.2: $\quad$ Dose response curve for KM-233 on U-87 MG .....................................16

Figure 2.3: Comparison of efficacies of KM-233 and $\Delta^{8}$-THC ................................17

Figure 2.4: Comparison of efficacies of KM-233 and BCNU ..................................18

Figure 2.5: In vivo efficacy of KM-233 against human U87 glioma in the murine

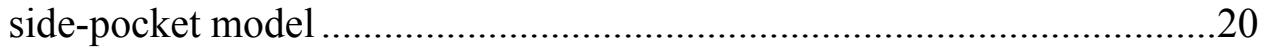

Figure 2.6: Tumor load in the U87 glioma model..................................................21

Figure 2.7: Dose-dependent reduction of U87 glioma after KM-233 treatment..........22

Figure 2.8: Western blots for cannabinoid receptors in human glioma cell lines ........24

Figure 2.9: $\quad$ C1'-dimethyl-aryl $\Delta^{8}$-tetrahydrocannabinol analogs screened against human glioblastoma cell lines...........................................................27

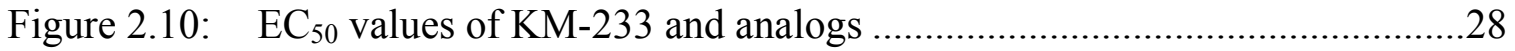

Figure 2.11: Effects of KM-233 on U-87 MG in the presence or absence of Ptx

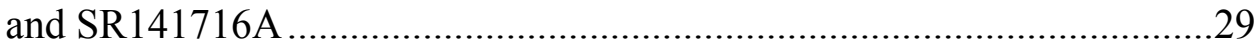

Figure 2.12: Functional activity of KM-233 at the CB1 and CB2 receptors ................30

Figure 2.13: pERK and pSTAT3 profiles of U-87 MG cells treated with KM-233 ......32

Figure 2.14: $\quad$ pMEK profile of U-87 MG cells treated with KM-233 ...........................33

Figure 2.15: $\quad$ pAKT profile of U-87 MG cells treated with KM-233.............................35

Figure 2.16: $\quad$ GGSK-3 $\beta$ and pP70S6K profiles of U-87 MG cells treated with

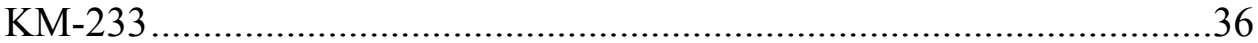

Figure 2.17: $\quad$ pBAD profile of U-87 MG cells treated with KM-233 .............................37

Figure 2.18: Cleaved caspase 3 profile of U-87 MG cells treated with KM-233 ..........38

Figure 2.19: Effects on membrane polarization in U-87 MG cells treated with

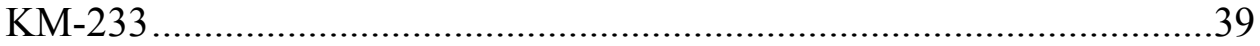

Figure 2.20: Effects on cytochrome C release in U-87 MG cells treated with KM-233

Figure 2.21: Effects on cytoskeleton structure in U-87 MG cells treated with

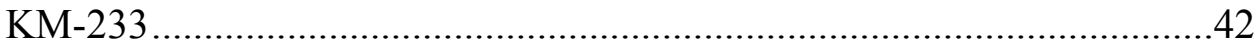

Figure 3.1: Design of the novel triaryl cannabinoid core ..........................................46

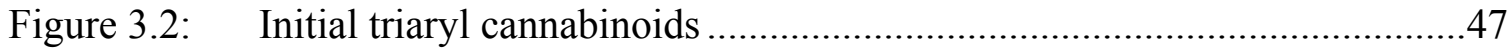

Figure 3.3: Nine triaryls used to develop SAR of this new class of cannabinoids.......50

Figure 3.4: Western blots for cannabinoid receptors in human glioma cell lines ........56

Figure 3.5: Effects of HB-I-172 on U-87 MG in the presence or absence of Ptx ........57

Figure 3.6: Functional activity of HB-I-172 at the CB1 and CB2 receptors...............59

Figure 3.7: Inefficient mixing of drug solutions with media in cytotoxicity assays ....60

Figure 3.8: $\quad$ pMEK1/2 profile of U-87 MG cells treated with HB-I-172 ....................63

Figure 3.9: $\quad$ pERK1/2 profile of U-87 MG cells treated with HB-I-172 ......................64

Figure 3.10: pAkt profile of U-87 MG cells treated with HB-I-172 ...........................66

Figure 3.11: Phospho-p70S6K profile of U-87 MG cells treated with HB-I-172 .........67

Figure 3.12: $\quad$ pGSK-3 $\beta$ profile of U-87 MG cells treated with HB-I-172 .....................68 
Figure 3.13: $\quad$ pSTAT3 profile of U-87 MG cells treated with HB-I-172 ......................69

Figure 3.14: p53 profile of U-87 MG cells treated with HB-I-172 ..............................70

Figure 3.15: Phospho-p53 profile of U-87 MG cells treated with HB-I-172 .................71

Figure 3.16: BAD phosphorylation of U-87 MG cells treated with HB-I-172 .............73

Figure 3.17: Caspase 3 activity profile of U-87 MG cells treated with HB-I-172 ........74

Figure 3.18: Caspase 9 activity of U-87 MG cells treated with HB-I-172 ....................75

Figure 3.19: Cleaved PARP profile of U-87 MG cells treated with HB-I-172 ..............76

Figure 3.20: Effects on membrane polarization in U-87 MG cells treated with HB-I-172 .................................................................................. 77

Figure 3.21: Effects on cytochrome $\mathrm{C}$ release in U-87 MG cells treated with

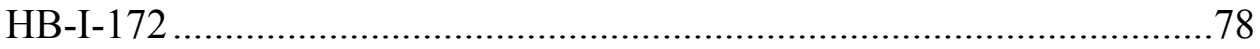

Figure 3.22: Activity of PARP in U-87 MG cells treated with HB-I-172 …...............79

Figure 3.23: Effects on cytoskeleton structure in U-87 MG cells treated with

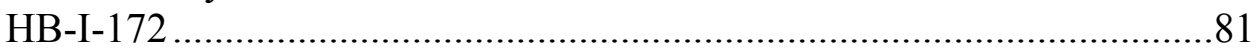

Figure 3.24: Proposed induction of apoptosis by HB-I-172 mediated through CB2 ....82

Figure 3.25: Proposed induction of apoptosis by HB-I-172 mediated through GPR55.....

Figure 3.26: Proposed induction of apoptosis by HB-I-172 initiated by ER stress mediated through CB2 ............................................................85

Figure 3.27: Proposed induction of autophagy followed by apoptosis by HB-I-172 initiated by ER stress mediated through CB2 ....

Figure 3.28: Entire proposed mechanism of glioma cell death by HB-I-172 mediated by CB2 and GPR55.

Figure 4.1: $\quad$ Structure of $\Delta^{8}$-THC with identification of its various structural domains 102

Figure 4.2: $\quad$ Representatives of various classes of non-classical cannabinoids...........104

Figure 4.3: Further representatives of various classes of non-classical

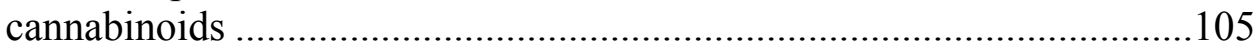

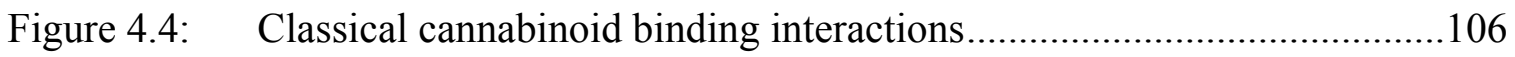

Figure 4.5: General design of pyrimidyl cannabinoids ........................................108

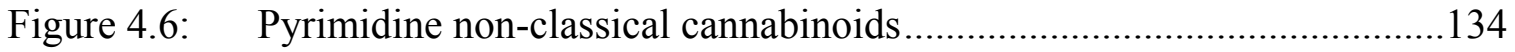

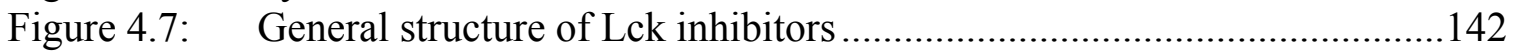

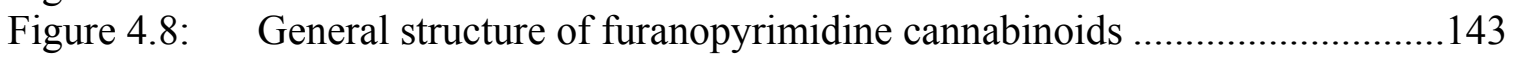

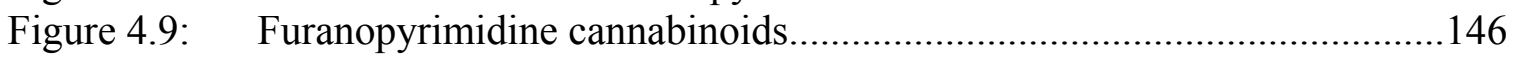

Figure 4.10: Furanopyrimidine cannabinoids screened against the human glioblastoma cell line T98G.

Figure 5.1: $\quad$ BB2 selective agonists used in preclinical studies as antinociceptive agents

Figure 5.2: $\quad$ Further CB2 selective agonists used in preclinical studies as antinociceptive agents 166

Figure 5.3: $\quad$ CB2 selective agonists used in preclinical studies as anti-inflammatory agents .......

Figure 5.4: Cannabinor (PRS-211,375)

Figure 5.5: Seven compounds initially screened for anti-inflammatory activity .......171

Figure 5.6: Anti-inflammatory study design 173 
Figure 5.7: Functional profiles of initial seven compounds screened for antiinflammatory activity ................................................................. 174

Figure 5.8: $\quad$ Eight compounds chosen for anti-inflammatory studies .........................176

Figure 5.9: Time-dependent release of IL-6 from THP-1 stimulated with LPS ........178

Figure 5.10: Effects of eight compounds on release of IFN- $\gamma$ from THP-1 in response to LPS and PGN

Figure 5.11: Effects of eight compounds on release of IL-12p70 from THP-1 in response to LPS and PGN.

Figure 5.12: Effects of eight compounds on release of IL-6 from THP-1 in response

to LPS and PGN.

Figure 5.13: Effects of eight compounds on release of GM-CSF and IL-1 $\beta$ from THP-1 in response to PGN

Figure 5.14: Effects of eight compounds on release of IL-10 and TNF- $\alpha$ from THP-1 in response to PGN

Figure 5.15: Effects of eight compounds on release of GM-CSF and IL-6 from HUVEC in response to TNF- $\alpha$.

Figure 5.16: Effects of eight compounds on release of IFN- $\gamma$, IL-12p70, and IL-2

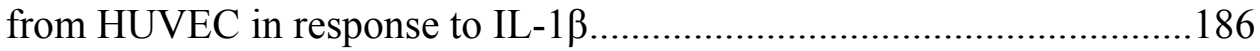

Figure 5.17: Effects of eight compounds on release of MIP-1 $\beta$ from A549 in response to TNF- $\alpha$ and IL- $1 \beta$

Figure 5.18: Effects of eight compounds on release of MIP-1 $\beta$ from HUVEC in response to TNF- $\alpha$ and IL- $1 \beta$

Figure 5.19: Effects of eight compounds on release of IP-10 from A549 and HUVEC in response to TNF- $\alpha$.

Figure 5.20: Effects of eight compounds on release of IL-8 from HUVEC in response to TNF- $\alpha$ and IL-1 $\beta$

Figure 5.21: Effects of eight compounds on release of MCP-1 from THP-1 in response to LPS.

Figure 5.22: Effects of eight compounds on release of MCP-1 from HUVEC in response to TNF- $\alpha$ and IL-1 $\beta$

Figure 5.23: Four compounds chosen for dose response study....

Figure 5.24: Examples of dose dependent reductions in chemokines produced by THP-1 after treatment with HB-I-172.

Figure 5.25: Examples of dose dependent reductions in chemokines produced by THP-1 after treatment with compound 83

Figure 5.26: CB2 inverse agonists screened as anti-inflammatory agents against human primary macrophages, epithelial, and endothelial cells . 198 


\section{List of Schemes}

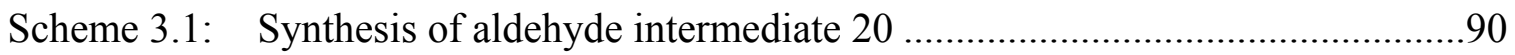

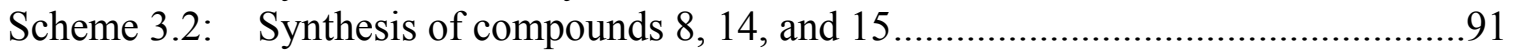

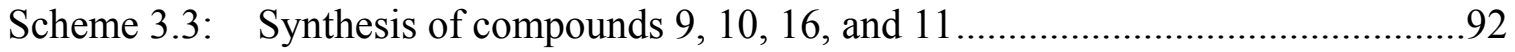

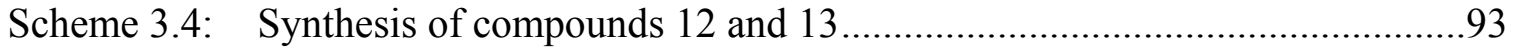

Scheme 4.1: Synthesis of hexahydro pyrimidine classical cannabinoids ....................110

Scheme 4.2: Synthesis of TBDMS protected hexahydro pyrimidine classical

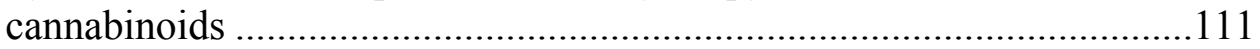

Scheme 4.3: Attempts at introduction of the hexahydro pyrimidine side chain...........112

Scheme 4.4: Synthesis of 4,6-dimethoxypyrimidine-2-carboxylate............................113

Scheme 4.5: Introduction of the side chain to 4,6-dimethoxypyrimidine-2-

carboxylate

Scheme 4.6: Attempts at dimethylation of the ketone intermediate............................115

Scheme 4.7: Further attempts at dimethylation of the ketone intermediate ................116

Scheme 4.8: Attempts at deprotection of the dimethoxy pyrimidine intermediates.....118

Scheme 4.9: Formation of nitrile intermediates from halides and isobutyronitrile......119

Scheme 4.10: Formation of nitrile intermediates from cyanides................................ 120

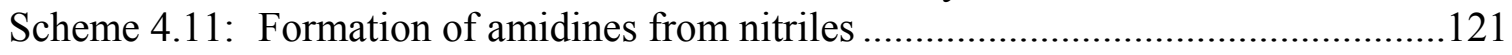

Scheme 4.12: Synthesis of dihydroxy pyrimidine intermediates ................................122

Scheme 4.13: Coupling of pyrimidine hexahydro classical cannabinoids .....................123

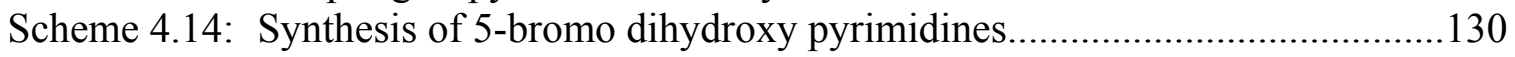

Scheme 4.15: Attempt at synthesis of pyrimidine triaryl compounds ..........................131

Scheme 4.16: Synthesis of pyrimidine nonclassical cannabinoids ...............................132

Scheme 4.17: Synthesis of 2-position substituted diethyl malonates ...........................133

Scheme 4.18: General synthesis of furanopyrimidine cannabinoids ..............................144

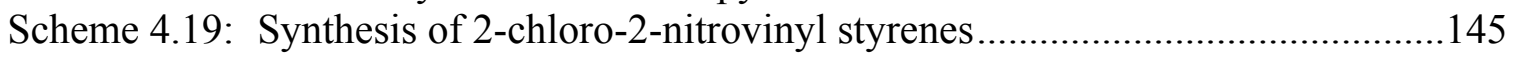




\section{List of Abbreviations}

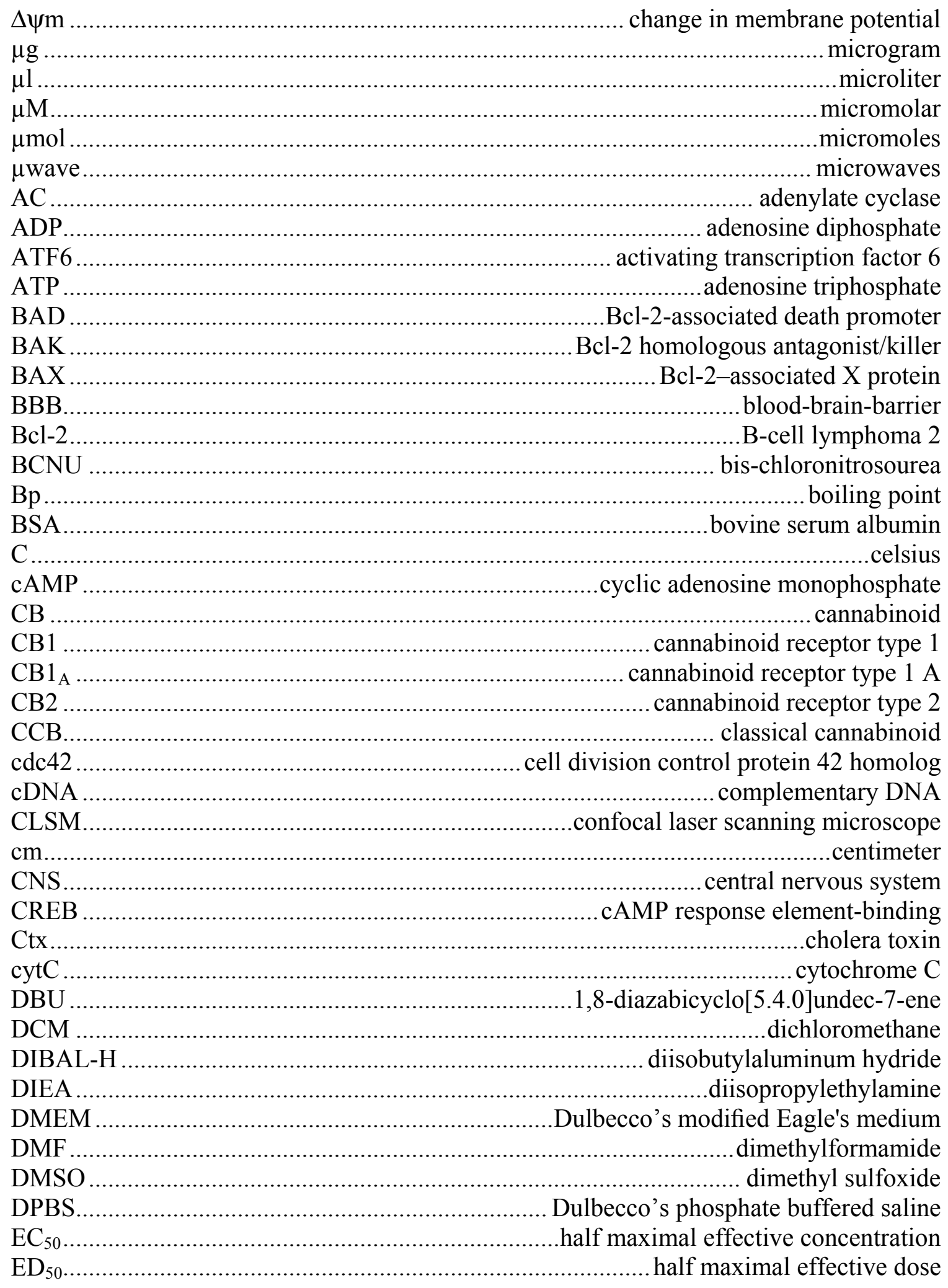


Egr1

ELF2 $\alpha$

Elk-1

EMEM

ER

ERK.

ESI.

EtOAc

EtOH

FAK

FAN

FBS

FCA

G

g.

G-CSF

G $\beta L$

GBM

GEF

gem

GFP

GM-CSF.

GPCR

GPR119

GPR35

GPR55

GSK-3 $\beta$

GTP

HPLC

hr

I.R

i.p.

i.v.

$\mathrm{IC}_{50}$

IFN- $\gamma$

IL

IP-10

JNK

$K_{\mathrm{d}}$.

$\mathrm{kDa}$

$\mathrm{kg}$........

KHMDS

$K_{\text {i }}$

Lck

Leu

$\log \mathrm{P}$ early growth response protein 1 E74-like factor 2 alpha

Ets like gene1

Eagle's minimal essential medium endoplasmic reticulum .extracellular signal-regulated kinase electrospray ionization . ethyl acetate .ethanol focal adhesion kinase factor associated with neutral SMase activation fetal bovine serum Freund's complete adjuvant guanine nucleotide-binding ..grams granulocyte colony-stimulating factor G-protein Beta-subunit like protein glioblastoma multiforme guanine nucleotide exchange factor geminal green fluorescent protein granulocyte-macrophage colony-stimulating factor G protein-coupled receptor G protein-coupled receptor 119 G protein-coupled receptor 35 G protein-coupled receptor 55 glycogen synthase kinase 3 beta .guanosine triphosphate high performance liquid chromatography hours infrared spectroscopy intraperitoneal intravenous half maximal inhibitory concentration interferon gamma interleukin $10 \mathrm{kDa}$ interferon-gamma-induced protein c-Jun N-terminal kinase dissociation constant ..kilodaltons ..kilograms .potassium bis(trimethylsilyl)amide dissociation constant leukocyte-specific protein tyrosine kinase leucine logarithm of octanol/water partition coefficient 
LPI

LPS

Lys

M.

$\mathrm{m} / \mathrm{z}$.

MAP

MCP-1

MEK

MeOD

$\mathrm{MeOH}$

Met

MIF

mg

$\mathrm{MHz}$

min

$\mathrm{ml}$

$\mathrm{mm}$

mmol

MRI

mRNA

mTOR

MS

$\mathrm{N}$

n.a.

n.d.

NBS

neg.

NFAT

NF-kB

ng.

NK

nM

nm

NMR

p53

P70S6K

p90RSK

PAK

pAkt.

PARP

pBAD

PBS

PCC.

pERK

PERK

PGN lysophosphatidylinsoitol lipopolysaccharide lysine molar mass-to-charge ratio mitogen-activated protein monocyte chemotactic protein-1 mitogen-activated protein kinase deuterated methanol methanol methionine macrophage migration inhibitory factor milligrams ..megahertz ..minutes milliliters millimeters millimoles magnetic resonance imaging messenger ribonucleic acid mammalian target of rapamycin mass spectroscopy normality not active not determined $\mathrm{N}$-bromosuccinimide negative nuclear factor of activated T-cells nuclear factor kappa-light-chain-enhancer of activated B cells nanograms natural killer nanomolar nanometer nuclear magnetic resonance spectroscopy protein 53 $70 \mathrm{kDa}$ ribosomal S6 kinase p90 ribosomal S6 kinase p21-activating kinase ..phospho-Akt poly (ADP-ribose) polymerase phospho-BAD phosphate buffered saline pyridinium chlorochromate .phospho-ERK protein kinase-like endoplasmic reticulum kinase peptidoglycan 


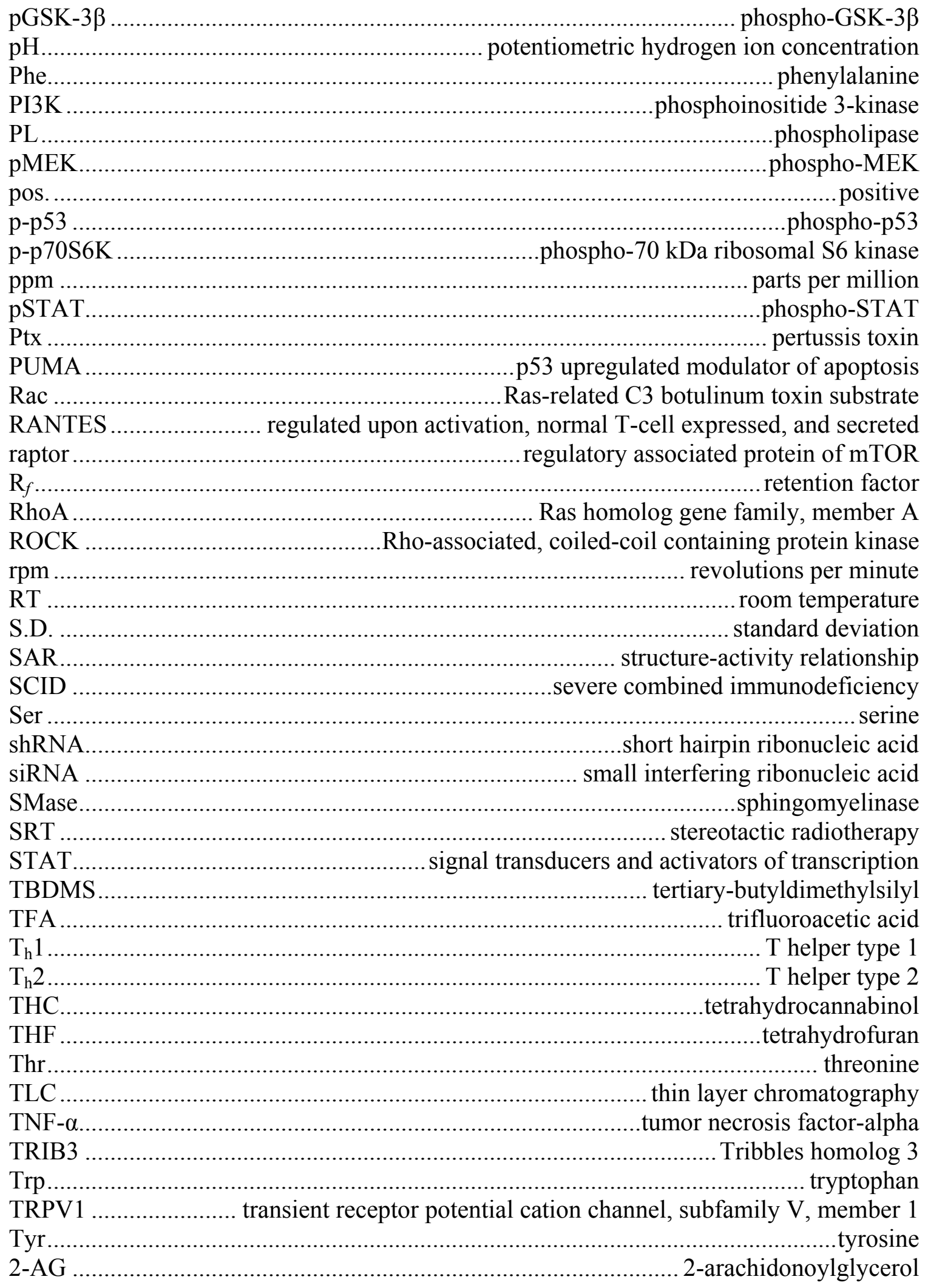




\section{Chapter 1: Introduction}

\subsection{History}

Cannabis sativa (marijuana) is one of the oldest known herbal drugs whose medicinal value has been exploited for centuries. The use of Cannabis sativa preparations for treatment of a variety of pathological conditions in ancient Indian, Chinese, and Egyptian civilizations is well-documented. ${ }^{1}$ Ancient literature is rich with therapeutic indications for Cannabis such as its usage as an analgesic, ${ }^{2}$ an anesthetic, ${ }^{3}$ an appetite stimulant, ${ }^{4}$ an anti-inflammatory agent, ${ }^{5}$ and as a treatment for hemorrhoids. ${ }^{1}$

Early in the nineteenth century there was a rapidly increasing interest in research of natural products, and several alkaloids such as morphine, cocaine, and strychnine were isolated from plant materials. The search for the psychoactive constituent of marijuana began during this period of rapid growth in the field of pharmacognosy. It was the common belief for many years that the principal active constituent of Cannabis sativa was in fact an alkaloid. In 1896, however, Marshall and coworkers isolated a mixture of terpenes from an ethereal extract of Indian hemp, ${ }^{6}$ which produced the same pharmacological effects that are observed with Cannabis sativa use. This provided the first evidence that the principal active compound was terpenoid in nature. In the early 1930s Cahn and coworkers isolated cannabinol (Figure 1.1) and determined a partial structure after conducting extensive chemical degradation studies. ${ }^{7}$ This discovery was quickly followed by the synthesis of several derivatives of cannabinol and $\Delta^{6 \mathrm{a}, 10 \mathrm{a}}$ tetrahydrocannabinol ( $\left.\Delta^{6 \mathrm{a}, 10 \mathrm{a}}-\mathrm{THC}\right)$ (Figure 1.1) by the Adams group in the $\mathrm{US}^{8}$ and the Todd group in Britain. ${ }^{9}$ The pharmacological activities of cannabinol and $\Delta^{6 \mathrm{a}, 10 \mathrm{a}}-\mathrm{THC}$, as tested by the Gayer areflexia test for suppression of corneal reflexes in the rabbit, were observed to be similar to those of the Cannabis extract, but were significantly less potent.

The isolation and structural characterization of the principal active constituent of Cannabis sativa, $\Delta^{9}$-tetrahydrocannabinol $\left(\Delta^{9}-\mathrm{THC}\right.$ ) (Figure 1.2), was reported in 1964 by Gaoni and Mechoulam. ${ }^{10}$ The activity of this cannabinoid was subsequently studied in a number of animal models. These studies ranged from evaluation of the cataleptic reaction in mice ${ }^{11}$ to the effects on motor activity and gross behavior in mice and rats. ${ }^{12}$ There was also extensive experimentation in rhesus monkeys ${ }^{12}$ which ultimately led to the pharmacological characterization of cannabinoids.

Following these reports, several other constituents of marijuana such as cannabidiol, cannabigerol, cannabichromene, cannabicyclol (Figure 1.2) and related cannabinoid acids were isolated and characterized. The structural characterization of $\Delta^{9}$ THC provided a springboard to stimulate interest in research on cannabinoids. This resulted in the design and synthesis of several structurally diverse classes of cannabinoids. Studies on the effects of various analogues in animal models showed a positive correlation between structure and biological activity indicating the possible involvement of a defined receptor for the cannabinoids. This variety of compounds then aided in the discovery and cloning of the cannabinoid receptors and the subsequent identification of their endogenous ligands. 


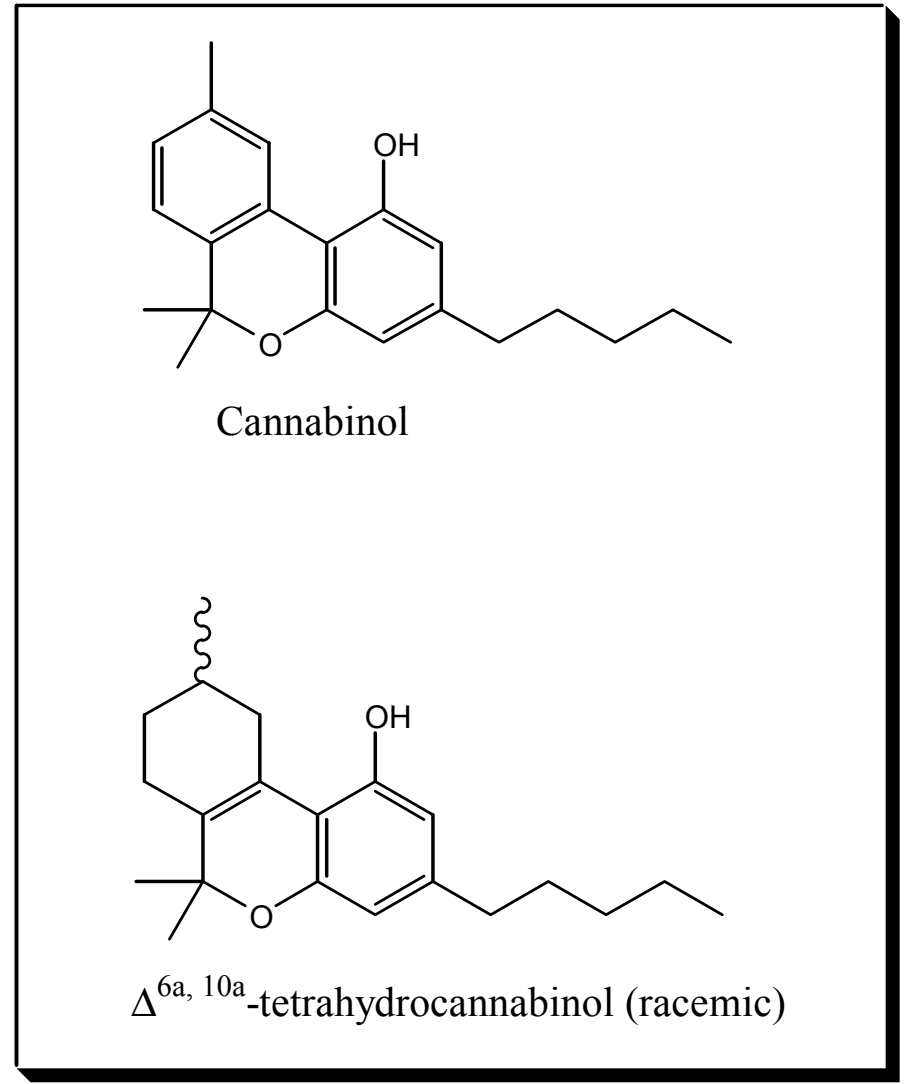

Figure 1.1: Structures of cannabinol and $\Delta^{6 \mathrm{a}, 10 \mathrm{a}}$-tetrahydrocannabinol 


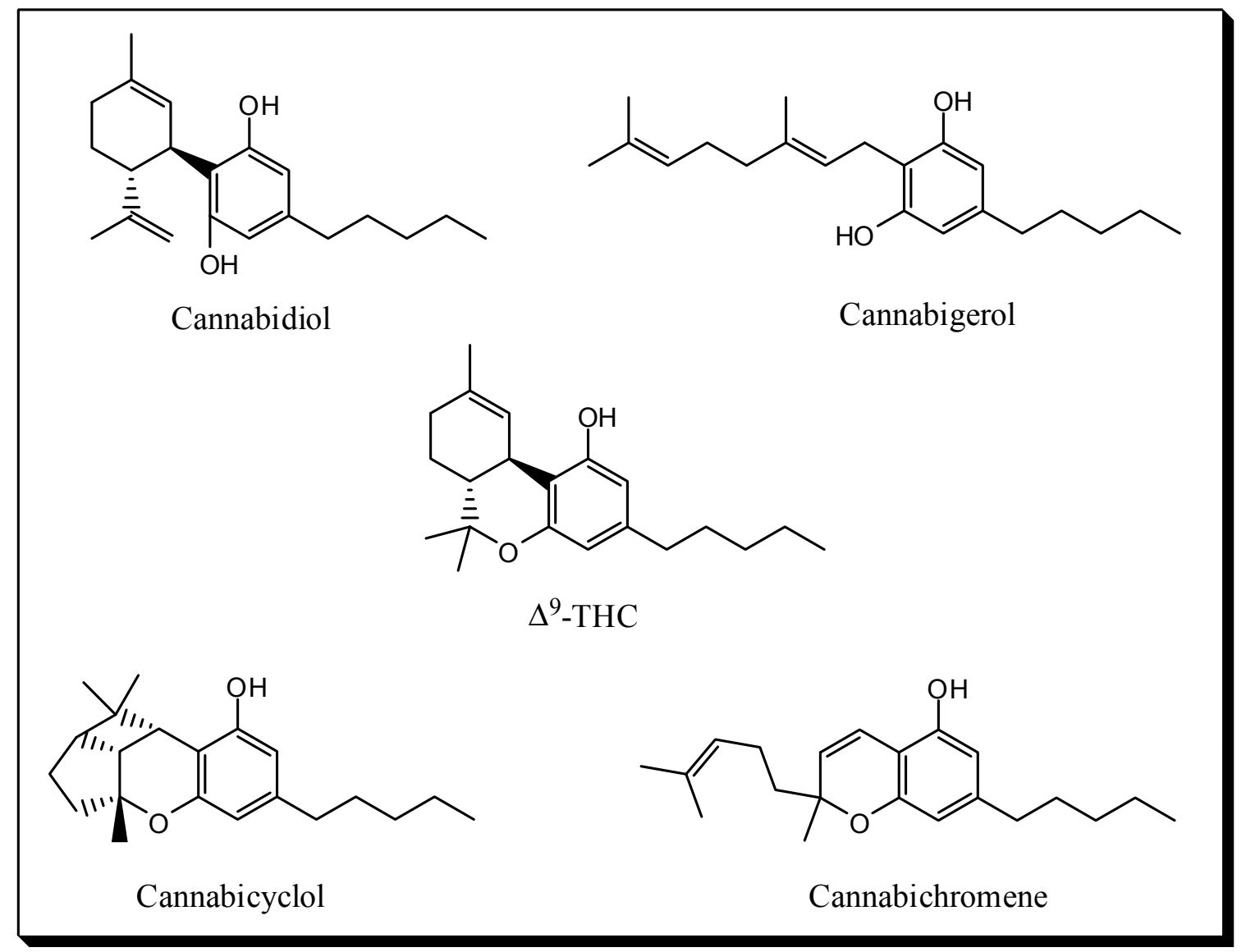

Figure 1.2: Structures of $\Delta^{9}-\mathrm{THC}$, cannabidiol, cannabigerol, cannabichromene, and cannabicyclol 


\subsection{Cannabinoid Receptors}

The beginning of the exponential growth seen in the field of cannabinoid research in the last 20 years can be traced to the identification, isolation and cloning of the cannabinoid receptors. After the discovery of $\Delta^{9}-\mathrm{THC}$, but before the discovery of the cannabinoid receptors, research on cannabinoids in the late 1970s and in the early 1980s was based on the assumption that the pharmacological effects attributed to cannabinoids were mediated not through specific receptors but by perturbation of cellular membranes. Due to their high lipophilicity, it was thought that cannabinoids behaved like general anesthetics in terms of their mechanism of action. ${ }^{13}$ However, the highly stereospecific activity associated with several cannabinoids ${ }^{14}$ suggested receptor mediated action. Then, in 1988, Devane and coworkers ${ }^{15}$ utilized a tritium labeled synthetic cannabinoid to tag specific sites in the brain and on neuronal cells. This then identified the presence of a specific cannabinoid receptor in the rat brain. This discovery was finally followed by the isolation of the cDNA of this receptor, its expression in Chinese hamster ovary (CHO) cells, and subsequent naming as the cannabinoid receptor type 1 (CB1). ${ }^{16}$ In 1993, a second cannabinoid receptor subtype, cannabinoid receptor type 2 (CB2), was isolated and cloned from the human promyelocytic cell line HL60 by Munro and coworkers. ${ }^{17}$ An amino terminal variant of the human $\mathrm{CB} 1$ receptor has also been reported ${ }^{18}$ in which, a 167 base portion of the CB1 coding sequence is spliced out of the mRNA. This amino terminal variant of the $\mathrm{CB} 1$ receptor was named the $\mathrm{CB} 1_{\mathrm{A}}$ receptor.

Whether there are additional cannabinoid receptors is still a hotly debated topic among several researchers in this field. This is, in part, due to the observation that the endogenous cannabinoid ligand, anandamide, induces spinal antinociception via a different mechanism than $\Delta^{9}-$ THC $^{19}$ In 2000, Di Marzo and coworkers carried out studies ${ }^{20}$ using transgenic mice ${ }^{21}$ that lack the $\mathrm{CB} 1$ receptor to evaluate the $\mathrm{CB} 1$ mediated effects of anandamide and $\Delta^{9}-\mathrm{THC}$. They found that in these knockout mice anandamide still produced CB1-like effects such as catalepsy, analgesia, and loss of spontaneous activity whereas $\Delta^{9}-\mathrm{THC}$ did not. This suggested the presence of a new cannabinoid receptor in the brain. Recent studies have also shown that a number of the cannabinoid ligands bind to the orphan receptor GPR55. ${ }^{22}$ This receptor is reported to be expressed in several tissues and might function in regulation of vascular tone and immune-cell migration. $^{22 \mathrm{c}}$ Anandamide is also known to act on other classes of receptors such as the vanilloid receptor TRPV1. Anandamide activates the vanilloid receptor and produces membrane currents or increases in intracellular calcium levels. ${ }^{23}$ The presence of these multiple sites for cannabinoid action (along with the possibility for yet undiscovered sites) with several signaling pathways and associated second messengers is consistent with the complex pharmacology associated with cannabinoids.

The cannabinoid receptors belong to the superfamily of G-protein coupled receptors ${ }^{11}$ (GPCRs) and are single polypeptides with seven transmembrane $\alpha$-helices, an extracellular, glycosylated N-terminus and intracellular C-terminus. The CB1 and CB2 receptor subtypes posses $68 \%$ amino acid identity within their transmembrane regions but only $44 \%$ identity throughout the entire peptide. The CB1 receptor is a $52.8 \mathrm{kDa}, 472$ amino acid polypeptide, which is found in both the CNS and periphery and in both neural and non-neural tissues. $\mathrm{CB} 1$ is coupled to inhibitory $\mathrm{G}_{\mathrm{i} / \mathrm{o}}$ proteins ${ }^{24}$ which bind to its 
third intracellular loop and its C-terminus. ${ }^{25}$ The amino acid residues in transmembrane regions 3, 5, and 6 of the $\mathrm{CB} 1$ receptor are believed to be involved in binding with cannabinergic ligands. ${ }^{26}$ Activation of CB1 causes inhibition of adenylate cyclase and a reduction in cAMP levels. Reduction of cAMP affects phosphorylation by protein kinase A which causes modulation of ion channels and other second messengers. Activation of voltage-sensitive outwardly rectifying $\mathrm{K}^{+}$channels, ${ }^{27}$ inwardly rectifying $\mathrm{K}^{+}$channels ${ }^{28}$ and inhibition of voltage gated N, L, P, and Q-type $\mathrm{Ca}^{2+}$ channels ${ }^{29}$ are observed in response to $\mathrm{CB} 1$ receptor activation.

The CB1 receptor is also known to regulate different members of the mitogenactivated protein kinase (MAP kinase) family of enzymes such as:
a. extracellular signal-regulated kinase $(\mathrm{ERK})^{30}$
b. c-Jun N-terminal kinase ${ }^{31}$
c. $\mathrm{p} 38 .^{31 \mathrm{~b}, 32}$

Activation of MAP kinases is associated with:
a. activation of immediate early genes like c-Fos and Egr1 (Krox-24) ${ }^{30 \mathrm{a}}$
b. activation of focal adhesion kinase (important for integrating cytoskeletal changes associated with signal transduction events)
c. activation of phospholipases (PL), PLA, PLC, and PLD ${ }^{33}$
d. activation of the $\mathrm{Na}^{+} / \mathrm{H}^{+}$exchanger ${ }^{34}$
e. activation of phospholipase $\mathrm{A}_{2}$, which in turn causes release of arachidonic acid leading to the synthesis of prostaglandins. ${ }^{35}$

There is also evidence of a second pathway initiated by $\mathrm{CB} 1$ receptor stimulation which is coupled with sphingomyelinase activation and subsequent release of ceramide. $^{30 \mathrm{c}}$ Ceramide then causes activation of the Raf-1/MAP kinase cascade ${ }^{36}$ and also carnitine palmitoyltransferase I in mitochondrial membranes to stimulate ketogenesis and fatty acid oxidation. ${ }^{37}$ This elevation in levels of intracellular ceramide have also been suggested to be one of the possible factors responsible for the apoptotic effects of cannabinoids seen on glioblastoma cells. ${ }^{38}$

The CB2 cannabinoid receptor, also referred to as the peripheral cannabinoid receptor, is a $41 \mathrm{kDa}, 360$ amino acid polypeptide that is predominantly expressed in the immune system. ${ }^{39}$ Its amino acid sequence is quite different from that of CB1, especially in the amino terminal domain where there is no significant conservation and which is significantly shorter in the $\mathrm{CB} 2$ receptor when compared to the $\mathrm{CB} 1$ receptor. The amino acid residues involved in ligand recognition are also found in the transmembrane domains 3,5 , and 6 for the $\mathrm{CB} 2$ receptor. 
Like the $\mathrm{CB} 1$ receptor, $\mathrm{CB} 2$ is also coupled to the inhibitory $\mathrm{G}_{\mathrm{i} / \mathrm{o}}$ proteins that bind to its third intracellular loop and C-terminus. Activation of the CB2 receptor also causes inhibition of adenylate cyclase and therefore a similar reduction in cAMP levels. Most of the known signaling pathways of CB2 are identical to those observed with CB1. However, unlike the $\mathrm{CB} 1$ receptor, the $\mathrm{CB} 2$ receptor has no effect on ion channels. ${ }^{28}$

After the discovery of the CB2 receptor it was believed that it was found only in the immune system and was completely absent from the CNS. ${ }^{40}$ Recently, however, CB2 expression has been confirmed in the brain on microglia, ${ }^{41}$ blood vessels, ${ }^{41}$ and on some neurons. ${ }^{42}$ CB2 is, however, expressed in much higher densities than CB1 in the immune system. The expression level of the CB2 gene in immune tissues is 10-100 times that of the $\mathrm{CB} 1$ gene. $^{43}$ In fact, the CB2 mRNA content of the spleen and tonsils is almost equivalent to the CB1 mRNA content in the CNS. ${ }^{43}$ This first led researchers to suggest that $\mathrm{CB} 2$ receptor might play an important role in immune function. It is no surprise then that many reports have been made over the years regarding the deleterious effects of marijuana smoking on host defense mechanisms where marijuana smokers were found to be more susceptible to bacterial and viral infections. ${ }^{44}$ Between transmembrane domains 1 and 7, including the loop regions, the CB2 receptor is only 44\% identical to the CB1 receptor, making it is possible to develop selective $\mathrm{CB} 2$ ligands to specifically target the immune system.

GPR55 was first identified and cloned by O'Dowd in $1999 .{ }^{45}$ It was later identified by an in silico screen as a putative cannabinoid receptor due to an amino acid sequence in the binding region that is similar. Research groups at the pharmaceutical companies Glaxo Smith Kline and Astra Zeneca then extensively characterized the receptor in the hope that it would be responsible for the blood pressure lowering effects of cannabinoids. GPR55 is indeed activated by endogenous, phyto-, and synthetic cannabinoids but GPR55 knockout mice generated at Glaxo Smith Kline showed no alteration in the cannabinoid induced blood pressure regulation after administration of the GPR55 agonist abnormal cannabidiol.

Many of the known CB1 and CB2 ligands also bind to GPR55. The receptor is activated by phytocannabinoids such as $\Delta^{9}-\mathrm{THC}$ and cannabidiol, ${ }^{22 \mathrm{c}}$ synthetic cannabinoids such as CP-55,940, and the endocannabinoids anandamide, 2arachidonoylglycerol, and nolandin ether. ${ }^{22 \mathrm{c}}$ Recent research suggests that lysophosphatidylinositol and its 2-arachidonoyl derivative may be the endogenous ligands for GPR $55^{46}$ and the receptor appears likely to be a possible target for treatment of inflammation and pain as with the other cannabinoid receptors. ${ }^{47}$

While the $\mathrm{CB} 1$ and $\mathrm{CB} 2$ receptors couple to $\mathrm{G}_{\alpha \mathrm{i} / \mathrm{o}}$ proteins, GPR55 is coupled to $\mathrm{G}_{\alpha 13}$ and activation of the receptor leads to stimulation of a number of GTPases such as Ras homolog gene family, member A (RhoA), cell division control protein 42 homolog (cdc42) and Ras-related C3 botulinum toxin substrate 1 (rac1). ${ }^{22 a}$ Members of this GTPase superfamily appear to regulate a diverse array of cellular events, including the control of cell growth, cytoskeletal reorganization, and the activation of protein kinases. ${ }^{48}$ Stimulation of GPR55 induces F-actin formation under the control of $\mathrm{G}_{\alpha 13}$, RhoA and Rho-associated, coiled-coil containing protein kinase (ROCK). ${ }^{46 \mathrm{~b}}$ GPR55 activation also 
induces intracellular calcium release and activation of the transcription factors nuclear factor kappa-light-chain-enhancer of activated B cells (NF-kB), nuclear factor of activated T-cells (NFAT) and cAMP response element-binding (CREB). ${ }^{46 \mathrm{~b}}$

The physiological role of GPR55 remains unclear. Mice with a target deletion of the GPR55 gene show no specific phenotype. ${ }^{49}$ GPR55 is has been found to be widely expressed in the brain, especially in the cerebellum. It is found in the gastrointestinal tract in the jejunum and ileum and also in the adrenal glands but is apparently not highly expressed elsewhere in the periphery. ${ }^{22 \mathrm{c}}$ Osteoblasts and osteoclasts have also been shown to express GPR55 and this has been shown to regulate bone cell function. ${ }^{50}$

\subsection{Cannabinoids as Anti-Glioma Agents}

Brain tumors are the second and fifth leading causes of cancer-related deaths in males and females ages 20 to 39 , respectively. ${ }^{51}$ The most prevalent form of adult primary CNS tumors is collectively referred to as glioma, and the most common, devastating, and high-grade glioma is glioblastoma multiforme (GBM) ${ }^{51-52}$ These tumors are classically thought to originate from supporting or glial cells of the CNS, and represent roughly fifty percent of all adult primary CNS tumors. Long term survival of patients with a GBM diagnosis with the best radiological, surgical, and anti-tumor drug therapy available is extremely rare. The historical median survival for GBM patients is 3 to 12 months depending on age and other prognostic factors. ${ }^{53}$

\subsubsection{Current Standard of Care for Glioblastoma}

CNS tumors in general are difficult to treat because of the complexities associated with removing cancerous cells from a background of normal brain tissue without introducing considerable neurological damage. Conventional therapy for malignant glioma consists of complete gross resection followed by radiation, either with or without chemotherapy. Radiation is indicated because surgical resection misses micro populations of cells that have migrated from the primary tumor site. However, currently accepted therapeutic adjuvants to surgery such as radiotherapy and chemotherapy provide only a minor improvement in the disease course and life expectancy of patients diagnosed with malignant glioma. ${ }^{54}$ The blood-brain-barrier (BBB) prevents the use of most chemotherapeutic agents; those that are available do not significantly impact the course of disease and often have unacceptable side effect profiles. ${ }^{55}$ Therefore, new drugs that adequately treat high-grade glioma of the brain and spinal cord are desperately needed if these tumors are going to be treated adequately in the future.

\subsubsection{Surgical Intervention}

Therapeutic treatment patterns for GBM have produced limited increases in overall survival over the last few decades. The advent of microneurosurgical techniques 
along with improvements in neuroanesthesia and perioperative care has resulted in remarkable advancement in the management of benign tumors of the brain. However, current surgical technique cannot adequately rid the surrounding brain tissue of microscopic foci of high-grade glioma cells that are not visible on the post-operative MRI scan. Although surgery is not sufficient for cure of glioma, it is a necessary component of a multi modality approach that includes radiation and chemotherapy in some instances. Goals of surgery for malignant glioma include: (1) establishment of a histological diagnosis; (2) reduction in tumor cell load leading to reduced intracranial pressure; and (3) possibly changing tumor kinetics.

\subsubsection{Radiotherapy}

Surgery alone has fallen short in providing any appreciable long-term increase in life expectancy for patients with high-grade gliomas. Radiotherapy directed at the gliomatous area around the surgical cavity is considered beneficial to survival outcome, and indeed postsurgical radiation has proven to be the most effective treatment currently available for improving survival. There is also mounting evidence to suggest that additional radiation, given in the form of brachytherapy or radiosurgery, at initial diagnosis as a "boost" to standard radiation or at tumor recurrence, may provide added improvement in survival outcome. ${ }^{56}$ With advancements in radiotherapy tools and techniques the practice of whole brain irradiation has somewhat fallen from favor as radiation therapy results in radionecrosis not only of rapidly dividing tumor cells, but also of surrounding healthy brain tissue. There are now two main radiotherapy delivery techniques: (1) external stereotactic radiotherapy (SRT) boost, administered either in one session or in fractions; and (2) brachytherapy boost by peri- or post-operative implantation of temporary catheters or permanent isotope seeds.

\subsubsection{Chemotherapy}

At present, most centers in the United States also employ adjuvant chemotherapy as part of a patient's initial treatment. Current conventional chemotherapy regimens for newly diagnosed malignant glioma include single-agent intravenous bis-chloroethylnitrosurea (BCNU; carmustine), single-agent oral procarbazine, or the PCV combination of procarbazine, chloroethyl-cyclohexylnitrosourea (CCNU) (lomustine), and vincristine. Since the 1980s, some but not all randomized studies have shown a small survival advantage for patients receiving one of these chemotherapy regimens in addition to surgery and RT. ${ }^{54 a, b, 57}$ A meta-analysis of published phase III randomized prospective studies showed a modest overall survival benefit and a higher proportion of long-term (> 24 months in some studies) survivors for patients with GBM who received adjuvant chemotherapy. ${ }^{58}$ The toxicity profile varies among these treatments, with myelosuppression being the most frequent dose-limiting effect. ${ }^{55,57}$ Factors limiting the use of chemotherapy are that there are no strong predictors of chemosensitivity among individual patients and the proper timing of upfront chemotherapy in relation to SRT for newly diagnosed tumors has never been studied rigorously. Empirically administered 
adjuvant chemotherapy generally is begun during or shortly after completion of SRT. Despite a variety of drugs and treatment schedules, the median time to tumor progression for patients with GBM is 6 to 8 months, and the overall survival remains around 12 months. To date there has been no proven prolongation of time to progression or survival for patients with malignant glioma when other drugs are added to the "standard" BCNU or PCV chemotherapeutic regimens, including cisplatin, ${ }^{59}$ carboplatin, ${ }^{60}$ dibromodulcitol, ${ }^{61}$ mercaptopurine, ${ }^{62}$ or 6-thioguanine. ${ }^{63}$

\subsubsection{Cannabinoids as Chemotherapeutic Agents for the Treatment of High- Grade Gliomas}

Investigational research related to the use of cannabinoids in oncology was primarily restricted to anti-emesis prior to the late 1980s. Although limited studies on Lewis lung cancer and leukemia predate this time, active research on the antineoplastic potential of cannabinoids gained increased attention following the discovery of the CB1 and CB2 receptors. Cannabinoids have now been shown to induce apoptosis in rhabdomyosarcoma, ${ }^{64}$ colon, ${ }^{65}$ prostate $^{66}$ and skin carcinomas, ${ }^{67}$ glioma, ${ }^{32,68}$ neuroblastoma, ${ }^{68 \mathrm{~b}}$ and lymphoma/leukemia. ${ }^{69}$ Furthermore, a decrease in tumor size and/or cell growth inhibition has been reported in breast, ${ }^{66 \mathrm{~b}, 70}$ uterus, ${ }^{71}$ and lung cancers, ${ }^{72}$ and in thyroid epithelioma. ${ }^{73}$ The ability of cannabinoids to modulate the cell survival and death pathways in neoplasia is extremely significant from a drug development perspective. Specifically, clinically investigated cannabinoids, in contrast to conventional cancer chemotherapies, do not exhibit the generalized toxic effects, are well tolerated by patients, and have the ability to penetrate the blood brain barrier. This fledgling strategy of cancer treatment has the potential of decreasing the often severe side effects associated with most antineoplastic agents. The future development of cannabinoids as peripheral antineoplastic agents holds great promise, however possibly the greatest impact in cancer therapy is the unprecedented opportunity for the development of antineoplastic agents capable of treating malignancies in the CNS.

The CNS and associated BBB is arguably the most difficult target organ for which drugs are developed. Despite the development of an arsenal of clinically utilized CNS drugs, e.g. anti-depressants and anti-psychotics, highly effective chemotherapeutics for the treatment of brain cancers such as glioblastoma remain elusive. One of the principle factors hindering this field of drug development is the fact that many of the otherwise efficacious natural product related antineoplastic agents are substrates for the anti-porter proteins that are abundant in the CNS microvasculature. The established permeability of classical cannabinoids (CCBs) through the BBB combined with the demonstrated in vitro anti-glioma activity of several cannabinoid ligands offers an unprecedented opportunity for drug development. Often such in vitro data does not translate to in vivo efficacy; however our lab and others have demonstrated the therapeutic potential of CCBs. Specifically, KM-233 and JWH-133 were highly effective in halting the progression of human brain tumors in mice. ${ }^{68 a}, 74$ This is significant in that JWH-133 and, to a lesser extent KM-233, are selective CB2 receptor ligands and a recent report shows that primary human glioblastomas express significant levels of CB2 receptors. ${ }^{75}$ This study was conducted prior to the emergence of GPR55 in the CB field 
and as such expression levels of GPR55 were not evaluated. As stated earlier, GPR55 is widely expressed in the CNS and increased GPR55 expression is consistent with our Western blot analysis of GBM cell lines (see Chapter 2, Figure 2.8). Validation of GPR55 as a target for CB based anti-neoplastic agents offers a significant advancement in therapies targeted at GBM.

Further support for targeting the cannabinoid system comes from a pilot clinical study using THC, a non-selective partial agonist of the CB receptors; there was a slight improvement in survival in patients with recurrent brain cancers. ${ }^{76}$ In this study THC was administered intratumorally which may have, in part, reduced the overall efficacy of the treatment. Specifically, in our side flank model, administration of KM-233 intraperitoneally (i.p.) resulted in a greater reduction of tumor load compared to intratumor injection. ${ }^{74 \mathrm{~b}}$ The development of cannabinoids as anti-glioma agents represents a promising field of research into the treatment of one of the most malignant forms of cancer whose current treatment is either ineffective or only palliative.

\subsection{Cannabinoids as Anti-Inflammatory Agents}

It has been shown over the last forty years, initially by examining marijuana users, then in animal studies, and finally in cell culture, that cannabinoids are proven immunomodulators. $^{77}$ It has been known for a much longer period of time, however, that marijuana does possess anti-inflammatory properties. In what is perhaps the earliest literature reference to Cannabis, it is described as an anti-inflammatory agent. The Chinese emperor Shen-nung (ca. 2000 B.C.), in a work called Pen-ts'ao Ching, noted many of the effects of Cannabis in humans. Among other properties, he claimed that Cannabis "undoes rheumatism", suggesting possible anti-inflammatory effects. ${ }^{78}$

\subsubsection{Cannabinoid Receptors in the Immune System}

The presence of a cannabinoid receptor in cells of the immune system was known before the discovery of CB2. In 1992, Kaminsky et al. reported the presence of the putative cannabinoid receptor on mouse splenic cells. ${ }^{79}$ This report was supported by the evidence of stereoselective immunomodulatory effects of cannabinoids on the splenic cells, specific binding of tritiated CP-55,940, and by the identification of mRNA for the receptor in the cells. Shortly after this discovery, the peripheral expression and distribution of CB1 was examined in human tissues. Messenger RNA for the receptor was identified in human spleen, tonsil, and peripheral blood leukocytes, ${ }^{80}$ although at much lower levels than in the brain. The expression patterns found in the human blood cells varied significantly by type. The order of expression level was found to be B cells $>$ natural killer (NK) cells $>$ polymorphonuclear neutrophils $>$ CD8+ T lymphocytes $>$ monocytes $>$ CD4+ T lymphocytes. CB1 mRNA at varying levels was also found in a number of monocytic and $\mathrm{T}$ and $\mathrm{B}$ leukemia cell lines, with the exception of Jurkat cells. On average, the B cell lines were found to have higher expression levels than the T cells. 
After the discovery of the CB2 receptor in 1993, it was shown to be the dominant cannabinoid receptor in the immune system with (at that time) no quantifiable expression in the brain. ${ }^{17,39 \mathrm{~b}}$ Human tissues were examined for expression levels of CB2 and it was found that levels of CB2 are 10-100X that of CB1 in human immune tissues. ${ }^{42 a, 81}$ It was shown by Galiegue and coworkers that the level of expression of CB2 in the spleen and tonsils was equivalent to the CB1 expression levels in the brain. ${ }^{43}$ Knowing the physiologic function of $\mathrm{CB} 1$ in the CNS, the researchers drew the conclusion that the CB2 receptor must be the major subtype responsible for the immunomodulatory effects of cannabinoids. The order of expression of CB2 in human blood cells was reported to be B cells $>$ NK cells $>$ monocytes $>$ polymorphonuclear neutrophils $>$ CD $8+\mathrm{T}$ lymphocytes $>$ CD4 + T lymphocytes. Human cell lines derived from myeloid, monocytic, and lymphoid lineages were shown to have the same rank order of CB2 density as determined by mRNA levels. The high level of mRNA in the B lymphocytes also correlates with receptor expression on the cell surface. This was confirmed by Lynn and Herkenham using tritiated CP-55,940. ${ }^{82}$ The CB2 receptor has also been verified to be present on dendritic cells. ${ }^{83}$ Its presence on these potent antigen-presenting cells suggests a possible role for cannabinoids to modulate antigen presentation. More recently, the presence of $\mathrm{CB} 2$ has been reported in the CNS. Here, the CB2 receptor plays a possible role in inflammation due to the high expression levels found in the macrophage-like cell type microglia. ${ }^{84}$

With the recent identification of GPR55 as a putative cannabinoid receptor, its role in cannabinoid mediated immunomodulatory effects has not been as well established as with CB1 and CB2. While GPR55 has not been shown to be present in peripheral blood leukocytes, it has been identified in the spleen. ${ }^{22 c}$ One possibly significant role for GPR55 in immune modulation is in the CNS. In a recent report by Pietr et al., the authors claim to have discovered the presence of mRNA for GPR55 in a primary culture of mouse microglia and also in the mouse microglial cell line BV-2. ${ }^{47 \mathrm{~d}, 85}$ The possible role of this receptor in inflammation in this cell type is supported by the observation of altered levels of mRNA for the GPR55 receptor upon exposure to various inflammatory stimuli. Pietr and coworkers reported that stimulation of primary microglia or BV-2 with lipopolysaccharide (LPS) caused a concentration-dependent decrease in mRNA levels. Differing results were seen, however, utilizing stimulation with interferon gamma (IFN$\gamma$ ) which caused a slight decrease in GPR55 mRNA levels in primary culture but an increase in the BV-2 cell line. ${ }^{47 \mathrm{~d}}$ This observation is similar to the reported variation in $\mathrm{CB} 2$ expression on microglia based on activation state. ${ }^{86}$

\subsubsection{Cannabinoid Effects on Immunity}

The effects elicited by cannabinoids on the immune system are mediated by the downstream signaling cascades of the cannabinoid receptors. This is achieved mainly by the down-regulation of cAMP formation by inhibition of adenylate cyclase (AC)

activity. ${ }^{87}$ It has been shown that lymphocyte activation and cytokine transcription in macrophages is regulated by AC activity and the cAMP cascade. ${ }^{88}$ This is supported by the fact that cAMP analogs can stimulate or inhibit the immune response and can also antagonize the anti-inflammatory effects of cannabinoids. ${ }^{87}$ 
The signaling cascade involved in the anti-inflammatory effects of cannabinoids is probably not simply due to regulation of AC. This would explain why endogenous and exogenous cannabinoids can elicit different immunomodulatory effects.

Endocannabinoids seem to have effects that are very localized, short lived, and are stimulatory in nature. ${ }^{89}$ The endocannabinoid 2-arachidonylglycerol (2-AG), for example, has been shown to have immunostimulatory actions such as serving as a chemoattractant to myeloid leukemia cells, splenocytes, and microglia ${ }^{90}$ and has also been shown to induce both chemotaxis and chemokinesis on microglia. ${ }^{91}$ There is some evidence, however, that certain metabolically stable analogs of endocannabinoids can be immunosuppressive. An example of this is the anandamide analog 2-methylarachidonyl(2'-fluoroethyl) amide that has been shown to suppress immune response in a model of $\mathrm{T}$ lymphocyte function. ${ }^{92}$

Exogenous cannabinoids, on the other hand, have effects on the immune system that are more generalized in nature, persist for longer periods of time, and tend to be immunosuppressive. ${ }^{93}$ Both phytocannabinoids and synthetic cannabinoids have been shown to have immunosuppressive effects. THC, CP-55,940, and the classical cannabinoid analog HU-210 have all been shown to inhibit cell contact-dependent lysis of tumor cells by macrophages, to inhibit the ability of macrophages to process antigens, and to suppress the proliferation of both B and T lymphocytes. ${ }^{94}$ THC has also been shown to attenuate the proliferation and maturation of cytotoxic $\mathrm{T}$ lymphocytes and to suppress the activity of NK cells. ${ }^{94 a}$ Exogenous cannabinoids have also been reported to cause a switch in the $T$ helper $\left(T_{h}\right)$ cell profile from the $T_{h} 1$ phenotype to the $T_{h} 2$ phenotype by alteration of the cytokine / chemokine network. ${ }^{95}$ This switching of $\mathrm{T}$ helper function to the anti-inflammatory $\mathrm{T}_{\mathrm{h}} 2$ phenotype is of particular interest due to the possible therapeutic implications in autoimmune diseases. For example, blocking the $T_{h} 1$ cytokines and enhancing the $\mathrm{T}_{\mathrm{h}} 2$ pathways has shown promise in several animal models of inflammatory diseases such as experimental autoimmune encephalomyelitis, ${ }^{81,96}$ experimental arthritis, ${ }^{97}$ and experimental autoimmune hepatitis. ${ }^{98}$ Exogenous cannabinoids have also been shown to inhibit immune cell migration and recruitment, ${ }^{99}$ in contrast to the effects of endocannabinoids such as 2-AG.

The indirect effects that cannabinoids exert on immune function through alterations in the cytokine / chemokine network have been extensively studied. ${ }^{77}$ Cytokines and chemokines are a large family of proteins that are produced by various cells of the immune system. Their function is to alter the state or the activity of other cells in the immune system or to direct the movement of immune cells to sites where they are needed. Cytokines and chemokines can be broadly classified as proinflammatory or anti-inflammatory depending upon whether their overall effect on the immune response is stimulatory or suppressive, respectively. Much of the original work in this field was with the classical cannabinoid THC and many results were confounding. For example, THC has been shown to increase the processing and release of the proinflammatory cytokine interleukin-1 (IL-1) in vitro ${ }^{100}$ and IL-1 along with proinflammatory tumor necrosis factor-alpha (TNF- $\alpha$ ) and IL-6 in vivo. ${ }^{101}$ On the other hand, THC has also been shown to suppress the acute phase proinflammatory cytokines such as TNF- $\alpha$, for example, in both human and mouse macrophage cultures. ${ }^{77}$ 
With the nearly ubiquitous expression of the cannabinoid receptors (especially CB2) in the immune system it is clear why they are ever increasing targets for the treatment of inflammatory disorders. Along with the previously mentioned cells, cannabinoids have also been shown to have immunomodulatory effects on other cell types such as mast cells,${ }^{5,102}$ neutrophils, ${ }^{103}$ astrocytes, ${ }^{104}$ glia, ${ }^{95 \mathrm{c}, 105}$ and neurons. ${ }^{95 \mathrm{~d}, 106}$ Levels of many other cytokines have been reported to be effected by cannabinoids such as decreases in the proinflammatory proteins IFN- $\gamma$, IL-2, and IL- $12^{5}$ and increases in the anti-inflammatory factors IL-4 and IL-10. ${ }^{107}$ Much of the current work with cannabinoids in this field is focused on CB2 selective compounds which are void of the psychotropic effects mediated by the CB1 receptor which could limit their usefulness. Current studies on CB2 selective ligands as anti-inflammatory agents will be covered in Chapter 5. 


\section{Chapter 2: Anti-Glioma Activity of KM-233 and Analogs}

\subsection{Introduction}

Due to the increasing attention given to the endocannabinoid system as a target for the development of antineoplastic agents, we began to evaluate the anti-cancer potential of our novel cannabinoid ligands. The initial antineoplastic work performed in our laboratory was by Dr. Mathangi Krishnamurthy for her doctoral dissertation. She prepared a novel series of C1'-dimethyl-aryl $\Delta^{8}$-tetrahydrocannabinol analogs and evaluated the compounds for CB1 and CB2 binding. ${ }^{108}$ The compound which exhibited the highest degree of CB2 selectivity was (6aR,10aR)-6,6,9-trimethyl-3-(2-phenylpropan2-yl)-6a,7,10,10a-tetrahydro-6H-benzo[c]chromen-1-ol; designated KM-233 1 (Figure 2.1). This classical cannabinoid exhibited high affinity for the $\mathrm{CB} 1$ and $\mathrm{CB} 2$ receptors with binding affinity constants of $12.3 \mathrm{nM}$ and $0.91 \mathrm{nM}$, respectively. This corresponds to a CB1/CB2 ratio of 13.5. KM-233 showed higher affinities for both the CB1 and CB2 receptors compared to $\Delta^{8}-\mathrm{THC}\left(K_{\mathrm{i}}(\mathrm{CB} 1)=28.5 \mathrm{nM}, K_{\mathrm{i}}(\mathrm{CB} 2)=25.0 \mathrm{nM}\right)$ with 13 -fold higher selectivity for the $\mathrm{CB} 2$ receptor. Introduction of a phenyl ring in the classical cannabinoid side chain also resulted in an increase in $\log \mathrm{P}$ from 6.08 for $\Delta^{8}$-THC to 6.27 for KM-233, which suggests improved blood-brain-barrier (BBB) penetrating ability. Hence, this combination of lipophilicity and high CB2 affinity indicates that this compound would possess significant permeation through the blood-brain-barrier as well as good activity at the CB2 receptor and would be a viable candidate to screen for antiglioma activity.

The cytotoxic effects of KM-233 were evaluated against the human glioblastoma multiforme cell line U-87 MG. ${ }^{109}$ Cell death was analyzed after 24, 48 and 72 hours of exposure to the drug. KM-233 effectively killed glioma cells over a 3-day incubation period with maximum cytotoxic effects occurring at $5 \mu \mathrm{M}$ (concentrations above $5 \mu \mathrm{M}$ not shown) (Figure 2.2) and with the $\mathrm{EC}_{50}$ at $3.1 \mu \mathrm{M}$. The cytotoxic effect produced by KM233 was compared with that produced by $\Delta^{8}$-THC (Figure 2.3) using a $5 \mu \mathrm{M}$ dose of each drug. Although both drugs showed similar cytotoxic effects at the end of 3 days, KM233 was more effective than $\Delta^{8}$-THC over the first 48 hours (data not shown here). In summary, KM-233 was shown to be as efficacious as $\Delta^{8}$-THC as a chemotherapeutic agent and may have a more rapid onset of action than $\Delta^{8}$-THC.

Next, the cytotoxic effect of KM-233 was compared with that produced by BCNU, a standard glioblastoma chemotherapeutic agent. For these experiments, a $5 \mu \mathrm{M}$ dose of KM-233 was compared with escalating doses of BCNU. A $5 \mu \mathrm{M}$ dose of KM233 was far more cytotoxic than the highest dose of BCNU tested which was $300 \mu \mathrm{M}$ (well above the typical $5 \mu \mathrm{M}$ blood level following i.v. administration in humans) (Figure 2.4). This led to the conclusion that KM-233 is superior to BCNU as a chemotherapeutic agent for the treatment of glioblastoma in terms of its in vitro cytotoxic activity. 


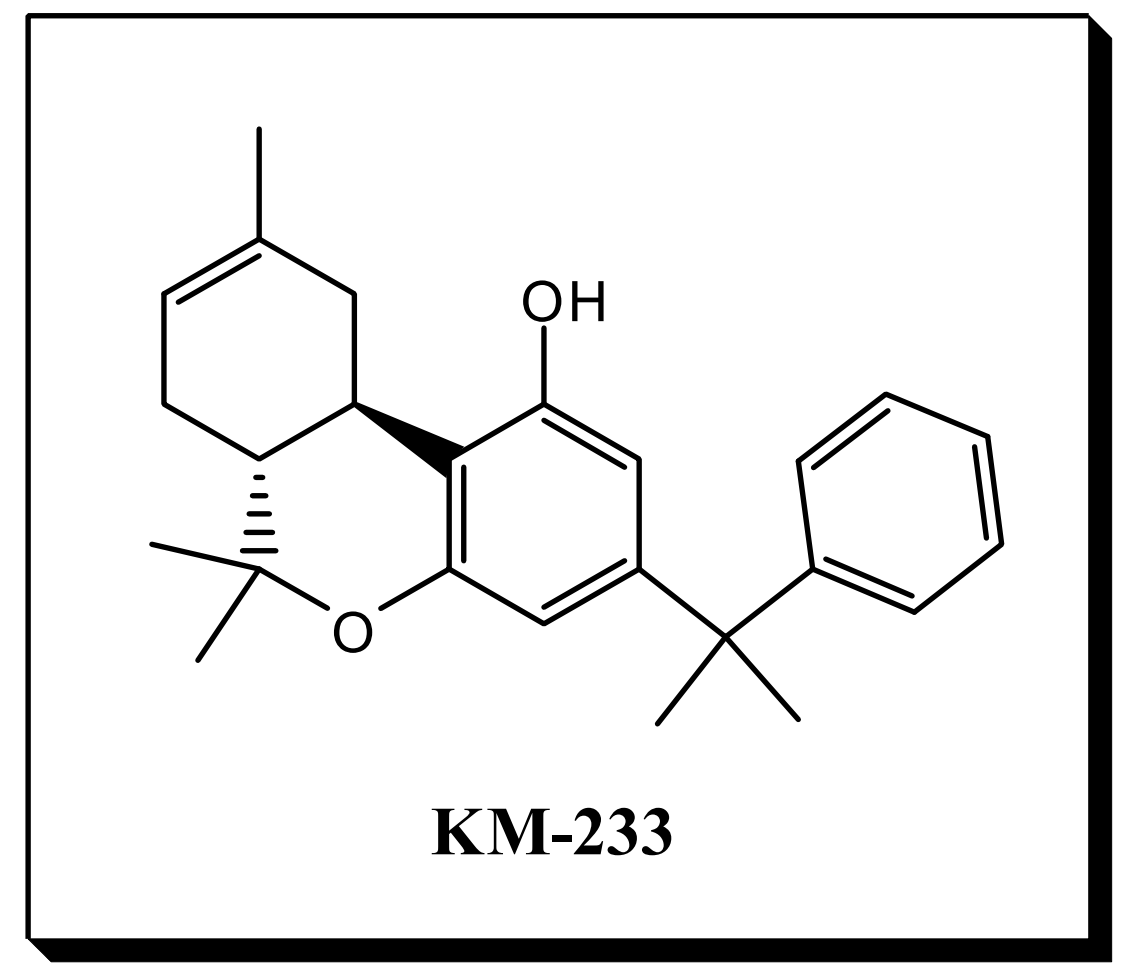

Figure 2.1: The structure of KM-233 1 

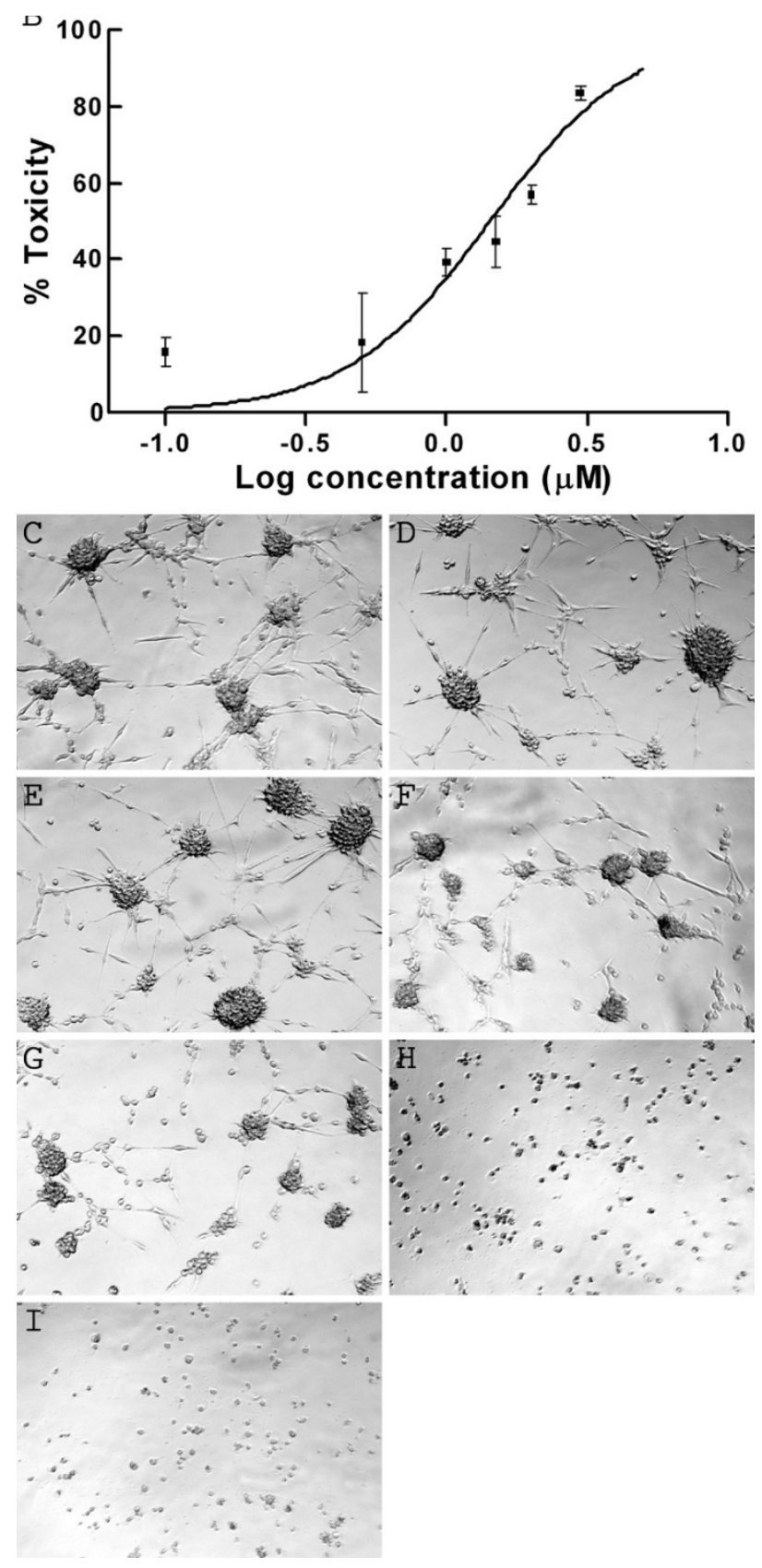

Figure 2.2: Dose response curve for KM-233 on U-87 MG

$\mathrm{C}$ represents untreated control, D represents untreated control with $0.5 \%$ DMSO, E represents drug treatment at concentration $0.1 \mu \mathrm{M}, \mathrm{F}$ represents drug treatment at $1 \mu \mathrm{M}$ concentration, $\mathrm{G}$ represents drug treatment at $2 \mu \mathrm{M}$ concentration, $\mathrm{H}$ represents drug treatment at $3 \mu \mathrm{M}$ concentration and I represents drug treatment at $5 \mu \mathrm{M}$ concentration. 


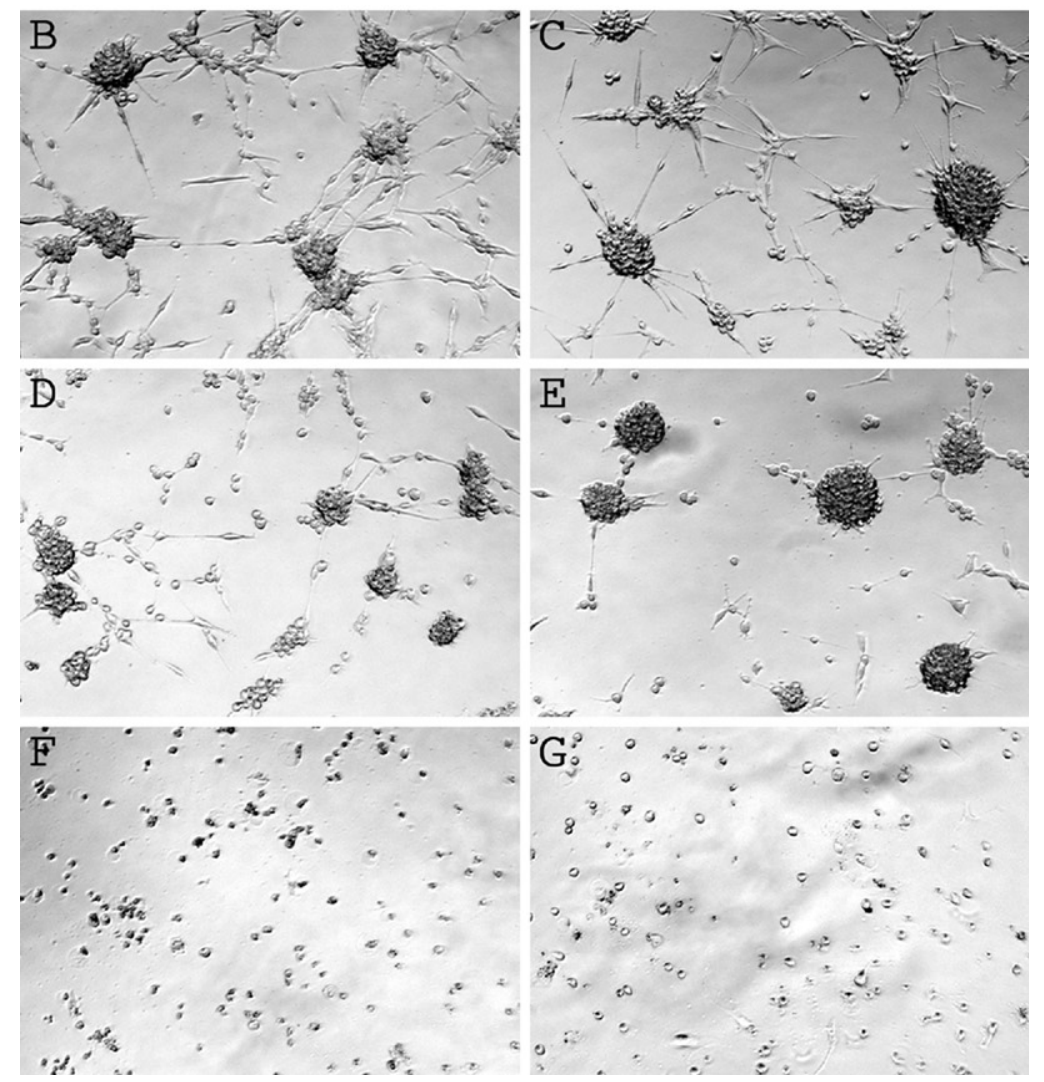

Figure 2.3: Comparison of efficacies of KM-233 and $\Delta^{\mathbf{8}}-\mathrm{THC}$

$\mathrm{B}$ represents untreated control, $\mathrm{C}$ represents untreated control with $0.5 \%$ DMSO, D represents effect after 1 day treatment with KM-233, E represents effect after 2 days treatment with KM-233, F represents effect after 3 days treatment with KM-233 and G represents effect after 3 days treatment with $\Delta^{\mathbf{8}}$-THC. 

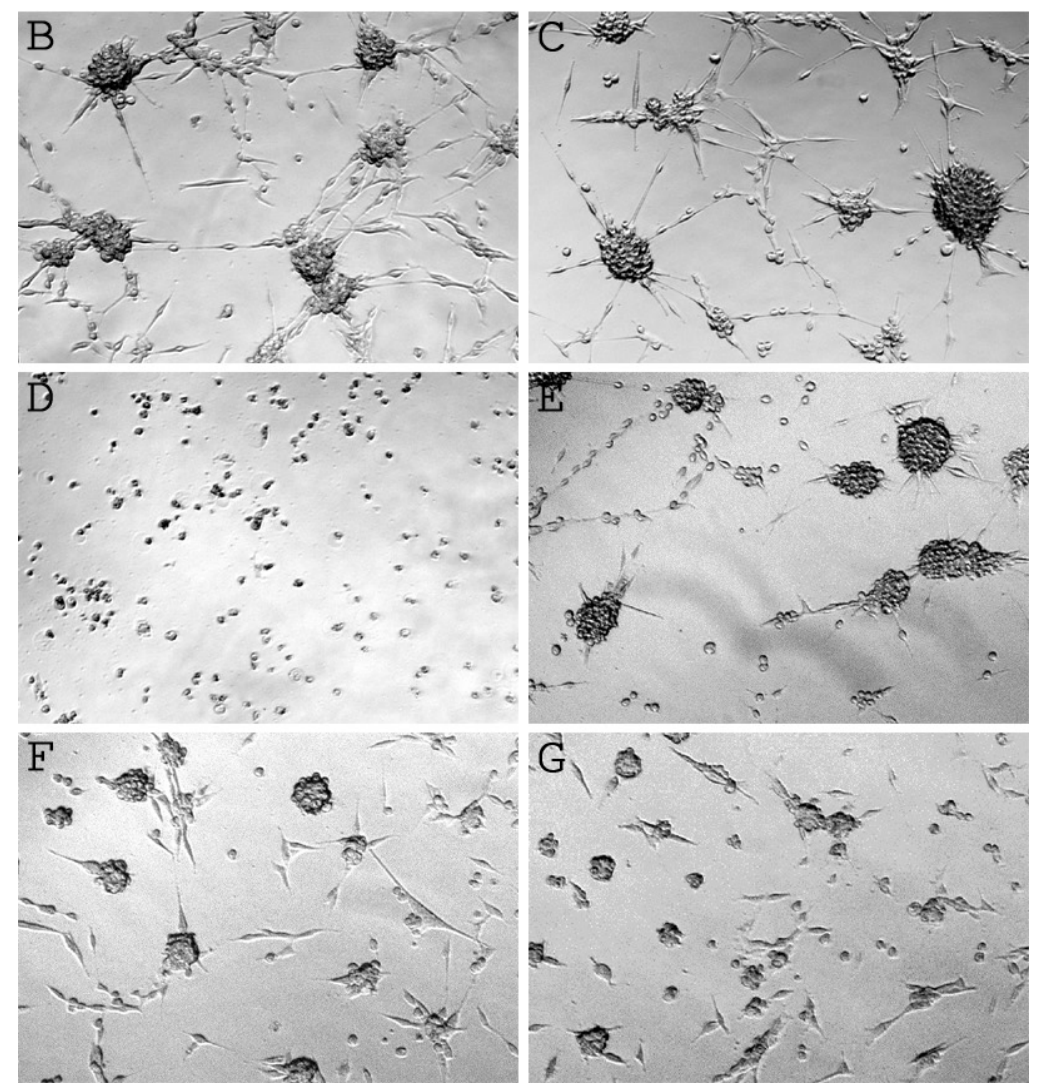

Figure 2.4: Comparison of efficacies of KM-233 and BCNU

$\mathrm{B}$ represents untreated control, $\mathrm{C}$ represents untreated control with $0.5 \%$ DMSO, D represents treatment with $5 \mu \mathrm{M} \mathrm{KM}-233$, E represents treatment with $100 \mu \mathrm{M} \mathrm{BCNU}, \mathrm{F}$ represents treatment with $200 \mu \mathrm{M} B C N U$ and G represents treatment with $300 \mu \mathrm{M} \mathrm{BCNU}$. 


\subsection{Murine Flank Model}

The in vitro efficacy of KM-233 was then translated into an animal model of glioblastoma to further evaluate its potential as a chemotherapeutic agent. The first in vivo model performed was a mouse flank model. The flank model was performed by the Duntsch group in the Department of Neurosurgery at the University of Tennessee Health Science Center. ${ }^{109}$ The mouse xenograft subcutaneous model was created by suspending $2 \times 10^{6}$ glioblastoma multiforme cells in PBS and injecting them subcutaneously into the flank of adult SCID mice. The cell line used was U-87 MG which had been previously transduced to express green fluorescent protein to aid in imaging of the tumor. After seeding, the tumors were allowed to develop for 5 days and the mice were then treated with KM-233 at a dose of $2 \mathrm{mg} / \mathrm{kg}$ either intratumorally or intraperitoneally every 12 hours for 15 days. Control groups were treated with vehicle at the same frequencies and routes of administration. The animals were sacrificed after 15 days of treatment and the tumors removed with the aid of epifluorescence microscopy.

Once the tumors were removed, they were weighed and the weights compared between the vehicle treated and the KM-233 treated groups. The groups treated with KM-233, both intratumorally and intraperitoneally, showed a reduction in tumor size when compared to the vehicle treated control groups (Figure 2.5). The group treated by the intraperitoneal route exhibited a greater reduction in tumor size from control when compared to the intratumorally treated group. Clinical monitoring and daily weights were similar between groups indicating no gross deleterious effects of KM-233 when administered locally or systemically. These results, while very promising, did not answer the question of whether or not KM-233 could cross the BBB and be a viable candidate for GBM therapy.

To further assess the ability of KM-233 to cross the BBB, intracranial model studies were carried out, also by the Duntsch group. The development of a chemotherapeutic agent against GBM that crosses the BBB provides distinct advantages to existing agents; 1) most existing agents used against GBM do not cross the BBB, and to achieve therapeutic levels they are generally administered directly into the tumor; and 2) direct administration may not target distant GBM foci whereas a systematically active agent should "bathe" the brain in drug thus targeting distant foci. Therefore, the ability of KM-233 to cross the blood brain barrier and affect a reduction in tumor load was studied in the orthotopic U87 model of GBM.

Tumors were induced in SCID mice by direct intracranial inoculation of human GFP-expressing U87 glioma cells. Five days following tumor cell inoculation, animals were assigned randomly to treatment groups and injected intraperitoneally twice a day for 20 subsequent days with vehicle, $2,4,8$, or $12 \mathrm{mg} / \mathrm{kg}$ of $\mathrm{KM}-233$ formulated in $100 \mu \mathrm{l}$ of saline containing $3 \%$ of Cremophor and $3 \%$ of ethanol. The animals were then sacrificed on the $25^{\text {th }}$ day $\left(20^{\text {th }}\right.$ day of treatment $)$ and the brains prepared for confocal laser scanning microscopy to measure tumor surface area using imaging software. As shown in Figures 2.6 and 2.7, treatment of KM-233 significantly reduced the average tumor load of each treatment group in a dose-dependent manner as compared to untreated and vehicle treated controls. Clinical monitoring and daily weights were similar between all controls and 
(a)
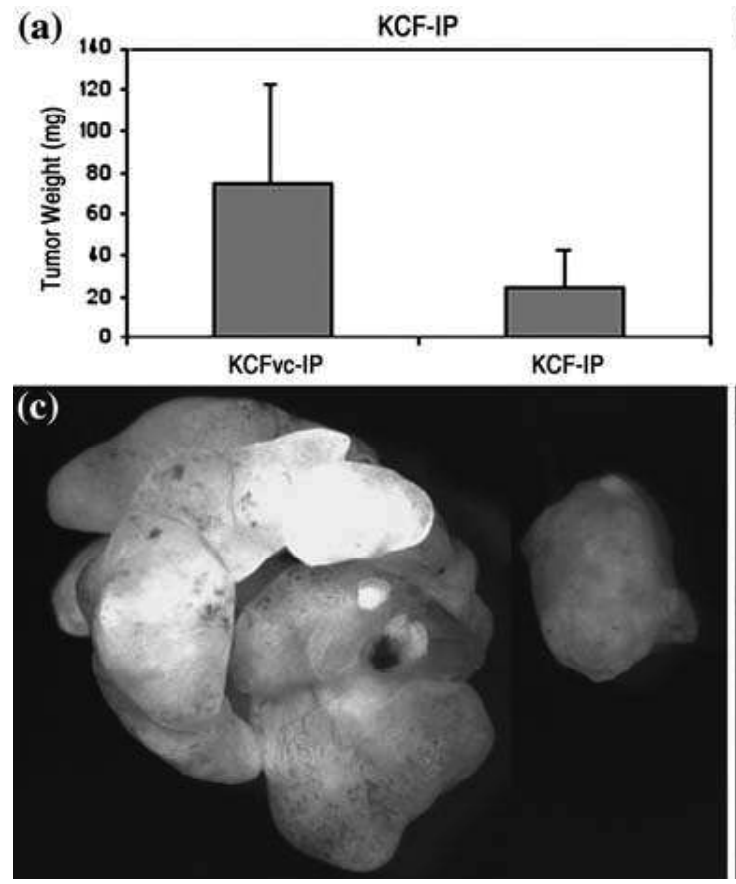

(b)

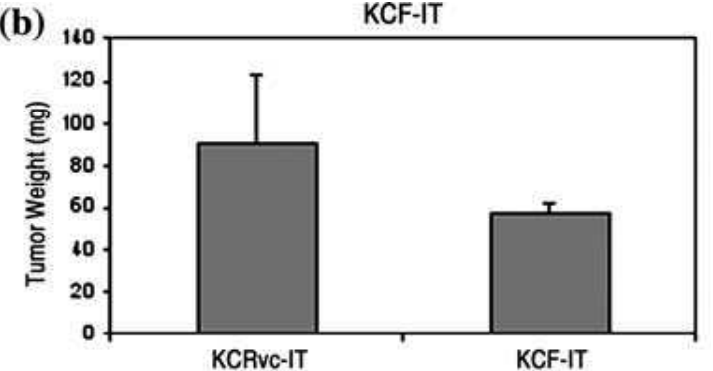

(d)

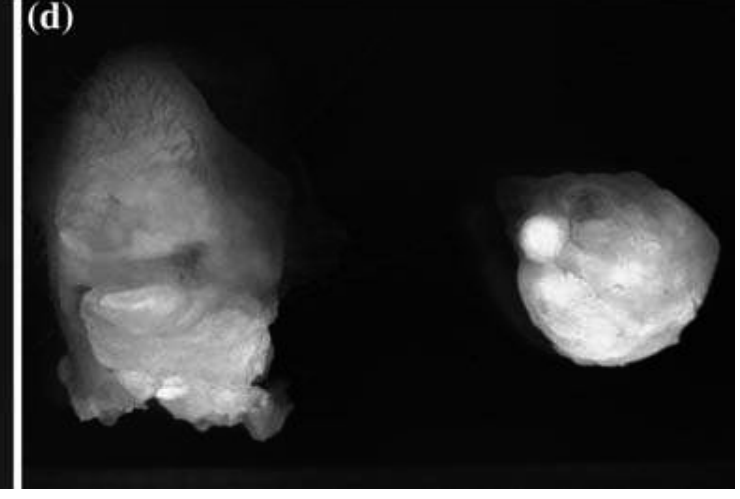

Figure 2.5: In vivo efficacy of KM-233 against human U87 glioma in the murine sidepocket model

Human glioma cells were inoculated into the flanks of nude mice ( $n=4$ per group). After 5 days of tumor cell incubation, groups were treated either by i.p. injection (a) or direct injection into the tumor (b) every $12 \mathrm{~h}$ for 15 subsequent days with vehicle or $2 \mathrm{mg} / \mathrm{kg}$ of KM-233. Panel c depicts an epifluorescence photomicrograph of the average size of tumors dissected from control animals vs. animals treated with i.p. injection KM-233 and panel d represents vehicle control vs. treated with intertumoral injection of KM-233. 


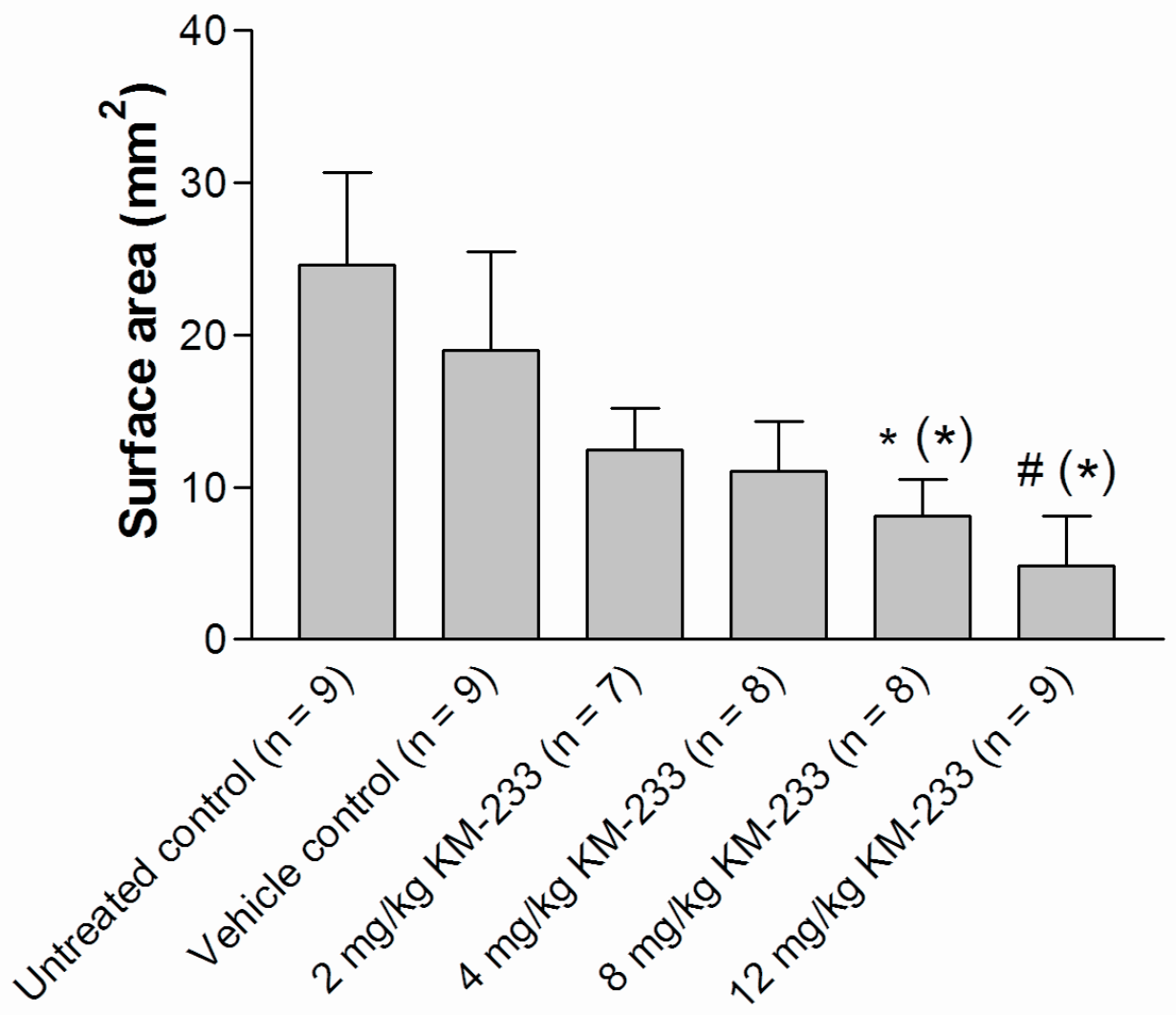

Figure 2.6: Tumor load in the U87 glioma model

Adult SCID mice bearing intracranial human U87 glioma were treated with KM-233 for 20 days. A one-way ANOVA of the untreated control vs. all treatments (symbols without parenthesis) and vehicle control vs. drug treatment (symbols with parenthesis) was conducted. ${ }^{*}=\mathrm{p}<0.05$ and $\#=\mathrm{p}<0.001$. 

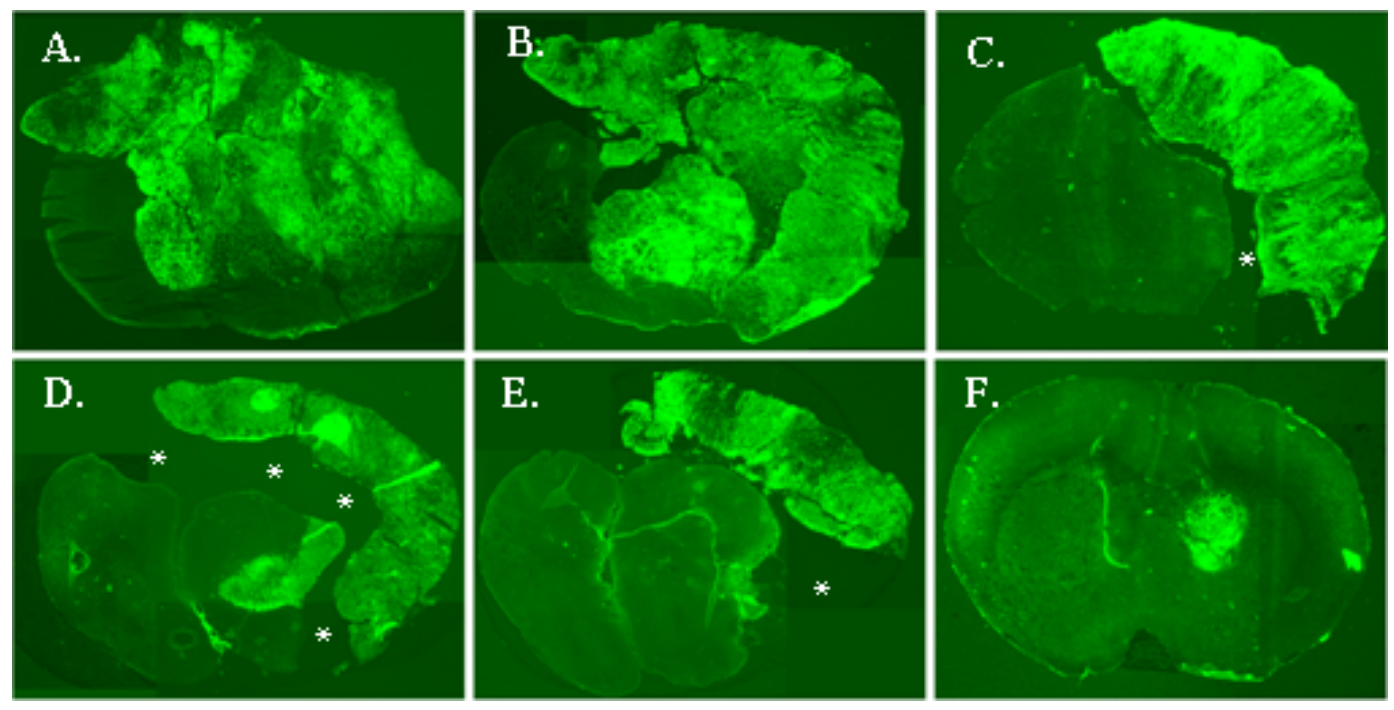

Figure 2.7: Dose-dependent reduction of U87 glioma after KM-233 treatment

Adult SCID mice bearing orthotopic human U87 glioma were treated with KM-233 for 20 days. Representative fluorescence micrographs showing tumor sizes in mouse brains where: (A) untreated control (B) vehicle control; (C) $2 \mathrm{mg} / \mathrm{kg} \mathrm{KM}-233$; (D) $4 \mathrm{mg} / \mathrm{kg} \mathrm{KM-}$ 233; (E) $8 \mathrm{mg} / \mathrm{kg} \mathrm{KM}-233$; and (F) $12 \mathrm{mg} / \mathrm{kg} \mathrm{KM}-233$. Brains were serial sectioned (asterisks indicate areas where tumor separated from normal brain tissue) and the region of the needle track identified. Pictures represent the largest fluorescent section within an animal brain where measurements were taken. 
treated groups. These studies in conjunction with the flank model described above suggest that KM-233 is safe and effective in reducing tumor load, even in the context of the BBB, and even when given systemically. Pathology on the organs of the study animals showed that there was no evidence of toxicity-related tissue damage due to KM233 administration. The results of this study demonstrated that KM-233 is a viable candidate for further development as an anti-glioma agent.

\subsection{Efficacy of KM-233 and Analogs against Multiple Glioma Lines}

To further evaluate the potential of KM-233 as a chemotherapeutic for the treatment of GBM, the drug was screened against four other human glioblastoma cell lines: LN-229, T98G, DBTRG-05MG, and MT-310 (a primary GBM cell line provided by the Department of Neurosurgery at the University of Tennessee Health Science Center). We hypothesized that KM-233 initiated GBM cell death by ligation to one or more of the $\mathrm{CB}$ receptors $\mathrm{CB} 1, \mathrm{CB} 2$, and/or GPR55. The first step in this study was to determine the relative expression levels of these receptors in these cell lines along with U-87 MG. Western blot analysis of the particulate fraction, membrane bound proteins, and cytosolic fraction of cell lysates is shown in Figure 2.8. Consistent with earlier reports, ${ }^{110}$ both $\mathrm{CB} 1$ and $\mathrm{CB} 2$ receptors are present in all the human GBM cell lines with $\mathrm{CB} 1$ showing a lower immunohistochemical staining relative to $\mathrm{CB} 2$. Interestingly, the GPR55 receptor exhibited a relative increased blotting in both the particulate and cytosolic fractions. The presence of GPR55 in the cytosolic fraction may indicate active synthesis of the protein or compartmental localization in the cytosol. The qualitative

analysis of the Western data was not interpreted in terms of absolute expression levels but was used to verify the presence or absence of each receptor type.

The similarities in receptor expression patterns found in each GBM cell line may account for the comparable efficacies of KM-233 in cytotoxicity assays against the human GBM cell lines U-87 MG, T98G, LN-229, DBTRG-O5MG, and MT310 (Table 2.1). The $\mathrm{EC}_{50}$ values were determined using escalating doses of $\mathrm{KM}-233$ and cell viability assayed 18 hours post treatment using the CCK-8 assay which utilizes the tetrazolium salt, WST-8 (2-(2-methoxy-4-nitrophenyl)-3-(4-nitrophenyl)-5-(2,4disulfophenyl)-2H-tetrazolium, monosodium salt), which produces a water-soluble formazan dye upon bioreduction in the presence of an electron carrier, 1-Methoxy PMS. WST-8 is bioreduced by cellular dehydrogenases to an orange formazan product that is soluble in tissue culture medium. The amount of formazan produced is directly proportional to the number of living cells. The $\mathrm{EC}_{50}$ for $\mathrm{KM}-233$ was comparable between the cell lines with a mean $\mathrm{EC}_{50}$ of $3.44 \mu \mathrm{M}$. In comparison, the clinically relevant anti-GBM agents $\mathrm{BCNU}$ and temozolomide were either ineffective or manifested high micromolar $\mathrm{EC}_{50} \mathrm{~s}$ (Table 2.1). Specifically, U87 and LN-229 were sensitive to BCNU after 18 hours of exposure with $\mathrm{EC}_{50} \mathrm{~s}$ of 223 and $178 \mu \mathrm{M}$, respectively. All cell lines responded to $\mathrm{BCNU}$ following 48 hours of exposure manifesting $\mathrm{EC}_{50} \mathrm{~S}$ ranging from 119 to $305 \mu \mathrm{M}$. In contrast, the $\mathrm{EC}_{50} \mathrm{~s}$ of $\mathrm{KM}-233$ ranged from 2.3 to $4.9 \mu \mathrm{M}$ and were effective in the 18 hour assay window. Temozolomide was inactive against the cell lines following 18 and 48 hours exposure 


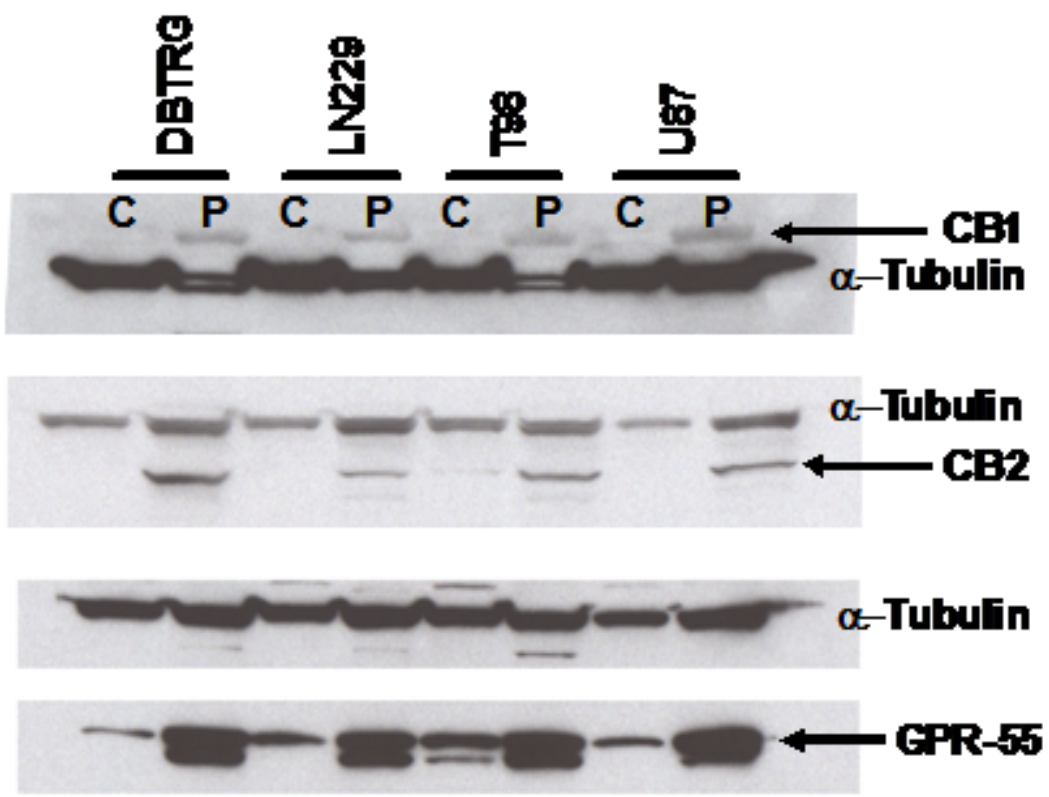

Figure 2.8: Western blots for cannabinoid receptors in human glioma cell lines

Western blots of the particulate (P) and cytosolic (C) fractions of whole cell lysates of U$87 \mathrm{MG}, \mathrm{T} 98 \mathrm{G}, \mathrm{LN}-229$, and DBTRG-O5MG. All blots are indexed to the structural protein $\alpha$-tubulin, cell lysates were divided into 3 aliquots and run on separate gels for each receptor. 
Table 2.1: $\mathrm{EC}_{50}$ values for $\mathrm{KM}-233$ and $\mathrm{BCNU}$ against human $\mathrm{GBM}$ cell lines

\begin{tabular}{|c|c|c|c|}
\hline Cell line & $\begin{array}{c}\text { KM-233 } 18 \text { hrs } \\
\text { EC }_{50}(\mu M) \pm \\
\text { S.D. }\end{array}$ & $\begin{array}{l}\text { BCNU } 18 \text { hrs } \\
\text { EC }_{50}(\mu M) \pm \\
\text { S.D. }\end{array}$ & $\begin{array}{c}\text { BCNU } 48 \text { hrs } \\
\text { EC }_{50}(\mu M) \pm \\
\text { S.D. }\end{array}$ \\
\hline U-87 MG & $3.08 \pm 0.30$ & $233 \pm 5.1$ & $156.0 \pm 2.4$ \\
\hline T98G & $4.68 \pm 0.45$ & $>1000$ & $305 \pm 1.2$ \\
\hline LN-229 & $2.26 \pm 0.32$ & $178 \pm 2.3$ & $119 \pm 4.2$ \\
\hline $\begin{array}{c}\text { DBTRG- } \\
\text { O5MG }\end{array}$ & $2.27 \pm 0.19$ & $>1000$ & $124 \pm 3.0$ \\
\hline MT310 & $4.92 \pm 1.08$ & n.d. & n.d. \\
\hline
\end{tabular}

Note: The $\mathrm{EC}_{50}$ values were obtained from three independent experiments run in triplicate. Abreviations: n.d. = not determined. 
(data not shown). Temozolomide is $\mathrm{pH}$ activated in vivo and the concentration of the active compound was not assessed and therefore these results are viewed with caution.

To further evaluate the KM-233 scaffold as an anti-GBM agent we screened a series of substituted aryl analogs (Figure 2.9) that displayed a broad range of binding affinities for the $\mathrm{CB} 1$ and $\mathrm{CB} 2$ receptors. ${ }^{111}$ The $\mathrm{CB} 1$ binding affinities ranged from 1.08 $\mathrm{nM}$ to $142.2 \mathrm{nM}$ while the $\mathrm{CB} 2$ affinities ranged from $0.27 \mathrm{nM}$ to $134.5 \mathrm{nM}$. The $\mathrm{CB} 1 / \mathrm{CB} 2$ affinity ratios ranged from 0.23 to 13.5 yielding compounds with modest selectivity for the $\mathrm{CB} 1$ or $\mathrm{CB} 2$ receptor. To assess if receptor subtype selectively affected cytotoxicity in the GBM cell lines, the $\mathrm{EC}_{50}$ s for the 4-ethylphenyl 2, 4propylphenyl 3, 4-fluorophenyl 4, 4-chlorophenyl 5, 2-thiophenyl 6, and KM-233 were determined (Figure 2.10). This study indicates that receptor subtype selectivity does not affect cytotoxicity; however, due to the modest selectivity range it is not possible to identify a specific target, i.e. CB1, CB2, or GPR55 receptor, for KM-233 or the analogs.

\subsection{Receptors Involved in the Anti-Glioma Activity of KM-233}

To study the contribution(s) of the CB1, CB2, and/or GPR55 receptors to the cytotoxic effect of KM-233 we utilized pharmacological probes. The CB1 and CB2 receptors couple through $\mathrm{G}_{\alpha \mathrm{i}}$ whereas GPR55 couples via $\mathrm{G}_{\alpha 12 / 13}$. To assess the possible contribution of $\mathrm{CB} 1$ and/or $\mathrm{CB} 2$ to the mechanism of action of KM-233, cytotoxicity assays were carried out using U87 cells pre-treated 18 hours before the assay with 100, 200 , and $300 \mathrm{ng} / \mathrm{ml}$ of pertussis toxin (Ptx). The Ptx inactivates $\mathrm{G}_{\alpha \mathrm{i}}$ via catalytic ADPribosylation thus blocking G-protein-receptor interactions, whereas the $G_{\alpha 12 / 13}$ proteins are Ptx-resistant. Increasing concentrations of Ptx caused a 0.75 fold average decrease in the $\mathrm{EC}_{50}$ of KM-233 against human U87 cells (Figure 2.11). We have determined that $\mathrm{KM}-233$ is an agonist of the $\mathrm{CB} 1$ and an antagonist of the $\mathrm{CB} 2$ receptor (Figure 2.12), both of which signal through $\mathrm{G}_{\mathrm{\alpha i}}$. If KM-233 acted only through CB1 or CB2 it would, therefore, be predicted that Ptx would block the affects of KM-233 thus the results indicate that $\mathrm{CB} 1$ and $\mathrm{CB} 2$ do not initiate KM-233 anti-cancer activity.

The compound SR141716A is reported to be an antagonist of GPR55 that has antagonist/inverse agonist activity (depending on the type of assay) at the CB1 receptor. ${ }^{11,112}$ The dose response curve of KM-233 in the presence of $10 \mu \mathrm{M}$ SR141716A (Figure 2.11) shifted the curve to the right increasing the $\mathrm{EC}_{50} 7$ fold. The shift in the dose response curve suggests that GPR55 is a target of KM-233; however, additional pharmacological probes need to be employeed such as cholera toxin (Ctx, constitutively activates Gs), inhibition of the $\mathrm{G}_{\alpha 12 / 13}$-RhoA-ROCK pathway (possible GPR55 mediated pathway) with Y-27632, or decoupling $\mathrm{G}_{\alpha \mathrm{i}}$ with Ptx and screening GPR55 selective ligands such as O-1602 or lysophosphatidylinsoitol (LPI). These studies are ongoing but may not conclusively identify the mechanism(s) of KM-233 action. We have also begun to utilize shRNA to knock-down each of these receptors to compliment the pharmacological studies. Additionally, we are in the process of developing functional assays for the GPR55 receptor to aid in studying the pharmacology of KM-233 and related analogs. 


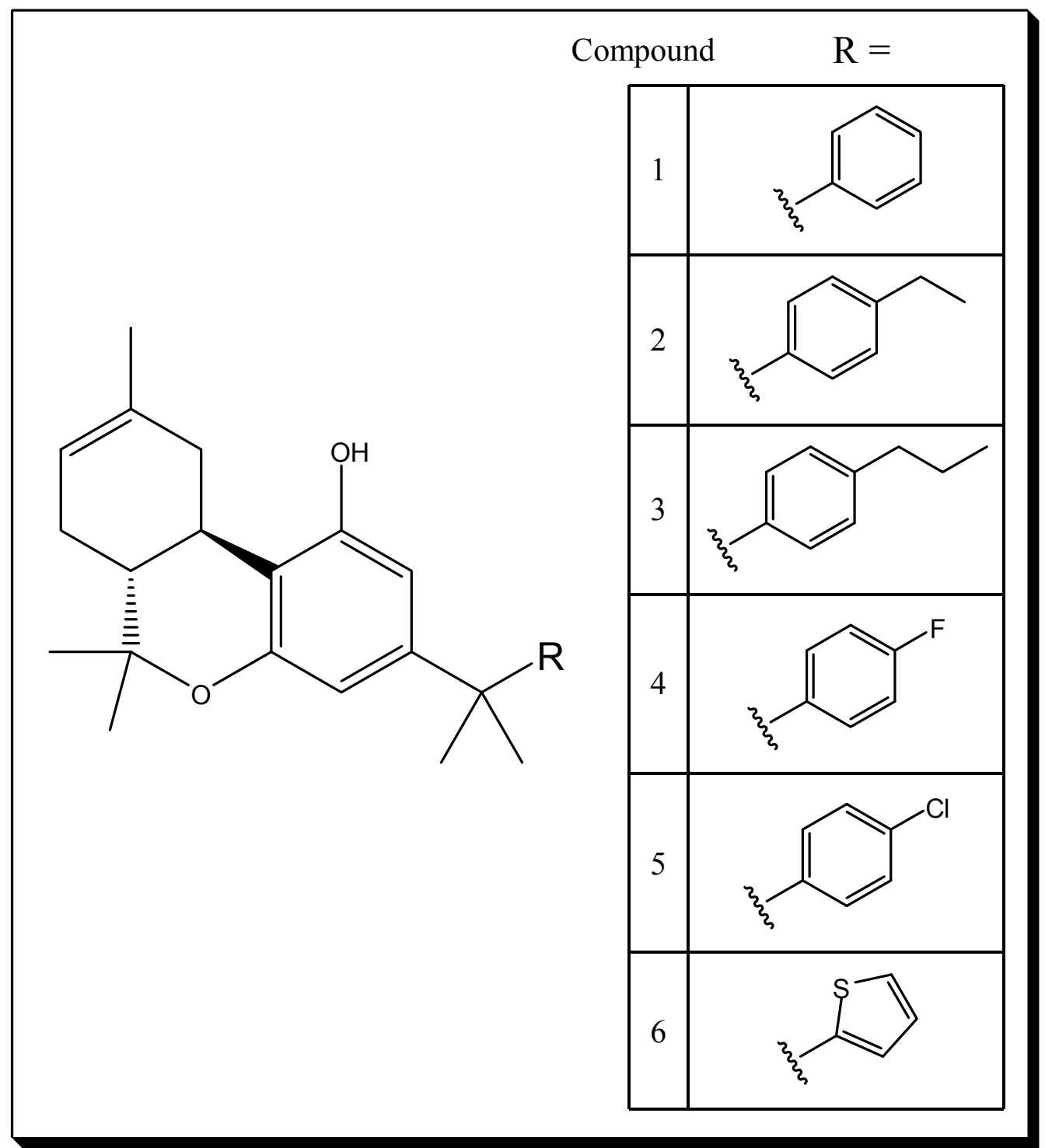

Figure 2.9: $C 1$-dimethyl-aryl $\Delta^{8}$-tetrahydrocannabinol analogs screened against human glioblastoma cell lines 


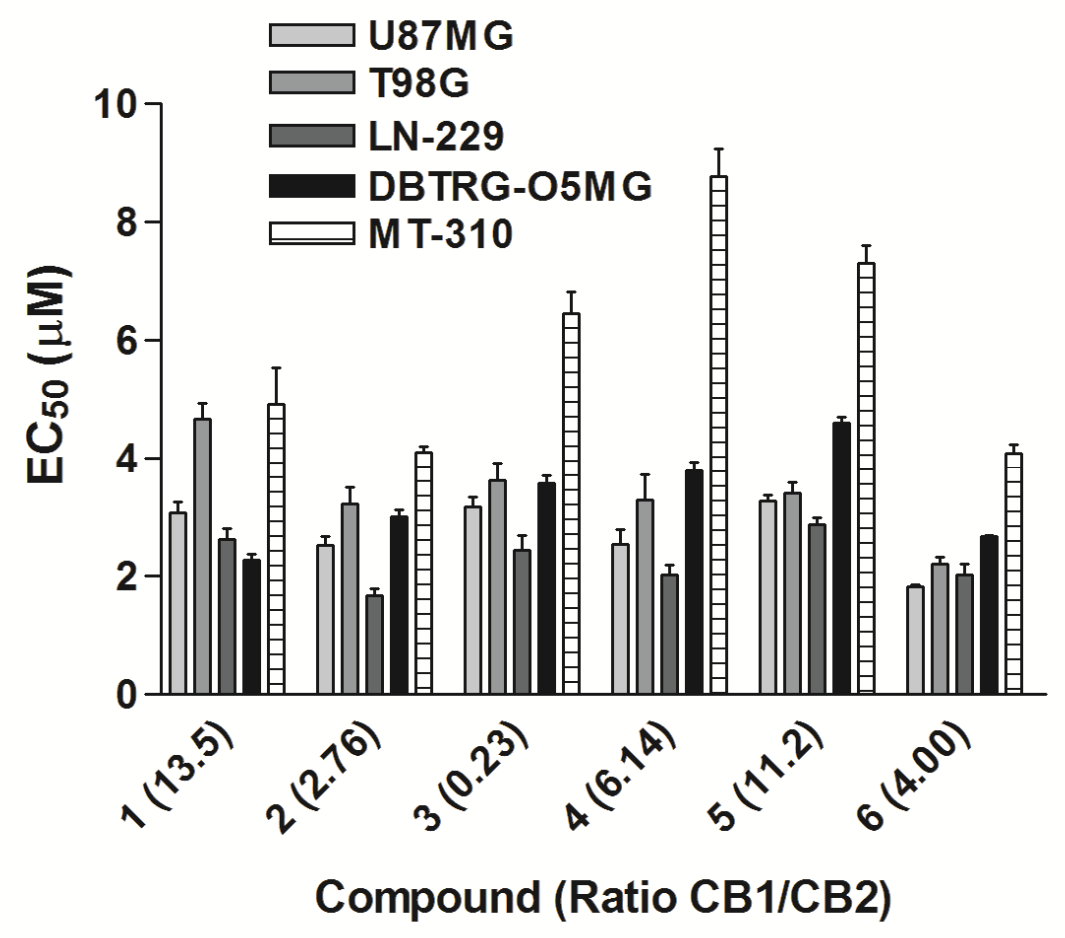

Figure 2.10: $\mathrm{EC}_{50}$ values of $\mathrm{KM}-233$ and analogs

Comparison of the $\mathrm{EC}_{50}$ values of $\mathrm{KM}-233$ and analogs as a function of the $\mathrm{CB} 1 / \mathrm{CB} 2 K_{\mathrm{i}}$ ratio in five human glioma cell lines. 


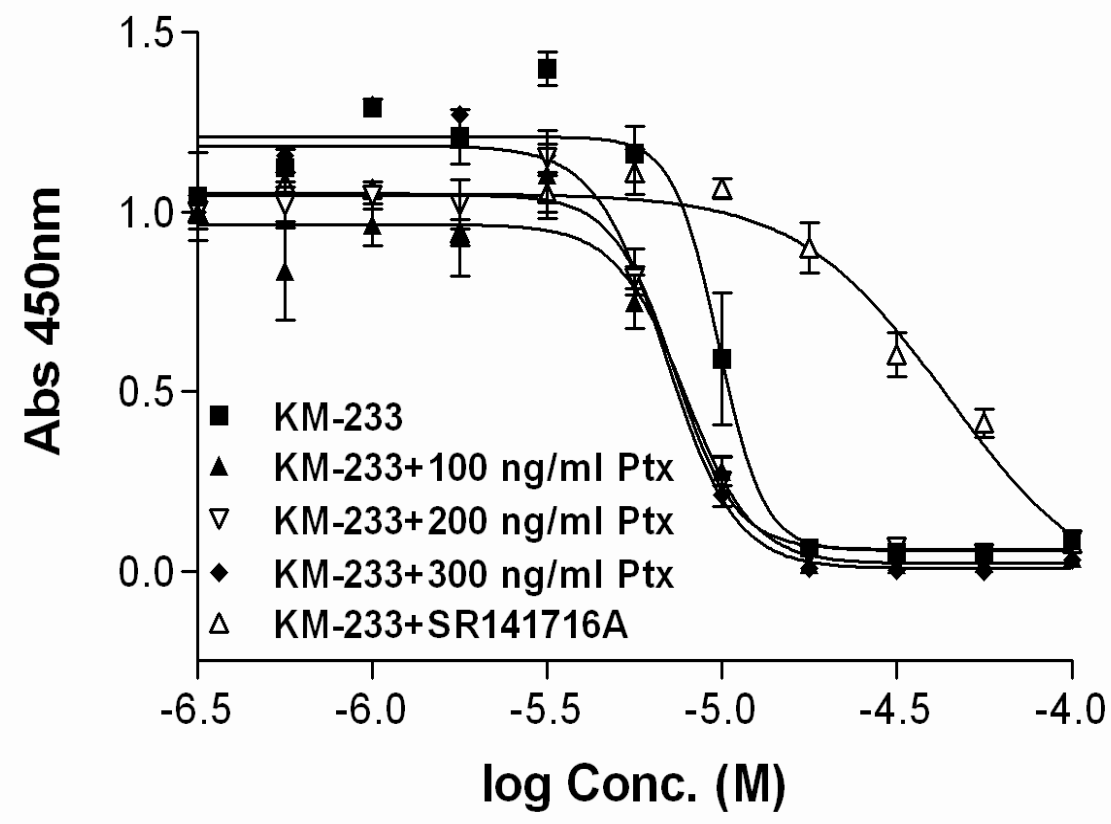

Figure 2.11: Effects of KM-233 on U-87 MG in the presence or absence of Ptx and SR141716A

Dose response curves for U-87 MG cells treated with KM-233 in the presence or absence of 100,200 , or $300 \mathrm{ng} / \mathrm{ml}$ Ptx or $10 \mu \mathrm{M}$ SR141716A. Data is derived from 3 independent experiments conducted in triplicate. 


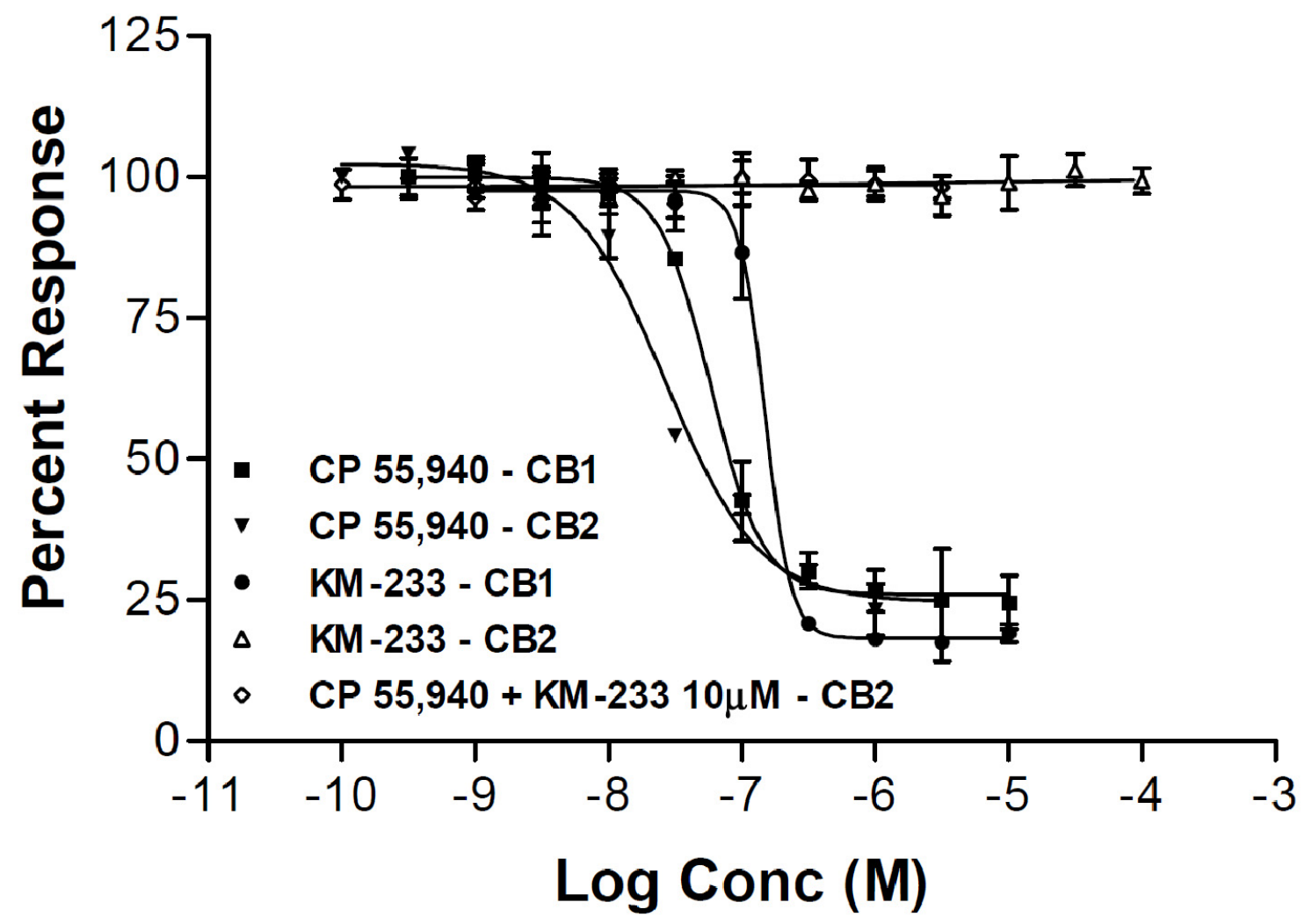

Figure 2.12: Functional activity of KM-233 at the $\mathrm{CB} 1$ and $\mathrm{CB} 2$ receptors KM-233 does not reverse cAMP production in the CB2 assay and abolishes the activity of CP-55,940 identifying KM-233 as an antagonist at CB2. 


\subsection{Mechanistic Studies to Determine the Anti-Glioma Activity of KM-233}

The second component of the mechanism(s) of human GBM cytotoxicity of KM233 is the downstream pathway(s) resulting in cell death. This is where I took over the study of the anti-glioma activity of KM-233. In order to elucidate the mechanism(s) by which KM-233 elicits anti-glioblastoma activity, we employed a number of techniques including epifluorescence microscopy and protein quantification using Meso Scale Discovery (MSD) MULTI-ARRAY® microplate technology. The MSD technology platform consists of 96- and 384-well microplates in which there are 1, 4, 7, or 10 electrodes coated with capture antibodies. Cell lysates are added to the wells of the plate and analytes are captured on the appropriate electrodes. The plates are then incubated with a secondary antibody that is conjugated to ruthenium (II) tris-bipyridine-(4methylsulfonate) NHS ester that luminesces when an electrical current is applied to each electrode within the well. The plates are imaged optically and the relative luminescent intensity of each sample is compared. We first conducted a preliminary screen of the effect(s) of KM-233 on pathways known to be over active in many cancers. Initially we examined total and phosphorylated ERK1/2, JNK, p38, and, Akt using MSD plates. The screen utilized U-87 MG cells treated with $\mathrm{KM}-233$ at the $\mathrm{EC}_{50}(8.7 \mu \mathrm{M})$ to evaluate signal cascade modulation over a 24 hour time course. It should be noted that the change in the $\mathrm{EC}_{50}$ from the previous experiments is due to modifications made in our cytotoxicity protocol which will be discussed in chapter 3 . The preliminary screen showed that ERK1/2 and Akt, but not JNK or p38, are downstream signals in U-87 MG cells following KM-233 exposure (data not shown). Based on these results we analyzed the kinetic profile of phosphorylation of MEK-ERK-STAT pathway using the MSD triplex plate for $\mathrm{pMEK} 1 / 2$, pERK1/2, and pSTAT3. A significant increase in pSTAT3 occurred 1 hour post drug treatment (Figure 2.13) which continued thru 18 hours followed by a rapid decline at 24 hours post drug treatment. The level of pERK1/2 (Figure 2.13) significantly decreased in 1 hour and increased from 12 to 18 hours then declined 24 hours post treatment. The decline in pSTAT3 is of particular interest due to its role in tumorigenesis, i.e. proliferation, angiogenesis, and inhibition of apoptosis, ${ }^{113}$ and its proposed role in immune tolerance in GBM patients, ${ }^{114}$ thus it provides a novel target in cancer therapy. ${ }^{113,115}$ This suggest that CBs may be a novel target for the regulation of STAT3 function via $\mathrm{CB} 1$ or $\mathrm{CB} 2$ regulation of $\mathrm{G}_{\alpha_{0}}{ }^{-\mathrm{c}-\mathrm{Src}^{116}}$ or via a GPR55 regulated $\mathrm{G}_{\alpha 12 / 13}$-RhoA-ERK-STAT3 pathway. ${ }^{117}$ The level of pMEK (Ser 217, 221) showed a significant decline at 6 hours (Figure 2.14), rebounded at 18 hours (though still lower than control), and then significantly decreased at 24 hours. The increases in pMEK, pERK (Thr 202, Tyr 204, Thr 185, Tyr 187), and pSTAT3 (Tyr705) in the 6 to 18 hours time interval may represent a pro-survival response to changes in pAkt and pBAD levels. The temporal increases in pMEK and pERK from 1 to 18 hours are generally consistent with the reported effects of HU-210 against U373MG and Neuro2a cell lines ${ }^{118}$ while the overall decrease is consistent with studies in C6 glioma cell lines. ${ }^{119}$ The activation is reported to originate from $\mathrm{CB} 1$ activation based on the inhibition of cytotoxicity by SR141716A; however, decoupling of the signal cascade by Ptx was not studied with HU-210 thus leaving open the question of GPR55 participation. Regardless of the molecular mechanism, the Ras-Raf-MEK-ERK pathway is a potential target for the development of anti-cancer agents. ${ }^{120}$ 


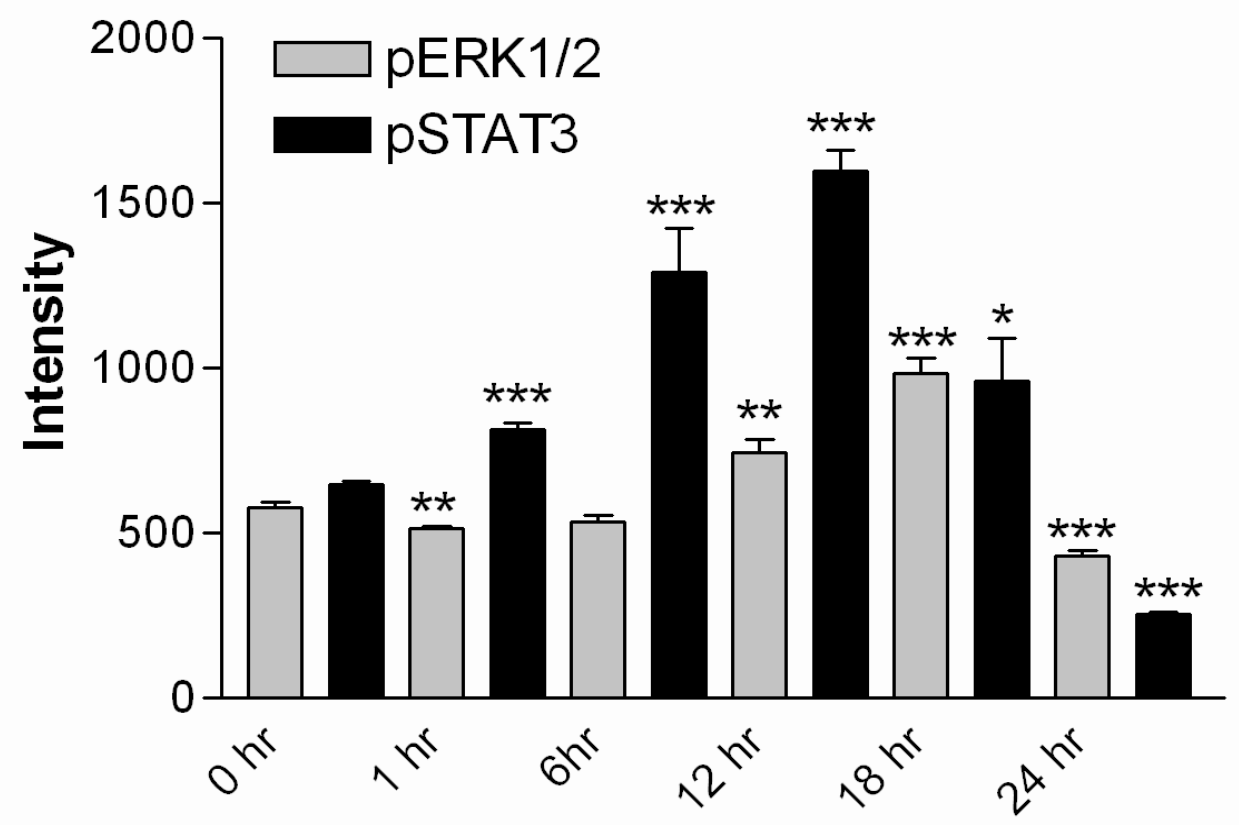

Figure 2.13: pERK and pSTAT3 profiles of U-87 MG cells treated with KM-233

U-87 MG cells were treated with KM-233 at the $\mathrm{EC}_{50}$ for the designated time intervals. Cells were scraped from the culture dishes, collected by centrifugation, lysed, and the protein concentration per lysate was determined. Total protein added to each well of the MSD plate was $20 \mu \mathrm{g}$. Aliquots from this experiment were used in all the following assays. All data points represent 3 independent determinations analyzed in duplicate. * = $\mathrm{p}<0.02, * *=\mathrm{p}<0.01$, and $* * *=\mathrm{p}<0.001$. 


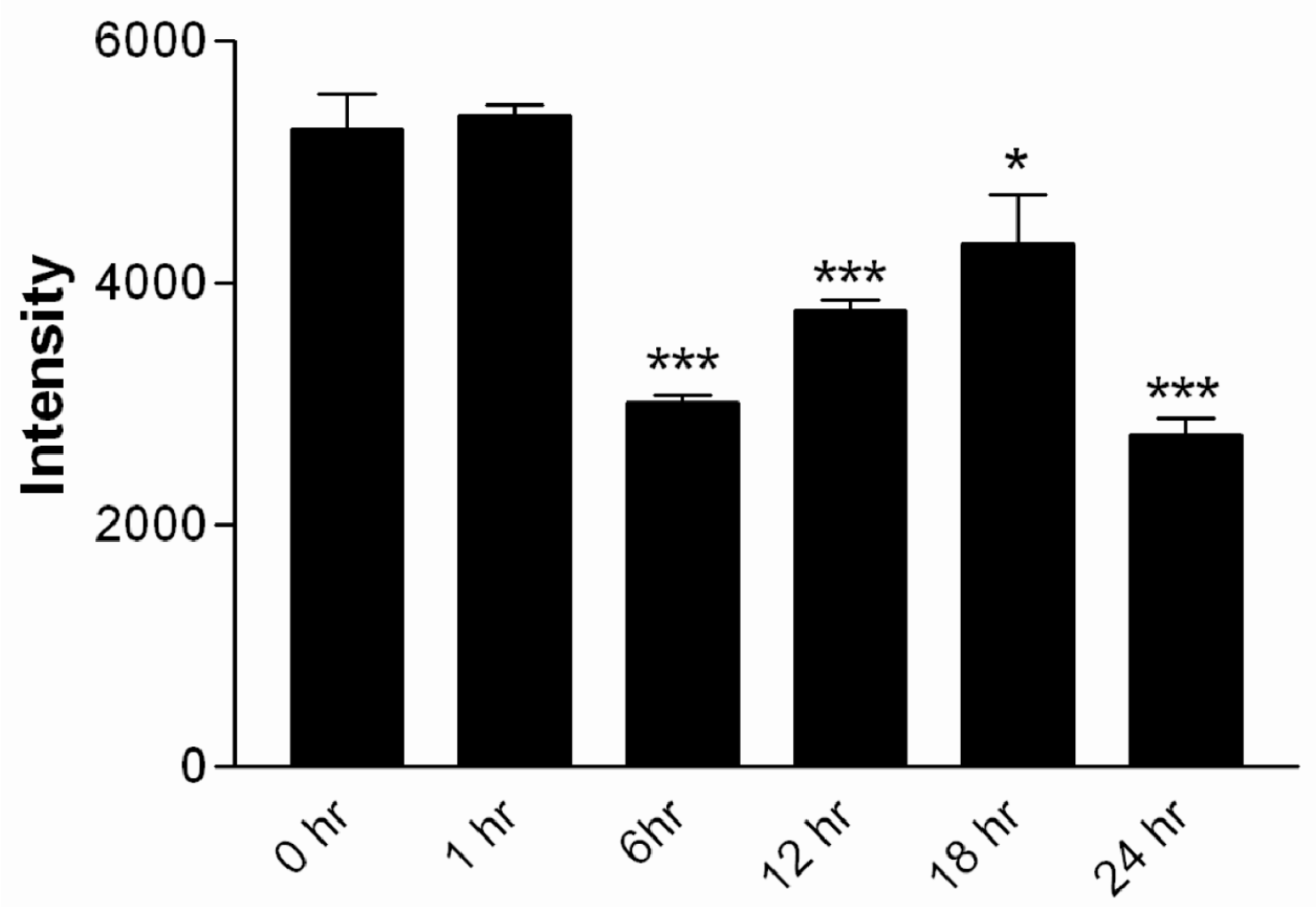

Figure 2.14: pMEK profile of U-87 MG cells treated with KM-233

U-87 MG cells were treated with KM-233 at the $\mathrm{EC}_{50}$ for the designated time intervals and treated as defined in Figure 2.13. All data points represent 3 independent

determinations analyzed in duplicate. $*=\mathrm{p}<0.02, * *=\mathrm{p}<0.01$, and $* * *=\mathrm{p}<0.001$. 
Interplay between the ERK1/2 and Akt pathways in C6 glioma cells was previously reported by Ellert-Miklaszewska et al. ${ }^{119}$ To assess the contribution of Akt to the signal cascade associated with KM-233 we utilized the pAkt-pGSK-3 $\beta$-pP70S6K MSD assay plate. Treatment of U87 cells with KM-233 triggers a significant and rapid decrease in pAkt beginning at 6 hours and continuing through 24 hours (Figure 2.15). Within this time interval a significant increase in glycogen synthase kinase $3 \beta$ (pGSK$3 \beta$, Ser9) and a decrease in 70-kDa ribosomal protein S6 kinase (pP70S6K, Ser 217/221) were manifested (Figure 2.16). The reduction in pAkt and pP70S6K are consistent with the recent report by Salazar et al. wherein U-87 MG cells were treated with THC. ${ }^{121}$ The inhibition of Akt in cancer therapy is well established; however, it has recently been shown that knock-down of p70S6K with siRNA inhibited U-251MG glioma cells by greater than 50 percent. The observed increase in pGSK-3 $\beta$ is consistent with the reported increase in apoptosis observed with GSK-3 $\beta$ activation ${ }^{122}$ although Kotliarova et al. reports that inhibition of GSK-3 $\beta$ induces cell death. ${ }^{123}$ In GBM cell lines it is generally reported that regulation of Akt pathways, as well as ERK signaling, is mediated through a $\mathrm{G}_{\alpha \mathrm{\alpha} / \mathrm{o}}$ and/or $\mathrm{G}_{\beta / \gamma}$ pathways; however, it is possible that a $\mathrm{G}_{\alpha 12 / 13}$-RhoA pathway, and by extension GPR55, may regulate the downstream activation/inactivation of the Akt and/or the ERK pathways. For example, $\mathrm{G}_{\alpha 12 / 13}$ activation of the PI3K-Akt axis has been proposed in neuroblastoma cells ${ }^{124}$ and a pathway involving Rho-ROCK signaling and ERK activation has been proposed in the human GBM line LN-19. ${ }^{125}$

The down-regulation of the pro-survival kinase cascades ERK1/2 and Akt raised the possibility that one downstream mediator may be the Bcl-2 family protein BAD. Specifically, phosphorylation of BAD by Akt and ERK has been previously reported; ${ }^{126}$ therefore, KM-233 may suppress phosphorylation of BAD resulting in heterodimerization with Bcl-2 death suppressors. To evaluate the effect of KM-233 on BAD phosphorylation status we utilized the MSD pBAD (Ser 112) plate. Treatment of U-87 MG cells with KM-233 caused a significant decrease in total pBAD (Figure 2.17), therefore it was expected that the decrease in pBAD would correlate with mitochondrial dysfunction. One manifestation would be cytochrome $C$ release and activation of caspase 3. To evaluate this component we employed the MSD apoptosis plate to screen cleaved caspase 3, cleaved PARP, and total- and phospho-p53. A significant increase in cleaved caspase 3 began at 12 hours with a rapid elevation at 18 hours post drug treatment (Figure 2.18). There were no changes in the levels of cleaved PARP or total/phosphop53 (data not shown).

The possible contribution of mitochondrial dysfunction to the mechanism of action of KM-233 was further evaluated using DePsipher ${ }^{\circledR}$ (Trevigen; Gaithersburg, MD) to evaluate the collapse of the mitochondrial membrane potential (Figure 2.19). Treatment of U87 cells with $3 \mu \mathrm{M}$ KM-233 resulted in a significant increase in mitochondrial membrane depolarization, as indicated by the inability of the fluorescent stain, DePsipher to form red fluorescent eximers inside mitochondria with an almost complete depolarization a 6 hours. Mitochondrial involvement was further verified by the observation of cytochrome $\mathrm{C}$ release in $\mathrm{U}-87 \mathrm{MG}$ after 18 hours of exposure to KM233 at the $\mathrm{EC}_{50}$ (Figure 2.20). 


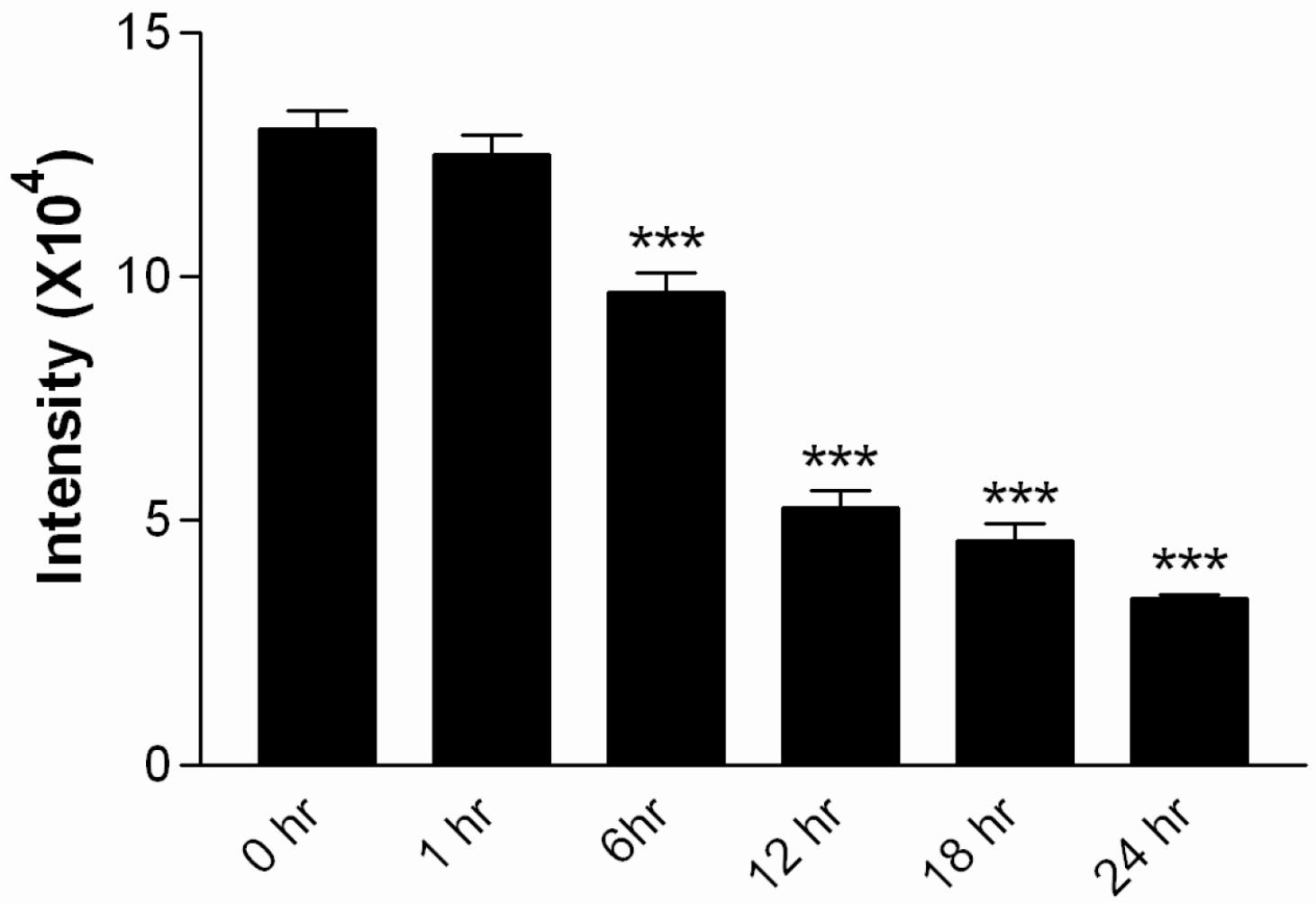

Figure 2.15: pAKT profile of U-87 MG cells treated with KM-233

U-87 MG cells were treated with KM-233 at the $\mathrm{EC}_{50}$ for the designated time intervals and treated as defined in Figure 2.13. All data points represent 3 independent determinations analyzed in duplicate. $* * *=p<0.001$. 


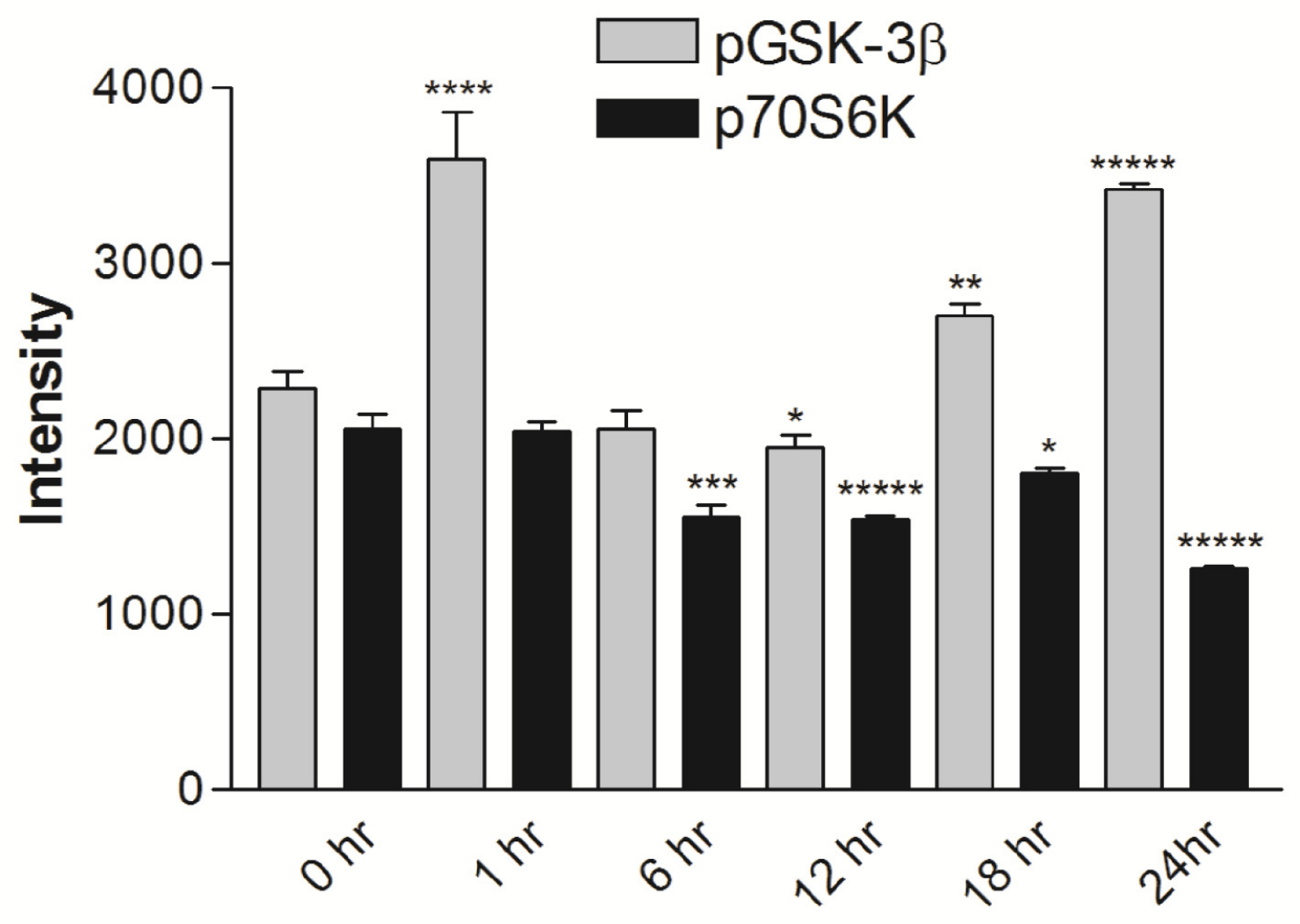

Figure 2.16: $p G S K-3 \beta$ and pP70S6K profiles of U-87 MG cells treated with KM-233

U-87 MG cells were treated with KM-233 at the $\mathrm{EC}_{50}$ for the designated time intervals and treated as defined in Figure 2.13. All data points represent 3 independent

determinations analyzed in duplicate. $*=\mathrm{p}<0.01, * *=\mathrm{p}<0.005, * * *=\mathrm{p}<0.001, * * * *$ $=\mathrm{p}<0.0005$, and $* * * * *=\mathrm{p}<0.00001$. 


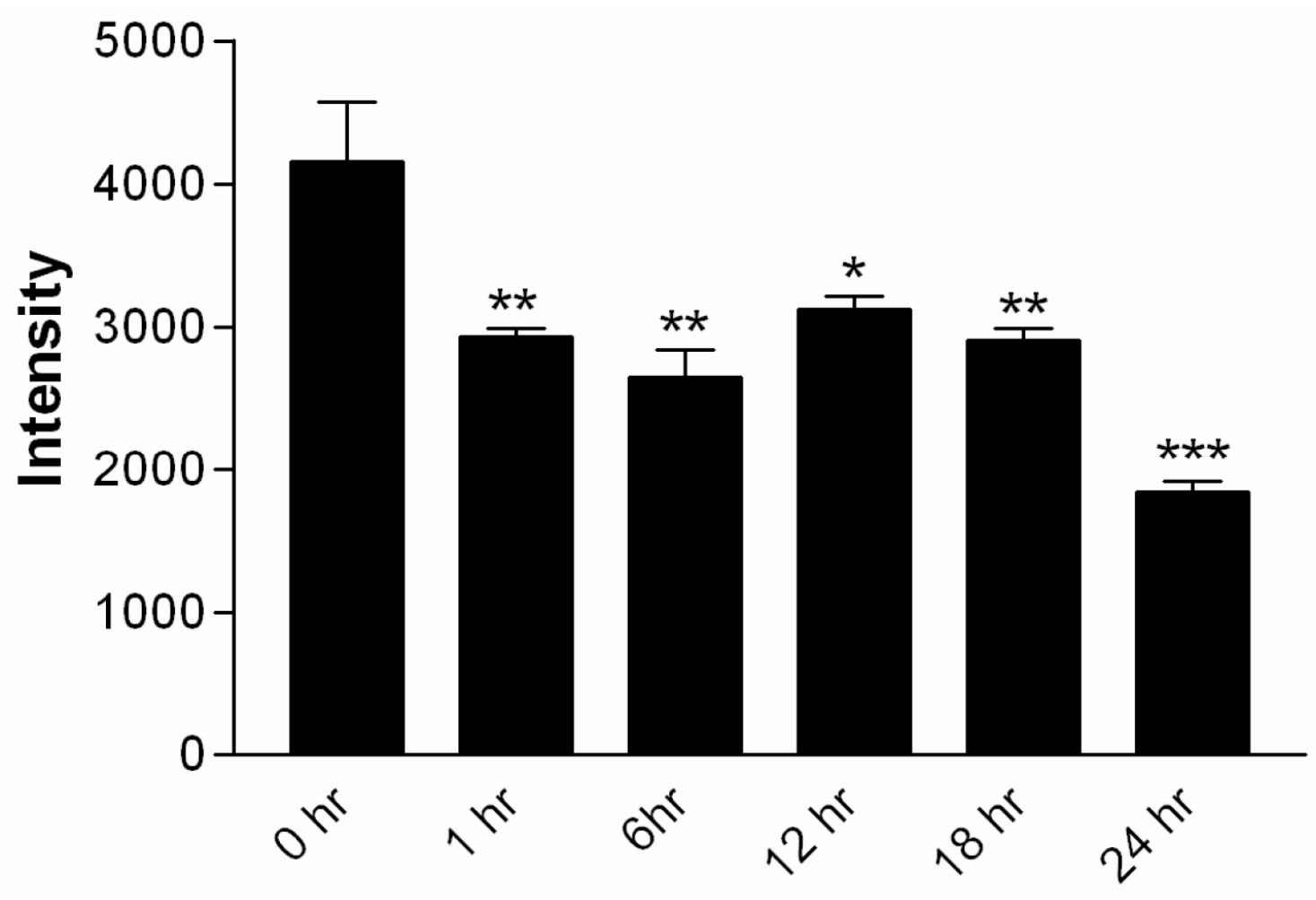

Figure 2.17: pBAD profile of $U-87$ MG cells treated with KM-233

U-87 MG cells were treated with KM-233 at the $\mathrm{EC}_{50}$ for the designated time intervals and treated as defined in Figure 2.13. All data points represent 3 independent

determinations analyzed in duplicate. $*=p<0.05, * *=p<0.01$, and ${ }^{* * *}=\mathrm{p}<0.001$. 


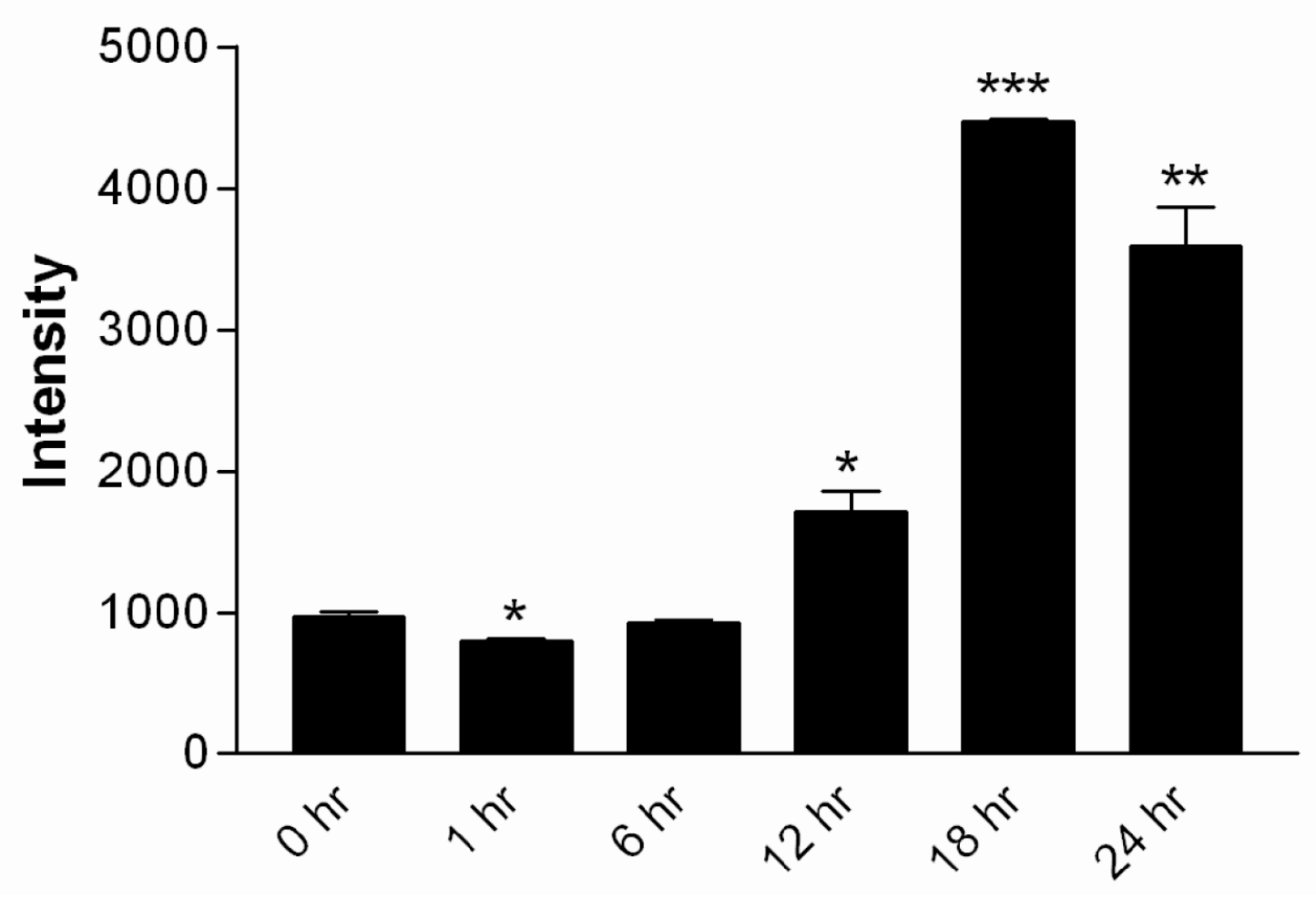

Figure 2.18: Cleaved caspase 3 profile of U-87 MG cells treated with KM-233

U-87 MG cells were treated with KM-233 at the $\mathrm{EC}_{50}$ for the designated time intervals and treated as defined in Figure 2.13. All data points represent 3 independent

determinations analyzed in duplicate. $*=p<0.05, * *=p<0.005$, and $* * *=p<0.001$. 

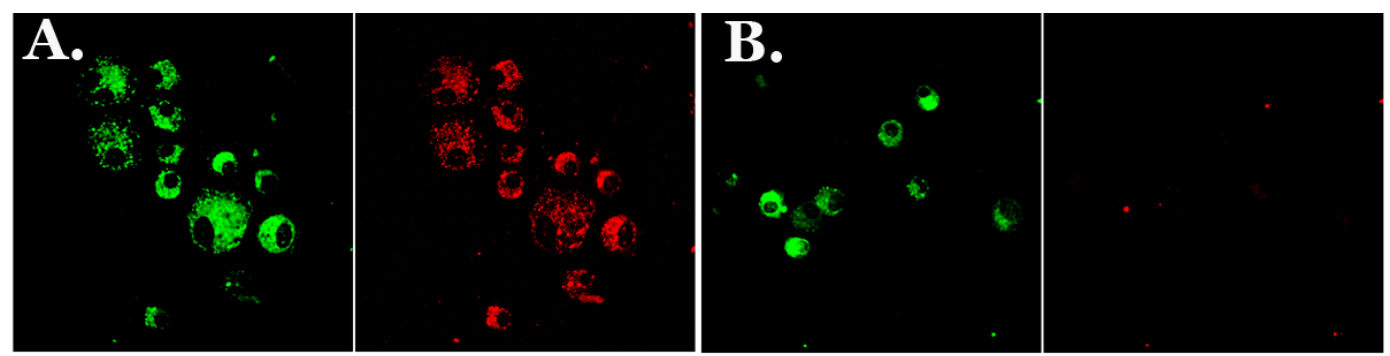

Figure 2.19: Effects on membrane polarization in U-87 MG cells treated with KM-233

Change in mitochondrial membrane polarization $(\Delta \psi m)$ with DePsipher ${ }^{\circledR}$ - U-87 MG cells were treated with $\mathrm{KM}-233$ at the $\mathrm{EC}_{50}$ for 6 hours then stained with dual fluorescent stain; Green fluorescence shifts to red emission following the formation of eximers within mitochondria as stain crosses polarized outer mitochondrial membrane. Mitochondrial depolarization by KM-233 inhibits DePsipher ${ }^{\circledR}$ permeability and subsequent red fluorescence. Images of U-87 MG cells were collected using $568 \mathrm{~nm}$ (Red) and 458 (Green) lines of a CLSM. Control (vehicle treated) cells are shown in the A panels and 6 hours post drug treatment is shown in the B panels. 


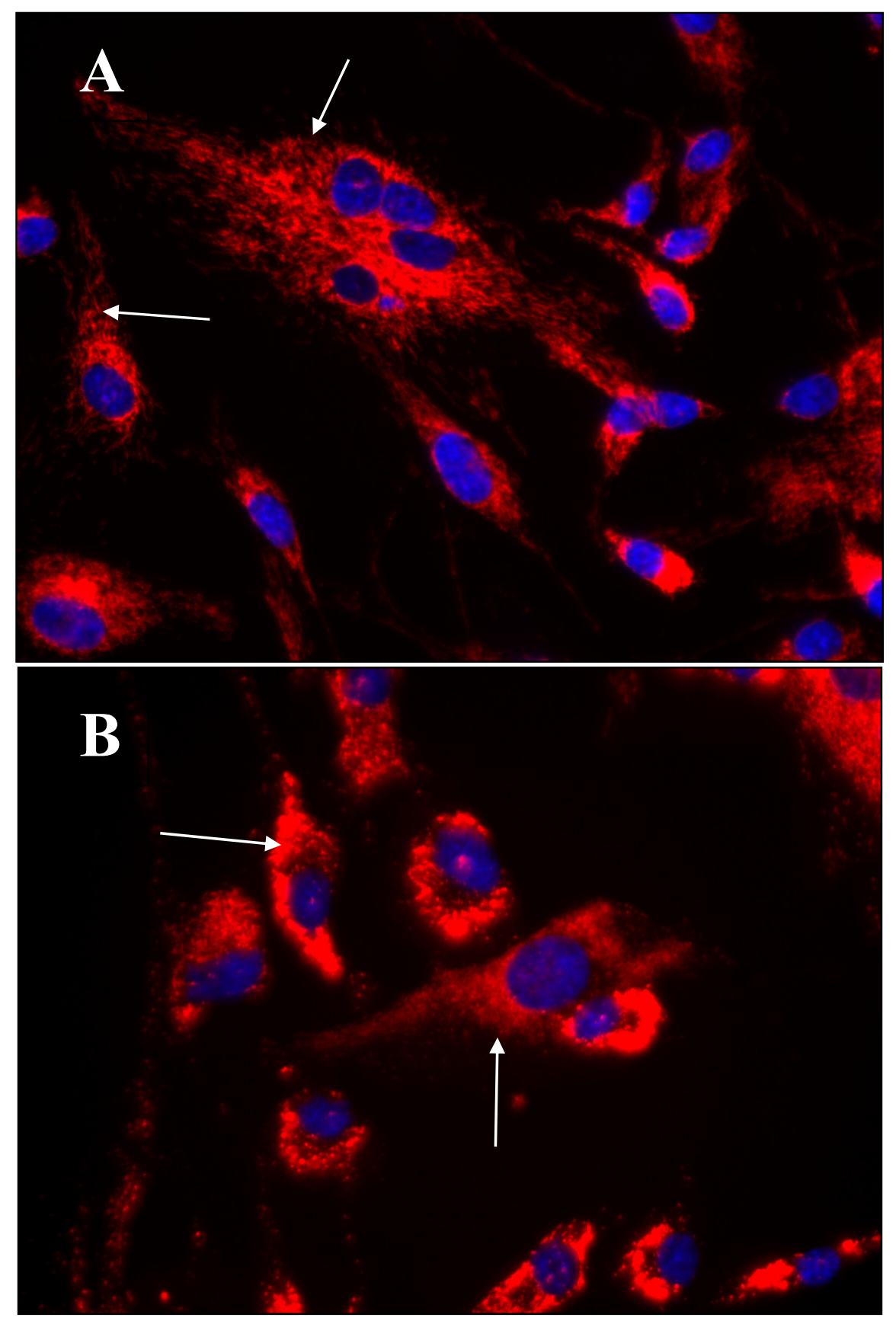

Figure 2.20: Effects on cytochrome C release in U-87 MG cells treated with KM-233

U-87 MG cells were treated with $\mathrm{KM}-233$ at the $\mathrm{EC}_{50}$ for 18 hours and evaluated for changes in localization of cytochrome $\mathrm{C}$ (red); nuclei are shown in blue. Control (vehicle treated) cells are shown in panel A and 18 hour drug treated cells are shown in panel B. Control cells exhibit localization of cytochrome $\mathrm{C}$ in the mitochondria (arrows) along the cytoskeletal network. KM-233 treated cells show a diffuse pattern of labeling (arrows) indicating that cytochrome $\mathrm{C}$ has been released from the mitochondria into the cytosol. 
We also conducted a microscopic study to assess the effects of KM-233 on cytoskeleton structure based on the known activity of RhoA and the possibility of a GPR55 initiated mechanism. At 18 hours post KM-233 treatment the cytoskeleton manifests profound contraction of F-actin and $\alpha$-tubulin (Figure 2.21). Though it is not possible to conclude a specific mechanism, the results provide a basis for further evaluating RhoA-ROCK contributions to cell death.

While many of the downstream signaling pathways have been identified, the receptor or receptors responsible for initiating the cascades are yet to be elucidated. We have utilized pharmacological knockouts in an effort to identify the target of KM-233 action. Pharmacological intervention has distinct limitations due to overlap in activity of known agonists and antagonists of the CB1, CB2, and GPR55 receptors. Our preliminary data suggest that GPR55 is the target of KM-233 however this cannot be confirmed without the selective knock-down of each CB receptor subtype. Knowledge of the target is critical for the development of a complete mechanism of action for the antiglioblastoma activity of KM-233. The contributions of each cannabinoid receptor are currently under investigation using shRNA stably transfected cell lines.

\subsection{Experimental Methods}

\subsubsection{Cytotoxicity Assays}

Human glioblastoma cells U-87MG, T98G, LN-229, DBTRG-O5MG (American Type Culture Collection), and MT310 (primary GBM cell line, grade IV, a gift from Dr. Valery Kukekov, University of Tennessee Health Science Center, Department of Neurosurgery) were cultured in supplemented media according to the recommendations of the supplier at $37^{\circ} \mathrm{C}$ in an atmosphere of $5 \% \mathrm{CO}_{2}$ and $95 \%$ air. Cell lines were plated in 96 -well polystyrene flat-bottom plates $(7,500$ cells/well) at $70 \%$ confluency in a $100 \mu 1$ total volume of supplemented media as indicated, and incubated overnight at $37^{\circ} \mathrm{C}$ to allow for adherence. The media was then replaced with media containing $1 \%$ FBS and the cultures were inoculated with escalating amounts of drug; cell death was analyzed at 18h using the BioTek Synergy 2 Multidetection Microplate Reader (BioTek Instruments, Inc.). The percentage of viable cells present in the culture at each time point was calculated by comparing the absorbance value at $450 \mathrm{~nm}$ from the CCK-8 assay (Dojindo Molecular Technologies) for each condition with untreated control cells. All assays were conducted per manufacturer's protocol. All described values represent the average of three data points per determination and three independent determinations.

\subsubsection{Cytotoxicity Assays with Pertussis Toxin}

U-87 MG cells were seeded in 96 -well polystyrene flat-bottom plates $(7,500$ cells/well) at $70 \%$ confluency in a $100 \mu \mathrm{l}$ total volume of complete growth media and incubated overnight at $37^{\circ} \mathrm{C}$ in an atmosphere of $5 \% \mathrm{CO}_{2}$ and $95 \%$ air to allow for 

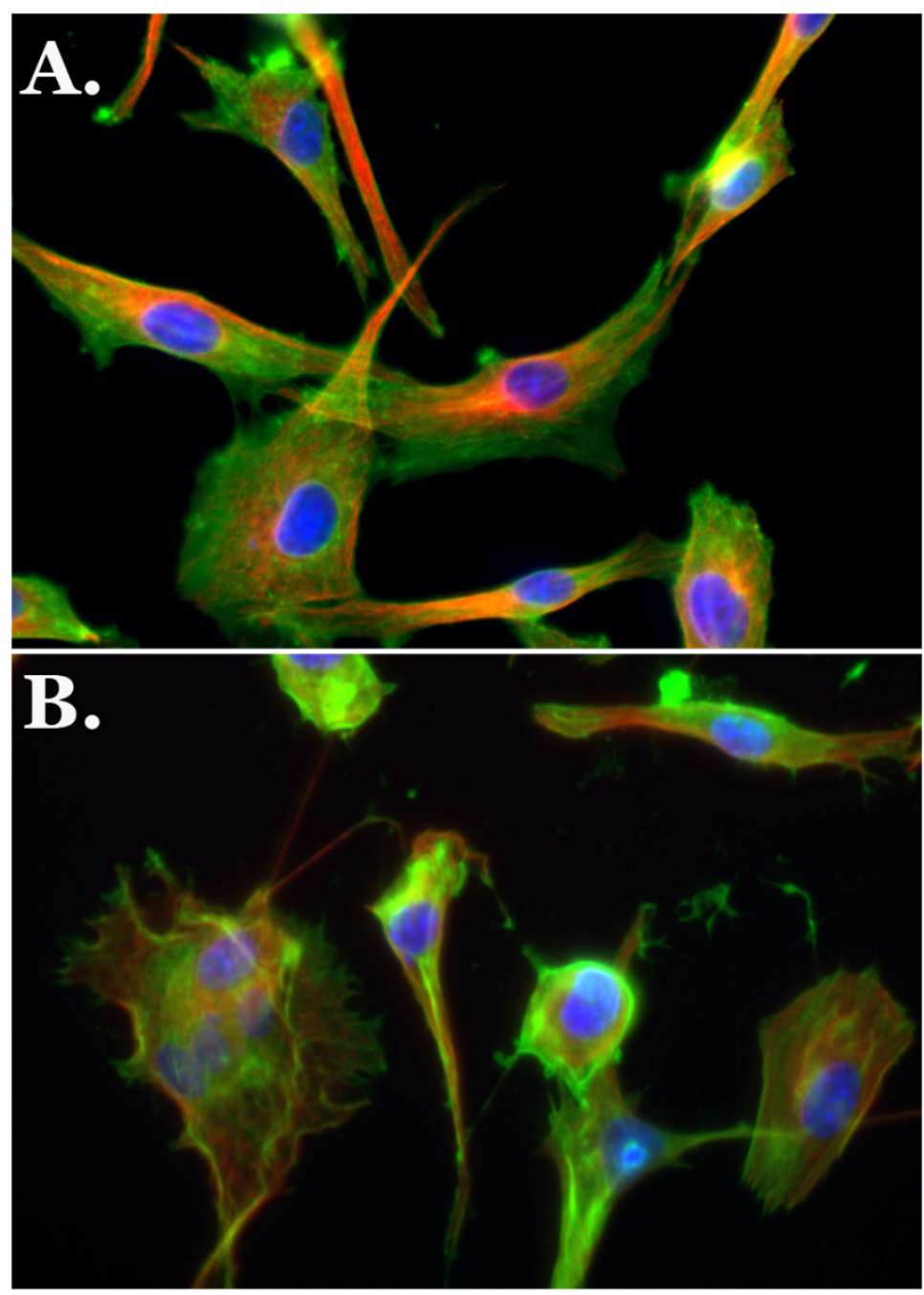

Figure 2.21: Effects on cytoskeleton structure in U-87 MG cells treated with KM-233 U-87 MG cells were treated with KM-233 at the $\mathrm{EC}_{50}$ and evaluated for changes in Factin (green) and $\alpha$-tubulin (red), nuclei are shown in blue. Control (vehicle treated) cells are shown in panel A and 18 hour drug treated cells are shown in panel B. KM-233 causes significant contraction of the cytoskeleton network as evidenced by the compact F-actin around the nucleus. 
adherence. The media was then replaced with complete growth media containing 0,100 , 200 , or $300 \mathrm{ng} / \mathrm{ml}$ pertussis toxin and the plates returned to the incubator for 18 hours. Drug solutions were prepared in DMSO at 100X concentration, and mixed 1:100 in media containing 1\% FBS to yield the desired concentration. After 18 hour incubation with or without Ptx, full serum media was removed and replaced with drug-media mixtures and incubated for an additional 18 hours. $10 \mu 1$ of CCK-8 reagent was added to each well to colorimetrically assess viability. After 2-4 hours of incubation with the CCK-8 dye, absorbance was read at 450nm using a BioTek Synergy 2 plate reader.

\subsubsection{CB1/CB2 Functional Assays}

HEK-293 cell lines stably transfected with a cyclic nucleotide-gated channel and either human CB1 or CB2 receptors (BD Biosciences, San Jose, CA USA) were seeded in poly-D-lysine coated 96-well plates at a density of 70,000 cells per well. Plates were incubated at $37^{\circ} \mathrm{C}$ in $5 \% \mathrm{CO}_{2}$ overnight prior to assay. Plates were then removed from the incubator and the complete growth medium (DMEM, 10\% FBS, 250 $\mu \mathrm{g} / \mathrm{ml} \mathrm{G418}$ and $1 \mu \mathrm{g} / \mathrm{ml}$ puromycin) was replaced with $100 \mu \mathrm{D}$ DEM containing $0.25 \% \mathrm{BSA}$. Next, $100 \mu 1$ membrane potential dye loading buffer (Molecular Devices) was added to each well. The plates were placed back into the incubator for 30 minutes and then the baseline fluorescence was read on a BioTek Synergy 2 multi-mode microplate reader with 540nm excitation and 590nm emission filters prior to drug addition. Drugs were added in $50 \mu 1$ DPBS containing 2.5\% DMSO, 1.25 $\mathrm{M}$ 5'-(N-ethylcarboxamido) adenosine and $125 \mu \mathrm{M}$ Ro 20-1724. Plates were then incubated at room temperature for 25 minutes and fluorescence measured again at 540nm excitation and 590nm emission.

\subsubsection{MSD Protein Quantification}

U-87 MG cells were plated in poly-D-lysine coated $10 \mathrm{~cm}$ Petri dishes at a density of $4 \times 10^{5}$ cells $/ \mathrm{ml}$ with $12 \mathrm{ml}$ of cell suspension added to each plate. The cells were grown overnight in EMEM media containing 10\% FBS. The following day the media was changed to EMEM media containing 1\% FBS and allowed to equilibrate for 24 hours. This step is essential to allow basal levels of signaling protein to re-equilibrate due to changes in the serum concentration. The media was then replaced at the appropriate time points with media containing $1 \%$ serum and $\mathrm{KM}-233$ at the $\mathrm{EC}_{50}$ or media containing 1\% serum and vehicle (DMSO). After the incubation period with KM233 or vehicle, the media was removed from the dishes and transferred to a $15 \mathrm{ml}$ centrifuge tube to collect any cells that were in suspension. Cells were then scraped from the surface of the dish and transferred into the same $15 \mathrm{ml}$ centrifuge tubes and pelleted at $1000 \mathrm{rpm}, 4^{\circ} \mathrm{C}$ for $5 \mathrm{~min}$. Supernatant was removed and $0.5 \mathrm{ml}$ lysis buffer was added to each pellet. The cells were suspended in the buffer and transferred to $1.5 \mathrm{ml}$ microcentrifuge tubes. These were incubated on ice for 30 minutes, being vortexed every 5 minutes. The lysates were then centrifuged at $10,000 \times \mathrm{g}, 4^{\circ} \mathrm{C}$ for 10 minutes to clear cellular debris from the lysate. The supernatant was removed and assayed for protein concentration using a Pierce BCA Protein Assay Kit (Thermo Scientific). The lysates 
were then diluted with complete lysis buffer to a concentration of $20 \mu \mathrm{g}$ of protein in $25 \mu \mathrm{l}$ volume. Signaling proteins were then analyzed using MSD phosphoprotein and intracellular marker multiplex kits which were imaged on a SECTOR Imager 2400 (Meso Scale Discovery) according to the manufacturer's instructions.

\subsubsection{Mitochondrial Membrane Potential Assay}

U-87 MG cells were grown in $75 \mathrm{~cm}^{2}$ flasks in complete growth medium until $80 \%$ confluent. At the appropriate time points, the media was removed and replaced with media containing $1 \% \mathrm{FBS}$ and either $\mathrm{KM}-233$ at the $\mathrm{EC}_{50}$ or vehicle (DMSO). After incubation with drug or vehicle, cells were harvested by trypsonization and pelleted by centrifugation. The cells were then dyed in suspension using DePsipher membrane potential dye according to the manufacturer's instructions. The cells were then placed on glass slides and covered with glass cover slips. The cells were then imaged using an Olympus BX50 microscope (Olympus America) fitted with a Bio-Rad MRC1024 confocal laser scanning system (Bio-Rad Laboratories).

\subsubsection{Microscopic Analysis of Cytoskeleton and Cytochrome C Release}

U-87 MG cells were plated in complete growth medium at a density of 15,000 cells/well in poly-D-lysine coated 96-well polystyrene tissue culture plates and allowed to attach over night at $37^{\circ} \mathrm{C}$ in an atmosphere of $5 \% \mathrm{CO}_{2} / 95 \%$ air. The media was then replaced with media containing $1 \%$ FBS and either $\mathrm{KM}-233$ at the $\mathrm{EC}_{50}$ or vehicle (DMSO). The cells were returned to the incubator and after 18 hours exposure to the drug or vehicle, media was aspirated and the cells fixed using 4\% paraformaldehyde. Cells were then stained by immunohistochemical methods using either a Cellomics Multiparameter Cell Death Detection Kit or a Cellomics Cytoskeletal Rearrangement Kit (Thermo Scientific) according to the manufacturer's instructions. Plates were then imaged using an Olympus IX71 inverted fluorescence microscope (Olympus America) fitted with a Retiga-SRV camera (QImaging).

\subsubsection{Synthesis of Compounds 1 - 6}

All chemicals and reagents were purchased from Sigma-Aldrich or Thermo Fisher Scientific Inc. Anhydrous solvents were prepared by distillation over sodium metal or calcium hydride prior to use. Reactions were carried out under dry conditions under an argon atmosphere. A Biotage SP1 system was used for flash chromatography purification. Routine NMR spectra were obtained on a Varian Inova $500 \mathrm{MHz}$ NMR and were consistent with the assigned structures. Routine mass spectra were determined on a Bruker ESQUIRE Ion Trap LC/MS system. Compounds 1 through $\mathbf{6}$ were prepared according to the methods reported by Krishnamurthy et al. ${ }^{108,111}$ and all spectral analyses of said compounds were in agreement with published data. 


\section{Chapter 3: Anti-Glioma Activity of HB-I-172 and Analogs}

\subsection{Introduction}

We have previously reported the synthesis of a small series of CB2 selective triaryl cannabinoids. ${ }^{127}$ These compounds were designed with the goal of producing cannabinoid ligands that would still elicit the desired physiological effects seen with other cannabinoids (antineoplastic activity, anti-inflammatory activity, etc.) but which would be void of the unwanted CNS side effects mediated by the CB1 receptor. These triaryl compounds were developed by merging structural aspects of two previously reported classes of cannabinoids. The design utilizes the $\mathrm{C} 1$ ' aryl substitution of the classical cannabinoid core previously reported by our laboratory with compounds such as KM-233 and the introduction of an aryl ring in place of the terpene $\mathrm{C}$ ring in a series of biaryl compounds reported by Makriyannis and co-workers as represented by the gemdimethyheptyl side chain analog compound $7^{128}$ (Figure 3.1). The design was developed with the hypothesis that the A ring of compounds 1 and 7 occupy the same region in the $\mathrm{CB} 1$ and $\mathrm{CB} 2$ ligand binding pockets.

In an effort to improve $\mathrm{CB} 2$ receptor affinity, the $\mathrm{C}$ ring of our triaryl cannabinoid core possesses a 3,5-dichloro substitution. This modification was based on the observation of Makriyannis that the 3,5-dichlo substitution pattern improves CB2 receptor affinity in the biaryl cannabinoids. ${ }^{128}$ In addition to the abovementioned structural modifications, three types of side chain linkers: hydroxyl, carbonyl, and gem dimethyl were utilized in the design and four compounds were synthesized and assayed for $\mathrm{CB} 1$ and $\mathrm{CB} 2$ receptor binding affinity (Figure 3.2).

The compounds designed were hypothesized to be selective at the CB2 receptor sub-type. This was based not only on the contribution of the 3,5-dichlorophenyl C ring but also on the fact that the compounds comprising the phenyl series, represented by KM233, were predominantly CB2 selective. We believed that the combined increase in electronic interactions of the substituents at both the $\mathrm{C} 3$ position of the phenolic A ring and the aryl $\mathrm{C}$ ring in the novel core would enhance the affinity and selectivity of these compounds to the cannabinoid receptor sub-type 2 .

The four compounds initially synthesized with the novel triaryl core show significant binding affinity to the CB2 cannabinoid receptor subtype (Table 3.1). The receptor subtype affinity $K_{\mathrm{i}}$ values ranged from 1.07 to $4.77 \mathrm{nM}$ at the CB2 receptor with 9-600 fold selectivity towards the CB2 receptor subtype. Although omission of the B ring from the $\mathrm{C} 10$ phenyl substituted classical core does not seem to affect the binding affinity to the $\mathrm{CB} 2$ cannabinoid receptor, there is a pronounced effect on the affinity for the $\mathrm{CB} 1$ receptor as evidenced by the new analogs 8-11. The extent to which the compounds show selectivity towards the CB2 receptor appears to depend on the substitution pattern on ring $\mathrm{A}$ and the type of side chain linker at the $\mathrm{C} 10$ position. Our binding affinity data show that compounds with a hydroxyl $\mathbf{8}$ and carbonyl linker (compounds 9 and 10) retain affinity for the CB2 receptor when compared to the gem dimethyl analog 11. The presence of the gem dimethyl group as a linker in compound 11 


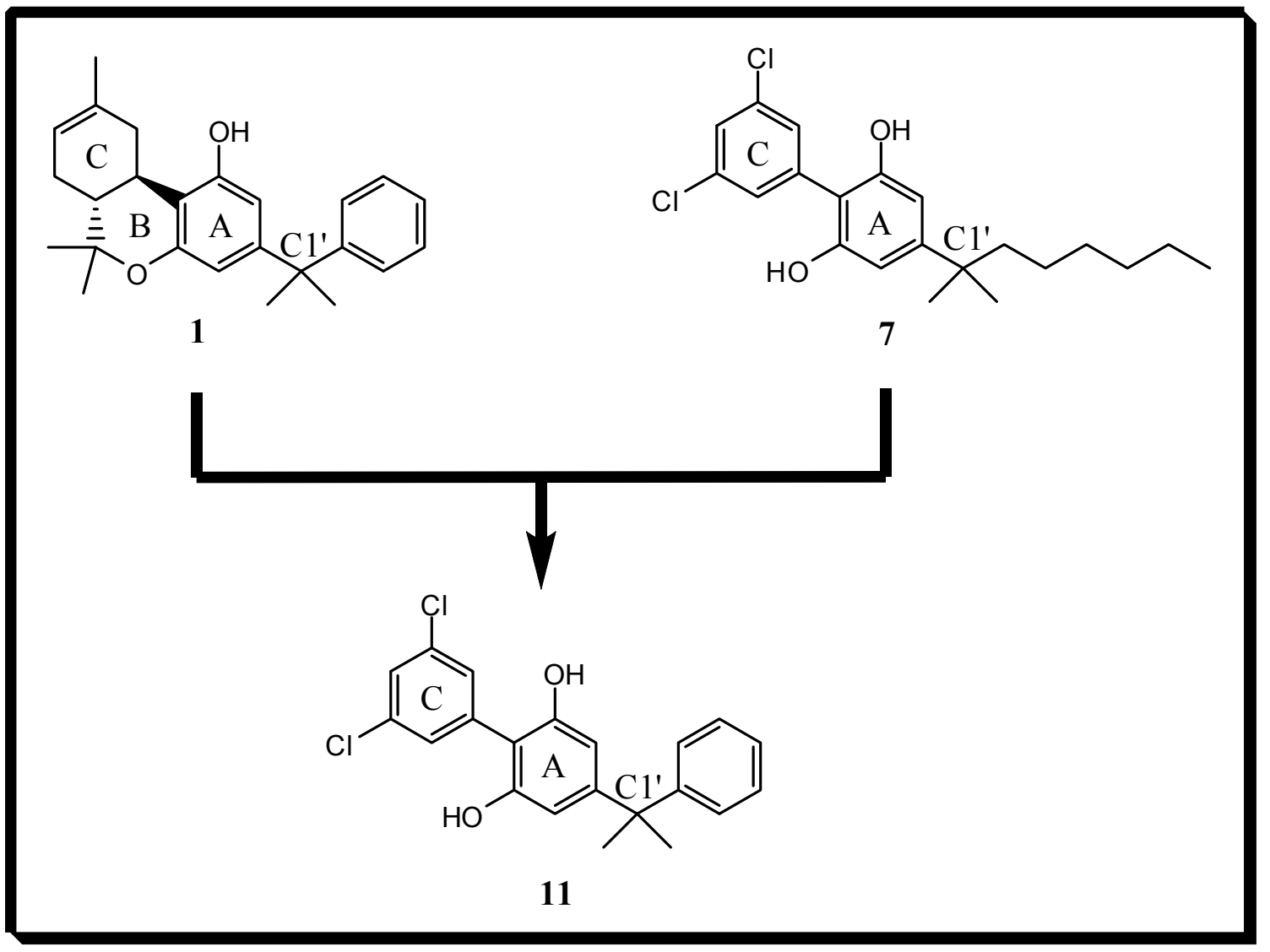

Figure 3.1: Design of the novel triaryl cannabinoid core

The design utilized elements from our $\mathrm{C} 1^{\prime}$ aryl $\Delta^{8}$ classical cannabinoid analogs 1 and the biaryl cannabinoids 7 reported by Makriyannis. 


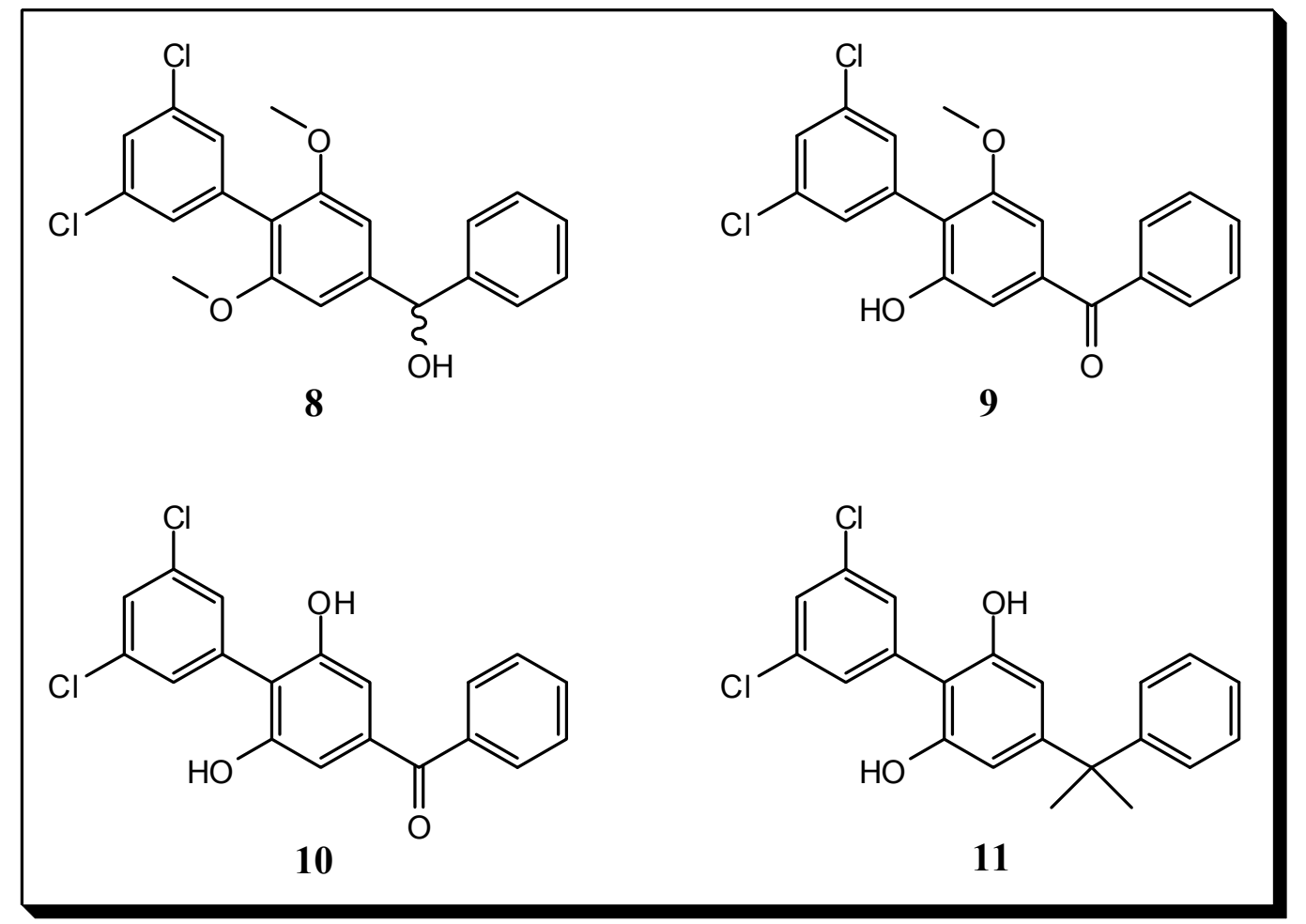

Figure 3.2: Initial triaryl cannabinoids

Initial novel triaryl cannabinoids synthesized and screened for receptor binding affinity. 
Table 3.1: Binding affinity $\left(K_{\mathrm{i}}\right)$ of compounds 8-11 for the $\mathrm{CB} 1$ and $\mathrm{CB} 2$ receptors compared to KM-233 1 and $\Delta^{8}$-THC

\begin{tabular}{cccc}
\hline Compound & $\mathbf{C B 1} \boldsymbol{K}_{\mathbf{i}}(\mathbf{n M})$ & $\mathbf{C B 2} \boldsymbol{K}_{\mathbf{i}}(\mathbf{n M})$ & $\mathbf{C B 1 / C B 2}$ Ratio \\
\hline$\Delta^{8}-\mathrm{THC}$ & $28.5( \pm 3.3)$ & $25.0( \pm 4.8)$ & 1.1 \\
$\mathrm{KM}-233 \mathbf{1}$ & $12.3( \pm 0.61)$ & $0.91( \pm 0.08)$ & 13.5 \\
$\mathbf{8}$ & $>1000$ & $1.66( \pm 0.38)$ & $>602.4$ \\
$\mathbf{9}$ & $>1000$ & $4.77( \pm 0.57)$ & $>209.6$ \\
$\mathbf{1 0}$ & $27.0( \pm 2.30)$ & $2.94( \pm 1.69)$ & 9.2 \\
$\mathbf{1 1}$ & $93.66( \pm 2.33)$ & $1.07( \pm 0.05)$ & 87.5 \\
\hline
\end{tabular}

Notes: Values are the mean of three experiments run in triplicate; standard deviation is given in parentheses. CB1/CB2 ratio is expressed as $K_{\mathrm{i}}$ for $\mathrm{CB} 1 / K_{\mathrm{i}}$ for CB2. 
results in a CB2 selective compound with a $K_{\mathrm{i}}$ value of $1.07 \mathrm{nM}$ at the $\mathrm{CB} 2$ receptor compared to $93.66 \mathrm{nM}$ at the $\mathrm{CB} 1$ receptor subtype $(\mathrm{CB} 1 / \mathrm{CB} 2=87.5)$. Compound 8 , which was initially viewed as a synthetic intermediate, gave perhaps the most significant finding. This compound showed high binding affinity for the CB2 receptor with a $K_{\mathrm{i}}$ of $1.66 \mathrm{nM}$ and exhibited a high 600 -fold selectivity for the CB2 receptor. The introduction of a C10 hydroxyl or carbonyl group in classical cannabinoids has not been extensively investigated; however, previous findings by our group ${ }^{108,129}$ and by Papahatjis and coworkers ${ }^{130}$ indicate that these $\mathrm{C} 10$ substituents reduce cannabinoid receptor affinity. Interestingly, binding affinity data for these first four compounds show that the carbonyl and hydroxyl substituents at the $\mathrm{C} 10$ position selectively decrease $\mathrm{CB} 1$ receptor affinity without altering CB2 receptor binding. The beneficial effect of the $\mathrm{C} 10$ equivalent hydroxyl, as well as the carbonyl, in CB2 receptor affinity may reflect that the removal of the pyran ring provides greater conformational flexibility thus allowing for more favorable receptor interactions at CB2, or that the triaryl compounds possess a different binding orientation relative to classical cannabinoids.

\subsection{SAR of Triaryl Cannabinoids}

To begin to develop an SAR (structure activity relationship) of this new triaryl class of cannabinoids, five more compounds were synthesized. Compounds 12-16 (Figure 3.3) completed the possible permutations of the three types of side chain linkers: hydroxyl, carbonyl, and gem-dimethyl, combined with the three substitution patterns on the A ring: dimethoxy, methoxy-hydroxy, and dihydroxy. These compounds were then screened for receptor binding affinity and functional activity at the CB1 and CB2 receptors.

After the remaining compounds were synthesized and evaluation of their affinity to the $\mathrm{CB} 1$ and $\mathrm{CB} 2$ receptors had begun, compound $\mathbf{1 0}$ was screened again. The results were contradictory to the previously published values. The $K_{\mathrm{i}}$ values for both CB1 and CB2 increased significantly from $27.0( \pm 2.30) n M$ to $6040( \pm 1477) n M$ and from $2.94( \pm$ $1.69) \mathrm{nM}$ to $487( \pm 101) \mathrm{nM}$, respectively. At first, it was suspected that there was some error in the more current assay technique or analysis. However, after performing several Scatchard analyses on the membrane preparations and screening several well characterized cannabinoids such as $\Delta^{8}$-THC and CP-55,940, the current analytical techniques were validated. The remaining three original compounds $(\mathbf{8}, \mathbf{9}$, and 11) were reevaluated for their receptor binding affinities and these values (along with compounds 12-16) are summarized in Table 3.2. There has been, as yet, no sufficient explanation as to the differences between the current $K_{\mathrm{i}}$ values and those previously reported.

A general SAR concerning the side chain linker and the substitutions on the A ring for this class of compounds can be easily developed from the binding data. First, the A ring must possess at least one hydroxyl group with two being ideal for maximum affinity at both the $\mathrm{CB} 1$ and $\mathrm{CB} 2$ receptors. The three dimethoxy analogs $8, \mathbf{1 4}$, and 15 showed very little binding at either receptor with $K_{\mathrm{i}}$ values above $10,000 \mathrm{nM}$ while the methoxy-hydroxy analogs 9 and 16 possessed detectable binding at the CB1 receptor with $K_{\mathrm{i}}$ values of $7235( \pm 3056) \mathrm{nM}$ and $206( \pm 130) \mathrm{nM}$ and at CB2 with values of 2439 


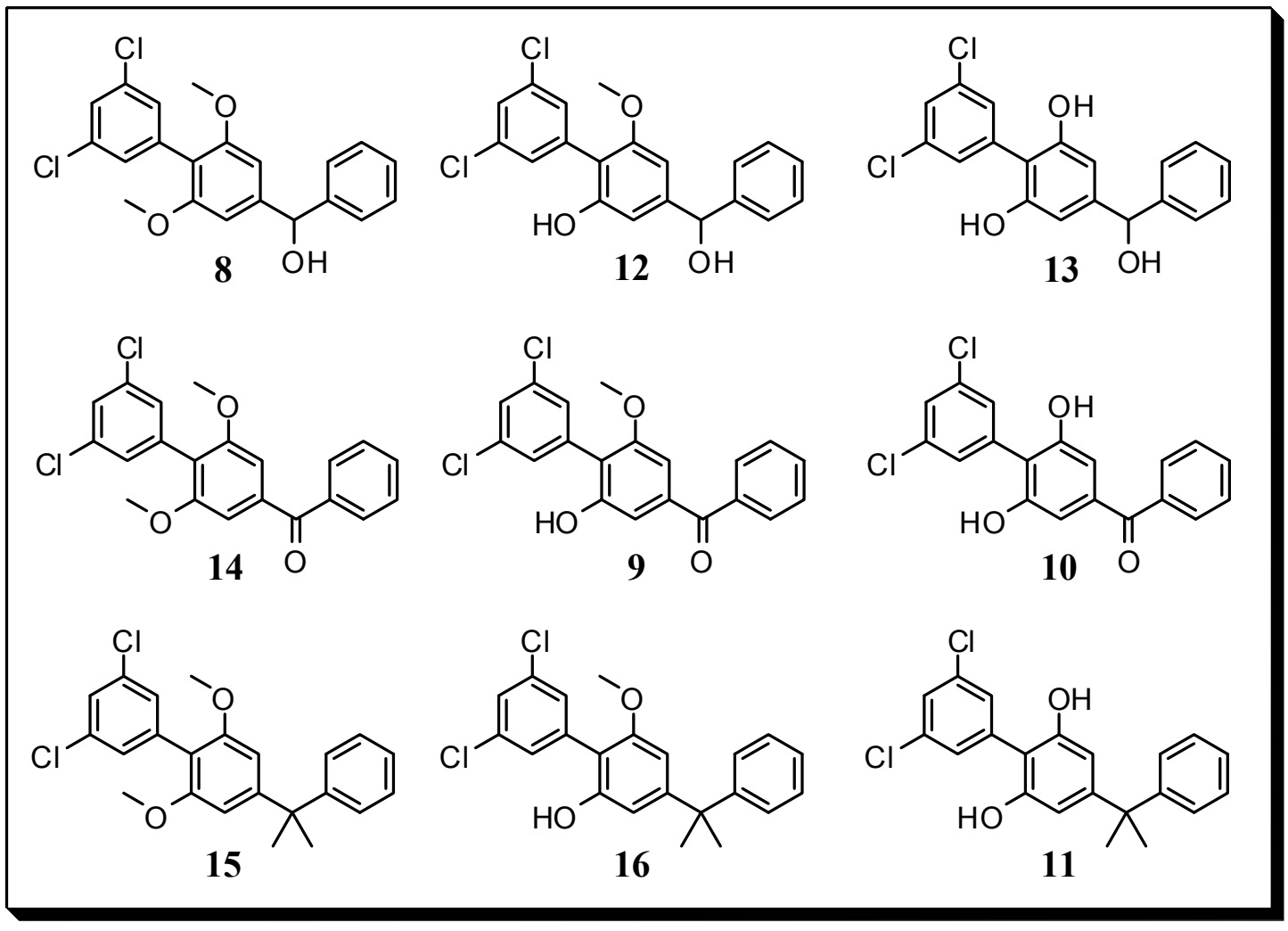

Figure 3.3: Nine triaryls used to develop SAR of this new class of cannabinoids 
Table 3.2: Binding affinity $\left(K_{\mathrm{i}}\right)$ of compounds 8-16 for the $\mathrm{CB} 1$ and $\mathrm{CB} 2$ receptors compared to KM-233 1 and $\Delta^{8}$-THC

\begin{tabular}{cccc}
\hline Compound & CB1 $\boldsymbol{K}_{\mathbf{i}}(\mathbf{n M})$ & $\mathbf{C B 2} \boldsymbol{K}_{\mathbf{i}}(\mathbf{n M})$ & $\mathbf{C B 1} / \mathbf{C B 2}$ Ratio \\
\hline$\Delta^{8}-\mathrm{THC}$ & $28.5( \pm 3.3)$ & $25.0( \pm 4.8)$ & 1.1 \\
$\mathrm{KM}-233 \mathbf{1}$ & $12.3( \pm 0.61)$ & $0.91( \pm 0.08)$ & 13.5 \\
$\mathbf{8}$ & $>10,000$ & $>10,000$ & n.d. \\
$\mathbf{9}$ & $7235( \pm 3056)$ & $2439( \pm 639)$ & 3.0 \\
$\mathbf{1 0}$ & $6040( \pm 1477)$ & $487( \pm 101)$ & 12.4 \\
$\mathbf{1 1}$ & $250( \pm 49)$ & $7.67( \pm 3.14)$ & 32.6 \\
$\mathbf{1 2}$ & $>10,000$ & $>10,000$ & n.d. \\
$\mathbf{1 3}$ & $>10,000$ & $3382( \pm 1389)$ & $>3.0$ \\
$\mathbf{1 4}$ & $>10,000$ & $>10,000$ & n.d. \\
$\mathbf{1 5}$ & $>10,000$ & $>10,000$ & n.d. \\
$\mathbf{1 6}$ & $206( \pm 130)$ & $66.0( \pm 17.2)$ & 3.1 \\
\hline
\end{tabular}

Notes: Values are the mean of three experiments run in triplicate; standard deviation is given in parentheses. CB1/CB2 ratio is expressed as $K_{\mathrm{i}}$ for CB1 / $K_{\mathrm{i}}$ for CB2. n.d. = not determined when there is no specific $K_{\mathrm{i}}$ that can be determined for either CB1 or CB2. 
( \pm 639$) \mathrm{nM}$ and $66.0( \pm 17.2) \mathrm{nM}$, respectively. The methoxy-hydroxy compound $\mathbf{1 2}$ which has a hydroxyl side chain linker, on the other hand, still possesses very low affinity for each receptor with $K_{\mathrm{i}}$ values above $10,000 \mathrm{nM}$. The three compounds possessing the dihydroxy substituted A ring were the best binders at each receptor subtype. Compound 11 which has the gem-dimethyl linker displayed the highest affinity for CB1 $\left(K_{\mathrm{i}}=250( \pm\right.$ $49) \mathrm{nM})$ and for $\mathrm{CB} 2\left(K_{\mathrm{i}}=7.67( \pm 3.14) \mathrm{nM}\right)$ while the hydroxyl side chain compound 13 possessed very low affinity for CB1 $\left(K_{\mathrm{i}}>10,000 \mathrm{nM}\right)$ and only very modest affinity for CB2 $\left(K_{\mathrm{i}}=3382( \pm 1389) \mathrm{nM}\right)$. Examining within each set of three compounds that have the same substitution pattern on the A ring: the dimethoxy $(\mathbf{8}, \mathbf{1 4}$, and 15), the methoxyhydroxy $(12,9,16)$, and the dihydroxy $(13,10$, and 11), it becomes clear that the side chain linker has a very profound effect on the binding affinity of the triaryl cannabinoids. The rank order for the highest affinity to both CB1 and CB2 is gem-dimethyl $>$ ketone $>$ hydroxyl. The negative effects on binding contributed by the hydroxyl linker is perhaps the most significant deviation from the originally reported data which showed that compound 8 retained high affinity for $\mathrm{CB} 2\left(K_{\mathrm{i}}=1.66( \pm 0.38) \mathrm{nM}\right)$. It is also worth noting that while all of the compounds with detectable affinity were CB2 selective, none were as selective as the previously reported values for compounds 8 and 9 (> 602.4 and $>$ 209.6, respectively). Compound 11 which showed the highest affinity for each receptor also has the highest level of CB2 selectivity with a $K_{\mathrm{i}}(\mathrm{CB} 1) / K_{\mathrm{i}}(\mathrm{CB} 2)$ ratio of 32.6. The next most $\mathrm{CB} 2$ selective compound was $\mathbf{1 0}$ with a ratio of 12.4 .

\subsection{Antineoplastic Activity of Triaryl Cannabinoids and Selection of HB-I-172}

Due to reported efficacy of cannabinoids against a wide range of cancers, ${ }^{72 a, 131}$ it was decided to screen the nine triaryl analogs against a panel of varied human neoplastic cell lines. The compounds were tested against human lung (H69AR), prostate (DU-145 and PC-3), colorectal (HCT-15), glioma (U-87 MG, LN-229, T98G, and DBTRG-05MG) and pancreatic (BxPC-3) cancer cell lines (Tables 3.3 and 3.4). The dimethoxy-ketone 14 and dimethoxy-dimethyl 15 analogs were quickly eliminated due to a lack of solubility in the cytotoxicity assay conditions. All but one of the remaining seven triaryls exhibited broad spectrum antineoplastic activity against all cell lines tested. Compound 13, the dihydroxy-hydroxy analog, was efficacious only against the PC-3 prostate carcinoma line and two glioblastoma lines: U-87 MG and LN-229. Even though compound 13 lacked broad spectrum activity, it proved to be potent against the susceptible cancers with $\mathrm{EC}_{50} \mathrm{~s}$ ranging from 4.63 to $5.10 \mu \mathrm{M}$. Compounds 8, 9, 10, 11, 12, and 16 were active against all cancers screened with $\mathrm{EC}_{50} \mathrm{~S}$ ranging from 0.30 to $6.34 \mu \mathrm{M}$. These results were unexpected considering the vast range in binding affinity of these compounds for the $\mathrm{CB} 1$ and $\mathrm{CB} 2$ receptors. This finding is evidence for the possibility that the antineoplastic activity of the triaryls is mediated through a receptor other than the cannabinoid receptor subtypes 1 and 2.

To begin to elucidate the antineoplastic mechanism of action of this new class of cannabinoids we needed to pick one lead compound for the studies. We decided to evaluate their metabolic stability in order to select a compound that could potentially be evaluated in vivo. Phase I metabolism studies were performed in the lab of Dr. Bernd Meibohm at the University of Tennessee Health Science Center with an in vitro method 
Table 3.3: Efficacy of three triaryl cannabinoids against a variety of human cancer cell lines

\begin{tabular}{|c|c|c|c|}
\hline Cell Line & $\begin{array}{c}\text { Compound } \\
8 \\
\mathrm{EC}_{50}(\mu \mathrm{M}) \pm \\
\text { S.D. } \\
\end{array}$ & $\begin{array}{c}\text { Compound } \\
12 \\
\mathrm{EC}_{50}(\mu \mathrm{M}) \pm \\
\text { S.D. } \\
\end{array}$ & $\begin{array}{c}\text { Compound } \\
13 \\
\mathrm{EC}_{50}(\mu \mathrm{M}) \pm \\
\text { S.D. } \\
\end{array}$ \\
\hline $\begin{array}{l}\text { DU-145 } \\
\text { prostate }\end{array}$ & $5.07( \pm 0.30)$ & $3.72( \pm 0.83)$ & n.a. \\
\hline $\begin{array}{c}\text { PC-3 } \\
\text { prostate }\end{array}$ & $3.47( \pm 1.36)$ & $4.08( \pm 0.82)$ & $4.63( \pm 0.63)$ \\
\hline $\begin{array}{l}\text { H69AR } \\
\text { lung }\end{array}$ & $6.30( \pm 0.19)$ & $5.16( \pm 0.53)$ & n.a. \\
\hline $\begin{array}{l}\text { HCT-15 } \\
\text { colorectal }\end{array}$ & $5.43( \pm 0.73)$ & $3.74( \pm 0.57)$ & n.a. \\
\hline $\begin{array}{c}\text { BxPC-3 } \\
\text { pancreatic }\end{array}$ & $3.28( \pm 1.21)$ & $2.49( \pm 0.28)$ & n.a. \\
\hline $\begin{array}{l}\text { U-87 MG } \\
\text { glioma }\end{array}$ & $4.67( \pm 0.23)$ & $3.13( \pm 0.31)$ & $4.94( \pm 0.11)$ \\
\hline $\begin{array}{l}\text { LN-229 } \\
\text { glioma }\end{array}$ & $2.57( \pm 0.19)$ & $1.93( \pm 0.09)$ & $5.10( \pm 0.18)$ \\
\hline $\begin{array}{l}\text { T98G } \\
\text { glioma }\end{array}$ & $5.20( \pm 0.69)$ & $5.29( \pm 2.39)$ & n.a. \\
\hline $\begin{array}{l}\text { DBTRG-05MG } \\
\text { glioma }\end{array}$ & n.a. & $5.03( \pm 1.10)$ & n.a. \\
\hline
\end{tabular}

Note: The $\mathrm{EC}_{50}$ values were obtained from three independent experiments run in triplicate; standard deviation is given in parentheses. Compounds 14 and 15 were not screened due to insolubility in assay conditions. Abreviations: n.a. $=$ not active. 
Table 3.4: Efficacy of four triaryl cannabinoids against a variety of human cancer cell lines

\begin{tabular}{|c|c|c|c|c|}
\hline Cell Line & $\begin{array}{c}\text { Compound } \\
9 \\
\mathrm{EC}_{50}(\mu \mathrm{M}) \pm \\
\text { S.D. } \\
\end{array}$ & $\begin{array}{c}\text { Compound } \\
10 \\
\mathrm{EC}_{50}(\mu \mathrm{M}) \pm \\
\text { S.D. } \\
\end{array}$ & $\begin{array}{c}\text { Compound } \\
16 \\
\mathrm{EC}_{50}(\mu \mathrm{M}) \pm \\
\text { S.D. } \\
\end{array}$ & $\begin{array}{c}\text { Compound } \\
11 \\
\mathrm{EC}_{50}(\mu \mathrm{M}) \pm \\
\text { S.D. } \\
\end{array}$ \\
\hline $\begin{array}{l}\text { DU-145 } \\
\text { prostate }\end{array}$ & $4.45( \pm 0.35)$ & $2.38( \pm 0.75)$ & $2.56( \pm 0.63)$ & $2.46( \pm 0.46)$ \\
\hline $\begin{array}{c}\text { PC-3 } \\
\text { prostate }\end{array}$ & $1.96( \pm 0.32)$ & $3.83( \pm 0.62)$ & $2.90( \pm 0.55)$ & $1.83( \pm 0.24)$ \\
\hline $\begin{array}{l}\text { H69AR } \\
\text { lung }\end{array}$ & $3.49( \pm 0.66)$ & $5.22( \pm 1.12)$ & $3.34( \pm 0.39)$ & $1.73( \pm 0.13)$ \\
\hline $\begin{array}{l}\text { HCT-15 } \\
\text { colorectal }\end{array}$ & $2.23( \pm 0.07)$ & $4.40( \pm 1.03)$ & $2.42( \pm 0.37)$ & $2.09( \pm 0.46)$ \\
\hline $\begin{array}{c}\text { BxPC-3 } \\
\text { pancreatic }\end{array}$ & $2.47( \pm 0.20)$ & $4.60( \pm 0.11)$ & $4.32( \pm 0.74)$ & $2.18( \pm 0.39)$ \\
\hline $\begin{array}{l}\text { U-87 MG } \\
\text { glioma }\end{array}$ & $2.01( \pm 0.27)$ & $2.90( \pm 0.27)$ & $2.68( \pm 0.74)$ & $1.20( \pm 0.58)$ \\
\hline $\begin{array}{l}\text { LN-229 } \\
\text { glioma }\end{array}$ & $1.29( \pm 0.40)$ & $4.63( \pm 1.10)$ & $1.14( \pm 0.08)$ & $0.30( \pm 0.06)$ \\
\hline $\begin{array}{l}\text { T98G } \\
\text { glioma }\end{array}$ & $3.15( \pm 0.75)$ & $4.25( \pm 0.26)$ & $2.34( \pm 0.49)$ & $3.69( \pm 0.74)$ \\
\hline $\begin{array}{l}\text { DBTRG-05MG } \\
\text { glioma }\end{array}$ & $6.20( \pm 3.33)$ & $6.34( \pm 4.10)$ & $4.73( \pm 1.94)$ & $2.18( \pm 1.92)$ \\
\hline
\end{tabular}

Note: The $\mathrm{EC}_{50}$ values were obtained from three independent experiments run in triplicate; standard deviation is given in parentheses. Compounds 14 and 15 were not screened due to insolubility in assay conditions. 
utilizing rat liver microsomal preparations. The criteria used for selecting acceptable compounds would be that at least $30 \%$ of the parent drug must remain after a 90 minute incubation with the microsomes. The results of that study narrowed the compounds to three potential candidates: 10, 12, and $\mathbf{1 3}$ with 45.3, 38.7, and 42.1\% remaining, respectively. Compound $\mathbf{1 3}$ was eliminated due to the previously mentioned lack of broad spectrum activity and compound $\mathbf{1 2}$ was dropped from consideration because of the presence of a chiral center in this molecule. The development of an asymmetric ligand with therapeutic potential requires efficacy and toxicology studies on each isomer along with the racemic mixture. The synthesis of each chirally pure isomer can be expensive or the attempted separation of the isomers from the mixture can be very difficult and time consuming. Therefore, compound 10 (henceforth named HB-I-172) was selected to proceed into studies to determine the mechanism of action of these compounds and to determine the receptor responsible for the antineoplastic efficacy.

\subsection{Receptors Involved in the Anti-Glioma Activity of HB-I-172}

Based on our previous experience with both in vitro and in vivo models of glioblastoma multiforme, ${ }^{109,111}$ it was decided to focus on this particular type of neoplasm for our further studies. We hypothesized that HB-I-172 initiated GBM cell death by ligation to one or more of the $\mathrm{CB}$ receptors $\mathrm{CB} 1, \mathrm{CB} 2$, and/or GPR55. The first step in our target receptor studies was to determine the relative expression levels of the receptors in the human GBM lines U-87 MG, T98G, LN-229, and DBTRG-O5MG. Western blot analysis of the particulate fraction (membrane bound proteins) and cytosolic fraction of

cell lysates is shown in Figure 3.4. Consistent with earlier reports, ${ }^{10 b, 132}$ both $\mathrm{CB} 1$ and $\mathrm{CB} 2$ receptors are present in all the human GBM cell lines with the $\mathrm{CB} 1$ showing a lower immunohistochemical staining relative to CB2. Interestingly, the GPR55 receptor exhibited a relative increased blotting in both the particulate and cytosolic fractions. The presence of GPR55 in the cytosolic fraction may indicate active synthesis of the protein or compartmental localization in the cytosol. The qualitative analysis of the Western data is not interpreted in terms of absolute expression levels but is used to verify the presence or absence of each receptor type. The similarities in receptor expression patterns found in each GBM cell line may account for the comparable efficacies of HB-I-172 against the four human GBM cell lines screened.

To study the contribution(s) of the CB1, CB2, and/or GPR55 receptors to the cytotoxic effect of HB-I-172 we utilized pharmacological probes. The CB1 and CB2 receptors couple through $\mathrm{G}_{\alpha \mathrm{i}}$ whereas GPR55 couples via $\mathrm{G}_{\alpha 12 / 13}$. To assess the possible contribution of CB1 and/or CB2 to the mechanism of action of HB-I-172, cytotoxicity assays were carried out using U87 cells pre-treated 8 hours before the assay with $100 \mathrm{ng} / \mathrm{ml}$ of pertussis toxin (Ptx). The Ptx inactivates $\mathrm{G}_{\alpha \mathrm{i}}$ via catalytic ADP-ribosylation thus blocking G-protein-receptor interactions, the $\mathrm{G}_{\alpha 12 / 13}$ proteins are Ptx-resistant. The decoupling of $\mathrm{CB} 1$ and $\mathrm{CB} 2$ from $\mathrm{G}_{\alpha \mathrm{i}}$ using Ptx caused an insignificant increase in $\mathrm{EC}_{50}$ of HB-I-172 against human U87 cells (Figure 3.5). We have determined that HB-I-172 is an antagonist of the CB1 receptor (binding affinity $\left.K_{\mathrm{i}}=6040( \pm 1477) \mathrm{nM}\right)$ and an inverse agonist $\left(\mathrm{EC}_{50}=361 \mathrm{nM}\right)$ of the $\mathrm{CB} 2$ receptor (binding affinity $K_{\mathrm{i}}=487$ 

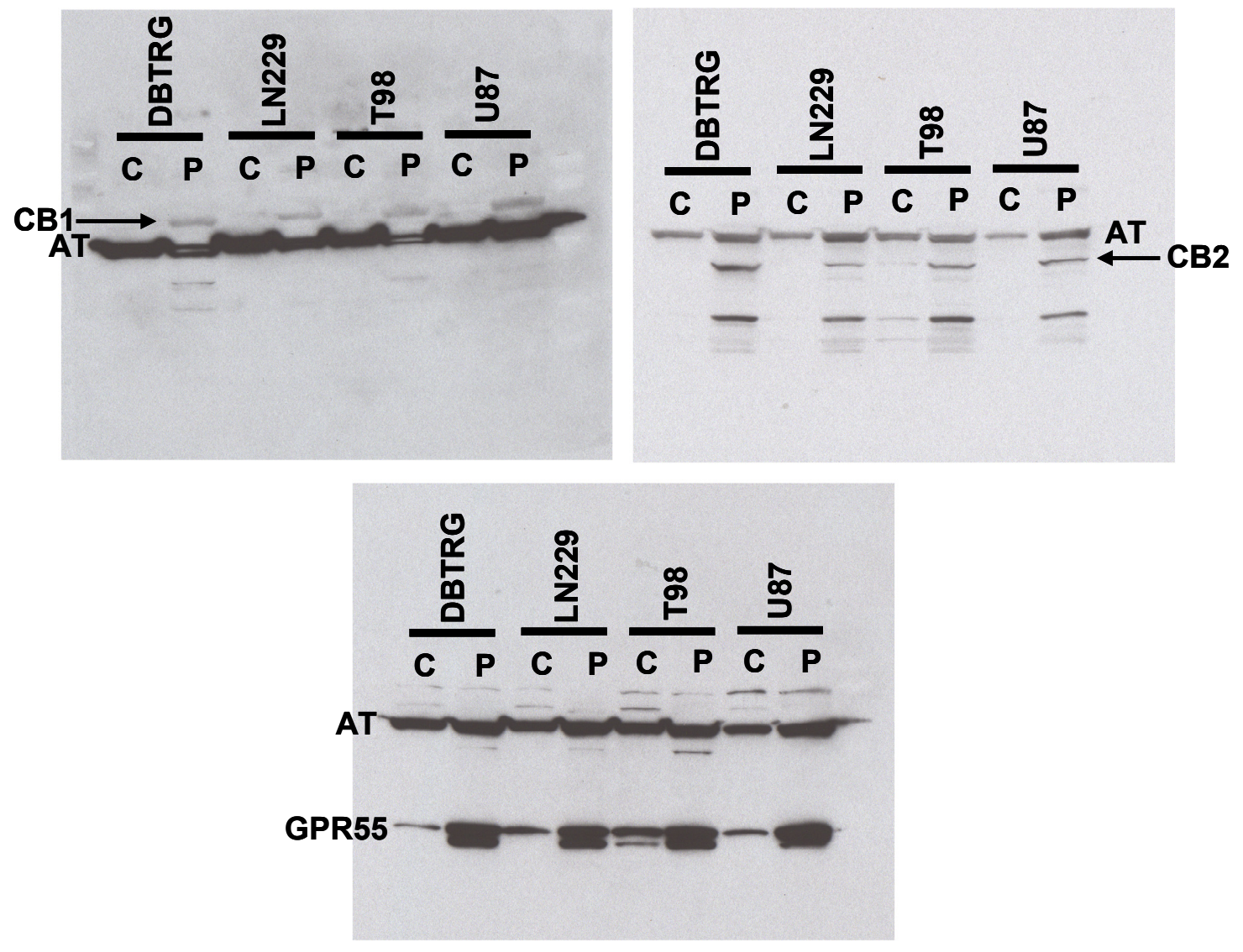

Figure 3.4: Western blots for cannabinoid receptors in human glioma cell lines

Western blots of the particulate $(\mathrm{P})$ and cytosolic $(\mathrm{C})$ fractions of whole cell lysates of U87 MG, T98G, LN-229, and DBTRG-O5MG. All blots are indexed to the structural protein $\alpha$-tubulin; cell lysates were divided into 3 aliquots and run on separate gels for each receptor. 


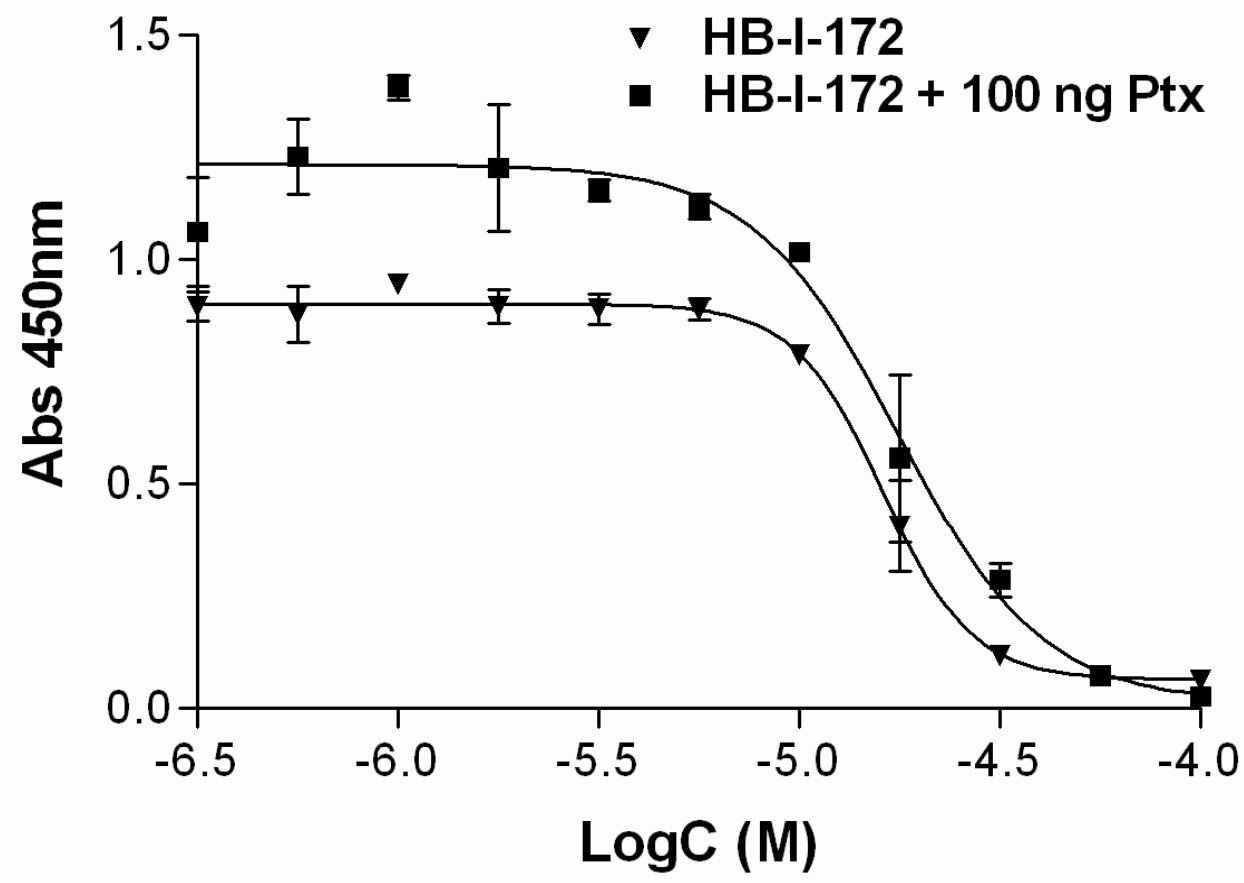

Figure 3.5: Effects of HB-I-172 on U-87 MG in the presence or absence of Ptx

Dose response curves for U-87 MG cells treated with HB-I-172 in the presence or absence of $100 \mathrm{ng} / \mathrm{ml}$ Ptx. The $\mathrm{EC}_{50}$ manifested a slight shift from 13.4 to $17.6 \mu \mathrm{M}$ in the presence of Ptx. Data is derived from 3 independent experiments conducted in triplicate. 
( \pm 101$) n M$ ) coupling through $\mathrm{G}_{\alpha \mathrm{i}}$ (Figure 3.6). If the antineoplastic activity is initiated by $\mathrm{CB} 1$ or CB2 it would be predicted that Ptx would block the effects of HB-I-172 thus the results indicate that $\mathrm{CB} 1$ and $\mathrm{CB} 2$ do not play a role in HB-I-172 anti-cancer activity.

Selective pharmacological inhibition of the three receptors is not a viable approach to identifying the target receptors. Specifically, the CB1 antagonist AM-251 is an agonist of GPR55, and SR141716A is reported to be either an agonist or antagonist of GPR55, depending on assay conditions. ${ }^{85}$ The selective CB2 antagonist/inverse agonist SR144528 to our knowledge has not been tested against GPR55; however, the inverse agonist activity at CB2 is the same as HB-I-172 and as such would not unambiguously help in identifying the target receptor. We are currently developing lines of U-87 MG that are stably expressing shRNA to knock-down each of the three receptors in an effort to overcome the limitations of pharmacological knock-downs.

\subsection{Comparison of HB-I-172 to Standard GBM Treatments}

During the course of a study utilizing immunohistochemical staining techniques to microscopically analyze the cytotoxic activities of other compounds prepared in our laboratory, it was discovered that the previously utilized method for determining cytotoxicity possessed an inherent error. In this method, cells were plated in 96-well polystyrene plates and allowed to attach overnight in an incubator. The complete growth media was then replaced with serum-free media $(100 \mu l)$ before drug addition. Small volumes of drug solutions $(1 \mu \mathrm{l})$ prepared in DMSO were then pipetted into the appropriate wells. The small ratio of drug solution to media did not allow for proper mixing and dilution of the drug and allowed for a much higher localized concentration of the test compounds in certain areas of the well. Figure 3.7 illustrates this finding. This micrograph is of LN-229 that has been treated with KM-233 at the $\mathrm{EC}_{50}$. It is evident that there is a disparity in the effects that the drug has on cells within this one field of view. In another set of experiments designed to evaluate the activity of caspase 3 in glioma cells treated with KM-233, we found that exposure to serum-free media during the drug incubation period increases the activity of caspase 3 separate and apart from any effect of the drug.

With the knowledge obtained from these two experiments, we developed a revised method for determining cytotoxicity of our compounds. The drug solutions are premixed in media containing $1 \%$ serum and added after the removal of the complete growth media. This allows for thorough mixing of the test compounds with the media and the small amount of serum reduces the interference with the basal metabolic activity observed in serum-free conditions.

We then screened HB-I-172 against U-87 MG and LN-229 utilizing the new method. We also tested two clinically relevant compounds, BCNU and temozolomide, under these conditions for comparison. We observed a 4.6 and 2.1 fold increase in the $\mathrm{EC}_{50}$ of HB-I-172 against U-87 MG and LN-229, respectively. In comparison, BCNU and temozolomide were either ineffective or manifested high micromolar $\mathrm{EC}_{50} \mathrm{~s}($ Table 3.5). Specifically, U87 and LN-229 were sensitive to BCNU after 18 hours of exposure 


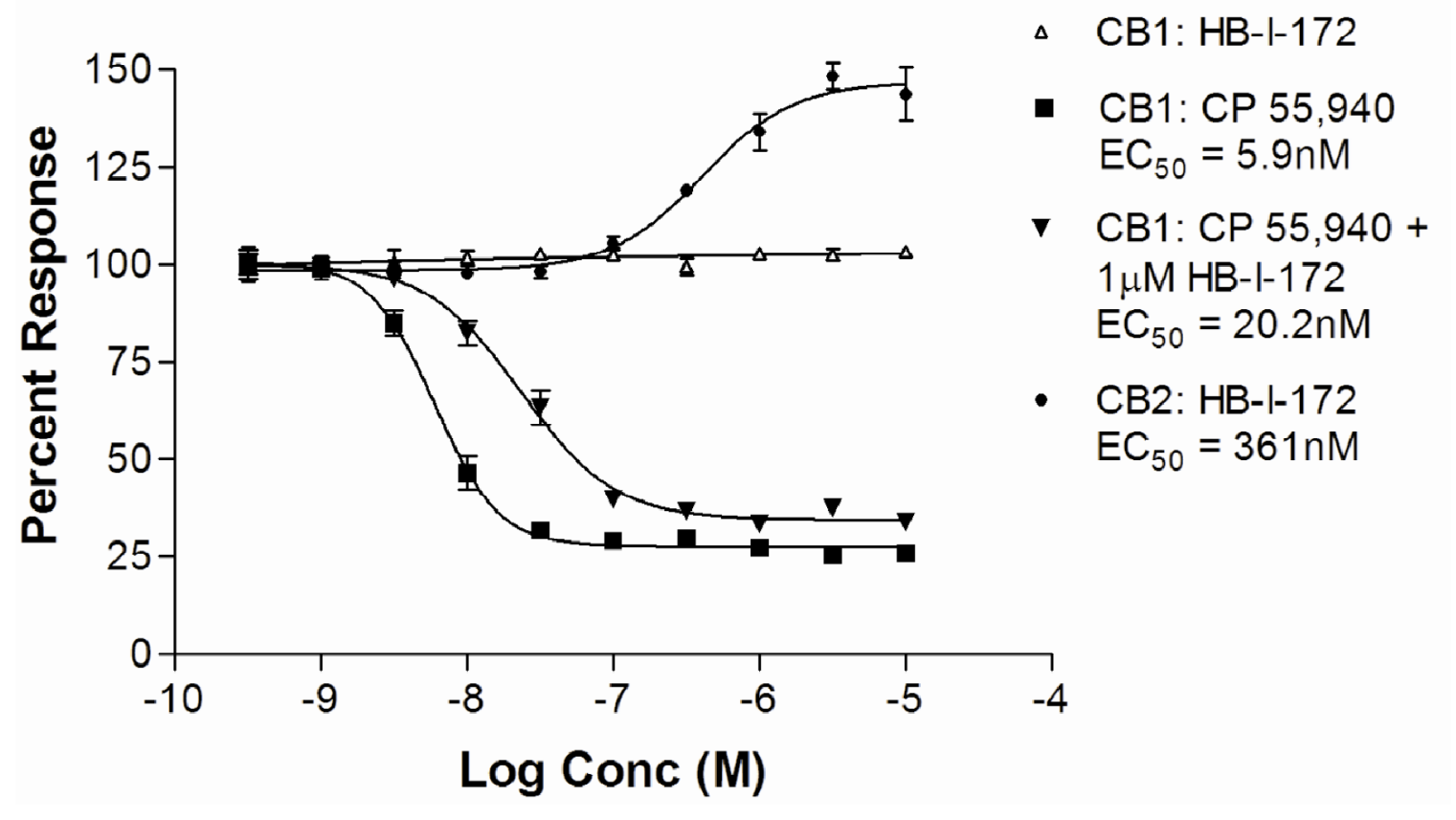

Figure 3.6: Functional activity of HB-I-172 at the $\mathrm{CB} 1$ and $\mathrm{CB} 2$ receptors

HB-I-172 does not reverse cAMP production in the CB1 ActOne $($ assay and shifts the response curve of CP-55,940 to the right identifying HB-I-172 as an antagonist at CB1. The increase in response in the $\mathrm{CB} 2$ assays is indicative of inverse agonist activity. 


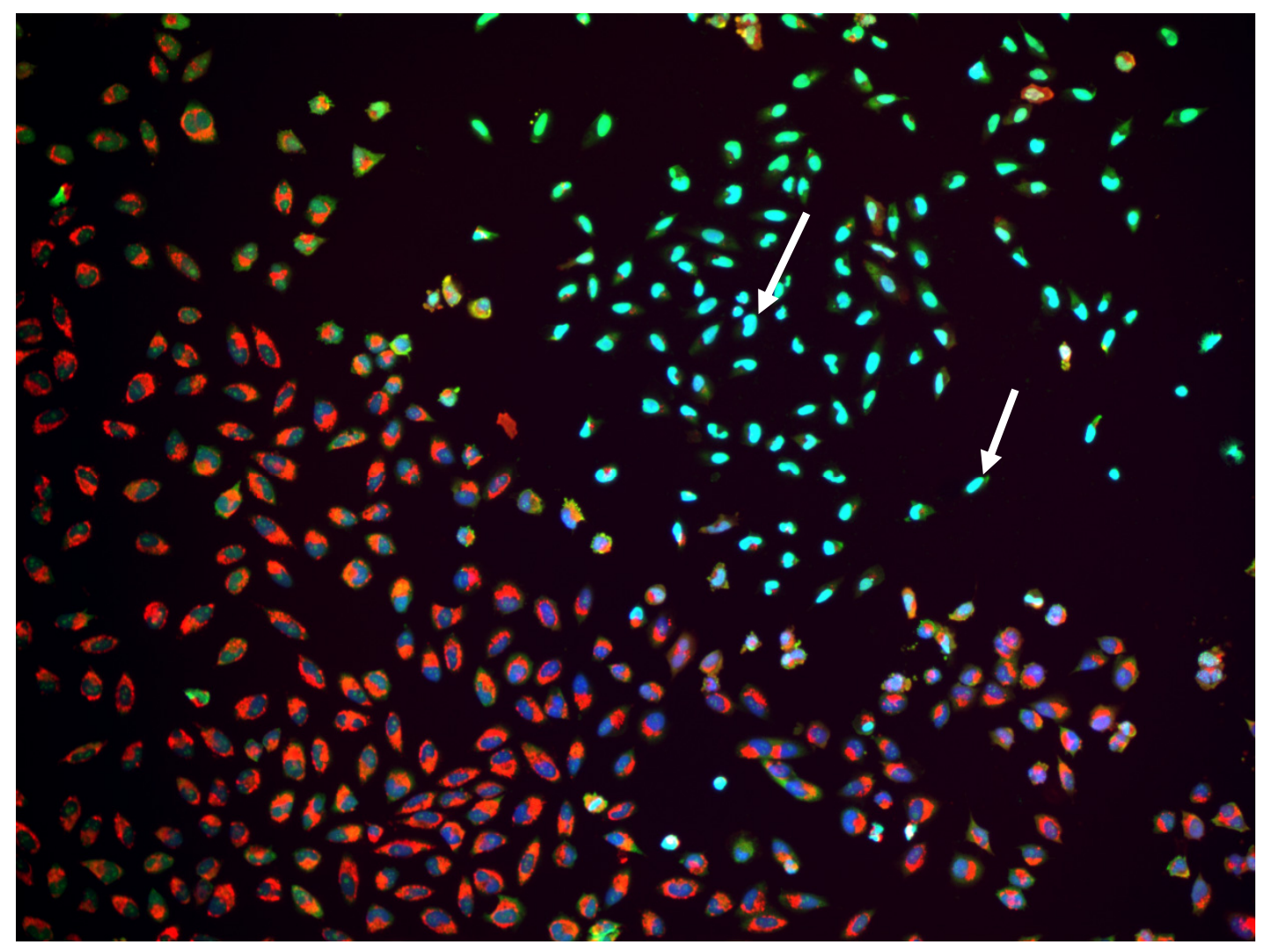

Figure 3.7: Inefficient mixing of drug solutions with media in cytotoxicity assays

Representative immunofluorescence micrograph of LN-229 GBM cells treated with KM233 at the $\mathrm{EC}_{50}$ imaged using a $10 \mathrm{X}$ objective. The labels are: cytochrome $\mathrm{C}$ (red), DAPI nuclear stain (blue), and whole cell stain (green). Cells with dysfunctional cell membranes show increased permeability so that the double stained nucleus appears cyan color (white arrows). The effects due to localized concentration of KM-233 can be easily observed in the upper right hand corner of the micrograph while the cells in the remainder of the image are relatively unaffected. 
Table 3.5: Efficacy of HB-I-172 against U-87 MG and LN-229

\begin{tabular}{lccc}
\hline & HB-I-172 18 hrs & BCNU 18 hrs & BCNU 48 hrs \\
Cell Line & $\begin{array}{c}\text { EC }_{\mathbf{5 0}}(\mu \mathrm{M}) \pm \\
\text { S.D. }\end{array}$ & $\begin{array}{c}\text { EC }_{\mathbf{5 0}}(\mu \mathrm{M}) \pm \\
\text { S.D. }\end{array}$ & $\begin{array}{c}\text { EC }_{\mathbf{5 0}}(\mu \mathrm{M}) \pm \\
\text { S.D. }\end{array}$ \\
\hline U-87 MG & $13.4( \pm 0.98)$ & $233( \pm 5.1)$ & $156.0( \pm 2.4)$ \\
LN-229 & $9.74( \pm 0.56)$ & $178( \pm 2.3)$ & $119( \pm 4.2)$ \\
\hline
\end{tabular}

Note: The $\mathrm{EC}_{50}$ values were obtained from three independent experiments run in triplicate; standard deviation is given in parentheses. 
with $\mathrm{EC}_{50} \mathrm{~s}$ of 223 and $178 \mu \mathrm{M}$, respectively. Following 48 hours of exposure to $\mathrm{BCNU}$ the $\mathrm{EC}_{50} \mathrm{~s}$ decreased to 156 and $119 \mu \mathrm{M}$. In contrast, the $\mathrm{EC}_{50} \mathrm{~S}$ of $\mathrm{HB}-\mathrm{I}-172$ were found to be 13.4 and $9.74 \mu \mathrm{M}$ for $\mathrm{U}-87 \mathrm{MG}$ and $\mathrm{LN}-229$, respectively, and were effective in the 18 hour assay window. Temozolomide was inactive against the cell lines following 18 and 48 hours exposure (data not shown). Although temozolomide is $\mathrm{pH}$ activated, the concentration of the active compound was not assessed and therefore these results are viewed with caution.

\subsection{Mechanistic Studies to Determine the Anti-Glioma Activity of HB-I-172}

\subsubsection{Methods and Results}

The second component of the mechanism(s) of human GBM cytotoxicity of HBI-172 is the downstream pathway resulting in cell death. To evaluate the downstream signal cascade(s) we utilized a combination of fluorescence microscopy and protein quantification using Meso Scale Discovery (MSD) MULTI-ARRAY ${ }^{\circledR}$ microplate technology. We first conducted a preliminary screen of the effect(s) of HB-I-172 on total and phosphorylated ERK1/2, JNK, p38, and, Akt using MSD plates. The screen utilized U-87 MG cells treated with HB-I-172 at the $\mathrm{EC}_{50}(13.4 \mu \mathrm{M})$ to evaluate signal cascade modulation over a 24 hour time course. The preliminary screen showed that ERK $1 / 2$ and Akt, but not JNK or p38, are downstream signals in U-87 MG cells following HB-I-172 exposure (data not shown). Based on these results we analyzed the kinetic profile of phosphorylation of the MEK-ERK-STAT pathway using the MSD triplex plate for pMEK1/2 (Ser217/221), pERK1/2 (Thr202/Tyr204 and Thr185/Tyr187), and pSTAT3 (Tyr705). Cells were cultured in $10 \mathrm{~cm}$ Petri dishes using media containing $1 \%$ serum for 24 hours to equilibrate intracellular signaling cascades. The media was replaced after equilibration with either media containing $1 \%$ serum and vehicle alone (DMSO) or media containing $1 \%$ serum and HB-I-172. The level of pMEK1/2 showed a significant decline in relative intensity 1 hour after the change in media in both control and drug treated cells (Figure 3.8). The control cells manifested a return to basal levels at 6 hours; however, HB-I-172 significantly reduced pMEK1/2 levels throughout the remainder of the experiment.

The levels of pERK1/2 exhibited a similar decline 1 hour post media change in both groups (Figure 3.9). Unlike pMEK1/2, the control group exhibited a significant increase in pERK $1 / 2$ at 6 hours that continued the remainder of the experiment. The effect of HB-I-172 on pERK1/2 however caused a significant and lasting decrease in relative levels. The Ras-Raf-MEK-ERK pathway is a potential target for the development of anti-cancer agents. ${ }^{120}$ The precise mechanism whereby HB-I-172 inhibits ERK phosphorylation remains to be determined. Due to the observed decrease in activated MEK, it must be further upstream in the signaling cascade. We do not believe that the response is due to Ras-Raf since we would predict an increase in pERK1/2 with decreased pAkt (see below), i.e. pAkt negatively regulates Raf thus an increase in pERK $1 / 2$ is predicted. ${ }^{133}$ 


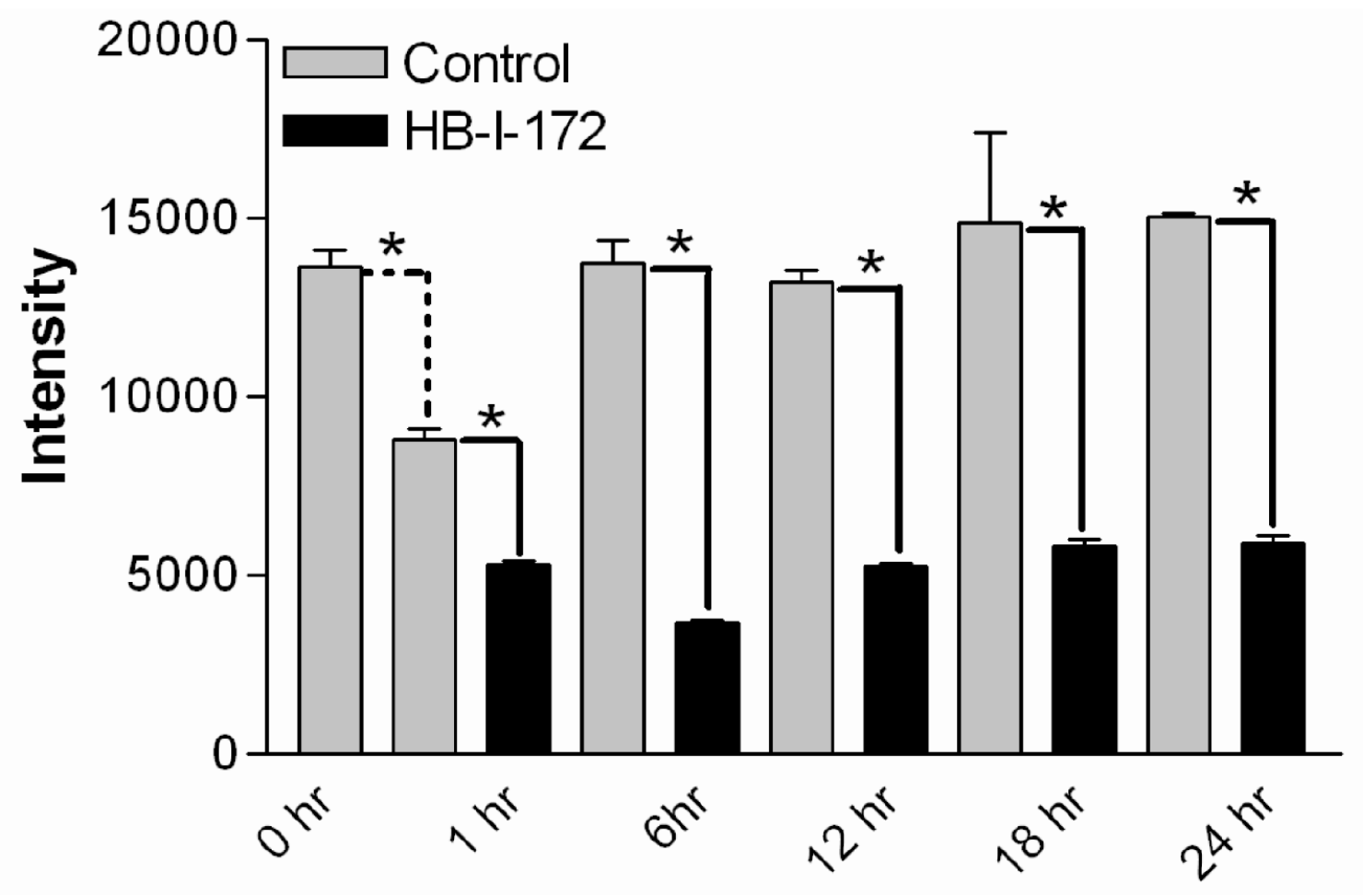

Figure 3.8: pMEK1/2 profile of U-87 MG cells treated with HB-I-172

U-87 MG cells were treated with HB-I-172 at the $\mathrm{EC}_{50}$ for the designated time intervals. Cell cultures were plated for 24 hours; the media changed to $1 \%$ serum for 24 hours to equilibrate intracellular pathways, then media was changed to either media containing $1 \%$ serum or media containing $1 \%$ serum plus HB-I-172. At the designated time intervals cells were scraped from the dishes, collected by centrifugation, the pellet lysed, and the protein concentration per lysate was determined. Total protein added to each well of the MSD plate was $20 \mu \mathrm{g}$. Aliquots from this experiment were used in all the following pathway assays. All data points represent 3 independent determinations analyzed in duplicate. Dashed lines indicate comparisons between control samples and solid lines between control and drug treated samples. ${ }^{*}=\mathrm{p}<0.001$. 


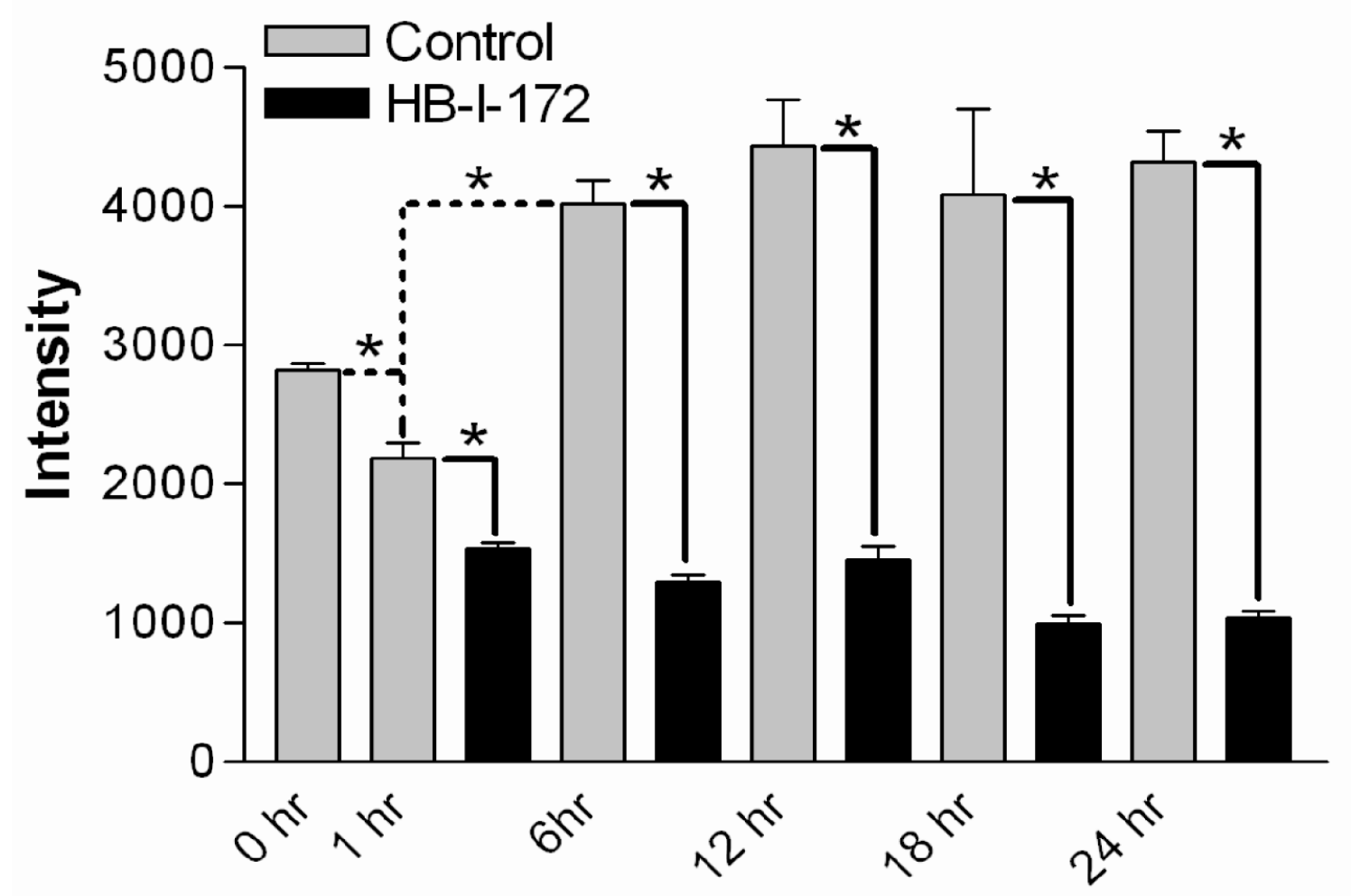

Figure 3.9: $\mathrm{pERK} 1 / 2$ profile of U-87 MG cells treated with HB-I-172

U-87 MG cells were treated with HB-I-172 at the $\mathrm{EC}_{50}$ for the designated time intervals. See legend of Figure 3.8 for experimental details. Dashed lines indicate comparisons between control samples and solid lines between control and drug treated samples. $*=p$ $<0.001$. 
Interplay between the ERK1/2 and Akt pathways in C6 glioma cells was previously reported by Ellert-Miklaszewska et al. ${ }^{119}$ To assess the contribution of Akt to the signal cascade associated with HB-I-172 we utilized the MSD Akt Signaling Panel which measures pAkt, pGSK-3 $\beta$, and p-p70S6K. Treatment of U-87 MG cells with HBI-172 triggers a significant and rapid decrease in pAkt (Ser 473) beginning at 1 hour and continuing through 24 hours (Figure 3.10). Within this time interval a decrease in phosphorylated 70-kDa ribosomal protein S6 kinase (p-p70S6K, Ser 217/221) (Figure 3.11) was observed along with a significant decrease in the Akt target glycogen synthase kinase 3 beta (pGSK-3 $\beta$, Ser 9) (Figure 3.12) at 1 and 6 hours followed by a highly significant increase in GSK-3 $\beta$ phosphorylation beginning at 18 hours post treatment.

The reductions in pAkt and p-p70S6K are consistent with the recent report by Salazar et al. wherein U-87 MG cells were treated with THC. ${ }^{134}$ The inhibition of Akt in cancer therapy is well established; however, it has recently been shown that knock-down of p70S6K with siRNA inhibited U-251 MG glioma cells by greater than 50 percent. The observed increase in pGSK-3 $\beta$ is consistent with the reported increase in apoptosis observed with GSK-3 $\beta$ activation ${ }^{122}$ although Kotliarova et al. report that inhibition of GSK-3 $\beta$ induces cell death. ${ }^{123}$ In GBM cell lines it is generally reported that regulation of Akt pathways, as well as ERK signaling, is mediated through a $\mathrm{G}_{\alpha \mathrm{\alpha} / \mathrm{o}}$ and/or $\mathrm{G}_{\beta / \gamma}$ pathway; however, it is possible that a $\mathrm{G}_{\alpha 12 / 13}-\mathrm{RhoA}$ pathway, and by extension GPR55, may regulate the downstream activation/inactivation of the Akt and/or the ERK pathways. For example, $\mathrm{G}_{\alpha 12 / 13}$ activation of the PI3K-Akt axis has been proposed in neuroblastoma cells ${ }^{124}$ and a pathway involving Rho-ROCK signaling and ERK activation in the human GBM line LN-19. ${ }^{125}$

The reduction in $\mathrm{pERK} 1 / 2$ could significantly impact the activity of cancer survival and gene expression pathways, for example p90RSK-BAD, p53, NF- $\kappa$ B, STAT1/3, Elk-1, and/or c-fos. Three of these pathways, p53, BAD, and STAT1/3 were evaluated using MSD technology. A significant decrease in pSTAT3 occurred at 1 hour in both treatment groups (Figure 3.13). The levels of pSTAT3 recovered by 12 hours; however, thereafter HB-I-172 caused a significant decrease in pSTAT3 levels relative to controls. The decline in pSTAT3 is of particular interest due its role in tumorigenesis, i.e. proliferation, angiogenesis, and inhibition of apoptosis, ${ }^{113}$ the proposed role in immune tolerance in glioblastoma patients, ${ }^{114}$ and provides a novel target in cancer therapy. ${ }^{113,115}$ This suggests that cannabinoid receptors may be a novel target for the regulation of STAT3 function via $\mathrm{CB} 1$ or $\mathrm{CB} 2$ regulation of $\mathrm{G}_{\alpha_{0}}-\mathrm{c}-\mathrm{Src}^{116}$ or via a GPR55 regulated $\mathrm{G}_{\alpha 12 / 13}$-RhoA-ERK-STAT3 pathway. ${ }^{117}$

The effect of HB-I-172 on the tumor suppressor protein p53 was determined using the MSD Multiplex Apoptosis Panel which measures levels of p53, p-p53 (Ser 15), cleaved PARP and cleaved caspase-3. Treatment of U-87 MG cells with HB-I-172 caused a significant increase in levels of both total p53 (Figure 3.14) and phospho-p53 (Figure 3.15) beginning 1 hour post treatment and continuing for the duration of the 24 hour experiment. This increase is probably a result of the decrease in ERK1/2 which suggests a negative regulation of p53 by ERK. This agrees with previous reports that also observed increases in p53 following suppression of ERK. ${ }^{135}$ This increase in p53 is 


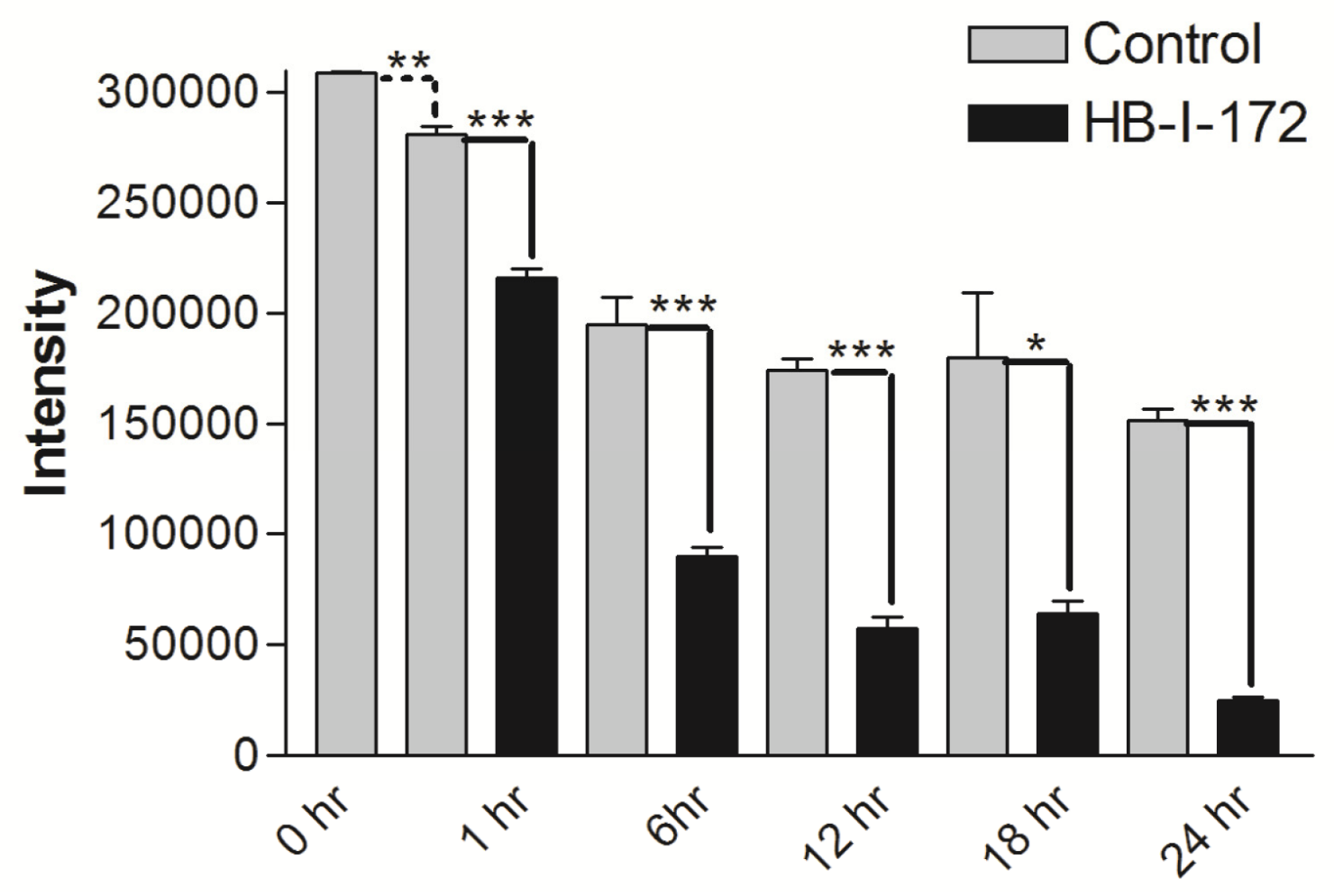

Figure 3.10: pAkt profile of U-87 MG cells treated with HB-I-172

U-87 MG cells were treated with HB-I-172 at the $\mathrm{EC}_{50}$ for the designated time intervals. See legend of Figure 3.8 for experimental details. Dashed lines indicate comparisons between control samples and solid lines between control and drug treated samples. $*=p$ $<0.005, * *=\mathrm{p}<0.0005, * * *=\mathrm{p}<0.00001$. 


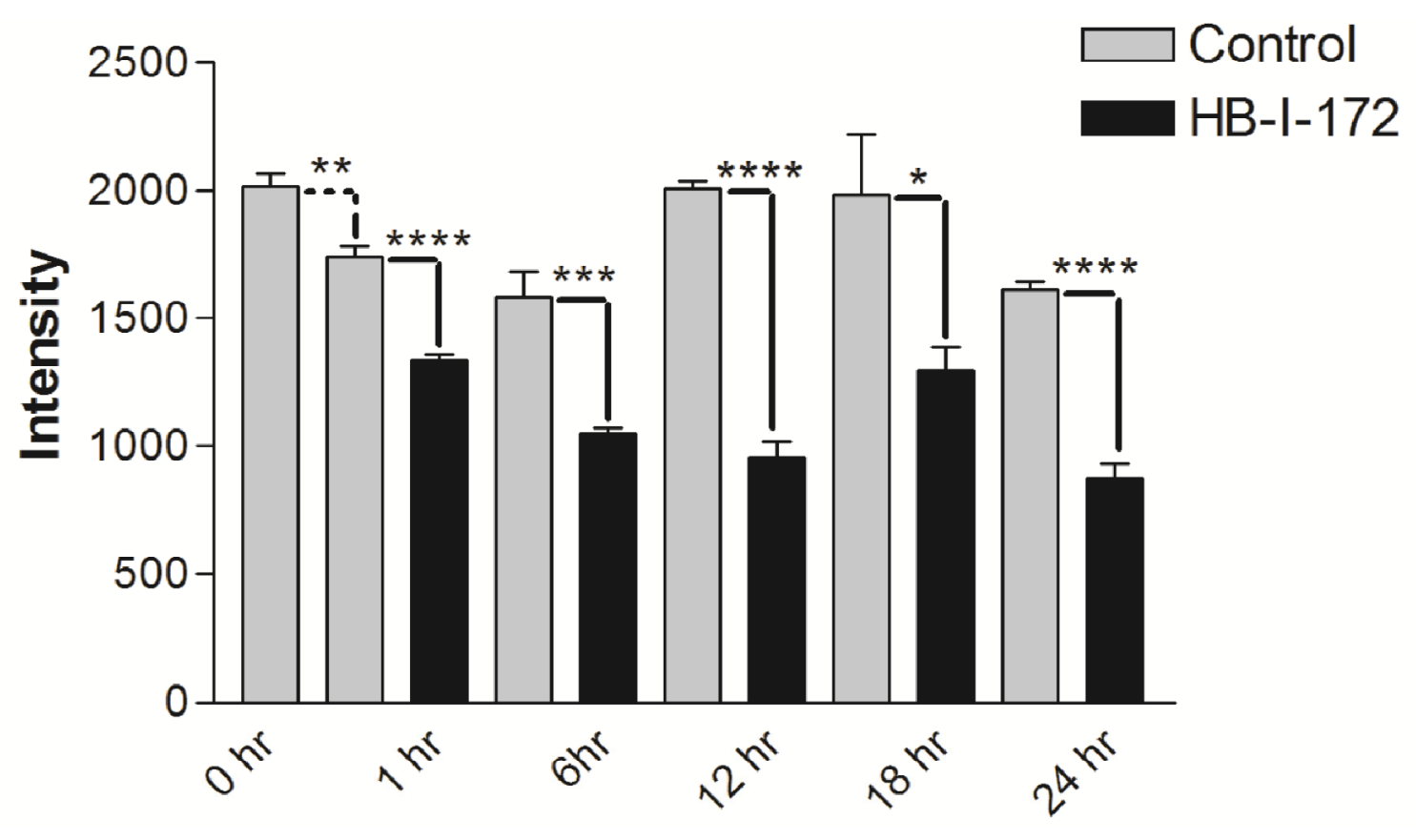

Figure 3.11: Phospho-p70S6K profile of U-87 MG cells treated with HB-I-172

U-87 MG cells were treated with HB-I-172 at the $\mathrm{EC}_{50}$ for the designated time intervals. See legend of Figure 3.8 for experimental details. Dashed lines indicate comparisons between control samples and solid lines between control and drug treated samples. $*=p$ $<0.05, * *=\mathrm{p}<0.005, * * *=\mathrm{p}<0.001, * * * *=\mathrm{p}<0.00001$. 


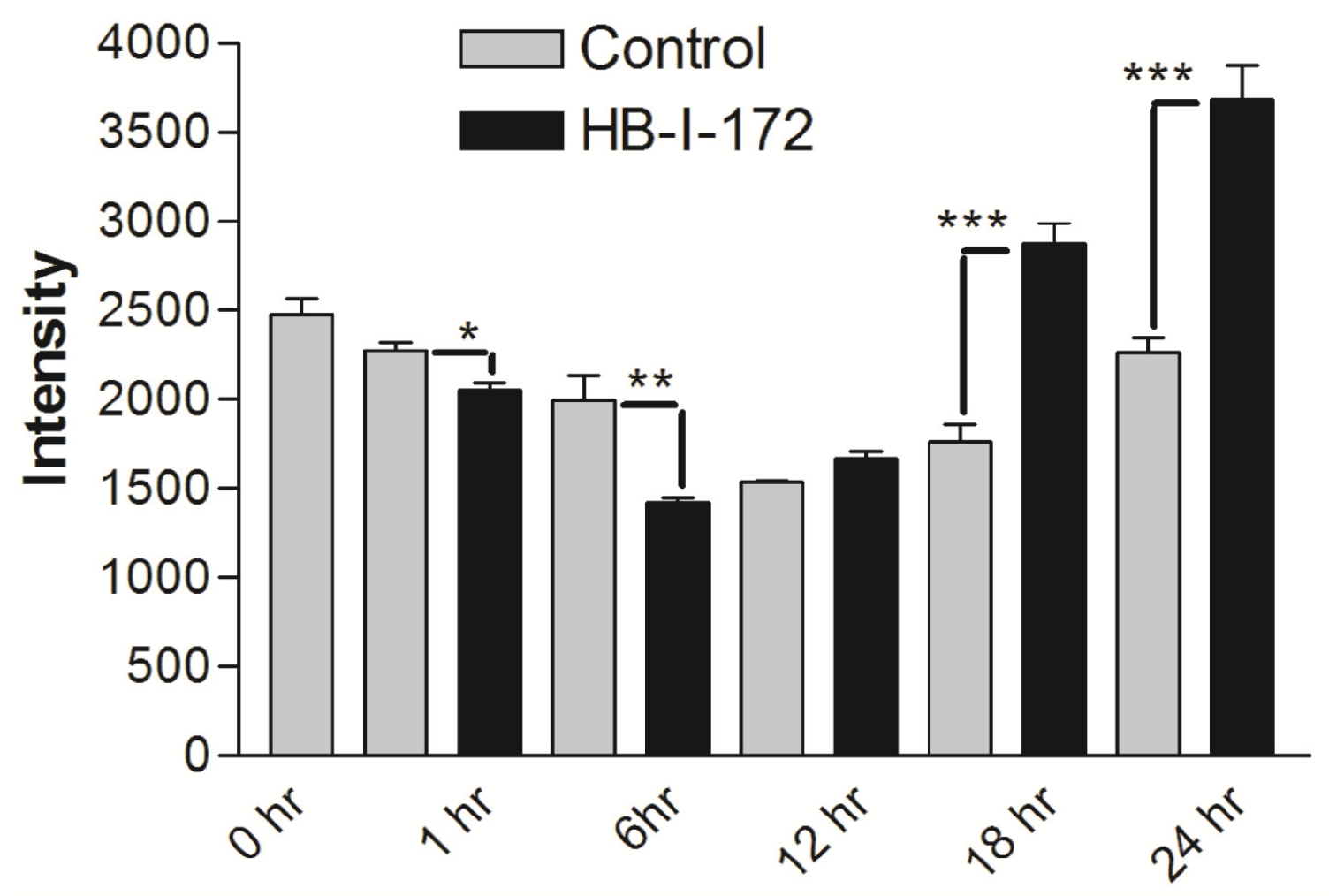

Figure 3.12: pGSK-3 $\beta$ profile of U-87 MG cells treated with HB-I-172

U-87 MG cells were treated with HB-I-172 at the $\mathrm{EC}_{50}$ for the designated time intervals. See legend of Figure 3.8 for experimental details. Solid lines indicate comparisons between control and drug treated samples. ${ }^{*}=\mathrm{p}<0.01,{ }^{*}=\mathrm{p}<0.001,{ }^{* * *}=\mathrm{p}<$ 0.0005 . 


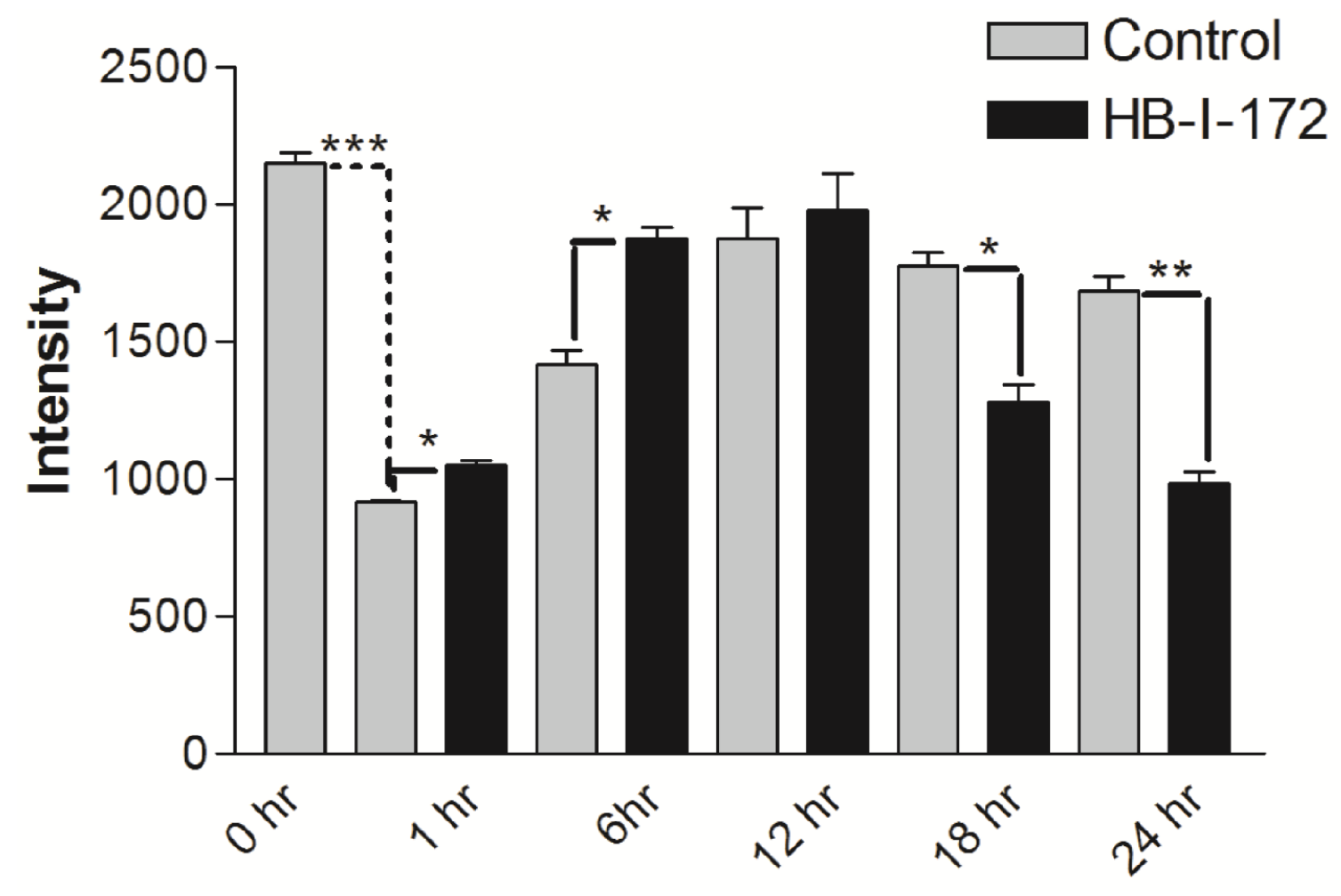

Figure 3.13: pSTAT3 profile of U-87 MG cells treated with HB-I-172

U-87 MG cells were treated with HB-I-172 at the $\mathrm{EC}_{50}$ for the designated time intervals. See legend of Figure 3.8 for experimental details. Dashed lines indicate comparisons between control samples and solid lines between control and drug treated samples. $*=p$ $<0.0005, * *=\mathrm{p}<0.000005, * * *=\mathrm{p}<0.00000005$. 


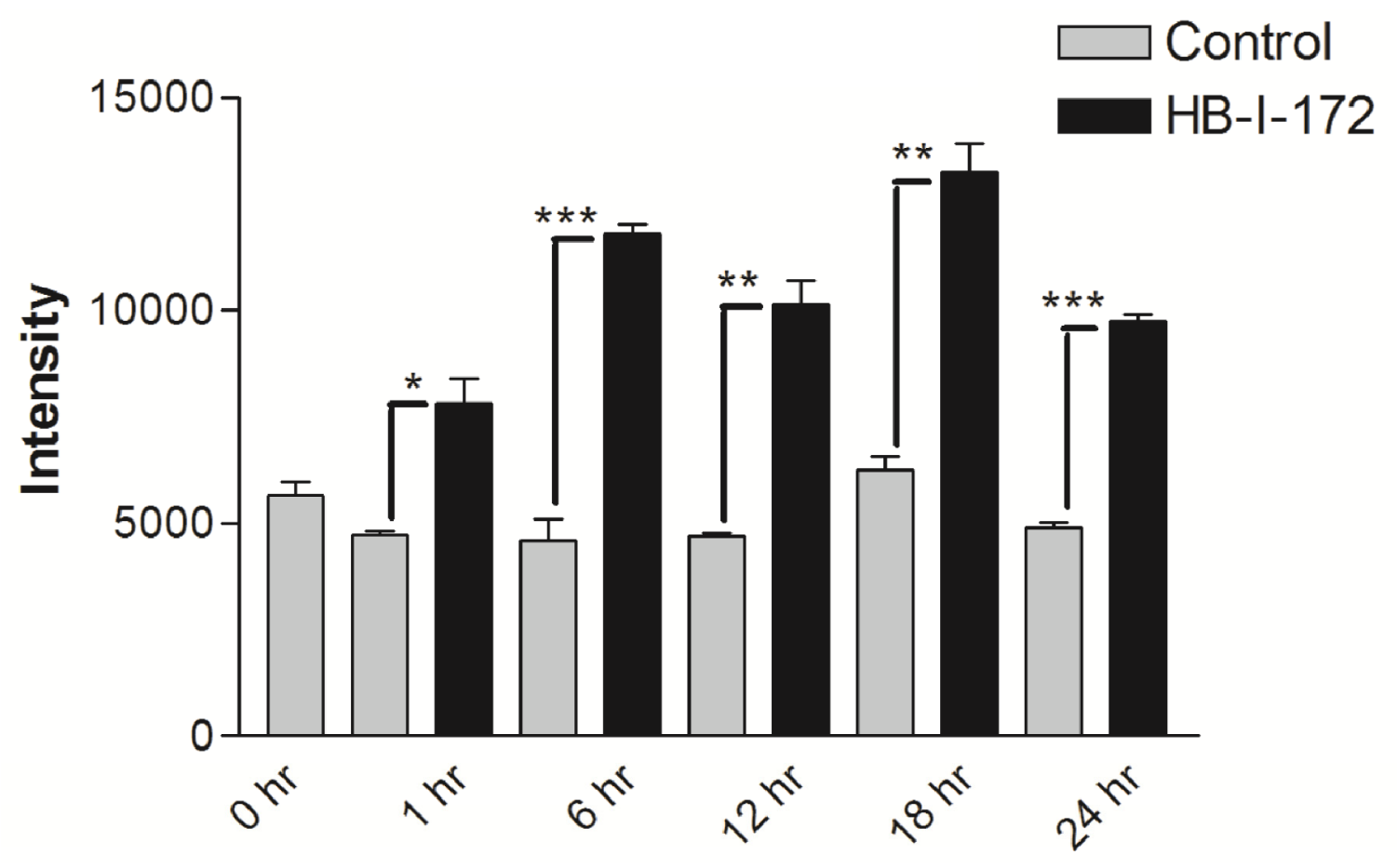

Figure 3.14: p53 profile of U-87 MG cells treated with HB-I-172

U-87 MG cells were treated with HB-I-172 at the $\mathrm{EC}_{50}$ for the designated time intervals. See legend of Figure 3.8 for experimental details. Solid lines indicate comparisons between control and drug treated samples. $*=p<0.005, * *=p<0.0001, * * *=p<$ 0.000001 . 


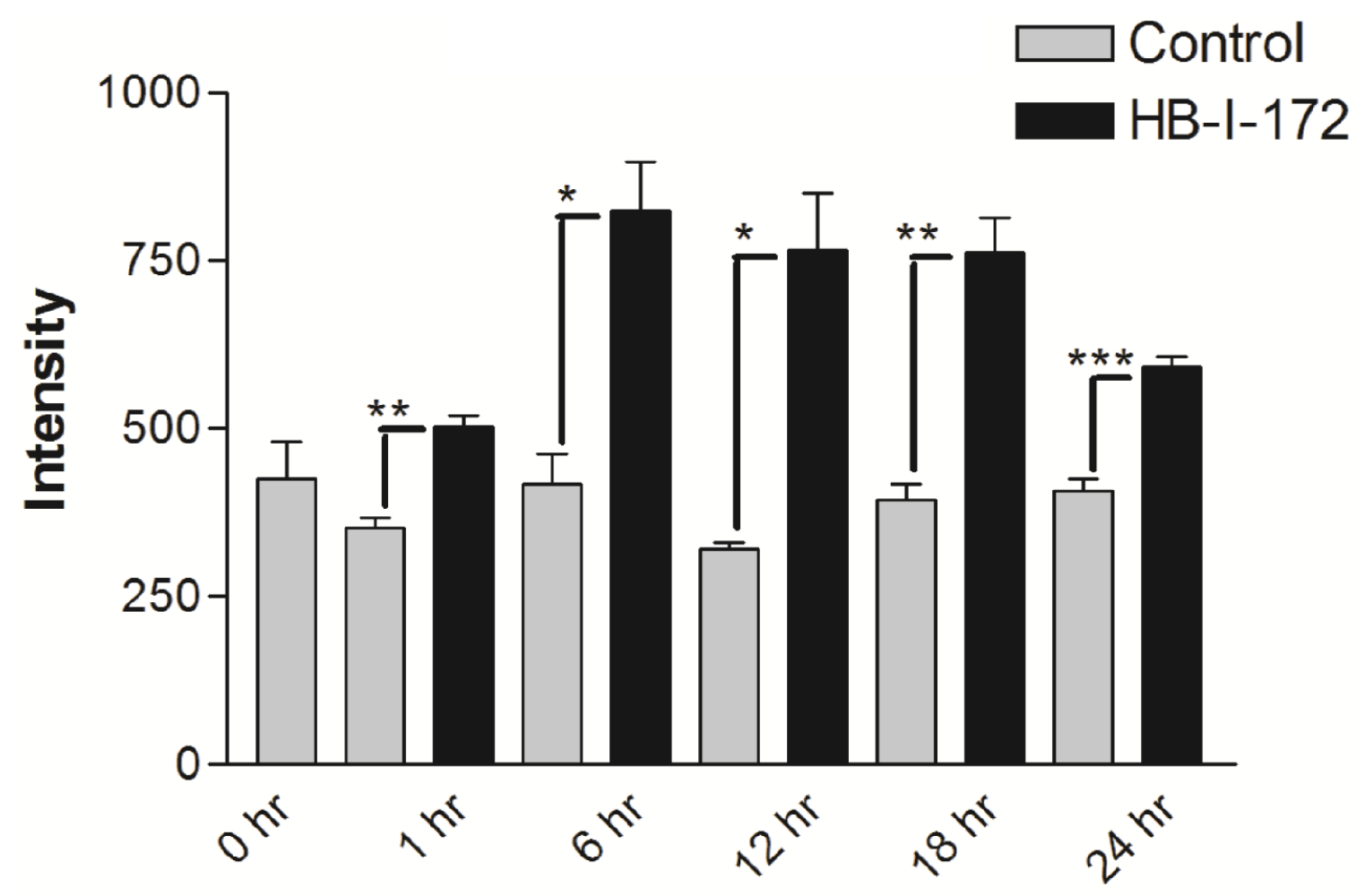

Figure 3.15: Phospho-p53 profile of U-87 MG cells treated with HB-I-172

U-87 MG cells were treated with HB-I-172 at the $\mathrm{EC}_{50}$ for the designated time intervals. See legend of Figure 3.8 for experimental details. Solid lines indicate comparisons between control and drug treated samples. $*=\mathrm{p}<0.005, * *=\mathrm{p}<0.001, * * *=\mathrm{p}<$ 0.0001 . 
of interest due to its role in suppression of tumor proliferation by initiating cellular senescence ${ }^{136}$ and its ability to initiate apoptosis. ${ }^{137}$

The down-regulation of the pro-survival kinase cascades ERK1/2 and Akt raised the possibility that one downstream mediator of glioblastoma cytotoxicity due to HB-I172 may be the Bcl-2 family protein BAD. Specifically, phosphorylation of BAD by Akt and ERK has been previously reported; ${ }^{126}$ therefore, HB-I-172 may suppress phosphorylation of BAD resulting in heterodimerization with $\mathrm{Bcl}-2$ death suppressors. To evaluate the effect of HB-I-172 on BAD phosphorylation status we utilized the MSD Phospho (Ser 112)/Total BAD assay. Treatment of U-87 MG cells with HB-I-172 caused a significant decrease in BAD phosphorylation at 6 hours post-exposure (Figure 3.16).

It was expected that the decrease in $\mathrm{pBAD}$ would correlate with mitochondrial dysfunction. One manifestation would be cytochrome $\mathrm{C}$ (cytC) release and activation of caspase 3. To evaluate this component we employed the previously mentioned MSD Apoptosis Panel. A rapid and significant increase in cleaved caspase 3 began at 6 hours post-drug treatment and persisted until the end of the experiment (Figure 3.17). The increase in caspase 3 activity is followed downstream by activation of caspase 9 twelve hours later at the 18 hour time point as determined by the Caspase-Glo® 9 Assay System from Promega (Figure 3.18). This probably accounts for the further increase in cleaved caspase 3 at the 24 hour time point as caspase 9 cleaves procaspase 3 into the active enzyme.

Coinciding with the increase in caspase 3 activity, the same rapid increase at 6 hours was observed in the level of cleaved PARP (Figure 3.19). The increase in cleaved PARP is of interest due to its ability to potentiate apoptosis by depleting the intracellular supply of ATP and also to independently initiate apoptosis by production of poly(ADPribose) which stimulates mitochondria to release apoptosis inducing factor. ${ }^{138}$

The possible contribution of mitochondrial dysfunction to the mechanism of action of HB-I-172 was further evaluated using DePsipher® (Trevigen; Gaithersburg, MD) to evaluate the collapse of the mitochondrial membrane potential (Figure 3.20). Treatment of LN-229 glioblastoma cells with HB-I-172 at the $\mathrm{EC}_{50}$ resulted in a significant increase in mitochondrial membrane depolarization, as indicated by the inability of the fluorescent stain DePsipher to form red fluorescent eximers inside mitochondria with a marked effect at 6 hours and an almost complete depolarization at 18 hours post-drug treatment. Mitochondrial involvement was further verified by the observation of cytochrome $\mathrm{C}$ release in U-87 MG after 6 hours of exposure to HB-I-172 at the $\mathrm{EC}_{50}$ as determined by immunohistochemical staining techniques (Figure 3.21). This same fluorescence photomicrograph also depicts cells with nuclei in which DNA has become fragmented and has begun to condense which is further evidence for an apoptotic mechanism of cell death.

The increased activity of PARP which was observed in the MSD assay was also confirmed by immunohistochemistry. In U-87 MG cells treated with HB-I-172 at the $\mathrm{EC}_{50}$, the presence of PARP can be seen in the nuclei 6 hours after drug exposure (Figure 3.22). The presence of PARP in the nuclei further confirms that there has been DNA fragmentation as one of its major roles is to repair DNA breaks. 


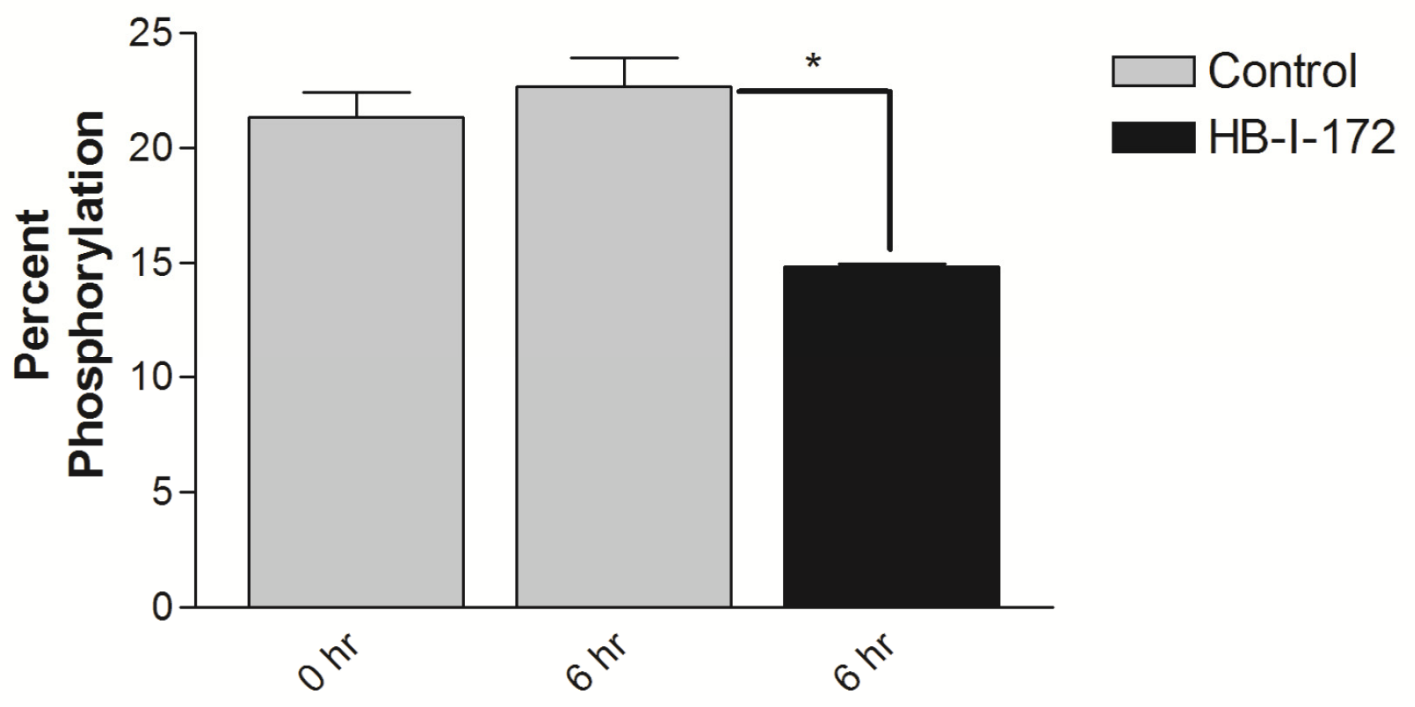

Figure 3.16: BAD phosphorylation of U-87 MG cells treated with HB-I-172

U-87 MG cells were treated with HB-I-172 at the $\mathrm{EC}_{50}$ for 6 hours. See legend of Figure 3.8 for experimental details. Solid line indicates comparison between control and drug treated samples. $*=\mathrm{p}<0.00005$. 


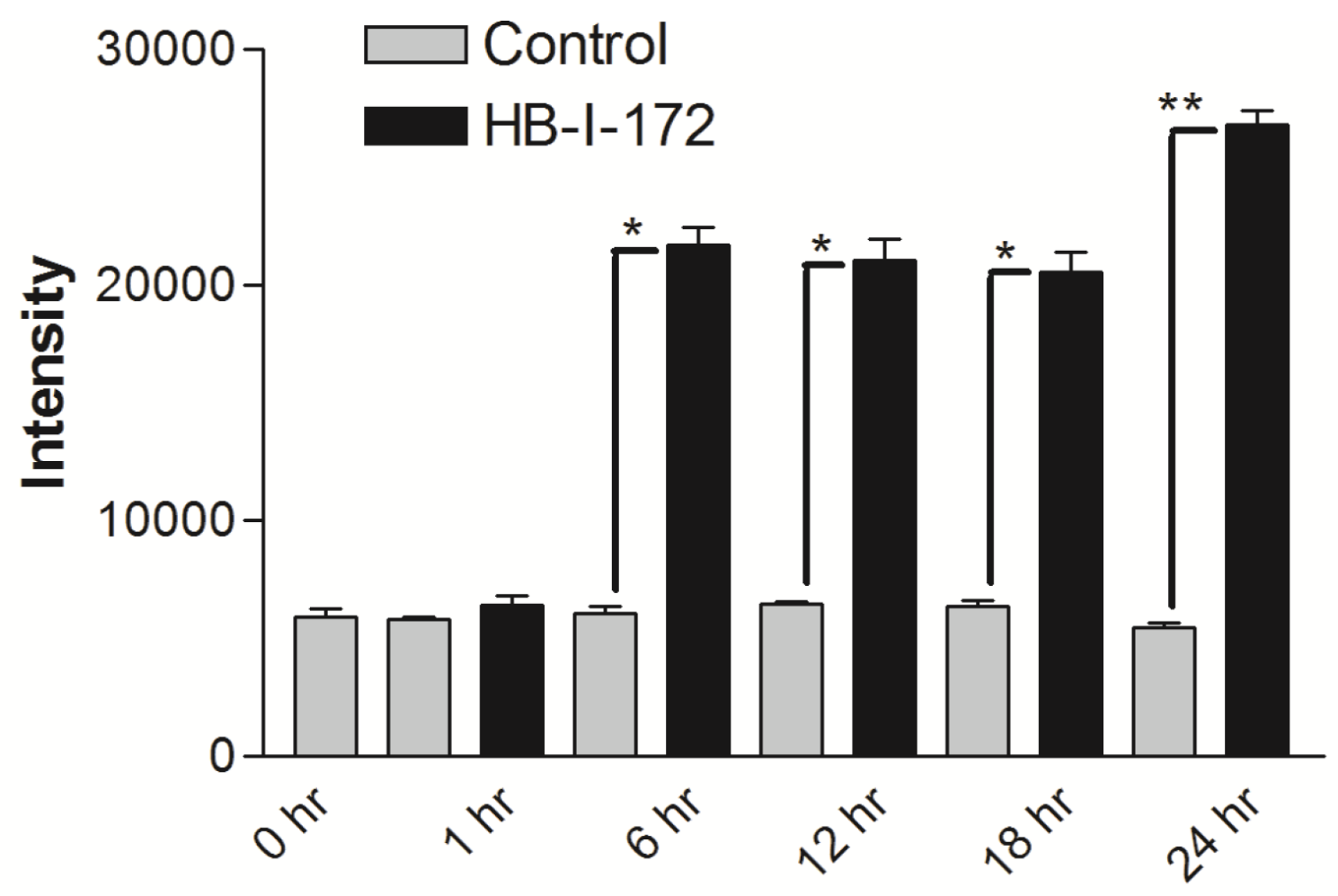

Figure 3.17: Caspase 3 activity profile of U-87 MG cells treated with HB-I-172

U-87 MG cells were treated with HB-I-172 at the $\mathrm{EC}_{50}$ for the designated time intervals. See legend of Figure 3.8 for experimental details. Solid lines indicate comparisons between control and drug treated samples. $*=p<0.000005, * *=p<0.000000005$. 


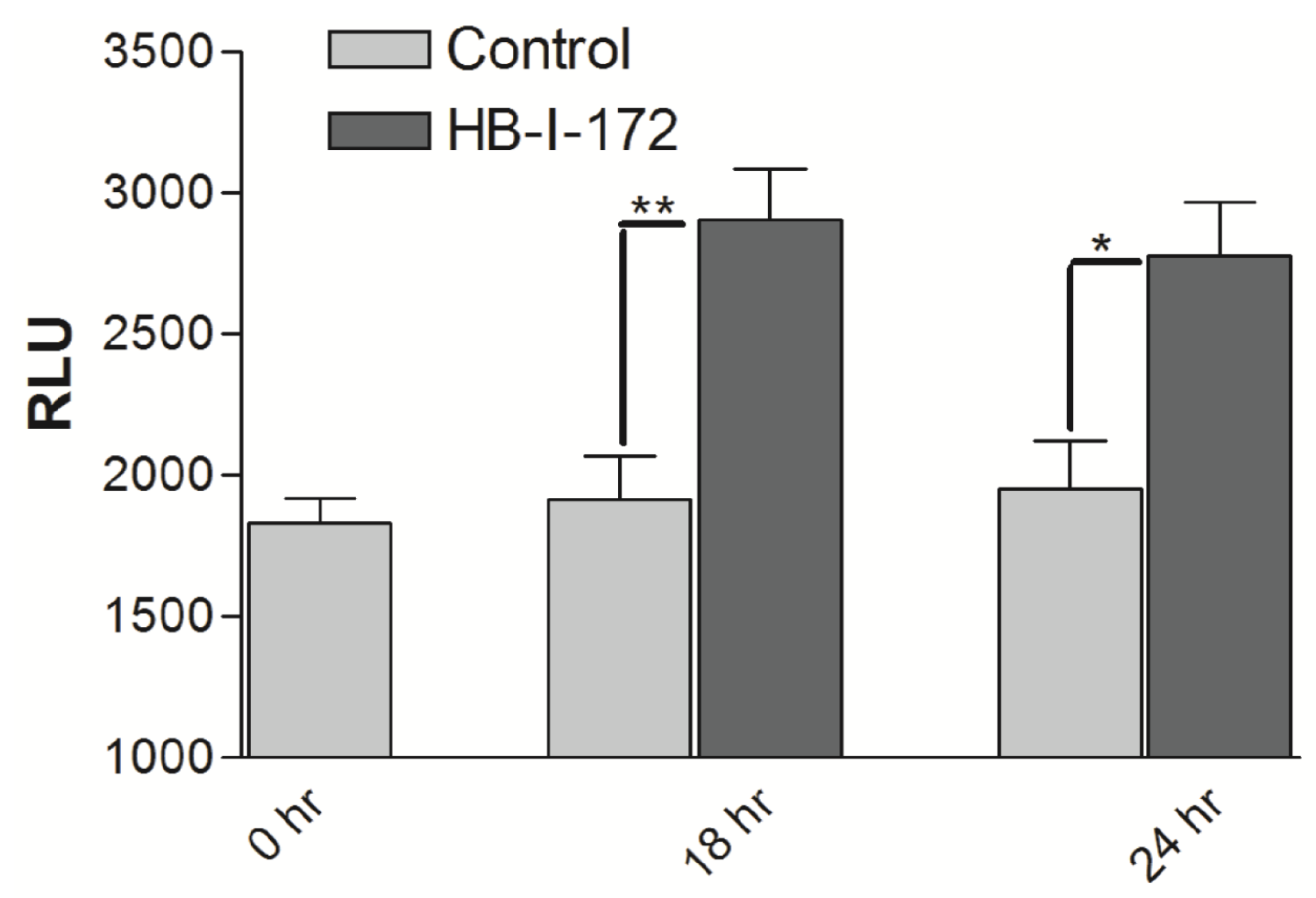

Figure 3.18: Caspase 9 activity of U-87 MG cells treated with HB-I-172

U-87 MG cells were treated with HB-I-172 at the $\mathrm{EC}_{50}$ for 18 and 24 hours. Cells were seeded in white, opaque $96-$ well culture plates at a density of 10,000 cells/well in a volume of $100 \mu 1$ media. The cells were allowed to attach overnight and the complete growth media was changed to media containing $1 \%$ serum for 24 hours to equilibrate intracellular signaling pathways, then media was changed to either media containing $1 \%$ serum or media containing $1 \%$ serum plus HB-I-172. At the designated time intervals $100 \mu 1$ of Caspase-Glo ${ }^{\circledR} 9$ Assay Reagent (Promega) was added to each well and the plate mixed on a rotary shaker for 30 seconds. After incubation at room temperature for 90 minutes the luminescence was read on a Biotek Synergy 2 plate reader. The luminescent signal is proportional to the amount of caspase 9 activity present. All data points represent 6 independent determinations. Solid lines indicate comparisons between control and drug treated samples. ${ }^{*}=\mathrm{p}<0.01, * *=\mathrm{p}<0.005$. 


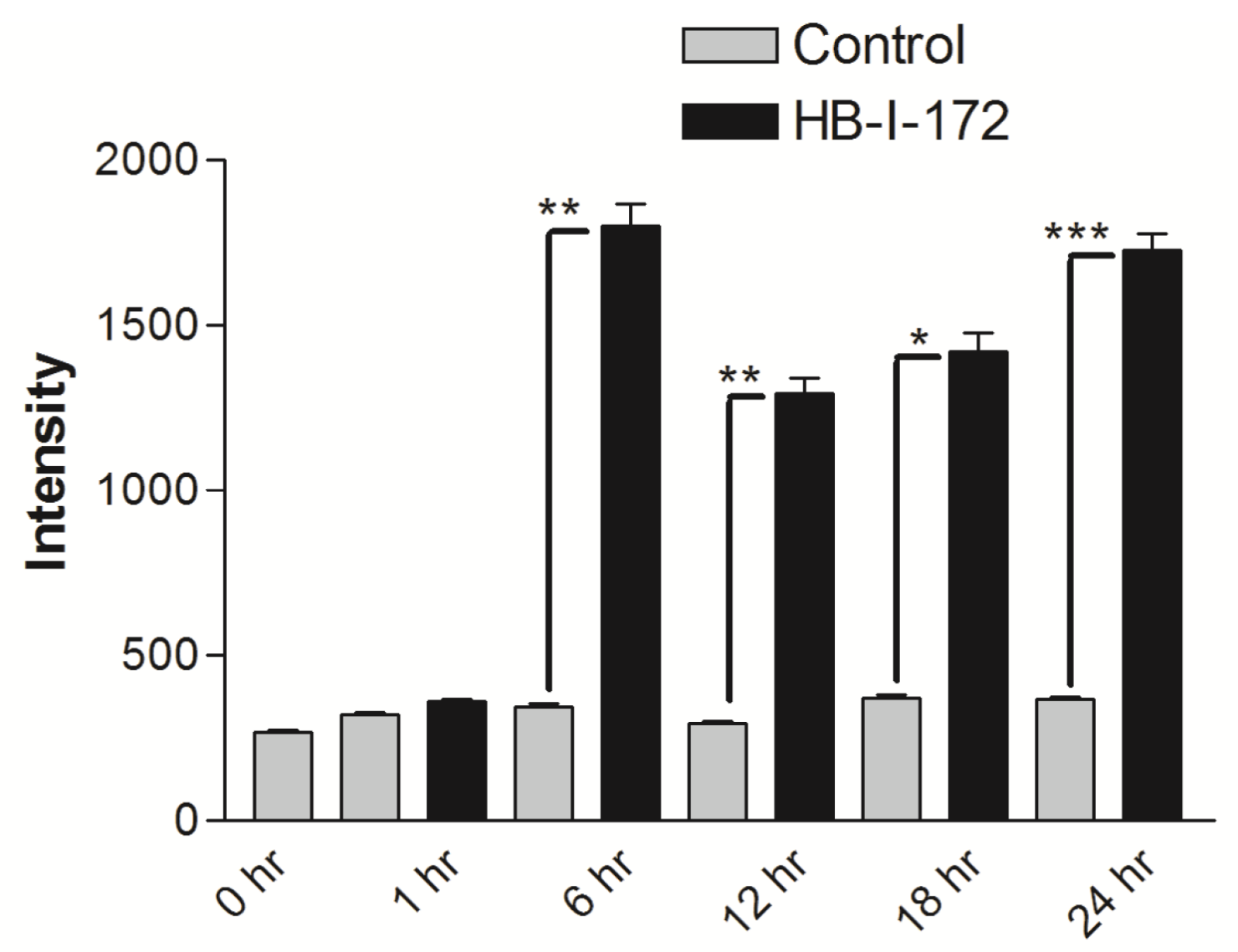

Figure 3.19: Cleaved PARP profile of U-87 MG cells treated with HB-I-172

U-87 MG cells were treated with HB-I-172 at the $\mathrm{EC}_{50}$ for the designated time intervals. See legend of Figure 3.8 for experimental details. Solid lines indicate comparisons between control and drug treated samples. $*=p<0.000001, * *=p<0.0000005, * * *=$ $\mathrm{p}<0.00000005$. 

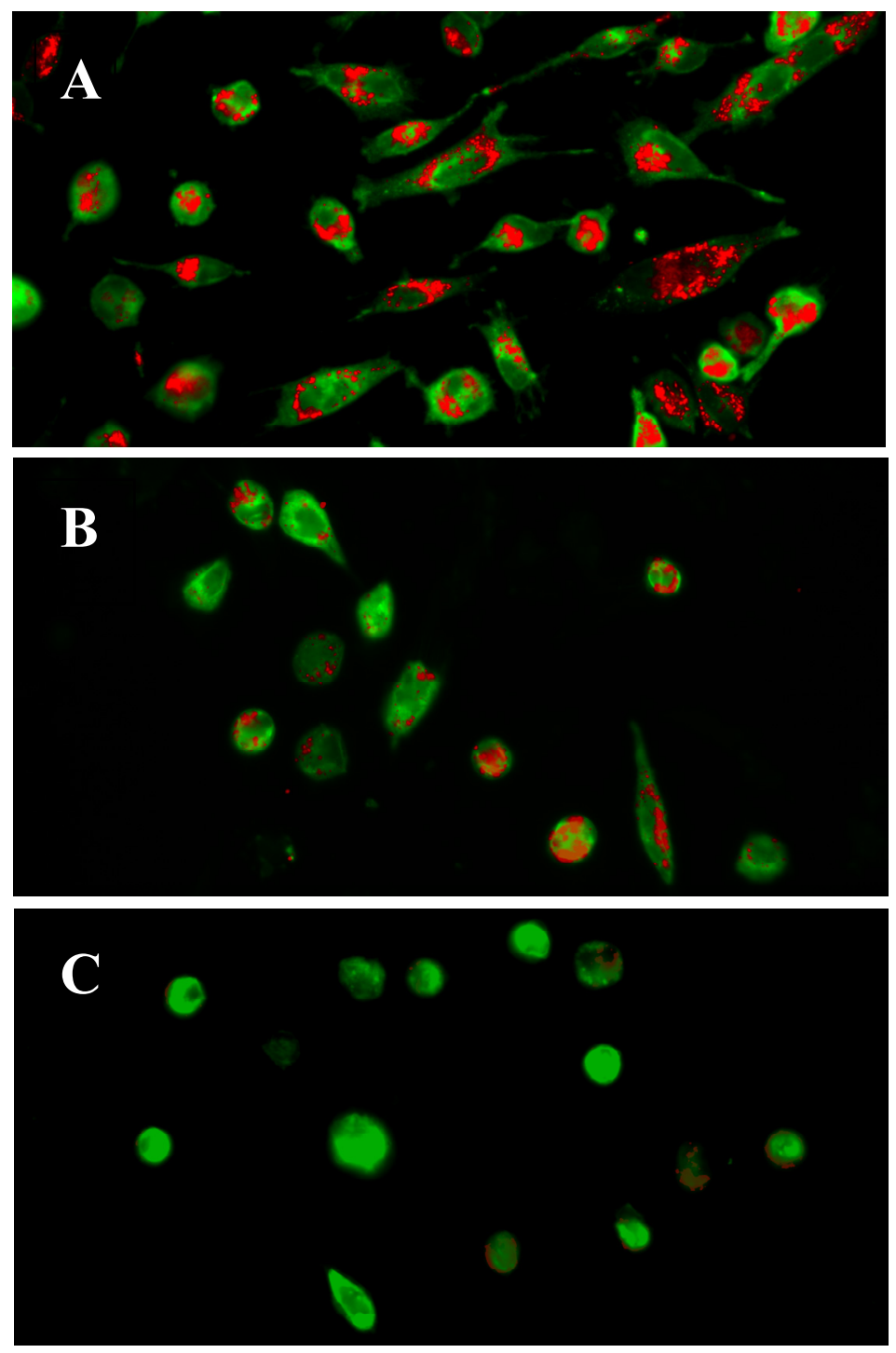

Figure 3.20: Effects on membrane polarization in U-87 MG cells treated with HB-I-172

Change in mitochondrial membrane polarization $(\Delta \psi \mathrm{m})$ with DePsipher ${ }^{\circledR}$ - U-87 MG cells were treated with HB-I-172 at the $\mathrm{EC}_{50}$ for 6 and 18 hours, and then stained with dual fluorescent stain; green fluorescence shifts to red emission following the formation of eximers within mitochondria as stain crosses polarized outer mitochondrial membrane. Mitochondrial depolarization by HB-I-172 inhibits DePsipher permeability and subsequent red fluorescence. U-87 MG cells were imaged using an Olympus IX71 inverted fluorescence microscope fitted with a Retiga-SRV camera. Control (vehicle treated) cells are shown in panel A, 6 hours post drug treatment in panel B, and 18 hours post drug treatment in panel $\mathrm{C}$. 

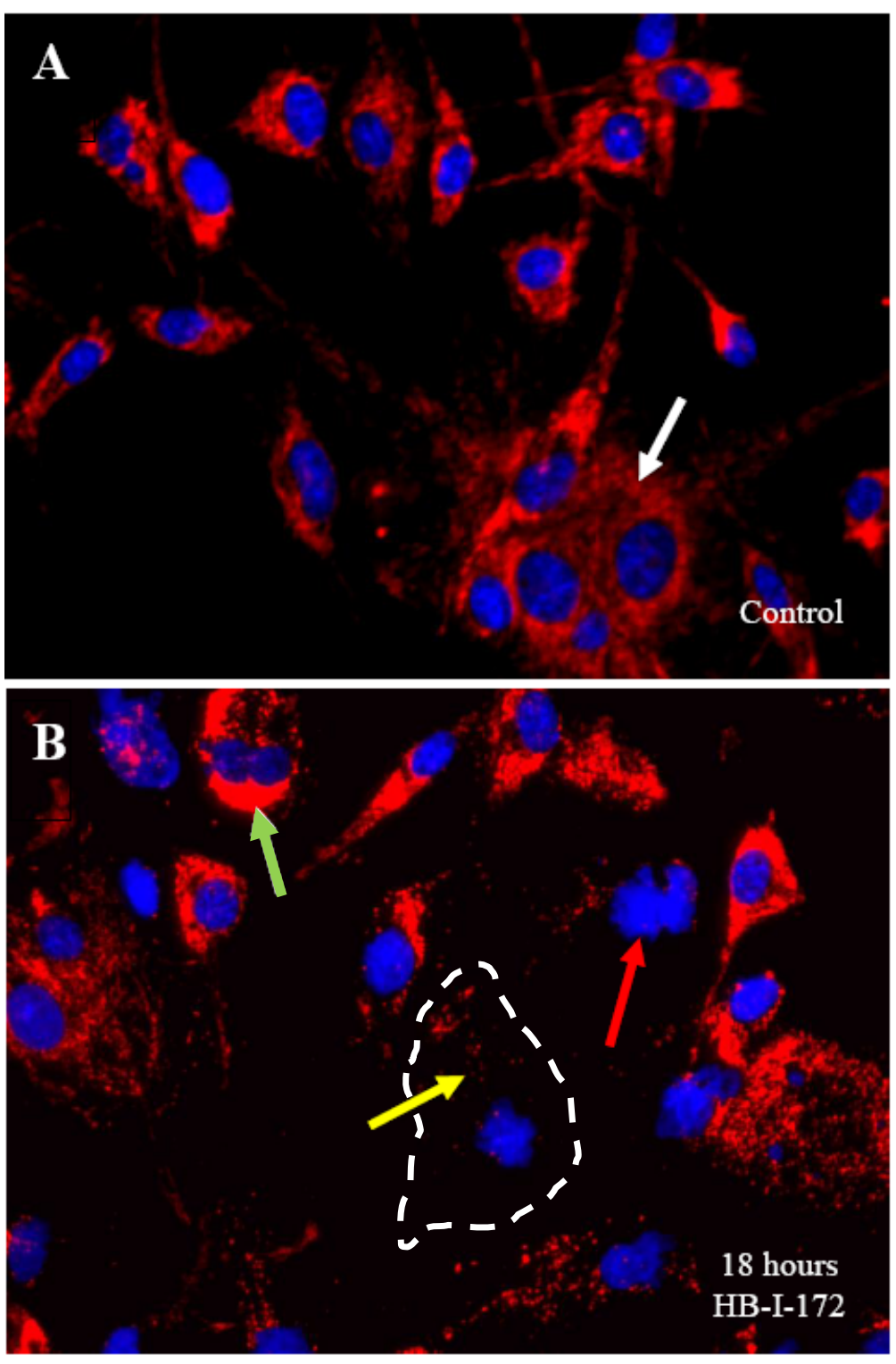

Figure 3.21: Effects on cytochrome $\mathrm{C}$ release in U-87 MG cells treated with HB-I-172

U-87 MG cells were treated with HB-I-172 at the $\mathrm{EC}_{50}$ for 18 hours and evaluated for changes in localization of cytochrome $\mathrm{C}$ (red); nuclei are shown in blue. Control (vehicle treated) cells are shown in panel A and 18 hour drug treated cells are shown in panel B. Control cells exhibit localization of cytochrome $\mathrm{C}$ in the mitochondria (white arrow) along the cytoskeletal network. HB-I-172 treated cells show a diffuse pattern of labeling (green arrow) indicating that cytochrome $\mathrm{C}$ has been released from the mitochondria into the cytosol. Some of the drug treated cells show a much fainter red labeling (yellow arrow, approximate cell outline represented by dashed white line) indicating depletion of cytochrome C. Some drug treated cells also show DNA fragmentation and condensation (red arrow). U-87 MG cells were imaged using an Olympus IX71 inverted fluorescence microscope fitted with a Retiga-SRV camera. 


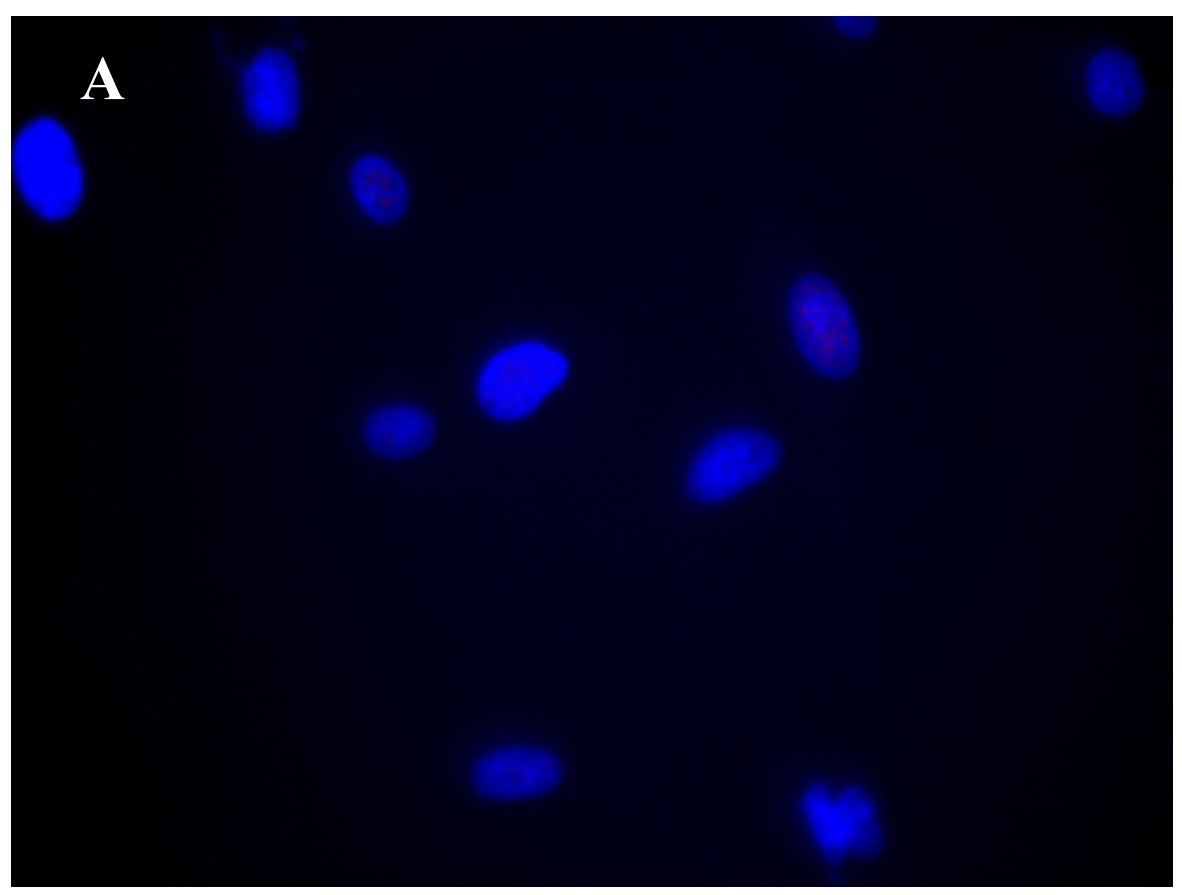

B

Figure 3.22: Activity of PARP in U-87 MG cells treated with HB-I-172

U-87 MG cells were treated with HB-I-172 at the $\mathrm{EC}_{50}$ and evaluated for activity of PARP (red) in the nuclei (blue). Control (vehicle treated) cells are shown in panel A and 6 hour drug treated cells are shown in panel B. HB-I-172 causes activation of PARP as seen by the red staining in the nuclei (white arrows). U-87 MG cells were imaged using an Olympus IX71 inverted fluorescence microscope fitted with a Retiga-SRV camera. 
We also conducted a microscopic study to assess the effects of HB-I-172 on cytoskeleton structure based on the known activity of RhoA and the possibility of a GPR55 initiated mechanism. At 18 hours post HB-I-172 treatment the cytoskeleton manifests profound contraction of F-actin and $\alpha$-tubulin (Figure 3.23). Though it is not possible to conclude a specific mechanism, this result provides a basis for further evaluating the possibility of RhoA-ROCK contributions to cell death.

\subsubsection{Proposed Mechanism of the Anti-Glioma Activity of HB-I-172}

The ability of HB-I-172 (and possibly the other triaryl cannabinoids) to block two major pro-survival signaling pathways, namely Raf1/MEK/ERK and PI3K/Akt, may account for its broad spectrum antineoplastic activity. Activation of these pathways is essential for cell survival and over-activation of each has been shown to be common in many forms of cancer. ${ }^{119}$ Constitutive Akt over-activation can many times be attributed to amplification of the Akt gene or mutations in components of the upstream signaling pathway. ${ }^{139}$ Akt plays a critical role in the regulation of cell survival and apoptosis by phosphorylation and inhibition of a number of proteins including GSK-3 $\beta^{140}$ and caspase9. ${ }^{141}$ The Raf1/MEK/ERK pathway has been shown to be over-active in nearly one-third of all human cancers. This pathway appears to play a large role in cell survival and transduction of differentiation and mitogenic signals. ${ }^{142}$ Both of these pro-survival pathways converge on the pro-apoptotic Bcl-2 protein BAD. Each pathway (Akt directly and ERK1/2 through p90 ribosomal S6 kinase (p90RSK)) phosphorylates and inhibits $\mathrm{BAD}$ which allows the pro-survival members of the Bcl-2 family to remain active and prevent apoptosis. Since each pathway is capable of inhibiting BAD, it would be predicted that inhibition of a single pathway would not be sufficient to bring about apoptosis. This has, in fact, been demonstrated in a recent report by Ellert-

Miklaszewska, Kaminska, and Konarska in which specific inhibition of each pathway in the C6 glioma cell line did not bring about any significant cell death. ${ }^{119}$ These two pathways share another common signaling protein, namely GSK-3 $\beta$. Akt and p90RSK both inhibit GSK-3 $\beta$ by phosphorylation. Inhibition of the two pathways leads to increases in the activity of GSK-3 $\beta$ which, in turn, activates the pro-apoptotic Bcl-2associated $X$ protein (BAX) which leads to loss of mitochondrial membrane potential. ${ }^{143}$ GSK-3 $\beta$ also up-regulates the transcription factor protein $53(\mathrm{p} 53)$ which leads to further up-regulation of BAX and also the pro-apoptotic Bcl-2 homologous antagonist/killer (BAK). ${ }^{144}$ By down-regulation of both the PI3K/Akt and Raf1/MEK/ERK pathways, HB-I-172 decreases the downstream phosphorylation of BAD and allows it to dimerize with Bcl-2 in the mitochondrial membrane leading to loss of membrane potential, release of cytochrome $\mathrm{C}$ and activation of the caspase cascade beginning with caspase 9 (Figure 3.24).

The down regulation of the two pro-survival pathways in glioblastoma by HB-I172 does not give clear evidence for the molecular target of the drug. We know that HB$\mathrm{I}-172$ is an inverse agonist at the CB2 receptor. Since CB2 signals through $\mathrm{G}_{\alpha \mathrm{i}}$, the effects of an agonist would lead to decreases in cAMP which would decrease the activity of protein kinase A (PKA) and up-regulate the Rafl/MEK/ERK pathway. An inverse agonist, on the other hand, would cause increases in cAMP which would increase the 

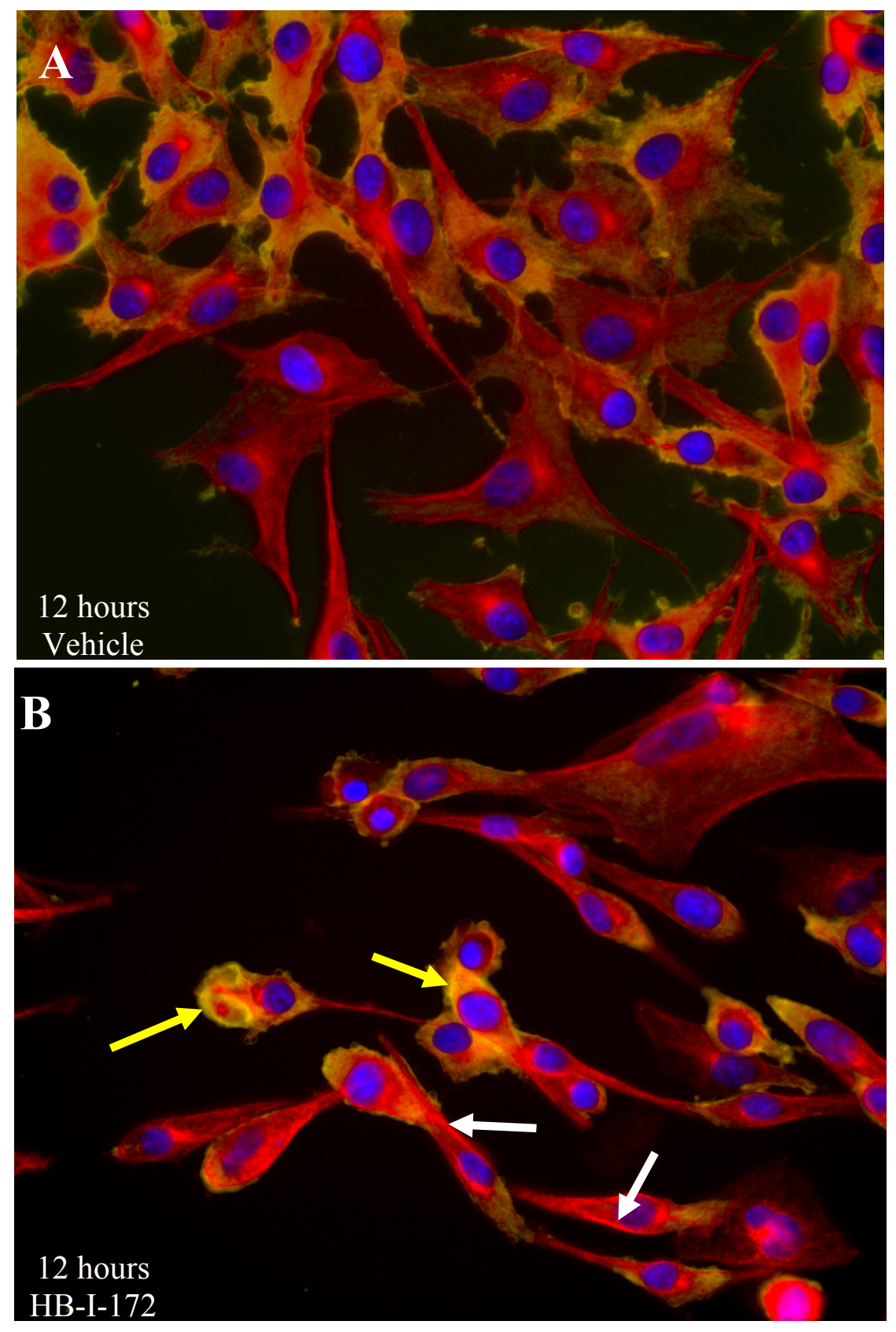

Figure 3.23: Effects on cytoskeleton structure in U-87 MG cells treated with HB-I-172

U-87 MG cells were treated with HB-I-172 and evaluated for changes in F-actin (yellow) and $\alpha$-tubulin (red), nuclei are shown in blue. Control (vehicle treated) cells are shown in panel A and 12 hour drug treated cells are shown in panel B. HB-I-172 causes significant contraction of the cytoskeleton network as evidenced by the compact F-actin (yellow arrows) and $\alpha$-tubulin (white arrows) around the nuclei. U-87 MG cells were imaged using an Olympus IX71 inverted fluorescence microscope fitted with a Retiga-SRV camera. 


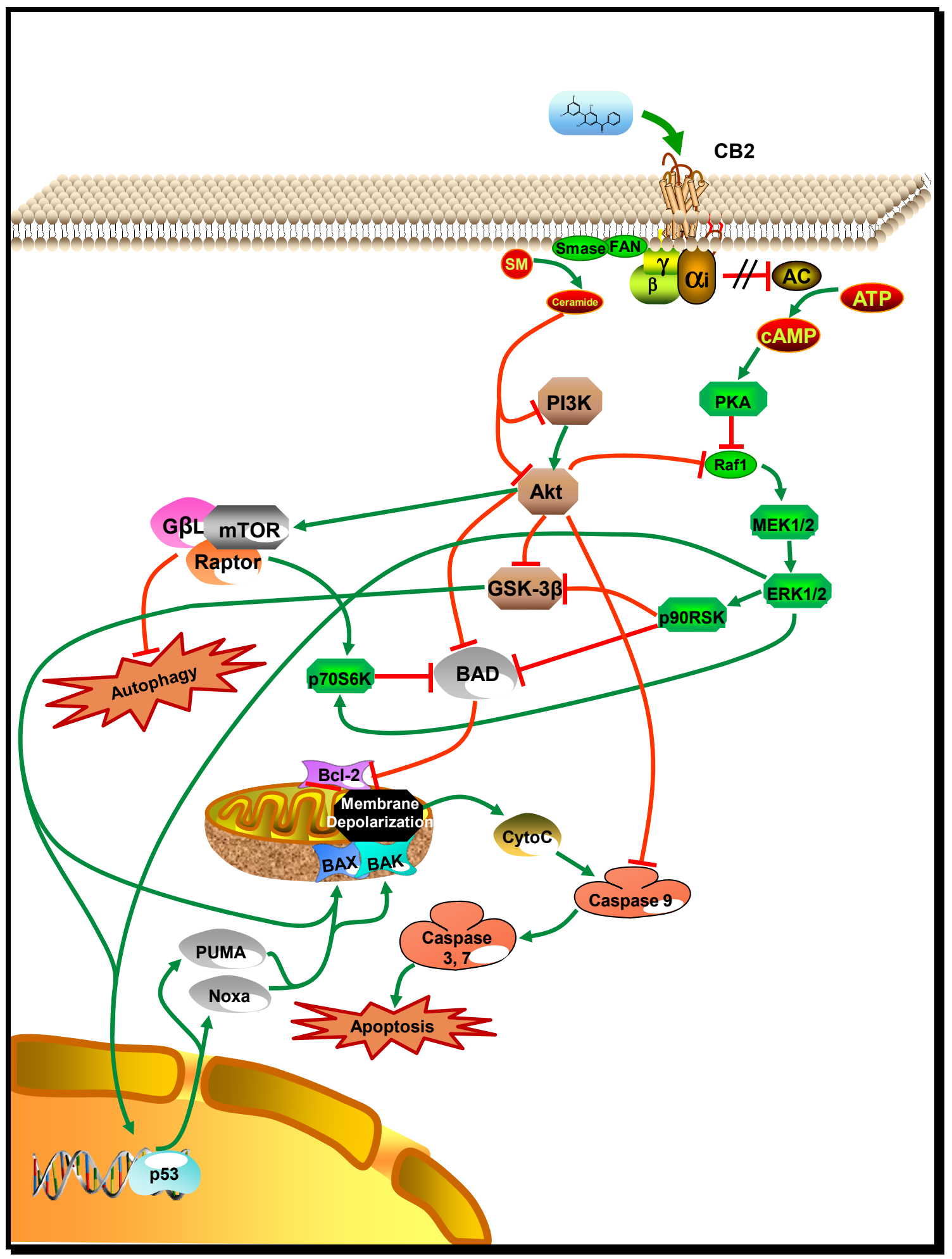

Figure 3.24: Proposed induction of apoptosis by HB-I-172 mediated through CB2 See text for definitions of abbreviations. 
activity of PKA and down-regulate Raf1/MEK/ERK. This correlates with our observed decreases in phosphorylated MEK1/2 and ERK1/2. The inhibition of the PI3K/Akt pathway can also be explained by a CB2 mediated mechanism. Ligation of the CB2 receptor can cause an intracelleular buildup of ceramide produced from sphingomyelin by the action of sphingomyelinase which is activated by factor associated with neutral sphingomyelinase (FAN) (Figure 3.24). The increase in ceramide inhibits the activation of both PI3K and $\mathrm{Akt}^{145}$ which, again, agrees with our data.

HB-I-172 acting through CB2 alone would however not account for the observed effects on the cytoskeleton of U-87 MG cells. The formation of stress fibers following treatment with HB-I-172 is better explained through a GPR55 mediated mechanism. GPR55 signals through $\mathrm{G}_{\alpha 12 / 13}$ which activates the Rho family of GTPases through the activity of guanine nucleotide exchange factors (GEFs). One of these GTPases, Rho A, then activates focal adhesion kinase (FAK) and Rho-associated, coiled-coil containing protein kinase (ROCK) which lead to cytoskeleton rearrangement and formation of stress fibers. ${ }^{146}$ GPR55 can also affect both the PI3K/Akt and Raf1/MEK/ERK pathways through the action of Ras on PI3K and Raf1. For this to be a viable explanation, HB-I172 would have to be an antagonist or an inverse agonist at GPR55 also. This would lead to decreases in the activities of Ras-related C3 botulinum toxin substrate $1(\operatorname{Rac} 1)$ and cell division control protein 42 homolog (cdc42) and downstream decreases in p21activated kinase (PAK) and Ras which would lead to decreases in the activity of PI3K and Raf1 (Figure 3.25).

The effects of either CB2 or GPR55 (or possibly both) on the two pro-survival pathways would be sufficient to initiate apoptosis in glioma cells via activation of BAD and GSK-3 $\beta$ by each pathway, the activation of caspase 9 by Akt, and the activation of p53 by both GSK-3 $\beta$ and ERK1/2. There, however, seems to be more to the puzzle than the inhibition of these two pathways. Our observation that caspase 3 is active approximately 12 hours before caspase 9 led us to look for alternative mechanisms for the activation of caspase 3 . One such possibility is through endoplasmic reticulum (ER) stress caused by the unfolded protein response (UPR). ER stress has been shown to initiate apoptosis in a mitochondria independent manner by activation of caspase 3 via caspase $12^{147}$ (Figure 3.26). ER stress can also initiate apoptosis via a mitochondria dependent pathway involving the release of calcium from the ER into the cytosol which causes the collapse of the mitochondrial membrane potential and leads to the release of cytochrome C. ${ }^{148}$ ER stress can play further roles in the initiation of apoptosis also. The UPR can lead to activation of the transcription factor $\mathrm{C} / \mathrm{EBP}$ homologous protein (CHOP) which inhibits the pro-survival Bcl-2 at the transcription level. ${ }^{149} \mathrm{We}$ observed increased translocation of active CHOP to the nuclei of HB-I-172 treated U-87 MG cells in as little as 3 hours. At the 3 hour time point, the percent of drug treated cells showing positive immunohistochemical staining for CHOP in the nucleus was nearly 8-fold higher than in control cells (data not shown). The number of CHOP positive cells in the drug treated group continued to grow throughout the length of the experiment and was 40 -fold higher than control at the 18 hour terminal time point. ER stress has also been shown to inhibit Akt by activation of the tribbles homolog 3 (TRIB3) pseudo-kinase by the transcriptional co-activator $\mathrm{p} 8 .{ }^{134}$ Much like ERK1/2, ER stress can increase the activity of the tumor suppressor p53 which leads to the transcription of p53-up-regulated 


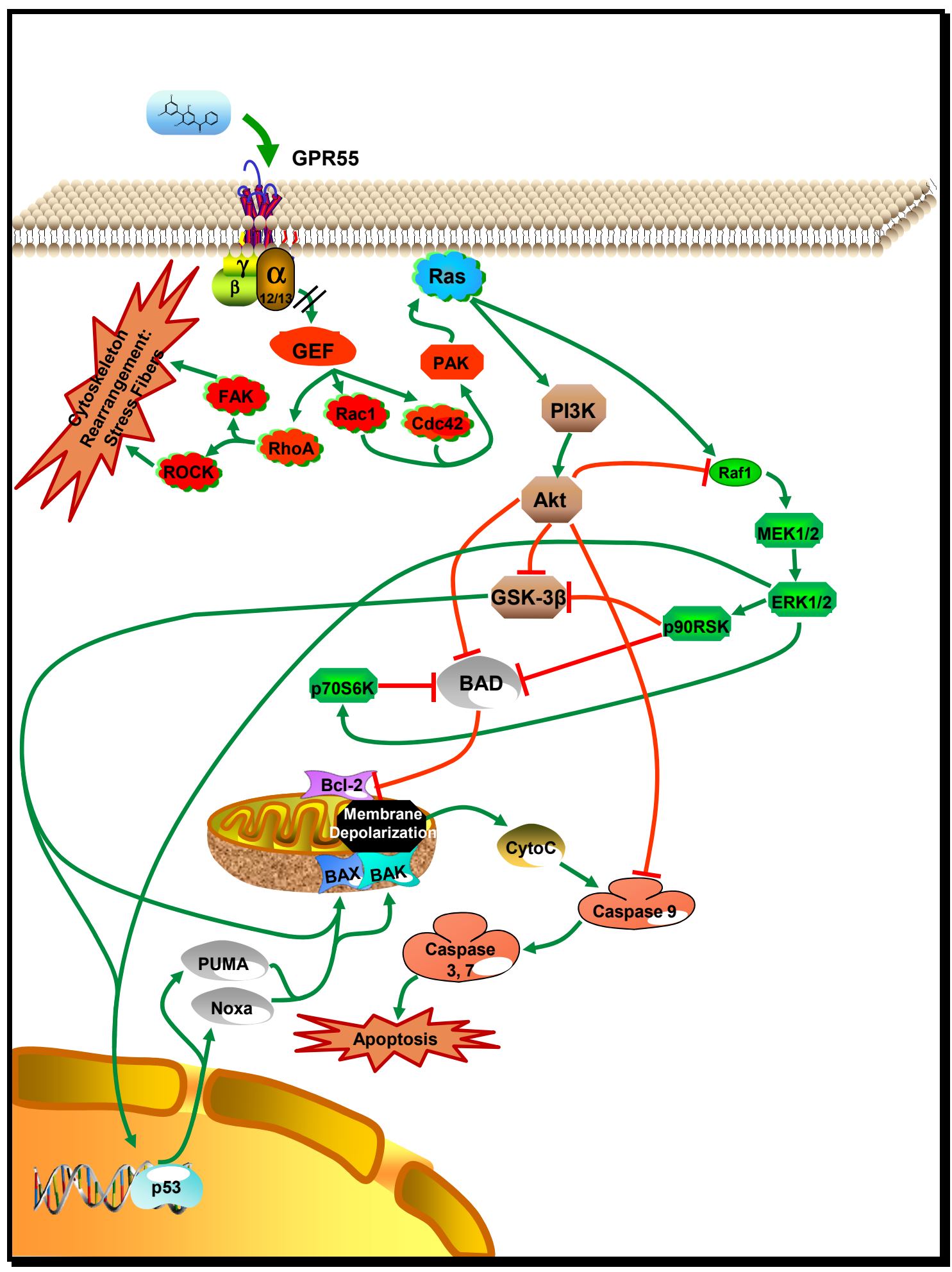

Figure 3.25: Proposed induction of apoptosis by HB-I-172 mediated through GPR55 See text for definitions of abbreviations. 


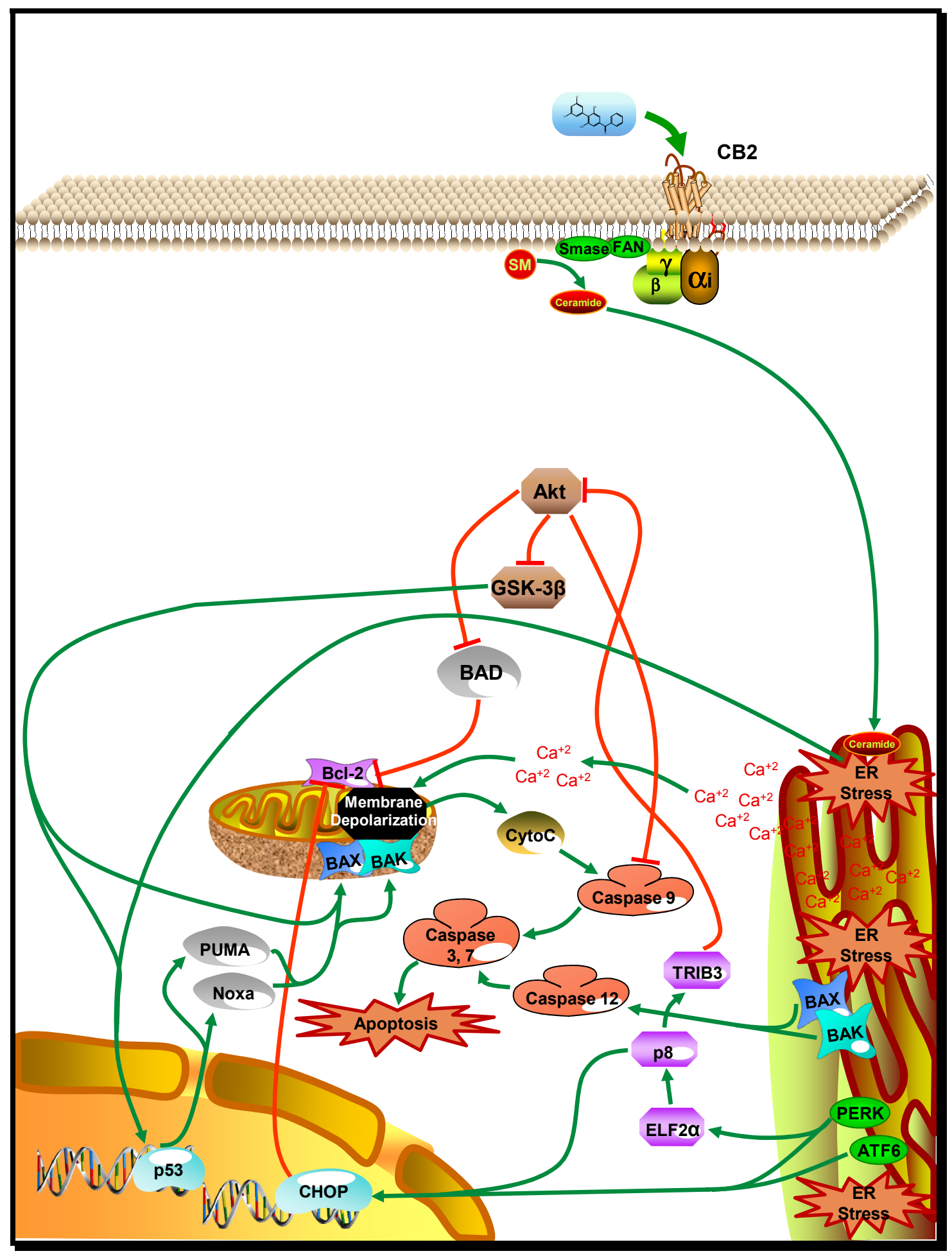

Figure 3.26: Proposed induction of apoptosis by HB-I-172 initiated by ER stress mediated through CB2

See text for definitions of abbreviations. 
modulator of apoptosis (PUMA) and Noxa which cause cytochrome $\mathrm{C}$ release from mitochondria through activation of BAX and BAK. ${ }^{150}$

ER stress has been shown to be caused by the accumulation of de novo synthesized ceramide in the ER. ${ }^{151}$ Considering the increase in ceramide synthesis caused by ligation of the $\mathrm{CB} 2$ receptor, this seems a reasonable pathway to consider. In the recent report by Salazar et al., THC was shown to induce ER stress in human glioma cells which led to autophagy preceding apoptosis. ${ }^{121}$ They determined that autophagy was initiated by the inhibition of Akt by TRIB3 which leads to the down-regulation of the autophagy inhibitor mammalian target of rapamycin complex 1 (mTORC1) (Figure 3.27) which consists of mTOR, regulatory associated protein of mTOR (Raptor), and mammalian LST8/G-protein $\beta$-subunit like protein $(\mathrm{G} \beta \mathrm{L})$. Though the authors were not able to determine the exact manner in which autophagy leads to apoptosis, they did determine that it is a necessary precursor to cannabinoid induced apoptotic cell death in glioblastoma. This, in fact, does fit with our data. The human glioblastoma cell line LN229 was treated with HB-I-172 at the cytotoxic $\mathrm{EC}_{50}$ and the cells were immunohistochemically stained for the microtubule associated protein 1 light chain 3 (LC3B) which associates into membranes of autophagosomes during autophagy. The number of cells positive for LC3B increased 6-fold over control in just 3 hours with a 20fold increase at the 18 hour terminal time point (data not shown). This corroborates the autophagy component of cannabinoid mediated glioma toxicity as reported by Salazar et al. The authors of the study however attributed the process to activation of the CB1 receptor. We do not believe that the CB1 receptor plays a role in the activity of HB-I-172 due to the previously mentioned finding that it is an antagonist at CB1. This same experiment needs to be repeated using U-87 MG to strengthen the evidence for autophagy in this mechanism of cell death.

I believe that there is clear evidence to support a mechanism of cell death in U-87 MG cells following HB-I-172 exposure that involves multiple targets such as CB2 and GPR55. The early intracellular events following drug exposure are most likely due to inverse agonism at the $\mathrm{CB} 2$ receptor. The increases in cAMP and ceramide following the ligation of CB2 by HB-I-172 would clearly lead to the down-regulation of the PI3K/Akt and Raf1/MEK/ERK pathways which is seen beginning at one hour post exposure. ER stress, as a result of ceramide production, is responsible for the activation of caspase 3 six hours before the observed activation of caspase 9. The case for ER stress is further supported by other events at earlier time points such as the activity of CHOP and the observance of autophagy as determined by LC3B staining at 3 hours. We believe that the observed changes in the cytoskeleton are due to GTPase activity resulting from ligation of the GPR55 receptor. The role that GPR55 plays in the down regulation of the PI3K/Akt and Rafl/MEK/ERK pathways remains to be determined. As previously stated, HB-I-172 could perhaps be an antagonist/inverse agonist at GPR55 which would lead to the down-regulation of Ras and the inhibition of the two pro-survival pathways. If HB-I-172 were a GPR55 agonist, an increase in the signaling of these two pathways would be expected. Cross talk and inhibition of signaling between the two receptors has been demonstrated following anandamide ligation. ${ }^{152}$ The complete proposed signaling pathway is shown in Figure 3.28. 


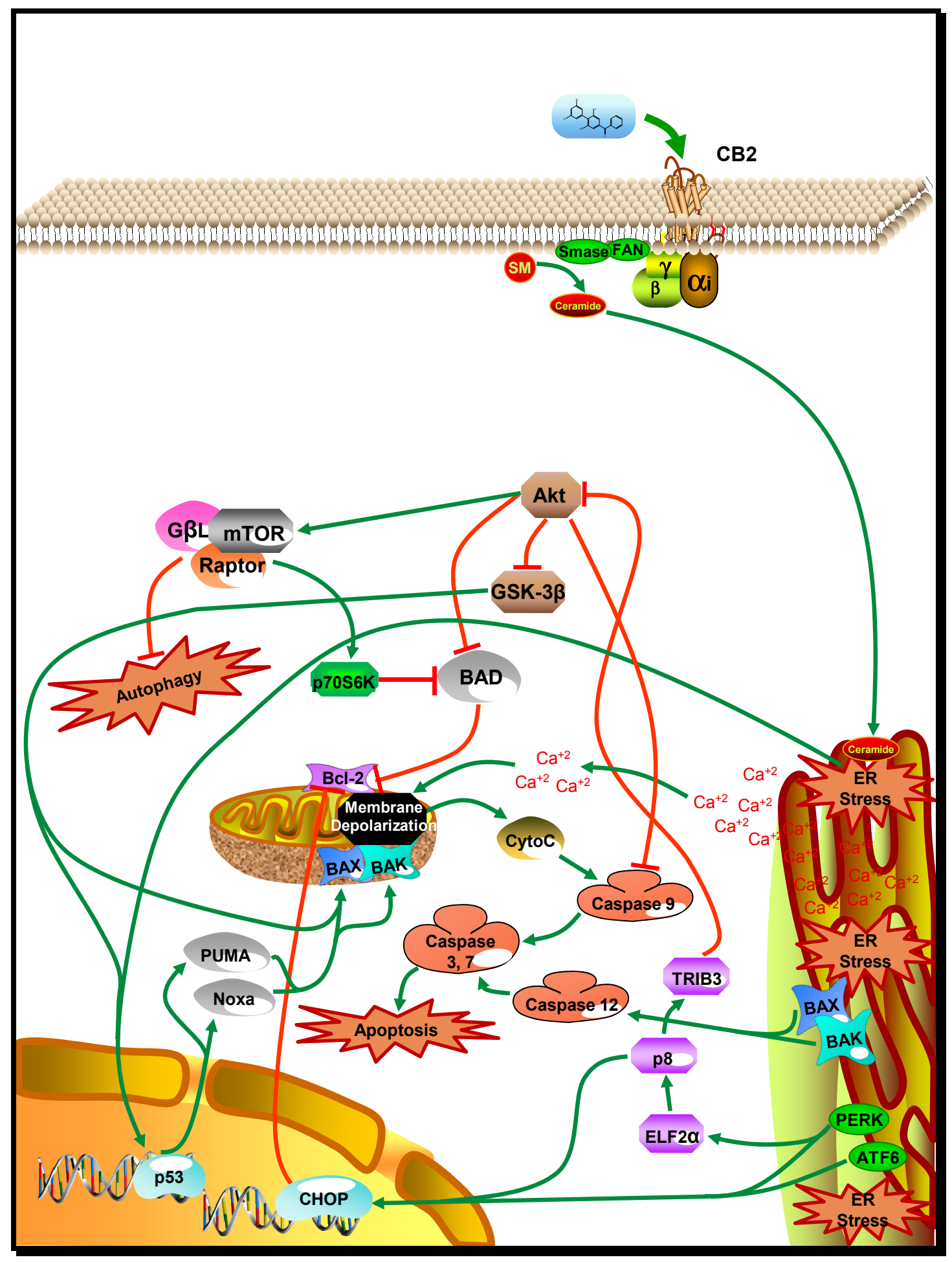

Figure 3.27: Proposed induction of autophagy followed by apoptosis by HB-I-172 initiated by ER stress mediated through CB2

See text for definitions of abbreviations. 


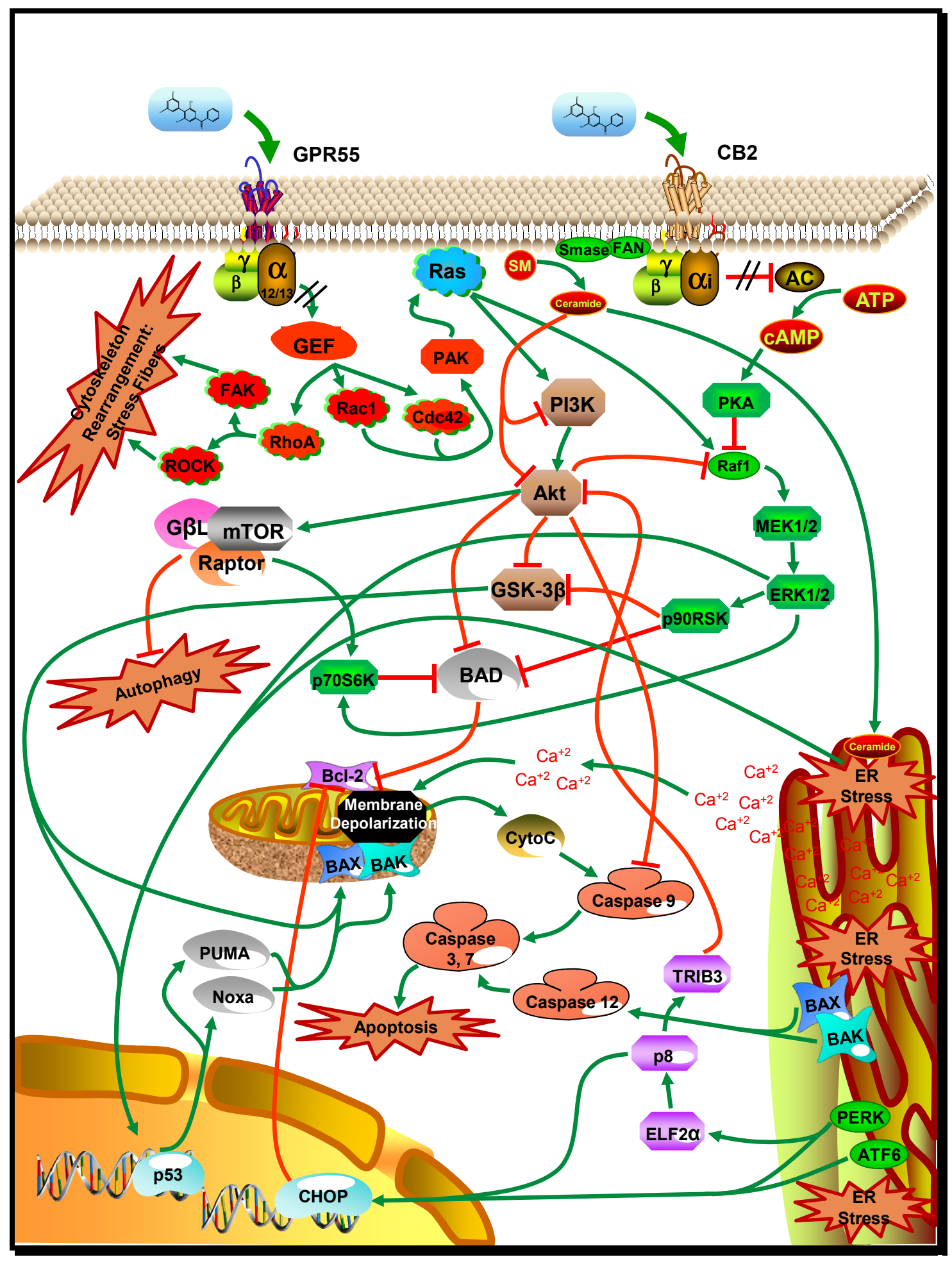

Figure 3.28: Entire proposed mechanism of glioma cell death by HB-I-172 mediated by CB2 and GPR55

See text for definitions of abbreviations. 
Much remains unanswered about the signaling cascade initiated by HB-I-172 in glioblastoma. We are currently in the process of preparing for a series of assays that will evaluate the activation of the GTPases RhoA and Rac1. This will give a clearer picture of the activity, or lack thereof, of GPR55 following exposure to HB-I-172. Selective inhibition of any of a number of signaling proteins in the proposed pathways either pharmacologically or by shRNA knock-down would give further insight into the importance of each segment of the cascade initiated by HB-I-172.

While many of the downstream signaling pathways have been identified, the receptor or receptors responsible for initiating the cascades are yet to be elucidated. We have utilized pharmacological knockouts in an effort to identify the target of HB-I-172 action. Pharmacological intervention has distinct limitations due to overlap in activity of known agonists and antagonists of the CB1, CB2, and GPR55 receptors. Our preliminary data suggests that both CB2 and GPR55 are both targets of HB-I-172 however this cannot be confirmed without the selective knock-down of each $\mathrm{CB}$ receptor subtype. Knowledge of the target is critical for the development of a complete mechanism of action for the anti-glioblastoma activity of HB-I-172. The contributions of each cannabinoid receptor are currently under investigation using shRNA stably transfected cell lines.

\subsection{Chemical Synthesis}

\subsubsection{General}

In order to synthesize the core of the triaryl compounds 8-16, and provide a route for future functionalization of this series, an efficient synthetic route was designed. Commercially available syringaldehyde $\mathbf{1 7}$ was selected to form the A ring in the triaryl series. The phenolic hydroxyl was activated for a Suzuki coupling by reaction with trifluoromethanesulfonic anhydride to yield intermediate 18 (Scheme 3.1). Formation of the biaryl ring system was carried out via a Suzuki coupling reaction with 3,5dichlorophenyl boronic acid 19 in the presence of tetrakis(triphenylphosphine) palladium, and potassium carbonate in toluene and water to yield the aldehyde intermediate $\mathbf{2 0}$ (Scheme 3.1). The resulting aldehyde was reacted with phenyl magnesium bromide 21 to obtain the racemic alcohol $\mathbf{8}$, which was oxidized using PCC, to yield the ketone $\mathbf{1 4}$. The ketone 14 was dimethylated using dimethylzinc and titanium (IV) chloride to yield compound 15 (Scheme 3.2). The mono- and di-deprotected analogs of 14 and 15 were prepared by reaction with $\mathrm{BBr}_{3}$ (Scheme 3.3). To prepare the equivalent analogs possessing the hydroxyl linker compounds $\mathbf{9}$ and $\mathbf{1 0}$ were reduced with sodium borohydride to give analogs 12 and 13 (Scheme 3.4).

All chemicals were obtained from Sigma Aldrich or Fisher Scientific Inc. Anhydrous solvents were obtained by distillation over either calcium hydride or metallic sodium and benzophenone. Final compounds and intermediates were purified using column chromatography on the Biotage SP1 system employing Flash column cartridges or by crystallization. NMR spectra were obtained on a Bruker $300 \mathrm{MHz}$ or Varian 


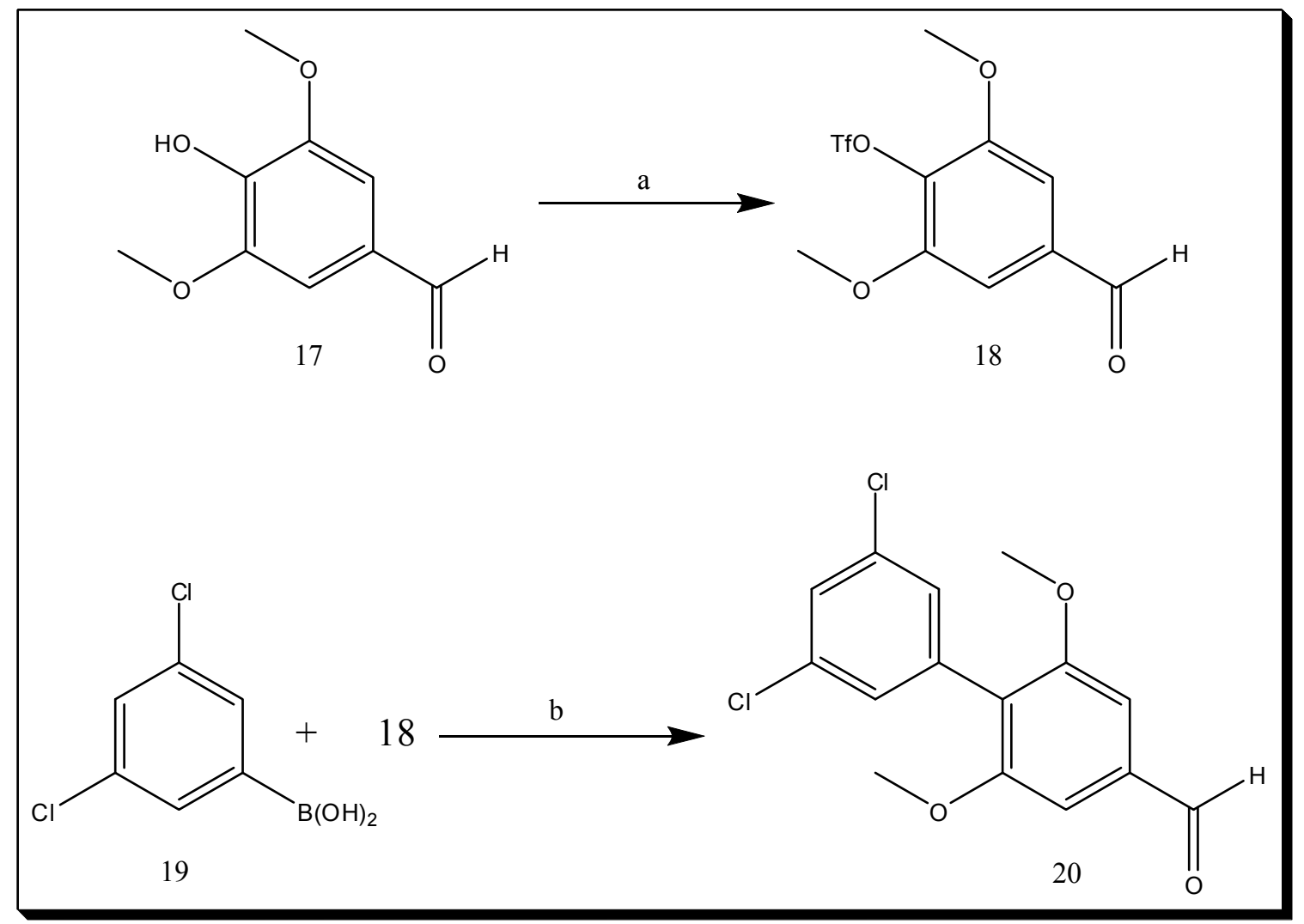

Scheme 3.1: Synthesis of aldehyde intermediate 20

(a) Triflic anhydride, 2,3,5 collidine, DCM, $-20^{\circ} \mathrm{C}, 2 \mathrm{hr}$; (b) $\left(\mathrm{PPh}_{3}\right)_{4} \mathrm{Pd}, \mathrm{K}_{2} \mathrm{CO}_{3}$, toluene / water, reflux, $18 \mathrm{hr}$. 


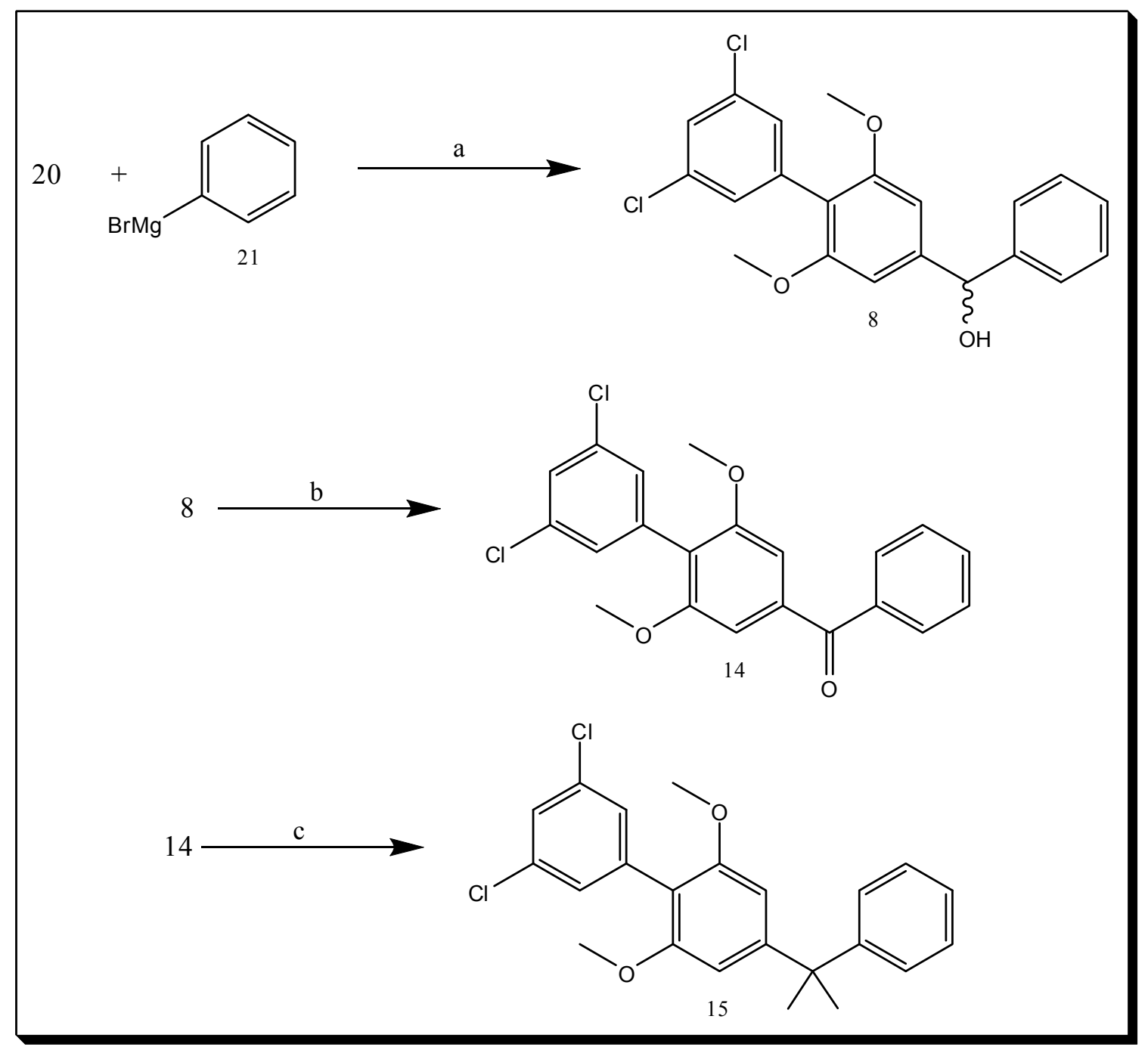

Scheme 3.2: Synthesis of compounds 8, 14, and 15

(a) THF, $0^{\circ} \mathrm{C}, 4 \mathrm{hr}-\mathrm{RT}$ overnight; (b) PCC, DCM, RT, 18 h; (c) $\mathrm{TiCl}_{4}, \mathrm{Zn}\left(\mathrm{CH}_{3}\right)_{2}, \mathrm{DCM}$, $-40^{\circ} \mathrm{C}, 2 \mathrm{hr}-\mathrm{RT}$ overnight. 


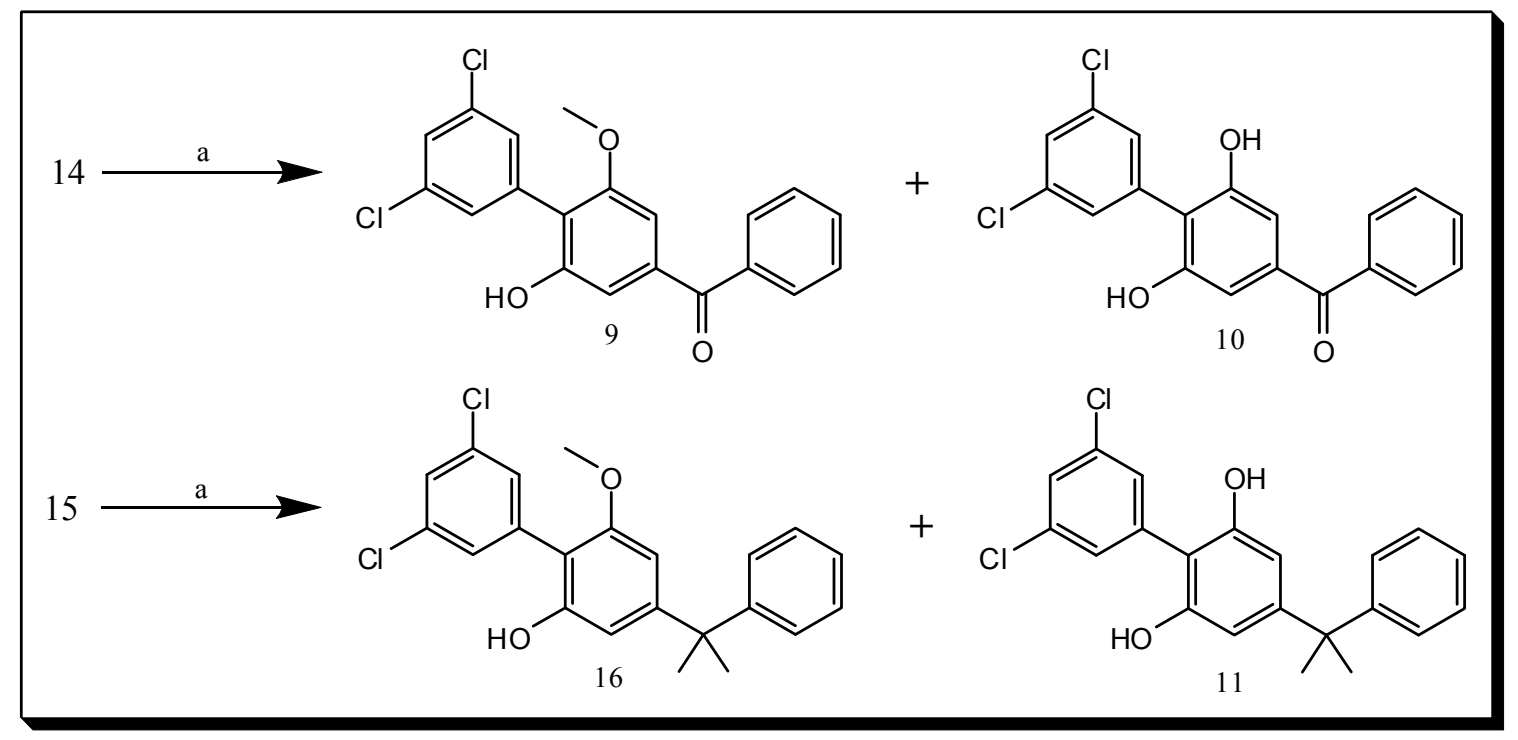

Scheme 3.3: Synthesis of compounds 9, 10, 16, and 11

(a) $\mathrm{BBr}_{3}, \mathrm{DCM},-78^{\circ} \mathrm{C}, 3 \mathrm{hr}-\mathrm{RT}$ overnight. 


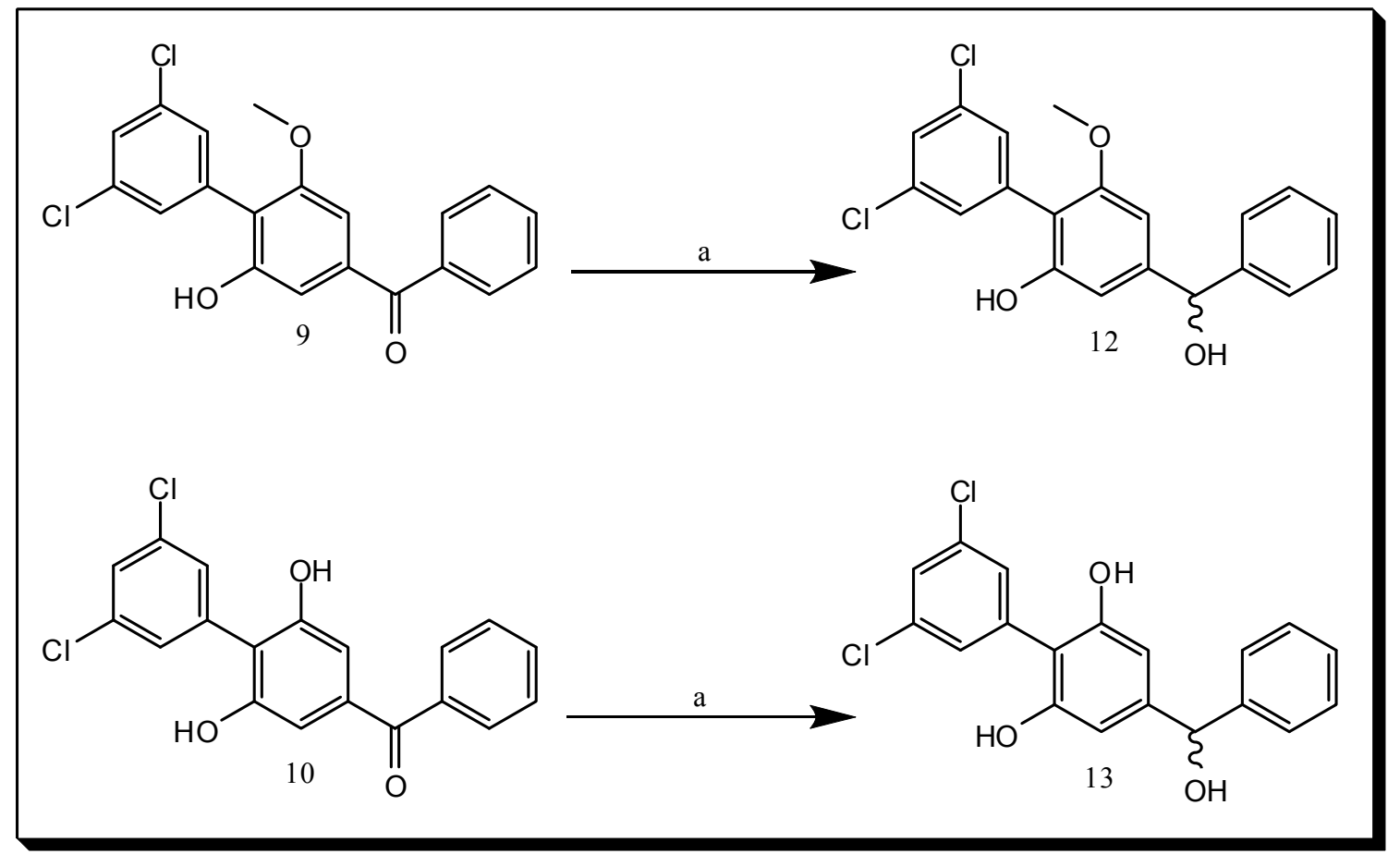

Scheme 3.4: Synthesis of compounds 12 and 13

(a) $\mathrm{NaBH}_{4}, \mathrm{MeOH}, 0^{\circ} \mathrm{C}-\mathrm{RT}, 2 \mathrm{hr}$. 
$500 \mathrm{MHz}$ Inova NMR. HPLC analysis of final products was carried out by gradient elution using water/acetonitrile (0.1\% TFA) with a gradient of 70\% (3min), 50\% (2min), and $10 \%(15 \mathrm{~min})$. A reverse-phase ODS Hypersil column, manufactured by Thermo Scientific, of dimensions $4.6 \times 150 \mathrm{~mm}$ with $5 \mu \mathrm{m}$ particle size was used for HPLC analyses.

\subsubsection{Synthesis of 4-formyl-2,6-dimethoxyphenyl trifluoromethanesulfonate} (18)

Syringaldehyde $17(10 \mathrm{~g}, 55 \mathrm{mmol})$ and $2,3,5$ collidine $(26.7 \mathrm{~g}, 28.7 \mathrm{ml}, 220 \mathrm{mmol})$ were dissolved in anhydrous DCM $(250 \mathrm{ml})$ under an atmosphere of argon,stirred, and cooled to $-20^{\circ} \mathrm{C}$ followed by the dropwise addition of triflic anhydride $(23.4 \mathrm{~g}, 14 \mathrm{ml}$, $83 \mathrm{mmol})$. The reaction was maintained at this temperature, with stirring, for 2 hours. The reaction was stopped by the addition of saturated aqueous $\mathrm{NaHCO}_{3}$. The layers were separated and the aqueous layer was extracted with DCM. The organic fractions were combined and washed with saturated aqueous $\mathrm{CuSO}_{4}$, water, and brine and dried over $\mathrm{Na}_{2} \mathrm{SO}_{4}$. The organic phase was concentrated and the product purified by column chromatography using $100 \%$ DCM as the mobile phase to yield a white to off-white solid. Yield: $14.14 \mathrm{~g}(82 \%)\left(\mathrm{R}_{f}=0.648 ; 100 \% \mathrm{DCM}\right) . \quad \mathrm{MS}: \mathrm{m} / \mathrm{z}(\mathrm{ESI}, \mathrm{pos})=.315.1[\mathrm{M}+$ $\mathrm{H}]^{+} .{ }^{1} \mathrm{H}$ NMR $\left(300 \mathrm{MHz}, \mathrm{CDCl}_{3}\right): \delta 9.93(\mathrm{~s}, 1 \mathrm{H}), 7.18(\mathrm{~s}, 2 \mathrm{H}), 3.99(\mathrm{~s}, 6 \mathrm{H}),{ }^{13} \mathrm{C} \mathrm{NMR}$ $\left(300 \mathrm{MHz}, \mathrm{CDCl}_{3}\right): \delta 190.52,153.48,136.15,121.00,116.52,106.18,56.88$.

\subsubsection{Synthesis of 3',5'-dichloro-2,6-dimethoxybiphenyl-4-carbaldehyde (20)}

Triflate $18(8 \mathrm{~g}, 25.5 \mathrm{mmol})$ along with 3,5 dichloro phenyl boronic acid $(7.25 \mathrm{~g}$, $38 \mathrm{mmol})$, Tetrakis(triphenylphosphine)palladium $(1.18 \mathrm{~g}, 1.02 \mathrm{mmol})$, and $\mathrm{K}_{2} \mathrm{CO}_{3}(7.05 \mathrm{~g}$, $51 \mathrm{mmol})$ were added to toluene $(100 \mathrm{ml})$ and water $(33 \mathrm{ml})$ in a round bottom flask fitted with a condenser. The reaction was heated to reflux for 18 hours. The reaction was then allowed to cool to room temperature and diluted with diethyl ether. The organic layer was separated and washed with saturated aqueous $\mathrm{NaHCO}_{3}$, water, and brine and then dried over $\mathrm{Na}_{2} \mathrm{SO}_{4}$. The organic phase was concentrated and the product was purified by column chromatography using an EtOAc / hexanes gradient to yield a white solid. Yield: $4.97 \mathrm{~g}(63 \%)\left(\mathrm{R}_{f}=0.402 ; 15 \%\right.$ EtOAc / hexanes). MS: $\mathrm{m} / z$ (ESI, pos. $)=333.1[\mathrm{M}+$ $\mathrm{Na}]^{+}$. ${ }^{1} \mathrm{H}$ NMR $\left(500 \mathrm{MHz}, \mathrm{CDCl}_{3}\right): \delta(\mathrm{ppm}) 9.98(\mathrm{~s}, 1 \mathrm{H}), 7.34(\mathrm{t}, 1 \mathrm{H}), 7.21(\mathrm{~d}, 2 \mathrm{H}), 7.15$ $(\mathrm{s}, 2 \mathrm{H}), 3.83(\mathrm{~s}, 6 \mathrm{H}) .{ }^{13} \mathrm{C} \mathrm{NMR}\left(500 \mathrm{MHz}, \mathrm{CDCl}_{3}\right): \delta(\mathrm{ppm}) 191.70,158.17,137.66$, $136.21,134.44,129.27,127.73,122.95,105.34,56.37$.

\subsubsection{Synthesis of (3',5'-dichloro-2,6-dimethoxybiphenyl-4- yl)(phenyl)methanol (8)}

Aldehyde 20 (4.94g, 15.9mmol) was dissolved in anhydrous THF (100ml), stirred under argon, and cooled to $0^{\circ} \mathrm{C}$. Phenyl $\mathrm{MgBr}$ (21) (3M solution, $1.67 \mathrm{ml}, 5 \mathrm{mmol}$ ) was then added by syringe and the reaction maintained with stirring at $0^{\circ} \mathrm{C}$ for 4 hours and 
then allowed to warm to room temperature overnight. The reaction mixture was then diluted with diethyl ether, washed with water and brine, and dried over $\mathrm{Na}_{2} \mathrm{SO}_{4}$. The organic phase was concentrated and the product was crystallized from EtOAc / hexanes to yield a white solid. Yield: $2.1 \mathrm{~g}(79 \%)\left(\mathrm{R}_{f}=0.341 ; 20 \%\right.$ EtOAc / hexanes). MS: $\mathrm{m} / \mathrm{z}$ $($ ESI, pos. $)=411.1[\mathrm{M}+\mathrm{Na}]^{+} .{ }^{1} \mathrm{H}$ NMR $\left(500 \mathrm{MHz}, \mathrm{CDCl}_{3}\right): \delta(\mathrm{ppm}) 7.43(\mathrm{~d}, 2 \mathrm{H}, \mathrm{J}=$ $7 \mathrm{~Hz}), 7.38(\mathrm{t}, 2 \mathrm{H}, \mathrm{J}=7.5 \mathrm{~Hz}), 7.31(\mathrm{t}, 1 \mathrm{H}, \mathrm{J}=7 \mathrm{~Hz}), 7.28(\mathrm{t}, 1 \mathrm{H}, \mathrm{J}=2 \mathrm{~Hz}), 7.20(\mathrm{~d}, 2 \mathrm{H}, \mathrm{J}=$ $1.5 \mathrm{~Hz}), 6.67(\mathrm{~s}, 2 \mathrm{H}), 5.85(\mathrm{~d}, 1 \mathrm{H}, \mathrm{J}=3 \mathrm{~Hz}), 3.72(\mathrm{~s}, 6 \mathrm{H}), 2.26(\mathrm{~d}, 1 \mathrm{H}, \mathrm{J}=3 \mathrm{~Hz}),{ }^{13} \mathrm{C}$ NMR $\left(500 \mathrm{MHz}, \mathrm{CDCl}_{3}\right): \delta(\mathrm{ppm}) 157.63,145.94,143.60,137.24,134.15,129.73,128.88$, $128.15,127.02,126.82,102.44,76.65,56.12$. HPLC retention time: $10.612 \mathrm{~min}$; purity $98.92 \%$.

\subsubsection{Synthesis of (3',5'-dichloro-2,6-dimethoxybiphenyl-4- yl)(phenyl)methanone (14)}

Alcohol 8 (2.1g, 5.4mmol), pyridinium chlorochromate $(2.33 \mathrm{~g}, 10.8 \mathrm{mmol})$ and Celite ${ }^{\circledR}(2.33 \mathrm{~g})$ were added to anhydrous $\mathrm{CH}_{2} \mathrm{Cl}_{2}$ and stirred under argon at room temperature for 18 hours. The reaction mixture was then filtered over a plug of silica gel and the filtrate was washed with saturated aqueous $\mathrm{NaHCO}_{3}$, water, and brine and then dried over $\mathrm{Na}_{2} \mathrm{SO}_{4}$. The organic phase was concentrated and the product was purified by column chromatography using an EtOAc / hexanes gradient to yield an off-white solid. Yield: $1.77 \mathrm{~g}(85 \%)\left(\mathrm{R}_{f}=0.448 ; 15 \%\right.$ EtOAc / hexanes). MS: $m / z($ ESI, pos. $)=409.1[\mathrm{M}$ $+\mathrm{Na}]^{+} .{ }^{1} \mathrm{H} \mathrm{NMR}\left(300 \mathrm{MHz}, \mathrm{CDCl}_{3}\right): \delta(\mathrm{ppm}) 7.86(\mathrm{~m}, 2 \mathrm{H}), 7.56(\mathrm{~m}, 4 \mathrm{H}), 7.34(\mathrm{t}, 1 \mathrm{H}, \mathrm{J}$ $=1.8 \mathrm{~Hz}), 7.25(\mathrm{t}, 1 \mathrm{H}, \mathrm{J}=2.4 \mathrm{~Hz}), 7.05(\mathrm{~s}, 2 \mathrm{H}), 3.75(\mathrm{~s}, 6 \mathrm{H}) .{ }^{13} \mathrm{C} \mathrm{NMR}(300 \mathrm{MHz}$, $\left.\mathrm{CDCl}_{3}\right): \delta 196.18,157.64,139.26,137.44,135.62,135.30,132.93,130.28,129.26$, $128.63,128.59,118.91,111.49,104.61,56.34$. HPLC retention time: $12.051 \mathrm{~min}$; purity $98.37 \%$.

\subsubsection{Synthesis of 3',5'-dichloro-2,6-dimethoxy-4-(2-phenylpropan-2- yl)biphenyl (15)}

Anhydrous $\mathrm{CH}_{2} \mathrm{Cl}_{2}$ was added to a round bottom flask, stirred under argon and cooled to $-40^{\circ} \mathrm{C}$ using a dry ice / acetonitrile bath. Titanium chloride $(1 \mathrm{M}, 30 \mathrm{ml}$, $30 \mathrm{mmol})$ was added by syringe followed by dimethyl zinc $(2 \mathrm{M}, 15 \mathrm{ml}, 30 \mathrm{mmol})$. This mixture was allowed to stir at $-40^{\circ} \mathrm{C}$ for 15 minutes. Ketone (14) $(1.95 \mathrm{~g}, 5 \mathrm{mmol})$ dissolved in anhydrous $\mathrm{CH}_{2} \mathrm{Cl}_{2}(100 \mathrm{ml})$ was then added by syringe and the reaction stirred at $-40^{\circ} \mathrm{C}$ for 2 hours and was then allowed to warm to room temperature overnight. The reaction was then quenched by the addition of ice. The organic layer was separated and washed with saturated aqueous $\mathrm{NaHCO}_{3}$, water, and brine and then dried over $\mathrm{Na}_{2} \mathrm{SO}_{4}$. The organic phase was concentrated and the product was purified by column chromatography using an EtOAc / hexanes gradient to yield a white solid. Yield: $1.9 \mathrm{~g}(95 \%)\left(\mathrm{R}_{f}=0.750 ; 15 \%\right.$ EtOAc / hexanes $) . \mathrm{MS}: \mathrm{m} / z(\mathrm{ESI}, \mathrm{pos})=.423.1[\mathrm{M}+\mathrm{Na}]^{+}$. ${ }^{1} \mathrm{H}$ NMR $\left(500 \mathrm{MHz}, \mathrm{CDCl}_{3}\right): \delta 7.43(\mathrm{~m}, 1 \mathrm{H}), 7.30(\mathrm{~m}, 3 \mathrm{H}), 7.27(\mathrm{t}, 1 \mathrm{H}, \mathrm{J}=1.5 \mathrm{~Hz}), 7.24$ $(\mathrm{d}, 1 \mathrm{H}, \mathrm{J}=2 \mathrm{~Hz}), 7.20(\mathrm{~m}, 2 \mathrm{H}), 6.48(\mathrm{~s}, 2 \mathrm{H}), 3.65(\mathrm{~s}, 6 \mathrm{H}), 1.72(\mathrm{~s}, 6 \mathrm{H}) .{ }^{13} \mathrm{C} \mathrm{NMR}$ $\left(500 \mathrm{MHz}, \mathrm{CDCl}_{3}\right): \delta 157.04,153.70,150.12,136.27,135.36,129.69,129.54,128.02$, 
126.84, 125.99, 112.49, 107.64, 102.75, 55.91, 43.50, 30.85, 30.73. HPLC retention time: $13.909 \mathrm{~min}$; purity $96.42 \%$.

\subsubsection{Synthesis of (3',5'-dichloro-2-hydroxy-6-methoxybiphenyl-4- yl)(phenyl)methanone (9) and (3',5'-dichloro-2,6-dihydroxybiphenyl-4- yl)(phenyl)methanone (10)}

Ketone 14 was dissolved in anhydrous $\mathrm{CH}_{2} \mathrm{Cl}_{2}(120 \mathrm{ml})$, stirred under argon, and cooled to $-78^{\circ} \mathrm{C}$ in a dry ice / acetone bath. Boron tribromide $(1 \mathrm{M}, 9 \mathrm{ml}, 9 \mathrm{mmol})$ was slowly added by syringe and the reaction was allowed to stir at $-78^{\circ} \mathrm{C}$ for 3 hours and then allowed to warm to room temperature and stirred an additional 16 hours. The reaction was then quenched by the addition of methanol and washed with saturated aqueous $\mathrm{NaHCO}_{3}$, water, and brine and then dried over $\mathrm{Na}_{2} \mathrm{SO}_{4}$. The organic phase was concentrated and the products were purified by column chromatography using an EtOAc / hexanes gradient to yield an off-white solid $\mathbf{9}$ and a reddish solid $\mathbf{1 0 .}$

9 Yield: $314 \mathrm{mg}(21 \%)\left(\mathrm{R}_{f}=0.390 ; 20 \%\right.$ EtOAc $/$ hexanes $) . \mathrm{MS}: \mathrm{m} / z(\mathrm{ESI}, \mathrm{neg})=$. $370.9[\mathrm{M}-\mathrm{H}]^{-} .{ }^{1} \mathrm{H}$ NMR $\left(500 \mathrm{MHz}, \mathrm{CDCl}_{3}\right): \delta 7.84(\mathrm{~d}, 2 \mathrm{H}, \mathrm{J}=8 \mathrm{~Hz}), 7.61(\mathrm{t}, 1 \mathrm{H}, \mathrm{J}=$ $8 \mathrm{~Hz}), 7.51(\mathrm{t}, 2 \mathrm{H}, \mathrm{J}=7.5 \mathrm{~Hz}), 7.42(\mathrm{~d}, 1 \mathrm{H}, \mathrm{J}=1.5 \mathrm{~Hz}), 7.30(\mathrm{~d}, 2 \mathrm{H}, \mathrm{J}=1.5 \mathrm{~Hz}), 7.04(\mathrm{~s}$, $1 \mathrm{H}), 7.00(\mathrm{~d}, 1 \mathrm{H}, \mathrm{J}=1.5 \mathrm{~Hz}), 5.19(\mathrm{~d}, 1 \mathrm{H}, \mathrm{J}=16 \mathrm{~Hz}), 3.79(\mathrm{~s}, 3 \mathrm{H}),{ }^{13} \mathrm{C} \mathrm{NMR}(500 \mathrm{MHz}$, $\left.\mathrm{CDCl}_{3}\right): \delta 196.18,157.64,153.36,139.26,137.44,135.62,135.30,132.93,130.28$, 129.26, 128.63, 128.59, 118.91, 111.49, 104.61, 56.34. HPLC retention time: $10.780 \mathrm{~min}$; purity $99.03 \%$.

10 Yield: $106 \mathrm{mg}(7 \%)\left(\mathrm{R}_{f}=0.235 ; 20 \%\right.$ EtOAc $/$ hexanes $) . \mathrm{MS}: \mathrm{m} / z(\mathrm{ESI}, \mathrm{neg})=$. $356.9[\mathrm{M}-\mathrm{H}]^{-} .{ }^{1} \mathrm{H}$ NMR $\left(500 \mathrm{MHz}, \mathrm{CDCl}_{3}\right): \delta 7.84(\mathrm{~d}, 2 \mathrm{H}, \mathrm{J}=7.5 \mathrm{~Hz}), 7.61(\mathrm{t}, 1 \mathrm{H}, \mathrm{J}=$ $7.5 \mathrm{~Hz}), 7.48(\mathrm{~m}, 3 \mathrm{H}), 7.37(\mathrm{~d}, 2 \mathrm{H}, \mathrm{J}=1.5 \mathrm{~Hz}), 7.02(\mathrm{~s}, 2 \mathrm{H}), 5.42(\mathrm{~s}, 2 \mathrm{H}),{ }^{13} \mathrm{C} \mathrm{NMR}$ $\left(500 \mathrm{MHz}, \mathrm{CDCl}_{3}\right): \delta 196.24,153.77,139.32,137.18,136.29,134.46,133.08,130.34$, $129.25,129.18,128.61,117.63,110.18$. HPLC retention time: $9.618 \mathrm{~min}$; purity $100 \%$.

\subsubsection{Synthesis of 3',5'-dichloro-6-methoxy-4-(2-phenylpropan-2-yl)biphenyl- 2-ol (16) and 3',5'-dichloro-4-(2-phenylpropan-2-yl)biphenyl-2,6-diol (11)}

Dimethyl compound 15 was reacted with boron tribromide $(1 \mathrm{M}, 9 \mathrm{ml}, 9 \mathrm{mmol})$ in anhydrous $\mathrm{CH} 2 \mathrm{Cl} 2(120 \mathrm{ml})$ in a manner similar to the synthesis of compounds $\mathbf{9}$ and $\mathbf{1 0}$. The products were purified by column chromatography using an EtOAc / hexanes gradient to yield a yellow resin $\mathbf{1 6}$ and a golden foam $\mathbf{1 1}$.

16 Yield: 290mg (17\%) $\left(\mathrm{R}_{f}=0.317 ; 10 \%\right.$ EtOAc / hexanes). MS: $m / z$ (ESI, pos.) $=409.2[\mathrm{M}+\mathrm{Na}]^{+} .{ }^{1} \mathrm{H} \mathrm{NMR}\left(500 \mathrm{MHz}, \mathrm{CDCl}_{3}\right): \delta 7.35(\mathrm{t}, 1 \mathrm{H}, \mathrm{J}=1.5 \mathrm{~Hz}), 7.29(\mathrm{~m}, 6 \mathrm{H})$, $7.20(\mathrm{~m}, 1 \mathrm{H}), 6.51(\mathrm{~d}, 1 \mathrm{H}, \mathrm{J}=1.5 \mathrm{~Hz}), 6.37(\mathrm{~d}, 1 \mathrm{H}, \mathrm{J}=1.5 \mathrm{~Hz}), 4.73(\mathrm{~s}, 1 \mathrm{H}), 3.63(\mathrm{~s}, 3 \mathrm{H})$, $1.69(\mathrm{~s}, 6 \mathrm{H}),{ }^{13} \mathrm{C} \mathrm{NMR}\left(500 \mathrm{MHz}, \mathrm{CDCl}_{3}\right): \delta 157.04,153.70,153.01,150.12,136.27$, 135.36, 129.69, 129.54, 128.02, 126.84, 125.99, 112.49, 107.64, 102.75, 55.91, 43.50, $30.85,30.73$. HPLC retention time: $11.716 \mathrm{~min}$; purity $99.48 \%$. 
11 Yield: 950mg (57\%) $\left(\mathrm{R}_{f}=0.167 ; 10 \%\right.$ EtOAc / hexanes). MS: $m / z$ (ESI, pos.) $=395.2[\mathrm{M}+\mathrm{Na}]^{+} .{ }^{1} \mathrm{H} \mathrm{NMR}\left(500 \mathrm{MHz}, \mathrm{CDCl}_{3}\right): \delta 7.40(\mathrm{t}, 1 \mathrm{H}, \mathrm{J}=2 \mathrm{~Hz}), 7.29(\mathrm{~m}$, $6 \mathrm{H}), 7.19(\mathrm{~m}, 1 \mathrm{H}), 6.40(\mathrm{~s}, 2 \mathrm{H}), 4.81(\mathrm{~s}, 2 \mathrm{H}), 1.65(\mathrm{~s}, 6 \mathrm{H}),{ }^{13} \mathrm{C} \mathrm{NMR}\left(500 \mathrm{MHz}, \mathrm{CDCl}_{3}\right): \delta$ $154.07,153.11,149.97,136.07,135.34,129.58,129.43,128.68,128.58,128.23,111.10$, 107.27, 43.16, 30.58. HPLC retention time: $10.271 \mathrm{~min}$; purity $96.81 \%$.

\subsubsection{Synthesis of 3',5'-dichloro-4-(hydroxy(phenyl)methyl)-6- methoxybiphenyl-2-ol (12)}

Ketone 9 (75mg, $0.2 \mathrm{mmol})$ was dissolved in $\mathrm{MeOH}(5 \mathrm{ml})$ and cooled to $0^{\circ} \mathrm{C}$. $\mathrm{NaBH}_{4}(9.5 \mathrm{mg}, 0.25 \mathrm{mmol})$ was slowly added with stirring. The reaction was then allowed to warm to room temperature and stirred for 2 hours. The solvent was then removed by rotary evaporation and the product was purified by column chromatography using an EtOAc / hexanes gradient to yield a white foam. Yield: $63 \mathrm{mg}(84 \%)\left(\mathrm{R}_{f}=\right.$ $0.361 ; 30 \%$ EtOAc / hexanes). MS: $m / z$ (ESI, neg.) $=372.8[\mathrm{M}-\mathrm{H}]{ }^{-} .{ }^{1} \mathrm{H}$ NMR $(300$ $\mathrm{MHz}, \mathrm{MeOD}): \delta 7.37(\mathrm{~m}, 6 \mathrm{H}), 7.25(\mathrm{~m}, 2 \mathrm{H}), 6.65(\mathrm{~s}, 1 \mathrm{H}), 6.62(\mathrm{~s}, 1 \mathrm{H}), 5.80(\mathrm{~d}, 1 \mathrm{H}, \mathrm{J}=$ $5 \mathrm{~Hz}), 4.83(\mathrm{~s}, 1 \mathrm{H}), 3.73(\mathrm{~s}, 3 \mathrm{H}), 2.25(\mathrm{~d}, 1 \mathrm{H}, \mathrm{J}=5.5 \mathrm{~Hz}) .{ }^{13} \mathrm{C} \mathrm{NMR}(500 \mathrm{MHz}, \mathrm{MeOD}): \delta$ $152.65,153.77,146.12,143.03,135.90,135.23,129.45,129.40,128.87,128.21,126.81$, 112.96, 106.47, 105.62, 76.09, 56.54. HPLC retention time: $9.989 \mathrm{~min}$; purity $94.88 \%$.

\subsubsection{Synthesis of 3',5'-dichloro-4-(hydroxy(phenyl)methyl)biphenyl-2,6-diol (13)}

Ketone 10 (33mg, 0.092mmol) was reacted with $\mathrm{NaBH}_{4}(4.8 \mathrm{mg}, 0.129 \mathrm{mmol})$ in $\mathrm{MeOH}(4 \mathrm{ml})$ in a manner similar to compound 12 . The product was purified by column chromatography using an EtOAc / hexanes gradient to yield a white solid. Yield: $18 \mathrm{mg}$ $(54 \%)\left(\mathrm{R}_{f}=0.441 ; 40 \%\right.$ EtOAc / hexanes $)$ MS: $m / z($ ESI, neg. $)=358.9[\mathrm{M}-\mathrm{H}]^{-} .{ }^{1} \mathrm{H}$ NMR (500 MHz, CDCl $): \delta 7.37(\mathrm{t}, 1 \mathrm{H}, \mathrm{J}=1.5 \mathrm{~Hz}), 7.33(\mathrm{~m}, 4 \mathrm{H}), 7.29(\mathrm{~m}, 1 \mathrm{H}), 7.25(\mathrm{~d}$, $2 \mathrm{H}, \mathrm{J}=1 \mathrm{~Hz}), 6.52(\mathrm{~s}, 2 \mathrm{H}), 5.67(\mathrm{~s}, 1 \mathrm{H}), 5.38(\mathrm{~s}, 2 \mathrm{H}), 2.71(\mathrm{~s}, 1 \mathrm{H}),{ }^{13} \mathrm{C} \mathrm{NMR}(500 \mathrm{MHz}$, $\left.\mathrm{CDCl}_{3}\right): \delta 153.77,146.12,143.03,135.90,135.23,129.45,129.40,128.87,128.21$, 126.81, 112.96, 106.47, 76.09. HPLC retention time: $9.046 \mathrm{~min}$; purity $96.05 \%$.

\subsection{Experimental Methods}

\subsubsection{Radioligand Binding Assays}

Binding affinity studies were carried out using cell membranes from HEK293 cells transfected with the human CB1 receptor $\left(K_{\mathrm{d}}\right.$ for tritiated CP-55,940 binding: 1.0 $\mathrm{nM})$ and membranes from HEK293 cells transfected with the human CB2 receptor $\left(K_{\mathrm{d}}\right.$ for tritiated CP-55,940 binding: $1.0 \mathrm{nM}$ ). Non-specific binding was determined using 10 $\mu \mathrm{M}$ WIN55, 212-2. Increasing concentrations of compounds to be tested were made ranging from $10^{-12} \mathrm{M}$ to $10^{-4} \mathrm{M}$ and were added in triplicate for each experiment and the 
individual molar $\mathrm{IC}_{50}$ values were determined using GraphPad Prism software. The corresponding $K_{\mathrm{i}}$ values for each drug were determined utilizing the Cheng-Prusoff equation and final data are presented as $K_{\mathrm{i}} \pm$ standard deviation of $\mathrm{n}=3$ experiments run in triplicate.

\subsubsection{Cytotoxicity Assays}

Human glioblastoma cells U-87MG, T98G, LN-229, DBTRG-O5MG (American Type Culture Collection) were cultured in supplemented media according to the recommendations of the supplier at $37^{\circ} \mathrm{C}$ in an atmosphere of $5 \% \mathrm{CO}_{2}$ and $95 \%$ air. Cell lines were plated in 96-well polystyrene flat-bottom plates $(7,500$ cells/well) at $70 \%$ confluency in a $100 \mu 1$ total volume of supplemented media as indicated, and incubated overnight at $37^{\circ} \mathrm{C}$ to allow for adherence. The media was then replaced with serum-free media and the cultures were treated with escalating amounts of drug and cell death was analyzed at 18h, using the BioTek Synergy 2 Multidetection Microplate Reader (BioTek Instruments, Inc.). The percentage of viable cells present in the culture at each time point was calculated by comparing the absorbance value at $450 \mathrm{~nm}$ from the CCK-8 assay (Dojindo Molecular Technologies) for each condition with untreated control cells. All assays were conducted per manufacturer's protocol. All described values represent the average of three data points per determination and three independent determinations.

\subsubsection{Cytotoxicity Assays with Pertussis Toxin}

U-87 MG cells were seeded in 96 -well polystyrene flat-bottom plates $(7,500$ cells/well) at $70 \%$ confluency in a $100 \mu \mathrm{l}$ total volume of complete growth media and incubated overnight at $37^{\circ} \mathrm{C}$ in an atmosphere of $5 \% \mathrm{CO}_{2}$ and $95 \%$ air to allow for adherence. The media was then replaced with complete growth media containing 0 or $100 \mathrm{ng} / \mathrm{ml}$ pertussis toxin and the plates returned to the incubator for 18 hours. Drug solutions were prepared in DMSO at 100X concentration, and mixed 1:100 in media containing 1\% FBS to yield the desired concentration. After 18 hour incubation with or without Ptx, full serum media was removed and replaced with drug-media mixtures and incubated for an additional 18 hours. $10 \mu \mathrm{L}$ of CCK-8 reagent was added to each well to colorimetrically assess viability. After $2-4$ hours of incubation with the CCK-8 dye, absorbance was read at 450nm using a BioTek Synergy 2 plate reader.

\subsubsection{CB1/CB2 Functional Assays}

HEK-293 cell lines stably transfected with a cyclic nucleotide-gated channel and either human CB1 or CB2 receptors (BD Biosciences, San Jose, CA USA) were seeded in poly-D-lysine coated 96-well plates at a density of 70,000 cells per well. Plates were incubated at $37^{\circ} \mathrm{C}$ in $5 \% \mathrm{CO}_{2}$ overnight prior to assay. Plates were then removed from the incubator and the complete growth medium (DMEM, 10\% FBS, $250 \mu \mathrm{g} / \mathrm{ml} \mathrm{G418}$ and $1 \mu \mathrm{g} / \mathrm{ml}$ puromycin) was replaced with $100 \mu \mathrm{L}$ DMEM containing $0.25 \%$ BSA. Next, 
$100 \mu \mathrm{L}$ membrane potential dye loading buffer (Molecular Devices) was added to each well. The plates were placed back into the incubator for 30 minutes and then the baseline fluorescence was read on a BioTek Synergy 2 multi-mode microplate reader with 540nm excitation and 590nm emission filters prior to drug addition. Drugs were added in $50 \mu \mathrm{L}$ DPBS containing $2.5 \%$ DMSO, $1.25 \mu \mathrm{M} 5$ '-(N-ethylcarboxamido) adenosine and $125 \mu \mathrm{M}$ Ro 20-1724. Plates were then incubated at room temperature for 25 minutes and fluorescence measured again at $540 \mathrm{~nm}$ excitation and $590 \mathrm{~nm}$ emission.

\subsubsection{MSD Protein Quantification}

U-87 MG cells were plated in poly-D-lysine coated $10 \mathrm{~cm}$ Petri dishes at a density of $4 \times 10^{5}$ cells $/ \mathrm{ml}$ with $12 \mathrm{ml}$ of cell suspension added to each plate. The cells were grown overnight in EMEM media containing 10\% FBS. The following day the media was changed to EMEM media containing 1\% FBS and allowed to equilibrate for 24 hours. This step is essential to allow basal levels of signaling protein to re-equilibrate due to changes in the serum concentration. The media was then replaced at the appropriate time points with media containing $1 \%$ serum and $\mathrm{KM}-233$ at the $\mathrm{EC}_{50}$ or media containing $1 \%$ serum and vehicle (DMSO). After the incubation period with KM233 or vehicle, the media was removed from the dishes and transferred to a $15 \mathrm{ml}$ centrifuge tube to collect any cells that were in suspension. Cells were then scraped from the surface of the dish and transferred into the same $15 \mathrm{ml}$ centrifuge tubes and pelleted at $1000 \mathrm{rpm}, 4^{\circ} \mathrm{C}$ for $5 \mathrm{~min}$. Supernatant was removed and $0.5 \mathrm{ml}$ lysis buffer was added to each pellet. The cells were suspended in the buffer and transferred to $1.5 \mathrm{ml}$ microcentrifuge tubes. These were incubated on ice for 30 minutes, being vortexed every 5 minutes. The lysates were then centrifuged at $10,000 \times \mathrm{g}, 4^{\circ} \mathrm{C}$ for 10 minutes to clear cellular debris from the lysate. The supernatant was removed and assayed for protein concentration using a Pierce BCA Protein Assay Kit (Thermo Scientific). The lysates were then diluted with complete lysis buffer to a concentration of $20 \mu \mathrm{g}$ of protein in $25 \mu \mathrm{l}$ volume. Signaling proteins were then analyzed using MSD phosphoprotein and intracellular marker multiplex kits which were imaged on a SECTOR Imager 2400 (Meso Scale Discovery) according to the manufacturer's instructions.

\subsubsection{Caspase 9 Assay}

U-87 MG cells were seeded in white, opaque 96-well culture plates at a density of $10,000 \mathrm{cells} / \mathrm{well}$ in a volume of $100 \mu 1$ complete growth media. The cells were allowed to attach overnight and the complete growth media was changed to media containing $1 \%$ serum for 24 hours to equilibrate intracellular signaling pathways, then media was changed to either media containing $1 \%$ serum or media containing $1 \%$ serum plus HB-I172. At the appropriate time intervals $100 \mu 1$ of Caspase-Glo® 9 Assay Reagent (Promega, prepared according to manufacturer's instructions) was added to each well and the plate mixed on a rotary shaker for 30 seconds. After incubation at room temperature for 90 minutes the luminescence was read on a Biotek Synergy 2 plate reader. The 
luminescent signal is proportional to the amount of caspase 9 activity present. All data points represent 6 independent determinations.

\subsubsection{Mitochondrial Membrane Potential Assay}

U-87 MG cells were seeded in poly-D-lysine coated 96-well plates at a density of 10,000 cells per well. At the appropriate time points, the media was removed and replaced with media containing $1 \% \mathrm{FBS}$ and either $\mathrm{HB}-\mathrm{I}-172$ at the $\mathrm{EC}_{50}$ or vehicle (DMSO). After incubation with drug or vehicle, cells were dyed using DePsipher membrane potential dye according to the manufacturer's instructions. Plates were then imaged using an Olympus IX71 inverted fluorescence microscope (Olympus America) fitted with a Retiga-SRV camera (QImaging).

\subsubsection{Microscopic Analysis Using Immunohistochemical Techniques}

U-87 MG cells were plated in complete growth medium at a density of 15,000 cells/well in poly-D-lysine coated 96 -well polystyrene tissue culture plates and allowed to attach over night at $37^{\circ} \mathrm{C}$ in an atmosphere of $5 \% \mathrm{CO}_{2} / 95 \%$ air. The media was then replaced with media containing $1 \% \mathrm{FBS}$ and either $\mathrm{HB}-\mathrm{I}-172$ at the $\mathrm{EC}_{50}$ or vehicle (DMSO). The cells were returned to the incubator and after the appropriate periods of exposure to the drug or vehicle, media was aspirated and the cells fixed using $4 \%$ paraformaldehyde. Cells were then stained by immunohistochemical methods using either a Cellomics Multiparameter Cell Death Detection Kit, Cellomics Cytoskeletal Rearrangement Kit, or Cellomics Cleaved PARP Activation Kit (Thermo Scientific) according to the manufacturer's instructions. Plates were then imaged using an Olympus IX71 inverted fluorescence microscope (Olympus America) fitted with a Retiga-SRV camera (QImaging). 


\section{Chapter 4: Design, Synthesis, and Testing of Novel Cannabinoid Ligands}

\subsection{Introduction}

Classical cannabinoids are tricyclic terpenoid compounds consisting of a benzopyran core structure that are either naturally occurring in the hemp plant (phytocannabinoids) or are synthetic analogs of naturally occurring compounds. $\Delta^{9}$-THC and its isomer $\Delta^{8}$-THC (Figure 4.1) are the prototypical members of this class of cannabinoids. They bind without selectivity to the CB1 and CB2 cannabinoid receptors and act as agonists or partial agonists. ${ }^{11}$ The classical cannabinoids are by far the most well studied of the cannabinoid compounds in terms of pharmacology and structure activity relationships (SAR) and have gained considerable attention over the years due to their potential indication in the treatment of several pathological conditions from inflammation to asthma, inflammatory bowel diseases, multiple sclerosis, Parkinson's disease, epilepsy, glaucoma, septic shock, hemorrhagic shock, and cancer.

SAR studies have determined that there are three pharmacophoric elements (Figure 4.1) in classical cannabinoids that are required for cannabimimetic activity. These include:
a. C-1 phenolic hydroxyl group
b. lipophilic C-3 alkyl side chain
c. trans ring junction at the $\mathrm{B}$ and $\mathrm{C}$ rings.

Many studies have been performed in an attempt to elucidate the SAR of the classical cannabinoids with respect to modifications of the tricyclic ring and side chain substituents at the C-3 position. Some of the different side chain modifications that have been reported thus far include the branched chain alkyls, ${ }^{153}$ unsaturated alkyls, ${ }^{130,154}$ and alkyls that contain a $11^{\prime}, 1^{\prime}$-cyclic functionality. ${ }^{155}$ Each of these modifications has been directed at the goal of producing compounds that have higher binding affinities for the cannabinoid receptors and also that are selective for one subtype over the other.

The potential for important therapeutic interventions from this class of compounds has provided the impetus to develop ligands that can elicit the desired physiological effects of $\Delta^{9}$-THC (mediated through CB2) without producing the unwanted CNS side effects. In the absence of X-ray crystal structures of cannabinoid receptors, medicinal chemists have relied on chemical diversifications to develop new ligands for CB1 and CB2. Structurally diverse compounds that probe the available space in the ligand binding pocket of the cannabinoid receptors have provided important insights into the structural requirements for binding as well as for activity.

This need for diversity in the cannabinoid chemical arsenal has led to the development of compounds belonging to diverse chemical classes, both classical and non-classical. The term "non-classical" was originally used to describe compounds that 


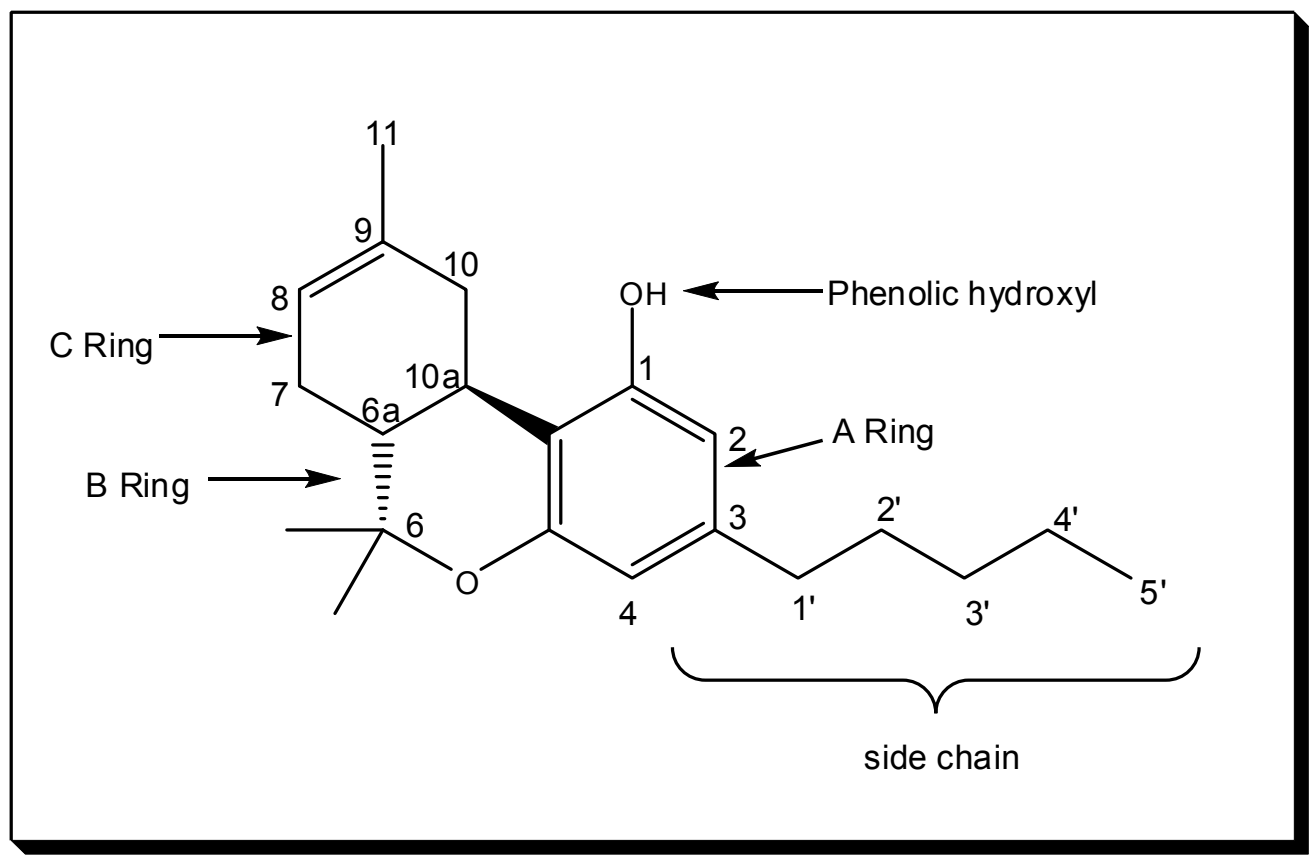

Figure 4.1: Structure of $\Delta^{8}$-THC with identification of its various structural domains 
lack the B ring of the classical cannabinoids and possess an alkyl side chain such as the compound CP-55,940 (Figure 4.2). The term is now more widely used to describe any compound which is a ligand that binds the cannabinoid receptors which lacks the basic tricyclic benzopyran ring system. An explosion of work into the non-classical arena has led to several new structural classes of cannabinoids. These new compounds can be classified broadly into 9 distinct chemical scaffolds (Figures 4.2 and 4.3):

a. Non-classical cannabinoids - exemplified by the CP-55,940 group of compounds. ${ }^{156}$

b. Aminoalkyl indoles - exemplified by the WIN 55,212-2 group of compounds. ${ }^{157}$

c. Biaryl pyrazoles - exemplified by SR14716A group of compounds. ${ }^{158}$

d. Biphenyls - exemplified by the biaryl-gem-dimethyl heptyl group of compounds. ${ }^{128}$

e. Triaryls - exemplified by HB-I-172. ${ }^{127}$

f. N-alkyl isatin acylhydrazones exemplified by MDA77. ${ }^{159}$

g. Benzimidazoles as exemplified by PF-03550096. ${ }^{160}$

h. N-arylamide oxadiazoles. ${ }^{161}$

i. Quinolone-3 carboxamides. ${ }^{162}$

j. Triaryl bis-sulfones. ${ }^{163}$

These compounds act via the $\mathrm{CB} 1$ or CB2 receptors (and possibly GPR55) and are agonists, antagonists, inverse agonists, or partial agonist/antagonists. They are collectively known as "cannabimimetic" agents, "cannabinergic" agents, or simply "cannabinoids." These structural classes of ligands display varying affinities as well as selectivity for the cannabinoid receptor subtypes, though most of the recent work has been in improving CB2 selectivity. Although all of these cannabinoids essentially bind to the same receptors and activate or deactivate the same downstream signaling cascade, it is not surprising that there is increasing evidence to suggest that they display different binding modes. It is believed that each structural class interacts with different critical amino acids in the binding pocket of each receptor. This may explain why the differing chemical classes display very different selectivities for CB1/CB2. This growing volume of knowledge can be exploited for the further tailoring of compounds that have specific binding affinity profiles.

The reported SAR studies of cannabinoid receptor ligands indicate that there are important amino acid residues in the transmembrane domains 3, 5, and 6 of the CB1 and the $\mathrm{CB} 2$ receptors that are involved in the discrimination of the cannabinergic ligands. ${ }^{164}$ Molecular modeling studies have also suggested the importance of specific amino acid residues in the $\mathrm{CB} 1$ receptor binding pocket that play a critical role in binding to classical cannabinoids (Figure 4.4). These residues are as follows: 


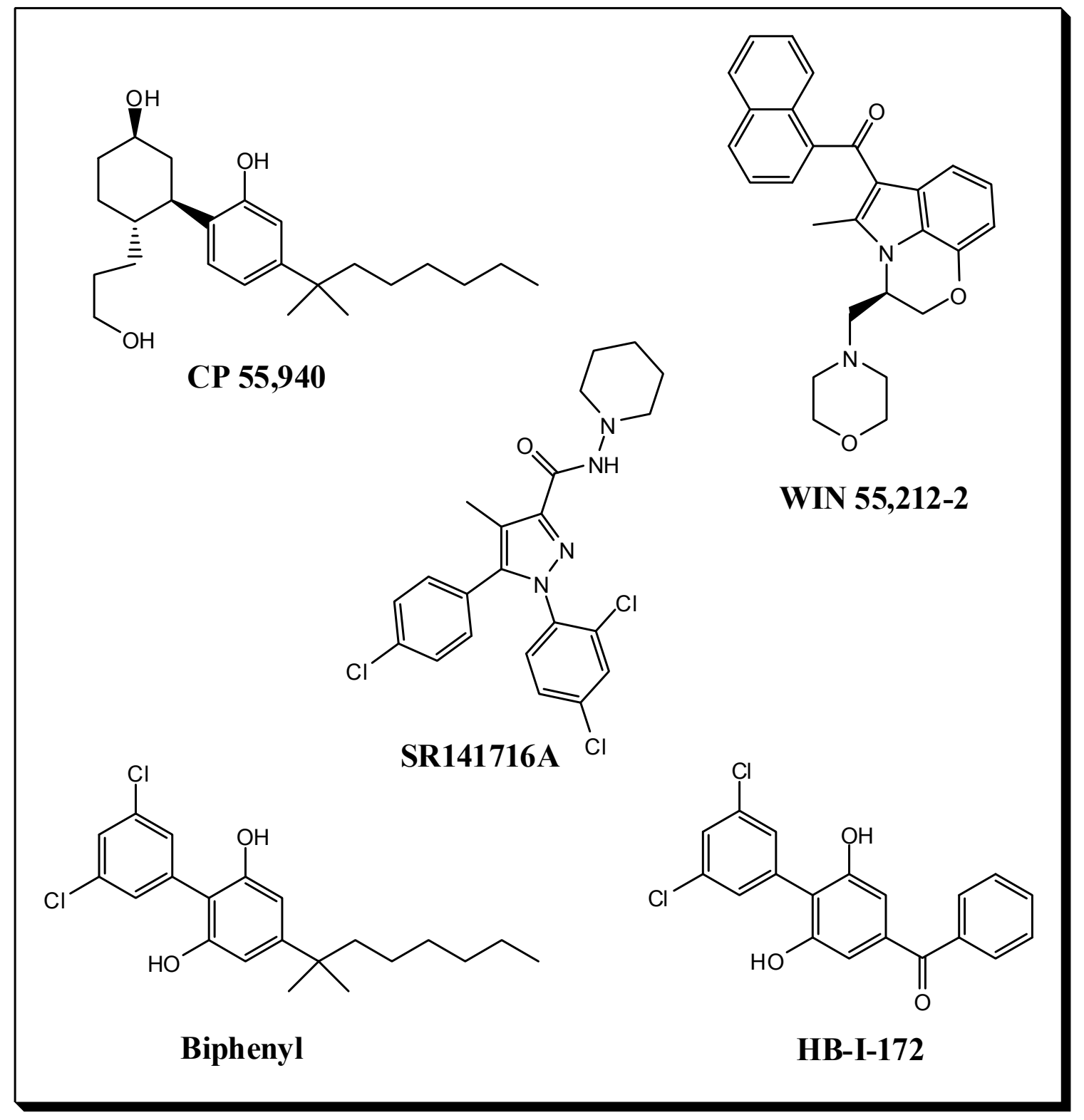

Figure 4.2: Representatives of various classes of non-classical cannabinoids 


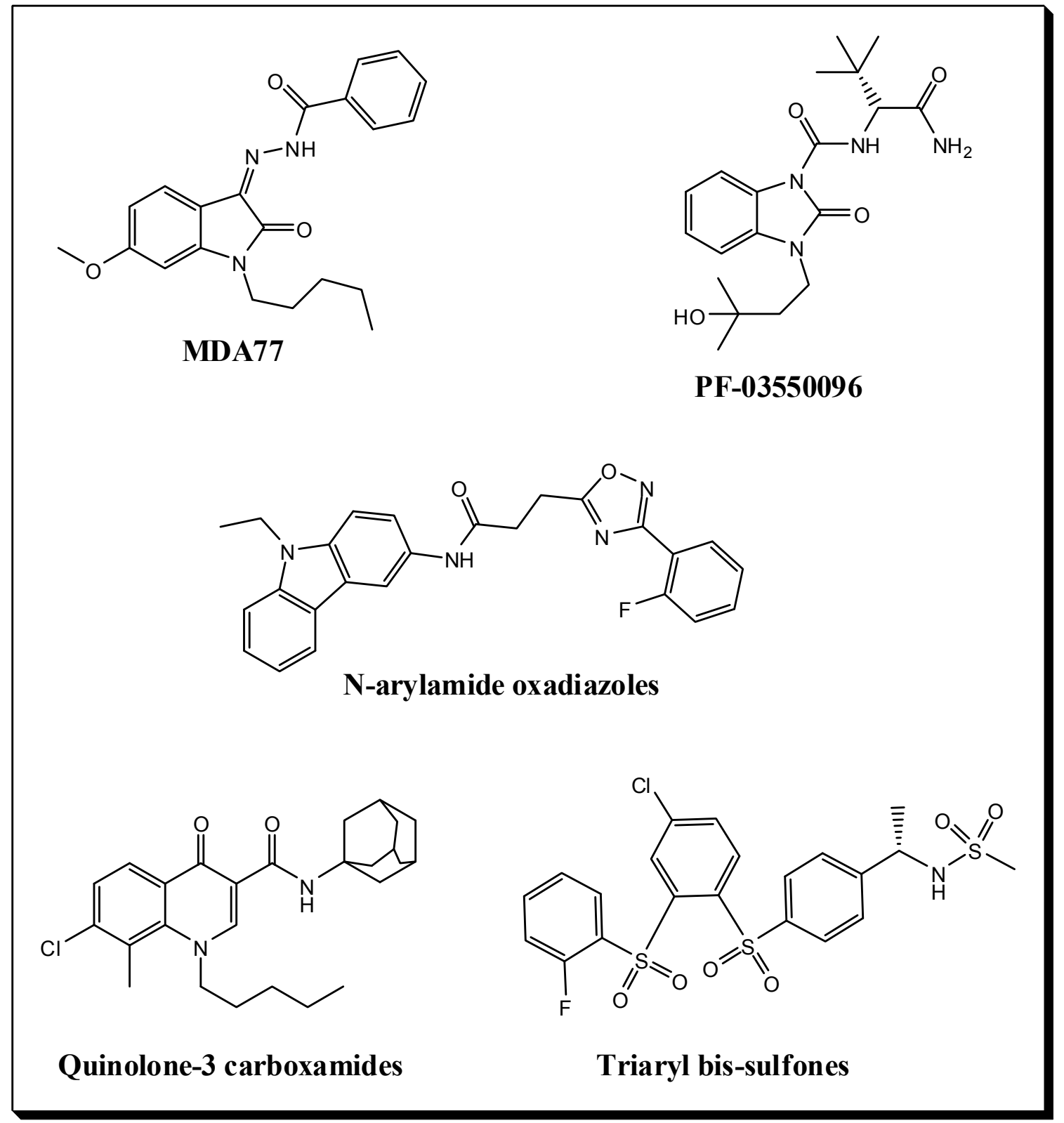

Figure 4.3: Further representatives of various classes of non-classical cannabinoids 


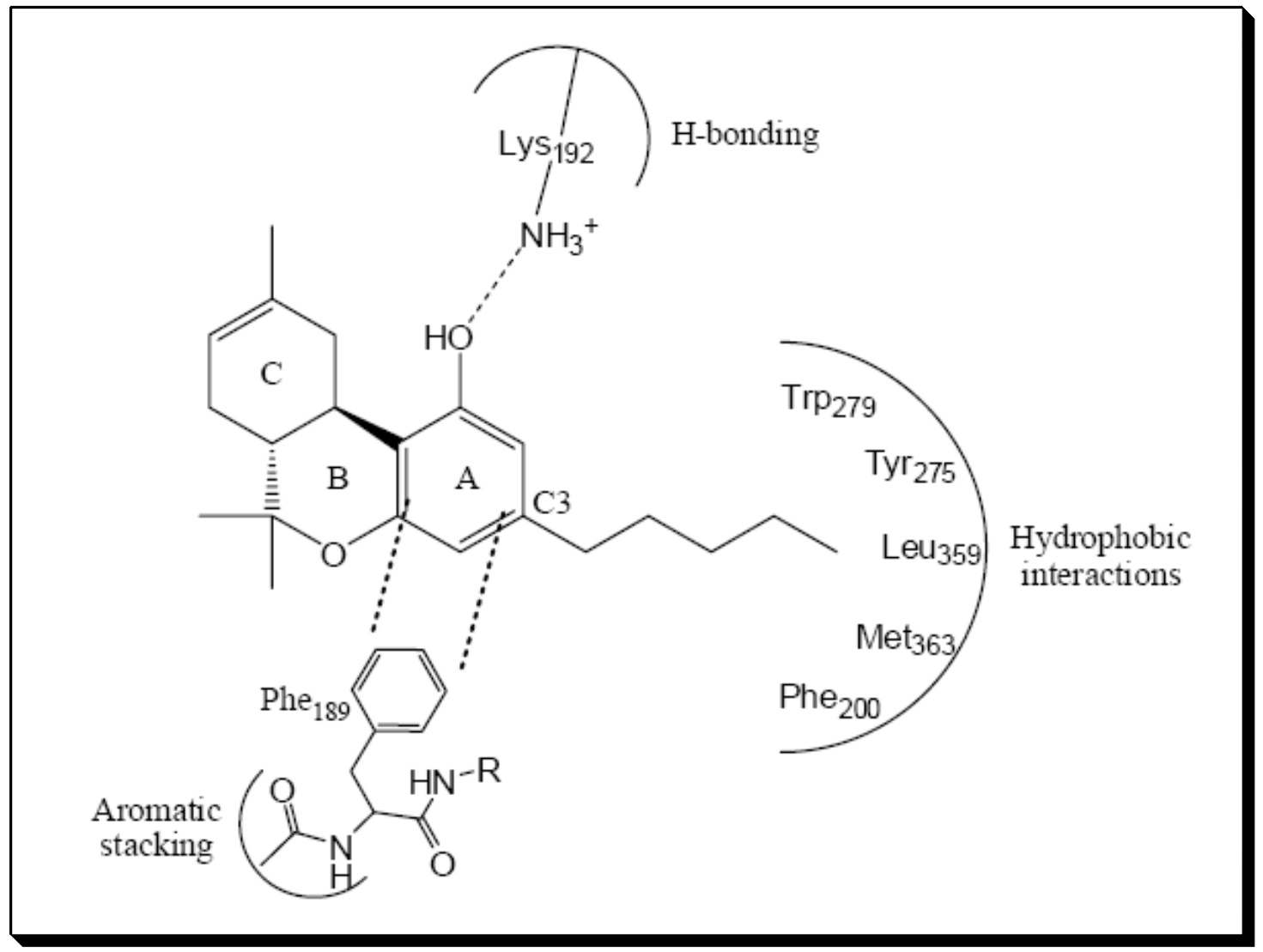

Figure 4.4: Classical cannabinoid binding interactions

Interactions between classical cannabinoids and key amino acid residues in the ligand binding pocket of the cannabinoid receptors. 
a. Lys 192 is a very important residue required for binding to classical cannabinoids. Due to the conformational flexibility of this residue, it can adopt an orientation that interacts with the phenolic hydroxyl in the A ring of classical cannabinoids. ${ }^{165}$

b. A predominantly hydrophobic pocket comprised of residues Tyr 275 , Trp 279 , Leu 359, Met 363, and Phe 200 is involved in binding to the C-3 side chain. ${ }^{166}$

c. Phe 189 lies in close proximity to the A ring of classical cannabinoids and may be involved in aromatic stacking interactions with the ring. ${ }^{166}$

While there is a sufficient understanding of binding interactions involved between ligands and the $\mathrm{CB} 1$ receptor, interactions at the $\mathrm{CB} 2$ receptor are not as well defined. It has been demonstrated that compounds lacking the phenolic hydroxyl of the A ring or having it occupied in an ether linkage, show enhanced affinity for CB2 as well as selectivity. ${ }^{167}$ The observations made with these modifications indicate the involvement of additional specific electronic and steric factors in ligand recognition at the CB2 receptor. It is also important to note that elimination of the $\mathrm{B}$ ring of the classical cannabinoid core with the biphenyl or triaryl core has resulted in compounds that are more CB2 selective also. ${ }^{127-128}$ Work in our laboratory has also demonstrated that classical cannabinoids with phenyl substitutions at the $\mathrm{C} 3$ position have affinities shifted toward CB2 selectivity. ${ }^{108}$

\subsection{Design and Synthesis of Novel Compounds}

\subsubsection{Rationale}

Upon careful review of past and current literature, it became clear that there had been one major design possibility that had been overlooked. In the efforts to design compounds that were more CB2 selective, no group had reported heteroaromatic modifications to the A ring of classical cannabinoids. Replacement of the phenyl ring with a pyrimidine could take advantage of the additional electronic interactions that are involved in ligand recognition at the CB2 receptor. A logical extension of this design was the addition of the heteroaromatic motif to the triaryl scaffold as well (Figure 4.5). It was also speculated that the addition of the pyrimidine to these cannabinoid structures could increase water solubility thereby making it easier to formulate these compounds into dosage forms. The decrease in lipophilicity could also limit the blood-brain barrier penetration of the cannabinoids which would make them ideal candidates as therapies for systemic inflammatory conditions. 


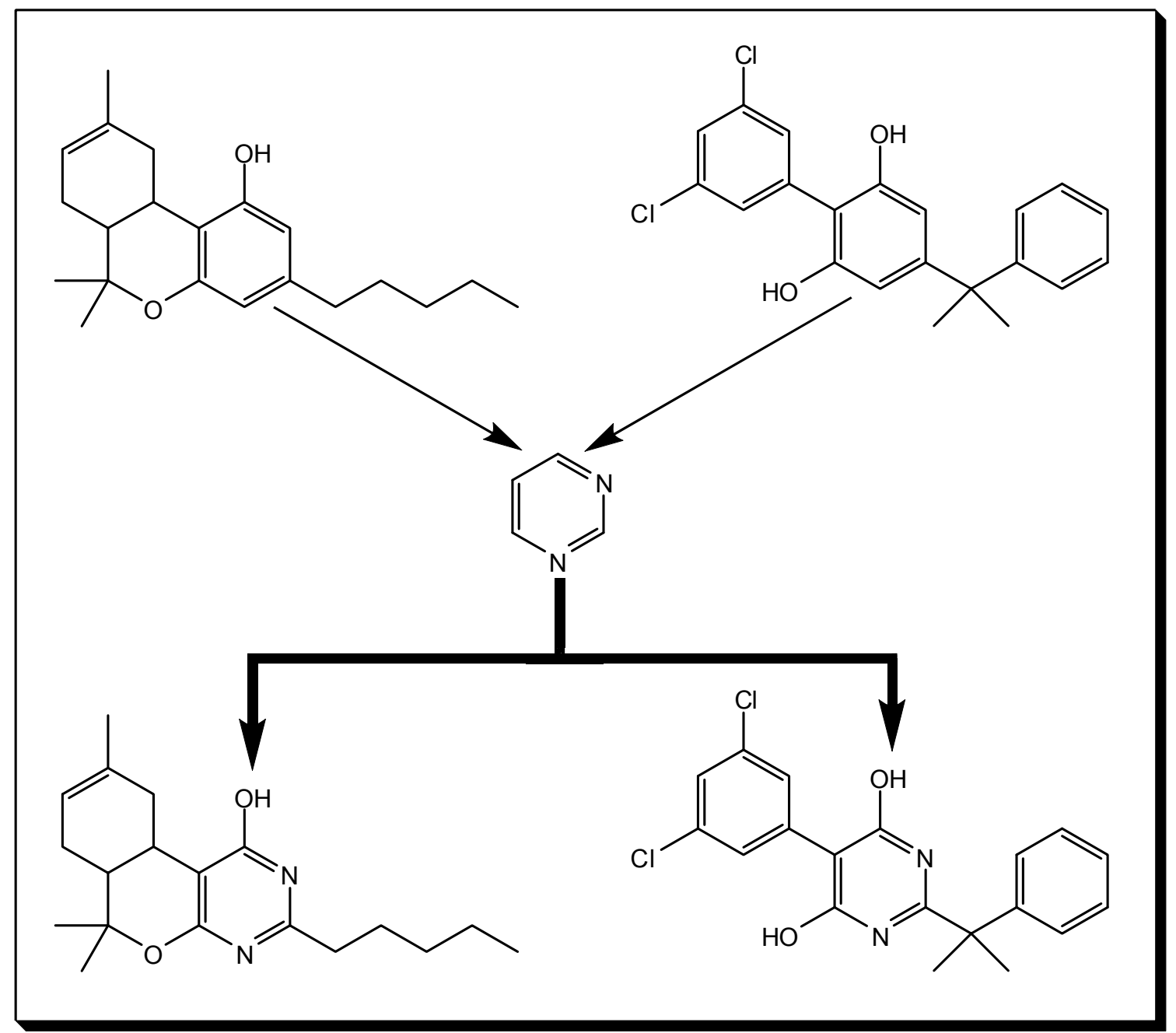

Figure 4.5: General design of pyrimidyl cannabinoids

Rationale for the pyrimidine heteroaromatic substitution in the classical cannabinoids and the triaryls. 


\subsubsection{Synthesis of Hexahydro Pyrimidine Classical Cannabinoids}

The hexadydro pyrimidine classical cannabinoids were prepared by reacting an intermediate pyrimidine with the appropriate terpene to generate the desired compounds according to the Scheme 4.1. The hexahydro compounds are synthesized utilizing a modification of the Diastereoselective Domino Knoevenagel Hetero-Diels-Alder Reaction described by Tietz. ${ }^{168}$ The ring formations are accomplished under microwave conditions with the appropriately substituted pyrimidine and citronellal.

Synthesis of the intermediate substituted pyrimidines proved to be more challenging. The first strategy was to form the hexahydro core with commercially available 4,6 dihydroxy pyrimidine which lacked the side chain (Scheme 4.2). The phenolic -OH would then be protected by a tert-butyldimethylsilyl (TBDMS) group. The addition of the side chain could then be accomplished by deprotonation of the 2 position of the pyrimidine and then reacting with an appropriate aldehyde to form the intermediate alcohol or lithiation of the same position by butyl lithium and N,N-dimethylaminoethanol and then reacting with the aldehyde to form the alcohol or a nitrile to form the ketone (Scheme 4.3). Neither of these methods was successful in formation of the side chain. An attempt to prime the 2 position of the pyrimidine as a Grignard substrate was also performed by reacting the lithiate with dimethylformamide to form an aldehyde in this position which could then be reacted with the appropriate magnesium halides (Scheme 4.3). This method was also unsuccessful.

The second strategy involved the synthesis of ethyl 4,6-dimethoxypyrimidine-2carboxylate from malononitrile, methanol, and ethyl chloroxoacetate (Scheme 4.4). ${ }^{169}$ This intermediate would then serve as the scaffold for building the side chain. The ester was then reduced to the aldehyde using diisobutylaluminum hydride (DIBAL-H) to prepare the molecule for the Grignard reaction (Scheme 4.5). The aldehyde was then reacted with an appropriate aldehyde and the resulting alcohol oxidized to the ketone using pyridinium chlorochromate (PCC) (Scheme 4.5). The ketone could then be dimethylated using tin chloride and dimethyl zinc according to the method employed by Krishnamurthy. ${ }^{108}$ This method, however led to the monomethyl alcohol as did methylation using trimethyl aluminum (Scheme 4.6). The same product was also formed when trimethyl aluminum was paired with trimethylsilyl trifluoromethanesulfonate in an attempt to make the alcohol a better leaving group to allow for the formation of the dimethyl linker ${ }^{170}$ (Scheme 4.7). An attempt to activate the alcohol was also made by converting it to the mesylate; ${ }^{171}$ this however led to the vinyl elimination product (Scheme 4.7).

It became clear that forming the dimethyl linker in the side chain would not be feasible using 4,6-dimethoxypyrimidine-2-carboxylate as the starting material. This would work, however, for the hydroxyl and ketone linker. Unfortunately, another problem arose. In order to form the hexahydro classical cannabinoids, citronellal must be reacted with the deprotected pyrimidine, not the dimethoxy intermediate. The differences between dimethoxy phenyl rings and pyrimidine rings caused difficulties here also. Previous methods employed by our laboratory to cleave the methyl ethers such as boron tribromide ${ }^{108,129}$ were unsuccessful in the deprotection of the dimethoxy pyrimidines. 


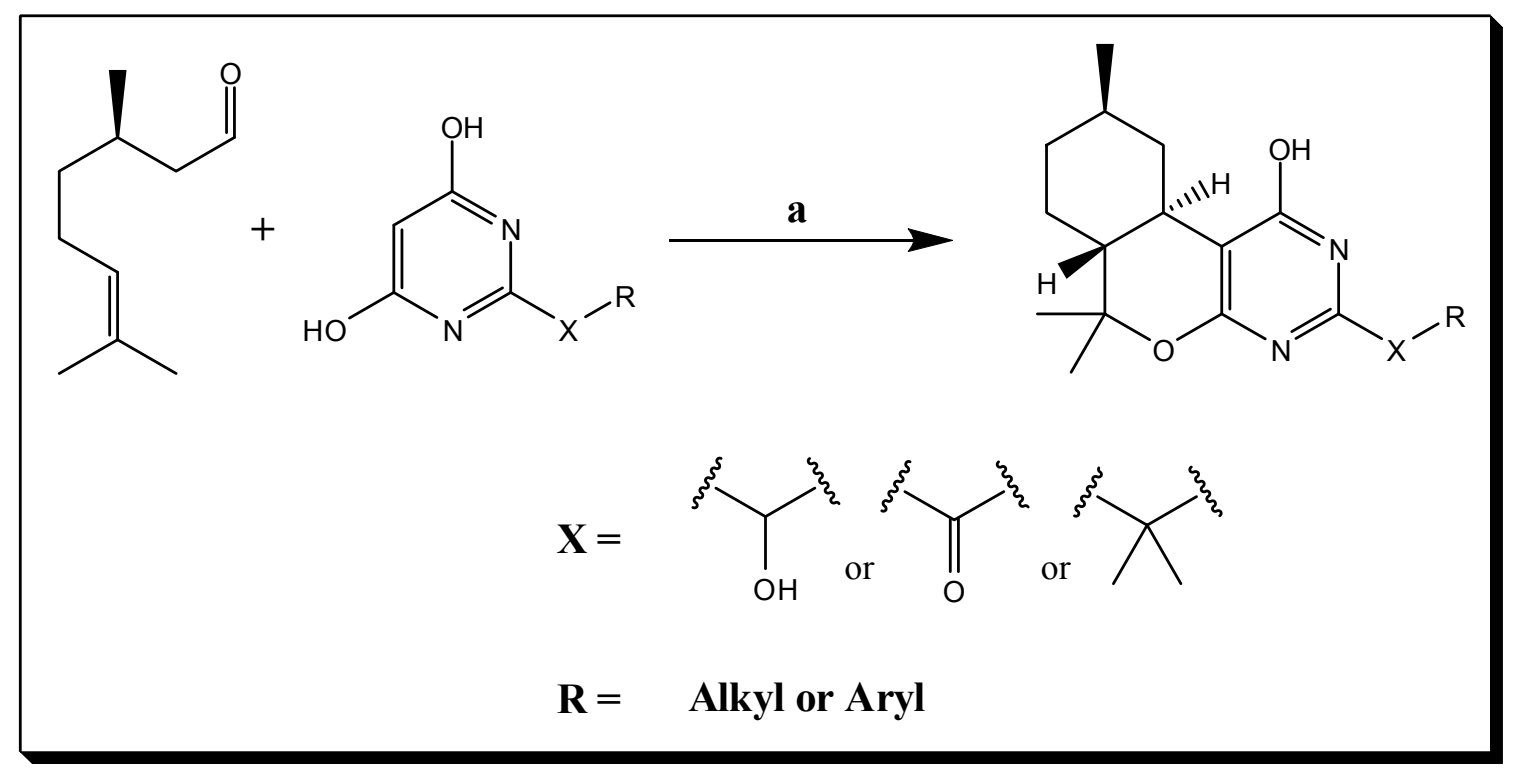

Scheme 4.1: Synthesis of hexahydro pyrimidine classical cannabinoids

Hexahydro pyrimidine classical cannabinoids are synthesized from R-citronellal and a substituted dihydroxy pyrimidine. (a) piperidine, pyrimidine, EtOH, $\mu$ wave, 200 watts, $130^{\circ} \mathrm{C}, 1 \mathrm{hr}$. 


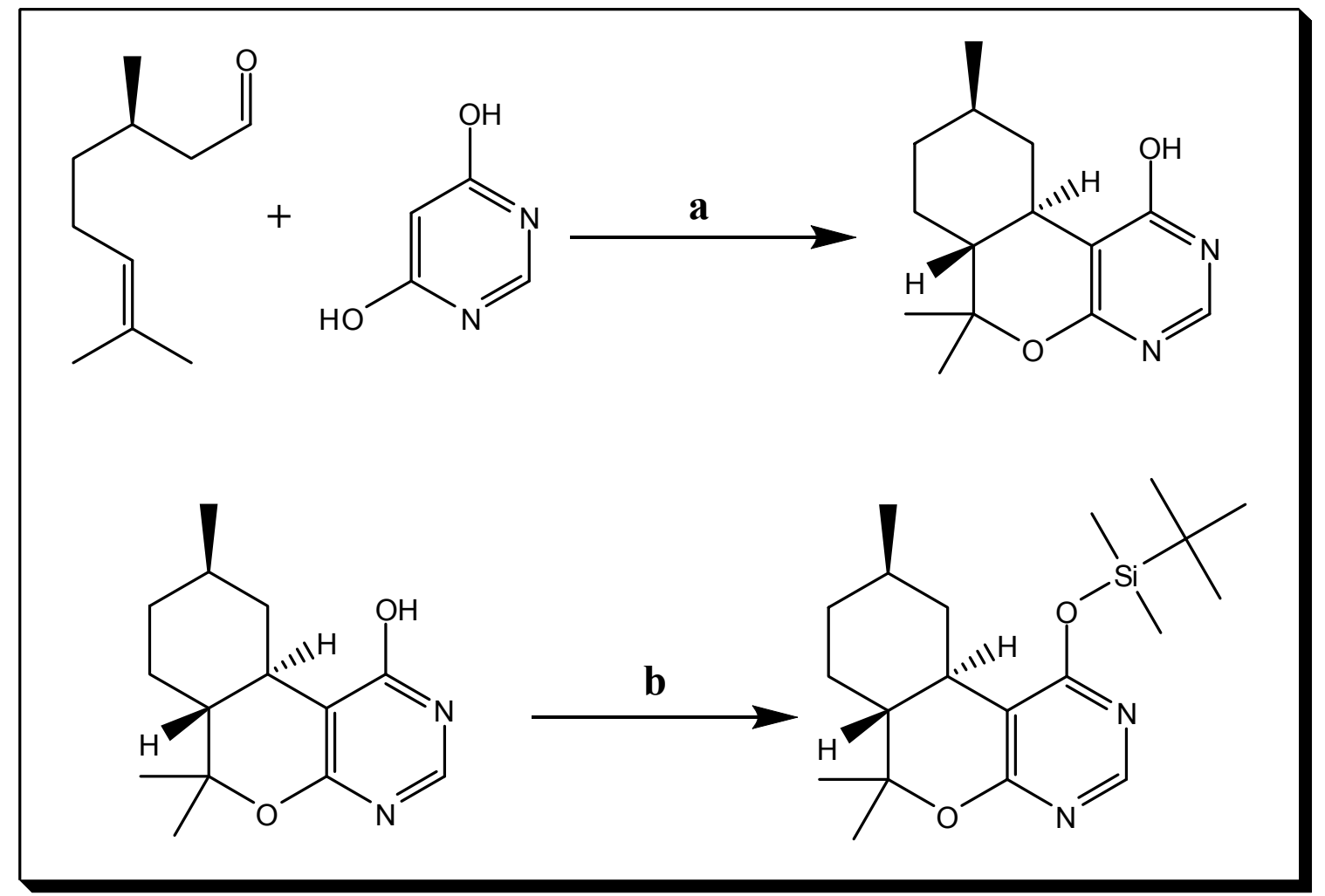

Scheme 4.2: Synthesis of TBDMS protected hexahydro pyrimidine classical cannabinoids

TBDMS protected hexahydro pyrimidine classical cannabinoids were synthesized from R-citronellal and substituted dihydroxy pyrimidine. (a) piperidine, pyrimidine, EtOH, $\mu$ wave, 200 watts, $130^{\circ} \mathrm{C}, 1 \mathrm{hr}$. (b) TBDMS-Cl, imidazole, $\mathrm{CH}_{3} \mathrm{CN}$, $\mu$ wave, 150 watts, $140^{\circ} \mathrm{C}, 2 \mathrm{hr}$. 


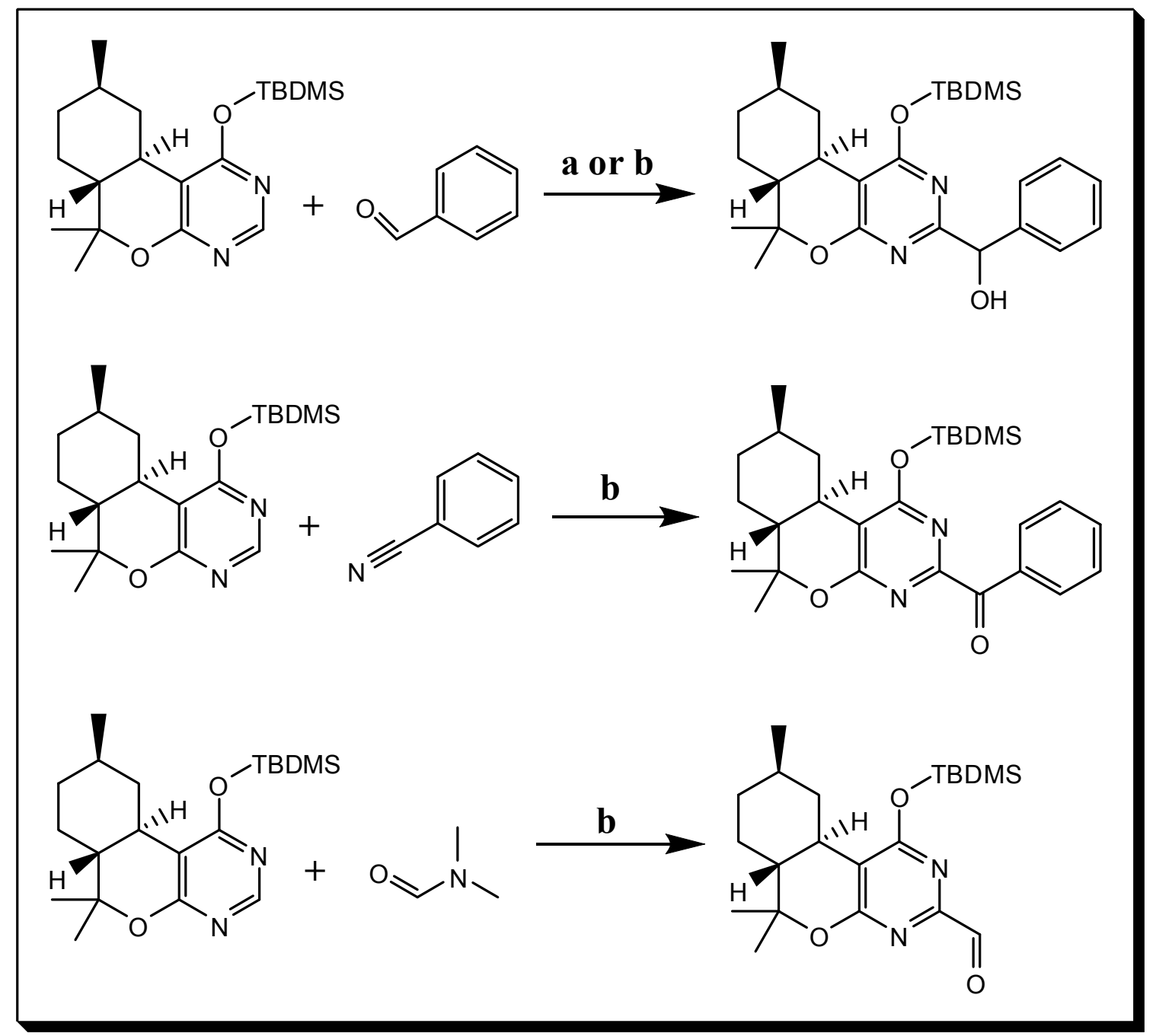

Scheme 4.3: Attempts at introduction of the hexahydro pyrimidine side chain

Failed attempts at introduction of the hexahydro pyrimidine side chain to protected hexahydro pyrimidine classical cannabinoids. (a) n-butyllithium, THF, $-78^{\circ} \mathrm{C}-\mathrm{RT}, 18 \mathrm{hr}$. (b) n-butyllithium, N,N dimethylaminoethanol, hexanes, THF, $-78^{\circ} \mathrm{C}-\mathrm{RT}, 18 \mathrm{hr}$. 


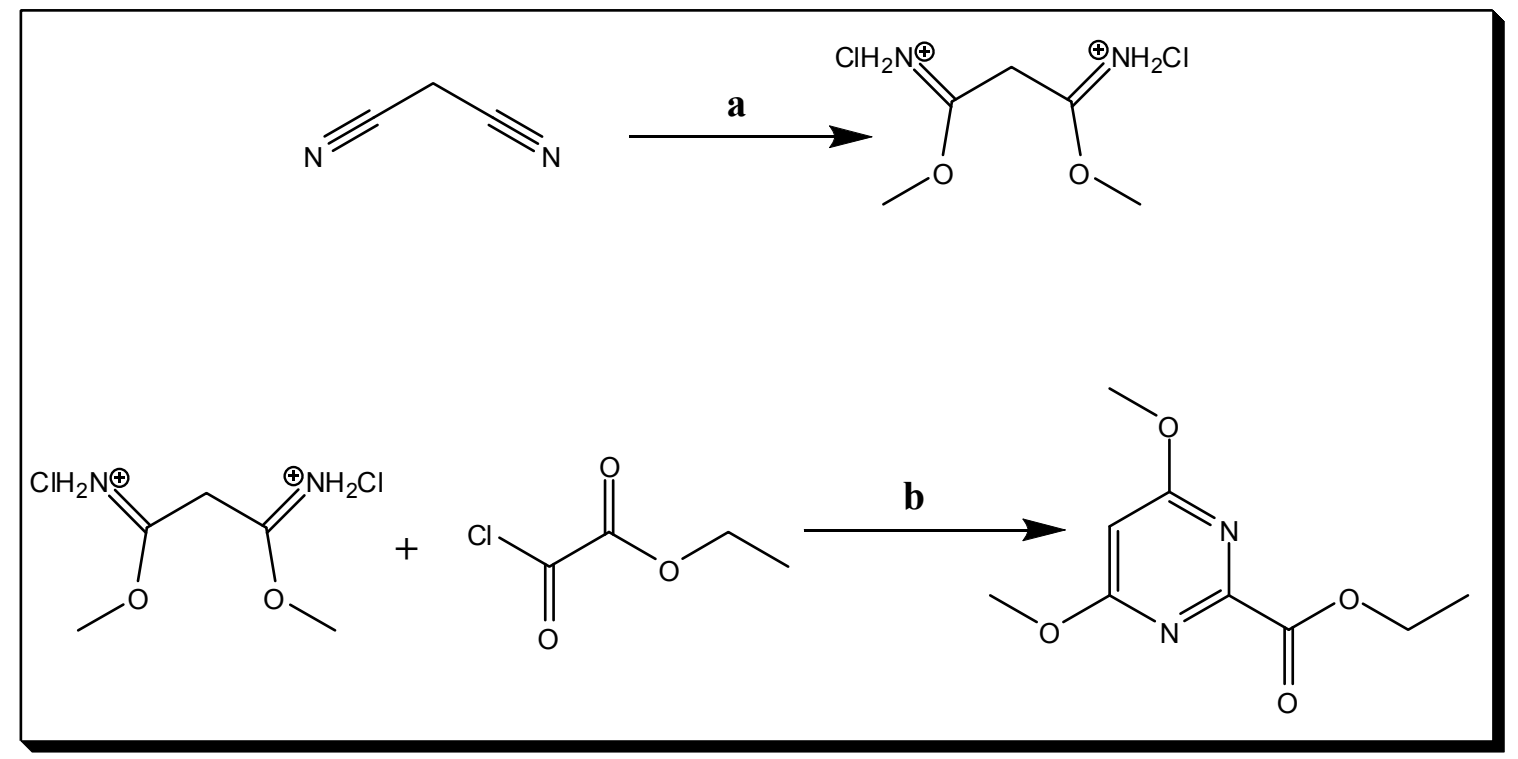

Scheme 4.4: Synthesis of 4,6-dimethoxypyrimidine-2-carboxylate

(a) methanol, $\mathrm{HCl}$ (gas), methyl acetate, $-18^{\circ} \mathrm{C}, 3 \mathrm{hr}$, cooled to $5^{\circ} \mathrm{C}$. (b) $\mathrm{DIEA}, \mathrm{CH}_{2} \mathrm{Cl}_{2}$, $40^{\circ} \mathrm{C}-\mathrm{RT}, 18 \mathrm{hr}$. 


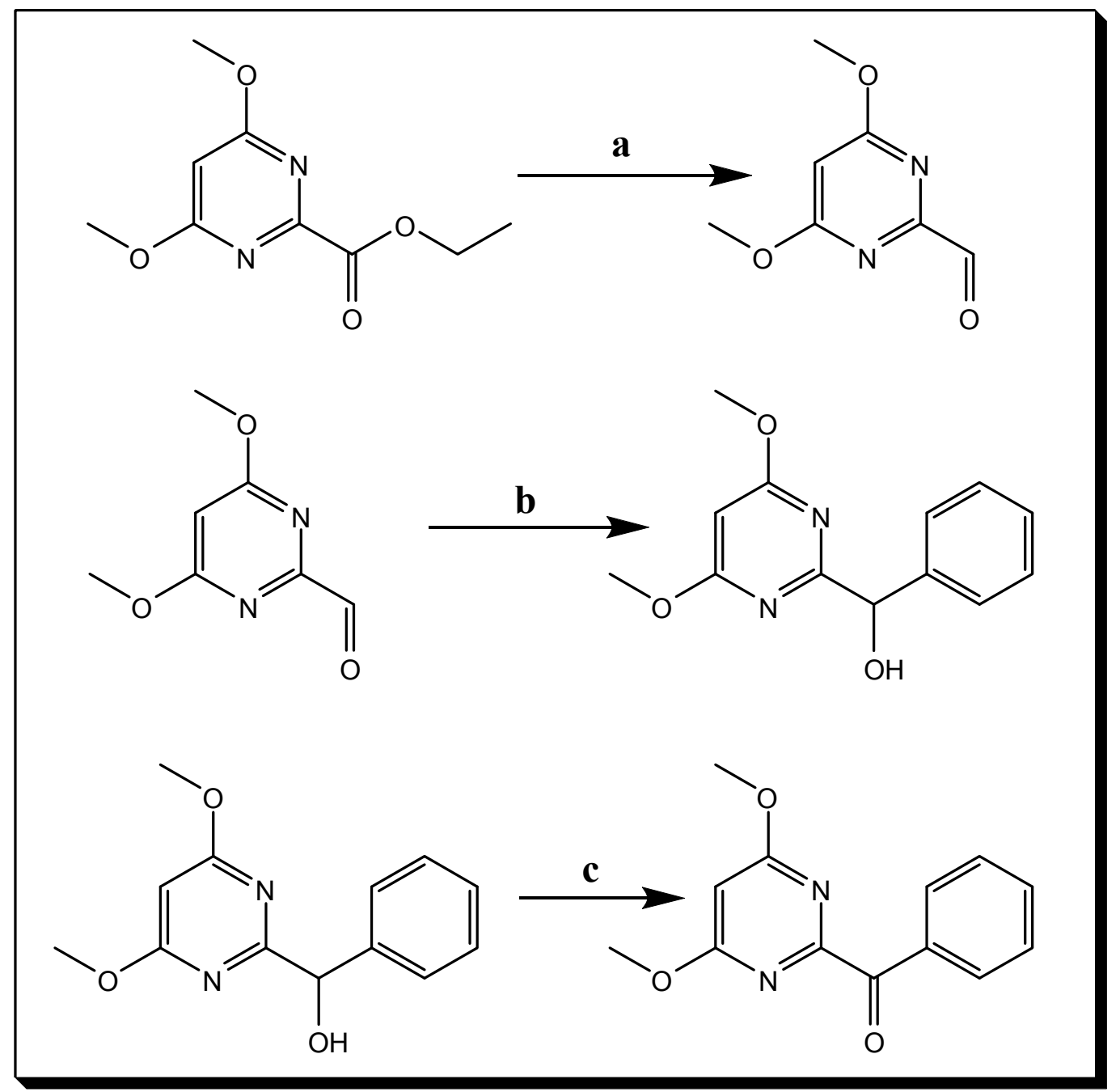

Scheme 4.5: Introduction of the side chain to 4,6-dimethoxypyrimidine-2-carboxylate

(a) DIBAL-H, toluene, $-78^{\circ} \mathrm{C}-25 \mathrm{~min}, \mathrm{HCl} 0^{\circ} \mathrm{C}$. (b) Phenyl $\mathrm{MgBr}$, THF, $0^{\circ} \mathrm{C}-4 \mathrm{hr}$, RT - 18hr. (c) PCC, Celite, $\mathrm{CH}_{2} \mathrm{Cl}_{2}$, RT, $18 \mathrm{hr}$. 


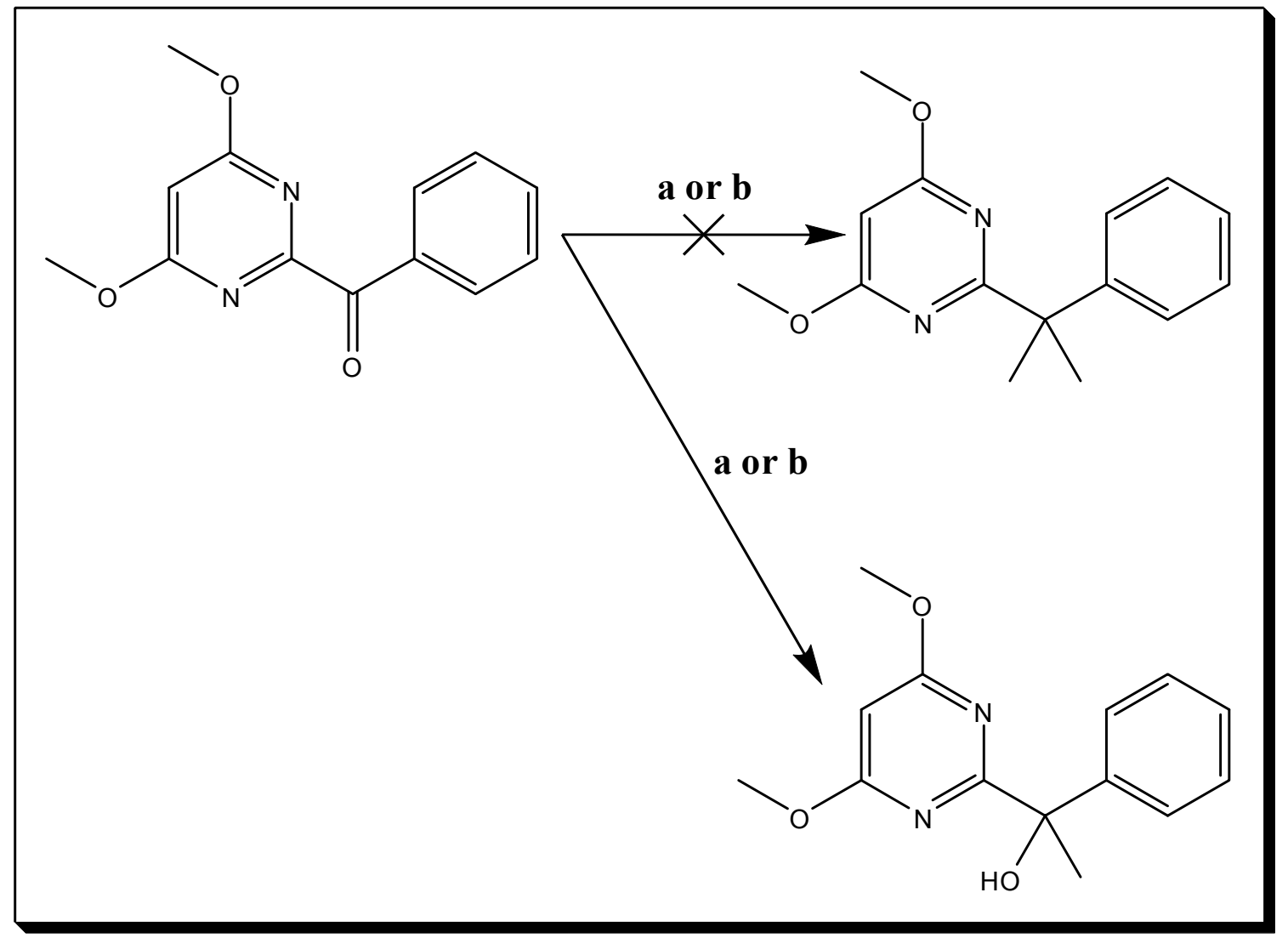

Scheme 4.6: Attempts at dimethylation of the ketone intermediate

(a) $\mathrm{TiCl}_{4}, \mathrm{Zn}\left(\mathrm{CH}_{3}\right)_{2}, \mathrm{CH}_{2} \mathrm{Cl}_{2},-40^{\circ} \mathrm{C}-2 \mathrm{hr}, \mathrm{RT}-16 \mathrm{hr}$. (b) $\mathrm{Me}_{3} \mathrm{Al}, \mathrm{CH}_{2} \mathrm{Cl}_{2}, 0^{\circ} \mathrm{C}-1 \mathrm{hr}, \mathrm{RT}$ $-16 \mathrm{hr}$. 


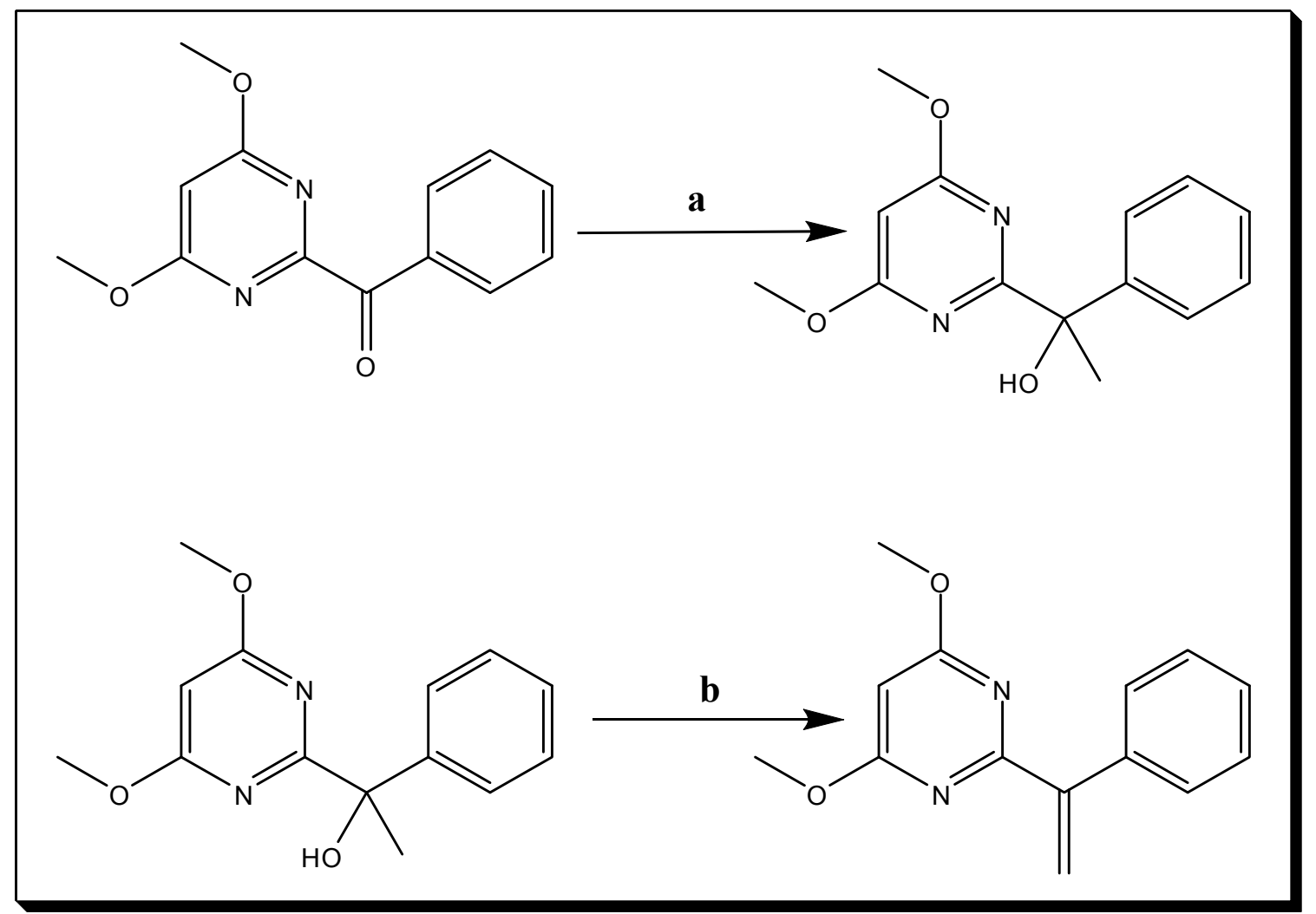

Scheme 4.7: Further attempts at dimethylation of the ketone intermediate $\mathrm{Me}_{3} \mathrm{Al}, \mathrm{TMSOSO}_{2} \mathrm{CF}_{3}, \mathrm{CH}_{2} \mathrm{Cl}_{2}, 0^{\circ} \mathrm{C}-1 \mathrm{hr}, \mathrm{RT}-16 \mathrm{hr}$. (b) $\mathrm{NaH}$, THF, $0^{\circ} \mathrm{C}-\mathrm{RT}, 1 \mathrm{hr}$, $40^{\circ} \mathrm{C}-3 \mathrm{hr}$, methane sulfonyl chloride, $\mathrm{RT}-1 \mathrm{hr}, 40^{\circ} \mathrm{C}-2 \mathrm{hr}$. 
Other methods were also employed: $\mathrm{KOH}, \mathrm{HBr}$, and methylmagnesium iodide. None of these proved successful (Scheme 4.8).

This combination of difficulties led to the abandonment of methods that had previously been employed successfully in our laboratory for synthesis of classical cannabinoid side chains. It was decided to form the side chain first which would contain a cyano group. This cyano group would be converted to the amidine which would be reacted with diethyl malonate to form the dihydroxy pyrimidine with the side chain in place.

The nitriles were formed either by reaction of a commercially available aryl or alkyl halide with isobutyronitrile in the presence of potassium bis(trimethylsilyl)amide (KHMDS) (Scheme 4.9) ${ }^{172}$ or by dimethylation of a commercially available aryl or alkyl nitrile with methyl iodide in the presence of KHMDS (Scheme 4.10). ${ }^{173}$ The amidines are then formed from the nitriles by Garigipati's reaction utilizing methylchloroaluminum amide formed in situ from trimethylaluminum and ammonium chloride (Scheme 4.11). ${ }^{174}$ This method, which only provided moderate yields of around $40 \%$, was chosen due to the known unreactivity of hindered nitriles in the more common Pinner reaction. The limited availability of anhydrous ammonia at that time also made the Pinner reaction less feasible. One major drawback of this method is that it requires an extensive two day purification procedure to separate the amidines from residual ammonium chloride.

The amidines were to then be cyclized into dihydroxy pyrimidines by reacting with diethyl malonate. This reaction was first attempted in the presence of N,Ndiisopropylethylamine (DIEA). This method did not lead to formation of the desired product and it was speculated that perhaps DIEA was not a strong enough base to drive the reaction forward. Sodium ethoxide was chosen as the base and commercially available solid sodium ethoxide was purchased and tried in the reaction. This also did not lead to formation of the product. The reaction was also attempted under solvent-free conditions and also under microwave irradiation. Each of these efforts remained unfruitful. Successful formation of the pyrimidines was finally achieved by in situ formation of fresh sodium ethoxide to deprotonate the amidines (Scheme 4.12). ${ }^{175}$ Diethyl malonate was then added and the reactions heated to reflux overnight. The solid sodium chloride was then filtered out and the filtrate was diluted with water and acidified to $\mathrm{pH} 2$ causing the dihydroxy pyrimidnes to precipitate.

The pyrimidine hexahydro classical cannabinoids were then formed by reaction of the appropriately substituted dihydroxy pyrimidines with R-citronellal under microwave conditions (Scheme 4.13). This Domino Knoevenagel Hetero-Diels-Alder method uses ethanol as the solvent and piperidine and pyridine as co-solvents and as the base to catalyze the coupling. The final compounds are easily purified using flash chromatography and are isolated in good yields of $\sim 80 \%$. The side chains selected for these novel structures were the dimethyl heptyl, dimethyl cyclohexyl, dimethyl phenyl, and the dimethyl 2-thiophene. These selections were made based on the known binding affinity afforded to classical cannabinoids by the linear dimethyl heptyl and by our groups findings that that the cyclohexyl has similar binding affinities to the linear alkyl and that aryl groups such as the phenyl and thiophenyl shift selectivity toward the CB2 receptor. $^{153 \mathrm{a}}$ 


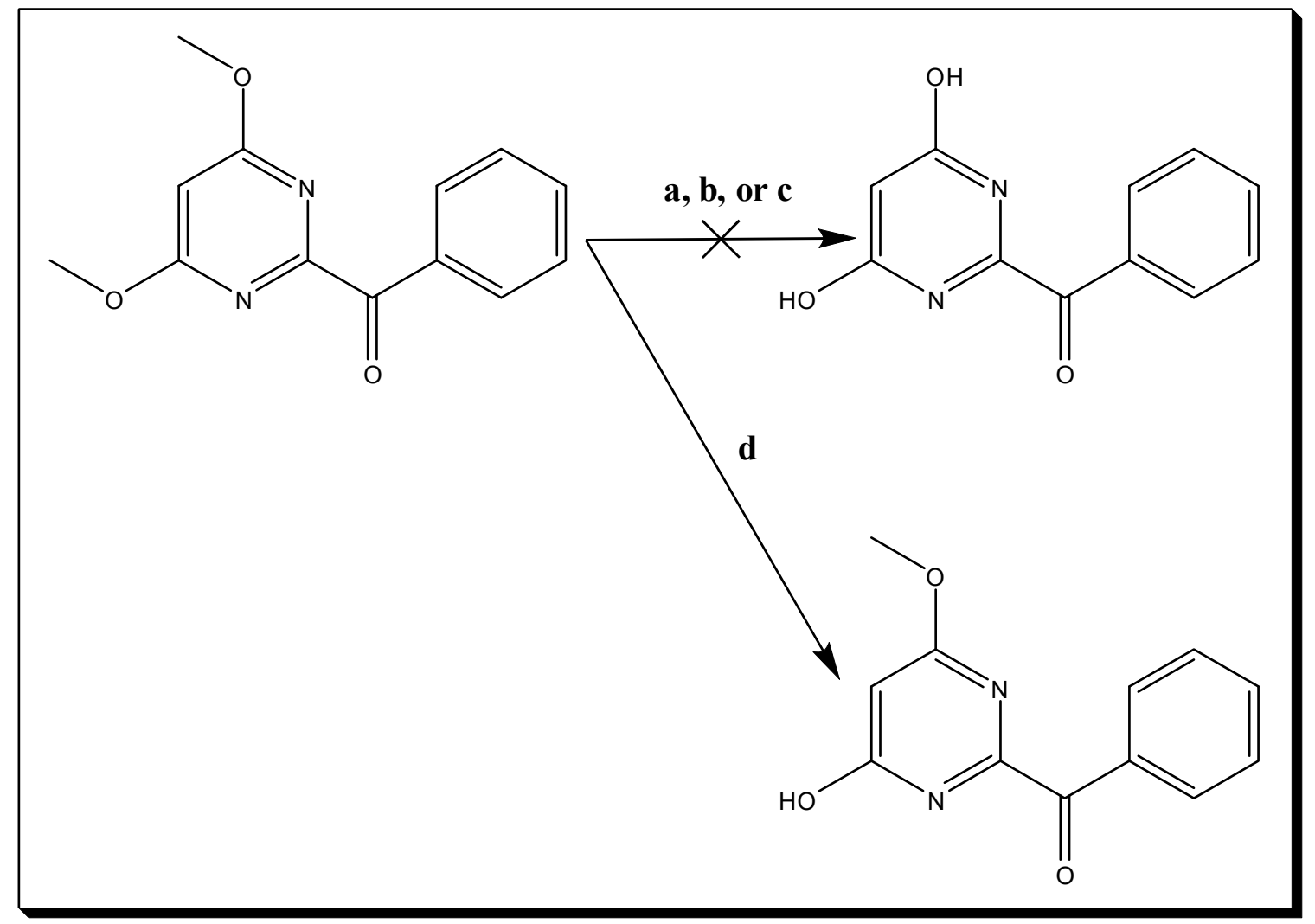

Scheme 4.8: Attempts at deprotection of the dimethoxy pyrimidine intermediates

(a) $\mathrm{BBr}_{3}, \mathrm{CH}_{2} \mathrm{Cl}_{2},-78^{\circ} \mathrm{C}-3 \mathrm{hr}, \mathrm{RT}-16 \mathrm{hr}$. (b) $\mathrm{HBr}-\mathrm{AcOH}(1: 3), 80^{\circ} \mathrm{C}, 1 \mathrm{~h}$, then saturated $\mathrm{NaHCO}_{3}$. (c) MeMgI, 1,4 dioxane, reflux, 16hr. (d) $\mathrm{KOH}, 1,4$ dioxane, reflux, $16 \mathrm{hr}$. 


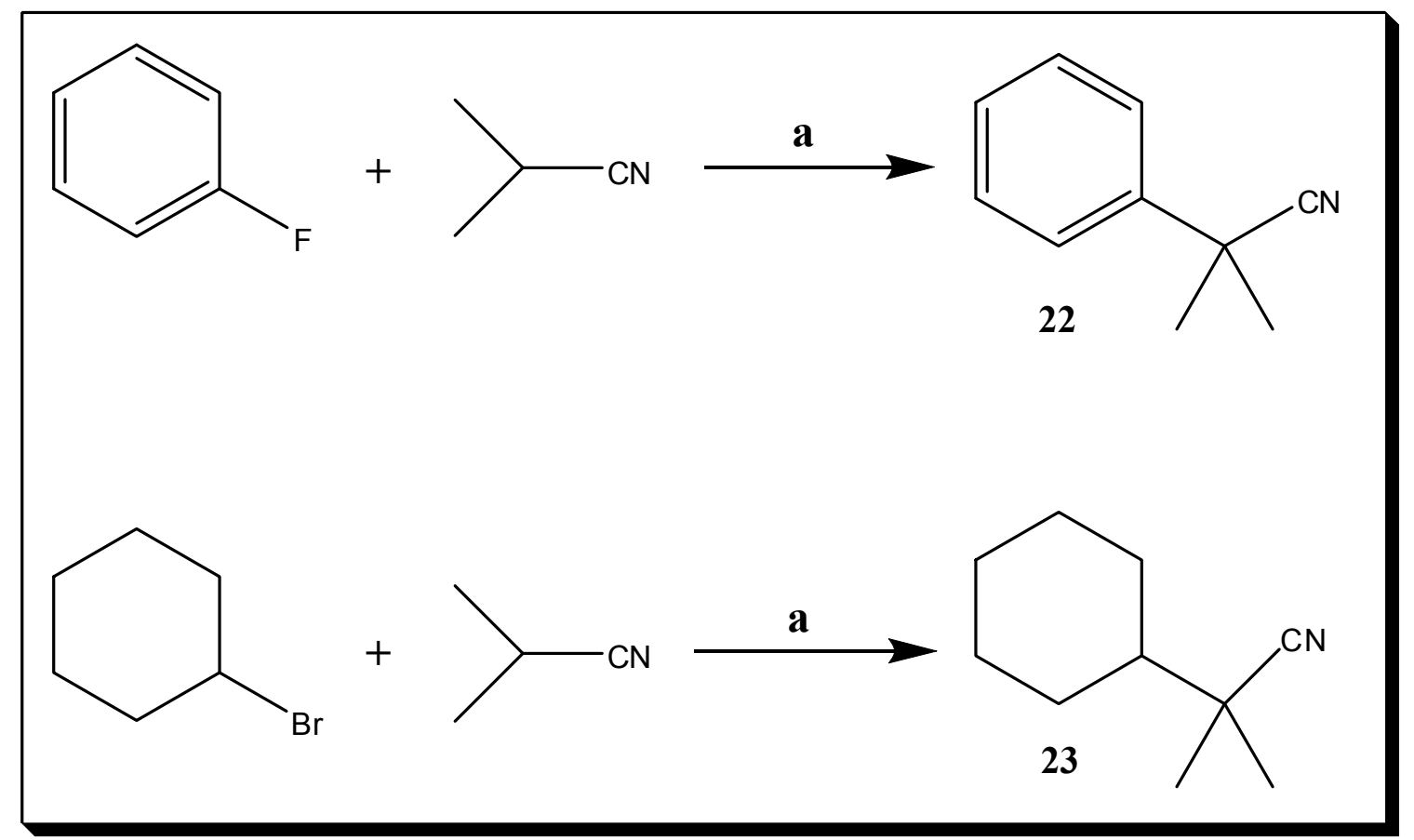

Scheme 4.9: Formation of nitrile intermediates from halides and isobutyronitrile

(a) $\mathrm{KHMDS}$, toluene, $80^{\circ} \mathrm{C}, 24 \mathrm{hr}$. 


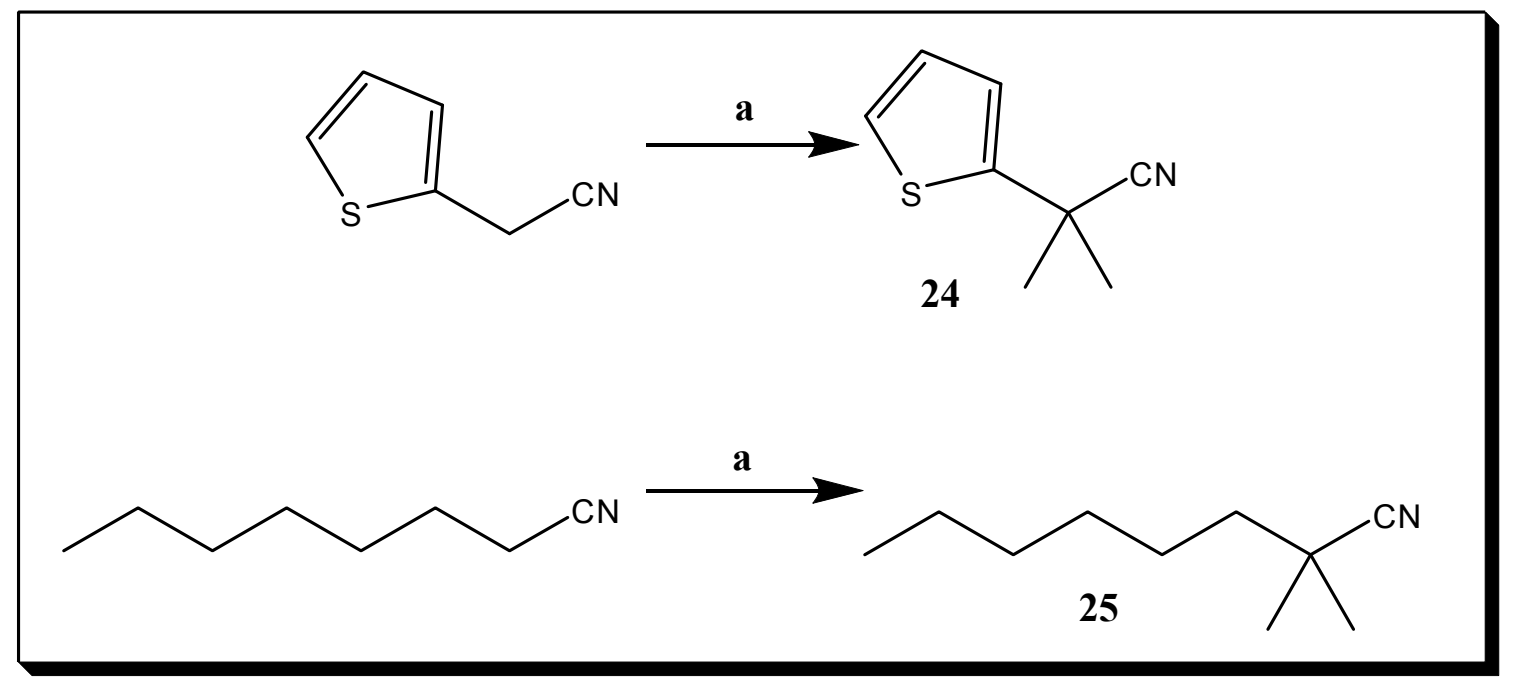

Scheme 4.10: Formation of nitrile intermediates from cyanides

(a) KHMDS, MeI, THF, $0^{\circ} \mathrm{C}, 10 \mathrm{~min}$. 


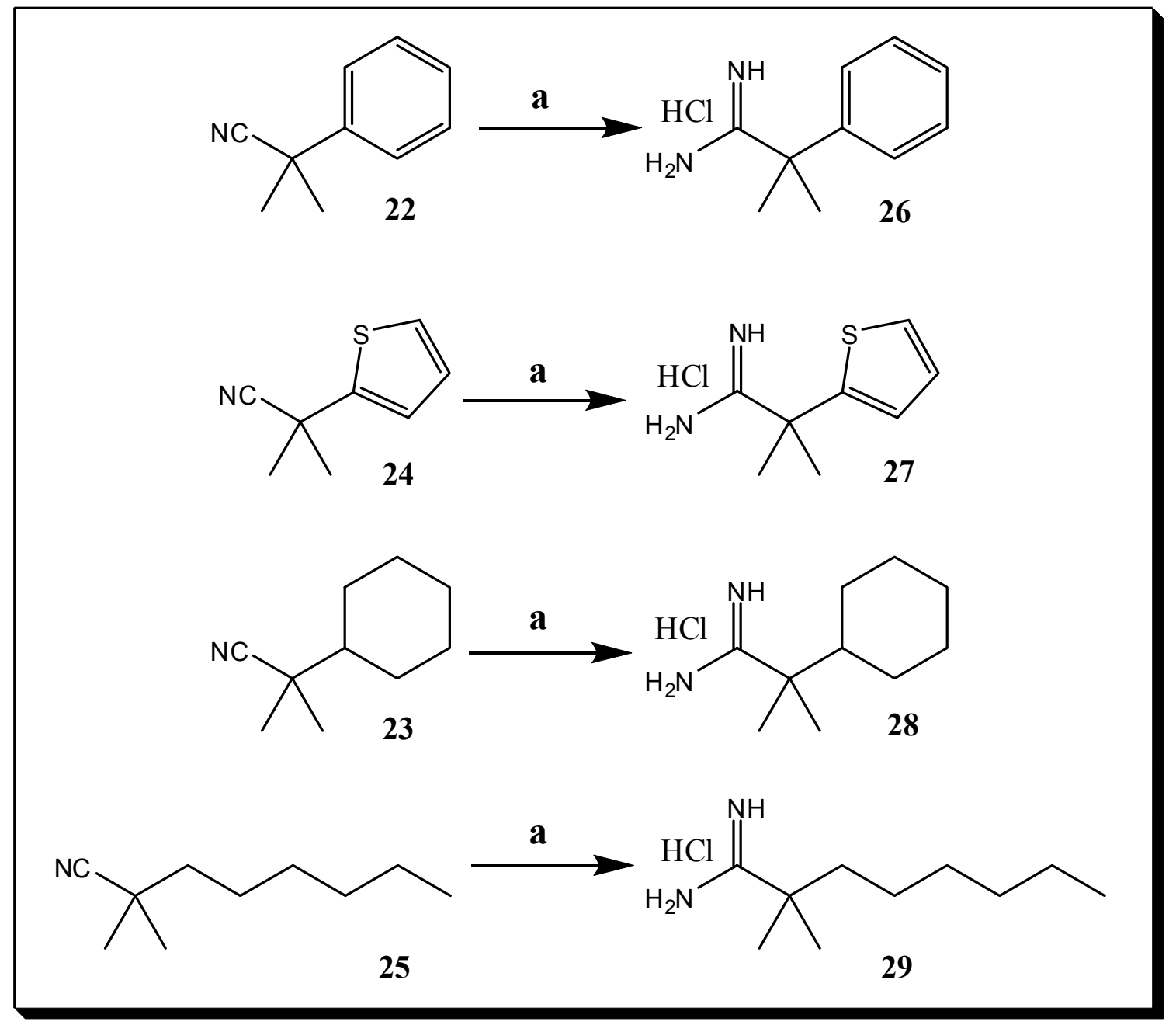

Scheme 4.11: Formation of amidines from nitriles

(a) $\mathrm{NH} 4 \mathrm{Cl}$, Me $3 \mathrm{Al}$, toluene, $0^{\circ} \mathrm{C}-\mathrm{RT}, 45 \mathrm{~min}$, reflux, $18 \mathrm{hr}$, then silica gel, $\mathrm{CHCl} 3, \mathrm{RT}$, $10 \mathrm{~min}$, then methanolic $\mathrm{HCl}$, diethyl ether, RT, overnight, then isopropanol:acetone 4:1, RT, overnight, then diethyl ether, RT. 


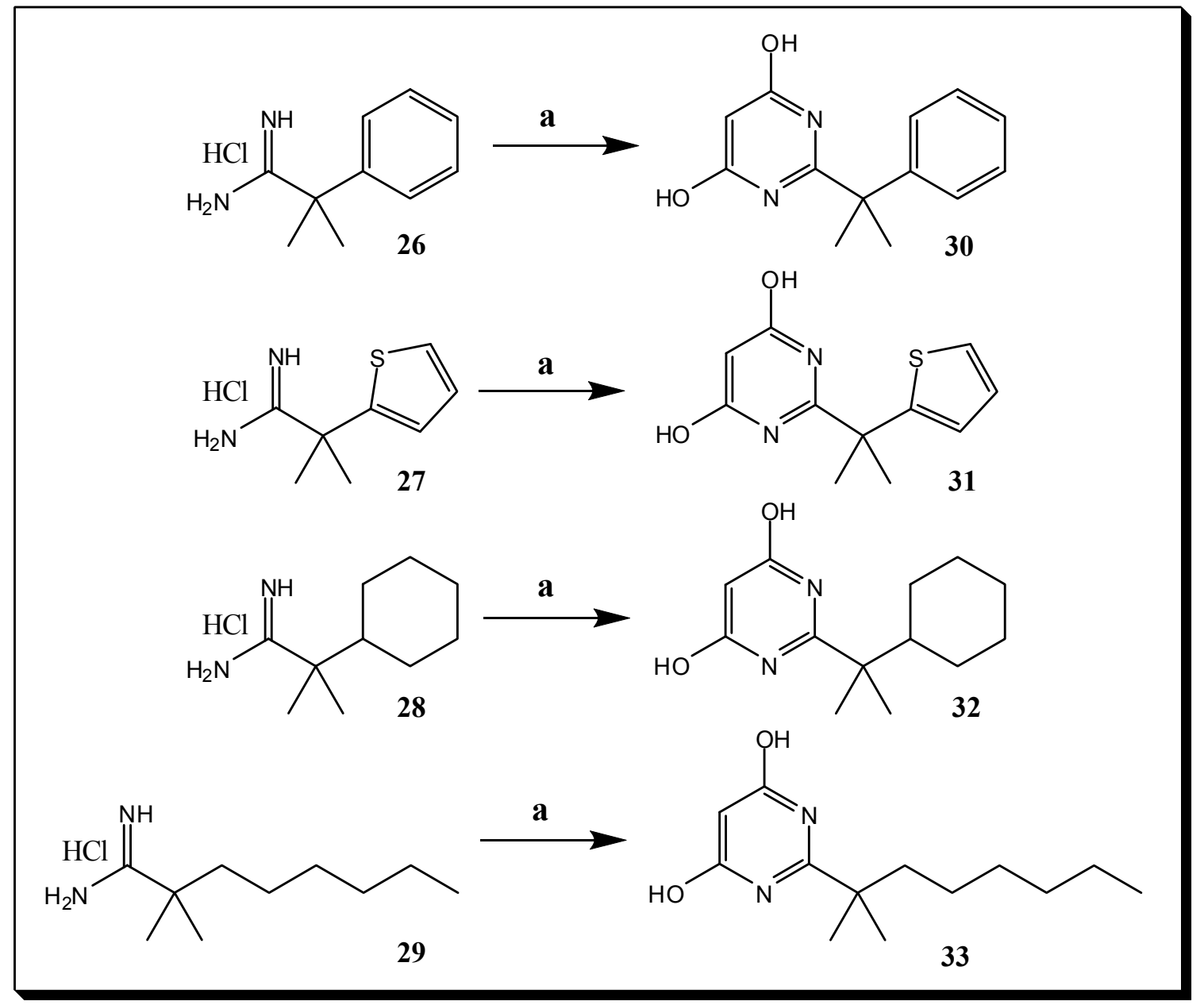

Scheme 4.12: Synthesis of dihydroxy pyrimidine intermediates

(a) Na metal, diethyl malonate, EtOH, reflux, $18 \mathrm{hr}$. 


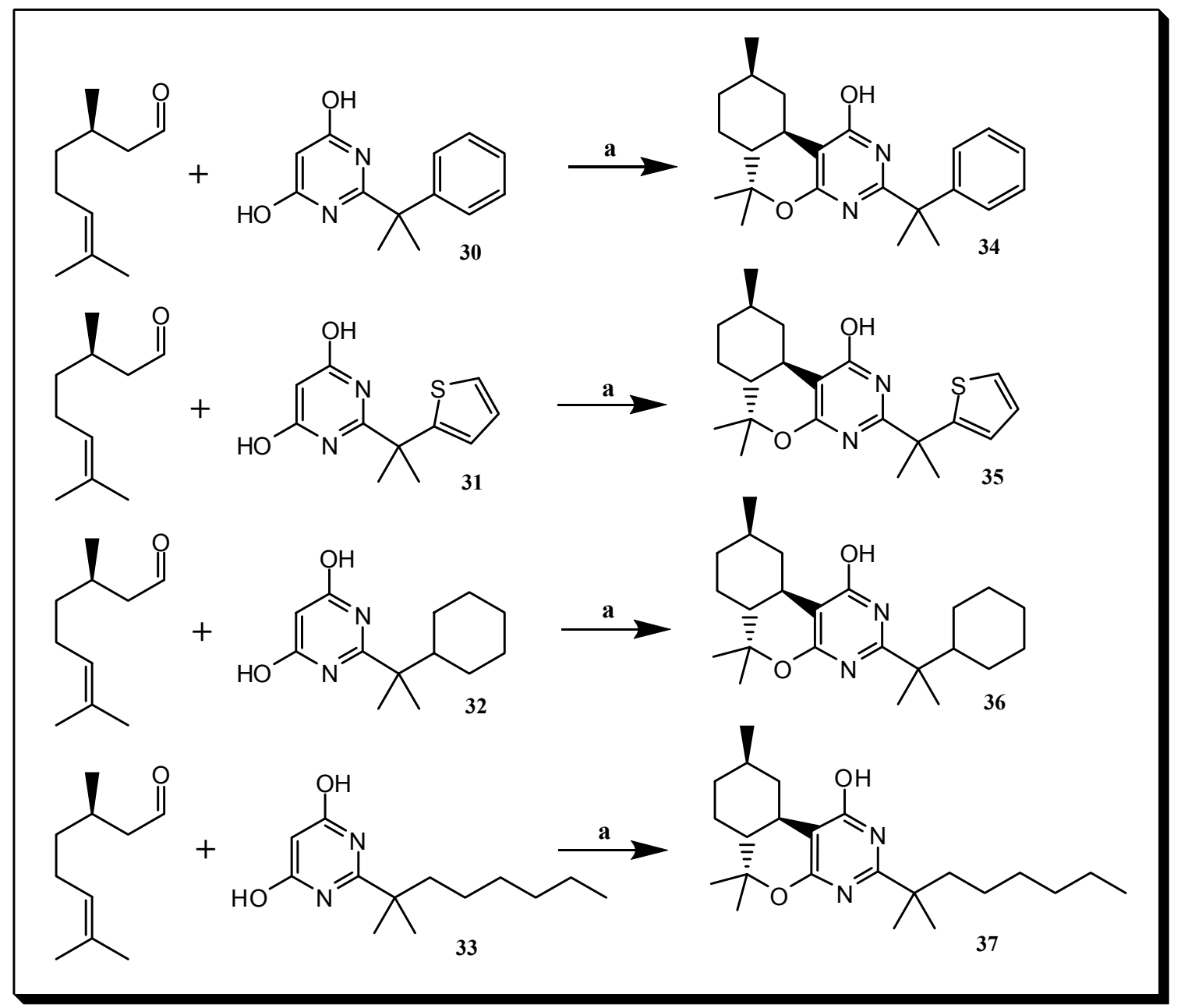

Scheme 4.13: Coupling of pyrimidine hexahydro classical cannabinoids

(a) piperidine, pyrimidine, EtOH , $\mu$ wave, 200 watts, $130^{\circ} \mathrm{C}, 1 \mathrm{hr}$. 
All chemicals were obtained from Sigma Aldrich or Fisher Scientific Inc. Anhydrous solvents were obtained by distillation over either calcium hydride or metallic sodium and benzophenone. Final compounds and intermediates were purified using column chromatography on the Biotage SP1 system employing Flash column cartridges or by crystallization. NMR spectra were obtained on a Bruker $300 \mathrm{MHz}$ or Varian $500 \mathrm{MHz}$ Inova NMR. HPLC analysis of final products was carried out by gradient elution using water/acetonitrile (0.1\% TFA) with a gradient of 70\% (3min), 50\% (2min), and $10 \%(15 \mathrm{~min})$. A reverse-phase ODS Hypersil column, manufactured by Thermo Scientific, of dimensions $4.6 \times 150 \mathrm{~mm}$ with $5 \mu \mathrm{m}$ particle size was used for HPLC analyses.

\subsubsection{Synthesis of 2-methyl-2-phenylpropanenitrile (22)}

To a solution of fluorobenzene $(5.85 \mathrm{~mL}, 62.4 \mathrm{mmol})$ in $100 \mathrm{~mL}$ of anhydrous toluene was added isobutyronitrile $(22.5 \mathrm{~mL}, 250 \mathrm{mmol})$ followed by $200 \mathrm{~mL}(100 \mathrm{mmol})$ of a $0.5 \mathrm{M}$ solution of KHMDS in toluene. The reaction was stirred at $80^{\circ} \mathrm{C}$ for 24 hours. The reaction was then allowed to cool to room temperature, diluted with diethyl ether, and washed with water and brine. The organic fraction was then dried over sodium sulfate and concentrated under reduced pressure. The product was purified by flash chromatography using an ethyl acetate/hexanes gradient to yield $4.57 \mathrm{~g}(50 \%)$ of the objective compound as a brown oil. MS: (ESI, pos.) $\mathrm{m} / z 168.0[\mathrm{M}+\mathrm{Na}]^{+}$, I.R. (neat) nitrile $2230 \mathrm{~cm}^{-1},{ }^{1} \mathrm{H}$ NMR $\left(500 \mathrm{MHz}, \mathrm{CDCl}_{3}\right): \delta(\mathrm{ppm}) 7.48(\mathrm{~d}, 2 \mathrm{H}), 7.39(\mathrm{t}, 2 \mathrm{H}), 7.31$ $(\mathrm{t}, 1 \mathrm{H}), 1.73(\mathrm{~s}, 6 \mathrm{H})$.

\subsubsection{Synthesis of 2-cyclohexyl-2-methylpropanenitrile (23)}

Prepared in a manner similar to compound $\mathbf{2 2}$ from bromo cyclohexane $(0.8 \mathrm{ml}$, $6.4 \mathrm{mmol})$ and isobutyronitrile $(2.34 \mathrm{ml}, 26 \mathrm{mmol})$ in the presence of KHMDS $(20 \mathrm{ml}$, $10 \mathrm{mmol}$ ). Purified via vacuum distillation (Bp $50-55^{\circ} \mathrm{C}$ at 1.1 torr). Yield: $84 \%$, MS: (ESI, pos.) $\mathrm{m} / z$ 174.1 $[\mathrm{M}+\mathrm{Na}]^{+}$, I.R. (neat) nitrile $2230 \mathrm{~cm}^{-1},{ }^{1} \mathrm{H}$ NMR $(500 \mathrm{MHz}$, $\left.\mathrm{CDCl}_{3}\right): \delta(\mathrm{ppm}) 1.5(\mathrm{~m}, 4 \mathrm{H}) 1.4-1.3(\mathrm{~m}, 12 \mathrm{H}), 0.9(\mathrm{~s}, 3 \mathrm{H})$.

\subsubsection{Synthesis of 2-methyl-2-(thiophen-2-yl)propanenitrile (24)}

To a solution of 2-(thiophen-2-yl) acetonitrile ( $1 \mathrm{~g}, 8.13 \mathrm{mmol})$ in $4 \mathrm{ml}$ anhydrous THF, KHMDS $\left(24.4 \mathrm{mmol}, 48.9 \mathrm{ml}, 0.5 \mathrm{M}\right.$ in toluene) was added at $0^{\circ} \mathrm{C}$. The mixture was allowed to stir for 3 minutes, after which a solution of $16.26 \mathrm{mmol}$ iodomethane $(1.13 \mathrm{ml}$ in $26 \mathrm{ml}$ anhydrous THF) was added slowly over a period of 10 minutes. The mixture was stirred for 5 minutes and monitored by TLC. Upon completion, the reaction was quenched with aqueous ammonium chloride. The organic phase was separated with ethyl acetate and dried over sodium sulfate. The product was purified via vacuum distillation. (bp $42^{\circ} \mathrm{C}$ at 1 torr) Yield: $89 \%$, MS: (ESI, pos.) $\mathrm{m} / z 174.1[\mathrm{M}+\mathrm{Na}]^{+}$, I.R. (neat) nitrile 
$2230 \mathrm{~cm}^{-1},{ }^{1} \mathrm{H}$ NMR (500 MHz, $\left.\mathrm{CDCl}_{3}\right): \delta(\mathrm{ppm}) 7.4 \mathrm{ppm}(\mathrm{d}, 1 \mathrm{H}), 7.2 \mathrm{ppm}(\mathrm{t}, 1 \mathrm{H})$, $7.0 \mathrm{ppm}(\mathrm{d}, 1 \mathrm{H}), 1.9 \mathrm{ppm}(\mathrm{s}, 6 \mathrm{H})$.

\subsubsection{Synthesis of 2,2-dimethyloctanenitrile (25)}

Prepared in a manner similar to compound $\mathbf{2 4}$ from octanenitrile and iodomethane in the presence of KHMDS. Purified via vacuum distillation (bp $50-55^{\circ} \mathrm{C}$ at 1.1 torr). Yield: $84 \%$, MS: (ESI, pos.) $\mathrm{m} / z 176.2[\mathrm{M}+\mathrm{Na}]^{+}$, I.R. (neat) nitrile $2230 \mathrm{~cm}^{-1},{ }^{1} \mathrm{H}$ NMR $\left(500 \mathrm{MHz}, \mathrm{CDCl}_{3}\right)$ : $\delta(\mathrm{ppm}) 1.5(\mathrm{~m}, 4 \mathrm{H}), 1.4-1.3(\mathrm{~m}, 12 \mathrm{H}), 0.9(\mathrm{~s}, 3 \mathrm{H})$.

\subsubsection{Synthesis of 2-methyl-2-phenylpropanimidamide hydrochloride (26)}

Ammonium chloride (1.06 g, 19.8mmol) was suspended in $10 \mathrm{ml}$ of anhydrous toluene, stirred under argon, and cooled to $0^{\circ} \mathrm{C}$. To this suspension was added $9.2 \mathrm{ml}$ (18.4 mmol) of a $2 \mathrm{M}$ solution of trimethylaluminum in toluene. The reaction was allowed to warm to room temperature and stirring was continued until the evolution of methane ceased ( 1 hour). Then, $1.6 \mathrm{~g}(11 \mathrm{mmol})$ of nitrile $22 \mathrm{in} 5 \mathrm{ml}$ of anhydrous toluene was added and the reaction was heated to $80^{\circ} \mathrm{C}$ for 18 hours. The reaction was then cooled to room temperature and slowly poured into a slurry of $5.5 \mathrm{~g}$ of silica gel in $18 \mathrm{ml}$ of chloroform and stirred for 5 minutes. The silica was filtered and washed with methanol. The filtrate and washings were combined and reduced to a volume of $\sim 5 \mathrm{ml}$ and refiltered. Then, $4 \mathrm{ml}(12 \mathrm{mmol})$ of a $3 \mathrm{~N}$ solution of methanolic $\mathrm{HCl}$ was added to the filtrate followed by $300 \mathrm{ml}$ of diethyl ether. After 16 hours of stirring at room temperature, a white precipitate formed was subsequently filtered. This crude solid was then added to $150 \mathrm{ml}$ of a 4:1 mixture of isopropanol-acetone and stirred for an additional 16 hours at room temperature. Undissolved ammonium chloride was then removed by filtration and the filtrate reduced to $\sim 5 \mathrm{ml}$. After the addition of $300 \mathrm{ml}$ of diethyl ether the mixture was stirred for $\sim 1$ hour. The white precipitate was then filtered and dried to yield $639 \mathrm{mg}(29 \%)$ of the objective compound as the hydrochloride salt. MS: (ESI, pos.) $\mathrm{m} / \mathrm{z}$ 163.0 $[\mathrm{M}+\mathrm{H}]^{+},{ }^{1} \mathrm{H}$ NMR (500 MHz, DMSO-d 6 ): $\delta$ (ppm) 9.27 (br.s, 2H), 8.71 (br.s, 2H), $7.41(\mathrm{~m}, 2 \mathrm{H}), 7.36(\mathrm{~m}, 2 \mathrm{H}), 7.32(\mathrm{~m}, 1 \mathrm{H}), 1.60(\mathrm{~s}, 6 \mathrm{H}){ }^{13} \mathrm{C}$ NMR $(500 \mathrm{MHz}$, DMSO-d $)_{6}: \delta$ (ppm) 175.91, 143.27, 128.71, 127.42, 125.65, 44.06, 26.31.

\subsubsection{Synthesis of 2-methyl-2-(thiophen-2-yl)propanimidamide hydrochloride (27)}

Prepared in a manner similar to compound 26 from nitrile $24(1.5 \mathrm{~g}, 9.9 \mathrm{mmol})$, ammonium chloride $(952 \mathrm{mg}, 17.8 \mathrm{mmol})$, and trimethylaluminum $(8.25 \mathrm{ml}, 16.5 \mathrm{mmol})$. Yield: 36\% (white solid) MS: (ESI, pos.) $m / z 169.0[\mathrm{M}+\mathrm{H}]^{+},{ }^{1} \mathrm{H}$ NMR (500 MHz, DMSO-d $\left.)_{6}\right): \delta(\mathrm{ppm}) 8.8$ (br.d, 3H), $7.53(\mathrm{dd}, 1 \mathrm{H}), 7.13(\mathrm{dd}, 1 \mathrm{H}), 7.04(\mathrm{dd}, 1 \mathrm{H}), 1.70$ (s, $1 \mathrm{H}){ }^{13} \mathrm{C}$ NMR (500 MHz, DMSO-d 6 ): $\delta$ (ppm) 174.75, 147.04, 127.18, 125.72, 125.48, 41.92, 27.20 . 


\subsubsection{Synthesis of 2-cyclohexyl-2-methylpropanimidamide hydrochloride (28)}

Prepared in a manner similar to compound 26 from nitrile $\mathbf{2 3}(6 \mathrm{~g}, 40 \mathrm{mmol})$, ammonium chloride $(3.92 \mathrm{~g}, 72 \mathrm{mmol})$, and trimethylaluminum $(33.3 \mathrm{ml}, 66.6 \mathrm{mmol})$. An exception to the procedure is that after the addition of methanolic HCL and diethyl ether, the solid that formed was ammonium chloride and the amidine remained in solution. The solid was removed and the solvent evaporated to yield the product as a highly viscous yellow resin. Yield: 70\%. MS: (ESI, pos.) $\mathrm{m} / z$ 169.1 $[\mathrm{M}+\mathrm{H}]^{+},{ }^{1} \mathrm{H}$ NMR $(500 \mathrm{MHz}$, DMSO-d $)_{6}$ ): $\delta$ (ppm) 8.84 (b.d., $\left.3 \mathrm{H}\right), 1.78(\mathrm{~m}, 3 \mathrm{H}), 1.57$ (m, 3H), $1.25(\mathrm{~s}, 2 \mathrm{H}), 1.19$ (t, $1 \mathrm{H}), 1.12(\mathrm{~s}, 6 \mathrm{H}), 0.97(\mathrm{~m}, 2 \mathrm{H}) .{ }^{13} \mathrm{C} \mathrm{NMR}\left(500 \mathrm{MHz}, \mathrm{DMSO}-\mathrm{d}_{6}\right): \delta(\mathrm{ppm}) 177.11$, $44.74,42.90,27.47,26.73,26.15,25.80,25.73,23.86,21.29$.

\subsubsection{Synthesis of 2,2-dimethyloctanimidamide hydrochloride (29)}

Prepared in a manner similar to compound 28 from nitrile $\mathbf{2 5}(1.5 \mathrm{~g}, 9.8 \mathrm{mmol})$, ammonium chloride $(957 \mathrm{mg}, 17.6 \mathrm{mmol})$, and trimethylaluminum $(8.15 \mathrm{ml}, 16.3 \mathrm{mmol})$. Product is a highly viscous yellow resin. Yield: 60.7\%. MS: (ESI, pos.) $m / z 171.1[\mathrm{M}+$ $\mathrm{H}]^{+},{ }_{1}^{1} \mathrm{H}$ NMR (500 MHz, DMSO-d ${ }_{6}$ ): $\delta$ (ppm) 8.87 (b.d., 4H), 1.56 (t, 2H), 1.3-1.0 (m, $14 \mathrm{H}), 0.85(\mathrm{t}, 3 \mathrm{H}) .{ }^{13} \mathrm{C}$ NMR (500 MHz, DMSO-d 6 ): $\delta$ (ppm) 176.63, 39.97, 39.62, $31.02,28.87,24.80,23.72,22.02,13.92$.

\subsubsection{Synthesis of 2-(2-phenylpropan-2-yl)pyrimidine-4,6-diol (30)}

Sodium metal (145mg, 6.29mmol) was reacted with $8 \mathrm{ml}$ of anhydrous ethanol. To this was added $500 \mathrm{mg}(2.52 \mathrm{mmol})$ of amidines hydrochloride $\mathbf{2 6}$ and the reaction was allowed to stir at room temperature for 5 minutes. Diethyl malonate $(400 \mathrm{mg}, 2.5 \mathrm{mmol}$ ) was then added and the reaction was heated to reflux overnight. The reaction was then cooled to room temperature and filtered. The solid was washed several times with absolute ethanol. The filtrate and washings were combined and diluted with 2 volumes of water and acidified with $5 \mathrm{~N} \mathrm{HCl}$ to a $\mathrm{pH}$ of $\sim 2$ and the product precipitated. The mixture was chilled and then filtered. The solid was washed several times with diethyl ether and then dried to yield $218 \mathrm{mg}$ (38\%) of an off-white solid. MS: (ESI, neg.) $\mathrm{m} / \mathrm{z}$ $228.9[\mathrm{M}-\mathrm{H}]^{-},{ }^{1} \mathrm{H}$ NMR (500 MHz, DMSO-d 6 ): $\delta$ (ppm) 11.35 (br.s, 2H), $7.33(\mathrm{t}, 2 \mathrm{H})$, $7.23(\mathrm{~m}, 3 \mathrm{H}), 5.19(\mathrm{~s}, 1 \mathrm{H}), 1.62(\mathrm{~s}, 6 \mathrm{H})$

\subsubsection{Synthesis of 2-(2-(thiophen-2-yl)propan-2-yl)pyrimidine-4,6- diol (31)}

Prepared in a manner similar to compound $\mathbf{3 0}$ from sodium metal (140mg, $6.11 \mathrm{mmol}$ ), amidine hydrochloride $27(500 \mathrm{mg}, 2.44 \mathrm{mmol})$, and diethyl malonate (388mg, 2.42mmol). Product is an off-white solid. Yield: $185 \mathrm{mg}$ (32\%). MS: (ESI, 
neg.) $\mathrm{m} / \mathrm{z} 235.1[\mathrm{M}-\mathrm{H}]^{-},{ }^{1} \mathrm{H}$ NMR (500 MHz, DMSO-d 6 ): $\delta$ (ppm) 11.39 (br.s, $\left.2 \mathrm{H}\right), 7.41$ $(\mathrm{d}, 1 \mathrm{H}), 6.99(\mathrm{~d}, 1 \mathrm{H}), 6.97(\mathrm{t}, 1 \mathrm{H}), 5.2(\mathrm{~s}, 1 \mathrm{H}), 1.72(\mathrm{~s}, 6 \mathrm{H})$.

\subsubsection{Synthesis of 2-(2-cyclohexylpropan-2-yl)pyrimidine-4,6-diol (32)}

Prepared in a manner similar to compound 30 from sodium metal (140mg, $6.11 \mathrm{mmol}$ ), amidine hydrochloride $28(500 \mathrm{mg}, 2.44 \mathrm{mmol})$, and diethyl malonate (509mg, $3.18 \mathrm{mmol})$. Product is an off-white solid. Yield: $185 \mathrm{mg}$ (32\%). MS: (ESI, neg.) $m / z 235.1[\mathrm{M}-\mathrm{H}]^{-},{ }^{1} \mathrm{H}$ NMR (500 MHz, DMSO-d $\left.{ }_{6}\right): \delta$ (ppm) 11.41 (br.s, $\left.2 \mathrm{H}\right), 5.12$ $(\mathrm{s}, 1 \mathrm{H}), 1.80(\mathrm{t}, 1 \mathrm{H}), 1.69(\mathrm{~d}, 2 \mathrm{H}), 1.38(\mathrm{~s}, 1 \mathrm{H}), 1.72(\mathrm{~d}, 2 \mathrm{H}), 1.19-1.02(\mathrm{~m}, 4 \mathrm{H}), 1.15(\mathrm{~s}$, $6 \mathrm{H}), 0.94(\mathrm{dd}, 2 \mathrm{H})$.

\subsubsection{Synthesis of 2-(2-methyloctan-2-yl)pyrimidine-4,6-diol (33)}

Prepared in a manner similar to compound $\mathbf{3 0}$ from sodium metal (111mg, $4.84 \mathrm{mmol}$ ), amidine hydrochloride 29 (400 $\mathrm{mg}, 1.93 \mathrm{mmol}$ ), and diethyl malonate (309mg, 1.93mmol). Product is a light peach colored solid. Yield: $39 \%$ MS: (ESI, neg.) $m / z 236.8[\mathrm{M}-\mathrm{H}]^{-},{ }^{1} \mathrm{H}$ NMR (500 MHz, DMSO-d ${ }_{6}$ ): $\delta$ (ppm) 11.33 (br.s, 2H), 5.13 (s, $1 \mathrm{H}), 1.62(\mathrm{~m}, 2 \mathrm{H}), 1.22(\mathrm{~s}, 6 \mathrm{H}), 1.20(\mathrm{~m}, 6 \mathrm{H}), 1.04(\mathrm{~m}, 2 \mathrm{H}), 0.83(\mathrm{t}, 3 \mathrm{H})$.

\subsubsection{Synthesis of (6aS,9R,10aR)-6,6,9-trimethyl-3-(2- phenylpropan-2-yl)-6a,7,8,9,10,10a-hexahydro-6H-isochromeno[3,4- d]pyrimidin-1-ol (34)}

Dihydroxy pyrimidine 30 (100 $\mathrm{mg}, 0.434 \mathrm{mmol}$ ) was added to $3 \mathrm{ml}$ of absolute ethanol in a $10 \mathrm{ml}$ microwave reaction vessel. To this was also added $66 \mu 1$ of pyridine and $3 \mu$ l of piperidine followed by (R)-(+)-citronellal $(201 \mathrm{mg}, 235 \mu 1,1.3 \mathrm{mmol})$. The reaction vessel was then sealed and irradiated at 200 watts to $130^{\circ} \mathrm{C}$ for 1 hour. The reaction mixture was then concentrated and the product purified by flash chromatography using a methanol/methylene chloride gradient to yield $180 \mathrm{mg}(69 \%)$ of the objective product as an off-white solid. MS: (ESI, neg.) $m / z 365.0[\mathrm{M}-\mathrm{H}]$, , ${ }^{1} \mathrm{H}$ NMR $(500 \mathrm{MHz}$, DMSO-d $_{6}$ ): $\delta 11.43(\mathrm{~s}, 1 \mathrm{H}), 7.32(\mathrm{q}, 2 \mathrm{H}, \mathrm{J}=7.5 \mathrm{~Hz}), 7.21(\mathrm{~m}, 3 \mathrm{H}), 3.06(\mathrm{~d}, 1 \mathrm{H}, \mathrm{J}=$ $12.5 \mathrm{~Hz}), 2.16(\mathrm{td}, 1 \mathrm{H}, \mathrm{J}=11 \mathrm{~Hz}$ and $2.5 \mathrm{~Hz}), 1.76(\mathrm{~m}, 2 \mathrm{H}), 1.56(\mathrm{~m}, 11 \mathrm{H}), 1.37(\mathrm{~s}, 3 \mathrm{H})$, $1.01(\mathrm{~m}, 2 \mathrm{H}), 0.87(\mathrm{~d}, 3 \mathrm{H}, \mathrm{J}=6.5 \mathrm{~Hz}), 0.48(\mathrm{q}, 1 \mathrm{H}, \mathrm{J}=12 \mathrm{~Hz}) .{ }^{13} \mathrm{C} \mathrm{NMR}(500 \mathrm{MHz}$, DMSO-d ${ }_{6}$ ): $\delta$ (ppm) 163.44, 163.23, 145.75, 128.27, 128.23, 126.46, 126.06, 125.86, $99.47,79.98,47.47,44.48,36.86,34.83,33.44,31.81,27.19,27.05,26.90,22.40,19.46$. HPLC retention time: $10.631 \mathrm{~min}$; purity $100 \%$. 


\subsubsection{Synthesis of (6aS,9R,10aR)-6,6,9-trimethyl-3-(2-(thiophen-2- yl)propan-2-yl)-6a,7,8,9,10,10a-hexahydro-6H-isochromeno[3,4- d]pyrimidin-1-ol (35)}

Prepared in a manner similar to compound $\mathbf{3 4}$ from dihydroxy pyrimidine $\mathbf{3 1}$

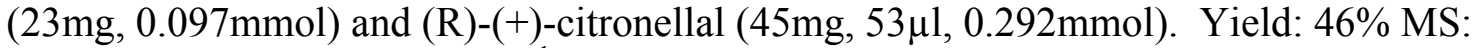
(ESI, neg.) $m / z 371.0[\mathrm{M}-\mathrm{H}]^{-},{ }^{1} \mathrm{H}$ NMR (500 MHz, DMSO-d 6 ): $\delta 11.52(\mathrm{~s}, 1 \mathrm{H}), 7.40(\mathrm{~d}$, $1 \mathrm{H}, \mathrm{J}=4.5 \mathrm{~Hz}), 6.97(\mathrm{~m}, 2 \mathrm{H}), 3.06(\mathrm{~d}, 1 \mathrm{H}, \mathrm{J}=12 \mathrm{~Hz}), 2.16(\mathrm{td}, 1 \mathrm{H}, \mathrm{J}=11.5 \mathrm{~Hz}$ and $2 \mathrm{~Hz})$, $1.70(\mathrm{~m}, 8 \mathrm{H}), 1.50(\mathrm{~m}, 2 \mathrm{H}), 1.36(\mathrm{~s}, 3 \mathrm{H}), 1.06(\mathrm{~m}, 5 \mathrm{H}), 0.87(\mathrm{~d}, 3 \mathrm{H}, \mathrm{J}=6.5 \mathrm{~Hz}), 0.47$ (q, $1 \mathrm{H}, \mathrm{J}=12 \mathrm{~Hz}) .{ }^{13} \mathrm{C}$ NMR $\left(500 \mathrm{MHz}, \mathrm{DMSO}-\mathrm{d}_{6}\right): \delta(\mathrm{ppm}) 163.46,163.07,150.12$, 126.70, 126.64, 124.86, 124.39, 99.63, 80.07, 47.42, 42.72, 36.79, 34.80, 33.42, 31.80, $28.08,28.00,27.02,26.87,22.40,19.43$. HPLC retention time: $10.580 \mathrm{~min}$; purity $98.04 \%$.

\subsubsection{Synthesis of (6aS,9R,10aR)-3-(2-cyclohexylpropan-2-yl)-6,6,9- trimethyl-6a,7,8,9,10,10a-hexahydro-6H-isochromeno[3,4- d]pyrimidin-1-ol (36)}

Prepared in a manner similar to compound $\mathbf{3 4}$ from dihydroxy pyrimidine $\mathbf{3 2}$ (100mg, 0.42mmol) and (R)-(+)-citronellal (194mg, 228 $\mu 1,1.26 \mathrm{mmol})$. Yield: 36\% MS: (ESI, neg.) $m / z 371.0[\mathrm{M}-\mathrm{H}]^{-},{ }^{1} \mathrm{H}$ NMR (500 MHz, DMSO-d $\left.{ }_{6}\right): \delta 11.52(\mathrm{~s}, 1 \mathrm{H}), 3.10(\mathrm{~d}$, $1 \mathrm{H}, \mathrm{J}=12.5 \mathrm{~Hz}), 2.16(\mathrm{td}, 1 \mathrm{H}, \mathrm{J}=11 \mathrm{~Hz}$ and $2.5 \mathrm{~Hz}), 1.73(\mathrm{~m}, 5 \mathrm{H}), 1.52(\mathrm{~m}, 2 \mathrm{H}), 1.34(\mathrm{~s}$, $3 \mathrm{H}), 1.26(\mathrm{~m}, 4), 1.10(\mathrm{~m}, 10 \mathrm{H}), 1.05(\mathrm{~s}, 3 \mathrm{H}), 0.96(\mathrm{~m}, 2 \mathrm{H}), 0.88(\mathrm{~d}, 3 \mathrm{H}, \mathrm{J}=6.5 \mathrm{~Hz}), 0.48$ $(\mathrm{q}, 1 \mathrm{H}, \mathrm{J}=12 \mathrm{~Hz}) .{ }^{13} \mathrm{C}$ NMR $\left(500 \mathrm{MHz}, \mathrm{DMSO}-\mathrm{d}_{6}\right): \delta(\mathrm{ppm}) 165.54,163.41,163.34$, 98.96, 79.78, 47.47, 44.61, 43.13, 36.97, 34.83, 33.45, 31.83, 27.17, 27.03, 26.90, 26.39, 26.06, 22.41, 22.05, 21.99, 19.41. HPLC retention time: $11.732 \mathrm{~min}$; purity $97.65 \%$.

\subsubsection{Synthesis of (6aS,9R,10aR)-6,6,9-trimethyl-3-(2-methyloctan- 2-yl)-6a,7,8,9,10,10a-hexahydro-6H-isochromeno[3,4-d]pyrimidin-1-ol (37)}

Prepared in a manner similar to compound $\mathbf{3 4}$ from dihydroxy pyrimidine $\mathbf{3 3}$ (100mg, 0.42mmol) and (R)-(+)-citronellal (194mg, 228 $\mu 1,1.26 \mathrm{mmol})$. Yield: 31\% MS: (ESI, neg.) $m / z 373.1[\mathrm{M}-\mathrm{H}]^{-},{ }^{1} \mathrm{H}$ NMR (500 MHz, DMSO-d 6 ): $\delta 11.57(\mathrm{~s}, 1 \mathrm{H}), 3.10(\mathrm{~d}$, $1 \mathrm{H}, \mathrm{J}=12.5 \mathrm{~Hz}), 2.16(\mathrm{td}, 1 \mathrm{H}, \mathrm{J}=11 \mathrm{~Hz}$ and $2.5 \mathrm{~Hz}), 1.75(\mathrm{~m}, 2 \mathrm{H}), 1.56(\mathrm{~m}, 6 \mathrm{H}), 1.34(\mathrm{~s}$, $3 \mathrm{H}), 1.18(\mathrm{~m}, 12 \mathrm{H}), 1.02(\mathrm{~m}, 5 \mathrm{H}), 0.89(\mathrm{~d}, 3 \mathrm{H}, \mathrm{J}=6.5 \mathrm{~Hz}), 0.82(\mathrm{t}, 3 \mathrm{H}, \mathrm{J}=7 \mathrm{~Hz}), 0.48(\mathrm{q}$, $1 \mathrm{H}, \mathrm{J}=12 \mathrm{~Hz}) .{ }^{13} \mathrm{C}$ NMR $\left(500 \mathrm{MHz}, \mathrm{DMSO}-\mathrm{d}_{6}\right): \delta(\mathrm{ppm}) 163.79,163.47,163.14,99.01$, 79.78, 47.52, 44.58, 40.14, 36.95, 34.85, 33.44, 31.82, 30.98, 29.00, 27.03, 26.89, 25.71, $25.59,23.87,22.42,22.02,19.37,13.91$. HPLC retention time: $12.503 \mathrm{~min}$; purity $95.12 \%$. 


\subsubsection{Synthesis of Pyrimidine Non-Classical Cannabinoids}

The first attempts at synthesis of the heteroaromatic triaryl compounds utilized reactions that were successfully employed in the creation of the carbocyclic triaryl compounds. ${ }^{127}$ The rationale was that the previously created dihydroxy pyrimidines (Compounds 30, 31, 32, and 33) could be activated for Suzuki coupling to introduce the aromatic $\mathrm{C}$ ring. This was accomplished by bromination of the dihydroxy pyrimidines in the 5 position by N-bromosuccinimide (Scheme 4.14). ${ }^{176}$ This brominated compound would then be a substrate for the palladium catalyzed Suzuki reaction. This reaction, however, was not successful in formation of the triaryl coupled products (Scheme 4.15).

With the experience gained from the successful formation of the dihydroxy pyrimidines, it was decided to form the final compounds by cyclization of the pyrimidine ring with the side chain and the aromatic $\mathrm{C}$ ring already in place. This could be accomplished in a reaction similar to the formation of the dihydroxy pyrimidines by reaction of an amidine with a substituted diethyl malonate (Scheme 4.16). The 2position substituted diethyl malonates can be formed by copper catalyzed coupling of an alkyl or aryl halide and diethyl malonate (Scheme 4.17). ${ }^{177}$ Six substituted malonates were reacted with four amidine hydrochlorides to successfully yield fifteen pyrimidine nonclassical cannabinoids (Figure 4.6). Some of the combinations of synthons would not react to yield a substituted pyrimidine product in every permutation. The purification of theses compounds was more involved than the dihydroxy pyrimidines which were precipitated by acidification. The pyrimidine nonclassical cannabinoids formed a gummy resin in the aqueous solution upon acidification. This was extracted with ethyl acetate, dried over sodium sulfate, concentrated by rotary evaporation, and purified using flash chromatography.

All chemicals were obtained from Sigma Aldrich or Fisher Scientific Inc. Anhydrous solvents were obtained by distillation over either calcium hydride or metallic sodium and benzophenone. Final compounds and intermediates were purified using column chromatography on the Biotage SP1 system employing Flash column cartridges or by crystallization. NMR spectra were obtained on a Bruker $300 \mathrm{MHz}$ or Varian $500 \mathrm{MHz}$ Inova NMR. HPLC analysis of final products was carried out by gradient elution using water/acetonitrile ( $0.1 \%$ TFA) with a gradient of $70 \%(3 \mathrm{~min}), 50 \%(2 \mathrm{~min})$, and $10 \%$ (15min). A reverse-phase ODS Hypersil column, manufactured by Thermo Scientific, of dimensions $4.6 \times 150 \mathrm{~mm}$ with $5 \mu \mathrm{m}$ particle size was used for HPLC analyses.

\subsubsection{Synthesis of diethyl 2-m-tolylmalonate (38)}

A two-necked round-bottomed flask was charged sequentially with $\mathrm{CuI}(0.114 \mathrm{~g}$, $0.6 \mathrm{mmol})$, 2-picolinic acid (0.148 g, $1.2 \mathrm{mmol})$, and $\mathrm{CsCO}_{3}(5.89 \mathrm{~g}, 18 \mathrm{mmol})$. The flask was evacuated and back filled with nitrogen 3 times. Anhydrous 1,4-dioxane $(10 \mathrm{ml})$ was added volumetrically followed by distilled diethyl malonate $(1.9 \mathrm{ml}$, $12 \mathrm{mmol})$ and 3 -iodotoluene $(0.8 \mathrm{ml}, 6 \mathrm{mmol})$. The reaction was heated to $70^{\circ} \mathrm{C}$ for 30 hours. After monitoring the progress by TLC, the reaction was cooled to room 


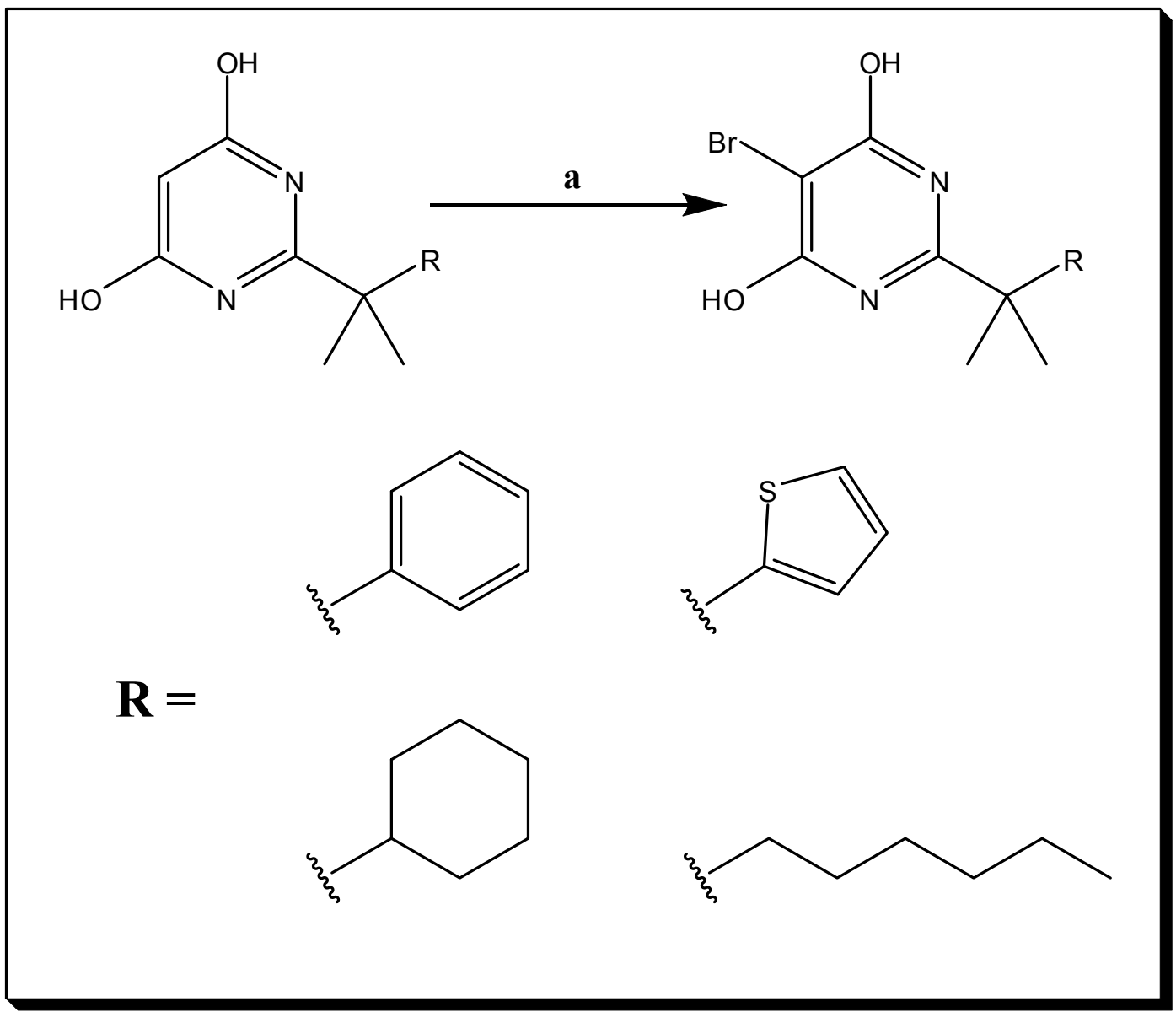

Scheme 4.14: Synthesis of 5-bromo dihydroxy pyrimidines

(a) NBS, DMF, RT, 40min. 


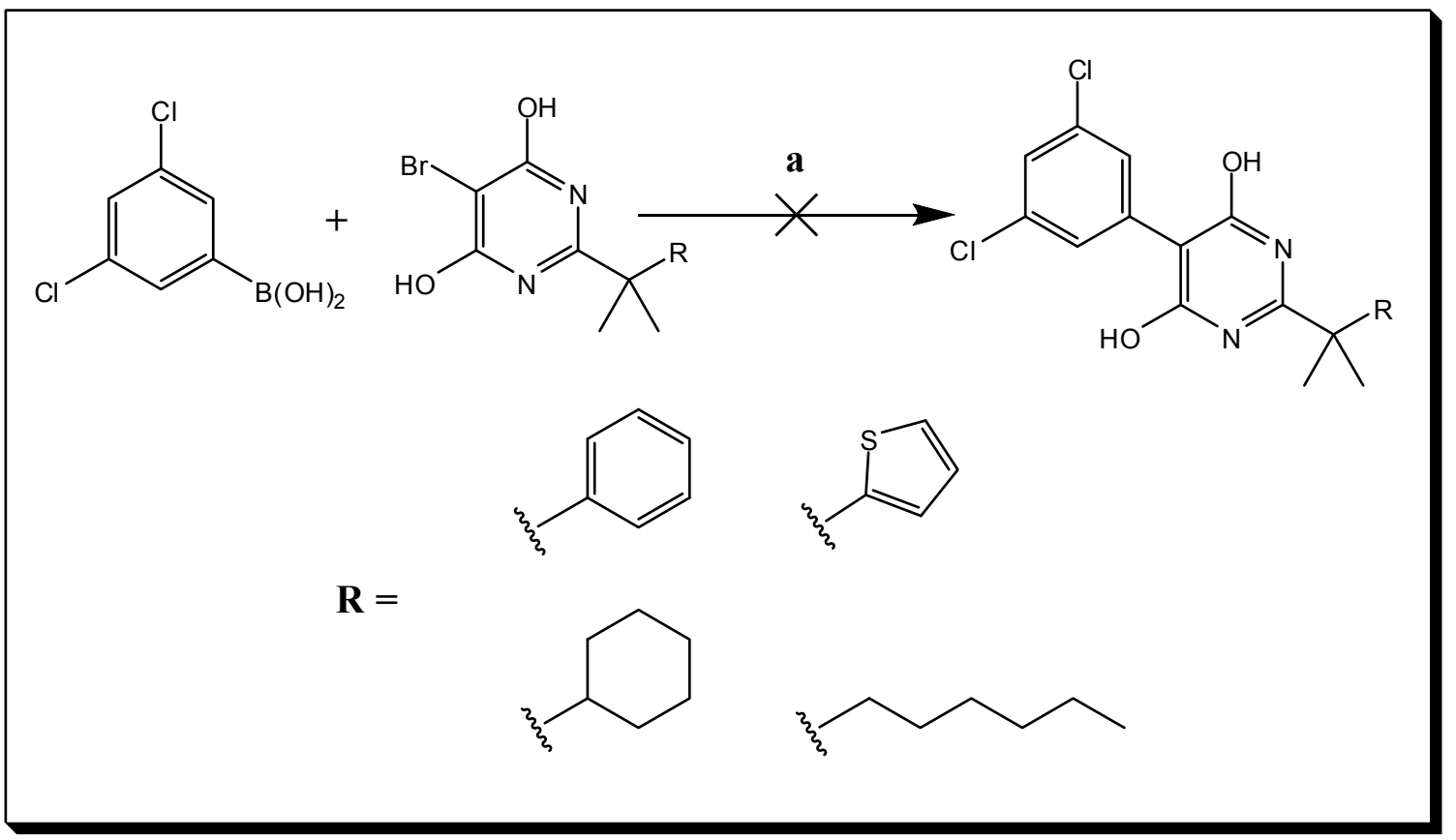

Scheme 4.15: Attempt at synthesis of pyrimidine triaryl compounds

Failed attempt at synthesis of pyrimidine triaryl compounds using palladium catalyzed Suzuki coupling. (a) $\left(\mathrm{PPh}_{3}\right)_{4} \mathrm{Pd}, \mathrm{K}_{2} \mathrm{CO}_{3}$, toluene / water, reflux, $18 \mathrm{hr}$. 


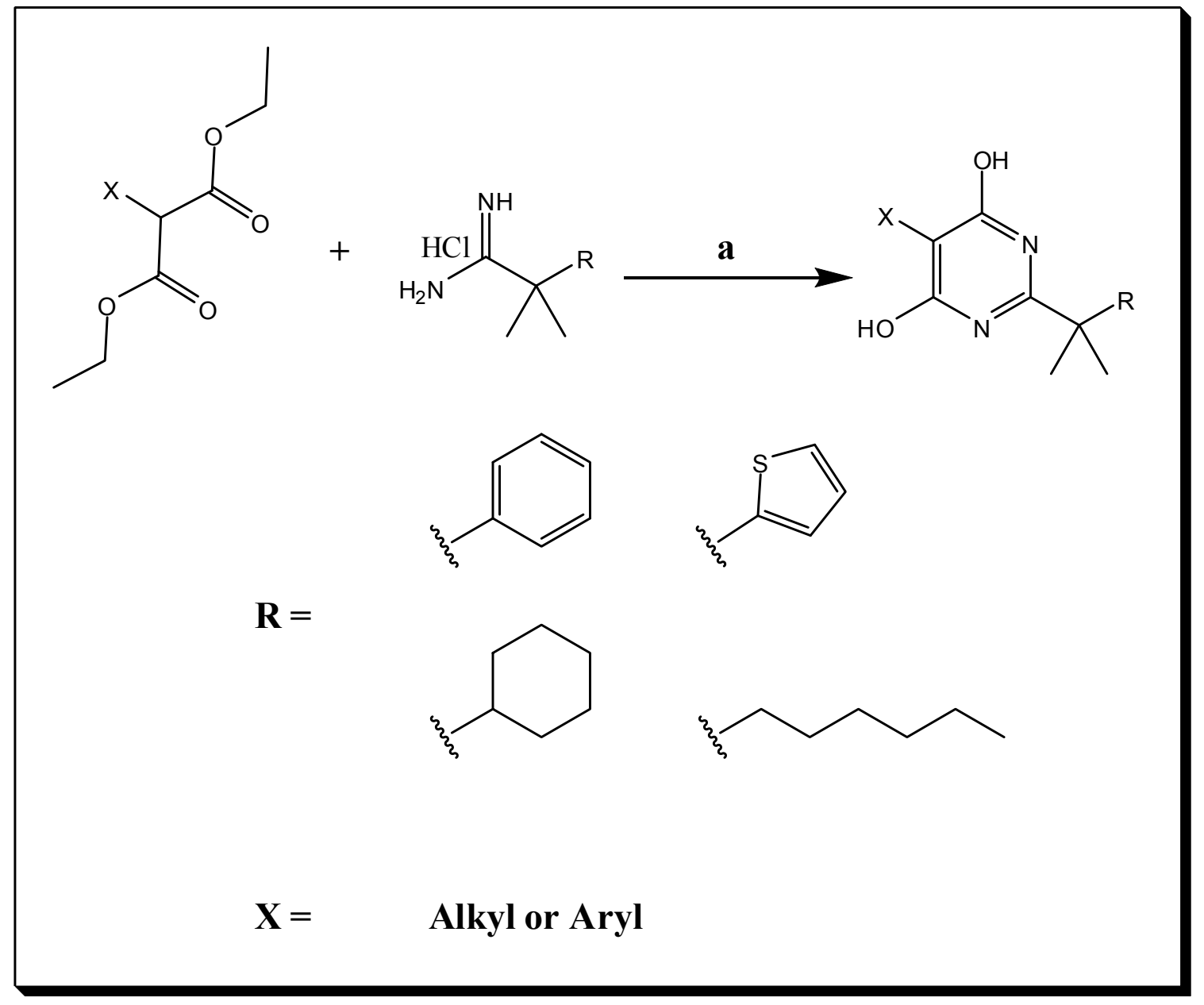

Scheme 4.16: Synthesis of pyrimidine nonclassical cannabinoids

(a) Na metal, EtOH, diethyl malonate, reflux, $18 \mathrm{hr}$. 


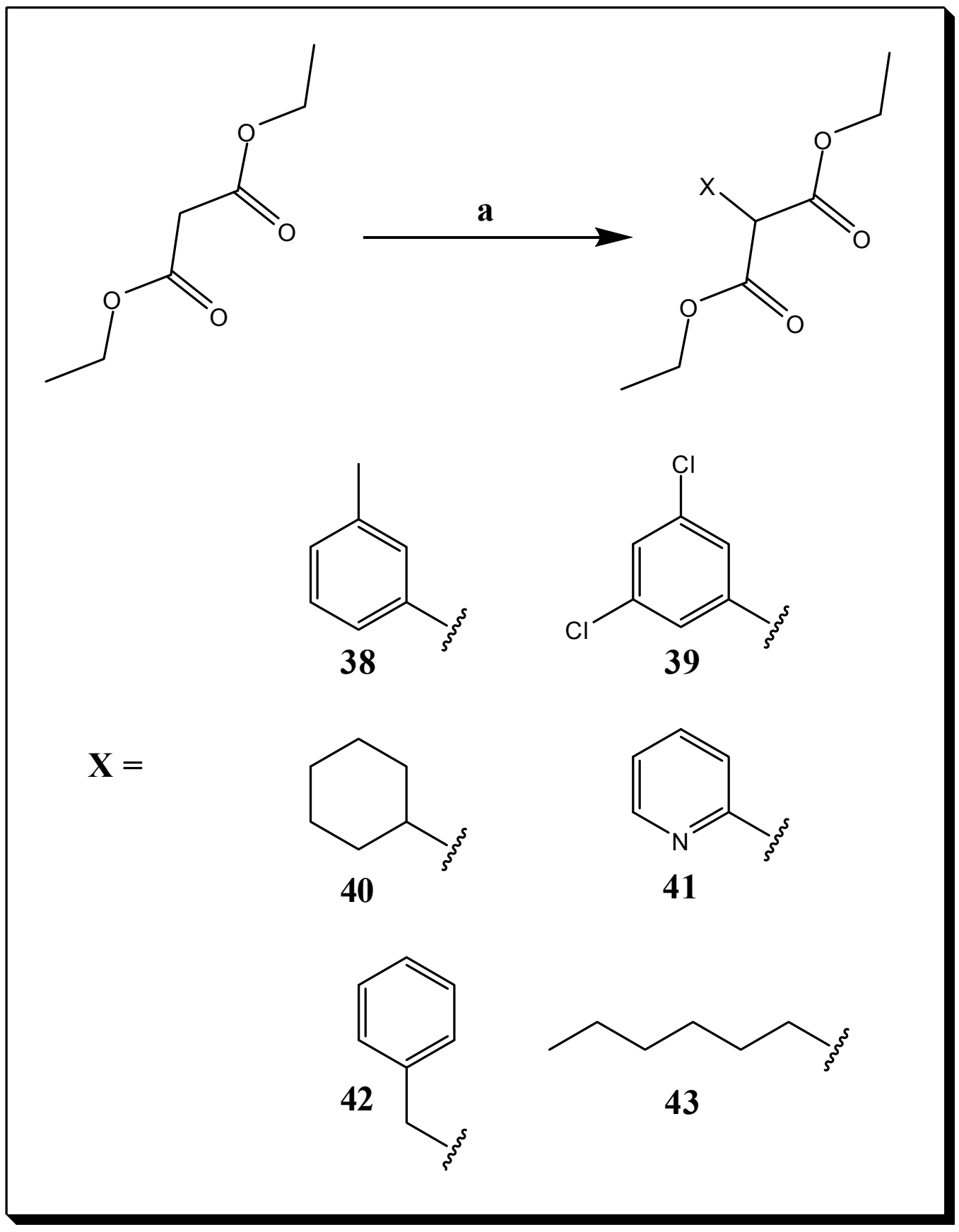

Scheme 4.17: Synthesis of 2-position substituted diethyl malonates

(a) $\mathrm{CuI}$, 2-picolinic acid, $\mathrm{CsCO}_{3}$, aryl or alkyl iodide, diethyl malonate, 1,4-dioxane, $70^{\circ} \mathrm{C}, 30 \mathrm{hr}$. 


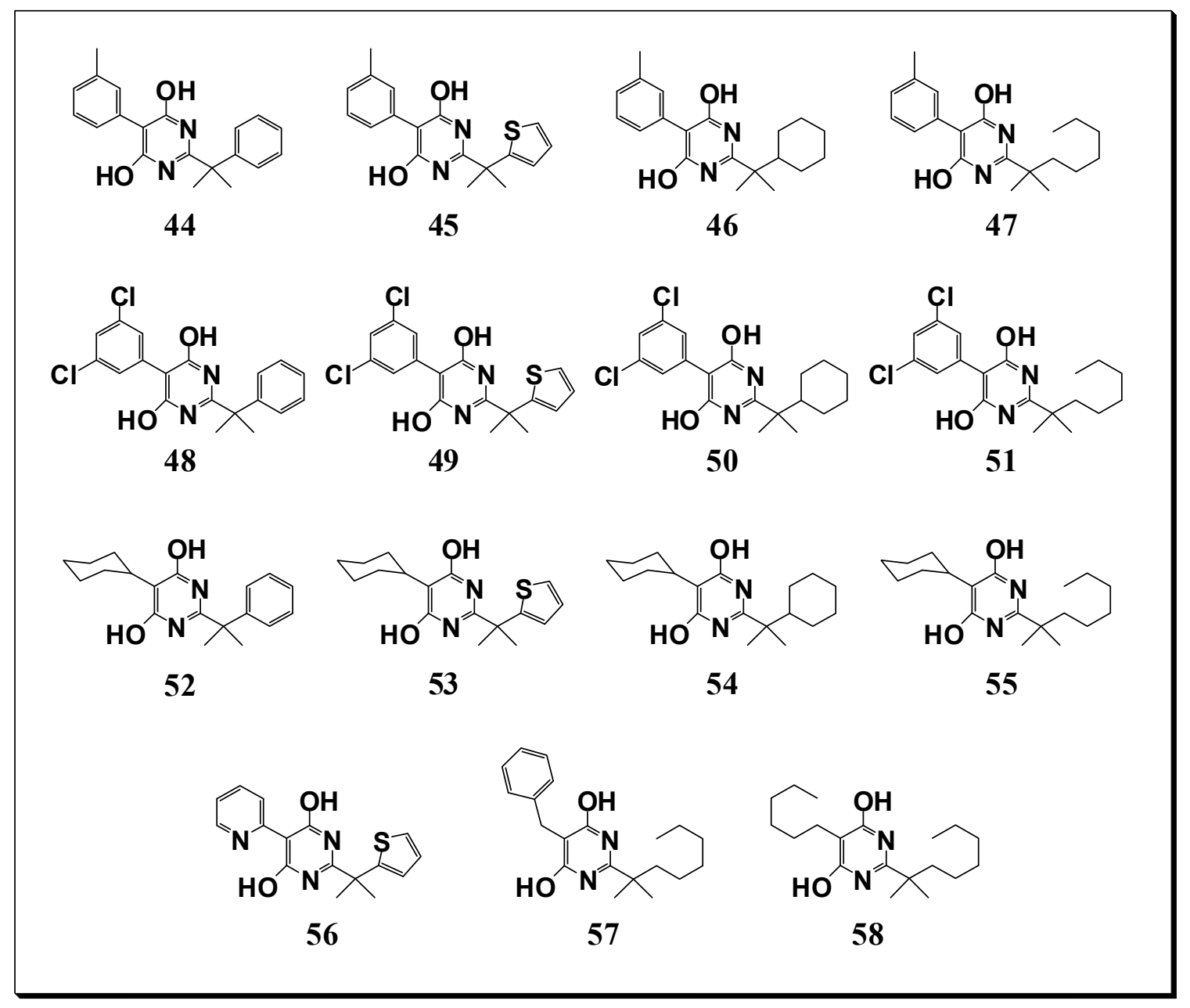

Figure 4.6: Pyrimidine non-classical cannabinoids 
temperature, diluted with ethyl acetate and washed with ammonium chloride. The organic phase was dried over sodium sulfate, concentrated, and the product purified by column chromatography using a 1:9 ethyl acetate/hexane mixture to yield the product as a colorless oil. Yield: $92 \%, \mathrm{R}_{\mathrm{f}}=0.48$ (ethyl acetate $/$ hexane $\left.=1: 9\right)$. MS: $($ ESI, pos.) $\mathrm{m} / \mathrm{z}$ $273.0[\mathrm{M}+\mathrm{Na}]^{+},{ }^{1} \mathrm{H}$ NMR $\left(500 \mathrm{MHz}, \mathrm{CDCl}_{3}\right): \delta(\mathrm{ppm})$ 7.12-7.3 (m, 4H), $4.62(\mathrm{~s}, 1 \mathrm{H})$, $4.20-4.28(\mathrm{~m}, 4 \mathrm{H}), 2.27$ (s, 3H), 1.22-1.25 (m, 6H).

\subsubsection{Synthesis of diethyl 2-(3,5-dichlorophenyl)malonate (39)}

Compound 39 was prepared in a manner similar to compound $\mathbf{3 8}$ from $\mathrm{CuI}$ (0.17 g, $0.89 \mathrm{mmol}), 2$-picolinic acid (0.22 g, $1.78 \mathrm{mmol}), \mathrm{CsCO}_{3}(8.7 \mathrm{~g}, 26.7 \mathrm{mmol})$, diethyl malonate $(2.9 \mathrm{ml}, 17.8 \mathrm{mmol})$, and 1,3-dichloro-5-iodobenzene $(2.4 \mathrm{~g}, 8.9 \mathrm{mmol})$. $\mathrm{R}_{\mathrm{f}}=0.41$ (ethyl acetate/hexane =1:9). MS: (ESI, pos.) $\mathrm{m} / z 328[\mathrm{M}+\mathrm{Na}]^{+},{ }^{1} \mathrm{H}$ NMR $\left(500 \mathrm{MHz}, \mathrm{CDCl}_{3}\right): \delta(\mathrm{ppm}) 7.34(\mathrm{~m}, 3 \mathrm{H}), 4.54(\mathrm{~s}, 1 \mathrm{H}), 4.25(\mathrm{~m}, 4 \mathrm{H}), 1.28(\mathrm{t}, 6 \mathrm{H})$.

\subsubsection{Synthesis of diethyl 2-cyclohexylmalonate (40)}

Compound $\mathbf{4 0}$ was prepared in a manner similar to compound $\mathbf{3 8}$ from $\mathrm{CuI}$ (0.114 g, $0.6 \mathrm{mmol})$, 2-picolinic acid (0.148 g, $1.2 \mathrm{mmol}), \mathrm{CsCO}_{3}(5.89 \mathrm{~g}, 18 \mathrm{mmol})$, diethyl malonate $(1.9 \mathrm{ml}, 12 \mathrm{mmol})$, and iodocyclohexane $(0.8 \mathrm{ml}, 6 \mathrm{mmol})$. Yield: $94 \%$, $\mathrm{R}_{\mathrm{f}}=0.59$ (ethyl acetate/hexane $=1: 9$ ). MS: (ESI, pos.) $\mathrm{m} / z 265[\mathrm{M}+\mathrm{Na}]^{+},{ }^{1} \mathrm{H}$ NMR $\left(500 \mathrm{MHz}, \mathrm{CDCl}_{3}\right): \delta(\mathrm{ppm}) 4.22-4.14(\mathrm{~m}, 4 \mathrm{H}), 3.12(\mathrm{~d}, 1 \mathrm{H}), 2.14-2.05(\mathrm{~m}, 1 \mathrm{H}), 1.75-$ $1.63(\mathrm{~m}, 5 \mathrm{H}), 1.34-1.24(\mathrm{~m}, 8 \mathrm{H}), 1.20-1.20(\mathrm{~m}, 3 \mathrm{H})$.

\subsubsection{Synthesis of diethyl 2-(pyridin-2-yl)malonate (41)}

Compound $\mathbf{4 1}$ was prepared in a manner similar to compound $\mathbf{3 8}$ from $\mathrm{CuI}$ (0.114 g, $0.6 \mathrm{mmol}), 2$-picolinic acid $(0.148 \mathrm{~g}, 1.2 \mathrm{mmol}), \mathrm{CsCO}_{3}(5.89 \mathrm{~g}, 18 \mathrm{mmol})$, diethyl malonate $(1.9 \mathrm{ml}, 12 \mathrm{mmol})$, and 2-bromopyridine $(0.59 \mathrm{ml}, 6 \mathrm{mmol})$. Yield: $88 \%$, $\mathrm{R}_{\mathrm{f}}=0.32$ (ethyl acetate/hexane $=3: 7$ ). MS: (ESI, pos.) $\mathrm{m} / z 260[\mathrm{M}+\mathrm{Na}]^{+},{ }^{1} \mathrm{H}$ NMR $\left(500 \mathrm{MHz}, \mathrm{CDCl}_{3}\right): \delta(\mathrm{ppm}) 8.5(\mathrm{~d}, 1 \mathrm{H}), 7.7(\mathrm{~d}, 1 \mathrm{H}), 7.62-7.58(\mathrm{~m}, 1 \mathrm{H}), 7.18-7.12(\mathrm{~m}$, $1 \mathrm{H}), 4.80(\mathrm{~s}, 1 \mathrm{H}), 4.21-4.15(\mathrm{~m}, 4 \mathrm{H}), 1.21(\mathrm{t}, 6 \mathrm{H})$.

\subsubsection{Synthesis of diethyl 2-benzylmalonate (42)}

Compound $\mathbf{4 2}$ was prepared in a manner similar to compound $\mathbf{3 8}$ from $\mathrm{CuI}$ (0.114 g, $0.6 \mathrm{mmol}), 2$-picolinic acid $(0.148 \mathrm{~g}, 1.2 \mathrm{mmol}), \mathrm{CsCO}_{3}(5.89 \mathrm{~g}, 18 \mathrm{mmol})$, diethyl malonate $(1.9 \mathrm{ml}, 12 \mathrm{mmol})$, and benzyl bromide $(1.44 \mathrm{ml}, 6 \mathrm{mmol})$. Yield: $78 \%$. $\mathrm{R}_{\mathrm{f}}=0.52$ (ethyl acetate/hexane $\left.=1: 9\right)$. MS: (ESI, pos.) $m / z 273[\mathrm{M}+\mathrm{Na}]^{+},{ }^{1} \mathrm{H}$ NMR $\left(300 \mathrm{MHz}, \mathrm{CDCl}_{3}\right): \delta(\mathrm{ppm}) 7.24(\mathrm{~m}, 5 \mathrm{H}), 4.15(\mathrm{q}, 4 \mathrm{H}), 3.66(\mathrm{t}, 1 \mathrm{H}), 3.22(\mathrm{~d}, 2 \mathrm{H}), 1.18$ $(\mathrm{t}, 6 \mathrm{H})$. 


\subsubsection{Synthesis of diethyl 2-hexylmalonate (43)}

Compound $\mathbf{4 3}$ was prepared in a manner similar to compound 38 from $\mathrm{CuI}$ (0.019 g, 0.1mmol), 2-picolinic acid (0.025 g, $0.2 \mathrm{mmol}), \mathrm{CsCO}_{3}(0.977 \mathrm{~g}, 3 \mathrm{mmol})$, diethyl malonate $(0.3 \mathrm{ml}, 2 \mathrm{mmol})$, and 1-iodohexane $(0.15 \mathrm{ml}, 1 \mathrm{mmol})$. Yield: $91 \%, \mathrm{R}_{\mathrm{f}}=$ 0.44 (ethyl acetate/hexane =1:9). MS: (ESI, pos.) $m / z 267[\mathrm{M}+\mathrm{Na}]^{+},{ }^{1} \mathrm{H} \mathrm{NMR}(300$ $\left.\mathrm{MHz}, \mathrm{CDCl}_{3}\right): \delta(\mathrm{ppm}) 4.11-4.40(\mathrm{~m}, 4 \mathrm{H}), 331(\mathrm{t}, 1 \mathrm{H}), 1.80(\mathrm{~m}, 2 \mathrm{H}), 1.38(\mathrm{~m}, 2 \mathrm{H}), 1.1-$ $1.4(\mathrm{~m}, 12 \mathrm{H}), 0.8(\mathrm{t}, 3 \mathrm{H})$.

\subsubsection{Synthesis of 2-(2-phenylpropan-2-yl)-5-m-tolylpyrimidine-4,6- diol (44)}

Sodium metal $(115 \mathrm{mg}, 5 \mathrm{mmol})$ was reacted with $4 \mathrm{ml}$ of anhydrous ethanol. To this was added $400 \mathrm{mg}(2 \mathrm{mmol})$ of 2-methyl-2-phenylpropanimidamide hydrochloride 26 and the reaction was allowed to stir at room temperature for 5 minutes. Diethyl 2-mtolylmalonate $38(500 \mathrm{mg}, 2 \mathrm{mmol})$ was then added in $2 \mathrm{ml}$ of anhydrous ethanol and the reaction was heated to reflux overnight. The reaction was then cooled to room temperature and filtered. The solid was washed several times with absolute ethanol. The filtrate and washings were combined and diluted with 2 volumes of water and acidified with $5 \mathrm{~N} \mathrm{HCl}$ to a $\mathrm{pH}$ of $\sim 2$ and the product precipitated as a sticky resin. The product was extracted with ethyl acetate and dried over sodium sulfate. The organic phase was concentrated and the product purified on a Biotage SP-1 flash chromatography using a $\mathrm{MeOH} / \mathrm{CH}_{2} \mathrm{Cl}_{2}$ gradient. Yield: 39\% MS: (ESI, neg.) $\mathrm{m} / z$ z19.0 [M - H] ${ }^{-}$, ${ }^{1} \mathrm{H}$ NMR (500 MHz, DMSO-d ${ }_{6}$ ): $\delta$ (ppm) 11.50 (br.s, 2H), 7.36 (t, 2H, J = 7Hz), 7.30 (d, 2H, J = $7.5 \mathrm{~Hz}), 7.25(\mathrm{~m}, 3 \mathrm{H}), 7.19(\mathrm{t}, 1 \mathrm{H}, \mathrm{J}=7.5 \mathrm{~Hz}), 7.01(\mathrm{~d}, 1 \mathrm{H}, \mathrm{J}=7.5 \mathrm{~Hz}), 2.29(\mathrm{~s}, 3 \mathrm{H}), 1.68$ $(\mathrm{s}, 6 \mathrm{H}) .{ }^{13} \mathrm{C}$ NMR $\left(500 \mathrm{MHz}, \mathrm{DMSO}-\mathrm{d}_{6}\right): \delta(\mathrm{ppm}) 145.79,135.93,132.54,130.96$, $128.31,127.56,127.07,126.75,126.51,126.03,100.61,44.64,27.26,21.08$. HPLC retention time: $9.724 \mathrm{~min}$; purity $99.65 \%$.

\subsubsection{Synthesis of 2-(2-(thiophen-2-yl)propan-2-yl)-5-m- tolylpyrimidine-4,6-diol (45)}

Compound 45 was prepared in a similar manner to compound 44 from sodium metal (140mg, $6.11 \mathrm{mmol})$, EtOH (8ml), 2-methyl-2-(thiophen-2-yl)propanimidamide hydrochloride 27 (500 mg, 2.44mmol), and diethyl 2-m-tolylmalonate $\mathbf{3 8}(611 \mathrm{mg}$, 2.44mmol). Yield 4.3\%, MS: (ESI, neg.) $\mathrm{m} / \mathrm{z} 324.9[\mathrm{M}-\mathrm{H}]^{-},{ }^{1} \mathrm{H}$ NMR (500 MHz, DMSO-d $\left.\mathrm{d}_{6}\right): \delta(\mathrm{ppm}) 11.51$ (br.s, $\left.2 \mathrm{H}\right), 7.44(\mathrm{dd}, 1 \mathrm{H}, \mathrm{J}=5 \mathrm{~Hz}$ and $1 \mathrm{~Hz}), 7.23(\mathrm{~s}, 1 \mathrm{H}), 7.20$ $(\mathrm{q}, 2 \mathrm{H}, \mathrm{J}=8 \mathrm{~Hz}), 7.06(\mathrm{dd}, 1 \mathrm{H}, \mathrm{J}=3.5 \mathrm{~Hz}$ and $1.5 \mathrm{~Hz}), 7.00(\mathrm{~m}, 2 \mathrm{H}), 2.28(\mathrm{~s}, 3 \mathrm{H}), 1.70(\mathrm{~s}$, $6 \mathrm{H}) .{ }^{13} \mathrm{C}$ NMR $\left(500 \mathrm{MHz}, \mathrm{DMSO}-\mathrm{d}_{6}\right): \delta(\mathrm{ppm}) 150.33,136.00,132.46,130.96,127.57$, 127.14, 126.85, 126.77, 126.46, 124.91, 124.40, 123.41, 100.76, 42.88, 28.14, 21.11. HPLC retention time: $8.060 \mathrm{~min}$; purity $95.35 \%$. 


\subsubsection{Synthesis of 2-(2-cyclohexylpropan-2-yl)-5-m-tolylpyrimidine- 4,6-diol (46)}

Compound $\mathbf{4 6}$ was prepared in a similar manner to compound $\mathbf{4 4}$ from sodium metal (140mg, $6.11 \mathrm{mmol}), \mathrm{EtOH}(6 \mathrm{ml}), 2$-cyclohexyl-2-methylpropanimidamide hydrochloride $28(500 \mathrm{mg}, 2.44 \mathrm{mmol})$, and diethyl 2-m-tolylmalonate $38(796 \mathrm{mg}$, $3.18 \mathrm{mmol}$ ). Yield 21\%, MS: (ESI, neg.) $\mathrm{m} / \mathrm{z} 325[\mathrm{M}-\mathrm{H}]^{-},{ }^{1} \mathrm{H}$ NMR $(500 \mathrm{MHz}$, DMSO$\left.\mathrm{d}_{6}\right): \delta(\mathrm{ppm}) 11.55$ (br.s), $7.26(\mathrm{~s}, 1 \mathrm{H}) 7.23(\mathrm{~d}, 1 \mathrm{H}, \mathrm{J}=8 \mathrm{~Hz}), 7.17(\mathrm{t}, 1 \mathrm{H}, \mathrm{J}=7.5 \mathrm{~Hz}), 6.99$ $(\mathrm{d}, 1 \mathrm{H}, \mathrm{J}=7.5 \mathrm{~Hz}), 2.28(\mathrm{~s}, 3 \mathrm{H}), 1.87(\mathrm{t}, 1 \mathrm{H}, \mathrm{J}=12 \mathrm{~Hz}), 1.72(\mathrm{~d}, 2 \mathrm{H}, \mathrm{J}=12.5 \mathrm{~Hz}), 1.44(\mathrm{~d}$, $2 \mathrm{H}, \mathrm{J}=12 \mathrm{~Hz}), 1.20(\mathrm{~s}, 6 \mathrm{H}), 1.18$ to $1.06(\mathrm{~m}, 4 \mathrm{H}), 0.99(\mathrm{~m}, 2 \mathrm{H}) .{ }^{13} \mathrm{C} \mathrm{NMR}(500 \mathrm{MHz}$, DMSO-d 6 ): $\delta$ (ppm) 170.36, 165.93, 135.90, 132.80, 131.01, 127.60, 127.07, 126.63, 100.04, 59.77, 44.84, 43.29, 27.22, 26.41, 26.06, 22.11, 21.13, 20.78, 14.10. HPLC retention time: $10.095 \mathrm{~min}$; purity $99.23 \%$.

\subsubsection{Synthesis of 2-(2-methyloctan-2-yl)-5-m-tolylpyrimidine-4,6- diol (47)}

Compound $\mathbf{4 7}$ was prepared in a similar manner to compound $\mathbf{4 4}$ from sodium metal (111 mg, 4.83mmol), EtOH (6ml), 2,2-dimethyloctanimidamide hydrochloride 29 (400mg, 1.93mmol), and diethyl 2-m-tolylmalonate 38 (483mg, 1.93mmol). Yield 11.2\%, MS: (ESI, pos.) $\mathrm{m} / z 351.3[\mathrm{M}+\mathrm{Na}]^{+},{ }^{1} \mathrm{H}$ NMR (500 MHz, DMSO-d 6 ): $\delta(\mathrm{ppm})$ 11.58 (br.s, 2H), $7.26(\mathrm{~s}, 1 \mathrm{H}) 7.22(\mathrm{~d}, 1 \mathrm{H}, \mathrm{J}=7.5 \mathrm{~Hz}), 7.19(\mathrm{t}, 1 \mathrm{H}, \mathrm{J}=7.5 \mathrm{~Hz}), 7.01(\mathrm{~d}, 1 \mathrm{H}$, $\mathrm{J}=7.5 \mathrm{~Hz}), 2.91(\mathrm{~s}, 3 \mathrm{H}), 1.68(\mathrm{~m}, 2 \mathrm{H}), 1.28(\mathrm{~s}, 6 \mathrm{H}), 1.24$ to $1.07(\mathrm{~m}, 16 \mathrm{H}), 0.853(\mathrm{~m}$, $3 \mathrm{H}) .{ }^{13} \mathrm{C}$ NMR (500MHz, DMSO-d 6 ): $\delta$ (ppm) 179.57, 165.84, 136.39, 133.20, 131.47, $128.08,127.55,127.16,100.64,48.48,40.61,31.53,29.57,26.21,25.90,24.48,22.53$, 21.61, 14.39. HPLC retention time: $9.685 \mathrm{~min}$; purity $91.54 \%$.

\subsubsection{Synthesis of 5-(3,5-dichlorophenyl)-2-(2-phenylpropan-2- yl)pyrimidine-4,6-diol (48)}

Compound $\mathbf{4 8}$ was prepared in a similar manner to compound $\mathbf{4 4}$ from sodium metal $(115 \mathrm{mg}, 5 \mathrm{mmol})$, EtOH $(6 \mathrm{ml}), 2$-methyl-2-phenylpropanimidamide hydrochloride 26 (400mg, 2mmol), and diethyl 2-(3,5-dichlorophenyl)malonate 39 (610 $\mathrm{mg}, 2 \mathrm{mmol}$ ). Yield: 27\%; MS: (ESI, neg.) $\mathrm{m} / \mathrm{z} 372.9[\mathrm{M}-\mathrm{H}]^{-},{ }^{1} \mathrm{H}$ NMR (500 MHz, DMSO-d 6 ): $\delta$ (ppm) 12.48 (br.s, 2H), $7.61(\mathrm{~d}, 2 \mathrm{H}, \mathrm{J}=2 \mathrm{~Hz}), 7.49(\mathrm{~s}, 1 \mathrm{H}), 7.31(\mathrm{~m}, 2 \mathrm{H}), 7.27(\mathrm{~m}, 3 \mathrm{H})$, $1.68(\mathrm{~s}, 6 \mathrm{H}) .{ }^{13} \mathrm{C}$ NMR $\left(500 \mathrm{MHz}, \mathrm{DMSO}-\mathrm{d}_{6}\right): \delta(\mathrm{ppm}) 172.33,171.56,141.09,139.75$, 134.08, 133.23, 129.02, 128.97, 126.78, 126.73, 110.00, 45.28, 32.74. HPLC retention time: $9.335 \mathrm{~min}$; purity $92.38 \%$. 


\subsubsection{Synthesis of 5-(3,5-dichlorophenyl)-2-(2-(thiophen-2- yl)propan-2-yl)pyrimidine-4,6-diol (49)}

Compound 49 was prepared in a similar manner to compound 44 from sodium metal (113mg, 4.9mmol), EtOH (6ml), 2-methyl-2-(thiophen-2-yl)propanimidamide hydrochloride 27 (400 $\mathrm{mg}, 1.95 \mathrm{mmol})$, and diethyl 2-(3,5-dichlorophenyl)malonate 39 (595mg, 1.95mmol). Yield: 32\%; MS: (ESI, neg.) $\mathrm{m} / \mathrm{z} 378.8[\mathrm{M}-\mathrm{H}]^{-},{ }^{1} \mathrm{H}$ NMR (500 MHz, DMSO-d $\left.{ }_{6}\right): \delta(\mathrm{ppm}) 7.62(\mathrm{~s}, 2 \mathrm{H}), 7.48(\mathrm{~s}, 1 \mathrm{H}), 7.43(\mathrm{~d}, 1 \mathrm{H}, \mathrm{J}=5.5 \mathrm{~Hz}), 7.06(\mathrm{~d}$, $1 \mathrm{H}, \mathrm{J}=3.5 \mathrm{~Hz}), 6.99(\mathrm{t}, 1 \mathrm{H}, \mathrm{J}=5 \mathrm{~Hz}), 1.77(\mathrm{~s}, 6 \mathrm{H}) .{ }^{13} \mathrm{C} \mathrm{NMR}\left(500 \mathrm{MHz}, \mathrm{DMSO}-\mathrm{d}_{6}\right): \delta$ (ppm) 172.39, 171.61, 141.14, 139.83, 134.12, 133.24, 129.13, 128.96, 126.87, 126.71, 99.19, 39.41, 28.08. HPLC retention time: $9.335 \mathrm{~min}$; purity $90.19 \%$.

\subsubsection{Synthesis of 2-(2-cyclohexylpropan-2-yl)-5-(3,5- dichlorophenyl)pyrimidine-4,6-diol (50)}

Compound 50 was prepared in a similar manner to compound 44 from sodium metal (140mg, 6.1 mmol), EtOH (6ml), 2-cyclohexyl-2-methylpropanimidamide hydrochloride 28 (500 mg, 2.44mmol), and diethyl 2-(3,5-dichlorophenyl)malonate 39 (970mg, 3.18mmol). Yield: 7\%; MS: (ESI, neg.) $m / z 379.0[\mathrm{M}-\mathrm{H}]^{-},{ }^{1} \mathrm{H}$ NMR (500 MHz, DMSO-d $\left.{ }_{6}\right): \delta(\mathrm{ppm}) 7.69(\mathrm{~s}, 2 \mathrm{H}), 7.38(\mathrm{~s}, 1 \mathrm{H}), 1.87(\mathrm{~m}, 1 \mathrm{H}), 1.68(\mathrm{~m}, 3 \mathrm{H}), 1.44(\mathrm{~d}$, $2 \mathrm{H}, \mathrm{J}=11 \mathrm{~Hz}), 1.21(\mathrm{~s}, 6 \mathrm{H}), 1.12(\mathrm{~m}, 3 \mathrm{H}), 0.98(\mathrm{~m}, 2 \mathrm{H}) .{ }^{13} \mathrm{C}$ NMR $(500 \mathrm{MHz}, \mathrm{DMSO}-$ $\left.\mathrm{d}_{6}\right): \delta$ (ppm) 172.38, 171.61, 140.65, 135.68, 133.24, 128.46, 100.25, 59.77, 44.10, 42.98, $27.22,26.49,26.02,21.67,20.34,14.10$. HPLC retention time: $8.932 \mathrm{~min}$; purity $94.24 \%$.

\subsubsection{Synthesis of 5-(3,5-dichlorophenyl)-2-(2-methyloctan-2- yl)pyrimidine-4,6-diol (51)}

Compound 51 was prepared in a similar manner to compound 44 from sodium metal (111mg, 4.83mmol), EtOH (8ml), 2,2-dimethyloctanimidamide hydrochloride 29 (400mg, 1.93mmol), and diethyl 2-(3,5-dichlorophenyl)malonate 39 (589mg, $1.93 \mathrm{mmol})$. Yield: 14\%; MS: (ESI, neg.) $380.9 \mathrm{~m} / \mathrm{z}[\mathrm{M}-\mathrm{H}]^{-},{ }^{1} \mathrm{H}$ NMR (500 MHz, methanol-d $\left.{ }_{4}\right): \delta$ (ppm) $7.57(\mathrm{~s}, 2 \mathrm{H}), 7.24(\mathrm{~s}, 1 \mathrm{H}), 1.71(\mathrm{~m}, 2 \mathrm{H}), 1.34(\mathrm{~m}, 5 \mathrm{H}), 1.28(\mathrm{~s}, 6 \mathrm{H}), 1.20(\mathrm{~m}, 3 \mathrm{H})$, $0.88(\mathrm{t}, 3 \mathrm{H}, \mathrm{J}=6 \mathrm{~Hz}) .{ }^{13} \mathrm{C} \mathrm{NMR}\left(500 \mathrm{MHz}, \mathrm{DMSO}-\mathrm{d}_{6}\right): \delta(\mathrm{ppm}) 170.98,169.54,139.32$, $135.79,131.05,129.09,100.15,48.48,40.61,30.48,29.79,26.92,25.43,23.76,22.53$, 14.41. HPLC retention time: $9.132 \mathrm{~min}$; purity $96.84 \%$.

\subsubsection{Synthesis of 5-cyclohexyl-2-(2-phenylpropan-2-yl)pyrimidine- 4,6-diol (52)}

Compound 52 was prepared in a similar manner to compound $\mathbf{4 4}$ from sodium metal (145mg, 6.3mmol), EtOH (6ml), 2-methyl-2-phenylpropanimidamide hydrochloride $26(500 \mathrm{mg}, 2.5 \mathrm{mmol})$, and diethyl 2-cyclohexylmalonate $40(606 \mathrm{mg}$, 2.5mmol). Yield: 39\% (yellow waxy solid) MS: (ESI, neg.) $m / z 311.0[\mathrm{M}-\mathrm{H}]^{-},{ }^{1} \mathrm{H}$ 
NMR (500 MHz, DMSO-d 6 ): $\delta$ (ppm) 11.30 (br.s, 1H), 10.93 (br.s., 1H), 7.33 (m, 2H), $7.23(\mathrm{~m}, 3 \mathrm{H}), 2.72(\mathrm{tt}, 1 \mathrm{H}, \mathrm{J}=12.5 \mathrm{~Hz}$ and $3 \mathrm{~Hz}), 1.92(\mathrm{q}, 2 \mathrm{H}, \mathrm{J}=13 \mathrm{~Hz}), 1.70(\mathrm{~d}, 2 \mathrm{H}, \mathrm{J}=$ $12.5 \mathrm{~Hz}), 1.62(\mathrm{~s}, 7 \mathrm{H}), 1.38(\mathrm{~d}, 2 \mathrm{H}, \mathrm{J}=12 \mathrm{~Hz}), 1.18(\mathrm{~m}, 3 \mathrm{H}) .{ }^{13} \mathrm{C} \mathrm{NMR}(500 \mathrm{MHz}$, DMSO-d $_{6}$ ): $\delta$ (ppm) $164.35,145.90,128.28,126.46,126.02,104.19,44.34,33.28,28.87$, 27.25, 26.67, 25.75. HPLC retention time: $8.983 \mathrm{~min}$; purity $99.46 \%$.

\subsubsection{Synthesis of 5-cyclohexyl-2-(2-(thiophen-2-yl)propan-2- yl)pyrimidine-4,6-diol (53)}

Compound 53 was prepared in a similar manner to compound $\mathbf{4 4}$ from sodium metal (140mg, 6.1 mmol), EtOH (6ml), 2-methyl-2-(thiophen-2-yl)propanimidamide hydrochloride 27 (500mg, 2.44mmol), and diethyl 2-cyclohexylmalonate $40(591 \mathrm{mg}$, 2.44mmol). Yield 8\%, MS: (ESI, neg.) $\mathrm{m} / \mathrm{z} 316.9[\mathrm{M}-\mathrm{H}]^{-},{ }^{1} \mathrm{H}$ NMR $(500 \mathrm{MHz}$, DMSO-d 6 ): $\delta$ (ppm) 11.36 (br.s, 1H), 10.88 (br.s, $1 \mathrm{H}) 7.39$ (d, 1H, J = 5Hz), 7.01 (s, $1 \mathrm{H}), 6.96(\mathrm{t}, 1 \mathrm{H}, \mathrm{J}=3.5 \mathrm{~Hz}), 2.72(\mathrm{t}, 1 \mathrm{H}, \mathrm{J}=12 \mathrm{~Hz}), 1.91(\mathrm{q}, 2 \mathrm{H}, \mathrm{J}=12.5 \mathrm{~Hz}), 1.72(\mathrm{~s}$, $6 \mathrm{H}), 1.61(\mathrm{~d}, 1 \mathrm{H}, \mathrm{J}=10.5 \mathrm{~Hz}), 1.36(\mathrm{~d}, 2 \mathrm{H}, \mathrm{J}=12 \mathrm{~Hz}), 1.18(\mathrm{~m}, 3 \mathrm{H}) .{ }^{13} \mathrm{C} \mathrm{NMR}(500 \mathrm{MHz}$, DMSO-d $\left.{ }_{6}\right): \delta$ (ppm) 164.41, 126.72, 124.80, 124.29, 109.53, 104.31, 48.61, 42.60, 33.27, 28.80, 28.11, 26.63, 25.72. HPLC retention time: $8.934 \mathrm{~min}$; purity $100 \%$.

\subsubsection{Synthesis of 5-cyclohexyl-2-(2-cyclohexylpropan-2- yl)pyrimidine-4,6-diol (54)}

Compound $\mathbf{5 4}$ was prepared in a similar manner to compound $\mathbf{4 4}$ from sodium metal (140mg, $6.1 \mathrm{mmol})$, EtOH (6ml), 2-cyclohexyl-2-methylpropanimidamide hydrochloride $28(500 \mathrm{mg}, 2.44 \mathrm{mmol})$, and diethyl 2-cyclohexylmalonate $40(770 \mathrm{mg}$, $3.18 \mathrm{mmol}$ ). Yield 1.3\%, MS: (ESI, neg.) $\mathrm{m} / z 316.9[\mathrm{M}-\mathrm{H}]^{-},{ }^{1} \mathrm{H} \mathrm{NMR}(500 \mathrm{MHz}$, DMSO-d ${ }_{6}$ ): $\delta$ (ppm) 11.43 (br.s, $\left.1 \mathrm{H}\right), 10.64$ (br.s, $\left.1 \mathrm{H}\right) 2.72$ (tt, $1 \mathrm{H}, \mathrm{J}=12.5 \mathrm{~Hz}$ and $3.5 \mathrm{~Hz}), 1.91(\mathrm{~m}, 2 \mathrm{H}), 1.81(\mathrm{t}, 1 \mathrm{H}, \mathrm{J}=12 \mathrm{~Hz}), 1.71(\mathrm{~d}, 4 \mathrm{H}, \mathrm{J}=12 \mathrm{~Hz}), 1.61(\mathrm{~m}, 2 \mathrm{H}), 1.37$ $(\mathrm{d}, 2 \mathrm{H}, \mathrm{J}=12.5 \mathrm{~Hz}), 1.23$ to $0.90(\mathrm{~m}, 16 \mathrm{H}) .{ }^{13} \mathrm{C}$ NMR $\left(500 \mathrm{MHz}, \mathrm{DMSO}-\mathrm{d}_{6}\right): \delta$ (ppm) 164.44, 164.29, 103.69, 44.73, 42.98, 33.25, 28.94, 27.18, 26.68, 26.38, 26.06, 25.75, 22.11. HPLC retention time: $10.273 \mathrm{~min}$; purity $98.33 \%$.

\subsubsection{Synthesis of 5-cyclohexyl-2-(2-methyloctan-2-yl)pyrimidine- 4,6-diol (55)}

Compound 55 was prepared in a similar manner to compound $\mathbf{4 4}$ from sodium metal (111 mg, 4.84mmol), EtOH (8ml), 2,2-dimethyloctanimidamide hydrochloride 29 (400mg, 1.93mmol), and diethyl 2-cyclohexylmalonate 40 (468mg, 1.93mmol). Yield 4.5\%, MS: (ESI, neg.) $\mathrm{m} / z 319.0[\mathrm{M}-\mathrm{H}]^{-},{ }^{1} \mathrm{H}$ NMR (500 MHz, DMSO-d 6 ): 2.72 (tt, $1 \mathrm{H}$, $\mathrm{J}=12 \mathrm{~Hz}$ and $3 \mathrm{~Hz}), 1.92(\mathrm{~m}, 2 \mathrm{H}), 1.70(\mathrm{~d}, 2 \mathrm{H}, \mathrm{J}=12.5 \mathrm{~Hz}), 1.61(\mathrm{t}, 4 \mathrm{H}, \mathrm{J}=8.5 \mathrm{~Hz}), 1.37$ $(\mathrm{d}, 2 \mathrm{H}, \mathrm{J}=12 \mathrm{~Hz}), 1.23$ to $1.16(\mathrm{~m}, 16 \mathrm{H}), 1.04(\mathrm{~m}, 2 \mathrm{H}), 0.83(\mathrm{~m}, 4 \mathrm{H}) .{ }^{13} \mathrm{C}$ NMR (500MHz, DMSO-d 6 ): $\delta$ (ppm) 164.35, 163.82, 103.74, 40.26, 33.23, 31.03, 29.04, 28.90, 
$26.67,25.75,25.71,23.95,22.04,13.91$. HPLC retention time: $10.304 \mathrm{~min}$; purity $99.06 \%$.

\subsubsection{Synthesis of 5-(pyridin-2-yl)-2-(2-(thiophen-2-yl)propan-2- yl)pyrimidine-4,6-diol (56)}

Compound 56 was prepared in a similar manner to compound $\mathbf{4 4}$ from sodium metal (112mg, 4.88mmol), EtOH (6ml), 2-methyl-2-(thiophen-2-yl)propanimidamide hydrochloride 27 (400mg, 1.95mmol), and diethyl 2-(pyridin-2-yl)malonate 41 (463mg, $1.95 \mathrm{mmol}$ ). Yield 1\%, MS: (ESI, pos.) $\mathrm{m} / z 336.1[\mathrm{M}+\mathrm{Na}]^{+},{ }^{1} \mathrm{H} \mathrm{NMR}(500 \mathrm{MHz}$, DMSO-d $\left.)_{6}\right): \delta(\mathrm{ppm}) 9.13(\mathrm{~d}, 1 \mathrm{H}, \mathrm{J}=8.5 \mathrm{~Hz}) 8.57(\mathrm{~s}, 1 \mathrm{H}), 8.12(\mathrm{td}, 1 \mathrm{H}, \mathrm{J}=7.5 \mathrm{~Hz}$ and $1.5 \mathrm{~Hz}), 7.41(\mathrm{q}, 2 \mathrm{H}, \mathrm{J}=7.5 \mathrm{~Hz}), 7.59(\mathrm{~d}, 1 \mathrm{H}, \mathrm{J}=4), 6.98(\mathrm{t}, 1 \mathrm{H}, \mathrm{J}=4 \mathrm{~Hz}), 1.73(\mathrm{~s}, 6 \mathrm{H})$. HPLC retention time: $8.367 \mathrm{~min}$; purity $97.78 \%$.

\subsubsection{Synthesis of 5-benzyl-2-(2-methyloctan-2-yl)pyrimidine-4,6- diol (57)}

Compound $\mathbf{5 7}$ was prepared in a similar manner to compound $\mathbf{4 4}$ from sodium metal (111mg, 4.84mmol), EtOH (6ml), 2,2-dimethyloctanimidamide hydrochloride $\mathbf{2 9}$ (400mg, 1.93mmol), and diethyl 2-benzylmalonate 42 (483mg, $1.93 \mathrm{mmol})$. Yield $21 \%$ MS: (ESI, neg.) $\mathrm{m} / \mathrm{z} 327.0[\mathrm{M}-\mathrm{H}]^{-},{ }^{1} \mathrm{H}$ NMR (500 MHz, DMSO-d 6 ): $\delta$ (ppm) 11.34 (br.s), $7.21(\mathrm{~m}, 4 \mathrm{H}) 7.11(\mathrm{~m}, 1 \mathrm{H}), 3.57(\mathrm{~s}, 2 \mathrm{H}), 1.63(\mathrm{~m}, 2 \mathrm{H}), 1.22(\mathrm{~s}, 6 \mathrm{H}), 1.21$ to 1.19 $(\mathrm{m}, 6 \mathrm{H}), 1.04(\mathrm{~m}, 2 \mathrm{H}), 0.82(\mathrm{t}, 3 \mathrm{H}, \mathrm{J}=6.5 \mathrm{~Hz}) .{ }^{13} \mathrm{C}$ NMR $\left(500 \mathrm{MHz}, \mathrm{DMSO}-\mathrm{d}_{6}\right): \delta(\mathrm{ppm})$ 164.70, 141.19, 128.23, 127.97, 125.49, 99.07, 54.92, 40.37, 39.77, 31.02, 29.03, 27.81, 25.77, 23.97, 22.02, 13.91. HPLC retention time: $9.716 \mathrm{~min}$; purity $100 \%$.

\subsubsection{Synthesis of 5-hexyl-2-(2-methyloctan-2-yl)pyrimidine-4,6- diol (58)}

Compound 58 was prepared in a similar manner to compound $\mathbf{4 4}$ from sodium metal (140mg, 6.05mmol), EtOH (6ml), 2,2-dimethyloctanimidamide hydrochloride 29 (500mg, 2.42mmol), and diethyl 2-hexylmalonate $43(591 \mathrm{mg}, 2.42 \mathrm{mmol})$. Yield $9 \%$, MS: (ESI, neg.) $m / z 321.0[\mathrm{M}-\mathrm{H}]{ }^{-1},{ }^{1} \mathrm{H}$ NMR (500 MHz, DMSO-d 6 ): $\delta(\mathrm{ppm}) 11.47$ (br.s, $2 \mathrm{H}), 4.10(\mathrm{q}, 1 \mathrm{H}, \mathrm{J}=7.5 \mathrm{~Hz}) 3.29(\mathrm{t}, 1 \mathrm{H}, \mathrm{J}=7.5 \mathrm{~Hz}), 2.23(\mathrm{t}, 2 \mathrm{H}, \mathrm{J}=7.5 \mathrm{~Hz}), 1.71$ $(\mathrm{q}, 1 \mathrm{H}, \mathrm{J}=7 \mathrm{~Hz}), 1.62(\mathrm{~m}, 2 \mathrm{H}), 1.36(\mathrm{t}, 2 \mathrm{H}, \mathrm{J}=6.5 \mathrm{~Hz}), 1.24(\mathrm{~s}, 6 \mathrm{H}), 1.21$ to $1.16(\mathrm{~m}, 9 \mathrm{H})$, $1.02(\mathrm{~m}, 2 \mathrm{H}), 0.83(\mathrm{~m}, 6 \mathrm{H}) .{ }^{13} \mathrm{C}$ NMR $\left(500 \mathrm{MHz}, \mathrm{DMSO}-\mathrm{d}_{6}\right): \delta(\mathrm{ppm}) 170.52,169.50$, $164.69,163.88,99.72,60.59,51.39,40.40,31.27,31.06,29.06,28.73,28.34,27.58$, 26.62, 25.81, 23.99, 22.03, 13.97. HPLC retention time: $10.616 \mathrm{~min}$; purity $97.38 \%$. 


\subsubsection{Synthesis of Furanopyrimidine Cannabinoids}

With the successful synthesis of both the pyrimidine hexahydro classical cannabinoids and the pyrimidine non-classical cannabinoids, sights were set on expansion of the pyrimidine containing series of compounds. Upon review of the current medicinal chemistry literature, a series of 4-amino-5,6-biaryl-furo[2,3-d]pyrimidines that had been developed by Amgen Inc. as inhibitors of lymphocyte-specific kinase (Lck) ${ }^{178}$ caught our attention. The general structure of this series of compounds (Figure 4.7) has aspects of both a conformationally restricted pyrimidine non-classical cannabinoid and a ring-contracted pyrimidine classical cannabinoid in which the six-membered pyran ring has been replaced by a five-membered furan. These compounds lacked the side chain at the 2-position of the pyrimidine ring found in the previously described pyrimidine cannabinoids. This series of compounds contained two substituents on the furan ring: one being an unsubstituted phenyl and the other a substituted phenyl.

It was decided to design the furanopyrimidine with just one aryl substitution on the furan ring in order to form compounds that would occupy similar space to the previously described pyrimidine cannabinoids. These new compounds were also to be synthesized with a side chain present similar to the classical and non-classical pyrimidine cannabinoids (Figure 4.8). By adapting the chemistry of DiMauro et al. ${ }^{178 a}$ the furanopyrimidine cannabinoids were formed by the base catalysed cyclization of a substituted dihydroxy pyrimidine with a 2-chloro-2-nitrovinyl substituted aromatic ring (Scheme 4.18).

The substituted dihydroxy pyrimidines used were the same ones formed for the pyrimidine hexahydro classical cannabinoids (Scheme 4.12) from substituted amidines and diethyl malonate. The 2-chloro-2nitrovinyl styrenes were formed from substituted benzaldehydes, bromonitromethane, dimethylamine hydrochloride, and potassium fluoride in xylenes (Scheme 4.19). The reaction is fitted with a Dean-Stark trap to allow for azeotropic removal of water and heated to $125^{\circ} \mathrm{C}$ for 8 hours. The styrenes were then readily purified by flash column chromatography.

Four styrenes (phenyl, m-tolyl, 3,5-dichloro phenyl, and 2-thiophenyl) were cyclized with four substituted dihydroxy pyrimidines (dimethyl phenyl, dimethyl thiophenyl, dimethyl cyclohexyl, and dimethyl heptyl) to form sixteen new furanopyrimidine cannabinoids (Figure 4.9).

All chemicals were obtained from Sigma Aldrich or Fisher Scientific Inc. Anhydrous solvents were obtained by distillation over either calcium hydride or metallic sodium and benzophenone. Final compounds and intermediates were purified using column chromatography on the Biotage SP1 system employing Flash column cartridges or by crystallization. NMR spectra were obtained on a Bruker $300 \mathrm{MHz}$ or Varian $500 \mathrm{MHz}$ Inova NMR. HPLC analysis of final products was carried out by gradient elution using water/acetonitrile (0.1\% TFA) with a gradient of $70 \%(3 \mathrm{~min}), 50 \%(2 \mathrm{~min})$, and $10 \%(15 \mathrm{~min})$. A reverse-phase ODS Hypersil column, manufactured by Thermo Scientific, of dimensions $4.6 \times 150 \mathrm{~mm}$ with $5 \mu \mathrm{m}$ particle size was used for HPLC analyses. 


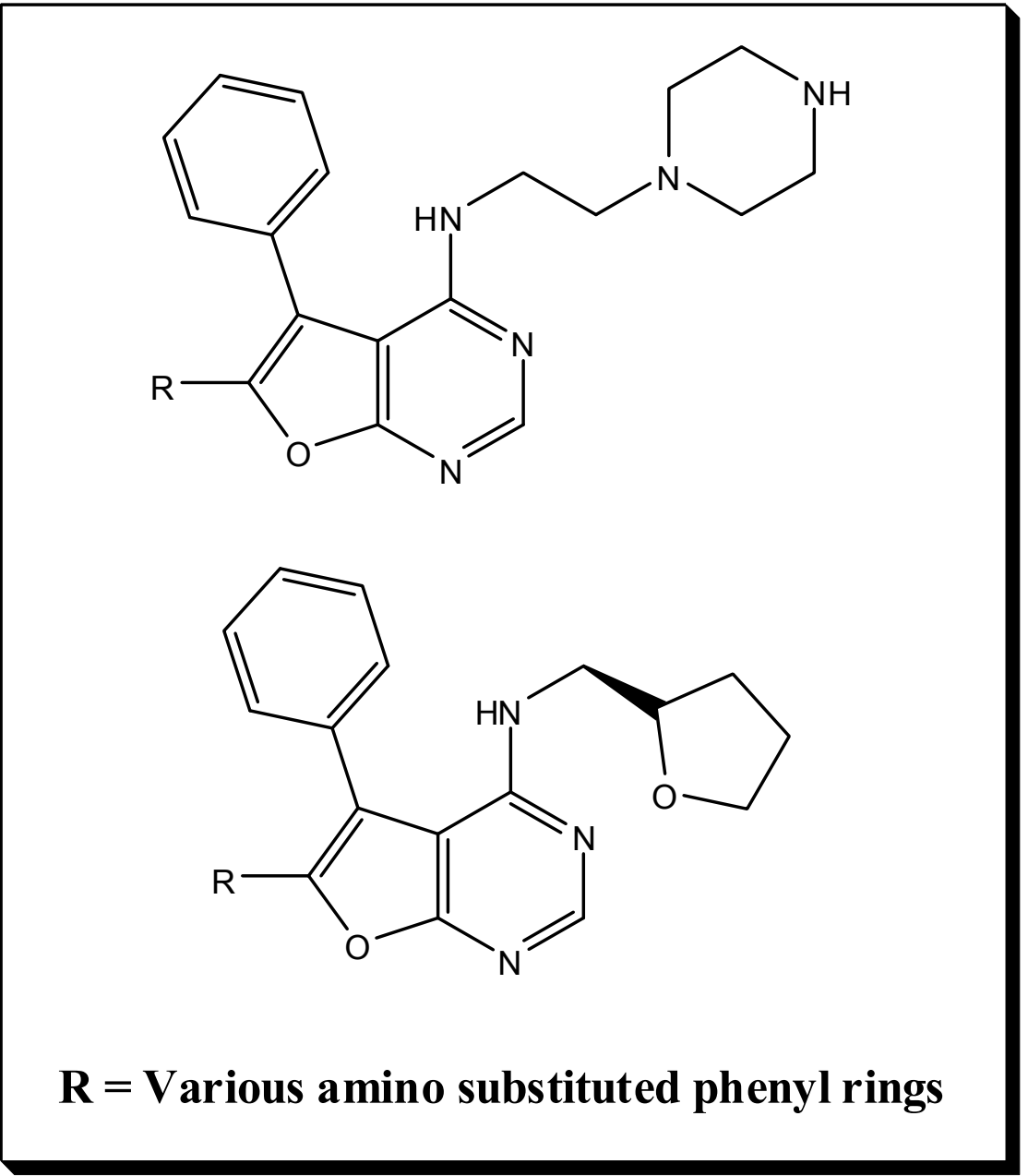

Figure 4.7: General structure of Lck inhibitors 


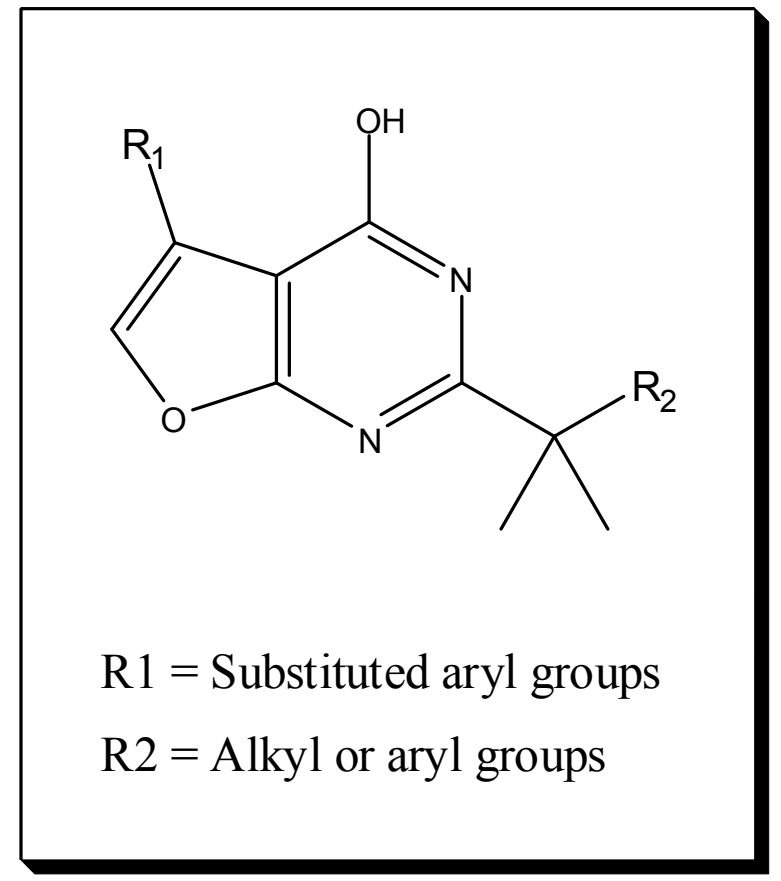

Figure 4.8: General structure of furanopyrimidine cannabinoids 


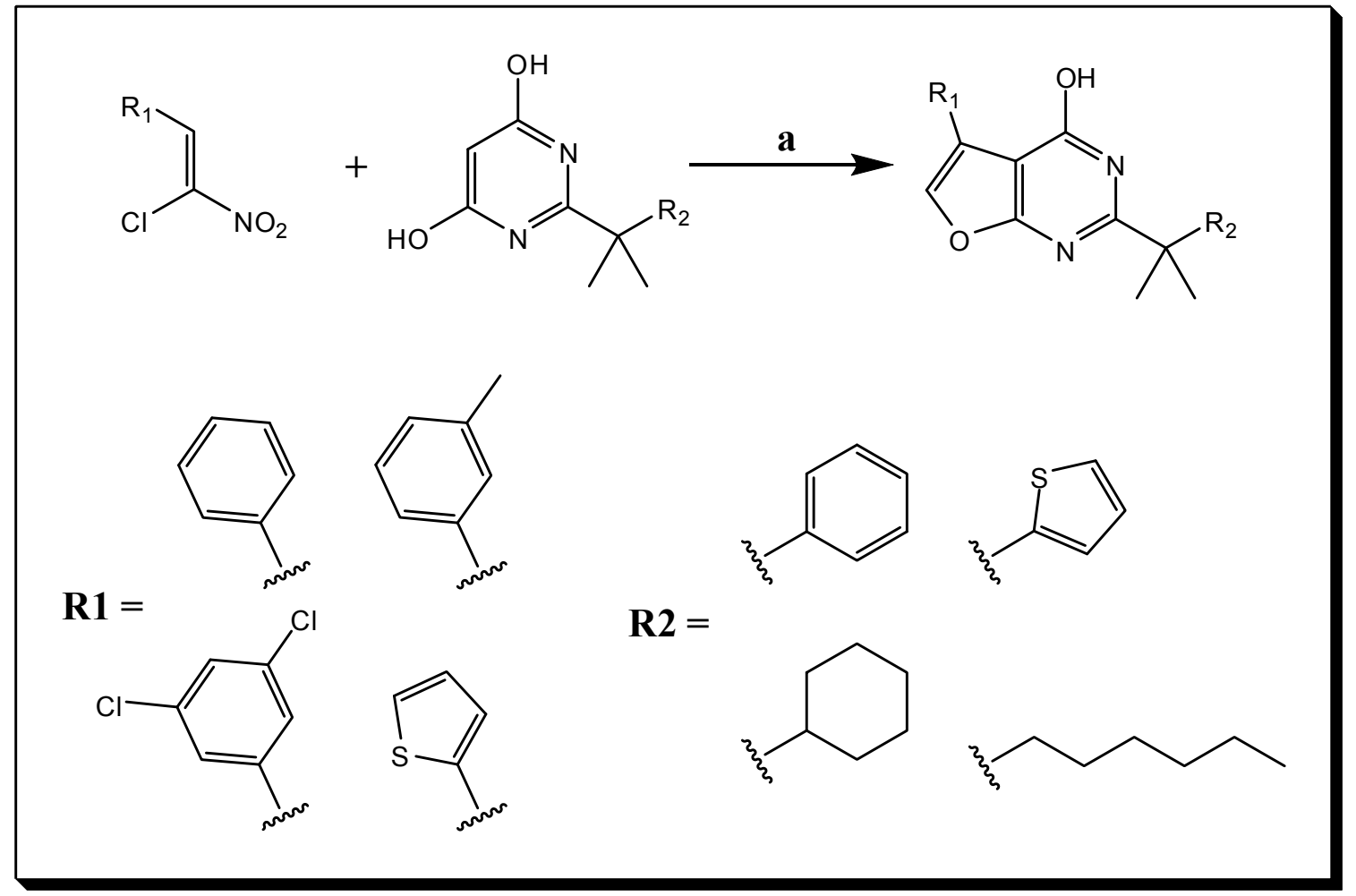

Scheme 4.18: General synthesis of furanopyrimidine cannabinoids

(a) DBU, acetonitrile, $\mu$ wave, 100 watts, $120^{\circ} \mathrm{C}, 15 \mathrm{~min}$. 


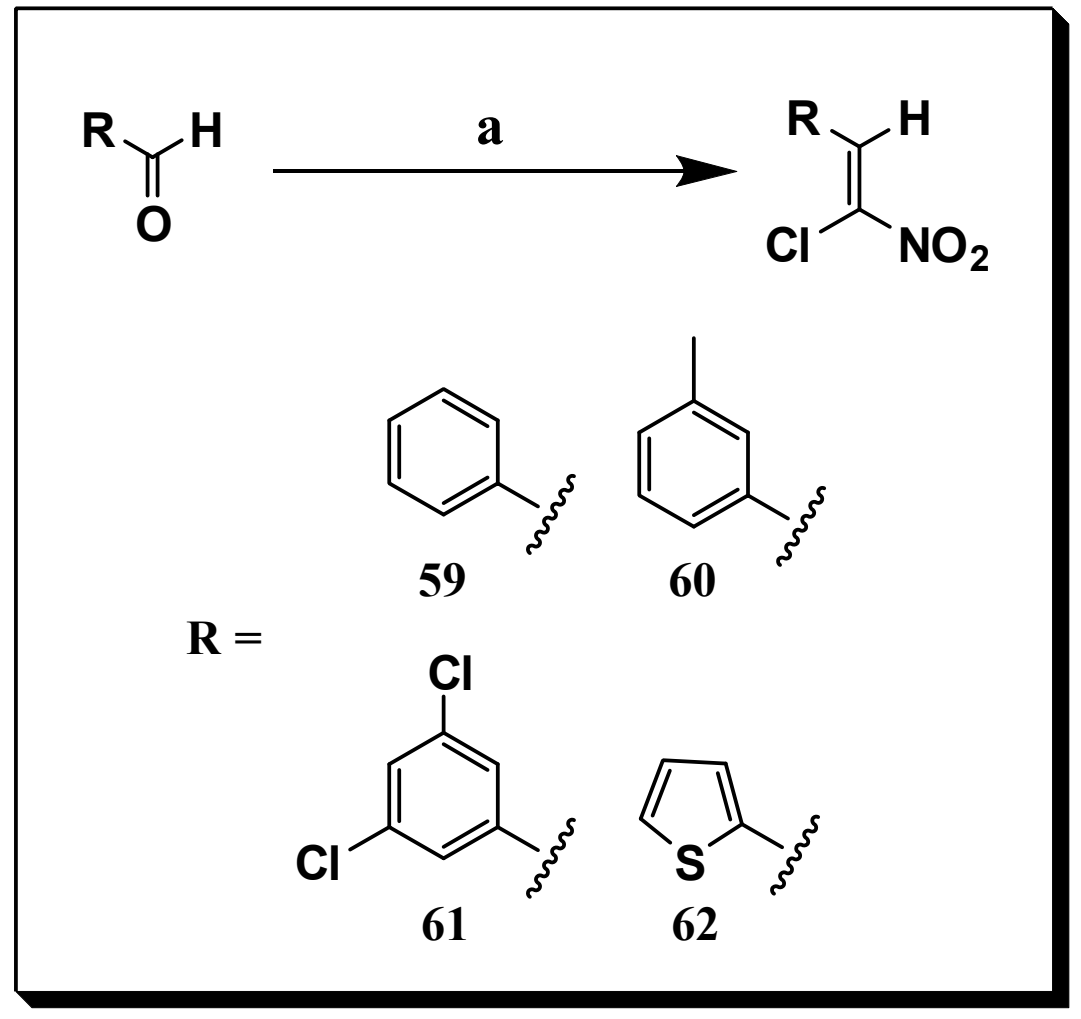

Scheme 4.19: Synthesis of 2-chloro-2-nitrovinyl styrenes

(a) bromonitromethane, dimethylamine hydrochloride, potassium fluoride, xylenes, $125^{\circ} \mathrm{C}, 8 \mathrm{hr}$. 


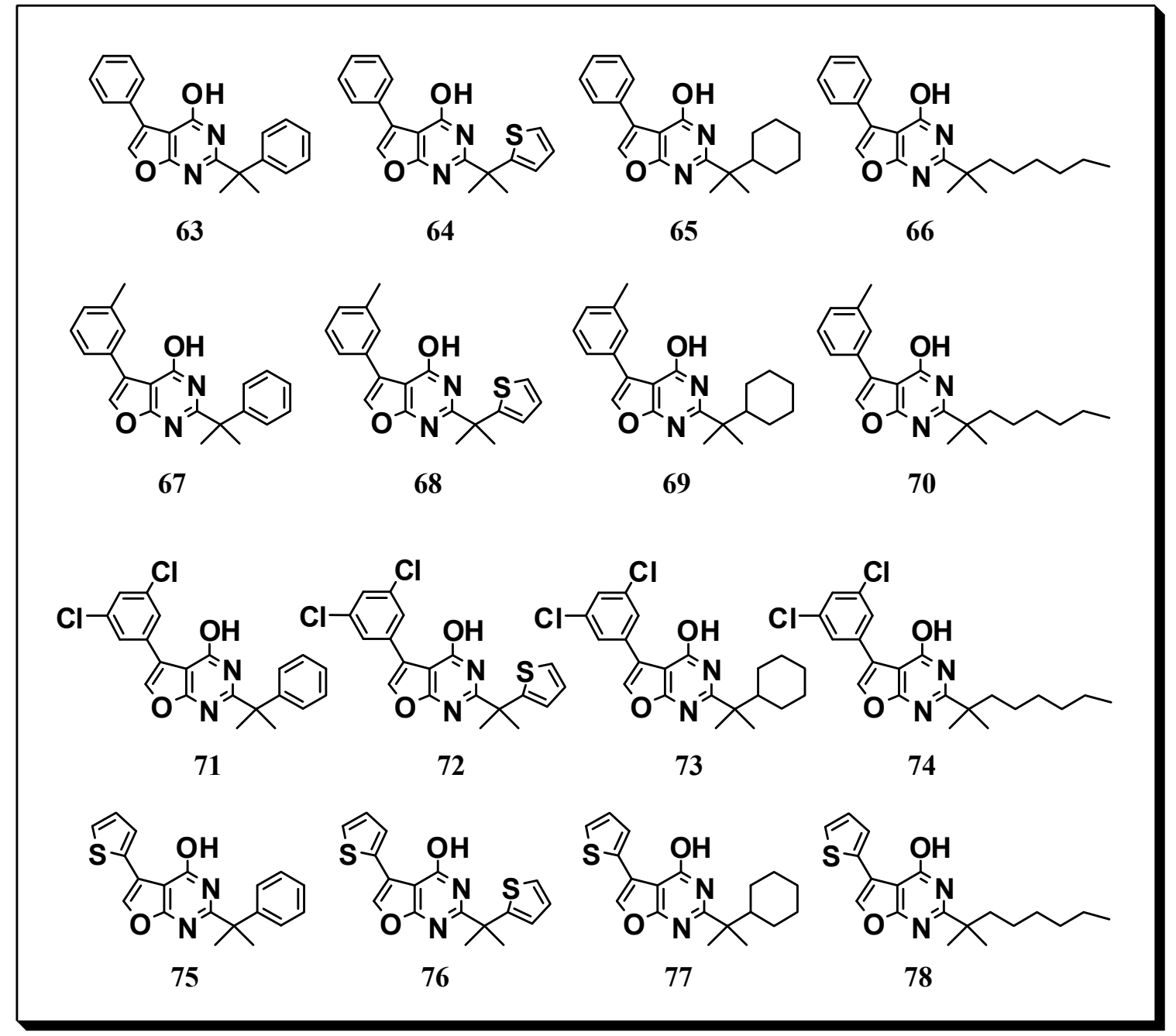

Figure 4.9: Furanopyrimidine cannabinoids 


\subsubsection{Synthesis of (2-chloro-2-nitrovinyl)benzene (59)}

Benzaldehyde (3.59g, $33.8 \mathrm{mmol})$, bromonitromethane $(9.0 \mathrm{~g}, 64.3 \mathrm{mmol})$, dimethylamine hydrochloride $(24.8 \mathrm{~g}, 304.2 \mathrm{mmol})$, potassium fluoride $(0.3 \mathrm{~g}, 5.08 \mathrm{mmol})$ and $\mathrm{m}$ - xylenes $(50 \mathrm{~mL})$ were combined in a $250 \mathrm{~mL}$ round bottomed flask fitted with a Dean-Stark trap. The mixture was heated at $125^{\circ} \mathrm{C}$ with azeotropic removal of water for $8 \mathrm{~h}$. The reaction mixture was concentrated and the residue was extracted with a mixture of 1:1 dicloromethane and water. After separation of the organic layer, the aqueous layer was extracted three times with dicloromethane. The combined organics were dried over anhydrous $\mathrm{Na}_{2} \mathrm{SO}_{4}$ and concentrated to a brown oil. The oily residue thus obtained was purified on a silica column (Biotage SP1 system) using a 1:9 ethyl acetate /hexane mixture to obtain the product as pale yellow needles $(4.8 \mathrm{~g}, 77 \%) . \mathrm{R}_{f}=0.66(1: 9$ ethyl acetate/hexane). ${ }^{1} \mathrm{H}$ NMR $\left(300 \mathrm{MHz}, \mathrm{CDCl}_{3}\right): \delta(\mathrm{ppm}) 8.40(\mathrm{~s}, 1 \mathrm{H}), 7.86-7.90(\mathrm{~m}, 2 \mathrm{H})$, 7.51-7.5 (m,3H).

\subsubsection{Synthesis of 1-(2-chloro-2-nitrovinyl)-3-methylbenzene (60)}

Compound 60 was prepared in a manner similar to compound 59. ${ }^{1} \mathrm{H}$ NMR (300 $\left.\mathrm{MHz}, \mathrm{CDCl}_{3}\right): \delta(\mathrm{ppm}) 8.38(\mathrm{~s}, 1 \mathrm{H}), 7.67(\mathrm{t}, 2 \mathrm{H}), 7.36-7.41(\mathrm{~m}, 2 \mathrm{H}), 2.45(\mathrm{~s}, 3 \mathrm{H})$.

\subsubsection{Synthesis of 1,3-dichloro-5-(2-chloro-2-nitrovinyl)benzene (61)}

Compound 61 was prepared in a manner similar to compound 59. ${ }^{1} \mathrm{H}$ NMR (300 $\left.\mathrm{MHz}, \mathrm{CDCl}_{3}\right): \delta(\mathrm{ppm}) 8.23(\mathrm{~s}, 1 \mathrm{H}), 7.74(\mathrm{~d}, 2 \mathrm{H}), 7.52(\mathrm{~s}, 1 \mathrm{H})$.

\subsubsection{Synthesis of 2-(2-chloro-2-nitrovinyl)thiophene (62)}

Compound 62 was prepared in a manner similar to compound 59. ${ }^{1} \mathrm{H}$ NMR (300 $\left.\mathrm{MHz}, \mathrm{CDCl}_{3}\right): \delta(\mathrm{ppm}) 8.65(\mathrm{~s}, 1 \mathrm{H}), 7.81(\mathrm{~d}, 1 \mathrm{H}), 7.69(\mathrm{~d}, 1 \mathrm{H}), 7.27-7.28(\mathrm{t}, 1 \mathrm{H})$.

\subsubsection{Synthesis of 5-phenyl-2-(2-phenylpropan-2-yl)furo[2,3- d]pyrimidin-4-ol (63)}

100mg (0.434mmol) of 2-(2-phenylpropan-2-yl)pyrimidine-4,6-diol 30 and $73 \mathrm{mg}$ $(0.4 \mathrm{mmol})$ of (2-chloro-2-nitrovinyl)benzene 59 were dissolved in $2 \mathrm{ml}$ acetonitrile in a $10 \mathrm{ml}$ microwave reaction vessel. $122 \mathrm{mg}(0.8 \mathrm{mmol})$ of 1,8-diazabicyclo[5.4.0] undec-7ene (DBU) was then added and the vessel sealed. The vessel was irradiated in a CEM LabMate microwave reactor (CEM, Matthews, NC USA) at 100 watts to $120^{\circ} \mathrm{C}$ for 15 minutes. The reaction mixture was then concentrated and the target compound purified by flash chromatography on a Biotage SP1 (Biotage, Charlottesville, VA USA) using a methylene chloride / methanol gradient. Yield: 42.3\%. MS: (ESI, neg) $\mathrm{m} / z 329.0$ [M $\mathrm{H}]^{-},{ }^{1} \mathrm{H}$ NMR (500 MHz, DMSO): $\delta$ (ppm) 12.03 (s, 1H), 8.24 (s, 1H), 7.96 (d, 2H, J = 
$7.5 \mathrm{~Hz}), 7.41(\mathrm{t}, 2 \mathrm{H}, \mathrm{J}=7.5 \mathrm{~Hz}), 7.33(\mathrm{q}, 3 \mathrm{H}, \mathrm{J}=7.5 \mathrm{~Hz}), 7.26(\mathrm{t}, 3 \mathrm{H}, \mathrm{J}=8 \mathrm{~Hz}), 1.71(\mathrm{~s}$, $6 \mathrm{H}) ;{ }^{13} \mathrm{C}$ NMR $\left(500 \mathrm{MHz}, \mathrm{CDCl}_{3}\right): \delta$ (ppm) 165.49, 164.18, 159.79, 145.77, 138.41, $130.22,128.37,128.32,127.94,126.57,129.17,123.49,120.92,44.98,27.53$. HPLC retention time: $10.185 \mathrm{~min}$; purity $100 \%$.

\subsubsection{Synthesis of 5-phenyl-2-(2-(thiophen-2-yl)propan-2-yl)furo[2,3- d]pyrimidin-4-ol (64)}

Compound 64 was prepared in a manner similar to compound 63 from 2-(2(thiophen-2-yl)propan-2-yl)pyrimidine-4,6-diol 31(100mg, 0.423 mmol), (2-chloro-2nitrovinyl)benzene 59 (73mg, $0.4 \mathrm{mmol})$, DBU $(122 \mathrm{mg}, 120 \mu 1,0.8 \mathrm{mmol})$, and $\mathrm{CH}_{3} \mathrm{CN}$ (2ml). Yield: 26.8\%. MS: (ESI, neg.) $m / z 335.0$ [M - H] $]^{-},{ }^{1} \mathrm{H}$ NMR (500 MHz, DMSO): $\delta(\mathrm{ppm}) 12.01(\mathrm{~s}, 1 \mathrm{H}), 8.24(\mathrm{~s}, 1 \mathrm{H}), 7.96(\mathrm{~d}, 2 \mathrm{H}, \mathrm{J}=7.5 \mathrm{~Hz}), 7.42(\mathrm{q}, 3 \mathrm{H}, \mathrm{J}=8 \mathrm{~Hz}), 7.32$ $(\mathrm{t}, 1 \mathrm{H}, \mathrm{J}=7 \mathrm{~Hz}), 7.04(\mathrm{~d}, 1 \mathrm{H}, \mathrm{J}=3 \mathrm{~Hz}), 6.99(\mathrm{t}, 1 \mathrm{H}, \mathrm{J}=4.5 \mathrm{~Hz}), 1.86(\mathrm{~s}, 6 \mathrm{H}) ;{ }^{13} \mathrm{C} \mathrm{NMR}$ $\left(500 \mathrm{MHz}, \mathrm{CDCl}_{3}\right): \delta$ (ppm) 165.38, 162.99, 159.67, 150.22, 138.60, 130.15, 128.38, 127.94, 127.62, 126.74, 124.98, 124.56, 123.50, 103.02, 43.16, 28.42. HPLC retention time: $10.136 \mathrm{~min}$; purity $100 \%$.

\subsubsection{Synthesis of 2-(2-cyclohexylpropan-2-yl)-5-phenylfuro[2,3- d]pyrimidin-4-ol (65)}

Compound 65 was prepared in a manner similar to compound 63 from 2-(2cyclohexylpropan-2-yl)pyrimidine-4,6-diol 32 (100mg, 0.423mmol), (2-chloro-2nitrovinyl)benzene 59 (73mg, 0.4mmol), DBU (122mg, $120 \mu 1,0.8 \mathrm{mmol})$, and $\mathrm{CH}_{3} \mathrm{CN}$ (2ml). Yield: 43.7\%. MS: (ESI, neg.) $m / z 335.0[\mathrm{M}-\mathrm{H}]^{-},{ }^{1} \mathrm{H}$ NMR (500 MHz, DMSO): $\delta(\mathrm{ppm}) 12.02(\mathrm{~s}, 1 \mathrm{H}), 8.19(\mathrm{~s}, 1 \mathrm{H}), 7.98(\mathrm{~d}, 2 \mathrm{H}, \mathrm{J}=8 \mathrm{~Hz}), 7.42(\mathrm{t}, 2 \mathrm{H}, \mathrm{J}=7.5 \mathrm{~Hz}), 7.32(\mathrm{t}$, $1 \mathrm{H}, \mathrm{J}=7 \mathrm{~Hz}), 1.94(\mathrm{t}, 1 \mathrm{H}, \mathrm{J}=11.5 \mathrm{~Hz}), 1.69$ (d, 2H, J = 12Hz), $1.60(\mathrm{~d}, 2 \mathrm{H}, \mathrm{J}=12 \mathrm{~Hz})$, $1.39(\mathrm{~d}, 2 \mathrm{H}, \mathrm{J}=12 \mathrm{~Hz}), 1.25(\mathrm{~s}, 6 \mathrm{H}), 1.07(\mathrm{~m}, 5 \mathrm{H}) ;{ }^{13} \mathrm{C} \mathrm{NMR}\left(500 \mathrm{MHz}, \mathrm{CDCl}_{3}\right): \delta$ (ppm) 165.58, 165.54, 159.89, 138.12, 130.29, 128.36, 127.91, 127.55, 123.41, 102.42, 44.92, 43.61, 27.19, 26.39, 26.01, 22.21. HPLC retention time: $11.245 \mathrm{~min}$; purity $99.45 \%$.

\subsubsection{Synthesis of 2-(2-methyloctan-2-yl)-5-phenylfuro[2,3- d]pyrimidin-4-ol (66)}

Compound 66 was prepared in a manner similar to compound 63 from 2-(2methyloctan-2-yl)pyrimidine-4,6-diol 33 (40mg, 0.168mmol), (2-chloro-2nitrovinyl)benzene 59 (28mg, 0.153mmol), DBU (47mg, 46.2 $\mu 1,0.308 \mathrm{mmol})$, and $\mathrm{CH}_{3} \mathrm{CN}$ (1ml). Yield: $32.6 \%$. MS: (ESI, neg.) $\mathrm{m} / \mathrm{z} 337[\mathrm{M}-\mathrm{H}]^{-},{ }^{1} \mathrm{H} \mathrm{NMR}(500 \mathrm{MHz}$, DMSO): $\delta$ (ppm) $12.12(\mathrm{~s}, 1 \mathrm{H}), 8.19(\mathrm{~s}, 1 \mathrm{H}), 7.98(\mathrm{~d}, 2 \mathrm{H}, \mathrm{J}=7.5 \mathrm{~Hz}), 7.42(\mathrm{t}, 2 \mathrm{H}, \mathrm{J}=$ $8 \mathrm{~Hz}), 7.32(\mathrm{t}, 1 \mathrm{H}, \mathrm{J}=7.5 \mathrm{~Hz}), 1.73(\mathrm{~m}, 2 \mathrm{H}), 1.31(\mathrm{~s}, 6 \mathrm{H}), 1.19(\mathrm{~m}, 6 \mathrm{H}), 1.06(\mathrm{~m} .2 \mathrm{H}), 0.8$ $(\mathrm{t}, 3 \mathrm{H}, \mathrm{J}=7 \mathrm{~Hz}) ;{ }^{13} \mathrm{C} \mathrm{NMR}\left(500 \mathrm{MHz}, \mathrm{CDCl}_{3}\right): \delta(\mathrm{ppm}) 165.65,164.91,159.91,138.14$, 
$130.28,128.36,127.90,127.55,112.34,102.44,40.44,40.33,30.98,29.06,25.90,23.94$, $22.01,13.88$. HPLC retention time: $11.846 \mathrm{~min}$; purity $100 \%$.

\subsubsection{Synthesis of 2-(2-phenylpropan-2-yl)-5-m-tolylfuro[2,3- d]pyrimidin-4-ol (67)}

Compound 67 was prepared in a manner similar to compound 63 from 2-(2phenylpropan-2-yl)pyrimidine-4,6-diol 30 (100mg, 0.434mmol), 1-(2-chloro-2nitrovinyl)-3-methylbenzene 60 (79mg, $0.4 \mathrm{mmol})$, DBU (122 $\mathrm{mg}, 120 \mu 1,0.8 \mathrm{mmol})$, and $\mathrm{CH}_{3} \mathrm{CN}(2 \mathrm{ml})$. Yield: 29.2\%. MS: (ESI, neg.) $\mathrm{m} / z 343.0[\mathrm{M}-\mathrm{H}]$ ], ${ }^{1} \mathrm{H}$ NMR $(500 \mathrm{MHz}$, DMSO): $\delta(\mathrm{ppm}) 11.99(\mathrm{~s}, 1 \mathrm{H}), 8.20(\mathrm{~s}, 1 \mathrm{H}), 7.79(\mathrm{~d}, 1 \mathrm{H}, \mathrm{J}=8 \mathrm{~Hz}), 7.74(\mathrm{~s}, 1 \mathrm{H}), 7.29(\mathrm{~m}$, $6 \mathrm{H}), 7.13(\mathrm{~d}, 1 \mathrm{H}, \mathrm{J}=7.5 \mathrm{~Hz}), 2.33(\mathrm{~s}, 3 \mathrm{H}), 1.71(\mathrm{~s}, 6 \mathrm{H}) ;{ }^{13} \mathrm{C} \mathrm{NMR}\left(500 \mathrm{MHz}, \mathrm{CDCl}_{3}\right): \delta$ (ppm) 165.44, 164.12, 159.71, 145.78, 138.31, 137.32, 130.10, 128.37, 128.32, 128.26, $128.23,126.57,126.16,125.30,123.58,102.95,44.96,27.53,21.11$. HPLC retention time: $10.569 \mathrm{~min}$; purity $100 \%$.

\subsubsection{Synthesis of 2-(2-(thiophen-2-yl)propan-2-yl)-5-m- tolylfuro[2,3-d]pyrimidin-4-ol (68)}

Compound 68 was prepared in a manner similar to compound 63 from 2-(2(thiophen-2-yl)propan-2-yl)pyrimidine-4,6-diol 31 (100mg, 0.434mmol), 1-(2-chloro-2nitrovinyl)-3-methylbenzene 60 (79mg, $0.4 \mathrm{mmol})$, DBU (122mg, $120 \mu \mathrm{l}, 0.8 \mathrm{mmol})$, and $\mathrm{CH}_{3} \mathrm{CN}(2 \mathrm{ml})$. Yield: $21.5 \%$. MS: (ESI, neg.) $\mathrm{m} / z 349.0[\mathrm{M}-\mathrm{H}]$ ], ${ }^{1} \mathrm{H}$ NMR $(500 \mathrm{MHz}$, DMSO): $\delta(\mathrm{ppm}) 12.07(\mathrm{~s}, 1 \mathrm{H}), 8.20(\mathrm{~s}, 1 \mathrm{H}), 7.78(\mathrm{~d}, 1 \mathrm{H}, \mathrm{J}=7.5 \mathrm{~Hz}), 7.73(\mathrm{~s}, 1 \mathrm{H}), 7.43$ $(\mathrm{d}, 1 \mathrm{H}, \mathrm{J}=5 \mathrm{~Hz}), 7.30(\mathrm{t}, 1 \mathrm{H}, \mathrm{J}=7.5 \mathrm{~Hz}), 7.14(\mathrm{~d}, 1 \mathrm{H}, \mathrm{J}=7.5 \mathrm{~Hz}), 7.03(\mathrm{~d}, 1 \mathrm{H}, \mathrm{J}=4 \mathrm{~Hz})$, $6.99(\mathrm{t}, 1 \mathrm{H}, \mathrm{J}=5 \mathrm{~Hz}), 2.33(\mathrm{~s}, 3 \mathrm{H}), 1.81(\mathrm{~s}, 6 \mathrm{H}) ;{ }^{13} \mathrm{C} \mathrm{NMR}\left(500 \mathrm{MHz}, \mathrm{CDCl}_{3}\right): \delta(\mathrm{ppm})$ $165.33,162.94,159.61,150.23,138.49,137.33,130.03$, 127.94, 128.39, 128.27, 129.74, $125.30,124.98,124.55,123.55,103.04,43.15,25.42,21.11$. HPLC retention time: $10.514 \mathrm{~min}$; purity $100 \%$.

\subsubsection{Synthesis of 2-(2-cyclohexylpropan-2-yl)-5-m-tolylfuro[2,3- d]pyrimidin-4-ol (69)}

Compound 69 was prepared in a manner similar to compound 63 from 2-(2cyclohexylpropan-2-yl)pyrimidine-4,6-diol 32 (100mg, 0.434mmol), 1-(2-chloro-2nitrovinyl)-3-methylbenzene 60 (79mg, $0.4 \mathrm{mmol})$, DBU (122mg, $120 \mu \mathrm{l}, 0.8 \mathrm{mmol})$, and $\mathrm{CH}_{3} \mathrm{CN}(2 \mathrm{ml})$. Yield: 30.8\%. MS: (ESI, neg.) $\mathrm{m} / z 349.0[\mathrm{M}-\mathrm{H}]^{-},{ }^{1} \mathrm{H}$ NMR $(500 \mathrm{MHz}$, DMSO): $\delta(\mathrm{ppm}) 11.95(\mathrm{~s}, 1 \mathrm{H}), 8.14(\mathrm{~s}, 1 \mathrm{H}), 7.80(\mathrm{~d}, 1 \mathrm{H}, \mathrm{J}=7.5 \mathrm{~Hz}), 7.54(\mathrm{~s}, 1 \mathrm{H}), 7.30$ $(\mathrm{t}, 1 \mathrm{H}, \mathrm{J}=7.5 \mathrm{~Hz}), 7.14(\mathrm{~d}, 1 \mathrm{H}, \mathrm{J}=7 \mathrm{~Hz}), 2.34(\mathrm{~s}, 3 \mathrm{H}), 1.93(\mathrm{t}, 1 \mathrm{H}, \mathrm{J}=12 \mathrm{~Hz}), 1.69(\mathrm{~d}, 2 \mathrm{H}$, $\mathrm{J}=12.5 \mathrm{~Hz}), 1.60(\mathrm{~d}, 1 \mathrm{H}, \mathrm{J}=12 \mathrm{~Hz}), 1.40(\mathrm{~d}, 2 \mathrm{H}, \mathrm{J}=12 \mathrm{~Hz}), 1.24(\mathrm{~s}, 6 \mathrm{H}), 1.05(\mathrm{~m}, 5 \mathrm{H})$; ${ }^{13} \mathrm{C}$ NMR $\left(500 \mathrm{MHz}, \mathrm{CDCl}_{3}\right): \delta(\mathrm{ppm}) 165.53,165.49,138.02,137.30,130.17,128.35$, $128.36,128.25,128.19,128.27,125.27,123.49,102.45,44.92,43.60,27.19,26.82$, $26.02,22.21,21.15$. HPLC retention time: $11.680 \mathrm{~min}$; purity $100 \%$. 


\subsubsection{Synthesis of 2-(2-methyloctan-2-yl)-5-m-tolylfuro[2,3- d]pyrimidin-4-ol (70)}

Compound 70 was prepared in a manner similar to compound 63 from 2-(2methyloctan-2-yl)pyrimidine-4,6-diol 33 (100mg, 0.42mmol), 1-(2-chloro-2-nitrovinyl)3-methylbenzene 60 (79mg, $0.4 \mathrm{mmol})$, DBU (122mg, $120 \mu \mathrm{l}, 0.8 \mathrm{mmol})$, and $\mathrm{CH}_{3} \mathrm{CN}$ (2ml). Yield: 36.4\%. MS: (ESI, neg.) $m / z 351[\mathrm{M}-\mathrm{H}]^{-},{ }^{1} \mathrm{H}$ NMR (500 MHz, DMSO): $\delta$ (ppm) $12.08(\mathrm{~s}, 1 \mathrm{H}), 8.15(\mathrm{~s}, 1 \mathrm{H}), 7.81(\mathrm{~d}, 1 \mathrm{H}, \mathrm{J}=7.5 \mathrm{~Hz}), 7.75(\mathrm{~s}, 1 \mathrm{H}), 7.30(\mathrm{t}, 1 \mathrm{H}, \mathrm{J}=$ $7.5 \mathrm{~Hz}), 7.14(\mathrm{~d}, 1 \mathrm{H}, \mathrm{J}=7.5 \mathrm{~Hz}), 2.34(\mathrm{~s}, 3 \mathrm{H}), 1.72(\mathrm{t}, 2 \mathrm{H}, \mathrm{J}=6.5 \mathrm{~Hz}), 1.31(\mathrm{~s}, 6 \mathrm{H}), 1.19$ (m. 6H), $1.06(\mathrm{~m} .2 \mathrm{H}), 0.81(\mathrm{t}, 3 \mathrm{H}, \mathrm{J}=6.5 \mathrm{~Hz}) ;{ }^{13} \mathrm{C} \mathrm{NMR}\left(500 \mathrm{MHz}, \mathrm{CDCl}_{3}\right): \delta(\mathrm{ppm})$ 165.60, 164.86, 159.85, 138.04, 137.31, 130.16, 128.34, 128.26, 128.20, 125.28, 123.48, $102.46,40.43,40.35,30.99,29.07,25.91,23.95,22.02,21.12,13.88$. HPLC retention time: $12.294 \mathrm{~min}$; purity $99.14 \%$.

\subsubsection{Synthesis of 5-(3,5-dichlorophenyl)-2-(2-phenylpropan-2- yl)furo[2,3-d]pyrimidin-4-ol (71)}

Compound 71 was prepared in a manner similar to compound 63 from 2-(2phenylpropan-2-yl)pyrimidine-4,6-diol 30 (100mg, $0.42 \mathrm{mmol}), 1,3$-dichloro-5-(2-chloro2-nitrovinyl)benzene 61 (101mg, $0.4 \mathrm{mmol})$, DBU (122mg, $120 \mu 1,0.8 \mathrm{mmol})$, and $\mathrm{CH}_{3} \mathrm{CN}$ (2ml). Yield: 20.4\%. MS: (ESI, neg.) $\mathrm{m} / z 397.0[\mathrm{M}-\mathrm{H}]^{-},{ }^{1} \mathrm{H}$ NMR (500 MHz, DMSO): $\delta(\mathrm{ppm}) 12.16(\mathrm{~s}, 1 \mathrm{H}), 8.51(\mathrm{~s}, 1 \mathrm{H}), 8.20(\mathrm{~s}, 2 \mathrm{H}), 7.56(\mathrm{~s}, 1 \mathrm{H}), 7.35(\mathrm{t}, 2 \mathrm{H}, \mathrm{J}=8 \mathrm{~Hz}), 7.27$ $(\mathrm{m}, 3 \mathrm{H}), 1.72(\mathrm{~s}, 6 \mathrm{H}) ;{ }^{13} \mathrm{C} \mathrm{NMR}\left(500 \mathrm{MHz}, \mathrm{CDCl}_{3}\right): \delta(\mathrm{ppm}) 165.62,164.63,159.83$, $145.68,140.20,134.13,133.81,128.34,126.90,126.61,126.25,126.17,121.12,102.55$, 45.06, 27.54. HPLC retention time: $11.468 \mathrm{~min}$; purity $97.36 \%$.

\subsubsection{Synthesis of 5-(3,5-dichlorophenyl)-2-(2-(thiophen-2- yl)propan-2-yl)furo[2,3-d]pyrimidin-4-ol (72)}

Compound 72 was prepared in a manner similar to compound 63 from 2-(2(thiophen-2-yl)propan-2-yl)pyrimidine-4,6-diol 31 (100mg, 0.423mmol), 1,3-dichloro-5-

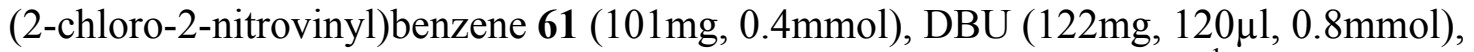
and $\mathrm{CH}_{3} \mathrm{CN}(2 \mathrm{ml})$. Yield: $32.9 \%$. MS: (ESI, neg.) $\mathrm{m} / z 404.9[\mathrm{M}-\mathrm{H}]^{-1},{ }^{1} \mathrm{H}$ NMR $(500$ MHz, DMSO): $\delta(\mathrm{ppm}) 12.22$ (s, 1H), $8.49(\mathrm{~s}, 1 \mathrm{H}), 8.18(\mathrm{~s}, 2 \mathrm{H}), 7.54(\mathrm{~s}, 1 \mathrm{H}), 7.44(\mathrm{~d}$, $1 \mathrm{H}, \mathrm{J}=5 \mathrm{~Hz}), 7.03(\mathrm{~d}, 1 \mathrm{H}, \mathrm{J}=3 \mathrm{~Hz}), 6.99(\mathrm{t}, \mathrm{H}, \mathrm{J}=4.5 \mathrm{~Hz}), 1.81(\mathrm{~s}, 6 \mathrm{H}) ;{ }^{13} \mathrm{C}$ NMR $(500$ $\mathrm{MHz}, \mathrm{CDCl}_{3}$ ): $\delta$ (ppm) 165.50, 163.42, 159.71, 150.08, 140.35, 134.13, 133.72, 126.92, $126.75,126.26,125.01,124.06,121.12,102.64,43.21,28.42$. HPLC retention time: $11.414 \mathrm{~min}$; purity $100 \%$. 


\subsubsection{Synthesis of 2-(2-cyclohexylpropan-2-yl)-5-(3,5- dichlorophenyl)furo[2,3-d]pyrimidin-4-ol (73)}

Compound 73 was prepared in a manner similar to compound 63 from 2-(2cyclohexylpropan-2-yl)pyrimidine-4,6-diol 32 (100mg, 0.423 mmol), 1,3-dichloro-5-(2chloro-2-nitrovinyl)benzene 61 (101mg, 0.4mmol), DBU (122mg, 120 $\mu 1,0.8 \mathrm{mmol})$, and $\mathrm{CH}_{3} \mathrm{CN}$ (2ml). Yield: 24.7\%. MS: (ESI, neg.) $\mathrm{m} / \mathrm{z} 403.0$ [M - H] ${ }^{-1}{ }^{1} \mathrm{H}$ NMR (500 MHz, $\left.\mathrm{CDCl}_{3}\right): \delta(\mathrm{ppm}) 10.91(\mathrm{~s}, 1 \mathrm{H}), 7.82(\mathrm{~s}, 2 \mathrm{H}), 7.66(\mathrm{~s}, 1 \mathrm{H}), 7.35(\mathrm{~s}, 1 \mathrm{H}), 1.71(\mathrm{~m}, 1 \mathrm{H}$,$) ,$ $1.64(\mathrm{~m}, 2 \mathrm{H}), 1.55(\mathrm{~d}, 1 \mathrm{H}, \mathrm{J}=10.5 \mathrm{~Hz}), 1.42(\mathrm{~d}, 2 \mathrm{H}, \mathrm{J}=6 \mathrm{~Hz}), 1.36(\mathrm{~s}, 6 \mathrm{H}), 1.00(\mathrm{~m}, 5 \mathrm{H})$; ${ }^{13} \mathrm{C} \mathrm{NMR}\left(500 \mathrm{MHz}, \mathrm{CDCl}_{3}\right): \delta(\mathrm{ppm}) 165.55,160.62,138.24,135.21,133.45,128.00$, $126.78,122.63,110.19,102.97,47.23,44.38,27.89,26.83,26.51,22.97$. HPLC retention time: $13.021 \mathrm{~min}$; purity $98.71 \%$.

\subsubsection{Synthesis of 5-(3,5-dichlorophenyl)-2-(2-methyloctan-2- yl)furo[2,3-d]pyrimidin-4-ol (74)}

Compound 74 was prepared in a manner similar to compound 63 from 2-(2methyloctan-2-yl)pyrimidine-4,6-diol 33 (100mg, 0.42mmol), 1,3-dichloro-5-(2-chloro2-nitrovinyl)benzene 61 (101mg, $0.4 \mathrm{mmol})$, DBU (122mg, $120 \mu 1,0.8 \mathrm{mmol})$, and $\mathrm{CH}_{3} \mathrm{CN}$ (2ml). Yield: 19.7\%. MS: (ESI, neg.) $m / z$ 405.1 [M - H] $]^{-},{ }^{1} \mathrm{H}$ NMR (500 MHz, CDCl3): $\delta(\mathrm{ppm}) 11.41(\mathrm{~s}, 1 \mathrm{H}), 7.82(\mathrm{~s}, 2 \mathrm{H}), 7.66(\mathrm{~s}, 1 \mathrm{H}), 7.35(\mathrm{~s}, 1 \mathrm{H}), 1.72(\mathrm{~m}, 2 \mathrm{H}), 1.43(\mathrm{~s}, 6 \mathrm{H})$, $1.14(\mathrm{~m}, 8 \mathrm{H}), 0.78(\mathrm{t}, 3 \mathrm{H}, \mathrm{J}=7 \mathrm{~Hz}) ;{ }^{13} \mathrm{C} \mathrm{NMR}\left(500 \mathrm{MHz}, \mathrm{CDCl}_{3}\right): \delta(\mathrm{ppm}) 166.90$, 166.04, 161.08, 138.29, 135.17, 133.80, 128.02, 126.76, 122.63, 102.98, 41.90, 41.24, $31.77,29.78,26.55,24.74,22.77,14.20$. HPLC retention time: $13.910 \mathrm{~min}$; purity $97.51 \%$.

\subsubsection{Synthesis of 2-(2-phenylpropan-2-yl)-5-(thiophen-2- yl)furo[2,3-d]pyrimidin-4-ol (75)}

Compound 75 was prepared in a manner similar to compound 63 from 2-(2phenylpropan-2-yl)pyrimidine-4,6-diol 30 (100mg, 0.434mmol), 2-(2-chloro-2nitrovinyl)thiophene 62 (76 mg, $0.4 \mathrm{mmol})$, DBU $(122 \mathrm{mg}, 120 \mu 1,0.8 \mathrm{mmol})$, and $\mathrm{CH}_{3} \mathrm{CN}$ (2ml). Yield: 36.1\%. MS: (ESI, neg.) $\mathrm{m} / z 335[\mathrm{M}-\mathrm{H}]^{-},{ }^{1} \mathrm{H}$ NMR (500 MHz, DMSO): $\delta$ (ppm) $12.07(\mathrm{~s}, 1 \mathrm{H}), 8.22(\mathrm{~s}, 1 \mathrm{H}), 7.97(\mathrm{~d}, 1 \mathrm{H}, \mathrm{J}=3.5 \mathrm{~Hz}), 7.50(\mathrm{~d}, 1 \mathrm{H}, \mathrm{J}=5 \mathrm{~Hz}), 7.34(\mathrm{t}$, $2 \mathrm{H}, \mathrm{J}=7.5 \mathrm{~Hz}), 7.25(\mathrm{~d}, 3 \mathrm{H}, \mathrm{J}=6.5 \mathrm{~Hz}), 7.10(\mathrm{t}, 1 \mathrm{H}, \mathrm{J}=5.5 \mathrm{~Hz}), 1.71(\mathrm{~s}, 6 \mathrm{H}) ;{ }^{13} \mathrm{C} \mathrm{NMR}$ $\left(500 \mathrm{MHz}, \mathrm{CDCl}_{3}\right): \delta$ (ppm) 165.23, 164.49, 159.60, 145.72, 137.31, 131.43, 128.32, 128.28, 127.72, 126.58, 126.17, 125.65, 117.49, 102.49, 45.03, 27.51. HPLC retention time: $10.219 \mathrm{~min}$; purity $99.69 \%$. 


\subsubsection{Synthesis of 5-(thiophen-2-yl)-2-(2-(thiophen-2-yl)propan-2- yl)furo[2,3-d]pyrimidin-4-ol (76)}

Compound 76 was prepared in a manner similar to compound 63 from 2-(2(thiophen-2-yl)propan-2-yl)pyrimidine-4,6-diol 31 (100mg, 0.423mmol), 2-(2-chloro-2nitrovinyl)thiophene 62 (76mg, $0.4 \mathrm{mmol})$, DBU (122mg, $120 \mu 1,0.8 \mathrm{mmol}$ ), and $\mathrm{CH}_{3} \mathrm{CN}$ (2ml). Yield: 19.2\%. MS: (ESI, neg.) $\mathrm{m} / z 341[\mathrm{M}-\mathrm{H}]^{-},{ }^{1} \mathrm{H}$ NMR (500 MHz, DMSO): $\delta$ (ppm) $12.15(\mathrm{~s}, 1 \mathrm{H}), 8.22(\mathrm{~s}, 1 \mathrm{H}), 7.96(\mathrm{~d}, 1 \mathrm{H}, \mathrm{J}=3 \mathrm{~Hz}), 7.51(\mathrm{~d}, 1 \mathrm{H}, \mathrm{J}=5 \mathrm{~Hz}), 7.44(\mathrm{~d}$, $1 \mathrm{H}, \mathrm{J}=5 \mathrm{~Hz}), 7.11(\mathrm{t}, 1 \mathrm{H}, \mathrm{J}=5 \mathrm{~Hz}), 7.03(\mathrm{~d}, 1 \mathrm{H}, \mathrm{J}=2.5 \mathrm{~Hz}), 6.99(\mathrm{t}, 1 \mathrm{H}, \mathrm{J}=4 \mathrm{~Hz}), 1.81(\mathrm{~s}$, $6 \mathrm{H}) ;{ }^{13} \mathrm{C}$ NMR $\left(500 \mathrm{MHz}, \mathrm{CDCl}_{3}\right): \delta(\mathrm{ppm}) 165.12,163.29,159.49,150.14,137.49$, $131.35,128.30,127.73,126.75,125.70,124.99,124.59,117.49,102.58,43.21,28.39$. HPLC retention time: $10.151 \mathrm{~min}$; purity $98.38 \%$.

\subsubsection{Synthesis of 2-(2-cyclohexylpropan-2-yl)-5-(thiophen-2- yl)furo[2,3-d]pyrimidin-4-ol (77)}

Compound 77 was prepared in a manner similar to compound 63 from 2-(2cyclohexylpropan-2-yl)pyrimidine-4,6-diol 32 (100mg, 0.423 mmol), 2-(2-chloro-2nitrovinyl)thiophene 62 (76mg, $0.4 \mathrm{mmol})$, DBU (122 $\mathrm{mg}, 120 \mu 1,0.8 \mathrm{mmol})$, and $\mathrm{CH}_{3} \mathrm{CN}$ (2ml). Yield: 40.2\%. MS: (ESI, neg.) $\mathrm{m} / z 341.0[\mathrm{M}-\mathrm{H}]^{-},{ }^{1} \mathrm{H}$ NMR (500 MHz, DMSO): $\delta(\mathrm{ppm}) 12.08(\mathrm{~s}, 1 \mathrm{H}), 8.18(\mathrm{~s}, 1 \mathrm{H}), 8.07(\mathrm{~d}, 1 \mathrm{H}, \mathrm{J}=3.5 \mathrm{~Hz}), 7.51(\mathrm{~d}, 1 \mathrm{H}, \mathrm{J}=4.5 \mathrm{~Hz}), 7.13$ $(\mathrm{t}, 1 \mathrm{H}, \mathrm{J}=5 \mathrm{~Hz}), 1.94(\mathrm{t}, 1 \mathrm{H}, \mathrm{J}=12 \mathrm{~Hz}), 1.70(\mathrm{~d}, 2 \mathrm{H}, \mathrm{J}=12.5 \mathrm{~Hz}), 1.62(\mathrm{~d}, 1 \mathrm{H}, \mathrm{J}=12 \mathrm{~Hz})$, $1.40(\mathrm{~d}, 2 \mathrm{H}, \mathrm{J}=12.5 \mathrm{~Hz}), 1.25(\mathrm{~s}, 6 \mathrm{H}), 1.02(\mathrm{~m}, 5 \mathrm{H}) ;{ }^{13} \mathrm{C} \mathrm{NMR}\left(500 \mathrm{MHz}, \mathrm{CDCl}_{3}\right): \delta$ (ppm) 165.91, 165.26, 159.71, 137.01, 131.53, 128.24, 127.71, 125.59, 117.40, 101.99, $44.95,43.68,27.19,26.38,26.01,22.19$. HPLC retention time: $11.285 \mathrm{~min}$; purity $100 \%$.

\subsubsection{Synthesis of 2-(2-methyloctan-2-yl)-5-(thiophen-2-yl)furo[2,3- d]pyrimidin-4-ol (78)}

Compound 78 was prepared in a manner similar to compound 63 from 2-(2methyloctan-2-yl)pyrimidine-4,6-diol 33 (100mg, 0.42mmol), 2-(2-chloro-2nitrovinyl)thiophene 62 (76mg, $0.4 \mathrm{mmol})$, DBU (122 $\mathrm{mg}, 120 \mu 1,0.8 \mathrm{mmol})$, and $\mathrm{CH}_{3} \mathrm{CN}$ (2ml). Yield: $12.3 \%$. MS: (ESI, neg.) $\mathrm{m} / z 343.0[\mathrm{M}-\mathrm{H}]^{-},{ }^{1} \mathrm{H}$ NMR (500 MHz, DMSO): $\delta(\mathrm{ppm}) 12.16(\mathrm{~s}, 1 \mathrm{H}), 8.71(\mathrm{~s}, 1 \mathrm{H}), 8.00(\mathrm{~d}, 1 \mathrm{H}, \mathrm{J}=3 \mathrm{~Hz}), 7.51(\mathrm{~d}, 1 \mathrm{H}, \mathrm{J}=5 \mathrm{~Hz}), 7.12(\mathrm{t}$, $1 \mathrm{H}, \mathrm{J}=4 \mathrm{~Hz}), 1.72(\mathrm{~m}, 2 \mathrm{H}), 1.30(\mathrm{~s}, 6 \mathrm{H}), 1.19(\mathrm{~m}, 6 \mathrm{H}), 1.05(\mathrm{~m}, 2 \mathrm{H}), 0.81(\mathrm{t}, 3 \mathrm{H}, \mathrm{J}=$ $6.5 \mathrm{~Hz}$ ); ${ }^{13} \mathrm{C}$ NMR (500 MHz, $\mathrm{CDCl}_{3}$ ): $\delta$ (ppm) 165.37, 165.24, 159.73, 137.04, 131.52, $128.26,127.72,125.60,117.41,102.01,40.50,40.35,30.99,29.06,25.08,23.94,22.02$, 13.89. HPLC retention time: $11.881 \mathrm{~min}$; purity $100 \%$.

\subsection{Testing of Novel Compounds}

Select compounds from each of the three series of novel pyrimidine cannabinoid ligands were tested for binding affinity to and functional activity at the CB1 and CB2 
receptors. Select compounds were also tested for efficacy as anti-glioblastoma agents. The complete series are currently undergoing receptor binding assays and functional activity screens in order to develop SARs for these new structural classes of cannabinoids.

\subsubsection{Pyrimidine Hexahydro Classical Cannabinoids}

All four of the pyrimidine hexahydro classical cannabinoids were screened for receptor binding affinity to the $\mathrm{CB} 1$ and $\mathrm{CB} 2$ receptors (Table 4.1). All compounds exhibited binding affinity to both receptors with the exception of the phenyl substituted 34 which showed very little affinity for the CB1 receptor $\left(K_{\mathrm{i}}>10,000 \mathrm{nM}\right)$. Compounds 34 and 35 were $>73.0$ and 26.9-fold selective for the CB2 receptor, respectively, which is greater than either KM-233 or HB-I-172. From this small series of compounds it would seem that aromatic side-chains (compounds 34 and 35) decrease binding affinity for both receptors yet increase CB2 selectivity. Compounds 36 and 37, which possess alkyl side-chains, displayed good binding affinity for both receptors yet lower CB2 selectivity ( 9.7 and 6.5, respectively). All compounds proved to be agonists at both the $\mathrm{CB} 1$ and $\mathrm{CB} 2$ receptor subtypes. Compounds 35, 36, and $\mathbf{3 7}$ were also tested against the human glioblastoma cell line LN-229. All three compounds showed efficacy which was comparable to HB-I-172 and KM-233 (Table 4.2).

\subsubsection{Pyrimidine Non-Classical Cannabinoids}

Compounds $47, \mathbf{4 8}, \mathbf{5 2}$, and $\mathbf{5 5}$ were assayed for receptor binding affinity at CB1 and CB2. The results of the binding assays are found in Table 4.3. Each of the four pyrimidine non-classical cannabinoids tested has very little affinity for the CB1 receptor with all four having a $K_{\mathrm{i}}>10,000$. They do, however, retain affinity for the CB2 receptor in varying degrees. Compound 55 (dimethyl heptyl side-chain with a cyclohexyl replacement of the A ring) possessed the greatest affinity for the CB2 receptor $\left(K_{\mathrm{i}}=43.3\right.$ $( \pm 19.6) \mathrm{nM})$ with a high CB2 selectivity ratio of $>230.9$-fold. Six of the compounds from this series were tested for functional activity at the cannabinoid receptors and were found to be agonists at CB2 with little or no agonist activity at CB1 (probably due to low affinity). Two exceptions were 47 and 55 which exhibited 59 and $68 \%$ agonist activity at CB1, respectively. Three compounds 47, 48, and $\mathbf{5 0}$ were tested against LN-229 and exhibited $\mathrm{EC}_{50} \mathrm{~s}(23.5 \mu \mathrm{M}-55.5 \mu \mathrm{M})$ slightly higher than HB-I-172 and KM-233, but much lower than BCNU (Table 4.4).

\subsubsection{Furanopyrimidine Cannabinoids}

Five furanopyrimidines were screened for receptor binfing affinity at the $\mathrm{CB} 1$ and $\mathrm{CB} 2$ receptors. All five compounds exhibited low affinity for both $\mathrm{CB} 1$ and $\mathrm{CB} 2\left(K_{\mathrm{i}}>\right.$ $10,000 \mathrm{nM})$. All sixteen compounds were screened for functional activity at the cannabinoid receptors also. Despite having low affinity, several compounds did exhibit 
Table 4.1: Binding affinity constants $\left(K_{\mathrm{i}}\right)$ for compounds 34-37 compared to $\Delta^{8}$-THC, KM-233, and HB-I-172

\begin{tabular}{cccc}
\hline Compound & CB1 $\boldsymbol{K}_{\mathbf{i}}(\mathbf{n M})$ & $\mathbf{C B 2} \boldsymbol{K}_{\mathbf{i}}(\mathbf{n M})$ & $\mathbf{C B 1} / \mathbf{C B 2}$ Ratio \\
\hline$\Delta^{8}-\mathrm{THC}$ & $28.5( \pm 3.3)$ & $25.0( \pm 4.8)$ & 1.1 \\
KM-233 & $12.3( \pm 0.61)$ & $0.91( \pm 0.08)$ & 13.5 \\
HB-I-172 & $6040( \pm 1477)$ & $487( \pm 101)$ & 12.4 \\
$\mathbf{3 4}$ & $>10,000$ & $137( \pm 13)$ & $>73.0$ \\
$\mathbf{3 5}$ & $1138( \pm 227)$ & $42.3( \pm 13.9)$ & 26.9 \\
$\mathbf{3 6}$ & $18.8( \pm 9.3)$ & $1.94( \pm 0.63)$ & 9.7 \\
$\mathbf{3 7}$ & $54.6( \pm 5.4)$ & $8.36( \pm 1.66)$ & 6.5 \\
\hline
\end{tabular}

Notes: Values are the mean of three experiments performed in triplicate; standard deviation is given in parentheses. $\mathrm{CB} 1 / \mathrm{CB} 2$ ratio is expressed as $K_{\mathrm{i}}$ for $\mathrm{CB} 1 / K_{\mathrm{i}}$ for CB2. 
Table 4.2: Efficacy of pyrimidine hexahydro classical cannabinoids compared to HB-I172, KM-233, and BCNU against LN-229

\begin{tabular}{cc}
\hline Compound & EC $_{\mathbf{5 0}}(\boldsymbol{\mu M}) \pm$ S.D. \\
\hline BCNU & $233( \pm 5.1)$ \\
HB-I-172 & $9.74( \pm 0.56)$ \\
KM-233 & $4.74( \pm 0.43)$ \\
$\mathbf{3 5}$ & $30.1( \pm 2.9)$ \\
$\mathbf{3 6}$ & $10.6( \pm 1.3)$ \\
$\mathbf{3 7}$ & $27.3( \pm 3.4)$ \\
\hline
\end{tabular}

Notes: Values are the mean of three experiments performed in triplicate; standard deviation is given in parentheses. 
Table 4.3: Binding affinity constants $\left(K_{\mathrm{i}}\right)$ for select pyrimidine non-classical cannabinoids compared to $\Delta^{8}$-THC, KM-233, and HB-I-172

\begin{tabular}{cccc}
\hline Compound & CB1 $\boldsymbol{K}_{\mathbf{i}}(\mathbf{n M})$ & $\mathbf{C B 2} \boldsymbol{K}_{\mathbf{i}}(\mathbf{n M})$ & $\mathbf{C B 1 / C B 2}$ Ratio \\
\hline$\Delta^{8}-\mathrm{THC}$ & $28.5( \pm 3.3)$ & $25.0( \pm 4.8)$ & 1.1 \\
KM-233 & $12.3( \pm 0.61)$ & $0.91( \pm 0.08)$ & 13.5 \\
HB-I-172 & $6040( \pm 1477)$ & $487( \pm 101)$ & 12.4 \\
$\mathbf{4 7}$ & $>10,000$ & $9673( \pm 566)$ & $>1.0$ \\
$\mathbf{4 8}$ & $>10,000$ & $130( \pm 38)$ & $>76.9$ \\
$\mathbf{5 2}$ & $>10,000$ & $621( \pm 158)$ & $>16.1$ \\
$\mathbf{5 5}$ & $>10,000$ & $43.3( \pm 19.6)$ & $>230.9$ \\
\hline
\end{tabular}

Notes: Values are the mean of three experiments performed in triplicate; standard deviation is given in parentheses. $\mathrm{CB} 1 / \mathrm{CB} 2$ ratio is expressed as $K_{\mathrm{i}}$ for $\mathrm{CB} 1 / K_{\mathrm{i}}$ for CB2. 
Table 4.4: Comparison of selected pyrimidine non-classical cannabinoids to HB-I-172, KM-233, and BCNU against LN-229

\begin{tabular}{cc}
\hline Compound & EC $_{\mathbf{5 0}}(\boldsymbol{\mu} \mathbf{M}) \pm$ S.D. \\
\hline BCNU & $233( \pm 5.1)$ \\
HB-I-172 & $9.74( \pm 0.56)$ \\
KM-233 & $4.74( \pm 0.43)$ \\
$\mathbf{4 7}$ & $55.5( \pm 4.3)$ \\
$\mathbf{4 8}$ & $23.5( \pm 2.9)$ \\
$\mathbf{5 0}$ & $51.8( \pm 6.4)$ \\
\hline
\end{tabular}

Notes: Values are the mean of three experiments performed in triplicate; standard deviation is given in parentheses. 
agonist activity. Compounds $\mathbf{6 6}$ and $\mathbf{7 8}$ proved to be agonists at $\mathrm{CB} 1$ and $\mathrm{CB} 2$ when screened at a concentration of $10 \mu \mathrm{M}$. Compounds $65,69,70,74,77$, and 78 showed agonist activity at only the $\mathrm{CB} 2$ receptor when tested at $10 \mu \mathrm{M}$. Five of the furanopyrimidines were tested against the human glioblastoma cell line T98G. When screened at a concentration of $10 \mu \mathrm{M}$, five of the compounds displayed cytotoxic activity against the GBM cell line (Figure 4.10). Interestingly, the cells had to experience a longer exposure to these compounds ( 72 hours) in order to manifest the toxic effects normally seen in 18 hours with other cannabinoid ligands.

\subsection{Experimental Procedures}

\subsubsection{Radioligand Binding Assays}

Binding affinity studies were carried out using cell membranes from HEK293 cells transfected with the human CB1 receptor ( $K_{\mathrm{d}}$ for tritiated CP-55,940 binding: 4.5 $\mathrm{nM})$ and membranes from HEK293 cells transfected with the human CB2 receptor $\left(K_{\mathrm{d}}\right.$ for tritiated CP-55,940 binding: $5.1 \mathrm{nM}$ ). Non-specific binding was determined using 10 $\mu \mathrm{M}$ WIN55, 212-2. Increasing concentrations of compounds to be tested were made ranging from $10^{-12} \mathrm{M}$ to $10^{-4} \mathrm{M}$ and were added in triplicate for each experiment and the individual molar $\mathrm{IC}_{50}$ values were determined using GraphPad Prism software. The corresponding $K_{\mathrm{i}}$ values for each drug were determined utilizing the Cheng-Prusoff equation and final data are presented as $K_{\mathrm{i}} \pm$ standard deviation of $\mathrm{n}=3$ experiments run in triplicate.

\subsubsection{Cytotoxicity Assays}

Human glioblastoma cells T98G and LN-229 (American Type Culture Collection) were cultured in supplemented media according to the recommendations of the supplier at $37^{\circ} \mathrm{C}$ in an atmosphere of $5 \% \mathrm{CO}_{2}$ and $95 \%$ air. Cell lines were plated in 96 -well polystyrene flat-bottom plates $(7,500$ cells/well $)$ at $70 \%$ confluency in a $100 \mu \mathrm{l}$ total volume of supplemented media as indicated, and incubated overnight at $37^{\circ} \mathrm{C}$ to allow for adherence. The media was then replaced with media containing $1 \%$ FBS and the cultures were treated with escalating amounts of drug and cell death was analyzed at $18 \mathrm{hr}$ or $72 \mathrm{hr}$, using the BioTek Synergy 2 Multidetection Microplate Reader (BioTek Instruments, Inc.). The percentage of viable cells present in the culture at each time point was calculated by comparing the absorbance value at $450 \mathrm{~nm}$ from the CCK-8 assay (Dojindo Molecular Technologies) for each condition with untreated control cells. All assays were conducted per manufacturer's protocol. All described values represent the average of three data points per determination and three independent determinations. 


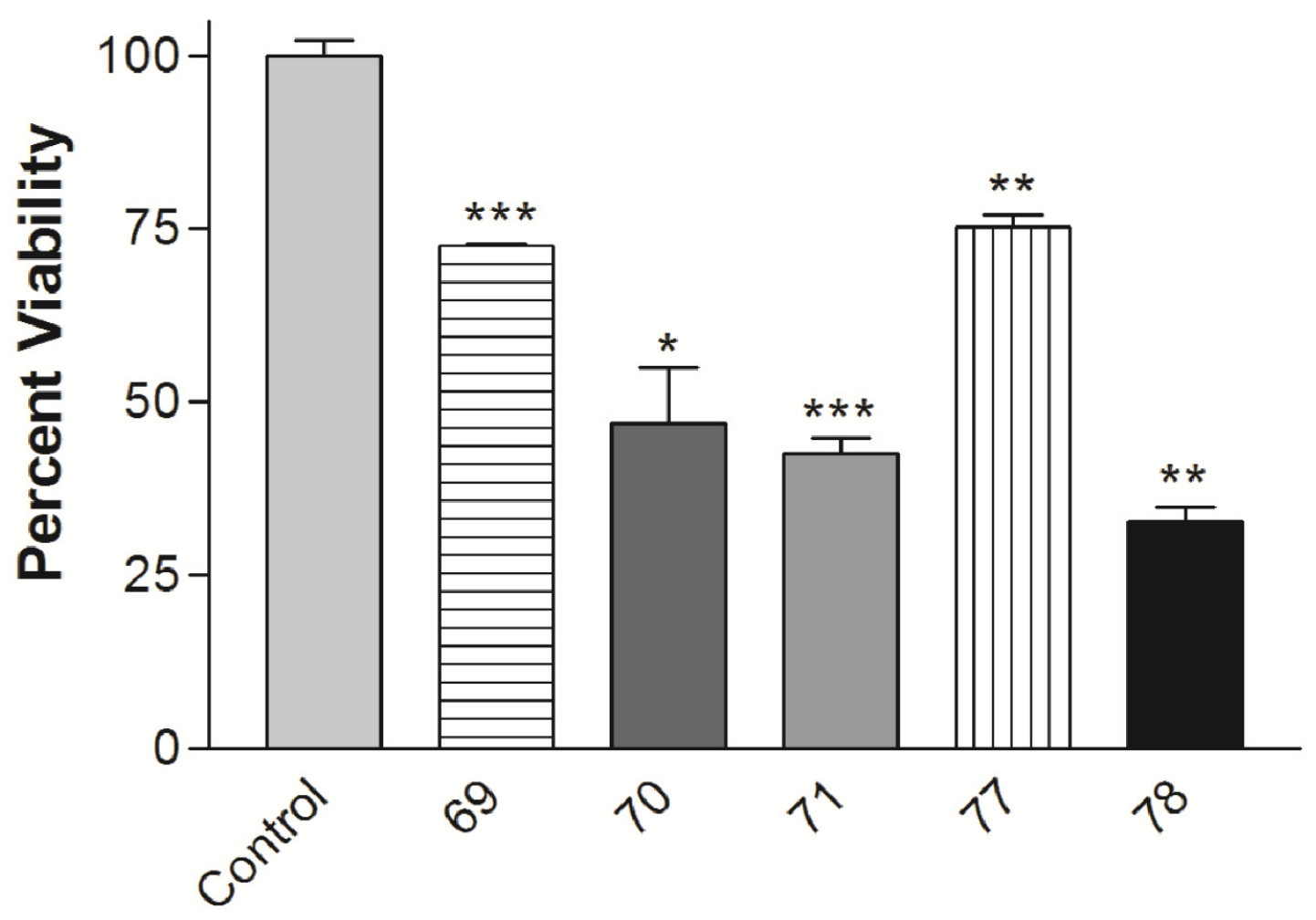

Figure 4.10: Furanopyrimidine cannabinoids screened against the human glioblastoma cell line T98G

All compounds were screened at $10 \mu \mathrm{M}$ for 48 hours. $*=\mathrm{p}<0.005, * *=\mathrm{p}<0.0005$, and $* * *=\mathrm{p}<0.00005$. 


\subsubsection{CB1/CB2 Functional Assays}

HEK-293 cell lines stably transfected with a cyclic nucleotide-gated channel and either human CB1 or CB2 receptors (BD Biosciences, San Jose, CA USA) were seeded in poly-D-lysine coated 96 -well plates at a density of 70,000 cells per well. Plates were incubated at $37^{\circ} \mathrm{C}$ in $5 \% \mathrm{CO}_{2}$ overnight prior to assay. Plates were then removed from the incubator and the complete growth medium (DMEM, 10\% FBS, $250 \mu \mathrm{g} / \mathrm{ml} \mathrm{G4} 18$ and $1 \mu \mathrm{g} / \mathrm{ml}$ puromycin) was replaced with $100 \mu \mathrm{L}$ DMEM containing $0.25 \%$ BSA. Next, $100 \mu \mathrm{L}$ membrane potential dye loading buffer (Molecular Devices) was added to each well. The plates were placed back into the incubator for 30 minutes and then the baseline fluorescence was read on a BioTek Synergy 2 multi-mode microplate reader with 540nm excitation and 590nm emission filters prior to drug addition. Drugs were added in $50 \mu \mathrm{L}$ DPBS containing 2.5\% DMSO, $1.25 \mu \mathrm{M} 5$ '-(N-ethylcarboxamido) adenosine and $125 \mu \mathrm{M}$ Ro 20-1724. Plates were then incubated at room temperature for 25 minutes and fluorescence measured again at 540nm excitation and 590nm emission. 


\section{Chapter 5: Cannabinoids as Anti-Inflammatory Agents}

\subsection{Introduction}

Pharmacologically, cannabinoids can be used to affect a variety of targets such as the central nervous system, the cardiovascular system, the immune system and/or endocrine system. More particularly, compounds possessing an affinity for either the $\mathrm{CB} 1$ or the $\mathrm{CB} 2$ receptor, and potentially GPR55, can act on the central nervous system or act as immunomodulators. In addition, these compounds are useful as anticancer agents, antiobesity agents, analgesics, myorelaxation agents and antiglaucoma agents. Such compounds can also be used for the treatment of thymic disorders, vomiting, various types of neuropathy, memory disorders, dyskinesia, migraine, multiple sclerosis, asthma, epilepsy, ischemia, orthostatic hypotension, osteoporosis, liver fibrosis, inflammation and irritable bowel disease, diabetes, and cardiac insufficiency.

However, certain cannabinoids such as $\Delta^{9}$-THC also affect normal neurotransmission, producing undesirable side effects such as drowsiness, impairment of monoamine oxidase function, and impairment of non-receptor mediated brain function. The addictive and psychotropic properties of some cannabinoids tend to limit their therapeutic value. The unwanted psychotropic effects are mediated through the CB1 receptor and are not observed upon activation of CB2. Many of the desirable effects associated with cannabinoids such as antinociception and anti-inflammation are mediated through the $\mathrm{CB} 2$ receptor.

Due to the effects mediated by CB2, it appears to be a promising target for the treatment of pain, inflammation, and neuro-inflammation. It is therefore no mystery as to why the current trend in the field is the development of CB2 selective compounds that will not activate the $\mathrm{CB} 1$ receptor and will thus be void of the unwanted psychotropic effects. The expression of CB2 was at one time thought to be limited to the periphery, being found mainly in the cells of the immune system. ${ }^{17,43}$ Recently, however, the CB2 receptor has also been shown to be present in the CNS in both perivascular microglial cells ${ }^{179}$ and in brainstem neurons. ${ }^{42 b} \mathrm{CB} 2$ also appears to be up-regulated in dorsal root ganglia and peripheral neurons following injury. ${ }^{180}$

CB2 has been validated as a potential target for the treatment of pain and neuroinflammation based on preclinical data using CB2 selective agonists and receptor knockout mice. Despite the challenge that lies in developing CB2 selective compounds that maintain activity and selectivity across species, several compounds have advanced into clinical development. 


\subsection{Evaluation of CB2 Agonists in Preclinical Models}

\subsubsection{Pain}

The ability of Cannabis to attenuate the pain response has been known for millennia in Asia. The medical use of marijuana was introduced to the West in 1839 by a physician serving with the British in India named W. B. O'Shaughnessy. He published a treatise expounding the usefulness of extracts of the plant in a wide variety of conditions including relieving the pain of rheumatism. The medical use of Cannabis grew rapidly in the late $19^{\text {th }}$ and early $20^{\text {th }}$ centuries. In the United States, the plant was even included in the National Formulary and Pharmacopoeia until 1941 when its use was virtually brought to a halt due to the Marihuana Tax Act of 1937. ${ }^{181}$ After the discovery of the cannabinoid receptors type I and II and their ligands, both exogenous and endogenous, research into the medical use of marijuana and cannabinoids has grown exponentially. Recently, the putative cannabinoid receptor GPR55 has been proposed as a target for the treatment of pain and inflammation, especially that of neuropathic origin. The largest body of work with cannabinoids in the field of antinociception, however, is with ligands that are selective for the $\mathrm{CB} 2$ receptor; therefore, the discussion will be limited to those compounds.

Several CB2 agonists such as GW405833 (Figure 5.1), a substituted indole derivative, have shown to be efficacious in preclinical models of neuropathic, inflammatory, and postoperative pain. GW405833 is a CB2 agonist with binding affinities of $3.92( \pm 1.58) n M$ for the human CB2 versus $4772( \pm 1676) n M$ for CB1 which translates into greater than 1000 -fold selectivity. ${ }^{182}$ As with many cannabinoids, binding affinity constants and selectivity ratios are found to be variable between species. When assayed against the rat cannabinoid receptors, GW405833 was found to be only 78-fold selective for CB2. Depending on the type of assay used, the compound appears to be a partial agonist at the human $\mathrm{CB} 2$ receptor ${ }^{182}$ and has also been reported to be a partial inverse agonist at both human and rat $\mathrm{CB} 2$ receptors. ${ }^{183}$

In a sciatic nerve partial ligation model of neuropathic pain, GW405833 showed a $63.3 \%$ reversal of pain at 1 hour following an i.p. dose of $10 \mathrm{mg} / \mathrm{kg}$ with an $\mathrm{ED}_{50}$ of $0.077 \mathrm{mg} / \mathrm{kg}$. This compound also exhibited a reversal of hyperalgesia with $\mathrm{ED}_{50} \mathrm{~s}$ of $2.58 \mathrm{mg} / \mathrm{kg}$ and $0.17 \mathrm{mg} / \mathrm{kg}$ in the hind paw surgical incision model and the Freund's complete adjuvant (FCA) model, respectively. The target was confirmed in a CB2 knockout mouse model in which the compound did not reverse inflammatory pain. GW405833 was also screened in a number of behavioral tests to determine if the compound would elicit unwanted effects due to interaction with CB1. The well established catalepsy assay, rotorod assay examining motor function, and tail flick and hot plate tests which test for acute analgesia were used in these studies. Only the highest dose tested $(100 \mathrm{mg} / \mathrm{kg})$ showed any positive results in these assays. This suggests that at higher doses GW405833 does signal through CB1, but not at lower doses. ${ }^{182}$

Data has been published by Abbott regarding two CB2 agonists, A-796260 ${ }^{183}$ and A-836339 ${ }^{184}$ (Figure 5.1). A-796260 is an indole derivative which possesses the same 2- 


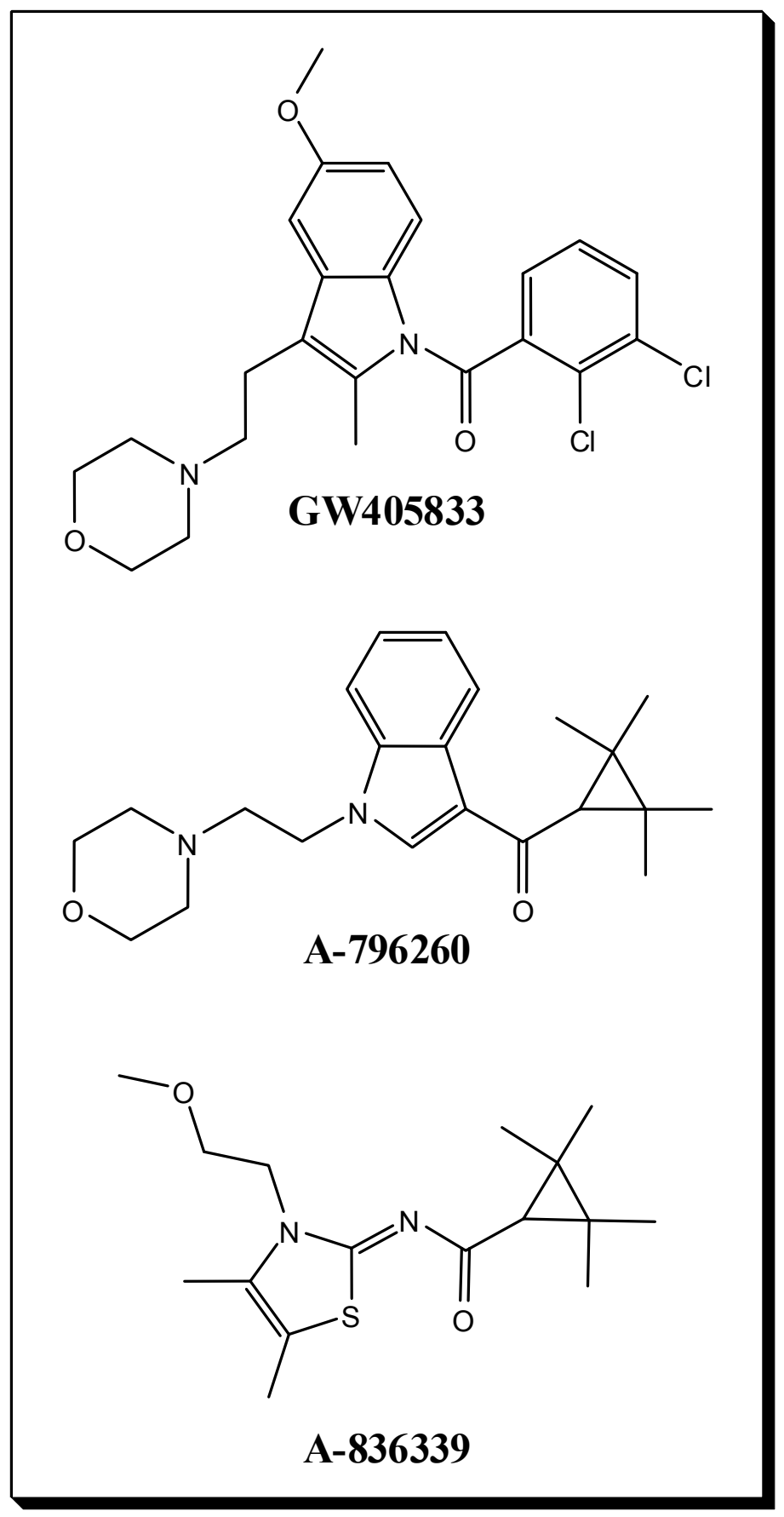

Figure 5.1: CB2 selective agonists used in preclinical studies as antinociceptive agents 
morpholinoethyl substitution as GW405833. It exhibits binding affinities for the human cannabinoid receptors of $845 \mathrm{nM}$ and $4.37 \mathrm{nM}$ for $\mathrm{CB} 1$ and $\mathrm{CB} 2$, respectively, which is a selectivity ratio of 193 -fold for the human CB2. Again, selectivity is lessened when assayed for affinity to the rat cannabinoid receptors. Assays obtained the values of $395 \mathrm{nM}$ and $13 \mathrm{nM}$ for rat $\mathrm{CB} 1$ and $\mathrm{CB} 2$, respectively, which is 30 -fold selective for the rat CB2 receptor. Functional selectivity of A-796260 for the CB2 receptor is greater than binding affinity selectivity for both the human and rat receptors (1380-fold and 175-fold, respectively).

Efficacy of A-796260 as an antinociceptive agent has been evaluated in the FCAinduced model of hyperalgesia using thermal stimulus. The compound exhibited dosedependent reduction in hyperalgesia following i.p. administration with an $\mathrm{ED}_{50}$ of $2.8 \mathrm{mg} / \mathrm{kg}$. In this particular model, the target was confirmed pharmacologically using the CB2 selective antagonists SR144528 ${ }^{185}$ and AM630. ${ }^{186}$ In each case, the analgesic effects of A-796260 were blocked. The CB1 selective antagonist SR141716A, however, had no effect on the analgesia produced by the compound. To evaluate the compound's ability attenuate postoperative pain, a skin incision model was performed. In this study, A-796260 was administered i.p. 1.5 hours following surgery; it dose-dependently lowered mechanical allodynia with an $\mathrm{ED}_{50}$ of $18 \mathrm{mg} / \mathrm{kg}$ and with a maximum efficacy of $68( \pm 7 \%)$ without the development of tolerance following repeated administration. In a study of neuropathic pain, the chronic constriction injury of the sciatic nerve model was used. In this study, A-796260 also reduced mechanical allodynia with an $\mathrm{ED}_{50}$ of $15 \mathrm{mg} / \mathrm{kg}$ with maximum efficacy of $66( \pm 9 \%)$. This compound has also shown efficacy in an osteoarthritis pain model and performed favorably compared to celecoxib. A796260 displayed $56 \%$ efficacy at a dose of $35 \mathrm{mg} / \mathrm{kg}$ while celecoxib was $62 \%$ efficacious using a $38 \mathrm{mg} / \mathrm{kg}$ dose. This compound was also screened for CNS effects and none were found up to a $35 \mathrm{mg} / \mathrm{kg}$ dose.

A-836339, a dimethylthiazole carboxamide derivative, displays similar binding affinities to A-796260. The $K_{\mathrm{i}}$ for binding affinity to the human CB2 receptor is $0.64 \mathrm{nM}$ and a selectivity of 425 -fold is observed over the human CB1. Affinity for the rat CB2 receptor is very similar to that of the human, $K_{\mathrm{i}}=0.76 \mathrm{nM}$, and again selectivity when compared to the CB1 receptor is lower than in the human receptors (189-fold). Like A$796260, \mathrm{~A}-836339$ is a full agonist at $\mathrm{CB} 2$ with an $\mathrm{EC}_{50}$ of $1.6 \mathrm{nM}$. Both compounds have also been shown to be full agonists at CB1 but, of course, much less potent.

A-836339 has been shown to be efficacious in the FCA-induced inflammatory pain model with an $\mathrm{ED}_{50}$ of $1.96 \mu \mathrm{mol} / \mathrm{kg}$ and has also shown efficacy in the skin incision model of postoperative pain with an $\mathrm{ED}_{50}$ of $12 \mu \mathrm{mol} / \mathrm{kg}$. The compound has also yielded promising results in a capsaicin-induced model of hyperalgesia and in the chronic constriction injury neuropathic pain model with $\mathrm{EC}_{50} \mathrm{~S}$ of $10.4 \mu \mathrm{mol} / \mathrm{kg}$ and $12.9 \mu \mathrm{mol} / \mathrm{kg}$, respectively. A-836339 was shown to have some CB1 mediated effects as determined by decreased horizontal locomotor activity following both $15 \mu \mathrm{mol} / \mathrm{kg}$ and $45 \mu \mathrm{mol} / \mathrm{kg}$ doses which was reversed by SR141716A treatment. Although A-796260 has been shown to have affinity for the $\delta$-opiod receptor, the antinociceptive effects of A-796260 and A836339 are not due to interaction with the $\mu$-opiod receptor as exemplified by the inability of naloxone to reverse the analgesic effects of either compound. 
The phenylamino pyrimidine GW842166X (Figure 5.2) is a clinical candidate belonging to GlaxoSmithKline which is being studied for its anti-inflammatory potential. The compound has been reported to be fully selective for CB2, both human and rat, with $\mathrm{EC}_{50} \mathrm{~s}$ of $63 \mathrm{nM}$ and $91 \mathrm{nM}$, respectively. The compound displayed no significant agonist activity at either human or rat CB1 receptors up to $30 \mu \mathrm{M} .{ }^{187}$ In the FCA-induced model of inflammatory pain, the compound was shown to be highly efficacious with an oral $\mathrm{ED}_{50}$ of $0.1 \mathrm{mg} / \mathrm{kg}$ with full reversal of hyperalgesia at an oral dose of $0.3 \mathrm{mg} / \mathrm{kg}$. As with A-796260, the development of tolerance was not observed within the timeframe of the study.

A dihydrobenzofuran developed at the MD Anderson Cancer Center, MDA7 (Figure 5.2), has shown to be another cross-species CB2 selective compound. ${ }^{159}$ The compound has a $K_{\mathrm{i}}$ of $422( \pm 123) \mathrm{nM}$ for human CB2 with no affinity for the human CB1 up to $10 \mu \mathrm{M}$. For rat cannabinoid receptors, the $K_{\mathrm{i}}$ values $2565( \pm 695) \mathrm{nM}$ for CB1 and $238( \pm 143) \mathrm{nM}$ for CB2. The compound was shown to be a weak partial agonist at the human $\mathrm{CB} 1$ at concentrations above $1 \mu \mathrm{M}$. The compound has produced promising results in attenuation of mechanical allodynia due to both paclitaxel and spinal nerve ligation. The authors also reported a transient antinociceptive effect from thermal stimulus following i.p. administration of a $10 \mathrm{mg} / \mathrm{kg}$ dose.

Pfizer has reported a dihydrobenzimidazole, PF-03550096 (Figure 5.2), that has also showed promise in preclinical research. ${ }^{188}$ The compound performed well in a rat colonic pain model utilizing 2,4,6-trinitrobenzene sulfonic acid as a gastric irritant. The authors report that the compound significantly suppressed visceral hypersensitivity in this model which was reversed by the CB2 antagonist SR144528.

\subsubsection{Neuro-Inflammation}

CB2 selective compounds have also begun to show promise as possible therapeutic options for the treatment of neuro-inflammatory diseases such as multiple sclerosis (MS), Parkinson's disease, Huntington's disease, Alzheimer's disease, and encephalitis. Compounds that do not have CB1 activity would not have been logical choices for treatment of these disorders before the discovery that $\mathrm{CB} 2$ is indeed expressed in the CNS.

A preclinical model that is used for the study of MS is experimental autoimmune encephalomyelitis. CB2 was found to be a legitimate target in this model by the fact that CB2 knockout mice have a more severe disease course upon induction than do wild-type mice. ${ }^{189}$ Furthermore, mice at the peak of disease development that are treated with the CB2 agonist HU-210 (Figure 5.3) are scored much lower on severity rating scales compared to vehicle treated controls. This efficacy is reported by the authors to be due to reduced microglia activation and reduction in the infiltration of myeloid cells. ${ }^{189 a} \mathrm{HU}-$ 210 is an analog of cannabidiol possessing the dimethyl heptyl side chain. Receptor binding affinity for the compound at human $\mathrm{CB} 1$ and $\mathrm{CB} 2$ is $>10 \mu \mathrm{M}$ and 22.7 ( \pm 3.9)nM, respectively. 


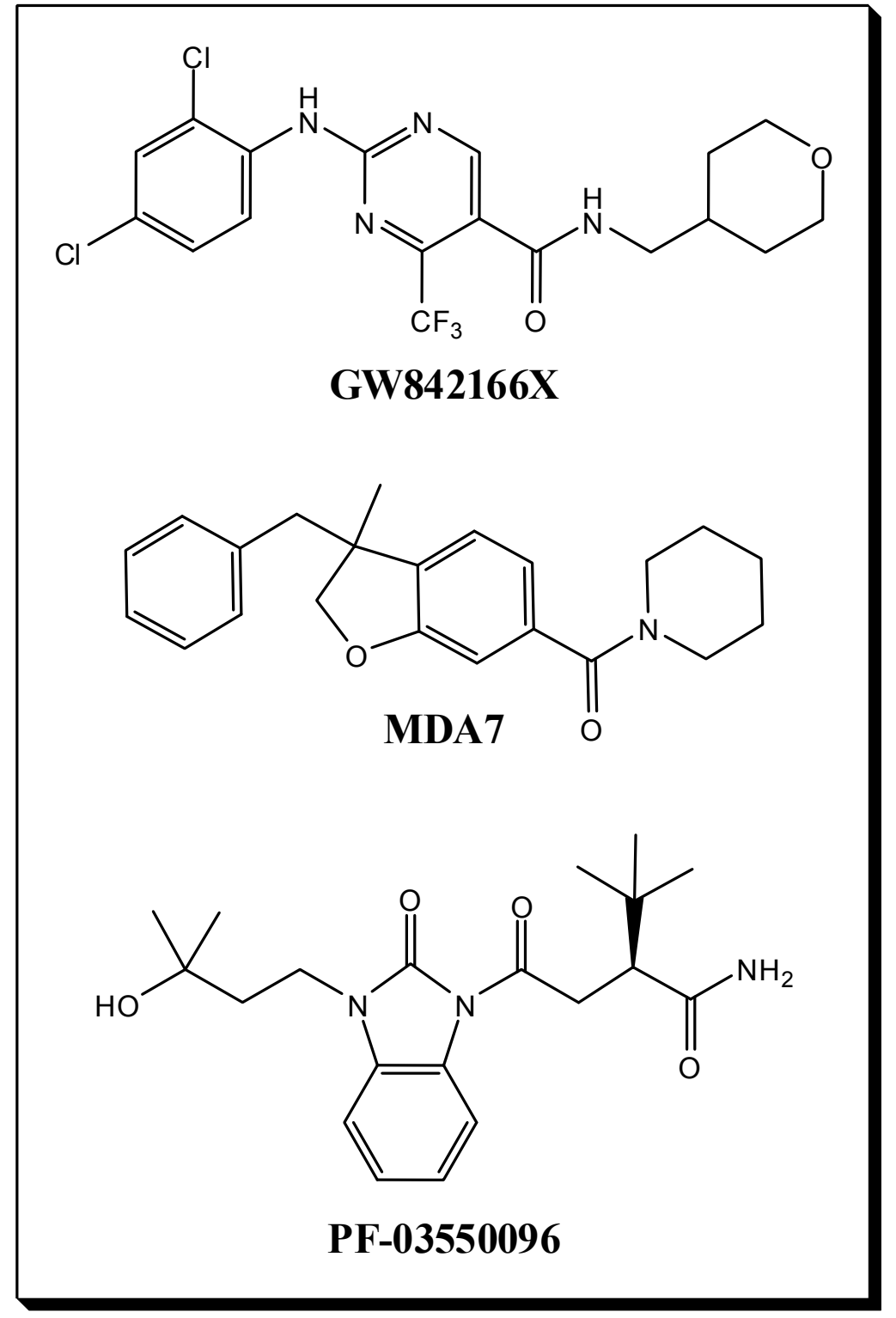

Figure 5.2: Further CB2 selective agonists used in preclinical studies as antinociceptive agents 


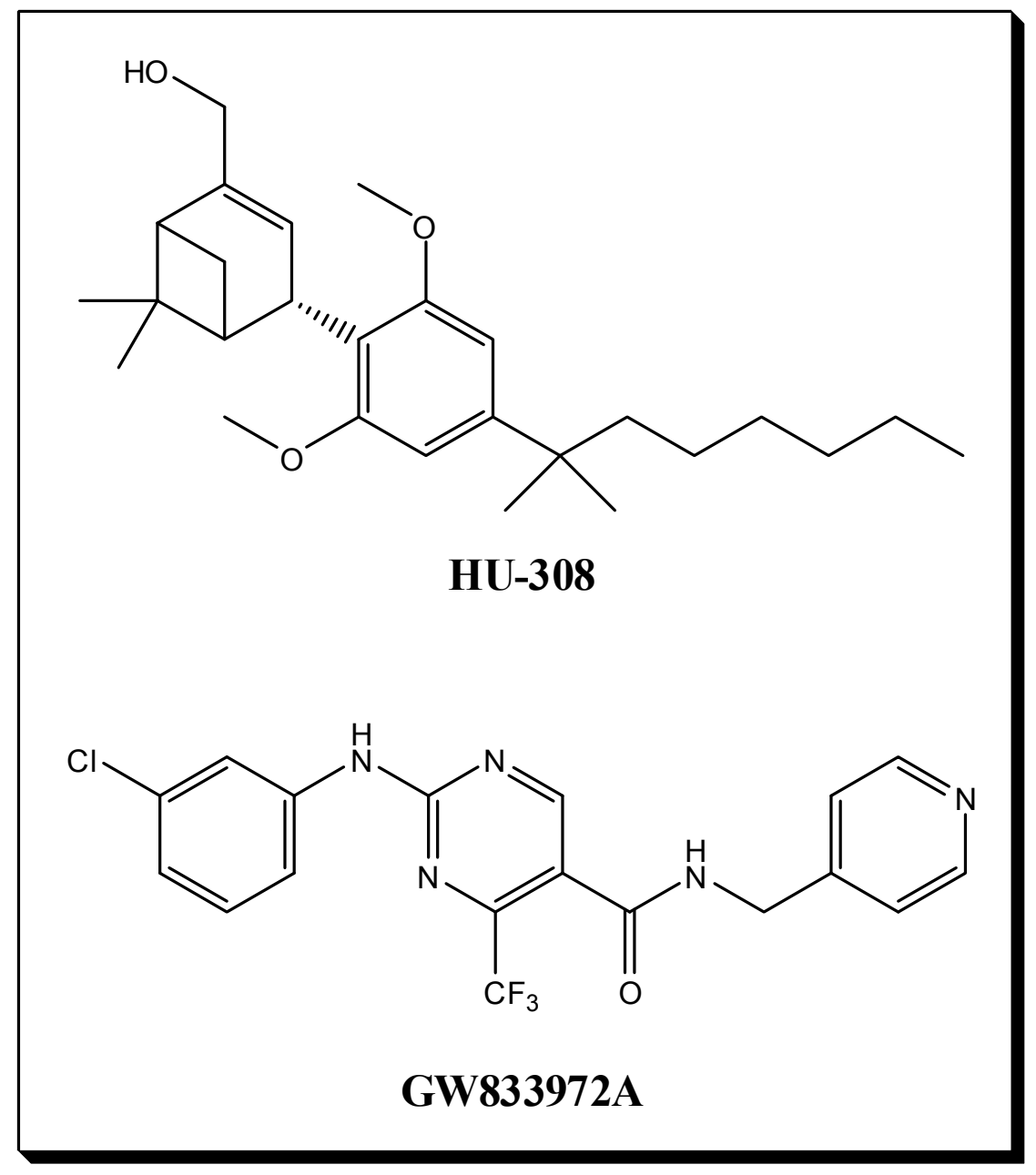

Figure 5.3: CB2 selective agonists used in preclinical studies as anti-inflammatory agents 


\subsubsection{Other Indications}

CB2 selective agonists have also shown promise for indications other than the inflammatory conditions mentioned above. GW833972A, another phenylamino pyrimidine (Figure 5.3), is a1000-fold selective CB2 agonist that has been shown to reduce citric acid-induced cough in a conscious guinea-pig model. ${ }^{190}$ The role of CB2 in the action of GW833972A was verified by pretreatment with antagonists for the CB1 and CB2 receptors. The antitussive effects were blocked by pretreatment with the CB2 selective antagonist SR144528 while the CB1 selective antagonist SR141716A had no effect.

HU-210 has been studied in other animal models of disease also. It has been reported that observed in vitro effects of HU-210 are suppression of trabecular osteoclastogenesis. ${ }^{191}$ This effect is reported to be due to inhibition of osteoclast precursors and also inhibition of receptor activator of $\mathrm{NF \kappa B}$ ligand. The authors of this report also tested this compound in a model of postmenopausal osteoporosis using ovarectomized mice. HU-210 lessened trabecular bone loss by $66 \%$ compared to untreated controls.

A potential role for $\mathrm{CB} 2$ agonists in the treatment of the chronic inflammatory disease atherosclerosis has also been identified. The presence of CB2 receptors was verified in both human and mouse atherosclerotic plaques. ${ }^{192}$ The non-selective cannabinoid agonist $\Delta^{9}$-THC showed significant inhibition of disease progression in a murine model of established atherosclerosis using apolipoprotein E knockouts. THC was dosed at $1 \mathrm{mg} / \mathrm{kg}$ orally once a day. ${ }^{192}$ This dose is below that which is usually associated with the CB1 mediated psychotropic effects of THC. The role of CB2 in this study was verified by the observation that the CB2 selective antagonist SR144528 blocks the therapeutic effect of THC. The authors also observed in vitro that THC blocks macrophage chemotaxis which is crucial for the formation of atherosclerotic plaques, an effect that was also blocked by SR144528.

\subsection{Evaluation of CB2 Agonists in Clinical Trials}

To date, there have been reports of three pharmaceutical companies entering clinical trials with CB2 agonists for the treatment of pain. ${ }^{193}$ Cannabinor, formerly known as PRS-211,375 (Figure 5.4) is a fumarate ester derivative of compounds similar to HU-308. In January 2007, Pharmos Corporation reported the results of a Phase IIa experimentally induced pain model performed with cannabinor. In this study the compound failed to meet the primary endpoint which was reduction of capsaicin-induced pain. ${ }^{194}$ In this study, however, researchers made the observation that, when compared to placebo, intravenous (i.v.) administration of cannabinor did have a significant effect in the reduction of both pressure-induced and heat-induced pain. In April of that same year, Pharmos reported the results of a separate Phase IIa trial with Cannabinor. The study was designed to examine the usefulness of i.v. cannabinor to attenuate pain following $3^{\text {rd }}$ molar dental extraction. The results were difficult to interpret. The lowest dose of $12 \mathrm{mg}$ demonstrated a significant analgesic effect while the higher doses of $24 \mathrm{mg}$ and $48 \mathrm{mg}$ 


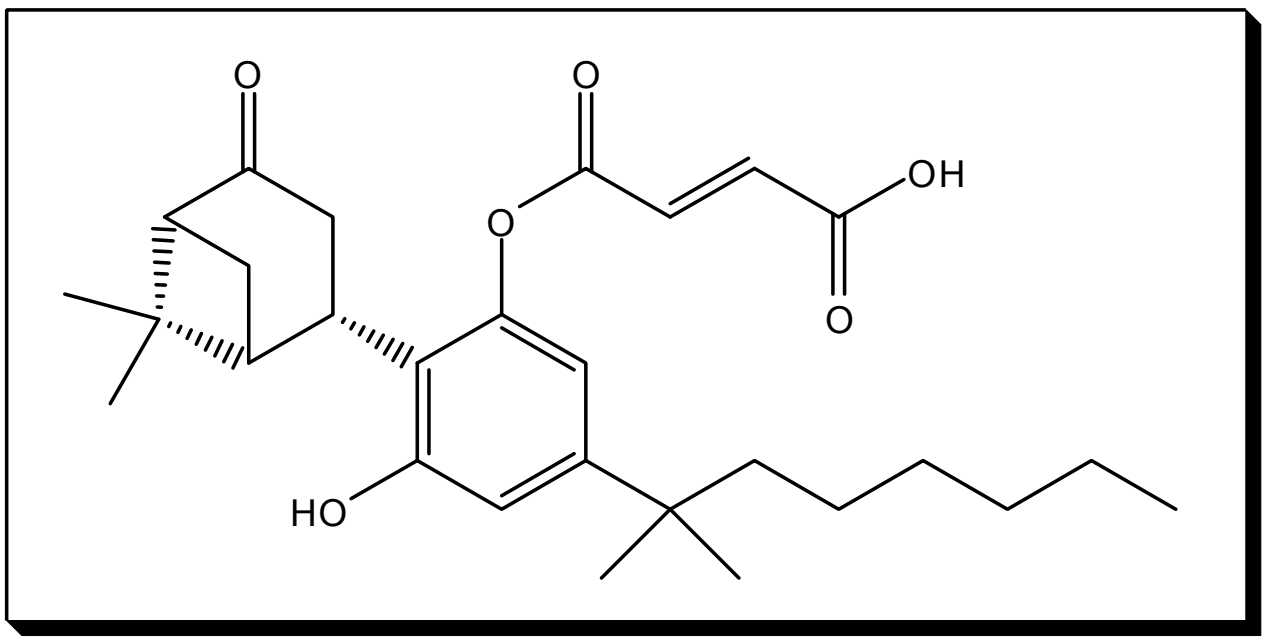

Figure 5.4: Cannabinor (PRS-211,375)

CB2 selective agonist studied in Phase II clinical trials. 
were not statistically efficacious. After this unexpected pattern of results the company decided to drop further development of i.v. cannabinor as an antinociceptive and retargeted the research with this compound to other possible indications and later dropped development all together. One positive note from each of these trials is that cannabinor was generally well tolerated with no serious adverse events reported. Pharmos currently has other CB2 selective compounds of undisclosed structure that are currently in preclinical studies for the conditions of neuropathic pain, autoimmune disease, and irritable bowel syndrome. ${ }^{195}$ GlaxoSmithKline has completed three Phase II clinical trials with GW842166. ${ }^{196}$ One trial was for analgesia following $3^{\text {rd }}$ molar extraction and the other two trials were examining the efficacy of the compound for treatment of osteoarthritis pain. The company has not reported the results of these studies and currently lists no cannabinoids in their list of pipeline drugs. ${ }^{197}$ In 2009, Glenmark Pharmaceuticals announced the completion of Phase I studies in Europe of GRC10693 which is a CB2 selective agonist ( $>4700$-fold) that possesses good oral bioavailability and is well tolerated. The company plans to pursue the compound for the treatment of neuropathic pain. $^{198}$

\subsection{Evaluation of Novel Cannabinoids as Anti-Inflammatory Agents}

\subsubsection{Initial Study}

In order to exploit the therapeutic potential of our cannabinoid ligands as possible anti-inflammatory agents, we designed a preliminary in vitro study to determine what effects we could observe. We chose to use cytokine and chemokine production as markers for inflammation. We chose six of our novel synthetic cannabinoids (along with

$\Delta^{8}$-THC) and screened them for anti-inflammatory activity against A-549 cells stimulated with tumor necrosis factor alpha (TNF- $\alpha)$. The six compounds chosen represented six different chemical classes (Figure 5.5). The previously described pyrimidine hexahydro classical cannabinoid $\mathbf{3 7}$ was selected along with a similar compound that is a pyridine hexahydro classical with the same dimethyl heptyl side chain 79 prepared in our laboratory by Dr. Suni Mustafa. The non-classical triaryl compounds were represented by HB-I-172, the pyrimidine nonclassical 50, and the equivalent pyridine non-classical 80 also prepared by Dr. Mustafa. The furanopyrimidine cannabinoids had not been synthesized at this point, but Dr. Mustafa had begun on a series of furanopyridines - one of which was included in this group 81. Cells were treated with vehicle, drug (at the toxic $\mathrm{EC}_{1}$ or $\mathrm{EC}_{10}$ ), TNF- $\alpha$, or a combination of TNF- $\alpha$ and drug. The cells were allowed to incubate in the presence of the stimulus or drug for periods of 4 or 18 hours. The media was then removed from the cells and flash frozen on dry ice. The samples were then shipped to an independent laboratory for analysis of excreted cytokines and chemokines.

The results of this preliminary study were quite promising. The efficacies of the compounds were evaluated by the levels of the excreted cytokines IL-1 $\beta$, IL-6, IL-8, granulocyte colony stimulating factor (G-CSF), monocyte chemotactic protein-1 (MCP1), macrophage migration inhibitory factor (MIF), and RANTES (Regulated upon 


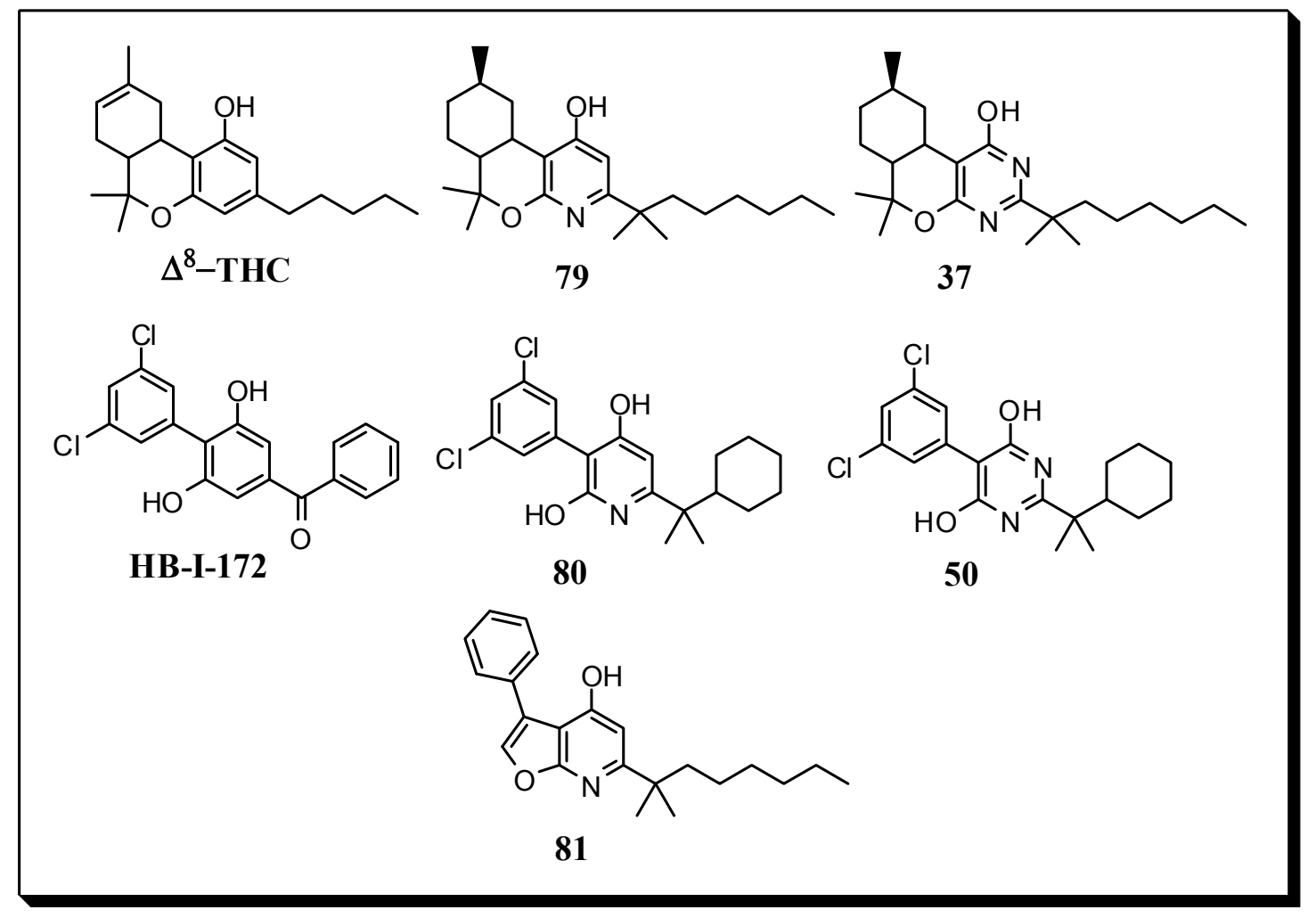

Figure 5.5: Seven compounds initially screened for anti-inflammatory activity 
Activation, Normal T-cell Expressed, and Secreted). All of the compounds screened exhibited an attenuation of the inflammatory response manifesting as a reduction in the excretion of one or more of the pro-inflammatory cytokines assayed for (data not shown).

This positive result prompted the design of a study to pick leads from the various chemical classes in our library to screen for anti-inflammatory activity. In order to select leads we first had to define criteria which we would use to make the selections. It was decided to use functional activity as the criterion with which to narrow the compounds. We would first screen a vast number of compounds from the library for functional activity at the $\mathrm{CB} 1$ and $\mathrm{CB} 2$ receptors. We would then select compounds that matched the functional profiles of the seven compounds screened in the initial study. The list of candidates would then be reduced based on solubility and/or toxicity data. We would then examine the cytokine and chemokine release profiles of human macrophage and dendritic cell lines challenged with lipopolysaccharide (LPS, to simulate a gram negative infection) or peptidoglycan (PGN, to simulate a gram positive infection) and human epithelial and endothelial cell lines challenged with TNF- $\alpha$ or IL-1 $\beta$. The candidates would then be screened for their ability to modulate the excretion of cytokines and chemokines in our in vitro models. The one or two compounds that proved to be most efficacious would then be moved into an in vivo inflammatory model such as bacteremia or sepsis. The overall design of the study is summarized in Figure 5.6.

\subsubsection{Selection of Compounds to Screen}

In order to select lead compounds from our chemically diverse library of cannabinoid ligands, it was necessary to first develop functional profiles for the initial seven compounds in order to develop the paradigm used for selection. It was presumed that since all seven compounds exhibited anti-inflammatory activity that they would each have similar functional activity patterns. This proved to be an incorrect assumption. To develop the functional profiles, we screened each compound in our functional assay platform for the CB1 and CB2 receptors at $10 \mu \mathrm{M}$. The assay determines a compound's ability to affect the alpha-agonist stimulated production of cAMP. Since CB1 and CB2 signal through $\mathrm{G}_{\alpha i}$, an agonist will decrease the amount of cAMP produced, and agonist will have no effect, and an inverse agonist will increase the production of cAMP. The profiles for each of the initial seven compounds are shown in Figure 5.7. The data is presented as a percentage of the cAMP production compared to a control. Higher agonist activity presents as a lower percentage. Lesser agonist activity or antagonism presents a percentage closer to 100 and inverse agonist activity will give percentages greater than 100. The compounds clearly have varied functional activity at CB1 and CB2. $\Delta^{8}-\mathrm{THC}$ along with compounds $\mathbf{3 7}$ and $\mathbf{7 9}$ has greater agonist activity at $10 \mu \mathrm{M}$ at the CB1 receptor than at CB2. In fact, THC appears to be an antagonist and possibly even an inverse agonist in our assay platform. HB-I-172, as previously described, is an antagonist at $\mathrm{CB} 1$ and an inverse agonist at $\mathrm{CB} 2$. The remaining compounds $(\mathbf{5 0}, \mathbf{8 0}$, and $\mathbf{8 1})$ exhibited very little if any agonist activity at CB1 and moderate agonist activity at CB2. The observed differences in the functional profiles of these compounds could point to the possibility that agonists of either the $\mathrm{CB} 1$ or $\mathrm{CB} 2$ receptors are efficacious in reducing the inflammatory response, or that possibly there is another target involved such as 


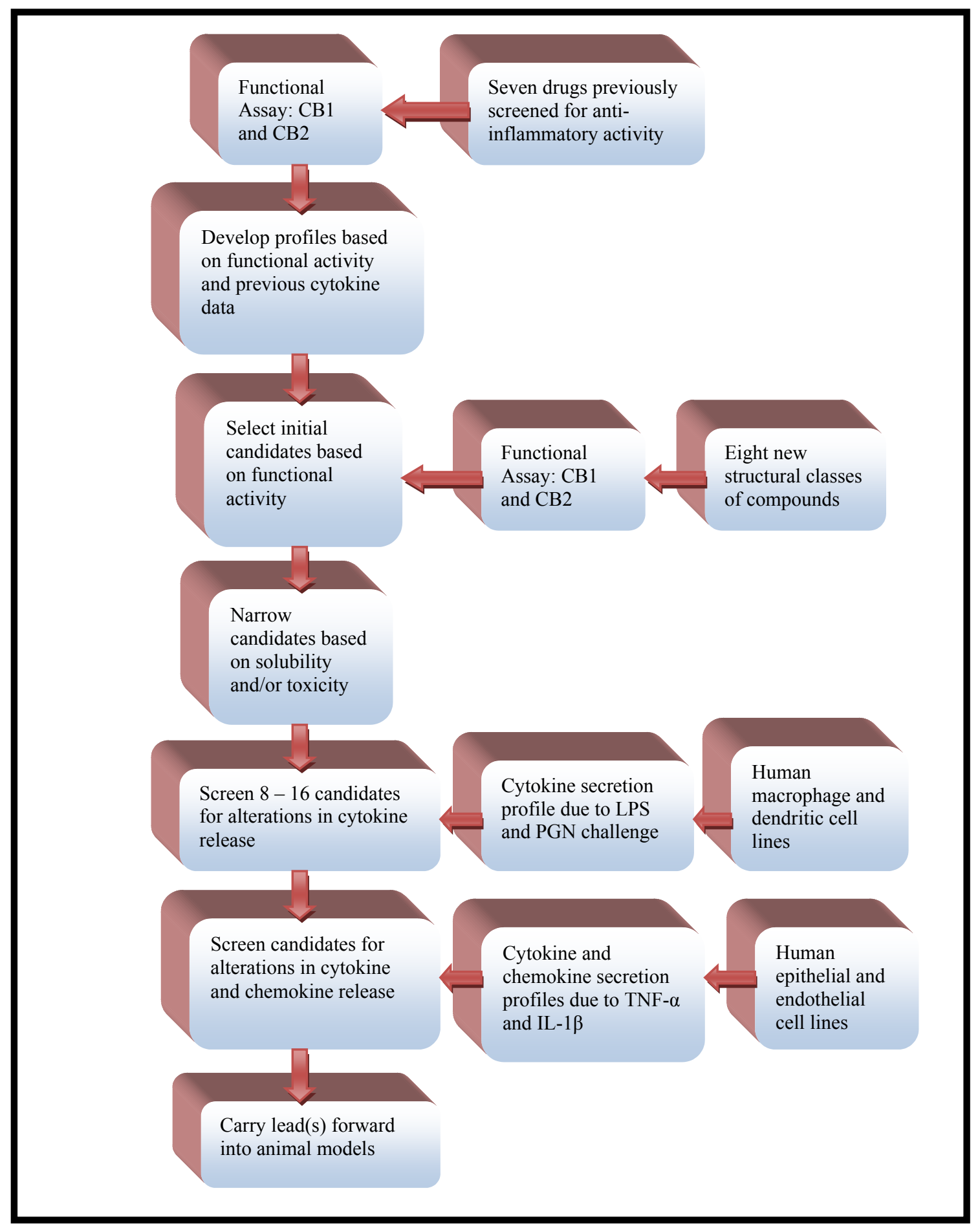

Figure 5.6: Anti-inflammatory study design 


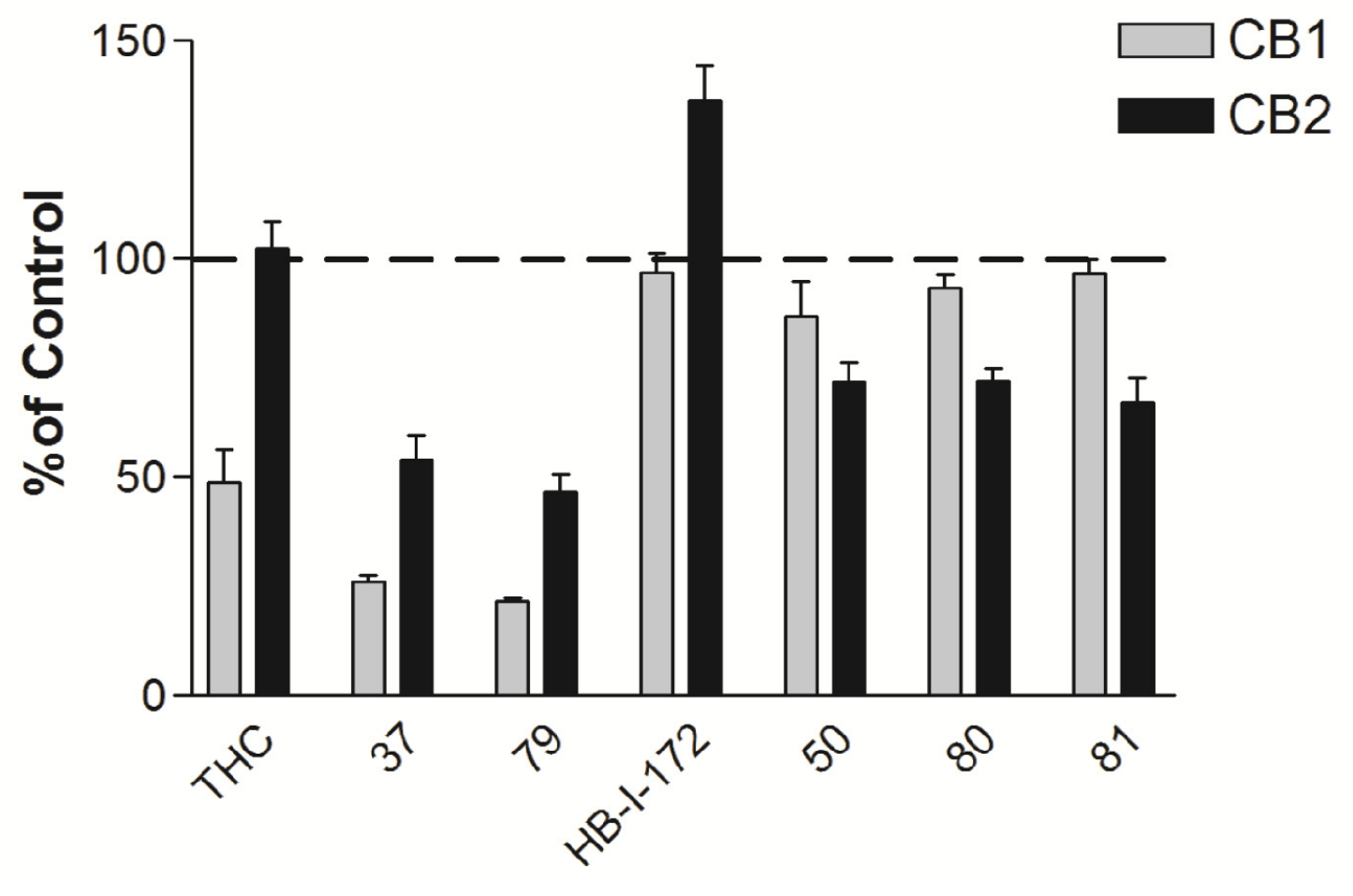

Figure 5.7: Functional profiles of initial seven compounds screened for antiinflammatory activity

Compounds were screened using the ActOne $\mathrm{O}$ whole cell functional assay for the $\mathrm{CB} 1$ and $\mathrm{CB} 2$ receptors. The assay measures a compound's ability to alter cAMP production stimulated by an alpha agonist. The lower the percentage of control, the greater ability of the compound to suppress the formation of cAMP; therefore, the greater the agonist activity at the given receptor. Percentages closer to 100 represent lower agonist activity or antagonist activity. Percentages above 100 represent activity of an inverse agonist. Data represents a minimum of three experiments run in triplicate. 
GPR55. The fact that HB-I-172 showed preliminary efficacy strengthens the possibility that $\mathrm{CB} 2$ inverse agonists may be viable candidates for the treatment of inflammatory disorders.

Since there was no clear pattern with which to use as a screening criterion, we chose to develop functional profiles for a large number of our compounds and select a number that had a variety of functional activities. Initially, we screened seventy-five compounds from eight distinct structural classes. The classes were the triaryls, pyridine triaryls, pyrimidine triaryls, pyridine hexahydros, pyrimidine hexahydros, benzofurans, furanopyridines, and the furanopyrimidines. Out of the seventy-five screened, forty-four compounds were carried forward. These compounds were then screened for toxicity against human dermal fibroblasts at 10,25 , and $50 \mu \mathrm{M}$ (data not shown). The compounds that exhibited the greatest toxicity at the lowest doses were eliminated. The remaining compounds were further narrowed based on solubility. The compounds that visibly precipitated at the $50 \mu \mathrm{M}$ concentration in the cytotoxicity assay were also eliminated. This narrowed down the field significantly and one compound was chosen from each class (Figure 5.8) to carry forward into the in vitro inflammation assays.

\subsubsection{Cytokine and Chemokine Release Profiles}

I initially decided to utilize a total of four cell lines in the anti-inflammatory study. Two cell lines were used to model cells of the immune system: THP-1 and KG-1. THP-1 is a human monocytic leukemia cell line that can be differentiated into a macrophage-like phenotype and KG-1 is a human lymphoblastic leukemia line that can be differentiated into a dendritic cell phenotype. Cells of the epithelial and endothelial lineages that also play roles in the inflammatory response were also to be studied. A-549, a human epithelial lung carcinoma cell line, was chosen to model the epithelial response and the human vascular endothelial cell line HUV-EC-C was selected to model the endothelial inflammatory response.

We decided to utilize the previously described MSD ${ }^{\circledR}$ technology to measure the cytokine and chemokine levels in-house. We selected two types of analysis plates for our studies. The first was the Human ProInflammatory 9-PlexTissue Culture Kit which measures the quantitative levels of nine cytokines, namely: IL-1 $\beta$, Il-2, IL-6, IL-8, IL-10, IL-12 p70, TNF- $\alpha$, IFN- $\gamma$, and GM-CSF (granulocyte-macrophage colony-stimulating factor). To measure chemokine production, the Human Chemokine 9-PlexTissue Culture Kit was chosen which assays for Eotaxin, Eotaxin-3, IL-8, IP-10 (10 kDa interferongamma-induced protein), MCP-1, MCP-4, MDC (macrophage-derived chemokine), MIP$1 \beta$ (macrophage inflammatory protein-1 beta), and TARC (thymus and activation regulated chemokine).

In order to test the ability of our assay design to detect the levels of excreted cytokines and chemokines, we initially collected time-course release profiles of each cell line challenged with two different stimuli. This would also give us information as to the optimum time point to use for screening our compounds. The THP-1 and KG-1 cell lines grow in suspension and must be transformed to attach and take on the macrophage and 


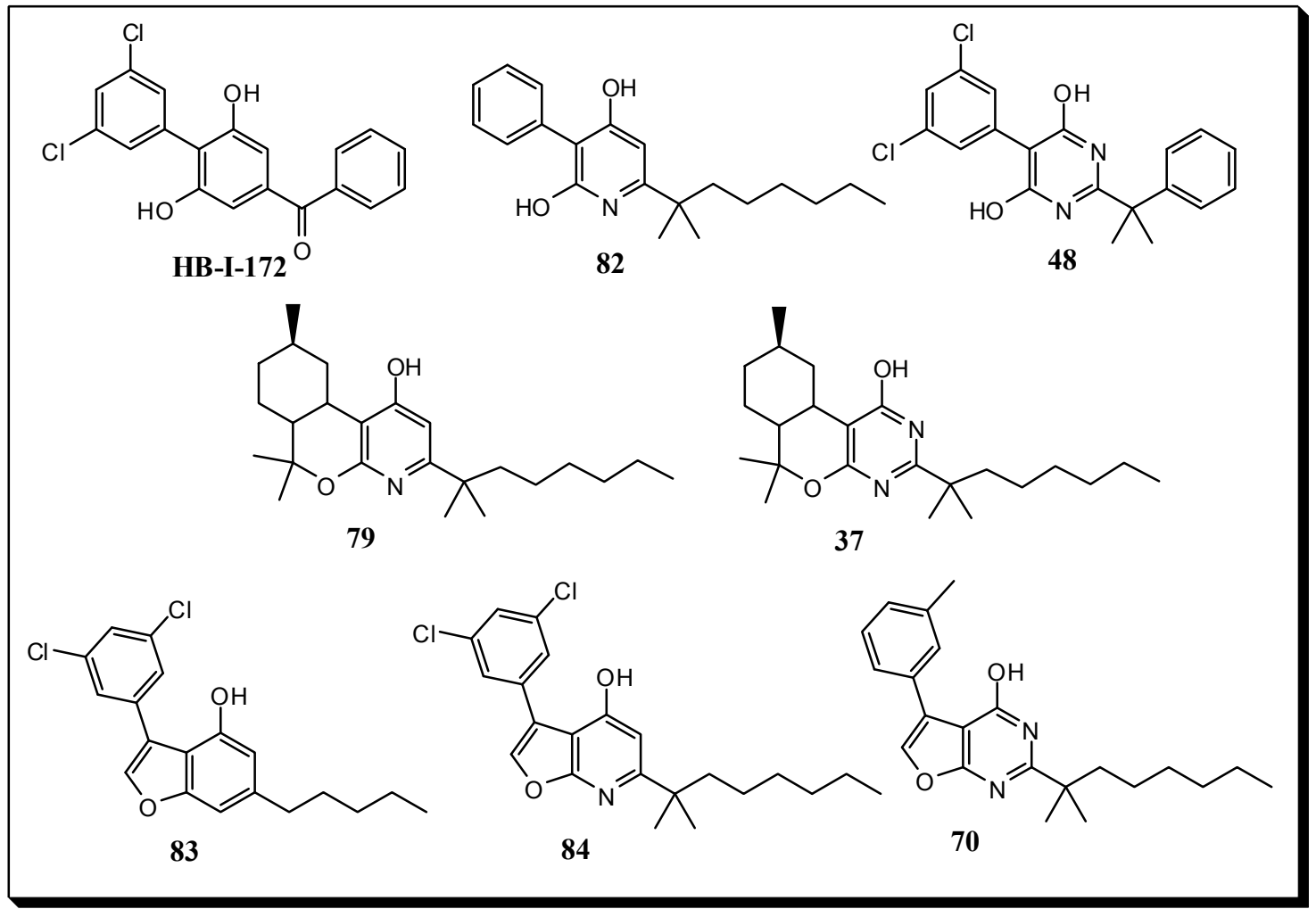

Figure 5.8: Eight compounds chosen for anti-inflammatory studies 
dendritic cell phenotypes, respectively. This is accomplished by incubating the cells in the presence of phorbol 12-myristate 13-acetate (PMA, 10ng/ml) and ionomycin $(500 \mathrm{ng} / \mathrm{ml})$ for a minimum of 5 days. The transformed cells, along with non-transformed THP-1 and KG-1 for comparison, were challenged with LPS or PGN at $1 \mu \mathrm{g} / \mathrm{ml}$ and the supernatant media was assayed at 10 time points from 1 to 24 hours post stimulus for the levels of the previously mentioned cytokines and chemokines. The same procedure was performed with A549 and HUV-EC-C stimulated with TNF- $\alpha$ and IL-1 $\beta$. A representative graph is shown in Figure 5.9. This data demonstrates the release of IL-6 from transformed and non-transformed THP-1 in response to LPS over the 24 hour experiment. The entire data set is included in Appendix A. From the time-course experiments we were able to validate the assay platform and to draw some conclusions that helped to shape the remainder of the experiments:

1. There is a detectable, time-dependent release of cytokines and chemokines from the human cell lines THP-1, KG-1, A549, and HUV-EC-C.

2. The cytokines and chemokines reached maximum or near maximum levels at 18 hours post stimulus.

3. THP-1 exhibited a more pronounced response than did KG-1.

4. LPS elicited a greater response from THP-1 and KG-1 than did PGN.

5. The responses elicited from A549 and HUV-EC-C from TNF- $\alpha$ and IL-1 $\beta$ were comparable.

\subsubsection{Screening of Initial Eight Compounds}

With the information gained from the cytokine/chemokine release profile experiments, the screening of the eight selected compounds was planned. Due to the lesser response of the transformed KG-1 cells, it was decided to use only the THP-1 cell line to model cells of the immune system and to continue with both A549 and HUV-ECC. Each of the eight compounds was screened for toxicity against the three cell lines, the $\mathrm{EC}_{50} \mathrm{~s}$ were determined, and the $\mathrm{EC}_{10} \mathrm{~S}$ calculated. The compounds were then screened for their abilities to attenuate the inflammatory response in THP-1 stimulated with LPS or PGN and in A549 and HUV-EC-C stimulated with TNF- $\alpha$ or IL-1 $\beta$. The drugs were screened at the $\mathrm{EC}_{10}$ or $25 \mu \mathrm{M}$ (whichever was less) and cytokine/chemokine levels were measured 18 hours post stimulus and drug treatment.

Each compound was capable of attenuating the inflammatory response on multiple cell lines as manifested by decreases in the levels of multiple secreted cytokines and chemokines. The entire data set from this series of experiments can be found in Appendix B. Significant effects were seen in a number of inflammatory markers; for example, in the transformed THP-1 cell line decreases in the cytokine IFN- $\gamma$ were observed for all compounds tested in the PGN group and five of the eight compounds tested in the LPS group (Figure 5.10). The significance of this effect is that IFN- $\gamma$ can 


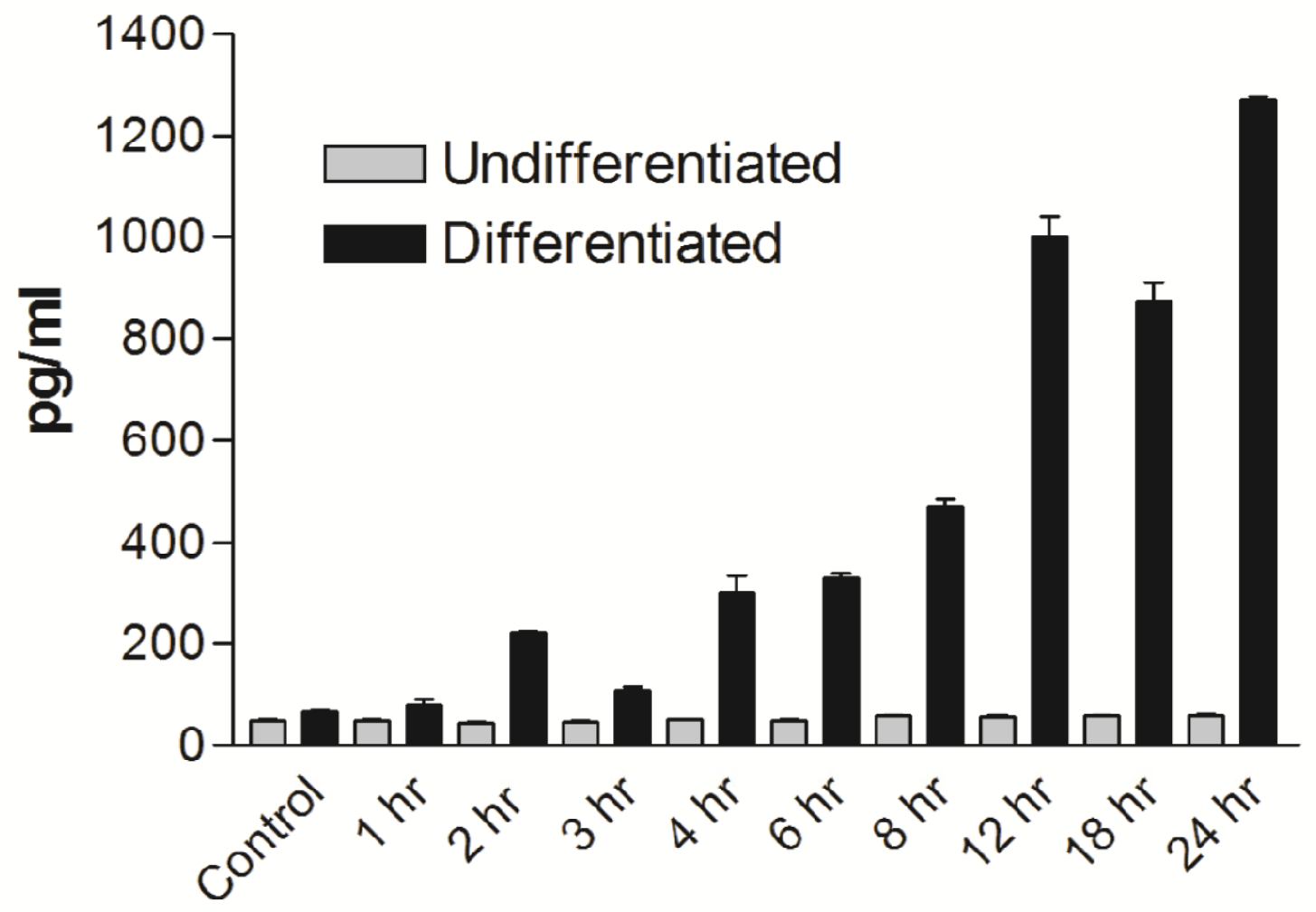

Figure 5.9: Time-dependent release of IL-6 from THP-1 stimulated with LPS 


\section{LPS}

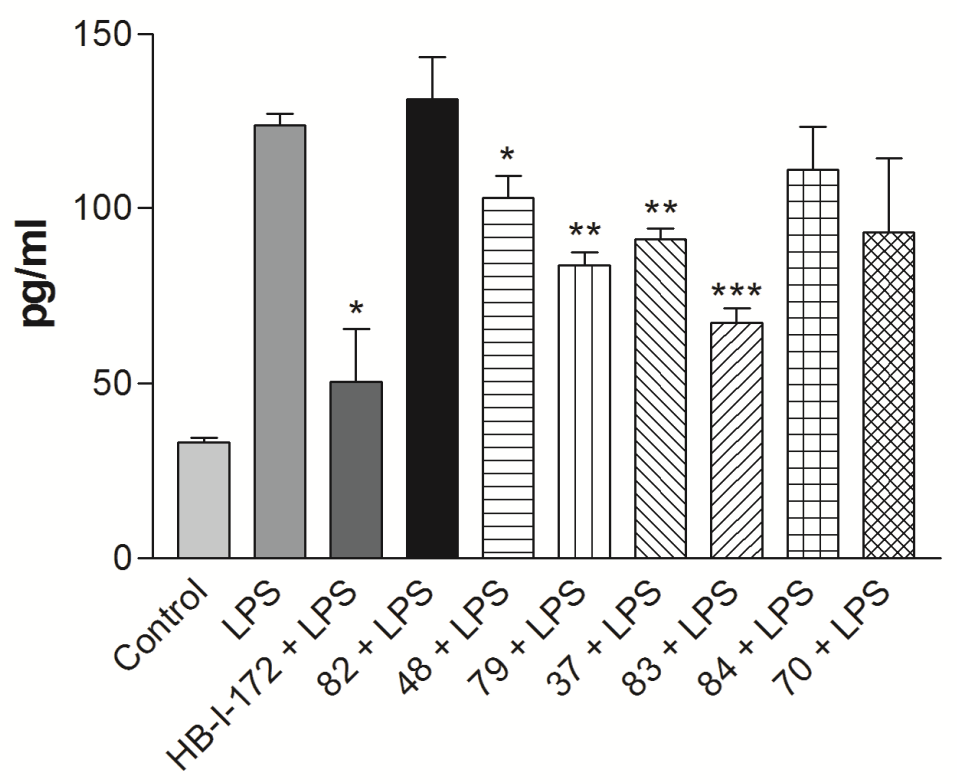

PGN

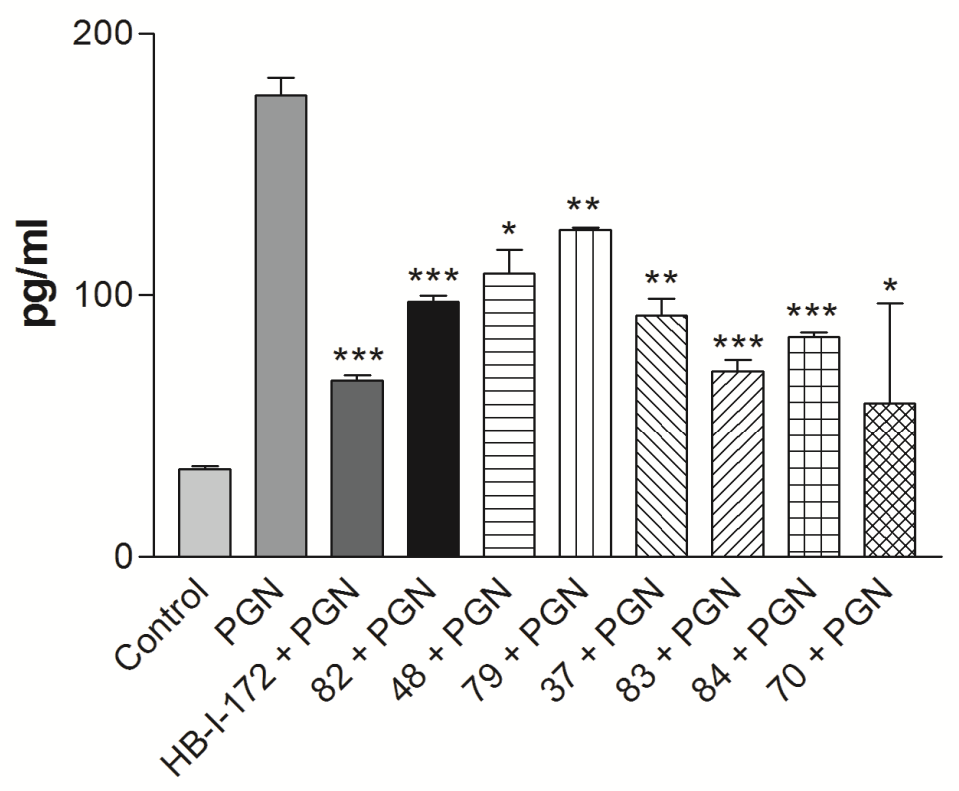

Figure 5.10: Effects of eight compounds on release of IFN- $\gamma$ from THP-1 in response to LPS and PGN

$*=\mathrm{p}<0.05, * *=\mathrm{p}<0.01$, and $* * *=\mathrm{p}<0.005$. 
further activate macrophages and lead to an increased synthesis and release of proinflammatory cytokines and chemokines. ${ }^{199}$ Significant results were also seen with THP1 in the levels of IL-12p70 and IL-6 produced in response to both LPS and PGN (Figures $\mathbf{5 . 1 1}$ and 5.12, respectively). IL- $12 p 70$ is a cytokine that can further activate macrophages; however, its main function is the activation of NK cells ${ }^{200}$ and differentiation of naïve T cells. ${ }^{201}$ Upon exposure to IL-12, T and NK cells are stimulated to produce and release the pro-inflammatory cytokines IFN- $\gamma$ and TNF- $\alpha$ which further perpetuate the inflammatory response. IL-6 is a major pro-inflammatory mediator produced mainly by macrophages that elicits a wide range of responses such as lymphocyte activation, increased antibody production, fever, and production of acute phase proteins by hepatocytes. Overproduction of IL-6 has also been implicated in a number of autoimmune disorders such as rheumatoid arthritis. ${ }^{202}$ Still further positive results were seen in the PGN stimulated THP-1 cells as evidenced by reductions in GMCSF, IL-1 $\beta$, and TNF- $\alpha$ and increases in the anti-inflammatory cytokine IL-10 (Figures 5.13 and 5.14). GM-CSF increases the production of granulocytes and monocytes and increases monocyte adhesion. ${ }^{203}$ TNF- $\alpha$ and IL- $1 \beta$ each produce a wide range of inflammatory responses such as increased expression of adhesion molecules on endothelial cells and increases in vascular permeability. ${ }^{204}$ The increased production of IL-10 following exposure to PGN and five of the eight compounds tested is of interest due to the anti-inflammatory role of IL-10 which inhibits the synthesis of proinflammatory mediators such as IFN- $\gamma$, IL-2, IL-3, TNF- $\alpha$ and GM-CSF. ${ }^{205}$

Attenuations in the production of cytokines were also observed in the HUV-EC-C endothelial cell line. Examples are reductions in GM-CSF and IL-6 in response to TNF- $\alpha$ (Figure 5.15) and reductions in IFN- $\gamma$, IL-12p70, and IL-2 in response to IL-1 $\beta$ (Figure 5.16). IL-2 is a cytokine normally produced by activated $T$ cells and stimulates the growth and differentiation of antigen-selected cytotoxic $\mathrm{T}$ cells and is required for the development of T cell immunologic memory. ${ }^{206}$ The significance of the lower levels of IL-2 produced by endothelial cells is not known.

There were also several noteworthy observations in the levels of chemokines following stimulus and drug exposure. For example, reductions in the production of MIP- $1 \beta$ were detected following both TNF- $\alpha$ and IL-1 $\beta$ challenge in both A549 (Figure 5.17) and HUV-EC-C (Figure 5.18). MIP-1 $\beta$ has many varied functions in the inflammatory process. It activates neutrophils, eosinophils, and basophils. It also attracts $\mathrm{NK}, \mathrm{T}$, and dendritic cells to sites of infection. ${ }^{207}$ It also induces the synthesis and release of other pro-inflammatory cytokines from fibroblasts and macrophages. ${ }^{208}$ MIP- $1 \beta$ is generally produced by macrophages and T cells, and as previously stated in regards to IL2 , the significance in the attenuation of the lower levels of MIP-1 $\beta$ produced from endothelial and epithelial cells cannot be speculated. Interestingly, the production of MIP-1 $\beta$ from the macrophage-like transformed THP-1 cell line was not significantly affected by any of the eight compounds tested. Significant reductions in the chemokine IP-10 were also observed for both A549 and HUV-EC-C after TNF- $\alpha$ challenge (Figure 5.19). IP-10 attracts monocytes/macrophages, $N K, T$, and dendritic cells to sites of infection and also promotes T cell adhesion to endothelial cells. ${ }^{209}$ The triaryl HB-I-172 was also found to decrease the production of IL-8 from HUV-EC-C stimulated with either TNF- $\alpha$ or IL-1 $\beta$ (Figure 5.20). IL-8 is another major pro-inflammatory mediator. 


\section{LPS}

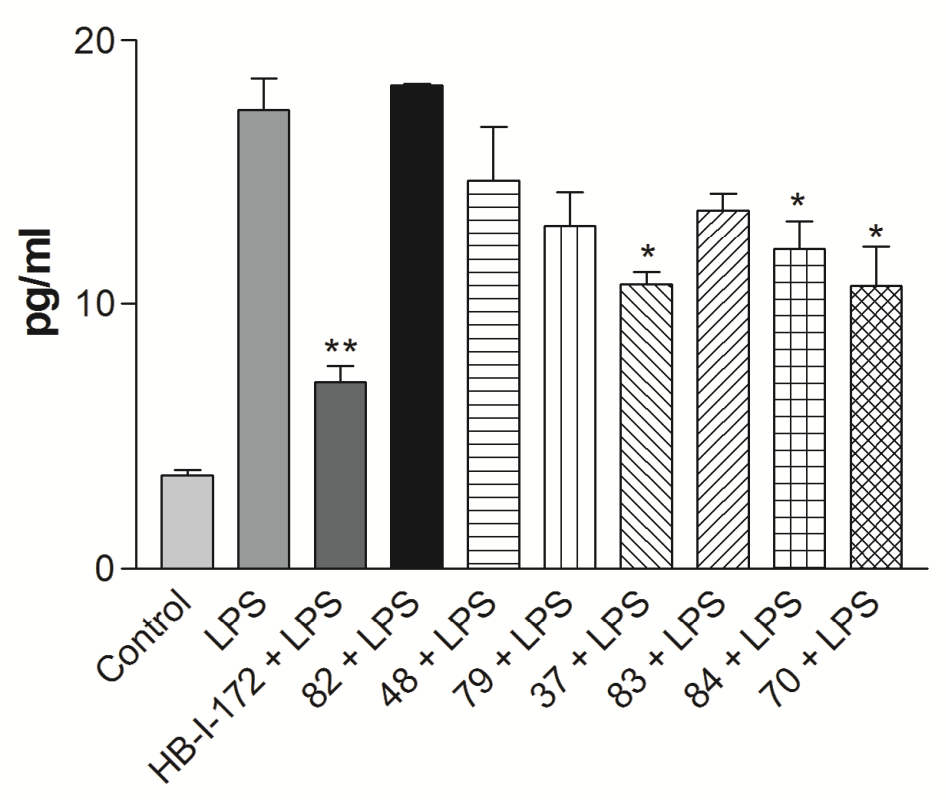

PGN

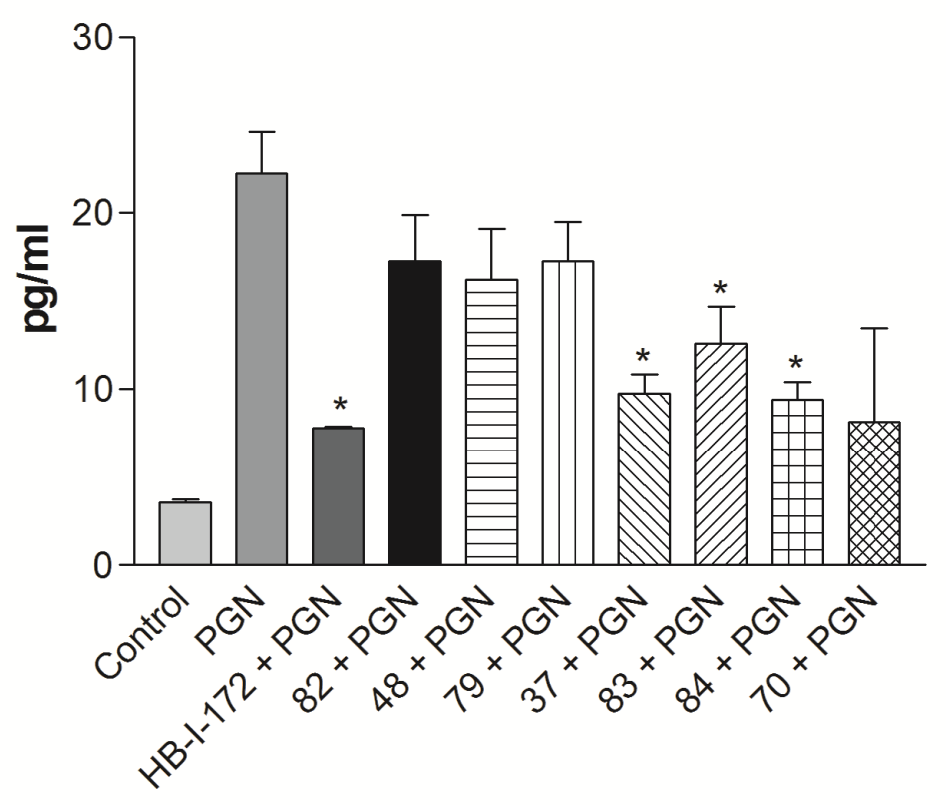

Figure 5.11: Effects of eight compounds on release of IL-12p70 from THP-1 in response to LPS and PGN

$*=\mathrm{p}<0.05$ and $* *=\mathrm{p}<0.01$ 


\section{LPS}

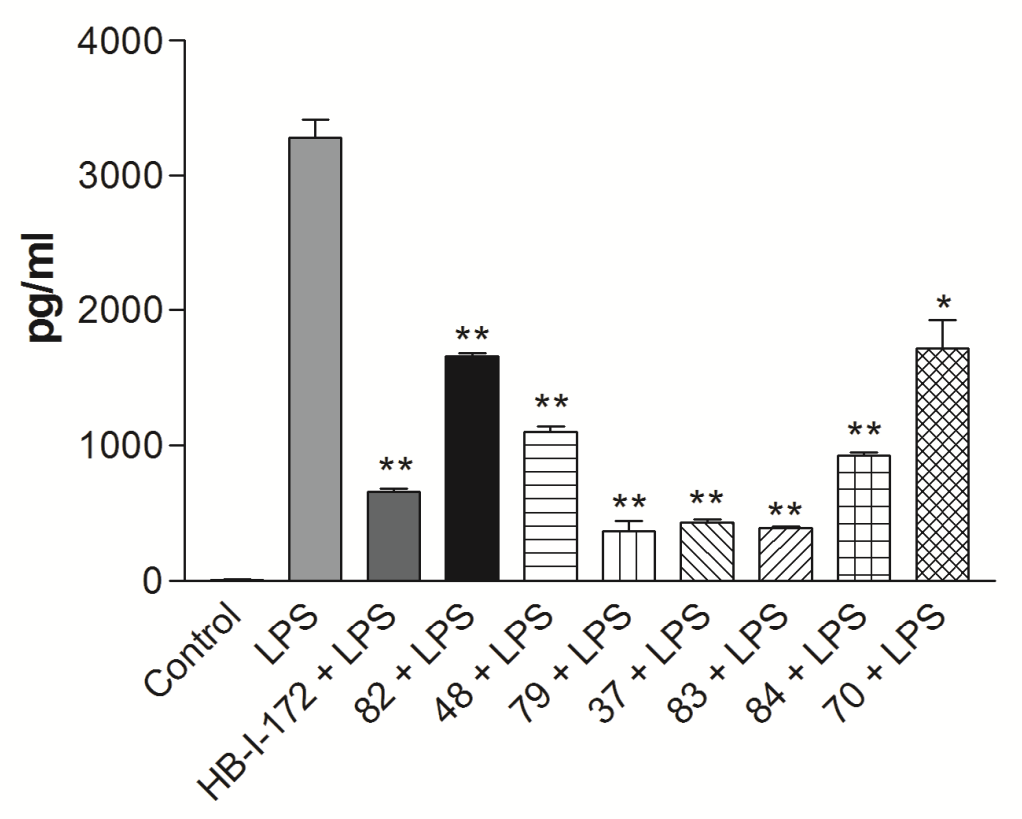

PGN

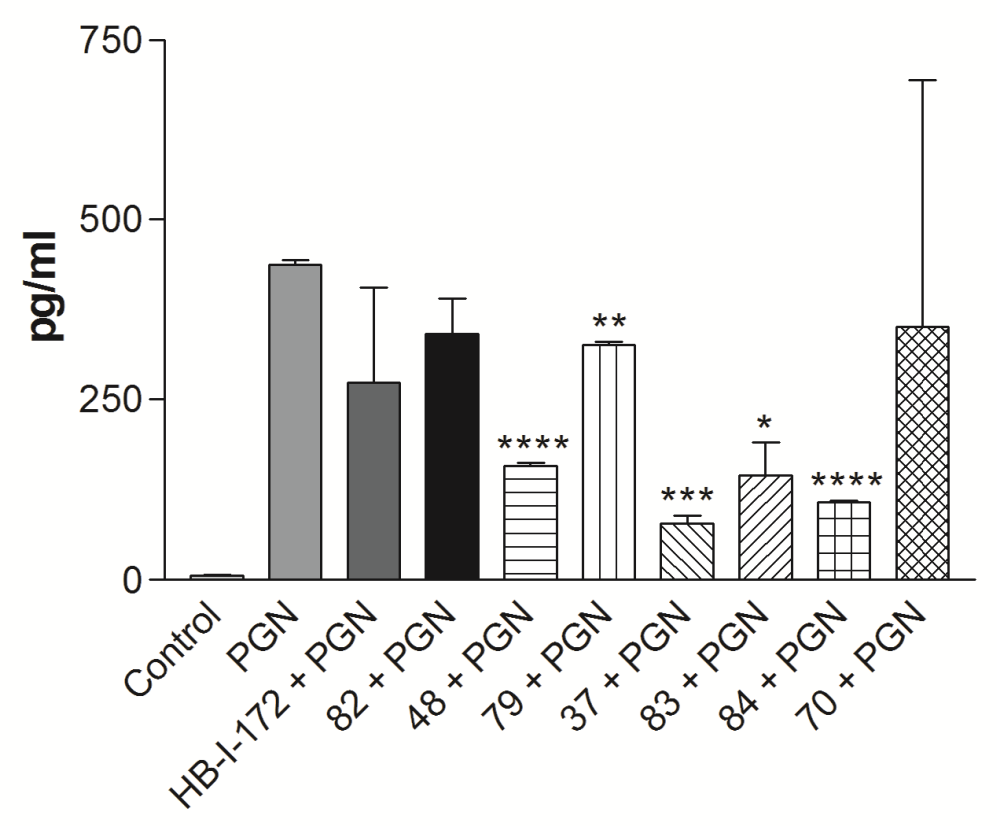

Figure 5.12: Effects of eight compounds on release of IL-6 from THP-1 in response to LPS and PGN

$$
*=\mathrm{p}<0.05, * *=\mathrm{p}<0.005, * * *=\mathrm{p}<0.001, \text { and } * * * *=\mathrm{p}<0.0005
$$




\section{GM-CSF}
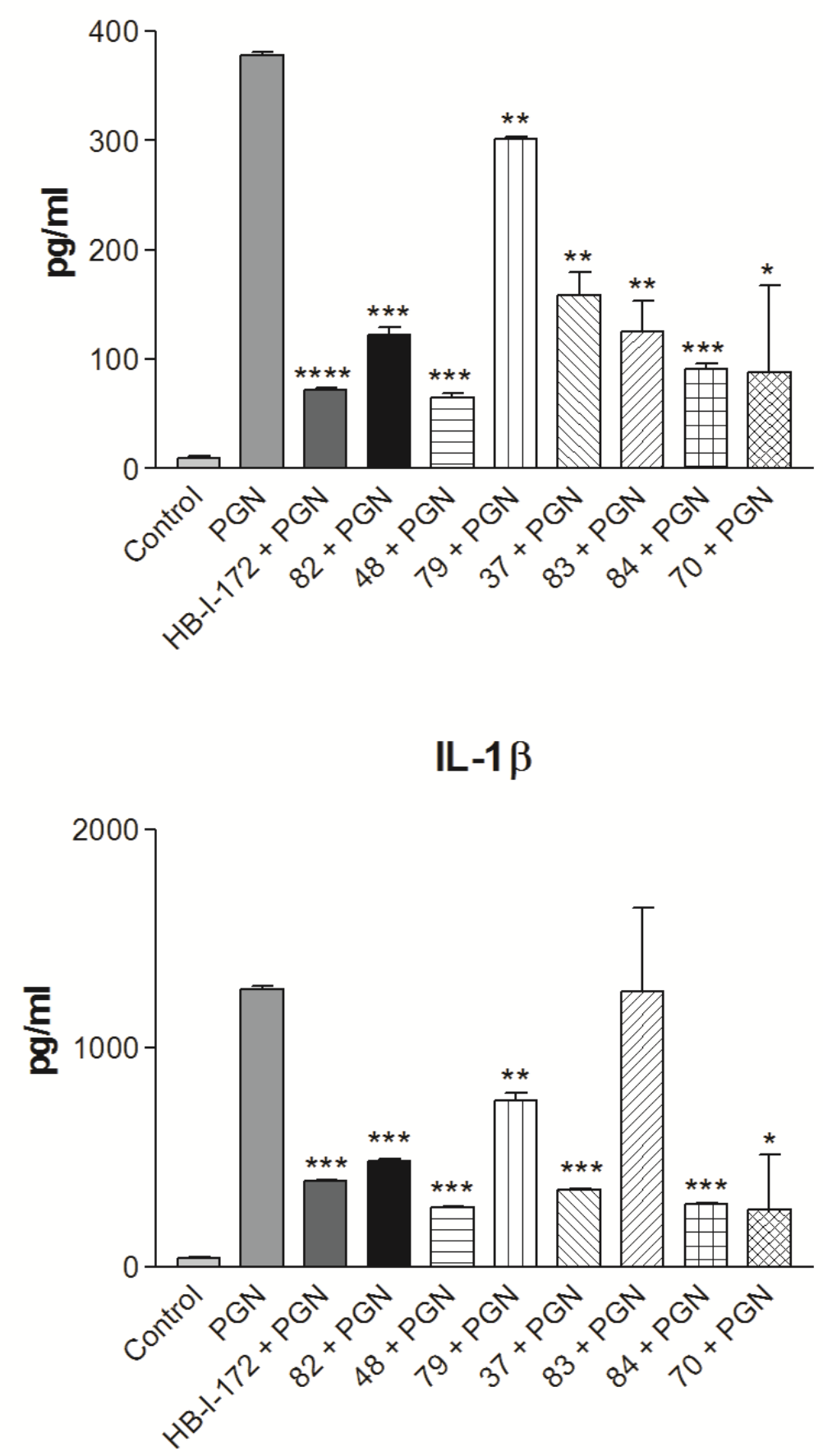

Figure 5.13: Effects of eight compounds on release of GM-CSF and IL- $1 \beta$ from THP-1 in response to PGN

$*=\mathrm{p}<0.05, * *=\mathrm{p}<0.005, * * *=\mathrm{p}<0.0005$, and $* * * *=\mathrm{p}<0.0001$. 


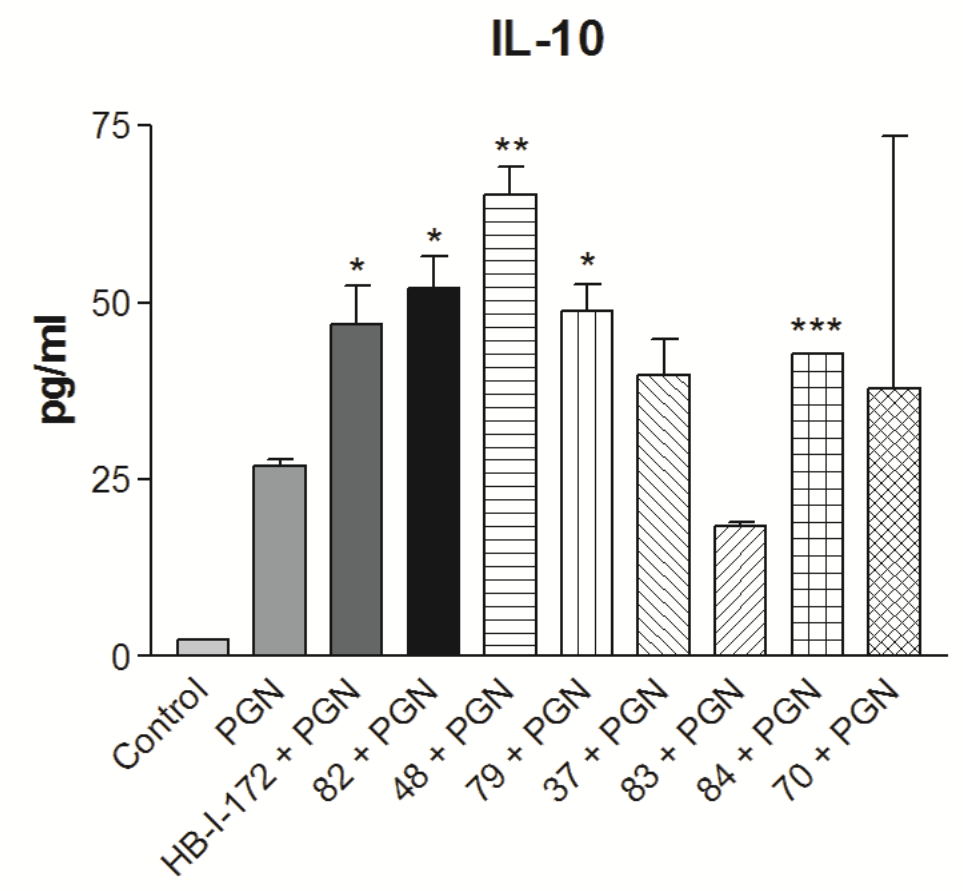

TNF- $\alpha$

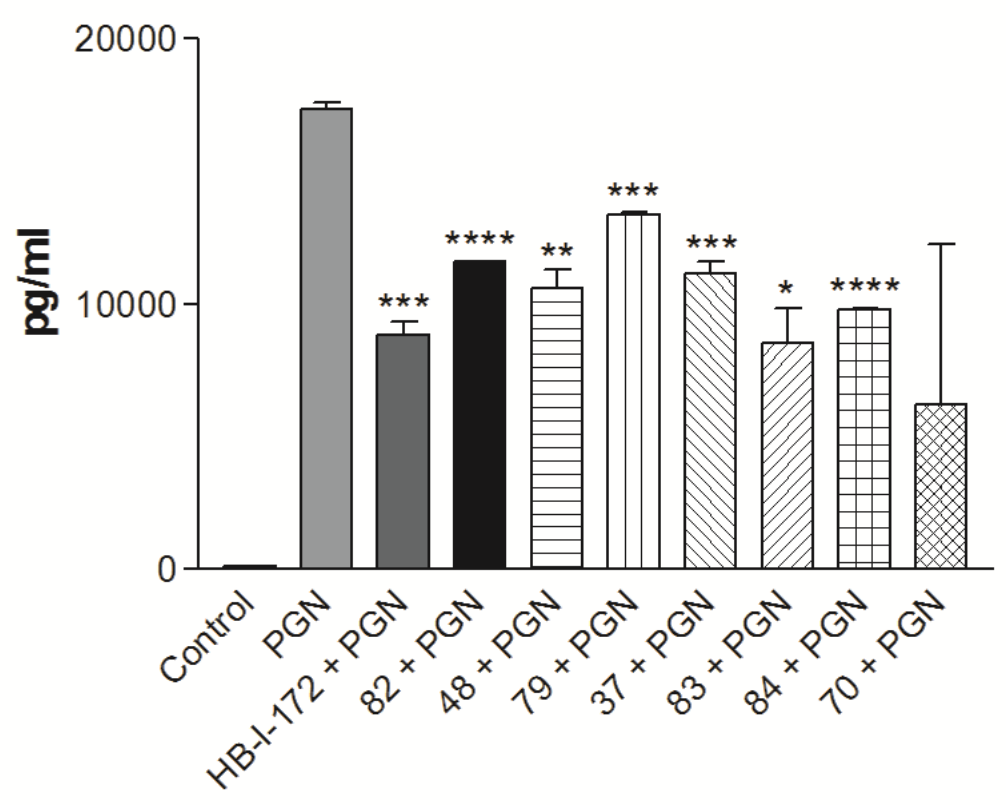

Figure 5.14: Effects of eight compounds on release of IL-10 and TNF- $\alpha$ from THP-1 in response to PGN

$*=\mathrm{p}<0.05, * *=\mathrm{p}<0.01, * * *=\mathrm{p}<0.005$, and $* * * *=\mathrm{p}<0.001$. 


\section{GM-CSF}

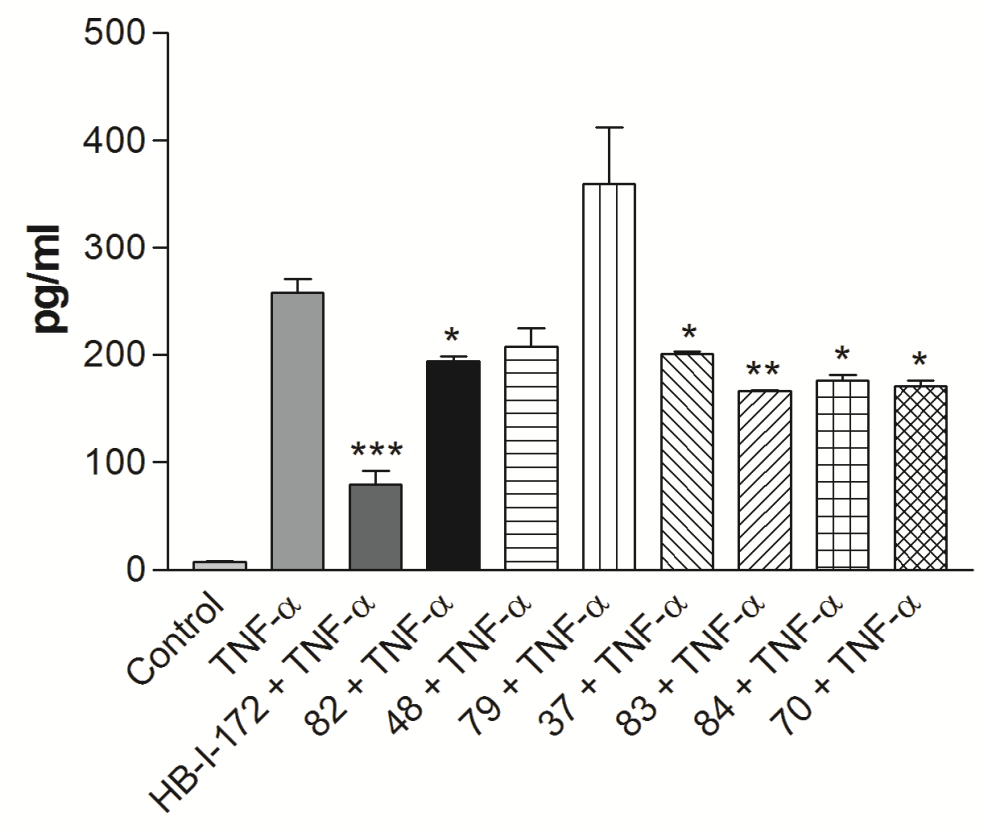

IL-6

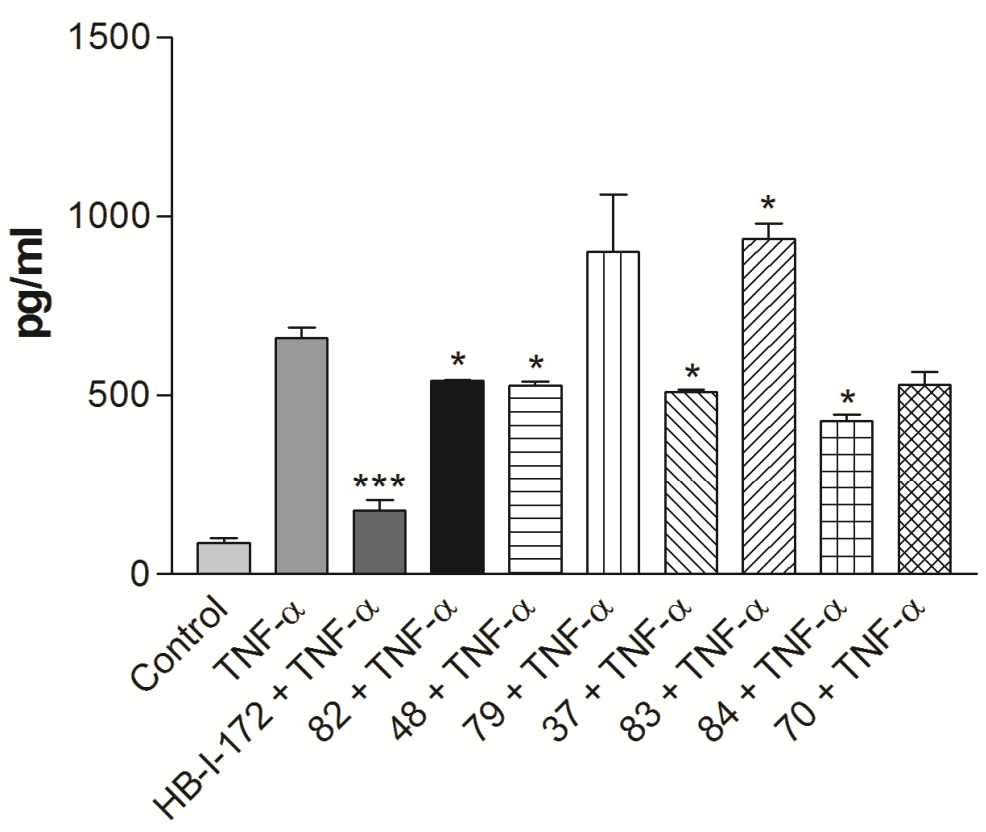

Figure 5.15: Effects of eight compounds on release of GM-CSF and IL-6 from HUVEC in response to TNF- $\alpha$

$$
*=\mathrm{p}<0.05, * *=\mathrm{p}<0.01 \text {, and } * * *=\mathrm{p}<0.005 \text {. }
$$


IFN $\gamma$

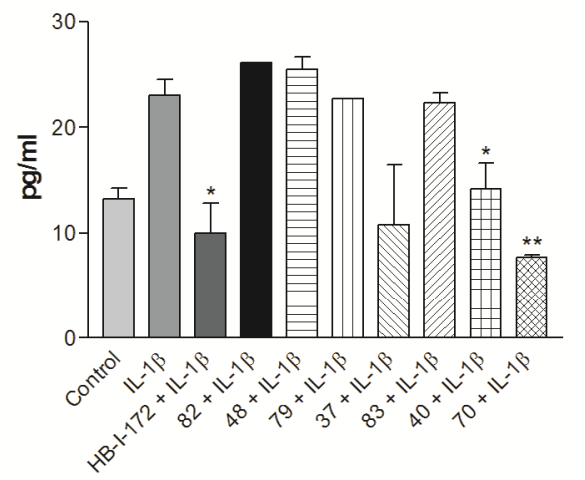

IL-12p70

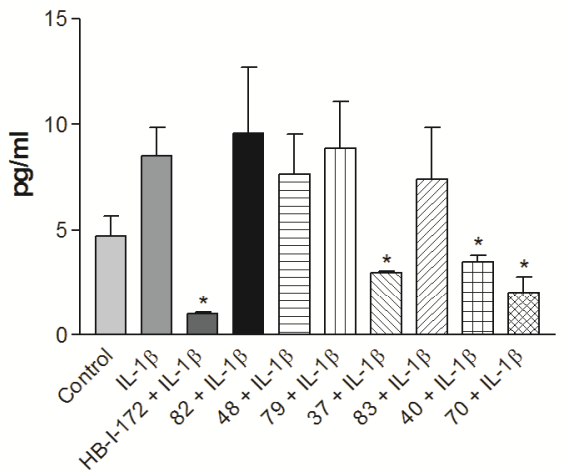

IL-2

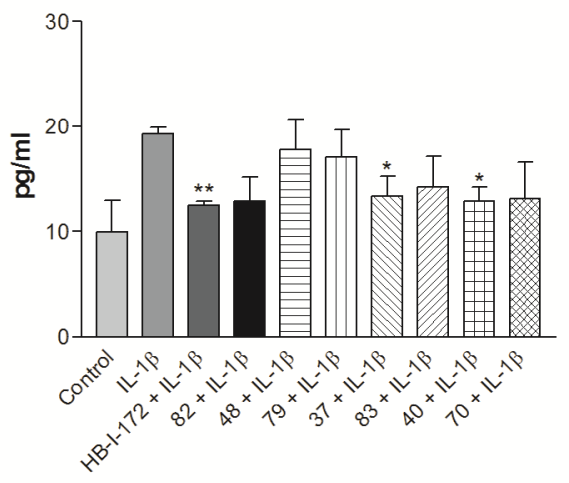

Figure 5.16: Effects of eight compounds on release of IFN- $\gamma$, IL-12p70, and IL-2 from HUVEC in response to IL-1 $\beta$

$$
*=\mathrm{p}<0.05 \text { and } * *=\mathrm{p}<0.005 \text {. }
$$


TNF- $\alpha$

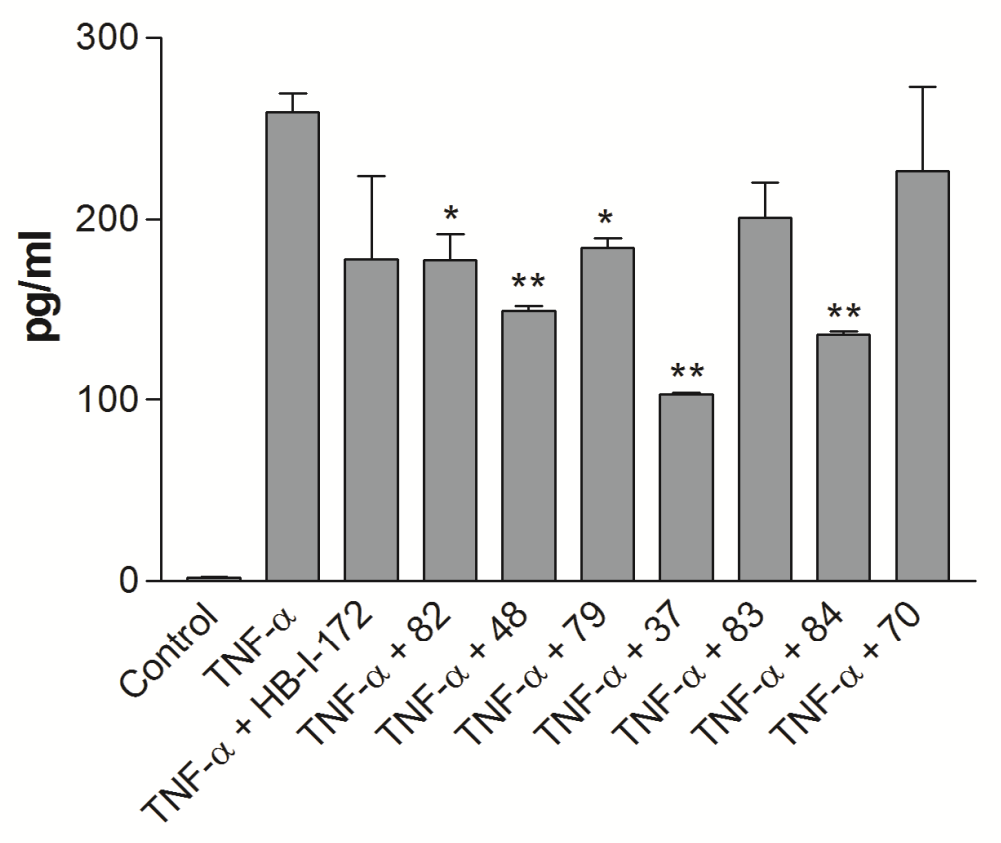

IL-1 $\beta$

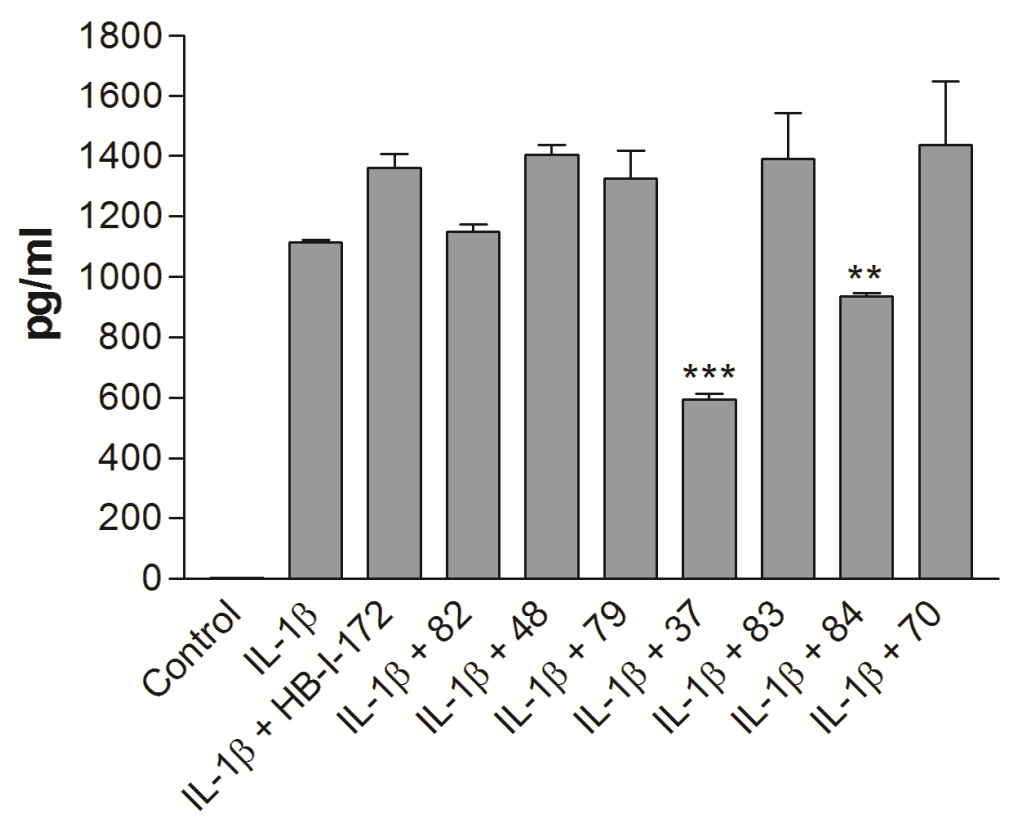

Figure 5.17: Effects of eight compounds on release of MIP-1 $1 \beta$ from A549 in response to TNF- $\alpha$ and IL- $1 \beta$

$$
*=\mathrm{p}<0.05, * *=\mathrm{p}<0.005 \text {, and } * * *=\mathrm{p}<0.001 \text {. }
$$


TNF- $\alpha$

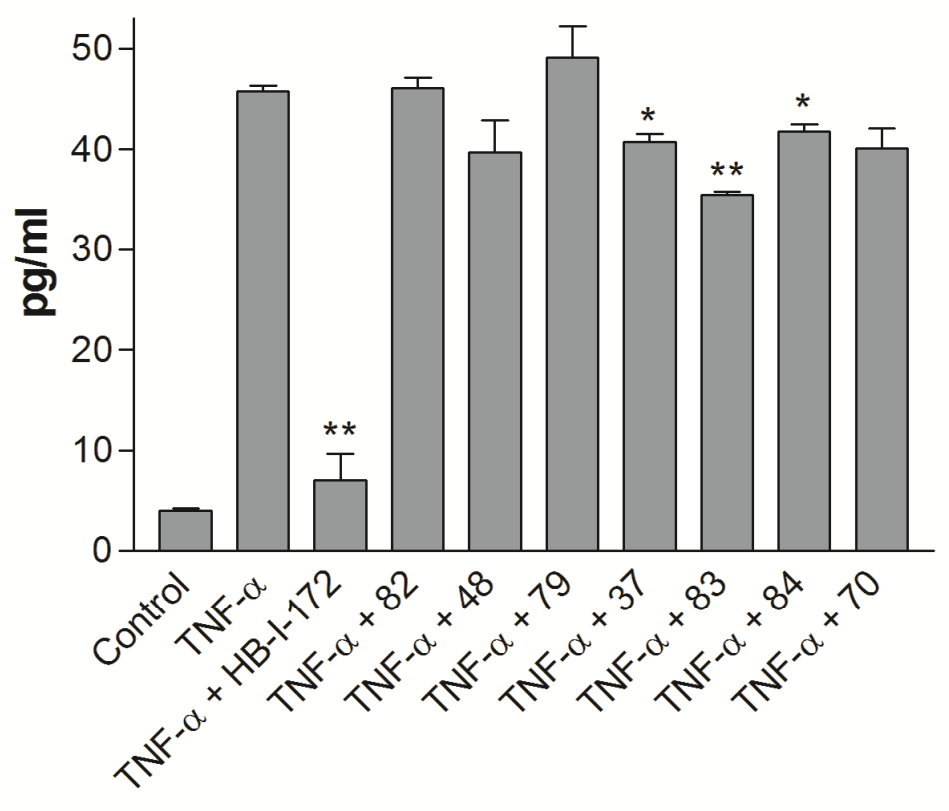

IL-1 $\beta$

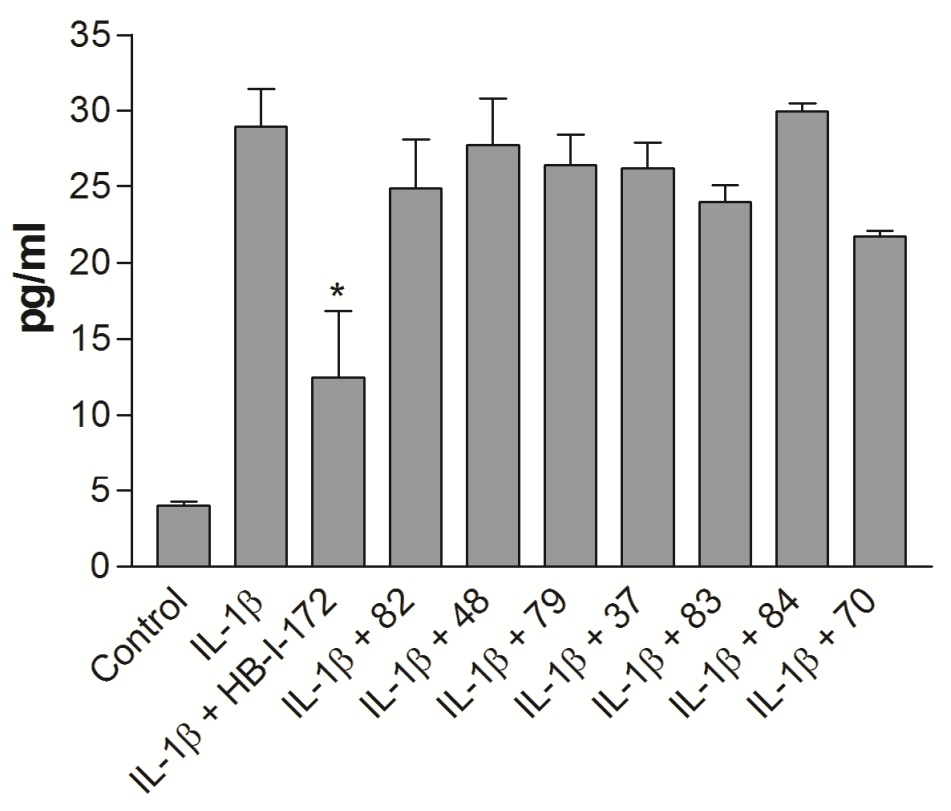

Figure 5.18: Effects of eight compounds on release of MIP- $1 \beta$ from HUVEC in response to TNF- $\alpha$ and IL- $1 \beta$

$*=\mathrm{p}<0.05$ and $* *=\mathrm{p}<0.005$ 
A549

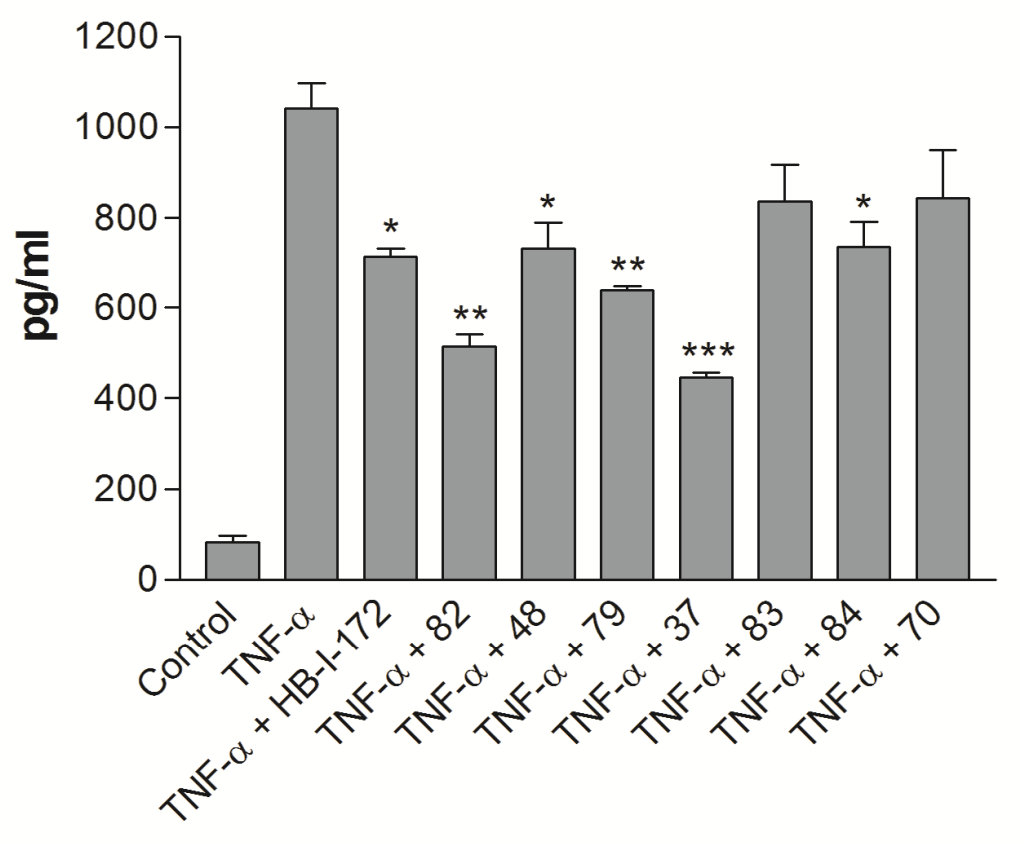

HUVEC

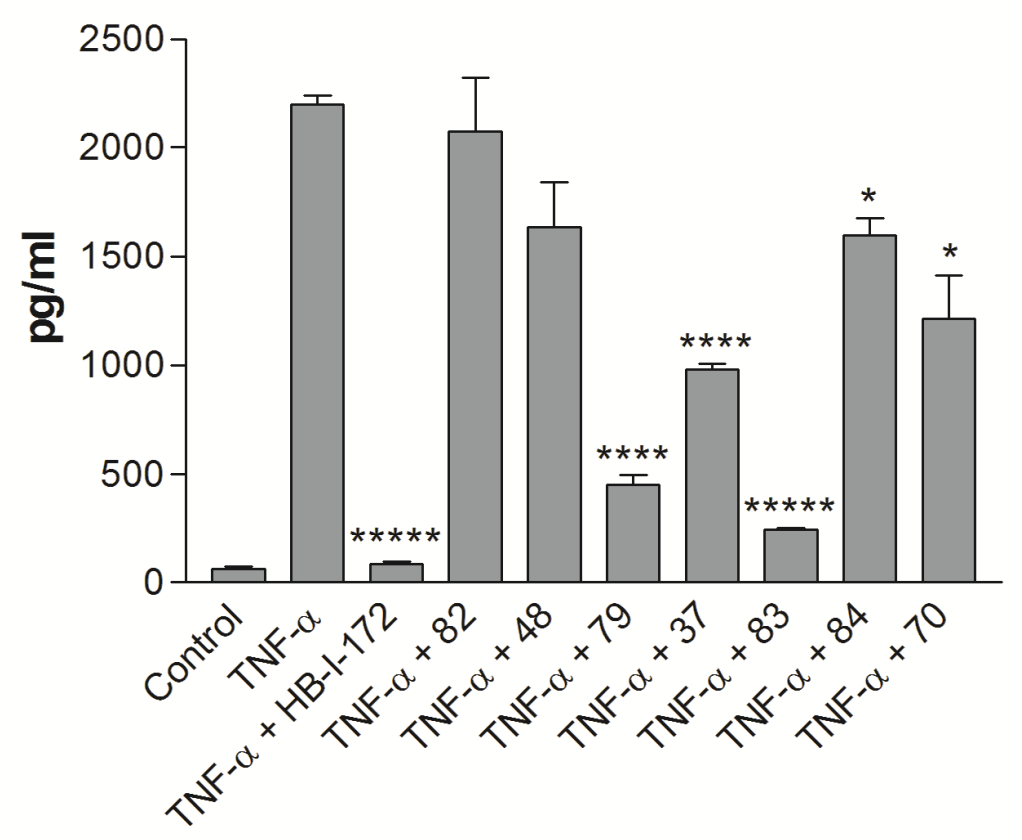

Figure 5.19: Effects of eight compounds on release of IP-10 from A549 and HUVEC in response to TNF- $\alpha$

$$
*=\mathrm{p}<0.05, * *=\mathrm{p}<0.01, * * *=\mathrm{p}<0.005, * * * *=\mathrm{p}<0.001, \text { and } * * * * *=\mathrm{p}<0.0005
$$


TNF- $\alpha$
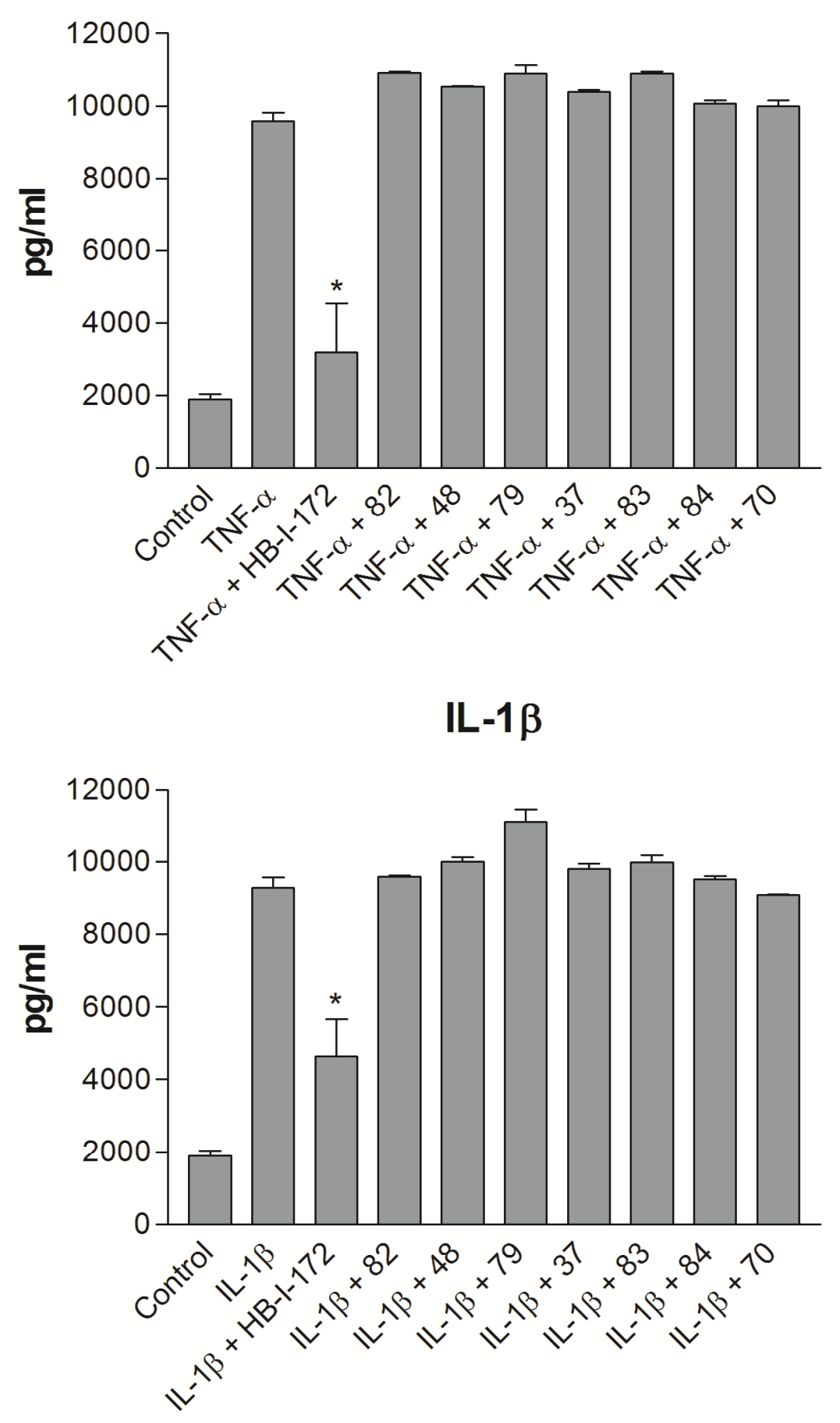

Figure 5.20: Effects of eight compounds on release of IL-8 from HUVEC in response to TNF- $\alpha$ and IL- $1 \beta$ $*=\mathrm{p}<0.05$. 
One of its main functions in the inflammatory response is to recruit neutrophils. Upon exposure to IL-8, neutrophils are mobilized and activated which is necessary for degranulation and the respiratory burst. ${ }^{210}$ Reductions in the chemokine MCP-1 were also observed in THP-1 (Figure 5.21) following LPS stimulation and in HUV-EC-C (Figure 5.22) following both TNF- $\alpha$ and IL-1 $\beta$ stimulation. MCP-1 activates macrophages, recruits monocytes, eosinophils, and basophils, and triggers histamine release from basophils. ${ }^{211}$ The action of this chemokine has been implicated in allergy, asthma, and arthritis, and a treatment that could effectively reduce the levels of this protein could be useful in those conditions. ${ }^{212}$

\subsubsection{Dose Response of Four Lead Compounds}

We decided to determine if the observed reductions in cytokines and chemokines due to the eight cannabinoids screened were dose dependant. The eight compounds were further narrowed down to four based on the compounds' overall effects of the cytokine and chemokine panels. The four that were chosen were the triaryl HB-I-172, the pyrimidyl and pyridyl hexahydros 37 and 79, and the benzofuran 83 (Figure 5.23). Two cell lines were chosen for the dose response study: THP-1 and HUV-EC-C and the compounds were screened in four escalating doses based on each compound's $\mathrm{EC}_{50}$ against each cell line. The highest concentration for each compound was the $\mathrm{EC}_{10}$ or $25 \mu \mathrm{M}$, whichever was lower.

Many of the drugs exhibited a dose dependent suppression of cytokine and chemokine release from THP-1 stimulated with LPS and HUV-EC-C stimulated with TNF- $\alpha$. The complete data set from this experiment may be found in Appendix C. Some examples of the dose dependent reduction are shown in Figures 5.24 and 5.25. The first graph depicts the dose dependent reduction in synthesis and secretion of Eotaxin and MCP-4 from THP-1 cells in response to HB-I-172 treatment post LPS exposure. The second graph shows the dose dependent reduction in Eotaxin-3 and TARC from THP-1 cells in response to treatment with the benzofuran 83. Interestingly, for some cytokines and chemokines, there was a greater reduction in levels due to the lowest concentration of certain drugs (see Appendix C).

\subsubsection{Further Evaluation of HB-I-172}

HB-I-172 was selected out of the four compounds screened due to its broad spectrum anti-inflammatory activity across the cell lines tested and also due to its novel inverse-agonist activity at the $\mathrm{CB} 2$ receptor coupled with being an antagonist at CB1. The previously described anti-inflammatory studies, with the exception of HUV-EC-C, utilized cancerous cell lines. The known abnormalities in the signaling cascades of cancers can overlap with the inflammatory pathways within the cell. For example the Akt and ERK pathways have been shown to be one signaling mechanism leading to NF$\kappa \beta$ translocation to the nucleus. ${ }^{213}$ 


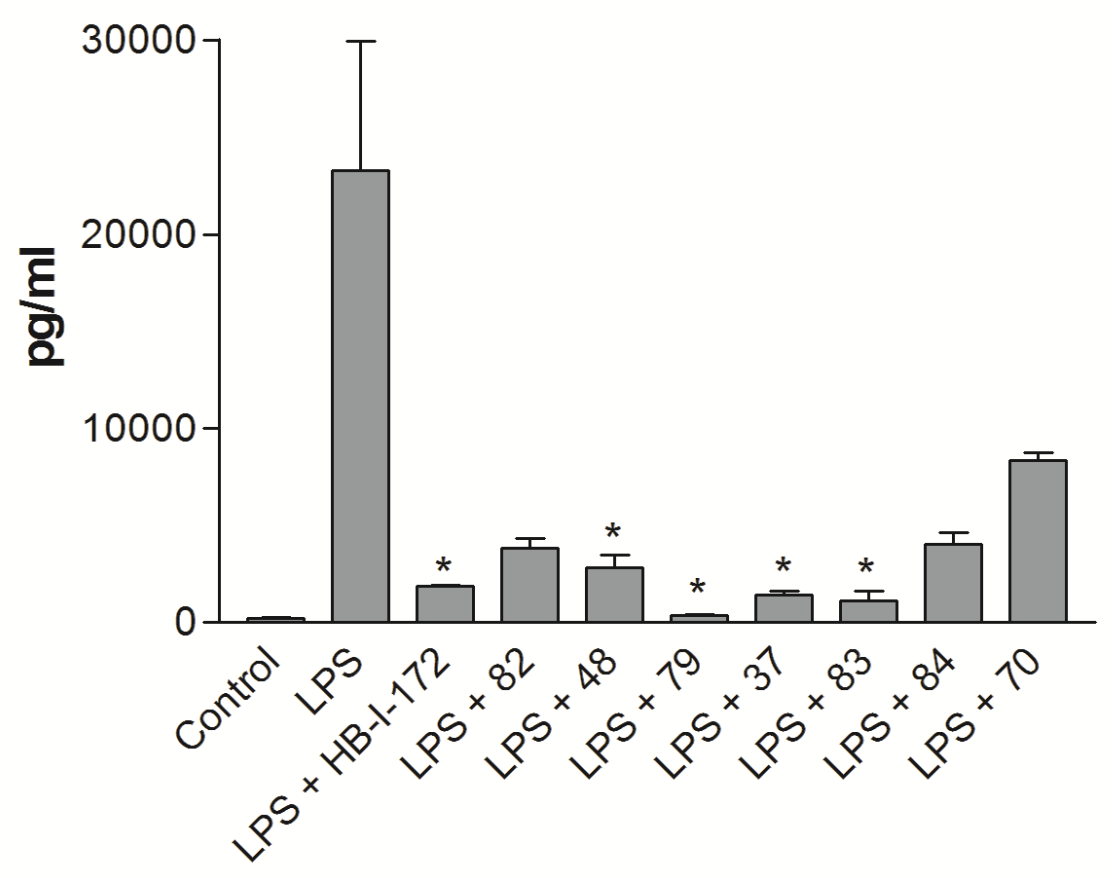

Figure 5.21: Effects of eight compounds on release of MCP-1 from THP-1 in response to LPS

$*=\mathrm{p}<0.05$. 
TNF- $\alpha$
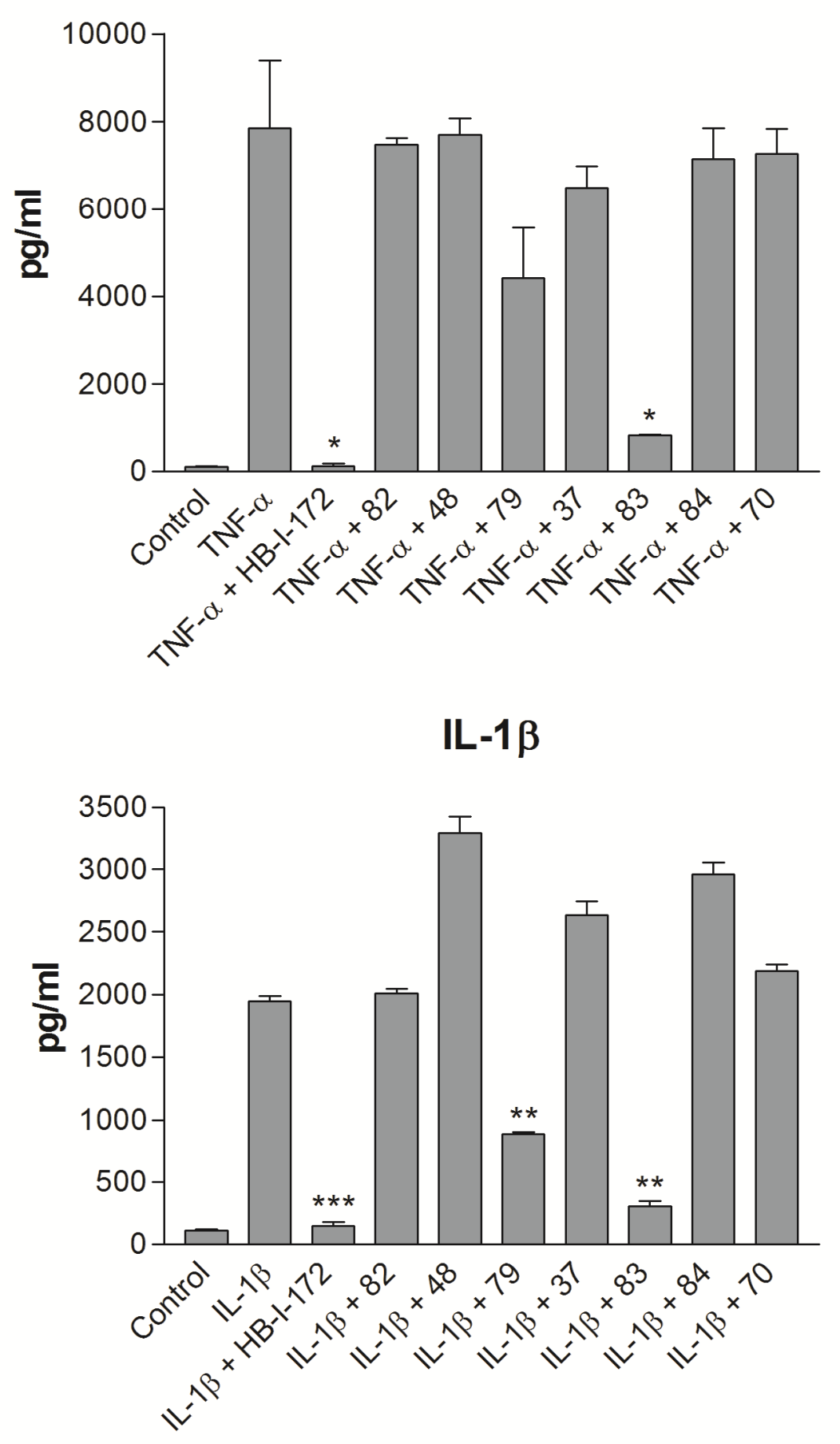

Figure 5.22: Effects of eight compounds on release of MCP-1 from HUVEC in response to TNF- $\alpha$ and IL- $1 \beta$

$*=\mathrm{p}<0.05, * *=\mathrm{p}<0.001$, and $* * *=\mathrm{p}<0.0005$. 


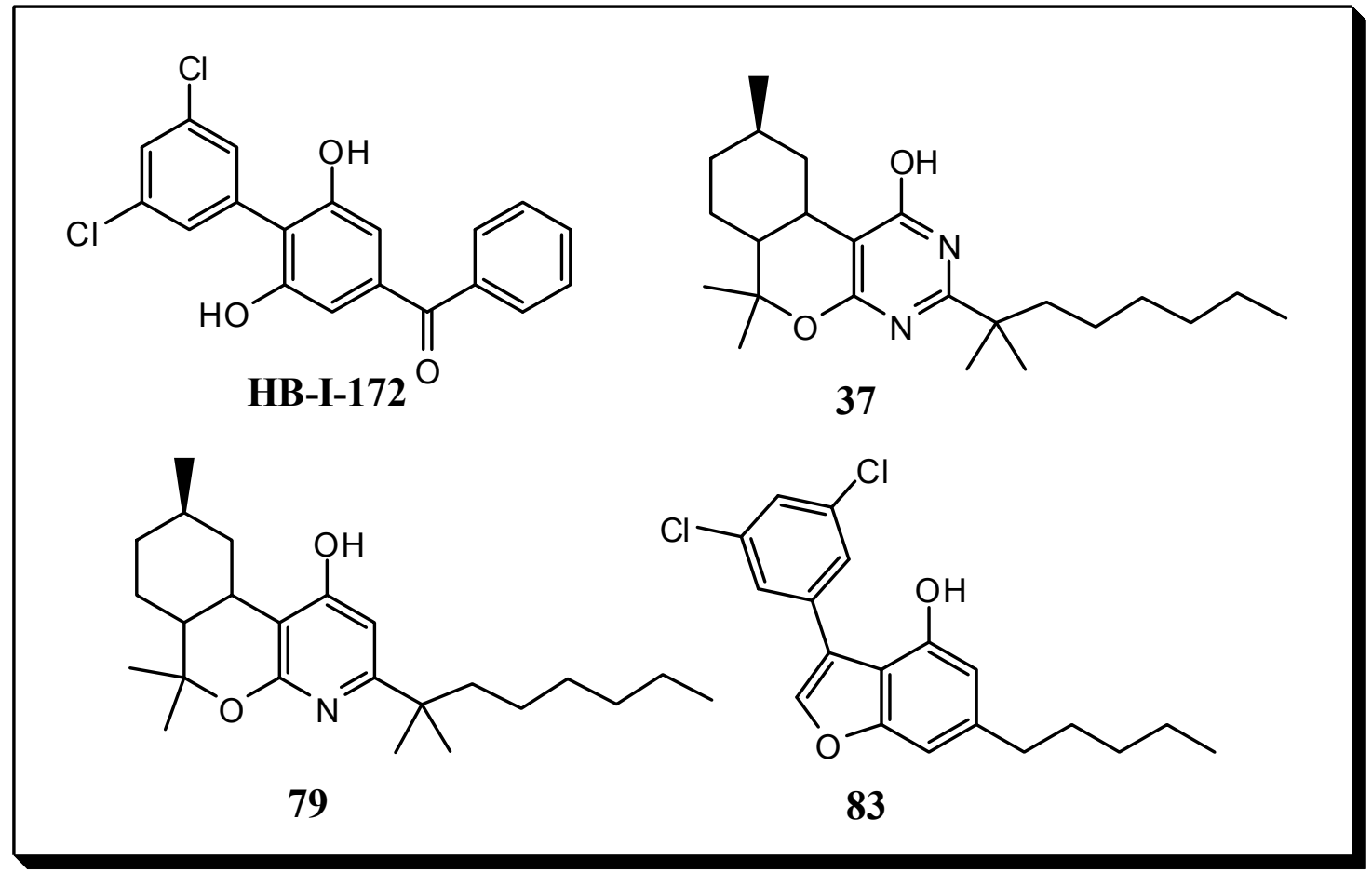

Figure 5.23: Four compounds chosen for dose response study 

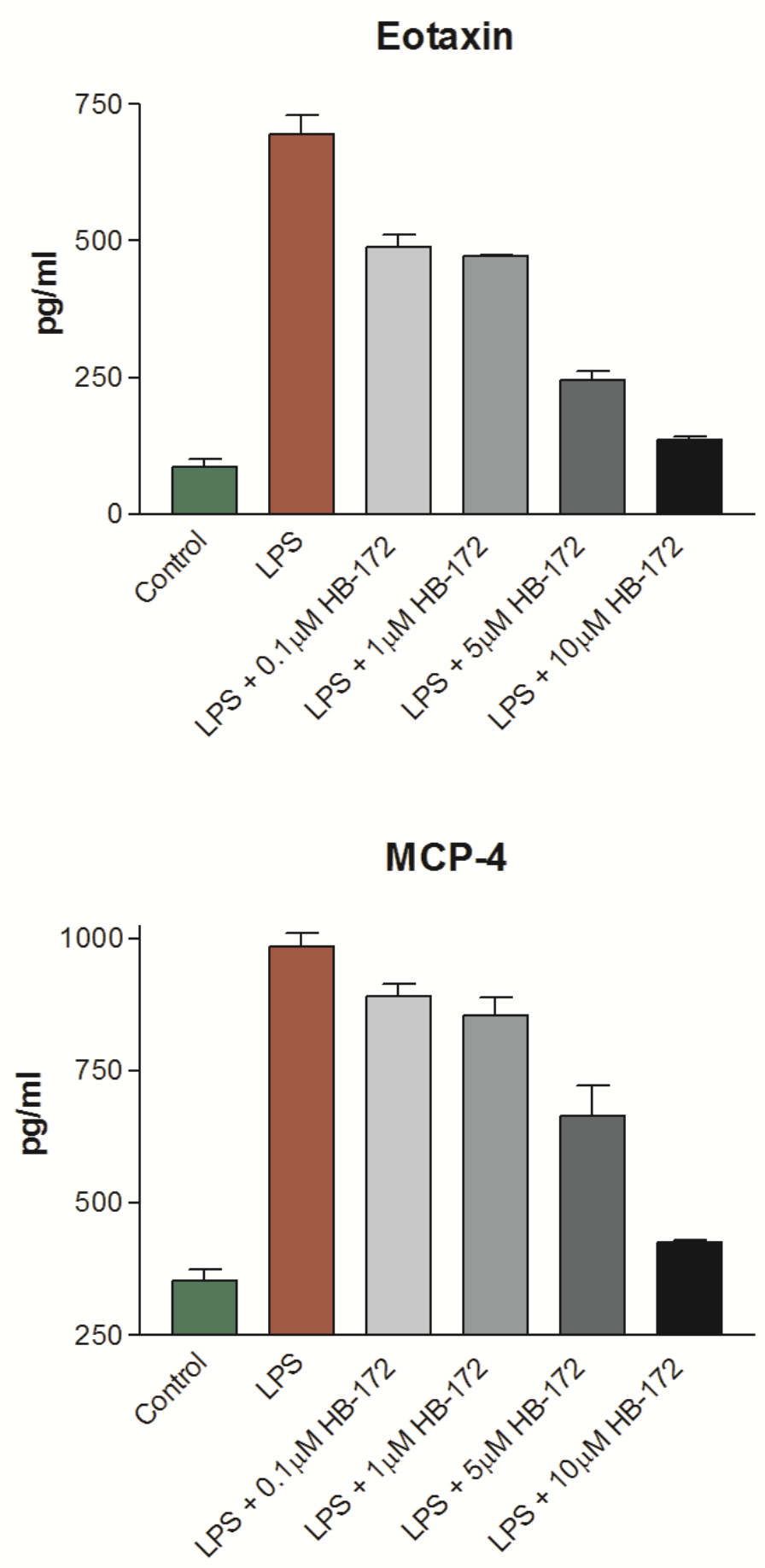

Figure 5.24: Examples of dose dependent reductions in chemokines produced by THP-1 after treatment with HB-I-172 


\section{Eotaxin-3}

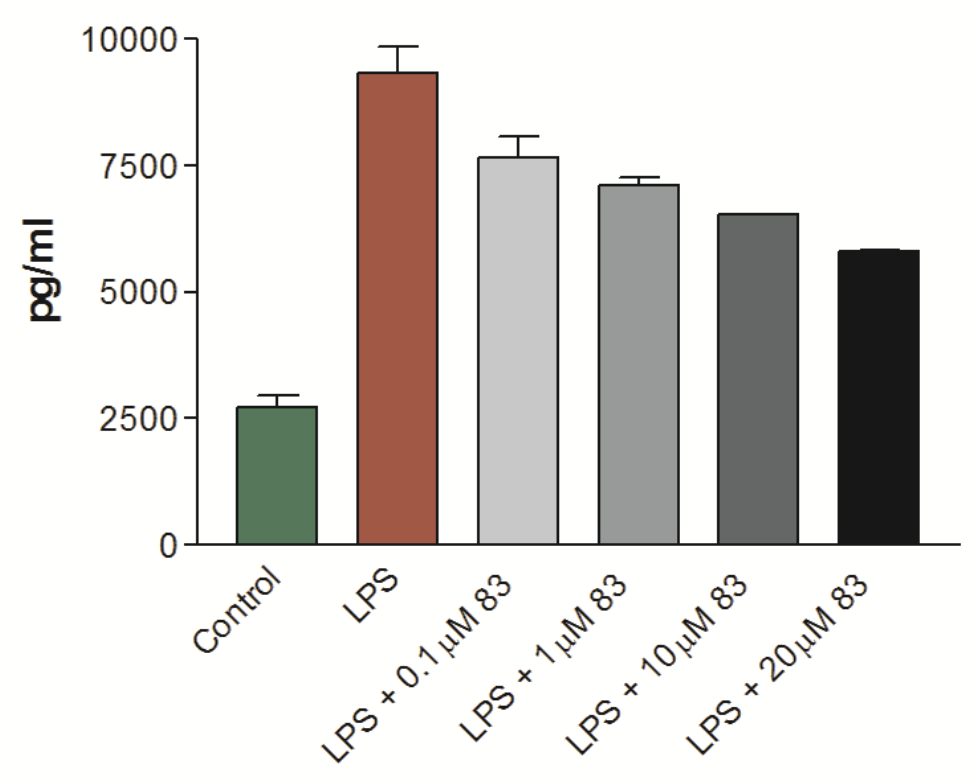

TARC

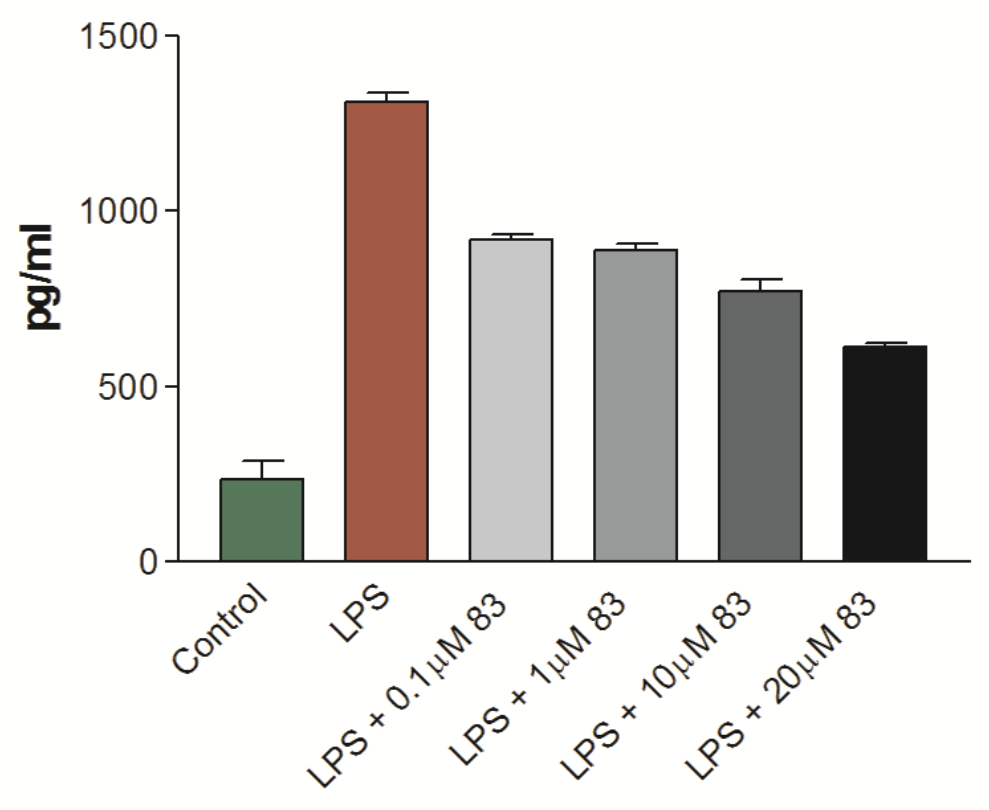

Figure 5.25: Examples of dose dependent reductions in chemokines produced by THP-1 after treatment with compound $\mathbf{8 3}$ 
In order to test HB-I-172 in a system that more closely resembles the responses of an in vivo environment, three human primary cell lines were used. HB-I-172 and the thiphenyl analog SMM-I-229 (Figure 5.26) were screened against human lung blood microvasculature endothelial cells (HMVEC-LBI, Lonza, Inc.), human small airway epithelial cells (SAEC, Lonza, Inc.), and human monocyte derived macrophages (AllCell, Inc.). SMM-I-229 possesses the same inverse agonist activity at CB2 as HB-I-172 and is also an antagonist at $\mathrm{CB} 1$.

Cytokine and chemokine release profiles were determined for the three cell lines, as with the initial experiments, and $\mathrm{EC}_{50} \mathrm{~s}$ were then determined for the two compounds against the cell lines. See Appendix D for the complete set of release profiles. The antiinflammatory assays were then carried out as before. The HMVEC-LBI and SAEC cell lines were stimulated with TNF- $\alpha$ and the primary macrophages were stimulated with LPS before drug treatment. The supernatant media was then assayed 18 hours later for levels of the same cytokines and chemokines. The complete set of graphs can be found in Appendix E. While the effects of HB-I-172 on the three primary cell lines were different than in the original cell lines tested, there were reductions in several of the proinflammatory cytokines and chemokines assayed for.

In order to test the possible contributions of cannabinoids in the treatment of neurological inflammatory disorders such as meningitis, Alzheimer's disease, Parkinson's disease, Huntington's disease, and neuropathic pain, we next tested HB-I172 and the CB1 agonist/CB2 antagonist classical cannabinoid KM-233 primary human microglia (Clonexpress) challenged with LPS. Again, the first step was to determine cytokine and chemokine release profiles for the cell line due to LPS exposure (Appendix F). The $\mathrm{EC}_{50} \mathrm{~S}$ were then determined for the two drugs against the cells and the assay performed as before. HB-I-172 reduced the levels of a majority of the cytokines and chemokines assayed including IFN- $\gamma$, IL-6, and MCP-1 which have all been found to play roles in a number of neuro-inflammatory conditions ${ }^{214}$ (Appendix G). KM-233 also performed well in this assay. There were, however, differences in the anti-inflammatory profile of the two drugs, probably due to the different functional activities at the cannabinoid receptors.

In order to strengthen the case for the use of CB2 inverse agonists as antiinflammatory agents, we chose to compare HB-I-172 to the clinically utilized antiinflammatory drug dexamethasone. HB-I-172 was compared to dexamethasone in the THP-1 cell line stimulated with LPS and in A549 and HMVEC-LBI stimulated with TNF- $\alpha$. In the majority of cytokines and chemokines assayed for, HB-I-172 performed as well or better than dexamethasone (See Appendix $\mathbf{H}$ for complete data set).

In conclusion, we have demonstrated that HB-I-172 is a viable candidate for development as an anti-inflammatory agent. The compound has exhibited in vitro reductions in the production and secretion of a wide range of cytokines and chemokines from a variety of cell types involved in the inflammatory process. Not only have we demonstrated that the compound is efficacious in standard inflammatory models using cancerous cell lines (THP-1, KG-1, and A549) but that this activity also translates into efficacy when normal human primary cells are utilized and when compared to a standard therapy. A number of future studies are planned to further evaluate the utility of HB-I- 


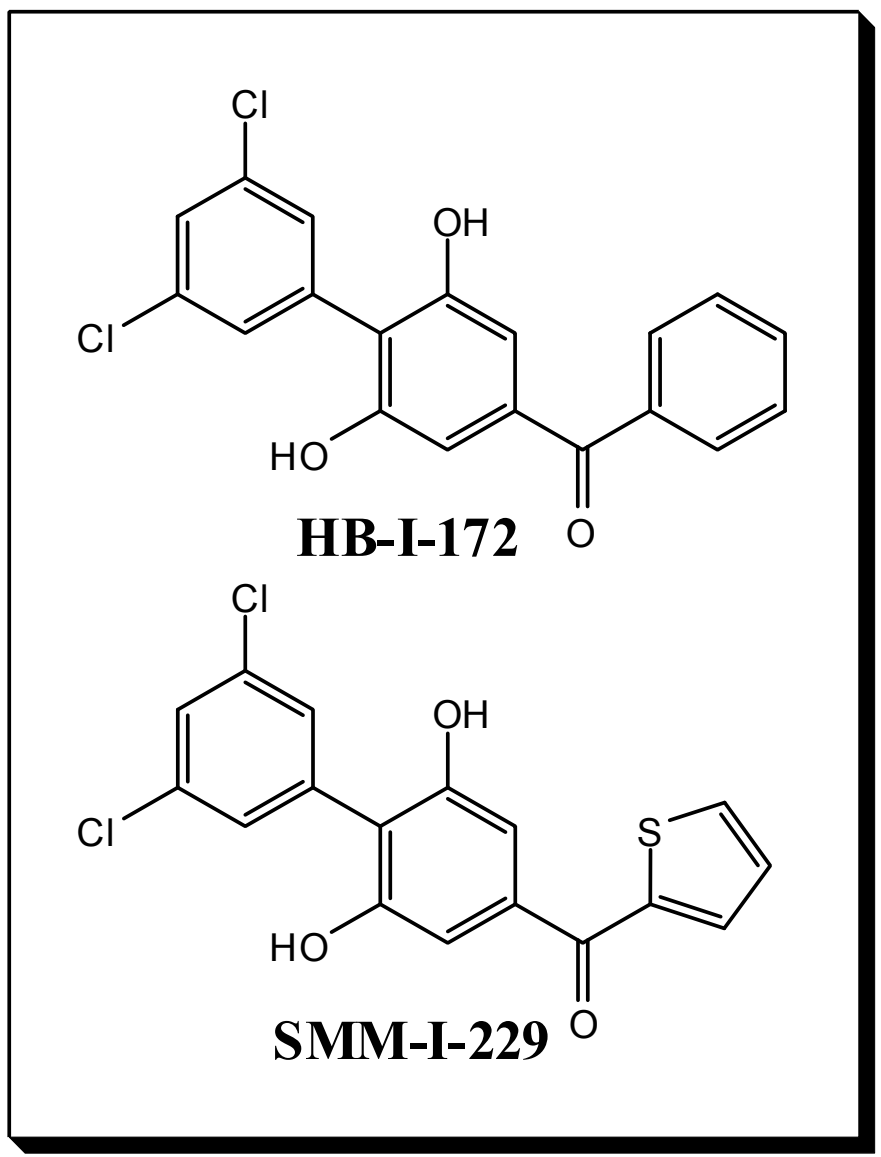

Figure 5.26: $\mathrm{CB} 2$ inverse agonists screened as anti-inflammatory agents against human primary macrophages, epithelial, and endothelial cells 
172 as an anti-inflammatory agent. These include an experiment to test the compound's ability to favorably modulate the immune response of $\mathrm{T}$ and $\mathrm{B}$ cells by observing the effects on antigen receptor-dependent activation using murine splenocytes, an in vivo endotoxemia model, and murine models of Huntington's disease, multiple sclerosis, and neuropathic pain. Future studies are also planned to determine the exact molecular target of HB-I-172 that is responsible for the anti-inflammatory activity and the signaling pathway that this activates. Knowledge gained from the work with HB-I-172 in the treatment of glioblastoma (Chapter 3) gives us some clues as to possible signaling mediators involved. As stated earlier, the Akt and ERK pathways have been shown to be one signaling mechanism that leads to NF- $\kappa \beta$ translocation to the nucleus during the inflammatory response. The profound effects of HB-I-172 on the PI3K/Akt and Raf1/MEK/ERK pathways in glioma may also be found in cells treated with the compound following inflammatory challenge. As observed in glioma, the effects of HBI-172 on STAT3 and GSK-3 $\beta$ may also play a role, as each of these proteins has been shown to be mediators of inflammation. ${ }^{215}$ We also believe that the possible interaction of HB-I-172 with the GPR55 receptor may contribute to its action as RhoA has been shown to contribute to NF- $\kappa \beta$-dependent proinflammatory gene transcription. ${ }^{216}$

\subsection{Experimental Methods}

\subsubsection{Transformation of THP-1 and KG-1}

Differentiation of Monocytes: To THP-1 human leukemia monocytes (ATCC \#TIB-202) or KG-1 human leukemia myeloblasts (ATCC \#CCL-246) in suspension was added phorbol 12-myristate 13-acetate (PMA Aldrich \#P1585) and ionomycin (Aldrich \#I0634), 10 and $500 \mathrm{ng} / \mathrm{ml}$ respectively, to induce differentiation into macrophage-like or dendritic-like cells, respectively. Cells were seeded at 30,000 cells/well in a 96-well polystyrene dish and allowed to incubate at $37^{\circ} \mathrm{C}$ in $5 \% \mathrm{CO} 2 / 95 \%$ air for $5-10$ days to complete transformation. Media was refreshed as needed until assay.

\subsubsection{Cytokine and Chemokine Assays}

A549 (ATCC \#CCL-185), HUV-EC-C (ATCC \#CRL-1730), HMVEC-LBI (Lonza \#CC-2815), SAEC (Lonza \#CC-2547), monocyte derived macrophages (AllCells \#PB-MDM-001F), microglia (Clonexpress \#HMG 030), or differentiated THP-1 or KG-1 cells were maintained according to suppliers recommendations and were seeded on 96well polystyrene plates at a density of $300,000 \mathrm{cells} / \mathrm{ml}(100 \mu \mathrm{L}$ per well) and incubated at $37^{\circ} \mathrm{C}$ in $5 \% \mathrm{CO} 2 / 95 \%$ air for 24 hours to allow cell attachment. Drug solutions were prepared in DMSO at 100x concentration and mixed 1:100 in media containing 1\% FBS to yield the desired concentration. Plates were then removed from the incubator and the complete growth media was replaced with $50 \mu \mathrm{L}$ media containing $1 \% \mathrm{FBS}$ and lipopolysaccharide or peptidoglycan at $1 \mu \mathrm{g} / \mathrm{ml}$ (for macrophages, microglia, or differentiated THP-1 or KG-1), or TNF- $\alpha(10 \mathrm{ng} / \mathrm{ml})$ or IL-1 $\beta(1 \mathrm{ng} / \mathrm{ml})$ in the case of 
A549, HUV-EC-C, HMVEC-LBI, or SAEC or without stimulus in the case of control wells. Cells were returned to the incubator for 1 hour before drug treatments. Drugmedia solutions were prepared at $2 \mathrm{x}$ desired final concentration in media containing $1 \%$ FBS and the appropriate stimulus at the previously mentioned concentration. Control media was also prepared which contained only vehicle (DMSO). $50 \mu \mathrm{L}$ of media containing drug or vehicle alone was then added to appropriate wells and the plates returned to the incubator for 18 hours. Media supernatants were then assayed for cytokines and chemokines using the Human ProInflammatory 9-PlexTissue Culture Kit (MSD \# K15007B-1) and the Human Chemokine 9-PlexTissue Culture Kit (MSD \# K15001B-1) according to the manufacturer's protocol. The plates were then imaged on the SECTOR Imager 2400 (Meso Scale Dscovery) according to the manufacturer's instructions. 


\section{List of References}

1. Mechoulam, R., Cannabinoids as Therapeutic Agents. CRC Press: Boca Raton, Florida, 1986.

2. (a) Mechoulam, R.; Hanus, L.; Fride, E., Towards Cannabinoid Drugs-Revisited. Elsevier: Amsterdam, Netherlands, 1998; (b) Walker, J. M.; Hohmann, A. G.; Martin, W. J.; Strangman, N. M.; Huang, S. M.; Tsou, K., The neurobiology of cannabinoid analgesia. Life Sci 1999, 65 (6-7), 665-73.

3. Kumar, R. N.; Chambers, W. A.; Pertwee, R. G., Pharmacological actions and therapeutic uses of cannabis and cannabinoids. Anaesthesia 2001, 56 (11), 105968.

4. Izzo, A. A.; Coutts, A. A., Cannabinoids and the digestive tract. Handb Exp Pharmacol 2005, 168, 573-98.

5. Croxford, J. L.; Yamamura, T., Cannabinoids and the immune system: potential for the treatment of inflammatory diseases? J Neuroimmunol 2005, 166 (1-2), 318.

6. Wood, T. B.; Newton Spivey, W. T.; Easterfield, T. H., Charas., The resin of Indian hemp. J Chem Soc 1896, 69, 539.

7. Cahn, R. S., Cannabis indica resin. Part IV. The synthesis of some 2:2-dimethyl dibenzopyrans and confirmation of the structure of cannabinol. J Chem Soc 1933, Pt 2, 1400-5.

8. (a) Adams, R.; Baker, B. R.; Wearn, R. B., Structure of cannabinol. III. Synthesis of cannabinol, 1-hydroxy-3-n-amyl-6,6,9-trimethyl-6-dibenzopyran. JACS 1940, 62 (8), 2204; (b) Adams, R.; Loewe, S., New analogs of tetrahydrocannabinol.XIX. JACS 1949, 71 (5), 1624.

9. Todd, A. R., Hashish. Experientia 1946, 2, 55.

10. Gaoni, Y.; Mechoulam, R., Isolation, structure and partial synthesis of an active constituent of hashish. JACS 1964, 86 (8), 1646.

11. Howlett, A. C.; Barth, F.; Bonner, T. I.; Cabral, G.; Casellas, P.; Devane, W. A.; Felder, C. C.; Herkenham, M.; Mackie, K.; Martin, B. R.; Mechoulam, R.; Pertwee, R. G., International Union of Pharmacology. XXVII. Classification of cannabinoid receptors. Pharmacol Rev 2002, 54 (2), 161-202.

12. (a) Mechoulam, R.; Shani, A.; Edery, H.; Grunfeld, Y., Chemical basis of hashish activity. Science 1970, 169 (945), 611-2; (b) Grunfeld, Y.; Edery, H.,

Psychopharmacological activity of the active constituents of hashish and some related cannabinoids. Psychopharmacologia 1969, 14 (3), 200-10.

13. Paton, W. D., Pharmacology of marijuana. Annu Rev Pharmacol 1975, 15, 191220.

14. Mechoulam, R.; Feigenbaum, J. J.; Lander, N.; Segal, M.; Jarbe, T. U.; Hiltunen, A. J.; Consroe, P., Enantiomeric cannabinoids: stereospecificity of psychotropic activity. Experientia 1988, 44 (9), 762-4.

15. Devane, W. A.; Dysarz, F. A., 3rd; Johnson, M. R.; Melvin, L. S.; Howlett, A. C., Determination and characterization of a cannabinoid receptor in rat brain. $\mathrm{Mol}$ Pharmacol 1988, 34 (5), 605-13. 
16. Matsuda, L. A.; Lolait, S. J.; Brownstein, M. J.; Young, A. C.; Bonner, T. I., Structure of a cannabinoid receptor and functional expression of the cloned cDNA. Nature 1990, 346 (6284), 561-4.

17. Munro, S.; Thomas, K. L.; Abu-Shaar, M., Molecular characterization of a peripheral receptor for cannabinoids. Nature 1993, 365 (6441), 61-5.

18. Shire, D.; Carillon, C.; Kaghad, M.; Calandra, B.; Rinaldi-Carmona, M.; Le Fur, G.; Caput, D.; Ferrara, P., An amino-terminal variant of the central cannabinoid receptor resulting from alternative splicing. J Biol Chem 1995, 270 (8), 3726-31.

19. Welch, S. P.; Eads, M., Synergistic interactions of endogenous opioids and cannabinoid systems. Brain Res 1999, 848 (1-2), 183-90.

20. Di Marzo, V.; Breivogel, C. S.; Tao, Q.; Bridgen, D. T.; Razdan, R. K.; Zimmer, A. M.; Zimmer, A.; Martin, B. R., Levels, metabolism, and pharmacological activity of anandamide in $\mathrm{CB}(1)$ cannabinoid receptor knockout mice: evidence for non- $\mathrm{CB}(1)$, non- $\mathrm{CB}(2)$ receptor-mediated actions of anandamide in mouse brain. J Neurochem 2000, 75 (6), 2434-44.

21. Zimmer, A.; Zimmer, A. M.; Hohmann, A. G.; Herkenham, M.; Bonner, T. I., Increased mortality, hypoactivity, and hypoalgesia in cannabinoid $\mathrm{CB} 1$ receptor knockout mice. Proc Natl Acad Sci U S A 1999, 96 (10), 5780-5.

22. (a) Lauckner, J. E.; Jensen, J. B.; Chen, H. Y.; Lu, H. C.; Hille, B.; Mackie, K., GPR55 is a cannabinoid receptor that increases intracellular calcium and inhibits M current. Proc Natl Acad Sci U S A 2008, 105 (7), 2699-704; (b) Pertwee, R. G., GPR55: a new member of the cannabinoid receptor clan? Br J Pharmacol 2007, 152 (7), 984-6; (c) Ryberg, E.; Larsson, N.; Sjogren, S.; Hjorth, S.; Hermansson, N. O.; Leonova, J.; Elebring, T.; Nilsson, K.; Drmota, T.; Greasley, P. J., The orphan receptor GPR55 is a novel cannabinoid receptor. Br J Pharmacol 2007, 152 (7), 1092-101.

23. (a) Zygmunt, P. M.; Petersson, J.; Andersson, D. A.; Chuang, H.; Sorgard, M.; Di Marzo, V.; Julius, D.; Hogestatt, E. D., Vanilloid receptors on sensory nerves mediate the vasodilator action of anandamide. Nature 1999, 400 (6743), 452-7;

(b) Smart, D.; Gunthorpe, M. J.; Jerman, J. C.; Nasir, S.; Gray, J.; Muir, A. I.; Chambers, J. K.; Randall, A. D.; Davis, J. B., The endogenous lipid anandamide is a full agonist at the human vanilloid receptor (hVR1). Br J Pharmacol 2000, 129 (2), 227-30.

24. Prather, P. L.; Martin, N. A.; Breivogel, C. S.; Childers, S. R., Activation of cannabinoid receptors in rat brain by WIN 55212-2 produces coupling to multiple G protein alpha-subunits with different potencies. Mol Pharmacol 2000, 57 (5), 1000-10.

25. Howlett, A. C.; Song, C.; Berglund, B. A.; Wilken, G. H.; Pigg, J. J., Characterization of $\mathrm{CB} 1$ cannabinoid receptors using receptor peptide fragments and site-directed antibodies. Mol Pharmacol 1998, 53 (3), 504-10.

26. Bramblett, R. D.; Panu, A. M.; Ballesteros, J. A.; Reggio, P. H., Construction of a 3D model of the cannabinoid CB1 receptor: determination of helix ends and helix orientation. Life Sci 1995, 56 (23-24), 1971-82.

27. Deadwyler, S. A., Cannabinoid Receptors. Academic Press: London, 1995.

28. Pertwee, R. G., Pharmacology of cannabinoid CB1 and CB2 receptors.

Pharmacol Ther 1997, 74 (2), 129-80. 
29. Mackie, K.; Hille, B., Cannabinoids inhibit N-type calcium channels in neuroblastoma-glioma cells. Proc Natl Acad Sci U S A 1992, 89 (9), 3825-9.

30. (a) Bouaboula, M.; Poinot-Chazel, C.; Bourrie, B.; Canat, X.; Calandra, B.; Rinaldi-Carmona, M.; Le Fur, G.; Casellas, P., Activation of mitogen-activated protein kinases by stimulation of the central cannabinoid receptor CB1. Biochem J 1995, 312 (2), 637-41; (b) Bouaboula, M.; Perrachon, S.; Milligan, L.; Canat, X.; Rinaldi-Carmona, M.; Portier, M.; Barth, F.; Calandra, B.; Pecceu, F.; Lupker, J.; Maffrand, J. P.; Le Fur, G.; Casellas, P., A selective inverse agonist for central cannabinoid receptor inhibits mitogen-activated protein kinase activation stimulated by insulin or insulin-like growth factor 1. Evidence for a new model of receptor/ligand interactions. J Biol Chem 1997, 272 (35), 22330-9; (c) Sanchez, C.; Galve-Roperh, I.; Rueda, D.; Guzman, M., Involvement of sphingomyelin hydrolysis and the mitogen-activated protein kinase cascade in the Delta9tetrahydrocannabinol-induced stimulation of glucose metabolism in primary astrocytes. Mol Pharmacol 1998, 54 (5), 834-43.

31. (a) Liu, J.; Gao, B.; Mirshahi, F.; Sanyal, A. J.; Khanolkar, A. D.; Makriyannis, A.; Kunos, G., Functional CB1 cannabinoid receptors in human vascular endothelial cells. Biochem J 2000, 346 (3), 835-40; (b) Rueda, D.; Galve-Roperh, I.; Haro, A.; Guzman, M., The CB(1) cannabinoid receptor is coupled to the activation of c-Jun N-terminal kinase. Mol Pharmacol 2000, 58 (4), 814-20.

32. Galve-Roperh, I.; Sanchez, C.; Cortes, M. L.; del Pulgar, T. G.; Izquierdo, M.; Guzman, M., Anti-tumoral action of cannabinoids: involvement of sustained ceramide accumulation and extracellular signal-regulated kinase activation. Nat Med 2000, 6 (3), 313-9.

33. Felder, C. C.; Joyce, K. E.; Briley, E. M.; Mansouri, J.; Mackie, K.; Blond, O.; Lai, Y.; Ma, A. L.; Mitchell, R. L., Comparison of the pharmacology and signal transduction of the human cannabinoid CB1 and CB2 receptors. Mol Pharmacol 1995, 48 (3), 443-50.

34. Bouaboula, M.; Bianchini, L.; McKenzie, F. R.; Pouyssegur, J.; Casellas, P., Cannabinoid receptor $\mathrm{CB} 1$ activates the $\mathrm{Na}+\mathrm{H}+$ exchanger $\mathrm{NHE}-1$ isoform via Gi-mediated mitogen activated protein kinase signaling transduction pathways. FEBS Lett 1999, 449 (1), 61-5.

35. Wartmann, M.; Campbell, D.; Subramanian, A.; Burstein, S. H.; Davis, R. J., The MAP kinase signal transduction pathway is activated by the endogenous cannabinoid anandamide. FEBS Lett 1995, 359 (2-3), 133-6.

36. Sanchez, C.; Rueda, D.; Segui, B.; Galve-Roperh, I.; Levade, T.; Guzman, M., The $\mathrm{CB}(1)$ cannabinoid receptor of astrocytes is coupled to sphingomyelin hydrolysis through the adaptor protein fan. Mol Pharmacol 2001, 59 (5), 955-9.

37. Blazquez, C.; Sanchez, C.; Daza, A.; Galve-Roperh, I.; Guzman, M., The stimulation of ketogenesis by cannabinoids in cultured astrocytes defines carnitine palmitoyltransferase I as a new ceramide-activated enzyme. J Neurochem 1999, 72 (4), 1759-68.

38. Guzman, M.; Galve-Roperh, I.; Sanchez, C., Ceramide: a new second messenger of cannabinoid action. Trends Pharmacol Sci 2001, 22 (1), 19-22.

39. (a) Pettit, D. A.; Anders, D. L.; Harrison, M. P.; Cabral, G. A., Cannabinoid receptor expression in immune cells. Adv Exp Med Biol 1996, 402, 119-29; (b) 
Schatz, A. R.; Lee, M.; Condie, R. B.; Pulaski, J. T.; Kaminski, N. E., Cannabinoid receptors $\mathrm{CB} 1$ and CB2: a characterization of expression and adenylate cyclase modulation within the immune system. Toxicol Appl Pharmacol 1997, 142 (2), 278-87.

40. Griffin, G.; Wray, E. J.; Tao, Q.; McAllister, S. D.; Rorrer, W. K.; Aung, M. M.; Martin, B. R.; Abood, M. E., Evaluation of the cannabinoid CB2 receptorselective antagonist, SR144528: further evidence for cannabinoid CB2 receptor absence in the rat central nervous system. Eur J Pharmacol 1999, 377 (1), 11725.

41. Ashton, J. C.; Friberg, D.; Darlington, C. L.; Smith, P. F., Expression of the cannabinoid CB2 receptor in the rat cerebellum: an immunohistochemical study. Neurosci Lett 2006, 396 (2), 113-6.

42. (a) Gong, J. P.; Onaivi, E. S.; Ishiguro, H.; Liu, Q. R.; Tagliaferro, P. A.; Brusco, A.; Uhl, G. R., Cannabinoid CB2 receptors: immunohistochemical localization in rat brain. Brain Res 2006, 1071 (1), 10-23; (b) Van Sickle, M. D.; Duncan, M.; Kingsley, P. J.; Mouihate, A.; Urbani, P.; Mackie, K.; Stella, N.; Makriyannis, A.; Piomelli, D.; Davison, J. S.; Marnett, L. J.; Di Marzo, V.; Pittman, Q. J.; Patel, K. D.; Sharkey, K. A., Identification and functional characterization of brainstem cannabinoid CB2 receptors. Science 2005, 310 (5746), 329-32.

43. Galiegue, S.; Mary, S.; Marchand, J.; Dussossoy, D.; Carriere, D.; Carayon, P.; Bouaboula, M.; Shire, D.; Le Fur, G.; Casellas, P., Expression of central and peripheral cannabinoid receptors in human immune tissues and leukocyte subpopulations. Eur J Biochem 1995, 232 (1), 54-61.

44. Klein, T., Cannabis and Immunity. The Health Effects of Cannabis. Centre for Addiction and Mental Health. Toronto, Canada, 1999.

45. $\quad$ Sawzdargo, M.; Nguyen, T.; Lee, D. K.; Lynch, K. R.; Cheng, R.; Heng, H. H.; George, S. R.; O'Dowd, B. F., Identification and cloning of three novel human G protein-coupled receptor genes GPR52, PsiGPR53 and GPR55: GPR55 is extensively expressed in human brain. Mol Brain Res 1999, 64 (2), 193-8.

46. (a) Oka, S.; Nakajima, K.; Yamashita, A.; Kishimoto, S.; Sugiura, T., Identification of GPR55 as a lysophosphatidylinositol receptor. Biochem Biophys Res Commun 2007, 362 (4), 928-34; (b) Henstridge, C. M.; Balenga, N. A.; Ford, L. A.; Ross, R. A.; Waldhoer, M.; Irving, A. J., The GPR55 ligand L-alphalysophosphatidylinositol promotes RhoA-dependent $\mathrm{Ca} 2+$ signaling and NFAT activation. FASEB J 2009, 23 (1), 183-93.

47. (a) Staton, P. C.; Hatcher, J. P.; Walker, D. J.; Morrison, A. D.; Shapland, E. M.; Hughes, J. P.; Chong, E.; Mander, P. K.; Green, P. J.; Billinton, A.; Fulleylove, M.; Lancaster, H. C.; Smith, J. C.; Bailey, L. T.; Wise, A.; Brown, A. J.; Richardson, J. C.; Chessell, I. P., The putative cannabinoid receptor GPR55 plays a role in mechanical hyperalgesia associated with inflammatory and neuropathic pain. Pain 2008, 139 (1), 225-36; (b) Kress, M.; Kuner, R., Mode of action of cannabinoids on nociceptive nerve endings. Exp Brain Res 2009, 196 (1), 79-88; (c) Ross, R. A., The enigmatic pharmacology of GPR55. Trends Pharmacol Sci 2009, 30 (3), 156-63; (d) Pietr, M.; Kozela, E.; Levy, R.; Rimmerman, N.; Lin, Y. H.; Stella, N.; Vogel, Z.; Juknat, A., Differential changes in GPR55 during microglial cell activation. FEBS Lett 2009, 583 (12), 2071-6. 
48. Ridley, A. J., Rho GTPases and actin dynamics in membrane protrusions and vesicle trafficking. Trends Cell Biol 2006, 16 (10), 522-9.

49. Johns, D. G.; Behm, D. J.; Walker, D. J.; Ao, Z.; Shapland, E. M.; Daniels, D. A.; Riddick, M.; Dowell, S.; Staton, P. C.; Green, P.; Shabon, U.; Bao, W.; Aiyar, N.; Yue, T. L.; Brown, A. J.; Morrison, A. D.; Douglas, S. A., The novel endocannabinoid receptor GPR55 is activated by atypical cannabinoids but does not mediate their vasodilator effects. Br J Pharmacol 2007, 152 (5), 825-31.

50. Whyte, L. S.; Ryberg, E.; Sims, N. A.; Ridge, S. A.; Mackie, K.; Greasley, P. J.; Ross, R. A.; Rogers, M. J., The putative cannabinoid receptor GPR55 affects osteoclast function in vitro and bone mass in vivo. Proc Natl Acad Sci U S A 2009, 106 (38), 16511-6.

51. Jemal, A.; Thomas, A.; Murray, T.; Thun, M., Cancer statistics, 2002. CA Cancer $J$ Clin 2002, 52 (1), 23-47.

52. Surawicz, T. S.; Davis, F.; Freels, S.; Laws, E. R., Jr.; Menck, H. R., Brain tumor survival: results from the National Cancer Data Base. J Neurooncol 1998, 40 (2), 151-60.

53. (a) Walker, M. D.; Alexander, E., Jr.; Hunt, W. E.; Leventhal, C. M.; Mahaley, M. S., Jr.; Mealey, J.; Norrell, H. A.; Owens, G.; Ransohoff, J.; Wilson, C. B.; Gehan, E. A., Evaluation of mithramycin in the treatment of anaplastic gliomas. $J$ Neurosurg 1976, 44 (6), 655-67; (b) Walker, M. D.; Green, S. B.; Byar, D. P.; Alexander, E., Jr.; Batzdorf, U.; Brooks, W. H.; Hunt, W. E.; MacCarty, C. S.; Mahaley, M. S., Jr.; Mealey, J., Jr.; Owens, G.; Ransohoff, J., 2nd; Robertson, J. T.; Shapiro, W. R.; Smith, K. R., Jr.; Wilson, C. B.; Strike, T. A., Randomized comparisons of radiotherapy and nitrosoureas for the treatment of malignant glioma after surgery. $N$ Engl J Med 1980, 303 (23), 1323-9; (c) Kelly, P. J.; Hunt, C., The limited value of cytoreductive surgery in elderly patients with malignant gliomas. Neurosurgery 1994, 34 (1), 62-6; discussion 66-7.

54. (a) Green, S. B.; Byar, D. P.; Walker, M. D.; Pistenmaa, D. A.; Alexander, E., Jr.; Batzdorf, U.; Brooks, W. H.; Hunt, W. E.; Mealey, J., Jr.; Odom, G. L.; Paoletti, P.; Ransohoff, J., 2nd; Robertson, J. T.; Selker, R. G.; Shapiro, W. R.; Smith, K. R., Jr.; Wilson, C. B.; Strike, T. A., Comparisons of carmustine, procarbazine, and high-dose methylprednisolone as additions to surgery and radiotherapy for the treatment of malignant glioma. Cancer Treat Rep 1983, 67 (2), 121-32; (b) DeAngelis, L. M.; Burger, P. C.; Green, S. B.; Cairncross, J. G., Malignant glioma: who benefits from adjuvant chemotherapy? Ann Neurol 1998, 44 (4), 691-5; (c) Fine, H. A.; Figg, W. D.; Jaeckle, K.; Wen, P. Y.; Kyritsis, A. P.; Loeffler, J. S.; Levin, V. A.; Black, P. M.; Kaplan, R.; Pluda, J. M.; Yung, W. K., Phase II trial of the antiangiogenic agent thalidomide in patients with recurrent high-grade gliomas. J Clin Oncol 2000, 18 (4), 708-15.

55. (a) Grant, R.; Liang, B. C.; Page, M. A.; Crane, D. L.; Greenberg, H. S.; Junck, L., Age influences chemotherapy response in astrocytomas. Neurology 1995, 45 (5), 929-33; (b) Nelson, D. F.; Diener-West, M.; Weinstein, A. S.; Schoenfeld, D.; Nelson, J. S.; Sause, W. T.; Chang, C. H.; Goodman, R.; Carabell, S., A randomized comparison of misonidazole sensitized radiotherapy plus BCNU and radiotherapy plus BCNU for treatment of malignant glioma after surgery: final report of an RTOG study. Int J Radiat Oncol Biol Phys 1986, 12 (10), 1793-800. 
56. Burton, E. C.; Prados, M. D., Malignant gliomas. Curr Treat Options Oncol 2000, 1 (5), 459-68.

57. Nelson, D. F.; Diener-West, M.; Horton, J.; Chang, C. H.; Schoenfeld, D.;

Nelson, J. S., Combined modality approach to treatment of malignant gliomas--reevaluation of RTOG 7401/ECOG 1374 with long-term follow-up: a joint study of the Radiation Therapy Oncology Group and the Eastern Cooperative Oncology Group. NCI Monogr 1988, (6), 279-84.

58. Fine, H. A.; Dear, K. B.; Loeffler, J. S.; Black, P. M.; Canellos, G. P., Metaanalysis of radiation therapy with and without adjuvant chemotherapy for malignant gliomas in adults. Cancer 1993, 71 (8), 2585-97.

59. (a) Boiardi, A.; Silvani, A.; Pozzi, A.; Farinotti, M.; Fariselli, L.; Broggi, G.; Salmaggi, A., Advantage of treating anaplastic gliomas with aggressive protocol combining chemotherapy and radiotherapy. J Neurooncol 1997, 34 (2), 179-85;

(b) Grossman, S.; O'Neill, A.; Grunnet, M.; Mehta, M.; Pearlman, J.; Wagner, H.; Gilbert, M.; Newton, H.; Hellman, R., Phase III study comparing three cycles of infusional $\mathrm{BCNU} /$ cisplatin followed by radiation with radiation and concurrent BCNU for patients with newly diagnosed supratentorial glioblastoma multiforme. Proc Am Soc Clin Oncol 2000, 19, 158a.

60. (a) Brandes, A. A.; Scelzi, E.; Zampieri, P.; Rigon, A.; Rotilio, A.; Amista, P.; Berti, F.; Fiorentino, M. V., Phase II trial with BCNU plus alpha-interferon in patients with recurrent high-grade gliomas. Am J Clin Oncol 1997, 20 (4), 364-7; (b) Levin, V. A.; Maor, M. H.; Thall, P. F.; Yung, W. K.; Bruner, J.; Sawaya, R.; Kyritsis, A. P.; Leeds, N.; Woo, S.; Rodriguez, L.; et al., Phase II study of accelerated fractionation radiation therapy with carboplatin followed by vincristine chemotherapy for the treatment of glioblastoma multiforme. Int $J$ Radiat Oncol Biol Phys 1995, 33 (2), 357-64; (c) Levin, V. A.; Yung, W. K.; Bruner, J.; Kyritsis, A.; Leeds, N.; Gleason, M. J.; Hess, K. R.; Meyers, C. A.; Ictech, S. A.; Chang, E.; Maor, M. H., Phase II study of accelerated fractionation radiation therapy with carboplatin followed by PCV for the treatment of anaplastic gliomas. Neurooncol 2000, 2, 278.

61. Hildebrand, J.; Sahmoud, T.; Mignolet, F.; Brucher, J. M.; Afra, D., Adjuvant therapy with dibromodulcitol and BCNU increases survival of adults with malignant gliomas. EORTC Brain Tumor Group. Neurology 1994, 44 (8), 147983.

62. Halperin, E. C.; Herndon, J.; Schold, S. C.; Brown, M.; Vick, N.; Cairncross, J. G.; Macdonald, D. R.; Gaspar, L.; Fischer, B.; Dropcho, E.; Rosenfeld, S.; Morowitz, R.; Piepmeier, J.; Hait, W.; Byrne, T.; Salter, M.; Imperato, J.; Khandekar, J.; Paleologos, N.; Burger, P.; Bentel, G. C.; Friedman, A., A phase III randomized prospective trial of external beam radiotherapy, mitomycin $\mathrm{C}$, carmustine, and 6-mercaptopurine for the treatment of adults with anaplastic glioma of the brain. CNS Cancer Consortium. Int J Radiat Oncol Biol Phys 1996, 34 (4), 793-802.

63. Prados, M. D.; Larson, D. A.; Lamborn, K.; McDermott, M. W.; Sneed, P. K.; Wara, W. M.; Chang, S. M.; Mack, E. E.; Krouwer, H. G.; Chandler, K. L.; Warnick, R. E.; Davis, R. L.; Rabbitt, J. E.; Malec, M.; Levin, V. A.; Gutin, P. H.; Phillips, T. L.; Wilson, C. B., Radiation therapy and hydroxyurea followed by the 
combination of 6-thioguanine and BCNU for the treatment of primary malignant brain tumors. Int J Radiat Oncol Biol Phys 1998, 40 (1), 57-63.

64. Oesch, S.; Walter, D.; Wachtel, M.; Pretre, K.; Salazar, M.; Guzmán, M.;

Velasco, G.; Schäfer, B. W., Cannabinoid receptor 1 is a potential drug target for treatment of translocation-positive rhabdomyosarcoma. Mol Cancer Ther 2009, 8 (7), 1838-45.

65. Cianchi, F.; Papucci, L.; Schiavone, N.; Lulli, M.; Magnelli, L.; Vinci, M. C.; Messerini, L.; Manera, C.; Ronconi, E.; Romagnani, P.; Donnini, M.; Perigli, G.; Trallori, G.; Tanganelli, E.; Capaccioli, S.; Masini, E., Cannabinoid receptor activation induces apoptosis through tumor necrosis factor alpha-mediated ceramide de novo synthesis in colon cancer cells. Clin Cancer Res. 2008, 14 (23), 7691-700.

66. (a) Mimeault, M.; Pommery, N.; Wattez, N.; Bailly, C.; Henichart, J. P., Antiproliferative and apoptotic effects of anandamide in human prostatic cancer cell lines: implication of epidermal growth factor receptor down-regulation and ceramide production. Prostate 2003, 56 (1), 1-12; (b) Melck, D.; De Petrocellis, L.; Orlando, P.; Bisogno, T.; Laezza, C.; Bifulco, M.; Di Marzo, V., Suppression of nerve growth factor Trk receptors and prolactin receptors by endocannabinoids leads to inhibition of human breast and prostate cancer cell proliferation.

Endocrinology 2000, 141 (1), 118-26; (c) Ruiz, L.; Miguel, A.; Diaz-Laviada, I., Delta9-tetrahydrocannabinol induces apoptosis in human prostate PC-3 cells via a receptor-independent mechanism. FEBS Lett 1999, 458 (3), 400-4.

67. Casanova, M. L.; Blazquez, C.; Martinez-Palacio, J.; Villanueva, C.; FernandezAcenero, M. J.; Huffman, J. W.; Jorcano, J. L.; Guzman, M., Inhibition of skin tumor growth and angiogenesis in vivo by activation of cannabinoid receptors. $J$ Clin Invest 2003, 111 (1), 43-50.

68. (a) Sanchez, C.; de Ceballos, M. L.; del Pulgar, T. G.; Rueda, D.; Corbacho, C.; Velasco, G.; Galve-Roperh, I.; Huffman, J. W.; Ramon y Cajal, S.; Guzman, M., Inhibition of glioma growth in vivo by selective activation of the $\mathrm{CB}(2)$ cannabinoid receptor. Cancer Res 2001, 61 (15), 5784-9; (b) Sanchez, C.; GalveRoperh, I.; Canova, C.; Brachet, P.; Guzman, M., Delta9-tetrahydrocannabinol induces apoptosis in C6 glioma cells. FEBS Lett 1998, 436 (1), 6-10; (c) Jacobsson, S. O.; Wallin, T.; Fowler, C. J., Inhibition of rat C6 glioma cell proliferation by endogenous and synthetic cannabinoids. Relative involvement of cannabinoid and vanilloid receptors. J Pharmacol Exp Ther 2001, 299 (3), 951-9; (d) Jacobsson, S. O.; Rongard, E.; Stridh, M.; Tiger, G.; Fowler, C. J., Serumdependent effects of tamoxifen and cannabinoids upon $\mathrm{C} 6$ glioma cell viability. Biochem Pharmacol 2000, 60 (12), 1807-13; (e) Fowler, C. J.; Jonsson, K. O.; Andersson, A.; Juntunen, J.; Jarvinen, T.; Vandevoorde, S.; Lambert, D. M.; Jerman, J. C.; Smart, D., Inhibition of C6 glioma cell proliferation by anandamide, 1-arachidonoylglycerol, and by a water soluble phosphate ester of anandamide: variability in response and involvement of arachidonic acid. Biochem Pharmacol 2003, 66 (5), 757-67; (f) Massi, P.; Vaccani, A.; Ceruti, S.; Colombo, A.; Abbracchio, M. P.; Parolaro, D., Antitumor effects of cannabidiol, a nonpsychoactive cannabinoid, on human glioma cell lines. J Pharmacol Exp Ther 2004, 308 (3), 838-45. 
69. McKallip, R. J.; Lombard, C.; Fisher, M.; Martin, B. R.; Ryu, S.; Grant, S.; Nagarkatti, P. S.; Nagarkatti, M., Targeting CB2 cannabinoid receptors as a novel therapy to treat malignant lymphoblastic disease. Blood 2002, 100 (2), 627-34.

70. (a) De Petrocellis, L.; Melck, D.; Palmisano, A.; Bisogno, T.; Laezza, C.; Bifulco, M.; Di Marzo, V., The endogenous cannabinoid anandamide inhibits human breast cancer cell proliferation. Proc Natl Acad Sci U S A 1998, 95 (14), 8375-80; (b) Melck, D.; Rueda, D.; Galve-Roperh, I.; De Petrocellis, L.; Guzman, M.; Di Marzo, V., Involvement of the cAMP/protein kinase A pathway and of mitogenactivated protein kinase in the anti-proliferative effects of anandamide in human breast cancer cells. FEBS Lett 1999, 463 (3), 235-40.

71. (a) Mon, M. J.; Jansing, R. L.; Doggett, S.; Stein, J. L.; Stein, G. S., Influence of delta9-tetrahydrocannabinol on cell proliferation and macromolecular biosynthesis in human cells. Biochem Pharmacol 1978, 27 (13), 1759-65; (b) Blevins, R. D.; Smith, D. P., Effects of delta-9-tetrahydrocannabinol on cultured HeLa cell growth and development. Growth 1980, 44 (2), 133-8.

72. (a) Munson, A. E.; Harris, L. S.; Friedman, M. A.; Dewey, W. L.; Carchman, R. A., Antineoplastic activity of cannabinoids. J Natl Cancer Inst 1975, 55 (3), 597 602; (b) Preet, A.; Ganju, R. K.; Groopman, J. E., Delta(9)-Tetrahydrocannabinol inhibits epithelial growth factor-induced lung cancer cell migration in vitro as well as its growth and metastasis in vivo. Oncogene 2008, 27 (3), 339-46.

73. Bifulco, M.; Laezza, C.; Portella, G.; Vitale, M.; Orlando, P.; De Petrocellis, L.; Di Marzo, V., Control by the endogenous cannabinoid system of ras oncogenedependent tumor growth. FASEB J 2001, 15 (14), 2745-7.

74. (a) Blázquez, C.; Salazar, M.; Carracedo, A.; Lorente, M.; Egia, A.; GonzálezFeria, L.; Haro, A.; Velasco, G.; Guzmán, M., Cannabinoids inhibit glioma cell invasion by down-regulating matrix metalloproteinase-2 expression. Cancer Res. 2008, 68 (6), 1945-52; (b) Duntsch, C.; Divi, M. K.; Jones, T.; Zhou, Q.; Krishnamurthy, M.; Boehm, P.; Wood, G.; Sills, A.; Moore, B. M., Safety and efficacy of a novel cannabinoid chemotherapeutic, KM-233, for the treatment of high-grade glioma. J. Neurooncol. 2006, 77 (2), 143-52.

75. Ellert-Miklaszewska, A.; Grajkowska, W.; Gabrusiewicz, K.; Kaminska, B.; Konarska, L., Distinctive pattern of cannabinoid receptor type II (CB2) expression in adult and pediatric brain tumors. Brain Res. 2007, 1137 (1), 161-9.

76. Guzmán, M.; Duarte, M. J.; Blázquez, C.; Ravina, J.; Rosa, M. C.; Galve-Roperh, I.; Sánchez, C.; Velasco, G.; González-Feria, L., A pilot clinical study of Delta9tetrahydrocannabinol in patients with recurrent glioblastoma multiforme. Br. J. Cancer 2006, 95 (2), 197-203.

77. Parolaro, D., Presence and functional regulation of cannabinoid receptors in immune cells. Life Sci 1999, 65 (6-7), 637-44.

78. Hui-Lin, L., The Origin and Use of Cannabis in Eastern Asia: Their Linguistic Cultural Implications. Cannabis and Culture. Mouton: Chicago, 1975.

79. Kaminski, N. E.; Abood, M. E.; Kessler, F. K.; Martin, B. R.; Schatz, A. R., Identification of a functionally relevant cannabinoid receptor on mouse spleen cells that is involved in cannabinoid-mediated immune modulation. Mol

Pharmacol 1992, 42 (5), 736-42. 
80. Bouaboula, M.; Rinaldi, M.; Carayon, P.; Carillon, C.; Delpech, B.; Shire, D.; Le Fur, G.; Casellas, P., Cannabinoid-receptor expression in human leukocytes. Eur $J$ Biochem 1993, 214 (1), 173-80.

81. Massi, P.; Vaccani, A.; Parolaro, D., Cannabinoids, immune system and cytokine network. Curr Pharm Des 2006, 12 (24), 3135-46.

82. Lynn, A. B.; Herkenham, M., Localization of cannabinoid receptors and nonsaturable high-density cannabinoid binding sites in peripheral tissues of the rat: implications for receptor-mediated immune modulation by cannabinoids. $J$ Pharmacol Exp Ther 1994, 268 (3), 1612-23.

83. Matias, I.; Pochard, P.; Orlando, P.; Salzet, M.; Pestel, J.; Di Marzo, V., Presence and regulation of the endocannabinoid system in human dendritic cells. Eur $J$ Biochem 2002, 269 (15), 3771-8.

84. (a) Stella, N., Endocannabinoid signaling in microglial cells. Neuropharmacology 2009, 56 (Suppl 1), 244-53; (b) Cabral, G. A.; Raborn, E. S.; Griffin, L.; Dennis, J.; Marciano-Cabral, F., CB2 receptors in the brain: role in central immune function. Br J Pharmacol 2008, 153 (2), 240-51; (c) Romero-Sandoval, E. A.; Horvath, R.; Landry, R. P.; DeLeo, J. A., Cannabinoid receptor type 2 activation induces a microglial anti-inflammatory phenotype and reduces migration via MKP induction and ERK dephosphorylation. Mol Pain 2009, 5, 25.

85. Sharir, H.; Abood, M. E., Pharmacological characterization of GPR55, a putative cannabinoid receptor. Pharmacol Ther 2010, 126 (3), 301-13.

86. (a) Carlisle, S. J.; Marciano-Cabral, F.; Staab, A.; Ludwick, C.; Cabral, G. A., Differential expression of the CB2 cannabinoid receptor by rodent macrophages and macrophage-like cells in relation to cell activation. Int Immunopharmacol 2002, 2 (1), 69-82; (b) Maresz, K.; Carrier, E. J.; Ponomarev, E. D.; Hillard, C. J.; Dittel, B. N., Modulation of the cannabinoid CB2 receptor in microglial cells in response to inflammatory stimuli. J Neurochem 2005, 95 (2), 437-45.

87. Koh, W. S.; Yang, K. H.; Kaminski, N. E., Cyclic AMP is an essential factor in immune responses. Biochem Biophys Res Commun 1995, 206 (2), 703-9.

88. Kaminski, N. E.; Koh, W. S.; Yang, K. H.; Lee, M.; Kessler, F. K., Suppression of the humoral immune response by cannabinoids is partially mediated through inhibition of adenylate cyclase by a pertussis toxin-sensitive G-protein coupled mechanism. Biochem Pharmacol 1994, 48 (10), 1899-908.

89. Cabral, G. A.; Griffin-Thomas, L., Cannabinoids as therapeutic agents for ablating neuroinflammatory disease. Endocr Metab Immune Disord Drug Targets 2008, 8 (3), 159-72.

90. (a) Klein, T. W.; Newton, C.; Larsen, K.; Lu, L.; Perkins, I.; Nong, L.; Friedman, H., The cannabinoid system and immune modulation. J Leukoc Biol 2003, 74 (4), 486-96; (b) Franklin, A.; Stella, N., Arachidonylcyclopropylamide increases microglial cell migration through cannabinoid CB2 and abnormal-cannabidiolsensitive receptors. Eur J Pharmacol 2003, 474 (2-3), 195-8; (c) Gokoh, M.; Kishimoto, S.; Oka, S.; Metani, Y.; Sugiura, T., 2-Arachidonoylglycerol, an endogenous cannabinoid receptor ligand, enhances the adhesion of HL-60 cells differentiated into macrophage-like cells and human peripheral blood monocytes. FEBS Lett 2005, 579 (28), 6473-8. 
91. Walter, L.; Franklin, A.; Witting, A.; Wade, C.; Xie, Y.; Kunos, G.; Mackie, K.; Stella, N., Nonpsychotropic cannabinoid receptors regulate microglial cell migration. J Neurosci 2003, 23 (4), 1398-405.

92. Kaplan, B. L.; Ouyang, Y.; Herring, A.; Yea, S. S.; Razdan, R.; Kaminski, N. E., Inhibition of leukocyte function and interleukin-2 gene expression by 2methylarachidonyl-(2'-fluoroethyl)amide, a stable congener of the endogenous cannabinoid receptor ligand anandamide. Toxicol Appl Pharmacol 2005, 205 (2), 107-15.

93. Cabral, G. A.; Staab, A., Effects on the immune system. Handb Exp Pharmacol 2005, (168), 385-423.

94. (a) Klein, T. W.; Kawakami, Y.; Newton, C.; Friedman, H., Marijuana components suppress induction and cytolytic function of murine cytotoxic $\mathrm{T}$ cells in vitro and in vivo. J Toxicol Environ Health 1991, 32 (4), 465-77; (b) BurnetteCurley, D.; Cabral, G. A., Differential inhibition of RAW264.7 macrophage tumoricidal activity by delta 9tetrahydrocannabinol. Proc Soc Exp Biol Med 1995, 210 (1), 64-76; (c) Coffey, R. G.; Yamamoto, Y.; Snella, E.; Pross, S., Tetrahydrocannabinol inhibition of macrophage nitric oxide production. Biochem Pharmacol 1996, 52 (5), 743-51; (d) McCoy, K. L.; Gainey, D.; Cabral, G. A., delta 9 -Tetrahydrocannabinol modulates antigen processing by macrophages. $J$ Pharmacol Exp Ther 1995, 273 (3), 1216-23.

95. (a) Zheng, Z. M.; Specter, S.; Friedman, H., Inhibition by delta-9tetrahydrocannabinol of tumor necrosis factor alpha production by mouse and human macrophages. Int J Immunopharmacol 1992, 14 (8), 1445-52; (b) Srivastava, M. D.; Srivastava, B. I.; Brouhard, B., Delta9 tetrahydrocannabinol and cannabidiol alter cytokine production by human immune cells.

Immunopharmacology 1998, 40 (3), 179-85; (c) Facchinetti, F.; Del Giudice, E.; Furegato, S.; Passarotto, M.; Leon, A., Cannabinoids ablate release of TNFalpha in rat microglial cells stimulated with lypopolysaccharide. Glia 2003, 41 (2), 1618; (d) Klegeris, A.; Bissonnette, C. J.; McGeer, P. L., Reduction of human monocytic cell neurotoxicity and cytokine secretion by ligands of the cannabinoid-type CB2 receptor. Br J Pharmacol 2003, 139 (4), 775-86.

96. (a) Racke, M. K.; Dhib-Jalbut, S.; Cannella, B.; Albert, P. S.; Raine, C. S.; McFarlin, D. E., Prevention and treatment of chronic relapsing experimental allergic encephalomyelitis by transforming growth factor-beta 1. J Immunol 1991, 146 (9), 3012-7; (b) Croxford, J. L.; Miller, S. D., Immunoregulation of a viral model of multiple sclerosis using the synthetic cannabinoid R+WIN55,212. J Clin Invest 2003, 111 (8), 1231-40.

97. (a) Mageed, R. A.; Adams, G.; Woodrow, D.; Podhajcer, O. L.; Chernajovsky, Y., Prevention of collagen-induced arthritis by gene delivery of soluble p75 tumour necrosis factor receptor. Gene Ther 1998, 5 (12), 1584-92; (b)

Triantaphyllopoulos, K. A.; Williams, R. O.; Tailor, H.; Chernajovsky, Y., Amelioration of collagen-induced arthritis and suppression of interferon-gamma, interleukin-12, and tumor necrosis factor alpha production by interferon-beta gene therapy. Arthritis Rheum 1999, 42 (1), 90-9. 
98. Hegde, V. L.; Hegde, S.; Cravatt, B. F.; Hofseth, L. J.; Nagarkatti, M.; Nagarkatti, P. S., Attenuation of experimental autoimmune hepatitis by exogenous and endogenous cannabinoids: involvement of regulatory T cells. Mol Pharmacol 2008, 74 (1), 20-33.

99. (a) Sacerdote, P.; Massi, P.; Panerai, A. E.; Parolaro, D., In vivo and in vitro treatment with the synthetic cannabinoid CP55, 940 decreases the in vitro migration of macrophages in the rat: involvement of both $\mathrm{CB} 1$ and $\mathrm{CB} 2$ receptors. J Neuroimmunol 2000, 109 (2), 155-63; (b) Raborn, E. S.; Marciano-Cabral, F.; Buckley, N. E.; Martin, B. R.; Cabral, G. A., The cannabinoid delta-9tetrahydrocannabinol mediates inhibition of macrophage chemotaxis to RANTES/CCL5: linkage to the CB2 receptor. J Neuroimmune Pharmacol 2008, 3 (2), 117-29.

100. Klein, T. W.; Friedman, H., Modulation of murine immune cell function by marijuana components. In Drugs of Abuse and Immune Function, Watson, R., Ed. CRC Press: Boca Raton, 1990; pp 87-111.

101. Klein, T. W.; Newton, C.; Widen, R.; Friedman, H., Delta 9-tetrahydrocannabinol injection induces cytokine-mediated mortality of mice infected with Legionella pneumophila. J Pharmacol Exp Ther 1993, 267 (2), 635-40.

102. De Filippis, D.; D'Amico, A.; Iuvone, T., Cannabinomimetic control of mast cell mediator release: new perspective in chronic inflammation. J Neuroendocrinol 2008, 20 (Suppl 1), 20-5.

103. (a) Deusch, E.; Kraft, B.; Nahlik, G.; Weigl, L.; Hohenegger, M.; Kress, H. G., No evidence for direct modulatory effects of delta 9-tetrahydrocannabinol on human polymorphonuclear leukocytes. J Neuroimmunol 2003, 141 (1-2), 99-103; (b) Kraft, B.; Wintersberger, W.; Kress, H. G., Cannabinoid receptor-independent suppression of the superoxide generation of human neutrophils (PMN) by CP55 940, but not by anandamide. Life Sci 2004, 75 (8), 969-77.

104. Sheng, W. S.; Hu, S.; Min, X.; Cabral, G. A.; Lokensgard, J. R.; Peterson, P. K., Synthetic cannabinoid WIN55,212-2 inhibits generation of inflammatory mediators by IL-1beta-stimulated human astrocytes. Glia 2005, 49 (2), 211-9.

105. Puffenbarger, R. A.; Boothe, A. C.; Cabral, G. A., Cannabinoids inhibit LPSinducible cytokine mRNA expression in rat microglial cells. Glia 2000, 29 (1), 58-69.

106. (a) Shohami, E.; Gallily, R.; Mechoulam, R.; Bass, R.; Ben-Hur, T., Cytokine production in the brain following closed head injury: dexanabinol (HU-211) is a novel TNF-alpha inhibitor and an effective neuroprotectant. J Neuroimmunol 1997, 72 (2), 169-77; (b) Molina-Holgado, F.; Lledo, A.; Guaza, C., Anandamide suppresses nitric oxide and TNF-alpha responses to Theiler's virus or endotoxin in astrocytes. Neuroreport 1997, 8 (8), 1929-33.

107. Molina-Holgado, F.; Molina-Holgado, E.; Guaza, C., The endogenous cannabinoid anandamide potentiates interleukin- 6 production by astrocytes infected with Theiler's murine encephalomyelitis virus by a receptor-mediated pathway. FEBS Lett 1998, 433 (1-2), 139-42.

108. Krishnamurthy, M.; Ferreira, A. M.; Moore, B. M., 2nd, Synthesis and testing of novel phenyl substituted side-chain analogues of classical cannabinoids. Bioorg Med Chem Lett 2003, 13 (20), 3487-90. 
109. Duntsch, C.; Divi, M. K.; Jones, T.; Zhou, Q.; Krishnamurthy, M.; Boehm, P.; Wood, G.; Sills, A.; Moore, B. M., 2nd, Safety and efficacy of a novel cannabinoid chemotherapeutic, KM-233, for the treatment of high-grade glioma. $J$ Neurooncol 2006, 77 (2), 143-52.

110. (a) Vaccani, A.; Massi, P.; Colombo, A.; Rubino, T.; Parolaro, D., Cannabidiol inhibits human glioma cell migration through a cannabinoid receptor-independent mechanism. Br J Pharmacol. 2005, 144 (8), 1032-6; (b) Aguado, T.; Carracedo, A.; Julien, B.; Velasco, G.; Milman, G.; Mechoulam, R.; Alvarez, L.; Guzman, M.; Galve-Roperh, I., Cannabinoids induce glioma stem-like cell differentiation and inhibit gliomagenesis. J Biol Chem 2007, 282 (9), 6854-62.

111. Krishnamurthy, M.; Gurley, S.; Moore, B. M., 2nd, Exploring the substituent effects on a novel series of C1'-dimethyl-aryl Delta8-tetrahydrocannabinol analogs. Bioorg Med Chem 2008, 16 (13), 6489-500.

112. Brown, A. J., Novel cannabinoid receptors. Br J Pharmacol 2007, 152 (5), 567 75.

113. Turkson, J.; Jove, R., STAT proteins: novel molecular targets for cancer drug discovery. Oncogene 2000, 19 (56), 6613-26.

114. Hussain, S. F.; Kong, L. Y.; Jordan, J.; Conrad, C.; Madden, T.; Fokt, I.; Priebe, W.; Heimberger, A. B., A novel small molecule inhibitor of signal transducers and activators of transcription 3 reverses immune tolerance in malignant glioma patients. Cancer Res 2007, 67 (20), 9630-6.

115. (a) Calo, V.; Migliavacca, M.; Bazan, V.; Macaluso, M.; Buscemi, M.; Gebbia, N.; Russo, A., STAT proteins: from normal control of cellular events to tumorigenesis. J Cell Physiol 2003, 197 (2), 157-68; (b) Fuh, B.; Sobo, M.; Cen, L.; Josiah, D.; Hutzen, B.; Cisek, K.; Bhasin, D.; Regan, N.; Lin, L.; Chan, C.; Caldas, H.; DeAngelis, S.; Li, C.; Li, P. K.; Lin, J., LLL-3 inhibits STAT3 activity, suppresses glioblastoma cell growth and prolongs survival in a mouse glioblastoma model. Br J Cancer 2009, 100 (1), 106-12.

116. Bromberg, K. D.; Ma'ayan, A.; Neves, S. R.; Iyengar, R., Design logic of a cannabinoid receptor signaling network that triggers neurite outgrowth. Science 2008, 320 (5878), 903-9.

117. Frias, M. A.; James, R. W.; Gerber-Wicht, C.; Lang, U., Native and reconstituted HDL activate Stat3 in ventricular cardiomyocytes via ERK1/2: role of sphingosine-1-phosphate. Cardiovasc Res 2009, 82 (2), 313-23.

118. (a) Galve-Roperh, I.; Rueda, D.; Gómez del Pulgar, T.; Velasco, G.; Guzmán, M., Mechanism of extracellular signal-regulated kinase activation by the $\mathrm{CB}(1)$ cannabinoid receptor. Mol Pharmacol. 2002, 62 (6), 1385-92; (b) Graham, E. S.; Ball, N.; Scotter, E. L.; Narayan, P.; Dragunow, M.; Glass, M., Induction of Krox-24 by endogenous cannabinoid type 1 receptors in Neuro2A cells is mediated by the MEK-ERK MAPK pathway and is suppressed by the phosphatidylinositol 3-kinase pathway. J Biol Chem 2006, 281 (39), 29085-95.

119. Ellert-Miklaszewska, A.; Kaminska, B.; Konarska, L., Cannabinoids downregulate $\mathrm{PI} 3 \mathrm{~K} / \mathrm{Akt}$ and Erk signalling pathways and activate proapoptotic function of Bad protein. Cell Signal 2005, 17 (1), 25-37.

120. Sridhar, S. S.; Hedley, D.; Siu, L. L., Raf kinase as a target for anticancer therapeutics. Mol Cancer Ther 2005, 4 (4), 677-85. 
121. Salazar, M.; Carracedo, A.; Salanueva, I. J.; Hernández-Tiedra, S.; Lorente, M.; Egia, A.; Vázquez, P.; Blázquez, C.; Torres, S.; García, S.; Nowak, J.; Fimia, G. M.; Piacentini, M.; Cecconi, F.; Pandolfi, P. P.; González-Feria, L.; Iovanna, J. L.; Guzmán, M.; Boya, P.; Velasco, G., Cannabinoid action induces autophagymediated cell death through stimulation of ER stress in human glioma cells. J Clin Invest. 2009, 119 (5), 1359-72.

122. (a) Beurel, E.; Jope, R. S., The paradoxical pro- and anti-apoptotic actions of GSK3 in the intrinsic and extrinsic apoptosis signaling pathways. Prog Neurobiol 2006, 79 (4), 173-89; (b) Woodgett, J. R., Physiological roles of glycogen synthase kinase-3: potential as a therapeutic target for diabetes and other disorders. Curr Drug Targets Immune Endocr Metabol Disord 2003, 3 (4), 28190.

123. Kotliarova, S.; Pastorino, S.; Kovell, L. C.; Kotliarov, Y.; Song, H.; Zhang, W.; Bailey, R.; Maric, D.; Zenklusen, J. C.; Lee, J.; Fine, H. A., Glycogen synthase kinase-3 inhibition induces glioma cell death through c-MYC, nuclear factorkappaB, and glucose regulation. Cancer Res 2008, 68 (16), 6643-51.

124. Shano, S.; Hatanaka, K.; Ninose, S.; Moriyama, R.; Tsujiuchi, T.; Fukushima, N., A lysophosphatidic acid receptor lacking the PDZ-binding domain is constitutively active and stimulates cell proliferation. Biochim Biophys Acta 2008, 1783 (5), 748-59.

125. Zohrabian, V. M.; Forzani, B.; Chau, Z.; Murali, R.; Jhanwar-Uniyal, M., Rho/ROCK and MAPK signaling pathways are involved in glioblastoma cell migration and proliferation. Anticancer Res 2009, 29 (1), 119-23.

126. (a) Datta, S. R.; Dudek, H.; Tao, X.; Masters, S.; Fu, H.; Gotoh, Y.; Greenberg, M. E., Akt phosphorylation of BAD couples survival signals to the cell-intrinsic death machinery. Cell 1997, 91 (2), 231-41; (b) Scheid, M. P.; Duronio, V., Dissociation of cytokine-induced phosphorylation of Bad and activation of PKB/akt: involvement of MEK upstream of Bad phosphorylation. Proc Natl Acad Sci U S A 1998, 95 (13), 7439-44.

127. Bhattacharjee, H.; Gurley, S. N.; Moore, B. M., 2nd, Design and synthesis of novel tri-aryl CB2 selective cannabinoid ligands. Bioorg Med Chem Lett 2009, 19 (6), 1691-3.

128. Makriyannis, A.; Lai, X.; Lu, D. Novel Biphenyl and Biphenyl-like compounds (US Patent WO 2004/017920 A2). WO 2004/017920 A2, 04/03/2004, 2004.

129. Nadipuram, A. K.; Krishnamurthy, M.; Ferreira, A. M.; Li, W.; Moore, B. M., Synthesis and testing of novel classical cannabinoids: exploring the side chain ligand binding pocket of the CB1 and CB2 receptors. Bioorg Med Chem 2003, 11 (14), 3121-32.

130. Papahatjis, D. P.; Kourouli, T.; Abadji, V.; Goutopoulos, A.; Makriyannis, A., Pharmacophoric requirements for cannabinoid side chains: multiple bond and C1'substituted delta 8-tetrahydrocannabinols. J Med Chem 1998, 41 (7), 1195-200.

131. (a) Michalski, C. W.; Oti, F. E.; Erkan, M.; Sauliunaite, D.; Bergmann, F.; Pacher, P.; Batkai, S.; Muller, M. W.; Giese, N. A.; Friess, H.; Kleeff, J., Cannabinoids in pancreatic cancer: correlation with survival and pain. Int J Cancer 2008, 122 (4), 742-50; (b) Preet, A.; Ganju, R. K.; Groopman, J. E., Delta9-

Tetrahydrocannabinol inhibits epithelial growth factor-induced lung cancer cell 
migration in vitro as well as its growth and metastasis in vivo. Oncogene 2008, 27 (3), 339-46; (c) Sarfaraz, S.; Afaq, F.; Adhami, V. M.; Mukhtar, H., Cannabinoid receptor as a novel target for the treatment of prostate cancer. Cancer Res 2005, 65 (5), 1635-41; (d) McKallip, R. J.; Nagarkatti, M.; Nagarkatti, P. S., Delta-9tetrahydrocannabinol enhances breast cancer growth and metastasis by suppression of the antitumor immune response. J Immunol 2005, 174 (6), 3281-9; (e) Ligresti, A.; Bisogno, T.; Matias, I.; De Petrocellis, L.; Cascio, M. G.; Cosenza, V.; D'Argenio, G.; Scaglione, G.; Bifulco, M.; Sorrentini, I.; Di Marzo, V., Possible endocannabinoid control of colorectal cancer growth.

Gastroenterology 2003, 125 (3), 677-87; (f) Parolaro, D.; Massi, P.; Rubino, T.; Monti, E., Endocannabinoids in the immune system and cancer. Prostaglandins Leukot Essent Fatty Acids 2002, 66 (2-3), 319-32.

132. Vaccani, A.; Massi, P.; Colombo, A.; Rubino, T.; Parolaro, D., Cannabidiol inhibits human glioma cell migration through a cannabinoid receptor-independent mechanism. Br J Pharmacol 2005, 144 (8), 1032-6.

133. Zimmermann, S.; Moelling, K., Phosphorylation and regulation of Raf by Akt (protein kinase B). Science 1999, 286 (5445), 1741-4.

134. Salazar, M.; Carracedo, A.; Salanueva, I. J.; Hernandez-Tiedra, S.; Lorente, M.; Egia, A.; Vazquez, P.; Blazquez, C.; Torres, S.; Garcia, S.; Nowak, J.; Fimia, G. M.; Piacentini, M.; Cecconi, F.; Pandolfi, P. P.; Gonzalez-Feria, L.; Iovanna, J. L.; Guzman, M.; Boya, P.; Velasco, G., Cannabinoid action induces autophagymediated cell death through stimulation of ER stress in human glioma cells. J Clin Invest 2009, 119 (5), 1359-72.

135. Sarfaraz, S.; Afaq, F.; Adhami, V. M.; Malik, A.; Mukhtar, H., Cannabinoid receptor agonist-induced apoptosis of human prostate cancer cells $\mathrm{LNCaP}$ proceeds through sustained activation of ERK1/2 leading to G1 cell cycle arrest. $J$ Biol Chem 2006, 281 (51), 39480-91.

136. (a) Zuckerman, V.; Wolyniec, K.; Sionov, R. V.; Haupt, S.; Haupt, Y., Tumour suppression by p53: the importance of apoptosis and cellular senescence. J Pathol 2009, 219 (1), 3-15; (b) d'Adda di Fagagna, F.; Reaper, P. M.; Clay-Farrace, L.; Fiegler, H.; Carr, P.; Von Zglinicki, T.; Saretzki, G.; Carter, N. P.; Jackson, S. P., A DNA damage checkpoint response in telomere-initiated senescence. Nature 2003, 426 (6963), 194-8; (c) Herbig, U.; Jobling, W. A.; Chen, B. P.; Chen, D. J.; Sedivy, J. M., Telomere shortening triggers senescence of human cells through a pathway involving ATM, p53, and p21(CIP1), but not p16(INK4a). Mol Cell 2004, 14 (4), 501-13; (d) Artandi, S. E.; DePinho, R. A., A critical role for telomeres in suppressing and facilitating carcinogenesis. Curr Opin Genet Dev 2000, 10 (1), 39-46.

137. (a) Lowe, S. W.; Cepero, E.; Evan, G., Intrinsic tumour suppression. Nature 2004, 432 (7015), 307-15; (b) Vousden, K. H.; Prives, C., Blinded by the light: the growing complexity of p53. Cell 2009, 137 (3), 413-31.

138. Yu, S. W.; Andrabi, S. A.; Wang, H.; Kim, N. S.; Poirier, G. G.; Dawson, T. M.; Dawson, V. L., Apoptosis-inducing factor mediates poly(ADP-ribose) (PAR) polymer-induced cell death. Proc Natl Acad Sci U S A 2006, 103 (48), 18314-9.

139. (a) Nicholson, K. M.; Anderson, N. G., The protein kinase B/Akt signalling pathway in human malignancy. Cell Signal 2002, 14 (5), 381-95; (b) Hill, M. M.; 
Hemmings, B. A., Inhibition of protein kinase B/Akt. implications for cancer therapy. Pharmacol Ther 2002, 93 (2-3), 243-51; (c) Bonneau, D.; Longy, M., Mutations of the human PTEN gene. Hum Mutat 2000, 16 (2), 109-22.

140. Pap, M.; Cooper, G. M., Role of glycogen synthase kinase-3 in the phosphatidylinositol 3-Kinase/Akt cell survival pathway. J Biol Chem 1998, 273 (32), 19929-32.

141. Cardone, M. H.; Roy, N.; Stennicke, H. R.; Salvesen, G. S.; Franke, T. F.; Stanbridge, E.; Frisch, S.; Reed, J. C., Regulation of cell death protease caspase-9 by phosphorylation. Science 1998, 282 (5392), 1318-21.

142. (a) Xia, Z.; Dickens, M.; Raingeaud, J.; Davis, R. J.; Greenberg, M. E., Opposing effects of ERK and JNK-p38 MAP kinases on apoptosis. Science 1995, 270 (5240), 1326-31; (b) Parrizas, M.; Blakesley, V. A.; Beitner-Johnson, D.; Le Roith, D., The proto-oncogene Crk-II enhances apoptosis by a Ras-dependent, Raf-1/MAP kinase-independent pathway. Biochem Biophys Res Commun 1997, 234 (3), 616-20.

143. Linseman, D. A.; Butts, B. D.; Precht, T. A.; Phelps, R. A.; Le, S. S.; Laessig, T. A.; Bouchard, R. J.; Florez-McClure, M. L.; Heidenreich, K. A., Glycogen synthase kinase-3beta phosphorylates Bax and promotes its mitochondrial localization during neuronal apoptosis. J Neurosci 2004, 24 (44), 9993-10002.

144. Qu, L.; Huang, S.; Baltzis, D.; Rivas-Estilla, A. M.; Pluquet, O.; Hatzoglou, M.; Koumenis, C.; Taya, Y.; Yoshimura, A.; Koromilas, A. E., Endoplasmic reticulum stress induces p53 cytoplasmic localization and prevents p53-dependent apoptosis by a pathway involving glycogen synthase kinase-3beta. Genes Dev 2004, 18 (3), 261-77.

145. Zundel, W.; Giaccia, A., Inhibition of the anti-apoptotic PI(3)K/Akt/Bad pathway by stress. Genes Dev 1998, 12 (13), 1941-6.

146. Targos, B.; Pomorski, P.; Krzeminski, P.; Baranska, J.; Redowicz, M. J.; Klopocka, W., Effect of Rho-associated kinase inhibition on actin cytoskeleton structure and calcium response in glioma C6 cells. Acta Biochim Pol 2006, 53 (4), 825-31.

147. Rutkowski, D. T.; Kaufman, R. J., A trip to the ER: coping with stress. Trends Cell Biol 2004, 14 (1), 20-8.

148. Filippin, L.; Magalhaes, P. J.; Di Benedetto, G.; Colella, M.; Pozzan, T., Stable interactions between mitochondria and endoplasmic reticulum allow rapid accumulation of calcium in a subpopulation of mitochondria. $J$ Biol Chem 2003, 278 (40), 39224-34.

149. McCullough, K. D.; Martindale, J. L.; Klotz, L. O.; Aw, T. Y.; Holbrook, N. J., Gadd153 sensitizes cells to endoplasmic reticulum stress by down-regulating Bcl2 and perturbing the cellular redox state. Mol Cell Biol 2001, 21 (4), 1249-59.

150. Li, J.; Lee, B.; Lee, A. S., Endoplasmic reticulum stress-induced apoptosis: multiple pathways and activation of p53-up-regulated modulator of apoptosis (PUMA) and NOXA by p53. J Biol Chem 2006, 281 (11), 7260-70.

151. (a) Carracedo, A.; Lorente, M.; Egia, A.; Blazquez, C.; Garcia, S.; Giroux, V.; Malicet, C.; Villuendas, R.; Gironella, M.; Gonzalez-Feria, L.; Piris, M. A.; Iovanna, J. L.; Guzman, M.; Velasco, G., The stress-regulated protein p8 mediates cannabinoid-induced apoptosis of tumor cells. Cancer Cell 2006, 9 (4), 301-12; 
(b) Ogretmen, B.; Hannun, Y. A., Biologically active sphingolipids in cancer pathogenesis and treatment. Nat Rev Cancer 2004, 4 (8), 604-16.

152. Waldeck-Weiermair, M.; Zoratti, C.; Osibow, K.; Balenga, N.; Goessnitzer, E.; Waldhoer, M.; Malli, R.; Graier, W. F., Integrin clustering enables anandamideinduced $\mathrm{Ca} 2+$ signaling in endothelial cells via GPR 55 by protection against CB1-receptor-triggered repression. J Cell Sci 2008, 121 (10), 1704-17.

153. (a) Huffman, J. W.; Liddle, J.; Duncan, S. G., Jr.; Yu, S.; Martin, B. R.; Wiley, J. L., Synthesis and pharmacology of the isomeric methylheptyl-delta8tetrahydrocannabinols. Bioorg Med Chem 1998, 6 (12), 2383-96; (b) Huffman, J. W.; Lainton, J. A. H.; Banner, W. K.; Duncan, S. G.; Jordan, R. D.; Yu, S.; Dai, D.; Martin, B. R.; Wiley, J. L.; Compton, D. R., Side chain methyl analogues of delta8-THC. Tetrahedron 1997, 53 (5), 1557-76.

154. (a) Busch-Petersen, J.; Hill, W. A.; Fan, P.; Khanolkar, A.; Xie, X. Q.; Tius, M. A.; Makriyannis, A., Unsaturated side chain beta-11hydroxyhexahydrocannabinol analogs. J Med Chem 1996, 39 (19), 3790-6; (b) Martin, B. R.; Jefferson, R.; Winckler, R.; Wiley, J. L.; Huffman, J. W.; Crocker, P. J.; Saha, B.; Razdan, R. K., Manipulation of the tetrahydrocannabinol side chain delineates agonists, partial agonists, and antagonists. $J$ Pharmacol Exp Ther 1999, 290 (3), 1065-79.

155. Papahatjis, D. P.; Nikas, S. P.; Andreou, T.; Makriyannis, A., Novel 1',1'-chain substituted Delta(8)-tetrahydrocannabinols. Bioorg Med Chem Lett 2002, 12 (24), 3583-6.

156. Compton, D. R.; Johnson, M. R.; Melvin, L. S.; Martin, B. R., Pharmacological profile of a series of bicyclic cannabinoid analogs: classification as cannabimimetic agents. J Pharmacol Exp Ther 1992, 260 (1), 201-9.

157. Casiano, F. M.; Arnold, R.; Haycock, D.; Kuster, J.; Ward, S. J., Putative aminoalkylindoles (AAI) antagonists. NIDA Res Monogr 1990, 105, 295-6.

158. Rinaldi-Carmona, M.; Barth, F.; Heaulme, M.; Shire, D.; Calandra, B.; Congy, C.; Martinez, S.; Maruani, J.; Neliat, G.; Caput, D.; et al., SR141716A, a potent and selective antagonist of the brain cannabinoid receptor. FEBS Lett 1994, 350 (2-3), 240-4.

159. Diaz, P.; Xu, J.; Astruc-Diaz, F.; Pan, H. M.; Brown, D. L.; Naguib, M., Design and synthesis of a novel series of $\mathrm{N}$-alkyl isatin acylhydrazone derivatives that act as selective cannabinoid receptor 2 agonists for the treatment of neuropathic pain. $J$ Med Chem 2008, 51 (16), 4932-47.

160. Page, D.; Balaux, E.; Boisvert, L.; Liu, Z.; Milburn, C.; Tremblay, M.; Wei, Z.; Woo, S.; Luo, X.; Cheng, Y. X.; Yang, H.; Srivastava, S.; Zhou, F.; Brown, W.; Tomaszewski, M.; Walpole, C.; Hodzic, L.; St-Onge, S.; Godbout, C.; Salois, D.; Payza, K., Novel benzimidazole derivatives as selective CB2 agonists. Bioorg Med Chem Lett 2008, 18 (13), 3695-700.

161. Cheng, Y.; Albrecht, B. K.; Brown, J.; Buchanan, J. L.; Buckner, W. H.; DiMauro, E. F.; Emkey, R.; Fremeau, R. T., Jr.; Harmange, J. C.; Hoffman, B. J.; Huang, L.; Huang, M.; Lee, J. H.; Lin, F. F.; Martin, M. W.; Nguyen, H. Q.; Patel, V. F.; Tomlinson, S. A.; White, R. D.; Xia, X.; Hitchcock, S. A., Discovery and optimization of a novel series of $\mathrm{N}$-arylamide oxadiazoles as potent, highly 
selective and orally bioavailable cannabinoid receptor 2 (CB2) agonists. $J$ Med Chem 2008, 51 (16), 5019-34.

162. Pasquini, S.; Botta, L.; Semeraro, T.; Mugnaini, C.; Ligresti, A.; Palazzo, E.; Maione, S.; Di Marzo, V.; Corelli, F., Investigations on the 4-quinolone-3carboxylic acid motif. 2. Synthesis and structure-activity relationship of potent and selective cannabinoid-2 receptor agonists endowed with analgesic activity in vivo. J Med Chem 2008, 51 (16), 5075-84.

163. Lavey, B. J.; Kozlowski, J. A.; Shankar, B. B.; Spitler, J. M.; Zhou, G.; Yang, D. Y.; Shu, Y.; Wong, M. K.; Wong, S. C.; Shih, N. Y.; Wu, J.; McCombie, S. W.; Rizvi, R.; Wolin, R. L.; Lunn, C. A., Optimization of triaryl bis-sulfones as cannabinoid-2 receptor ligands. Bioorg Med Chem Lett 2007, 17 (13), 3760-4.

164. Seltzman, H. H., Structure and receptor activity for classical cannabinoids. Curr Med Chem 1999, 6 (8), 685-704.

165. Huffman, J. W.; Yu, S.; Showalter, V.; Abood, M. E.; Wiley, J. L.; Compton, D. R.; Martin, B. R.; Bramblett, R. D.; Reggio, P. H., Synthesis and pharmacology of a very potent cannabinoid lacking a phenolic hydroxyl with high affinity for the CB2 receptor. J Med Chem 1996, 39 (20), 3875-7.

166. Salo, O. M.; Lahtela-Kakkonen, M.; Gynther, J.; Jarvinen, T.; Poso, A., Development of a 3D model for the human cannabinoid CB1 receptor. $J$ Med Chem 2004, 47 (12), 3048-57.

167. Gareau, Y.; Dufresne, C.; Gallant, M.; Rochette, C.; Sawyer, N.; Slipetz, D.; Tremblay, D.; Weech, P.; Metters, K.; Labelle, M., Structure activity relationships of tetrahydrocannabinol analogues on human cannabinoid receptors. Bioorg Med Chem Lett 1996, 6 (2), 189-94.

168. Tietze, L. F., Domino reactions in organic synthesis. Chem Rev 1996, 96 (1), 11536.

169. Eilingsfeld, H.; Patsch, M.; Scheuermann, H., Synthese von pyrimidinen aus malondiimidsäureestern. Chemische Berichte 1968, 101 (7), 2426-34.

170. Kim, C. U.; Misco, P. F.; Luh, B. Y.; Mansuri, M. M., 'Me(3)Al-Tmsoso(2)Cf(3) a new reagent for conversion of carbonyl to geminal dimethyl functionality regiospecific synthesis of alkylated a-ring of arotinoids. Tetrahedron Letters 1994, 35 (19), 3017-20.

171. Boucher, E.; Simard, M.; Wuest, J. D., Use of hydrogen-bonds to control molecular aggregation - behavior of dipyridones and pyridone-pyrimidones designed to form cyclic triplexes. Journal of Organic Chemistry 1995, 60 (5), 1408-12.

172. Caron, S.; Vazquez, E.; Wojcik, J. M., Preparation of tertiary benzylic nitriles from aryl fluorides. J Am Chem Soc 2000, 122 (4), 712-3.

173. Crich, D.; Xu, H. D.; Kenig, F., Total synthesis and structural verification of some novel branched alkanes with quaternary carbons isolated from diverse geological sources. Journal of Organic Chemistry 2006, 71 (13), 5016-9.

174. Moss, R. A.; Ma, W.; Merrer, D. C.; Xue, S., Conversion of obstinate nitriles to amidines by Garigipatis reaction. Tetrahedron Letters 1995, 36 (48), 8761-4.

175. Henze, H. R.; Clegg, W. J.; Smart, C. W., Researches on pyrimidines: certain derivatives of 2-methylpyrimidine. The Journal of Organic Chemistry 1952, 17 (10), 1320-7. 
176. Kulkarni, S. S. N.; Hauck, A., Discovery of heterobicyclic templates for novel metabotropic glutamate receptor subtype 5 antagonists. Bioorg Med Chem Lett 2007, 17 (11), 2987-91.

177. Yip, S. F.; Cheung, H. Y.; Zhou, Z.; Kwong, F. Y., Room-temperature coppercatalyzed alpha-arylation of malonates. Org Lett 2007, 9 (17), 3469-72.

178. (a) DiMauro, E. F.; Newcomb, J.; Nunes, J. J.; Bemis, J. E.; Boucher, C.; Buchanan, J. L.; Buckner, W. H.; Cheng, A.; Faust, T.; Hsieh, F.; Huang, X.; Lee, J. H.; Marshall, T. L.; Martin, M. W.; McGowan, D. C.; Schneider, S.; Turci, S. M.; White, R. D.; Zhu, X., Discovery of 4-amino-5,6-biaryl-furo[2,3d]pyrimidines as inhibitors of Lck: development of an expedient and divergent synthetic route and preliminary SAR. Bioorg Med Chem Lett 2007, 17 (8), 23059; (b) Buchanan, J. L.; Buckner, W. H.; Burkitt, S. A.; DiMauro, E. F.; Farthing, C. N.; Frenkel, A. D.; Harrison, M. J.; Kayser, F.; Liu, J.; Lively, S. E.; Marshall, T. L.; McGowan, D. C.; Sharma, R.; Shuttleworth, S. J.; Zhu, X., Furanopyrimidines (United States Patent Application 20060040961). 2006.

179. Nunez, E.; Benito, C.; Pazos, M. R.; Barbachano, A.; Fajardo, O.; Gonzalez, S.; Tolon, R. M.; Romero, J., Cannabinoid CB2 receptors are expressed by perivascular microglial cells in the human brain: an immunohistochemical study. Synapse 2004, 53 (4), 208-13.

180. (a) Anand, U.; Otto, W. R.; Sanchez-Herrera, D.; Facer, P.; Yiangou, Y.; Korchev, Y.; Birch, R.; Benham, C.; Bountra, C.; Chessell, I. P.; Anand, P., Cannabinoid receptor CB2 localisation and agonist-mediated inhibition of capsaicin responses in human sensory neurons. Pain 2008, 138 (3), 667-80; (b) Wotherspoon, G.; Fox, A.; McIntyre, P.; Colley, S.; Bevan, S.; Winter, J., Peripheral nerve injury induces cannabinoid receptor 2 protein expression in rat sensory neurons. Neuroscience 2005, 135 (1), 235-45.

181. Mikuriya, T. H., Marijuana in medicine: past, present and future. Calif Med 1969, $110(1), 34-40$.

182. Valenzano, K. J.; Tafesse, L.; Lee, G.; Harrison, J. E.; Boulet, J. M.; Gottshall, S. L.; Mark, L.; Pearson, M. S.; Miller, W.; Shan, S.; Rabadi, L.; Rotshteyn, Y.; Chaffer, S. M.; Turchin, P. I.; Elsemore, D. A.; Toth, M.; Koetzner, L.; Whiteside, G. T., Pharmacological and pharmacokinetic characterization of the cannabinoid receptor 2 agonist, GW405833, utilizing rodent models of acute and chronic pain, anxiety, ataxia and catalepsy. Neuropharmacology 2005, 48 (5), $658-72$.

183. Yao, B. B.; Hsieh, G. C.; Frost, J. M.; Fan, Y.; Garrison, T. R.; Daza, A. V.; Grayson, G. K.; Zhu, C. Z.; Pai, M.; Chandran, P.; Salyers, A. K.; Wensink, E. J.; Honore, P.; Sullivan, J. P.; Dart, M. J.; Meyer, M. D., In vitro and in vivo characterization of A-796260: a selective cannabinoid CB2 receptor agonist exhibiting analgesic activity in rodent pain models. Br J Pharmacol 2008, 153 (2), 390-401.

184. Yao, B. B.; Hsieh, G.; Daza, A. V.; Fan, Y.; Grayson, G. K.; Garrison, T. R.; El Kouhen, O.; Hooker, B. A.; Pai, M.; Wensink, E. J.; Salyers, A. K.; Chandran, P.; Zhu, C. Z.; Zhong, C.; Ryther, K.; Gallagher, M. E.; Chin, C. L.; Tovcimak, A. E.; Hradil, V. P.; Fox, G. B.; Dart, M. J.; Honore, P.; Meyer, M. D., Characterization of a cannabinoid CB2 receptor-selective agonist, A-836339 
[2,2,3,3-tetramethyl-cyclopropanecarboxylic acid [3-(2-methoxy-ethyl)-4,5dimethyl-3H-thiazol-(2Z)-ylidene]-amide], using in vitro pharmacological assays, in vivo pain models, and pharmacological magnetic resonance imaging. $J$ Pharmacol Exp Ther 2009, 328 (1), 141-51.

185. Rinaldi-Carmona, M.; Barth, F.; Millan, J.; Derocq, J. M.; Casellas, P.; Congy, C.; Oustric, D.; Sarran, M.; Bouaboula, M.; Calandra, B.; Portier, M.; Shire, D.; Breliere, J. C.; Le Fur, G. L., SR 144528, the first potent and selective antagonist of the CB2 cannabinoid receptor. J Pharmacol Exp Ther 1998, 284 (2), 644-50.

186. Hosohata, Y.; Quock, R. M.; Hosohata, K.; Makriyannis, A.; Consroe, P.; Roeske, W. R.; Yamamura, H. I., AM630 antagonism of cannabinoid-stimulated [35S]GTP gamma $\mathrm{S}$ binding in the mouse brain. Eur J Pharmacol 1997, 321 (1), R1-3.

187. Giblin, G. M.; O'Shaughnessy, C. T.; Naylor, A.; Mitchell, W. L.; Eatherton, A. J.; Slingsby, B. P.; Rawlings, D. A.; Goldsmith, P.; Brown, A. J.; Haslam, C. P.; Clayton, N. M.; Wilson, A. W.; Chessell, I. P.; Wittington, A. R.; Green, R., Discovery of 2-[(2,4-dichlorophenyl)amino]-N-[(tetrahydro-2H-pyran-4yl)methyl]-4-(trifluoromethyl)-5-pyrimidinecarboxamide, a selective CB2 receptor agonist for the treatment of inflammatory pain. $J$ Med Chem 2007, 50 (11), 2597-600.

188. (a) Kikuchi, A.; Ohashi, K.; Sugie, Y.; Sugimoto, H.; Omura, H., Pharmacological evaluation of a novel cannabinoid 2 (CB2) ligand, PF-03550096, in vitro and in vivo by using a rat model of visceral hypersensitivity. $J$ Pharmacol Sci 2008, 106 (2), 219-24; (b) Omura, H.; Kawai, M.; Shima, A.; Iwata, Y.; Ito, F.; Masuda, T.; Ohta, A.; Makita, N.; Omoto, K.; Sugimoto, H.; Kikuchi, A.; Iwata, H.; Ando, K., The SAR studies of novel CB2 selective agonists, benzimidazolone derivatives. Bioorg Med Chem Lett 2008, 18 (11), 3310-4.

189. (a) Maresz, K.; Pryce, G.; Ponomarev, E. D.; Marsicano, G.; Croxford, J. L.; Shriver, L. P.; Ledent, C.; Cheng, X.; Carrier, E. J.; Mann, M. K.; Giovannoni, G.; Pertwee, R. G.; Yamamura, T.; Buckley, N. E.; Hillard, C. J.; Lutz, B.; Baker, D.; Dittel, B. N., Direct suppression of CNS autoimmune inflammation via the cannabinoid receptor CB1 on neurons and CB2 on autoreactive T cells. Nat Med 2007, 13 (4), 492-7; (b) Palazuelos, J.; Davoust, N.; Julien, B.; Hatterer, E.; Aguado, T.; Mechoulam, R.; Benito, C.; Romero, J.; Silva, A.; Guzman, M.; Nataf, S.; Galve-Roperh, I., The CB(2) cannabinoid receptor controls myeloid progenitor trafficking: involvement in the pathogenesis of an animal model of multiple sclerosis. J Biol Chem 2008, 283 (19), 13320-9.

190. Belvisi, M. G.; Patel, H. J.; Freund-Michel, V.; Hele, D. J.; Crispino, N.; Birrell, M. A., Inhibitory activity of the novel CB2 receptor agonist, GW833972A, on guinea-pig and human sensory nerve function in the airways. Br J Pharmacol 2008, 155 (4), 547-57.

191. Ofek, O.; Karsak, M.; Leclerc, N.; Fogel, M.; Frenkel, B.; Wright, K.; Tam, J.; Attar-Namdar, M.; Kram, V.; Shohami, E.; Mechoulam, R.; Zimmer, A.; Bab, I., Peripheral cannabinoid receptor, CB2, regulates bone mass. Proc Natl Acad Sci U S A 2006, 103 (3), 696-701. 
192. Steffens, S.; Veillard, N. R.; Arnaud, C.; Pelli, G.; Burger, F.; Staub, C.; Karsak, M.; Zimmer, A.; Frossard, J. L.; Mach, F., Low dose oral cannabinoid therapy reduces progression of atherosclerosis in mice. Nature 2005, 434 (7034), 782-6.

193. Han, S.; Thatte, J.; Jones, R. M., Recent advances in the discovery of $\mathrm{CB}_{2}$ selective agonists. Annual Reports in Medicinal Chemistry 2009, 44, 227-46.

194. Pharmos Corporation, CB2 selective program: Cannabinor, http://www.pharmoscorp.com/development/cannabinor.html, accessed October 25, 2009.

195. Pharmos Corporation, CB2 selective program: second generation compounds, http://www.pharmoscorp.com/development/syn_cannabinoid.html, accessed October 25, 2009.

196. Clinical Trials.gov: a service of the U.S. National Institutes of Health, www.clinicaltrials.gov/ct2/results?term=gw842166 accessed January 14, 2009.

197. GlaxoSmithKline, Product development pipeline, http://www.gsk.com/investors/product pipeline/docs/GSK-product-pipeline-Feb2010.pdf, accessed March 4, 2010.

198. Glenmark Pharmaceuticals, Press release: Glenmark's molecule for neuropathic pain, osteoarthritis - GRC 10693, successfully completes Phase I trials, http:/www.glenmarkpharma.com/GLN_NWS/pdf/ Glenmarks_molecule_neuropathicpain_osteoarthritis_GRC10693_succes.pdf, accessed October 27, 2009.

199. Schroder, K.; Hertzog, P. J.; Ravasi, T.; Hume, D. A., Interferon-gamma: an overview of signals, mechanisms and functions. J Leukoc Biol 2004, 75 (2), 163 89.

200. Germann, T.; Rude, E., Interleukin-12. Int Arch Allergy Immunol 1995, 108 (2), 103-12.

201. O'Garra, A.; Murphy, K., Role of cytokines in determining T-lymphocyte function. Curr Opin Immunol 1994, 6 (3), 458-66.

202. Nishimoto, N., Interleukin-6 as a therapeutic target in candidate inflammatory diseases. Clin Pharmacol Ther 2010, 87 (4), 483-7.

203. Hamilton, J. A., GM-CSF in inflammation and autoimmunity. Trends Immunol 2002, 23 (8), 403-8.

204. Pober, J. S., Effects of tumour necrosis factor and related cytokines on vascular endothelial cells. Ciba Found Symp 1987, 131, 170-84.

205. Pestka, S.; Krause, C. D.; Sarkar, D.; Walter, M. R.; Shi, Y.; Fisher, P. B., Interleukin-10 and related cytokines and receptors. Annu Rev Immunol 2004, 22, 929-79.

206. Fazekas de St Groth, B.; Smith, A. L.; Higgins, C. A., T cell activation: in vivo veritas. Immunol Cell Biol 2004, 82 (3), 260-8.

207. Bystry, R. S.; Aluvihare, V.; Welch, K. A.; Kallikourdis, M.; Betz, A. G., B cells and professional APCs recruit regulatory T cells via CCL4. Nat Immunol 2001, 2 (12), 1126-32.

208. Wolpe, S. D.; Cerami, A., Macrophage inflammatory proteins 1 and 2: members of a novel superfamily of cytokines. FASEB J 1989, 3 (14), 2565-73. 
209. Miller, M. D.; Krangel, M. S., Biology and biochemistry of the chemokines: a family of chemotactic and inflammatory cytokines. Crit Rev Immunol 1992, 12 (1-2), 17-46.

210. Sherry, B.; Cerami, A., Small cytokine superfamily. Curr Opin Immunol 1991, 3 (1), 56-60.

211. (a) Carr, M. W.; Roth, S. J.; Luther, E.; Rose, S. S.; Springer, T. A., Monocyte chemoattractant protein 1 acts as a T-lymphocyte chemoattractant. Proc Natl Acad Sci U S A 1994, 91 (9), 3652-6; (b) Xu, L. L.; Warren, M. K.; Rose, W. L.; Gong, W.; Wang, J. M., Human recombinant monocyte chemotactic protein and other $\mathrm{C}-\mathrm{C}$ chemokines bind and induce directional migration of dendritic cells in vitro. J Leukoc Biol 1996, 60 (3), 365-71; (c) Conti, P.; Boucher, W.; Letourneau, R.; Feliciani, C.; Reale, M.; Barbacane, R. C.; Vlagopoulos, P.; Bruneau, G.; Thibault, J.; Theoharides, T. C., Monocyte chemotactic protein-1 provokes mast cell aggregation and [3H]5HT release. Immunology 1995, 86 (3), 434-40; (d) Bischoff, S. C.; Krieger, M.; Brunner, T.; Dahinden, C. A., Monocyte chemotactic protein 1 is a potent activator of human basophils. J Exp Med 1992, 175 (5), 1271-5.

212. (a) Rose, C. E., Jr.; Sung, S. S.; Fu, S. M., Significant involvement of CCL2 (MCP-1) in inflammatory disorders of the lung. Microcirculation 2003, 10 (3-4), 273-88; (b) Romagnani, S., Cytokines and chemoattractants in allergic inflammation. Mol Immunol 2002, 38 (12-13), 881-5.

213. Hennig, B.; Lei, W.; Arzuaga, X.; Ghosh, D. D.; Saraswathi, V.; Toborek, M., Linoleic acid induces proinflammatory events in vascular endothelial cells via activation of PI3K/Akt and ERK1/2 signaling. J Nutr Biochem 2006, 17 (11), 766-72.

214. Frank-Cannon, T. C.; Alto, L. T.; McAlpine, F. E.; Tansey, M. G., Does neuroinflammation fan the flame in neurodegenerative diseases? Mol Neurodegener 2009, 4, 47.

215. (a) Hodge, D. R.; Hurt, E. M.; Farrar, W. L., The role of IL-6 and STAT3 in inflammation and cancer. Eur J Cancer 2005, 41 (16), 2502-12; (b) Schaffer, B. A.; Bertram, L.; Miller, B. L.; Mullin, K.; Weintraub, S.; Johnson, N.; Bigio, E. H.; Mesulam, M.; Wiedau-Pazos, M.; Jackson, G. R.; Cummings, J. L.; Cantor, R. M.; Levey, A. I.; Tanzi, R. E.; Geschwind, D. H., Association of GSK3B with Alzheimer disease and frontotemporal dementia. Arch Neurol 2008, 65 (10), 1368-74; (c) Kim, H. S.; Kim, E. M.; Lee, J. P.; Park, C. H.; Kim, S.; Seo, J. H.; Chang, K. A.; Yu, E.; Jeong, S. J.; Chong, Y. H.; Suh, Y. H., C-terminal fragments of amyloid precursor protein exert neurotoxicity by inducing glycogen synthase kinase-3beta expression. FASEB J 2003, 17 (13), 1951-3.

216. (a) He, Y.; Xu, H.; Liang, L.; Zhan, Z.; Yang, X.; Yu, X.; Ye, Y.; Sun, L., Antiinflammatory effect of Rho kinase blockade via inhibition of NF-kappaB activation in rheumatoid arthritis. Arthritis Rheum 2008, 58 (11), 3366-76; (b) Wu, X.; Guo, R.; Chen, P.; Wang, Q.; Cunningham, P. N., TNF induces caspasedependent inflammation in renal endothelial cells through a Rho- and myosin light chain kinase-dependent mechanism. Am J Physiol Renal Physiol 2009, 297 (2), F316-26; (c) Shimada, H.; Rajagopalan, L. E., Rho-kinase mediates 
lysophosphatidic acid-induced IL-8 and MCP-1 production via p38 and JNK pathways in human endothelial cells. FEBS Lett 2010, 584 (13), 2827-32. 
Appendix A: Cytokine and Chemokine Release Profiles of THP-1, KG-1, A549, and HUV-EC-C 

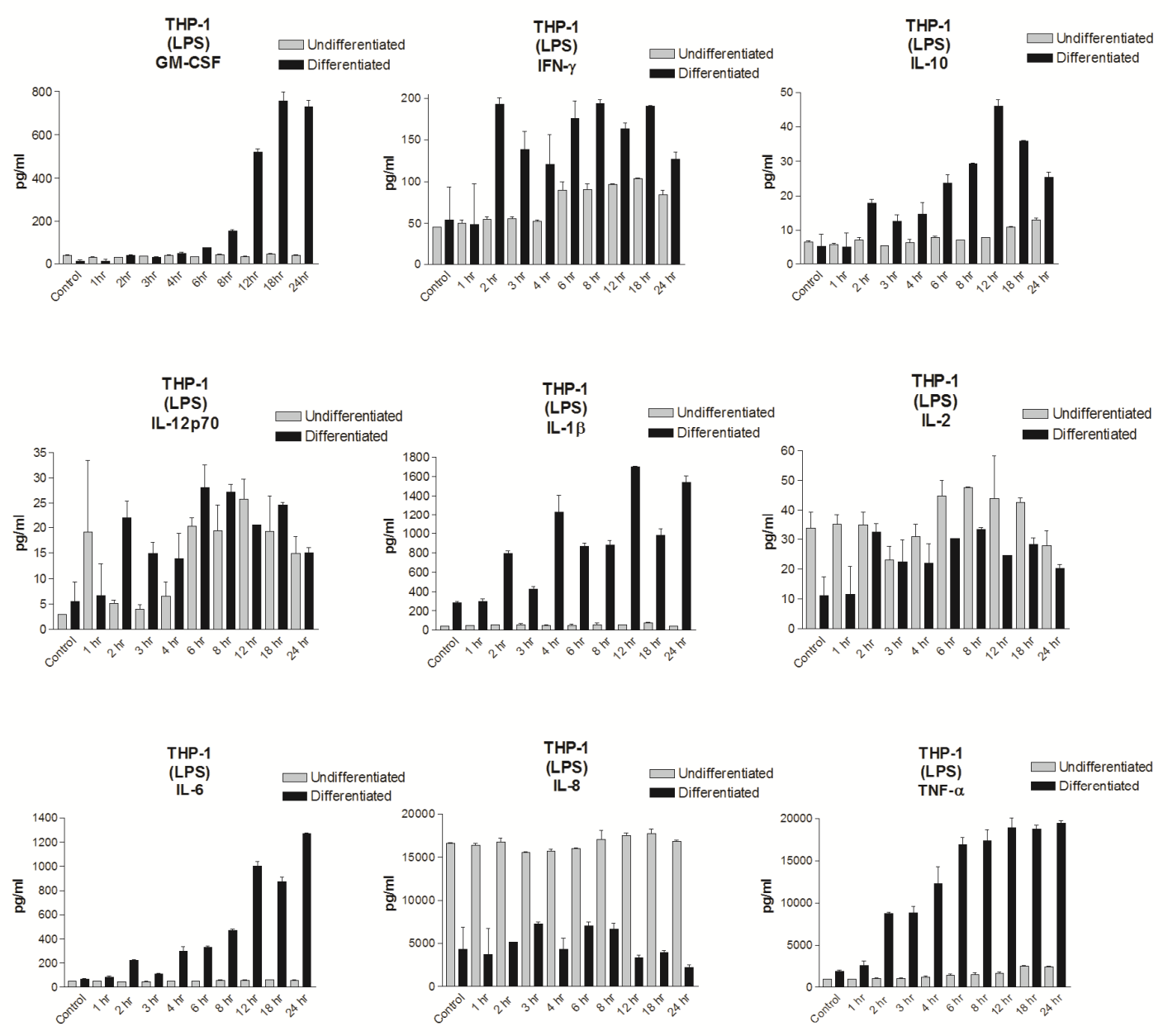

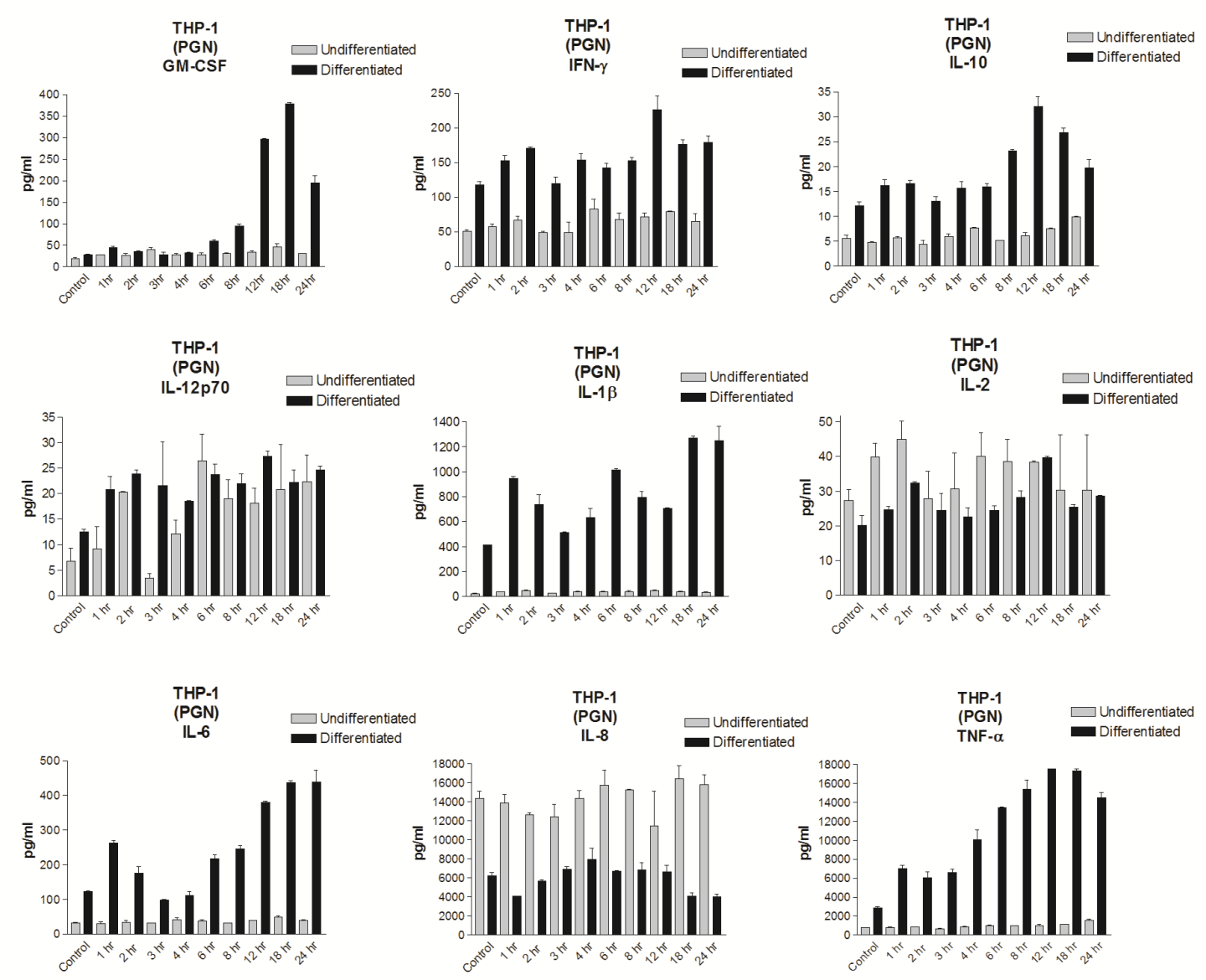

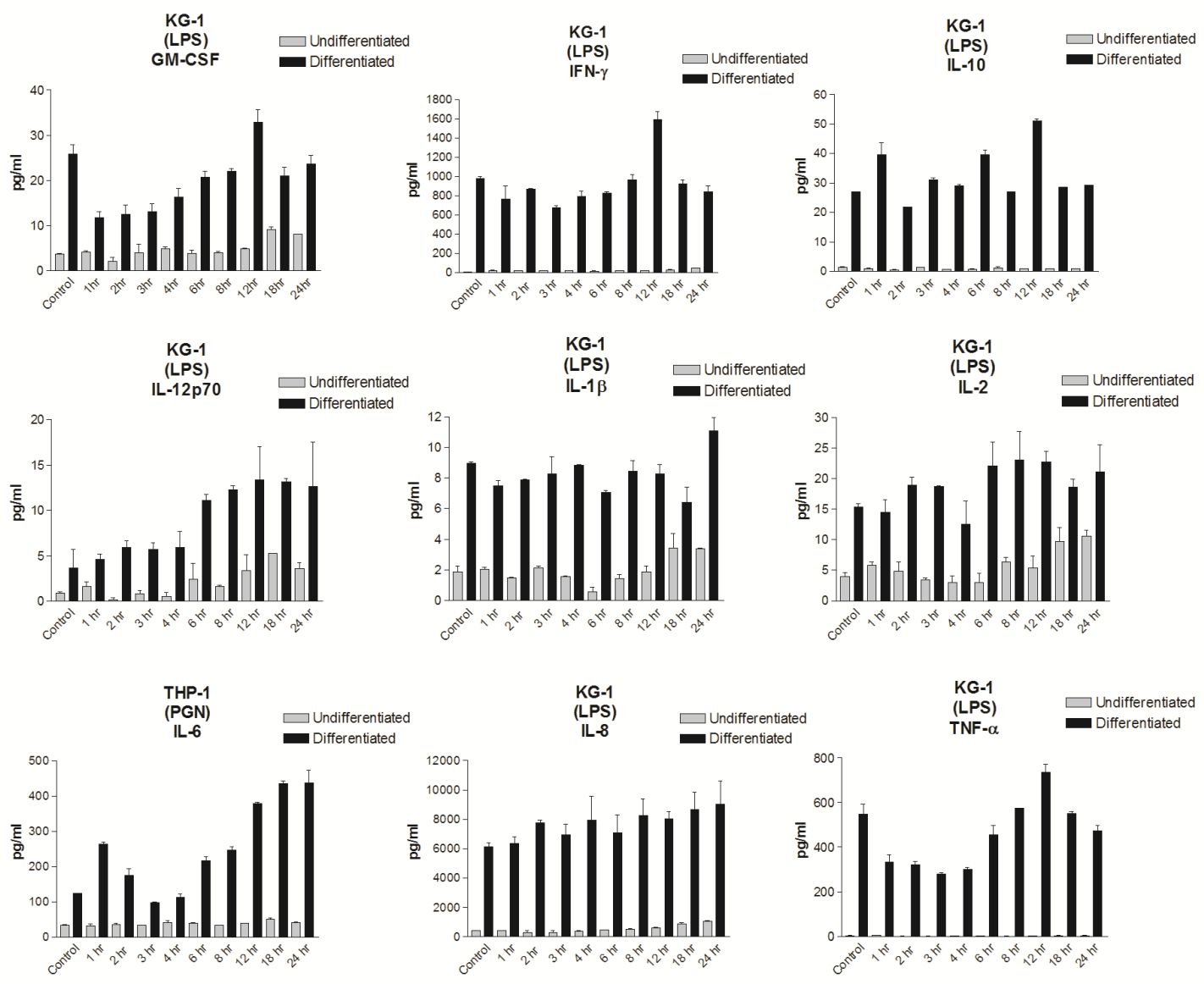

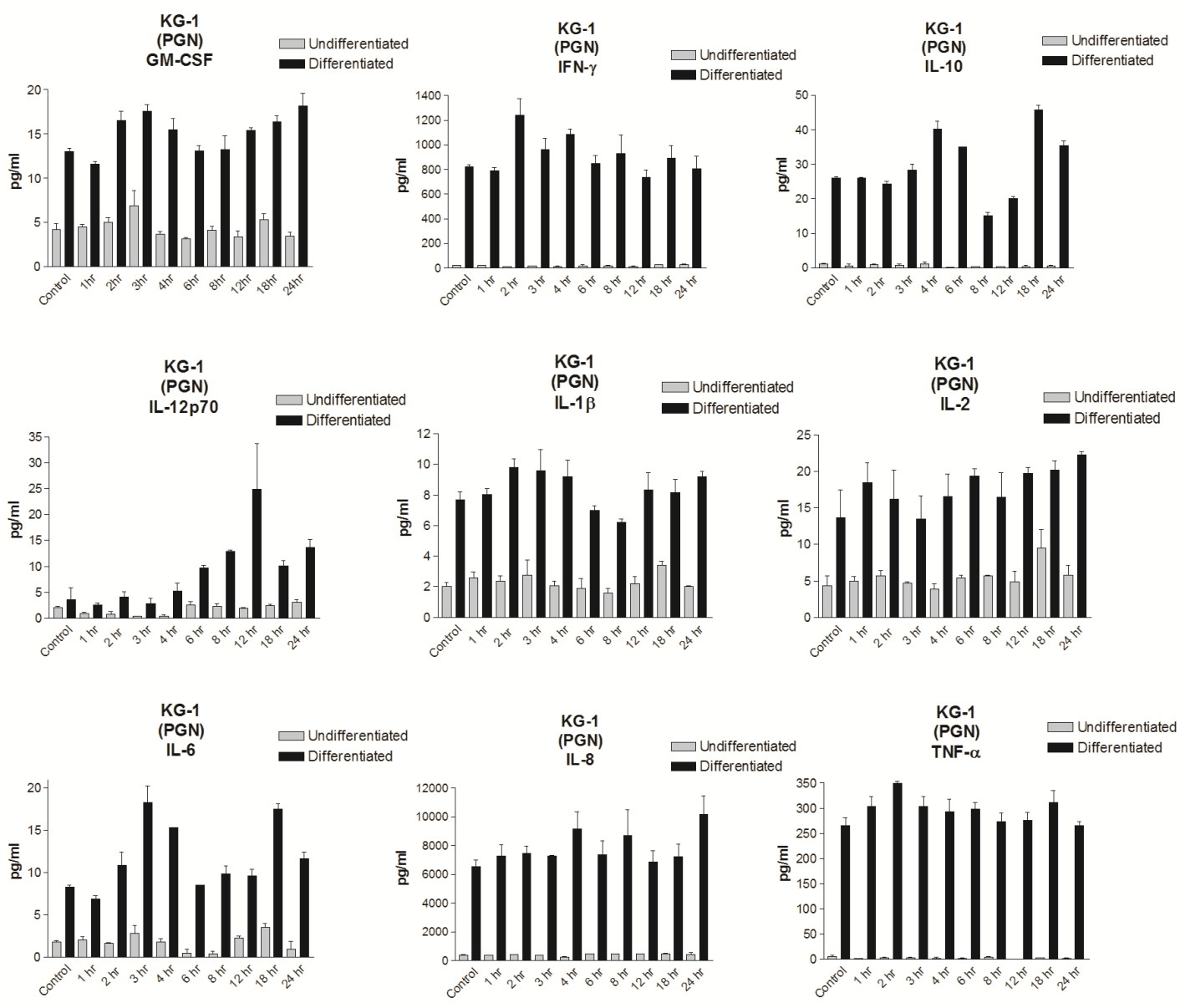

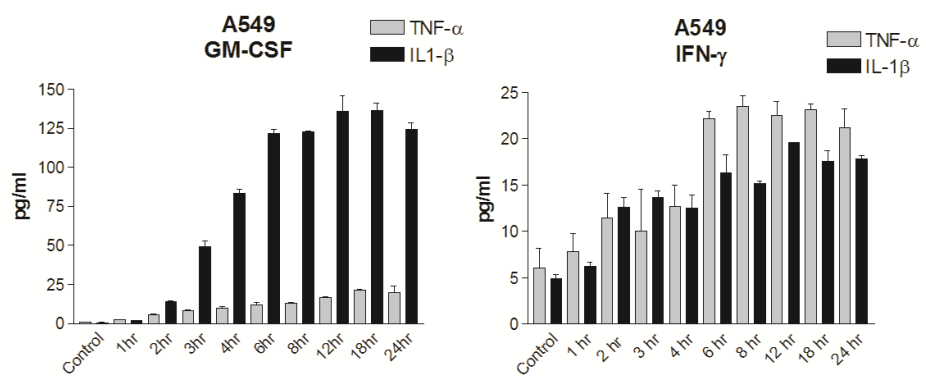

${ }_{\mathrm{IL}-10}^{\mathrm{A} 549} \square_{\mathrm{IL}-1 \beta}^{\mathrm{TNF}-\alpha}$
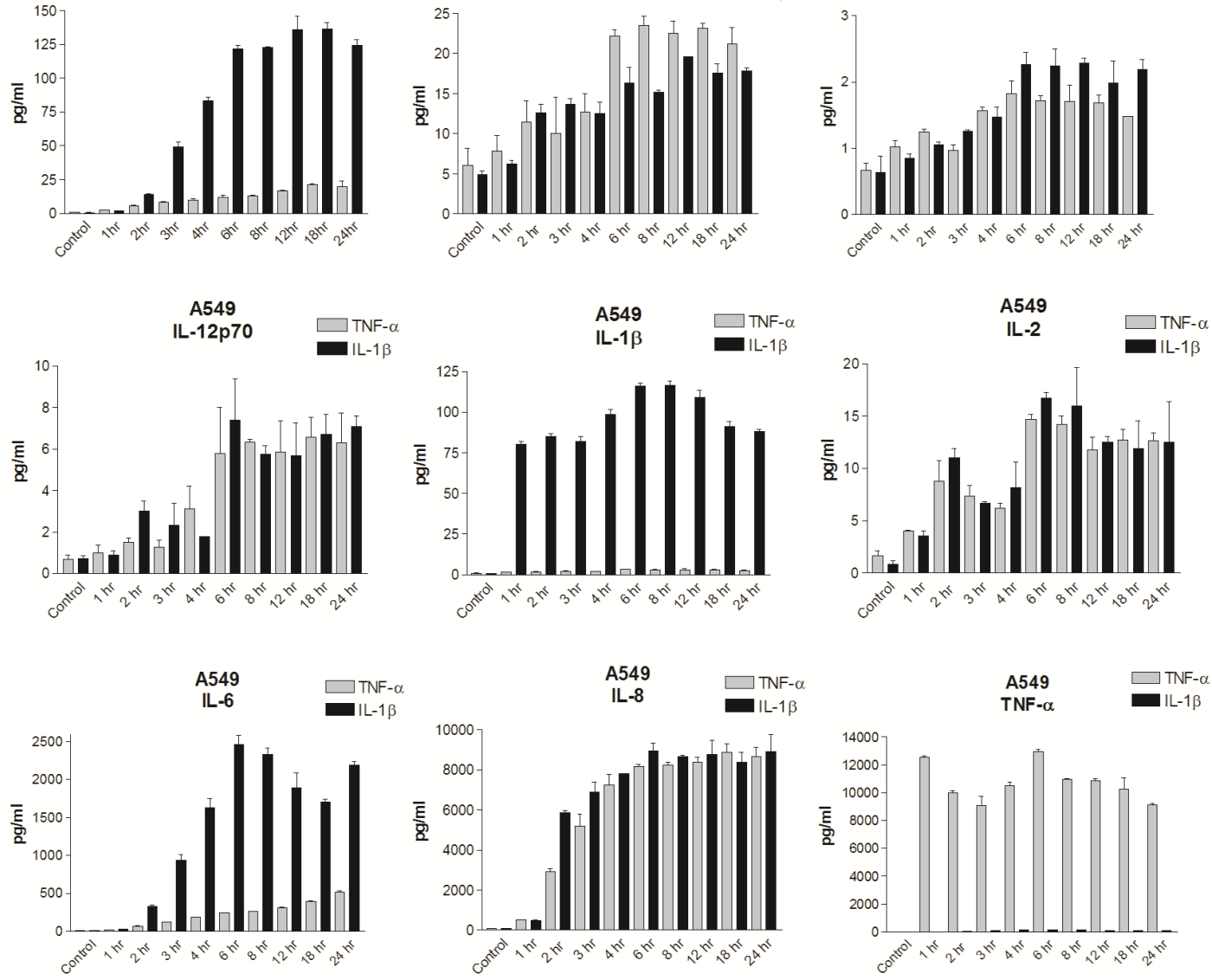

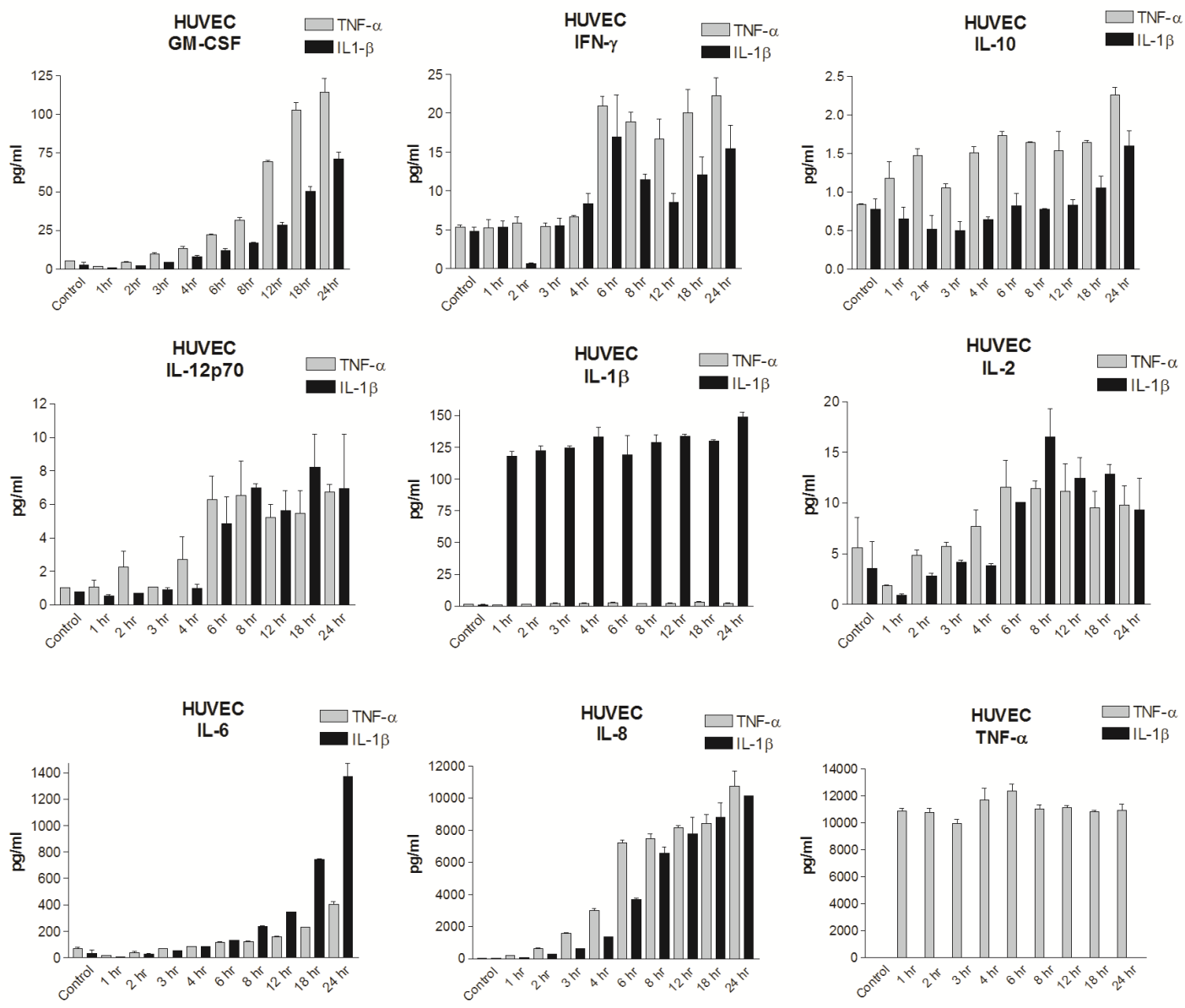

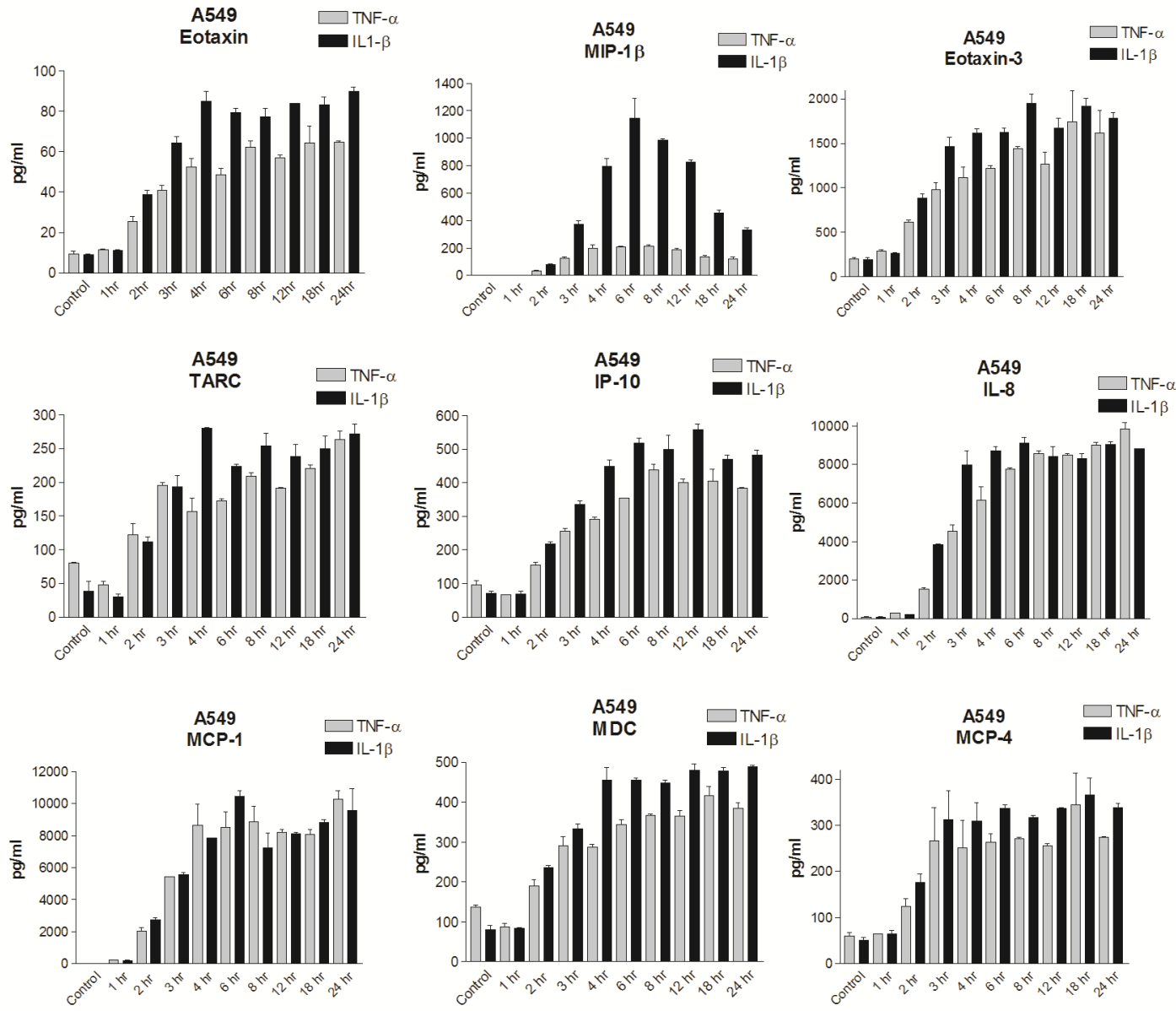

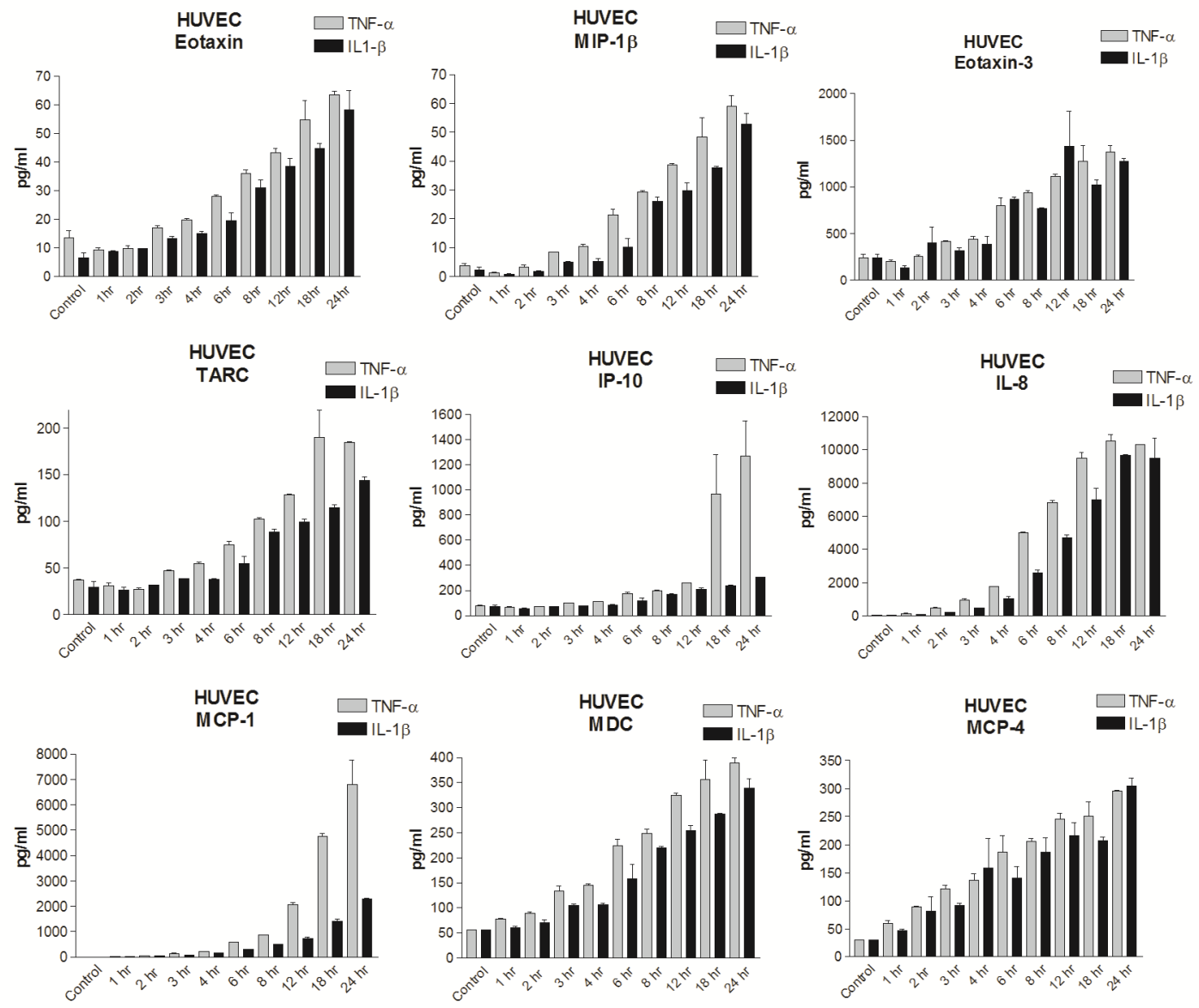
Appendix B: Effects of Eight Cannabinoids on Cytokine and Chemokine Release of THP-1, A549, and HUV-EC-C 

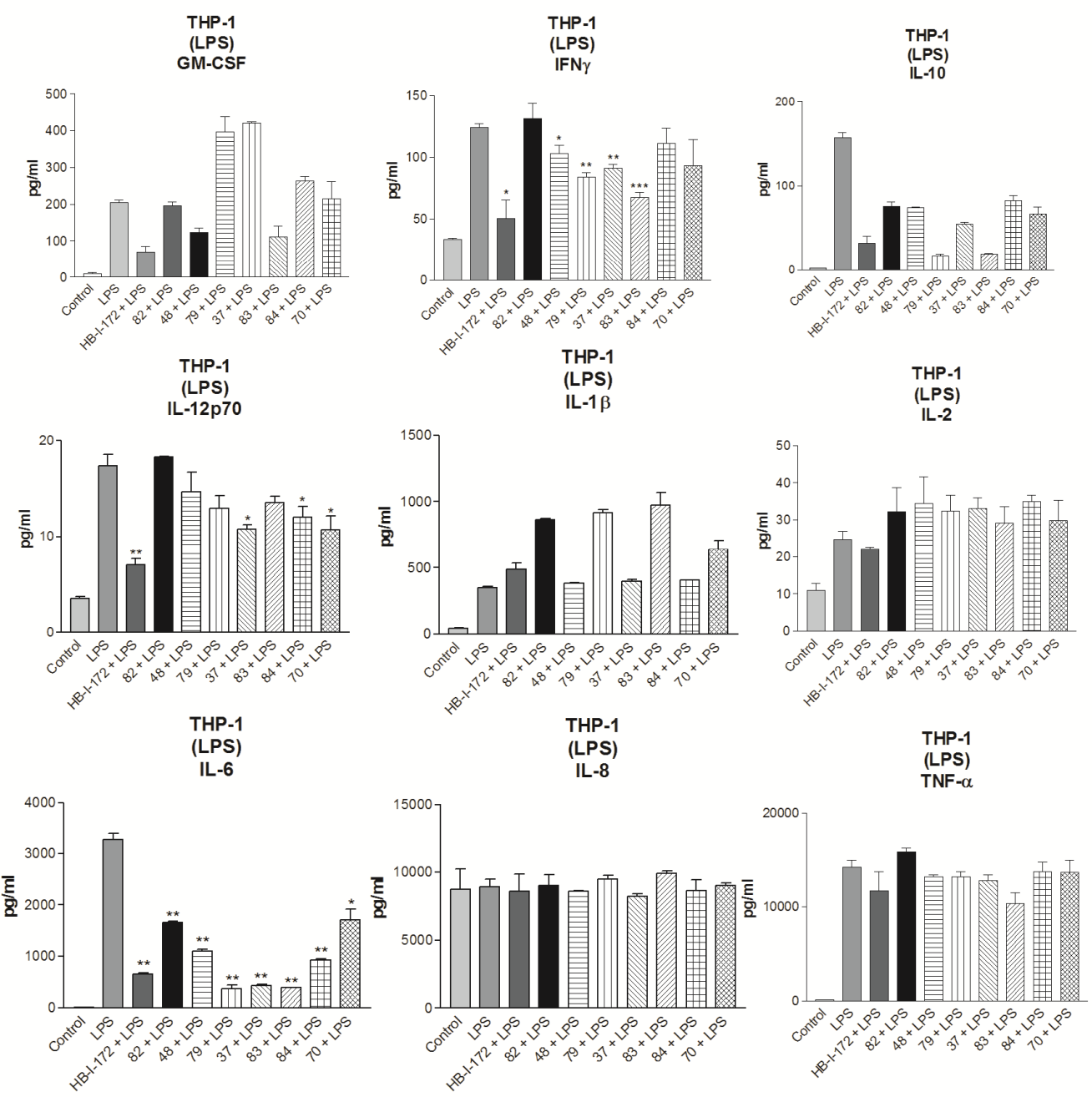


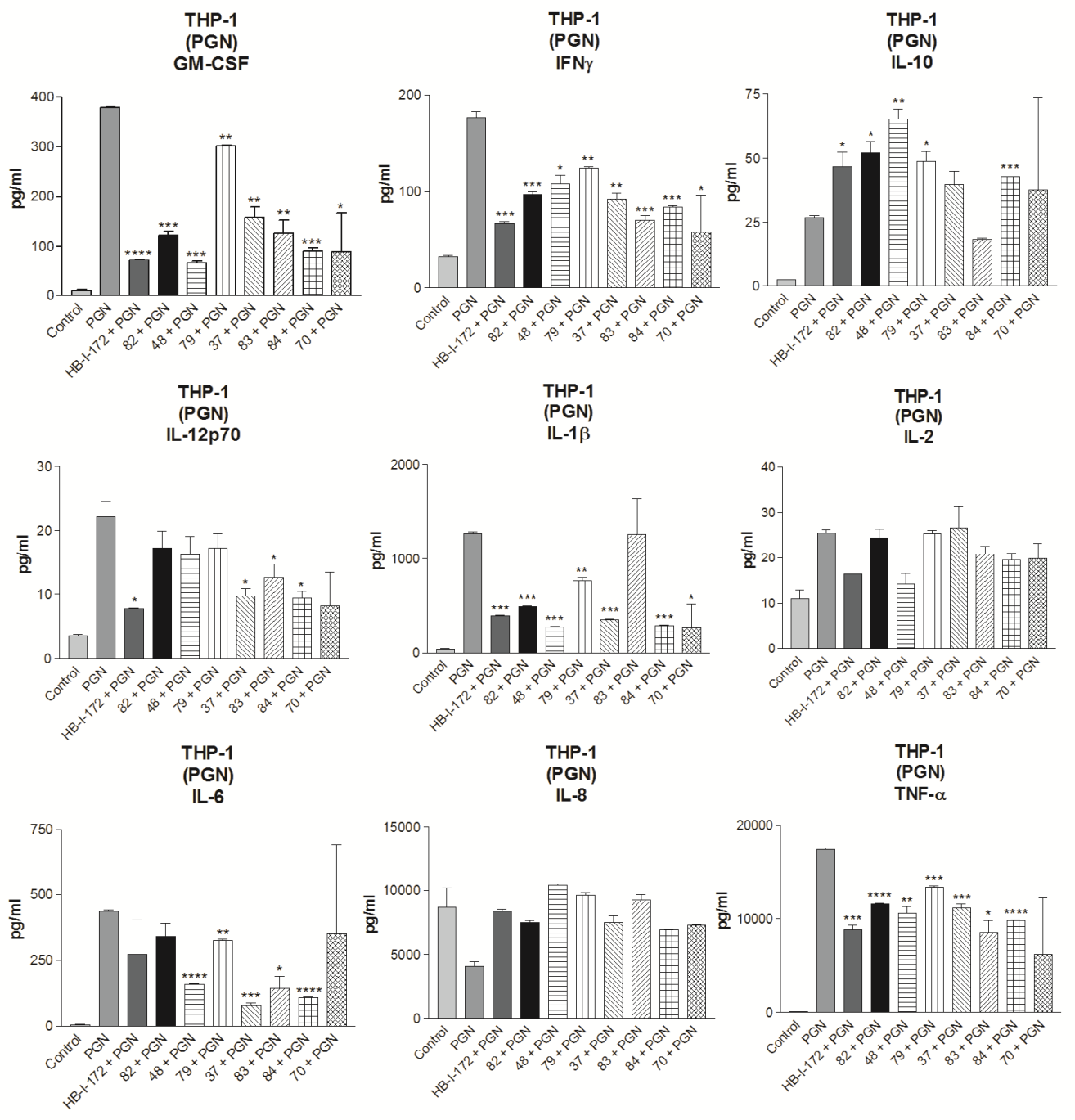



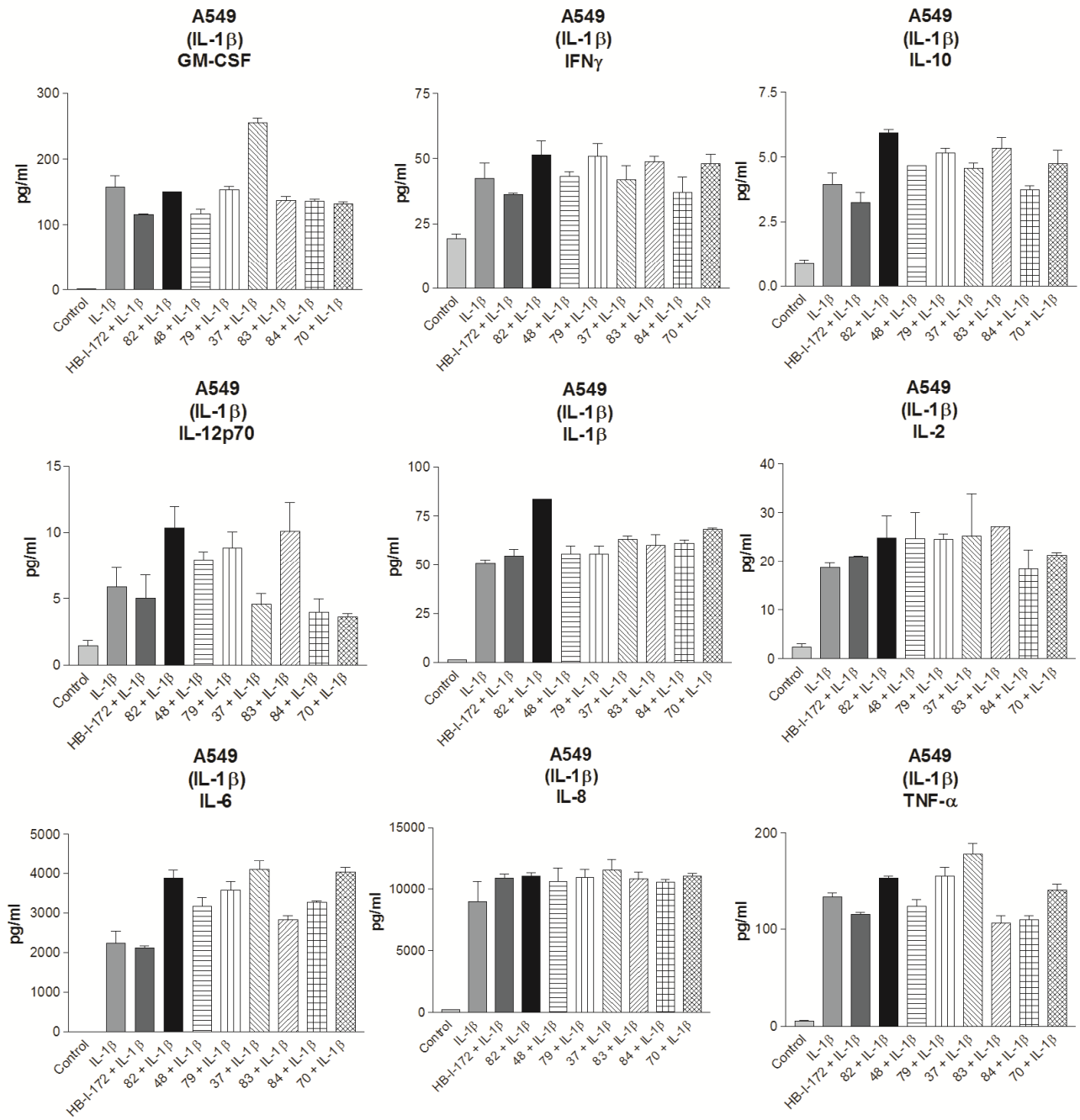

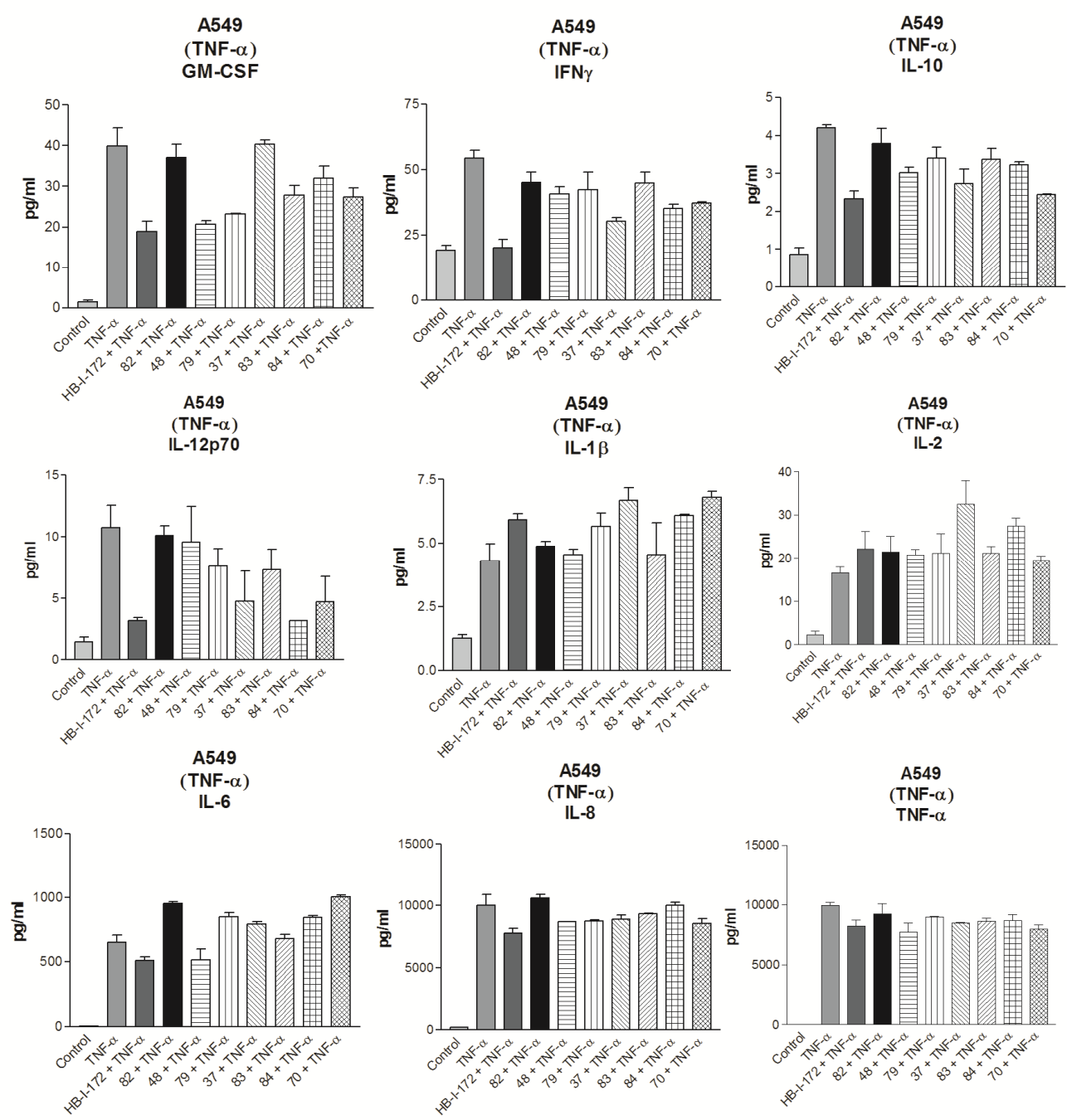

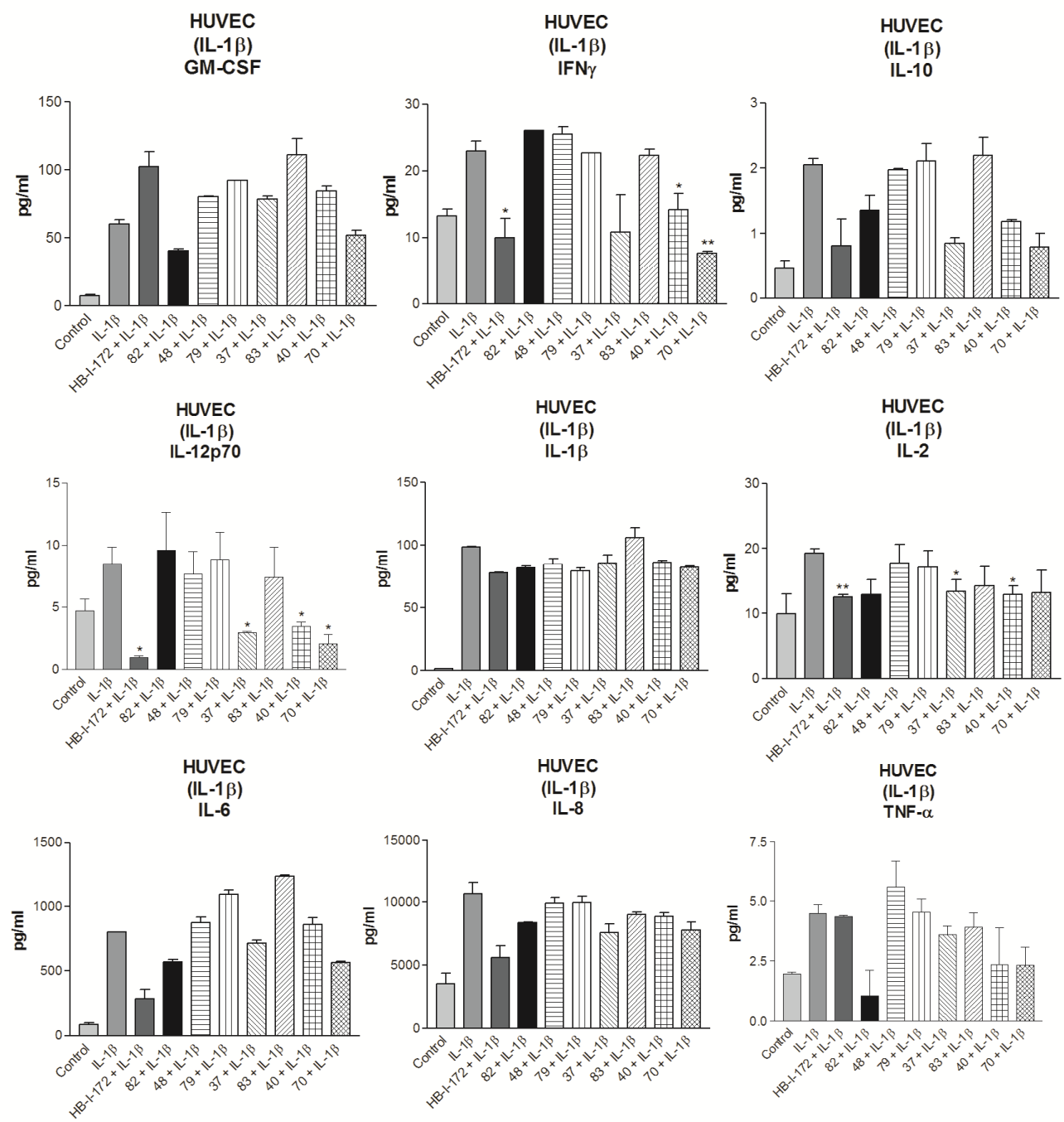

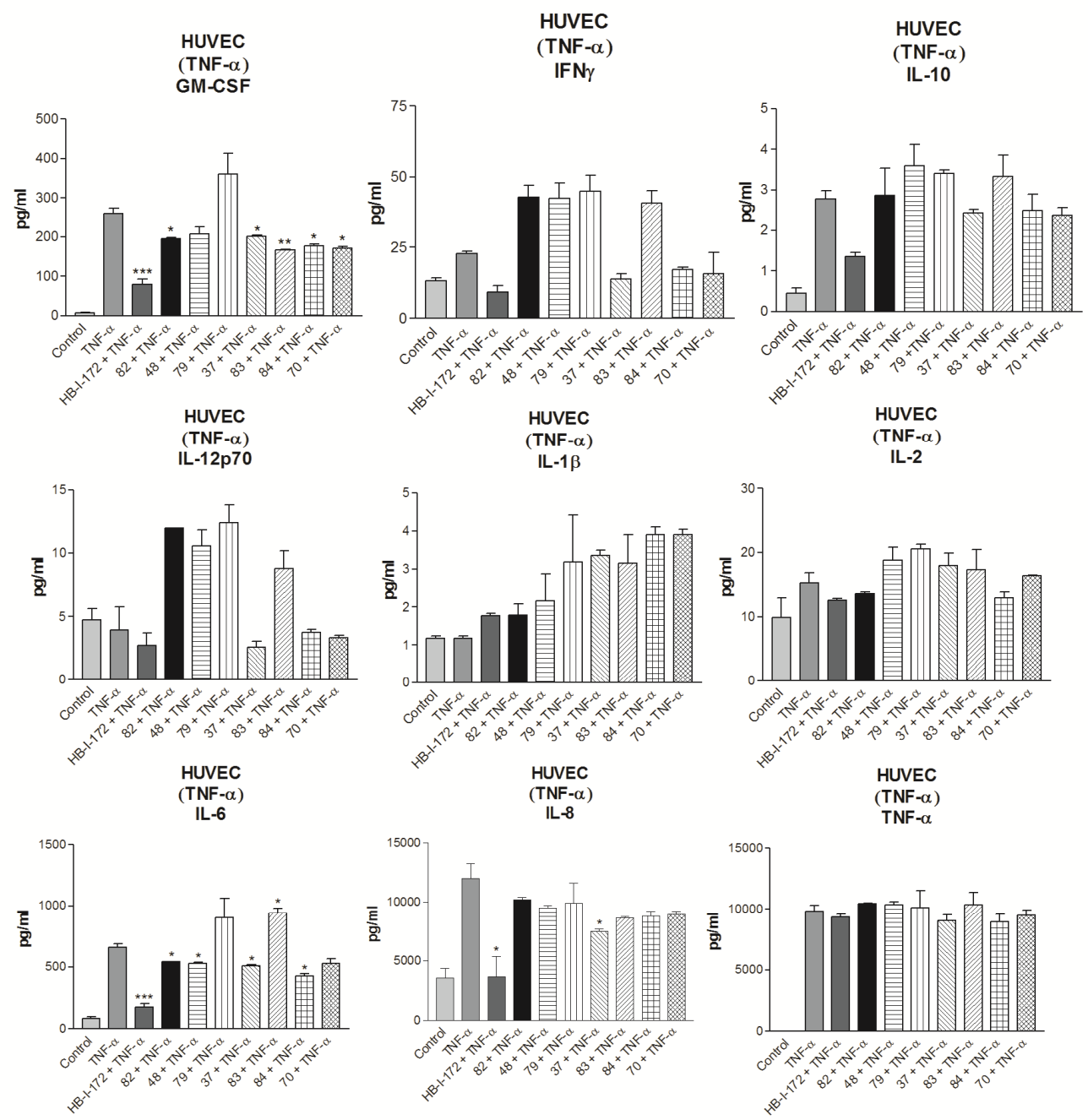

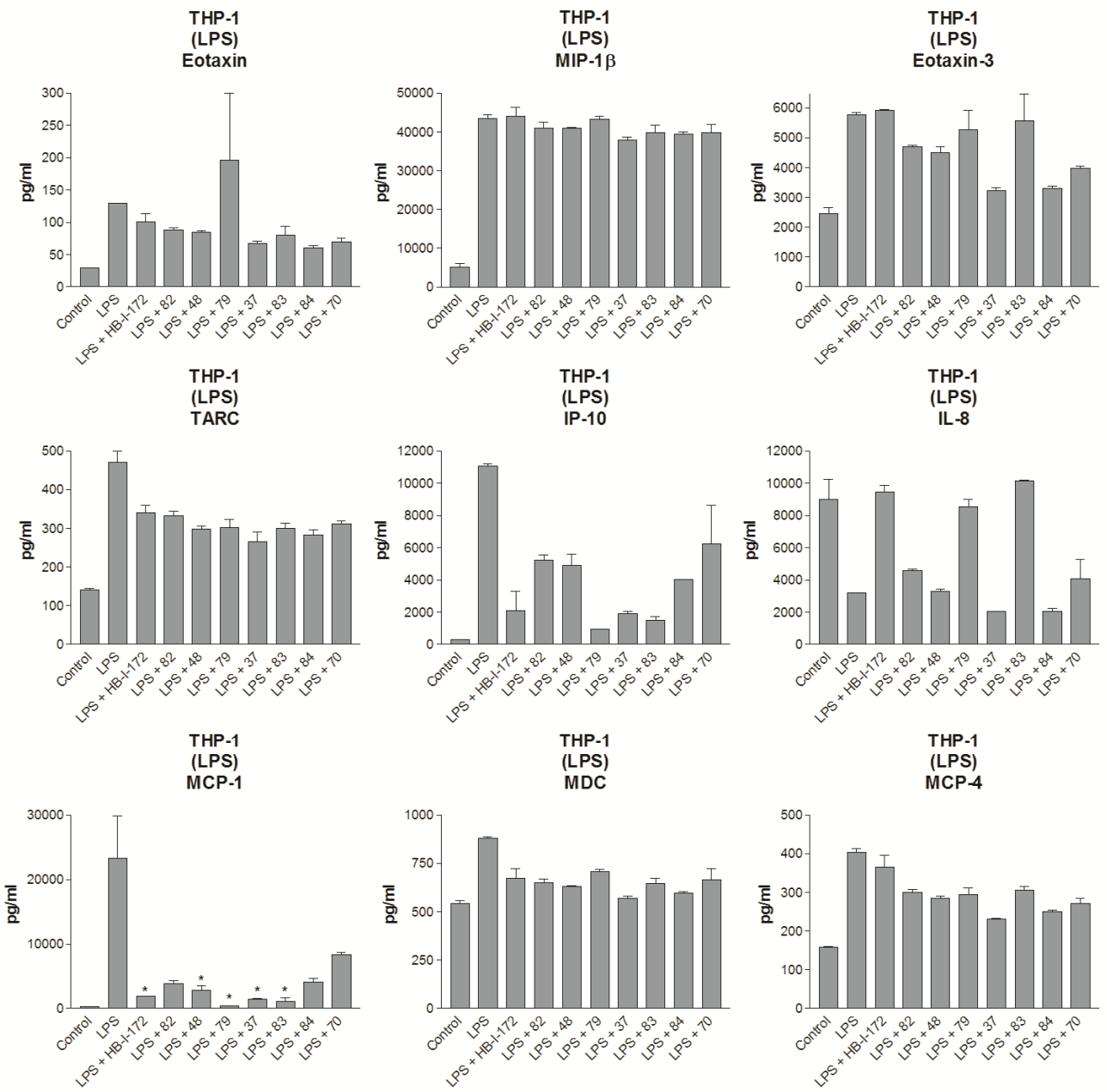

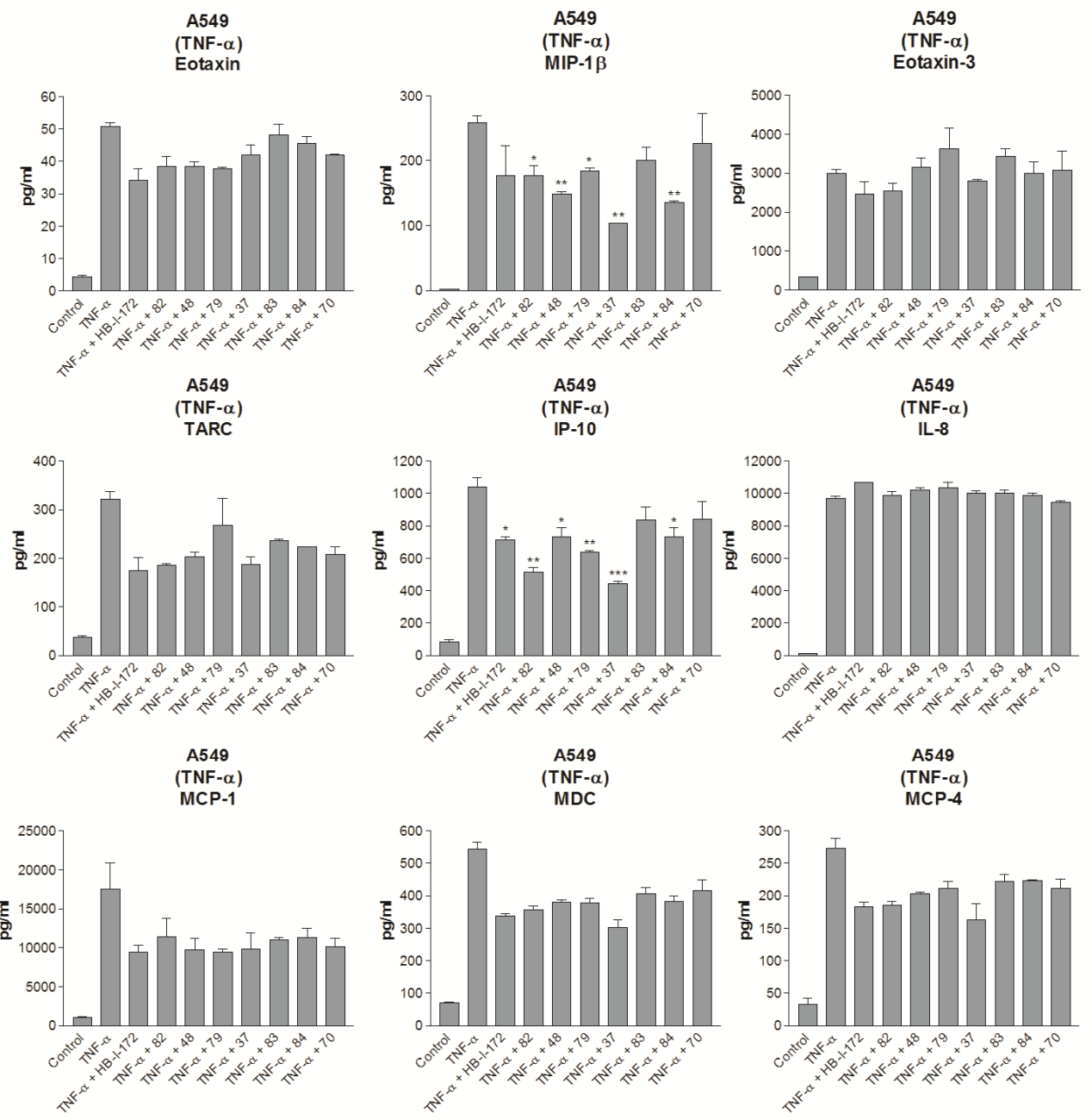

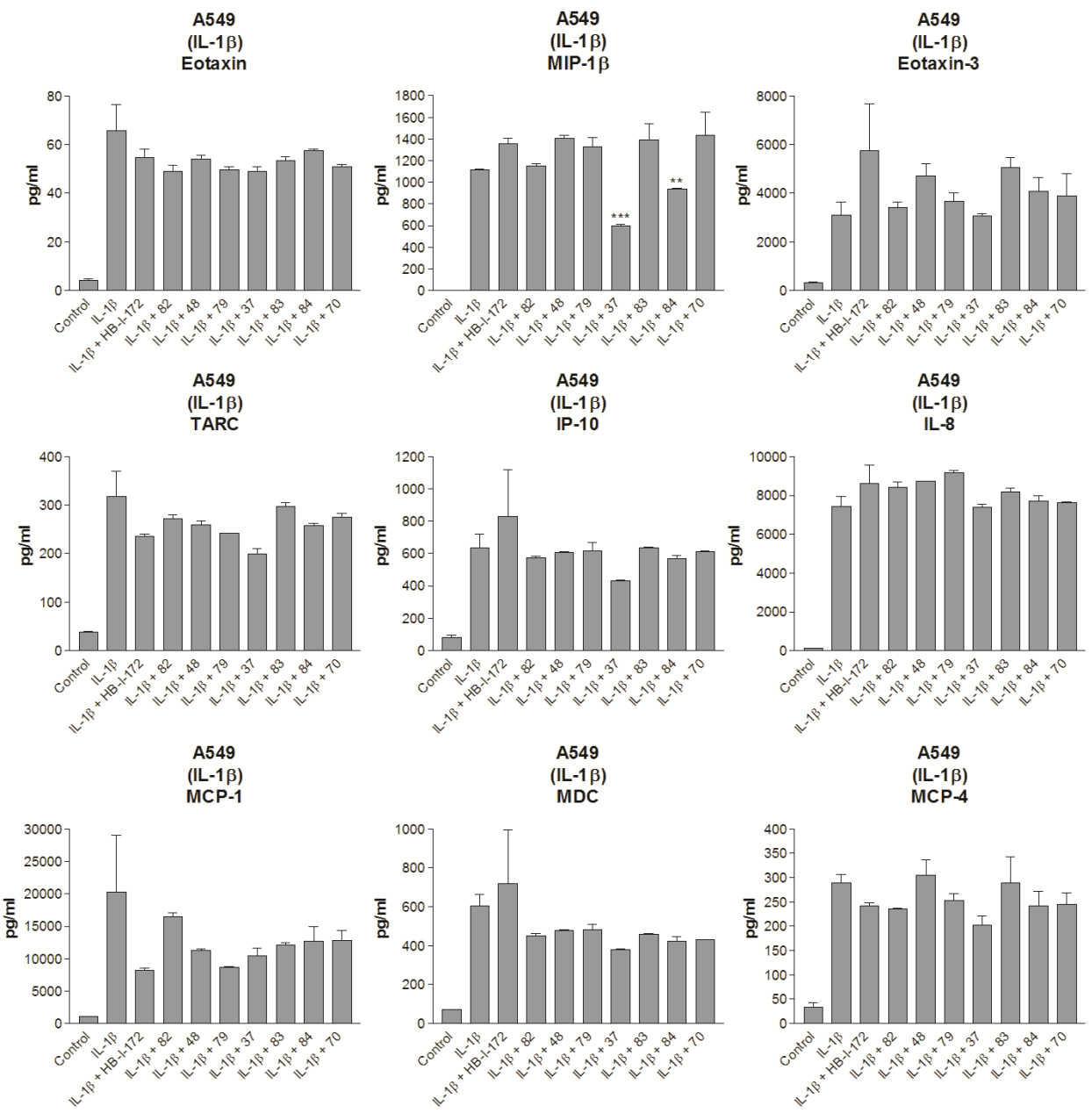

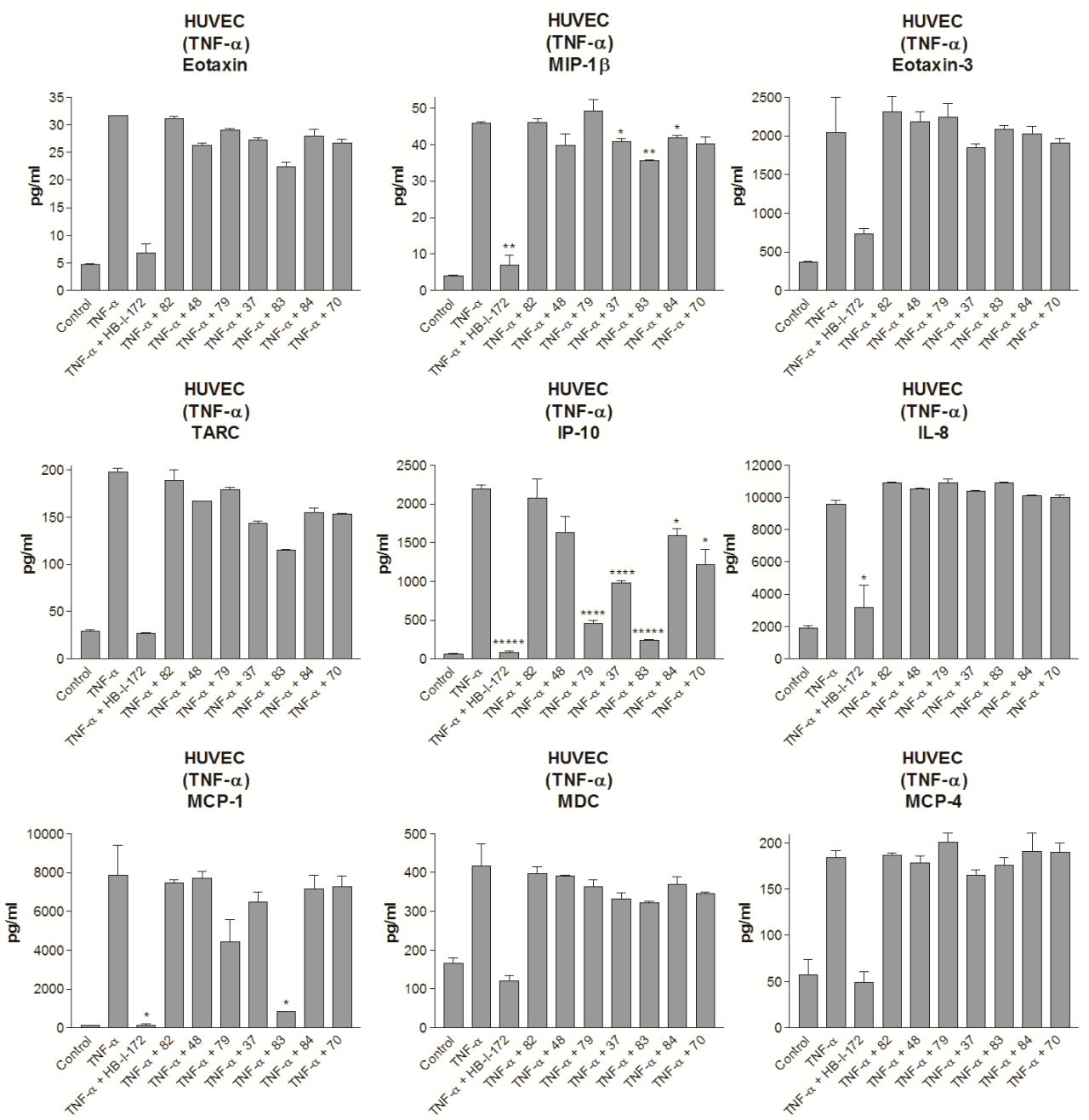

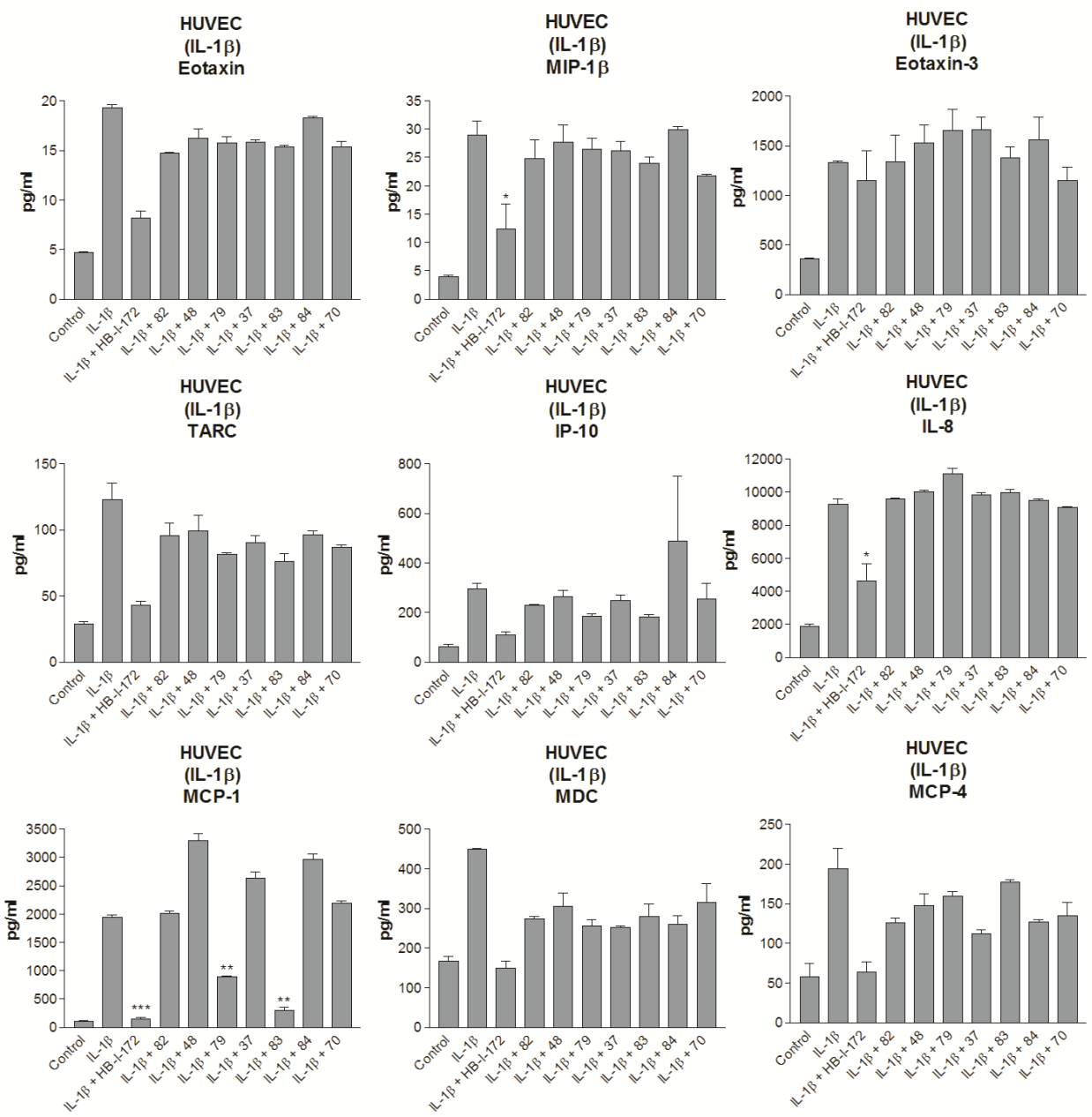
Appendix C: Dose Response of Four Cannabinoids on Cytokine and Chemokine Release of THP-1 and HUV-EC-C 

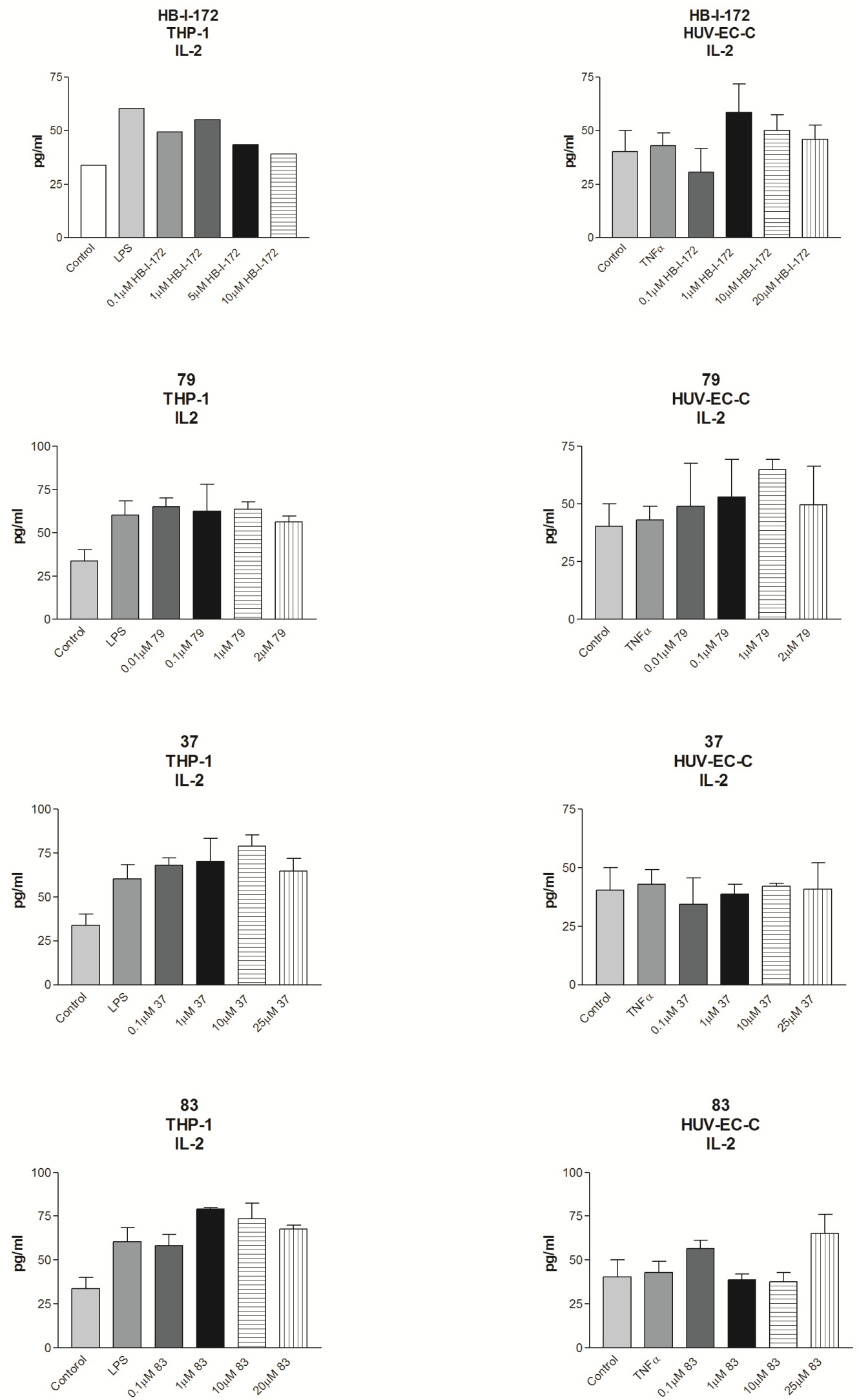

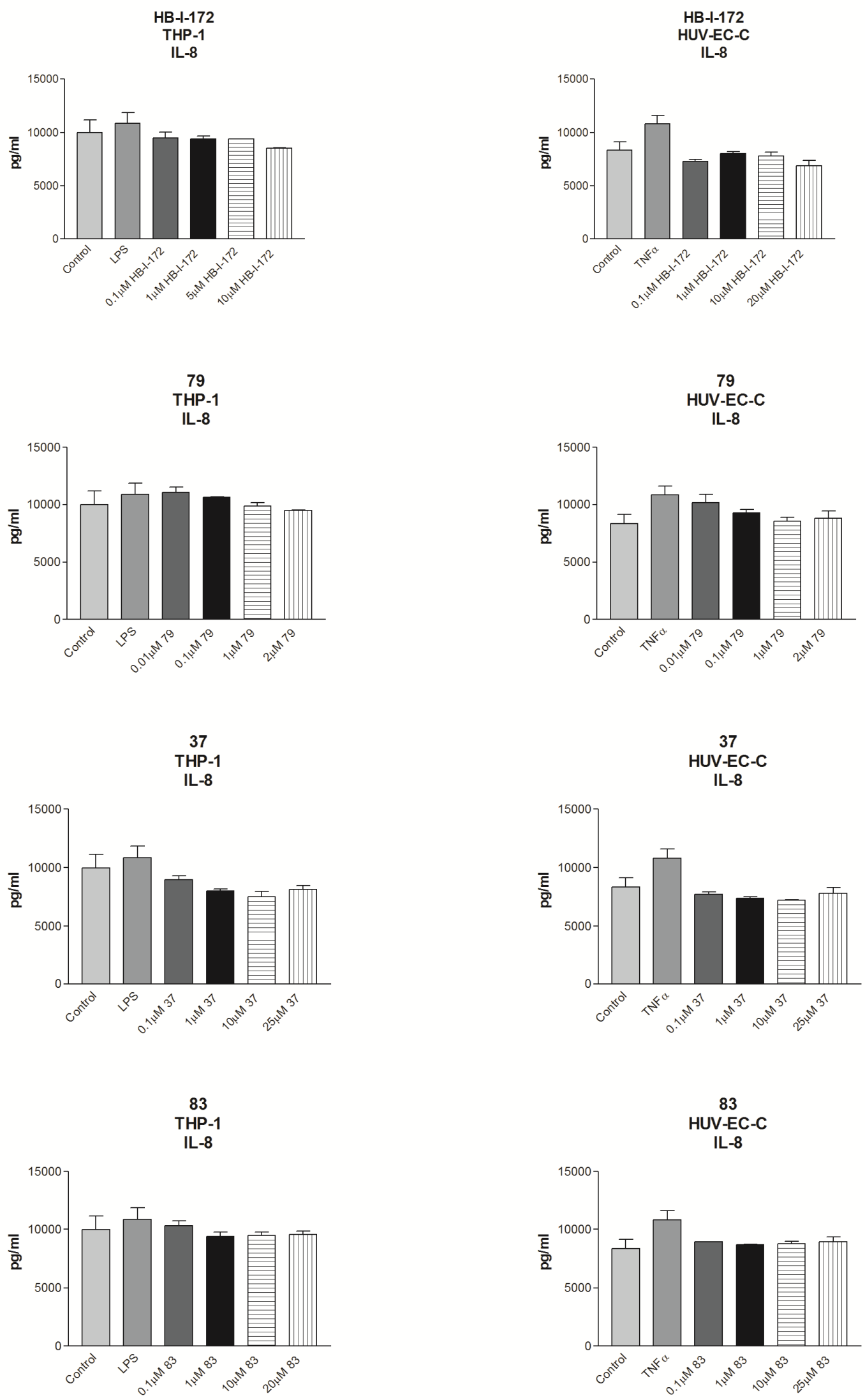

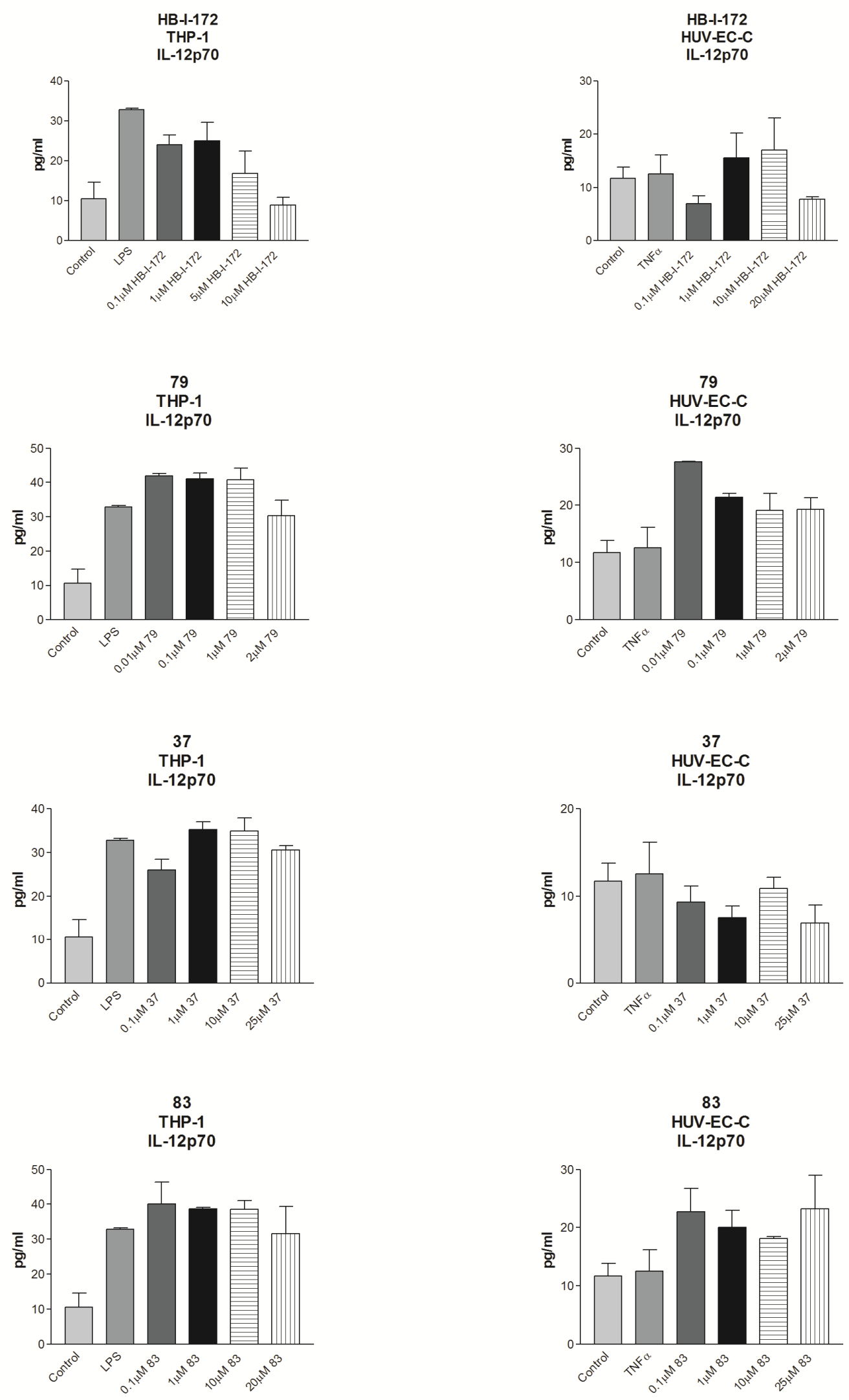

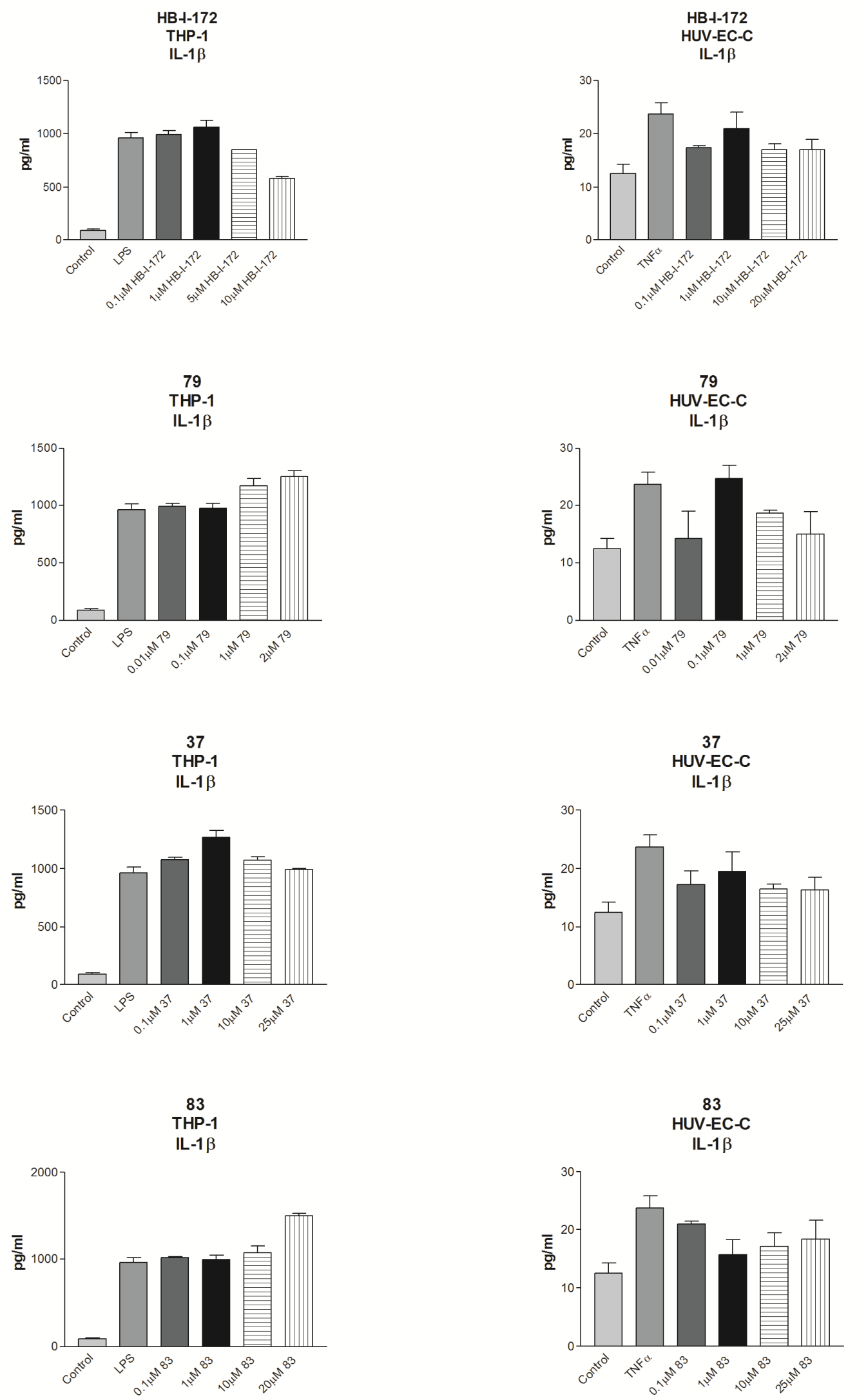

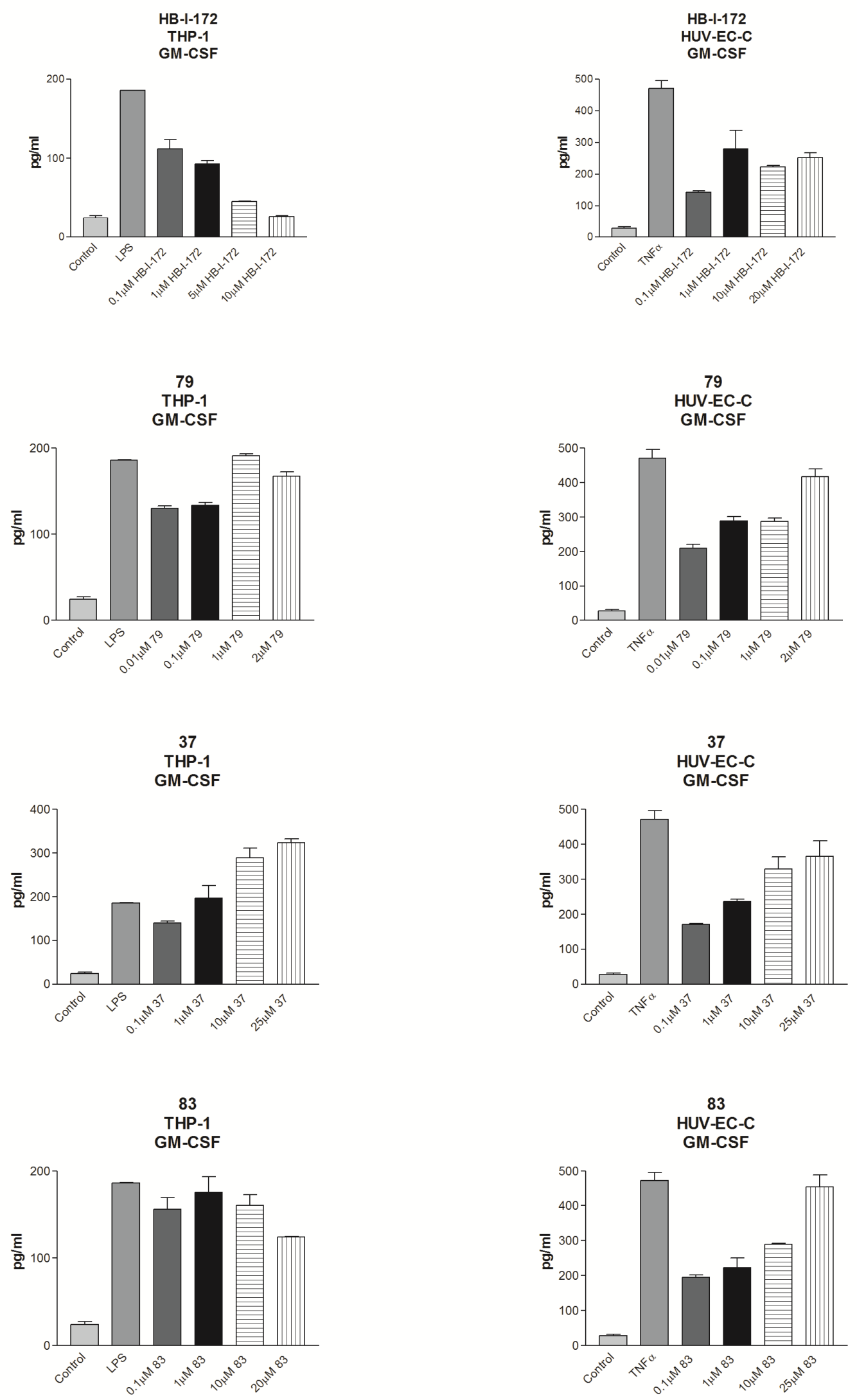

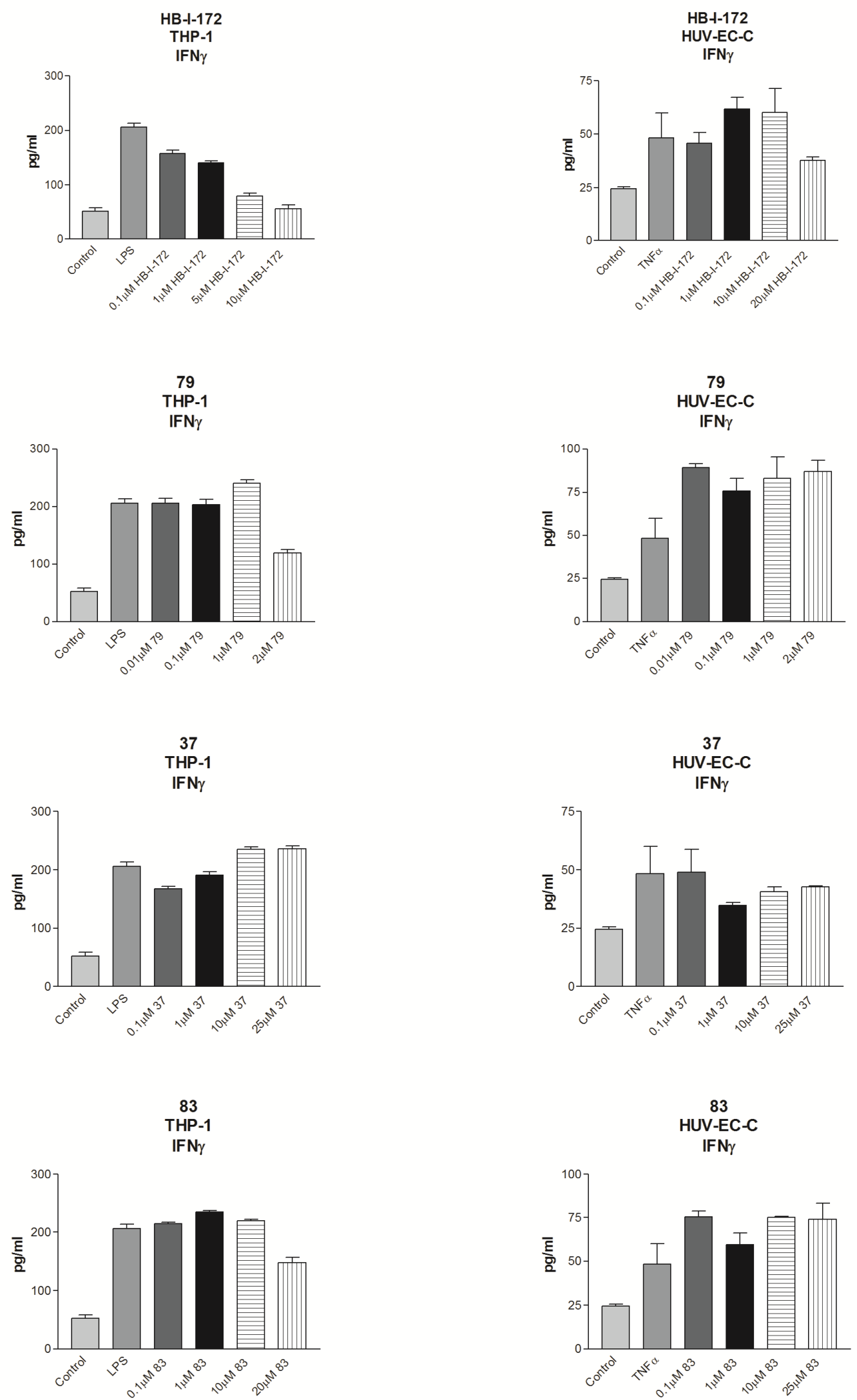

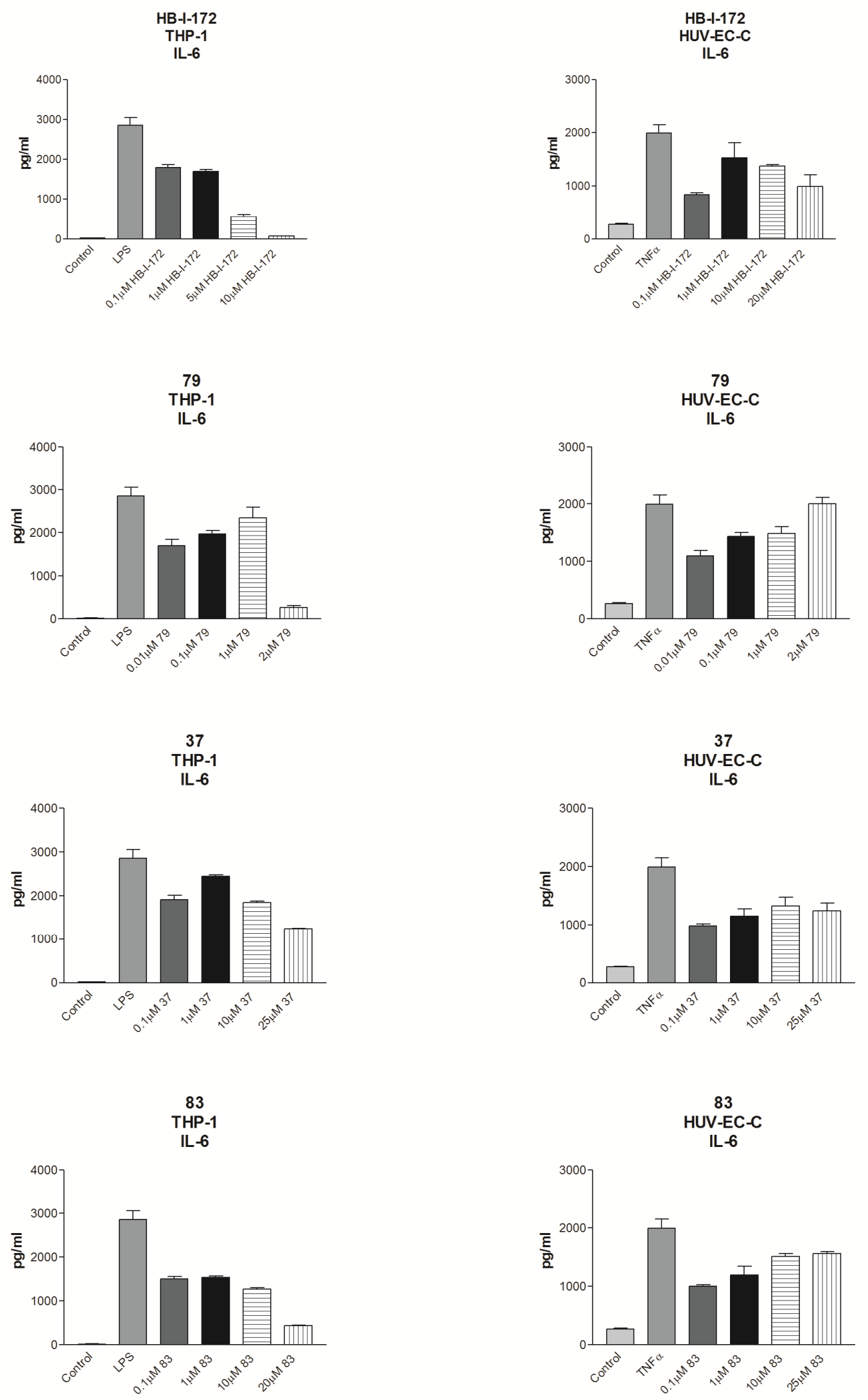

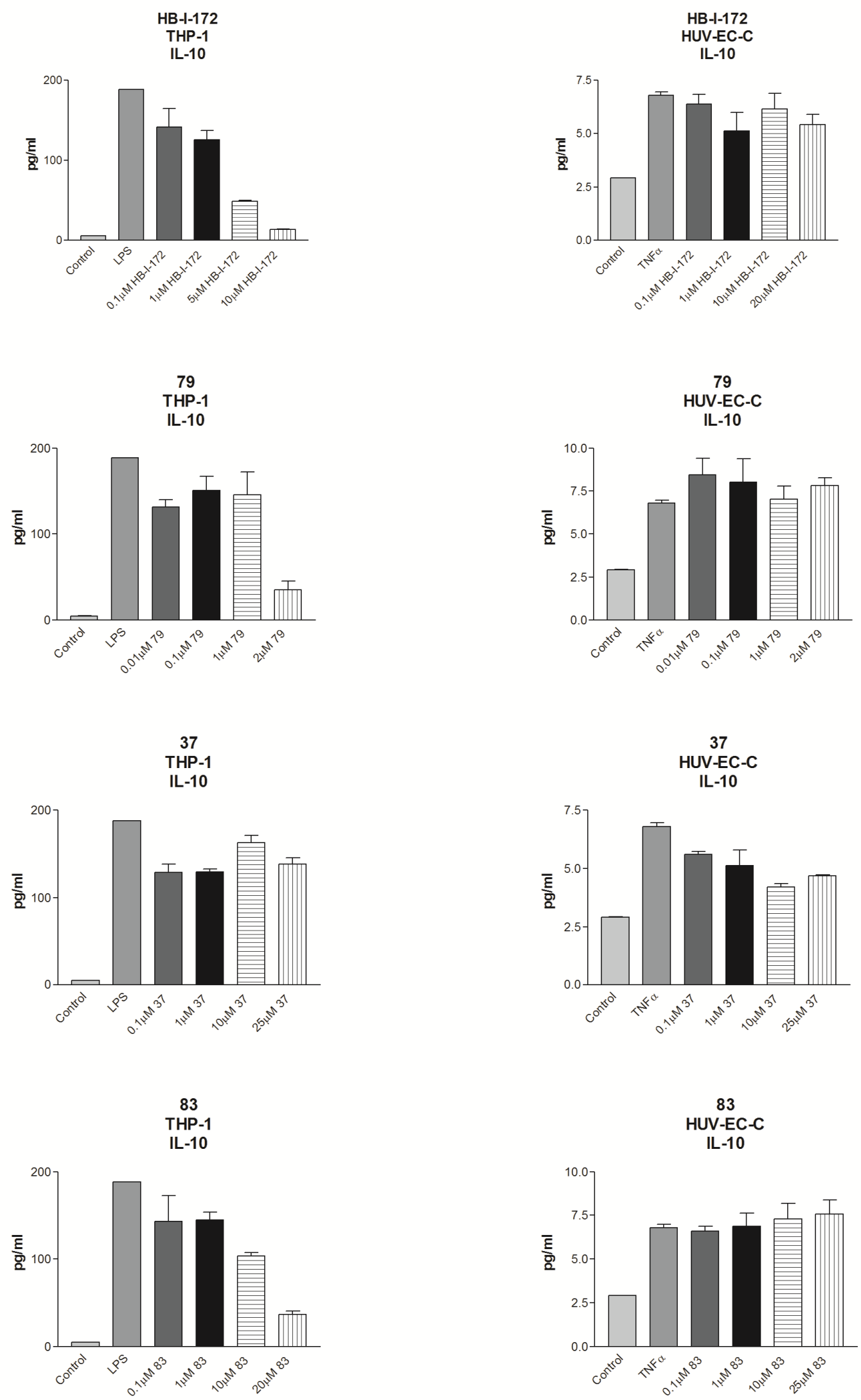

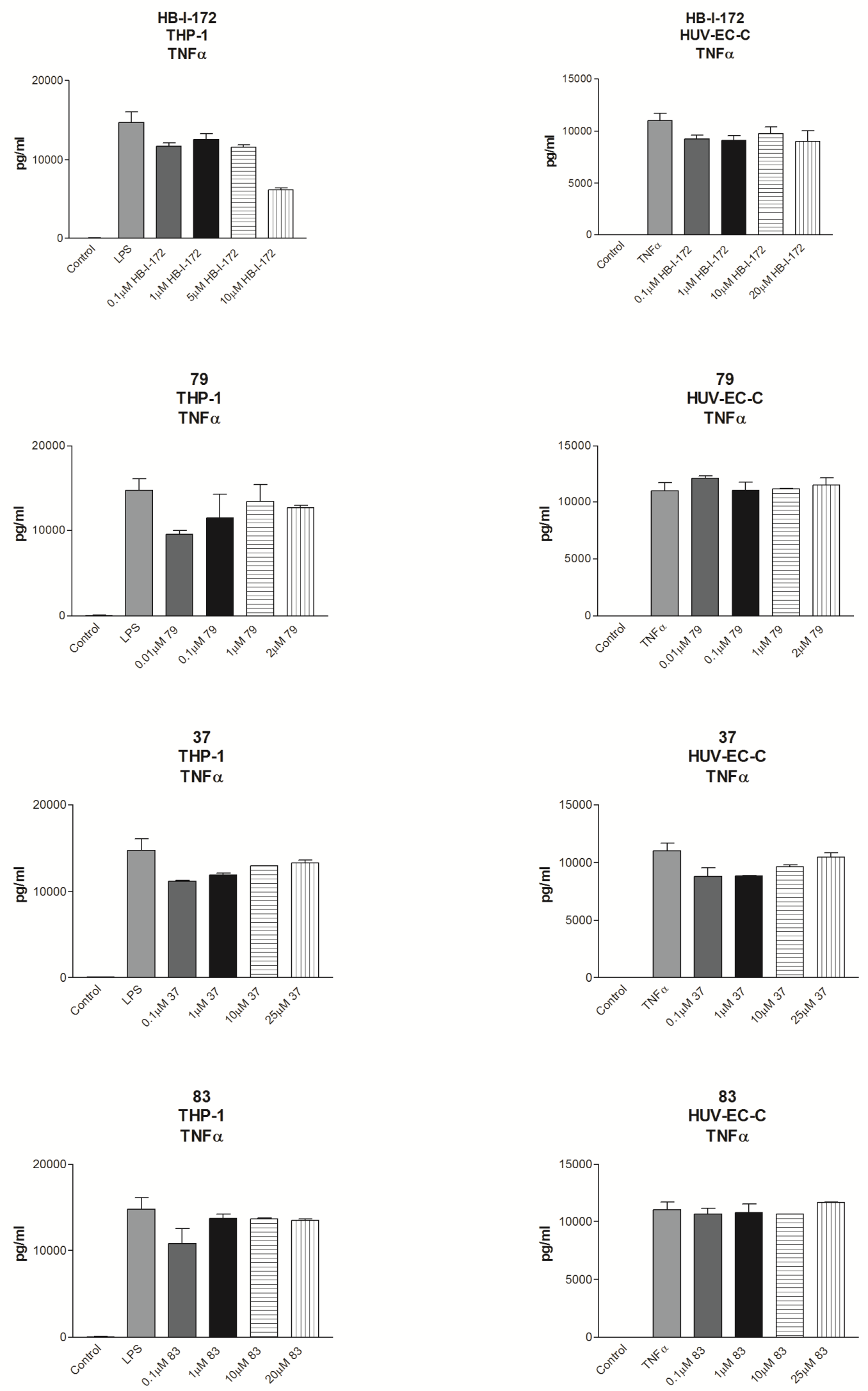

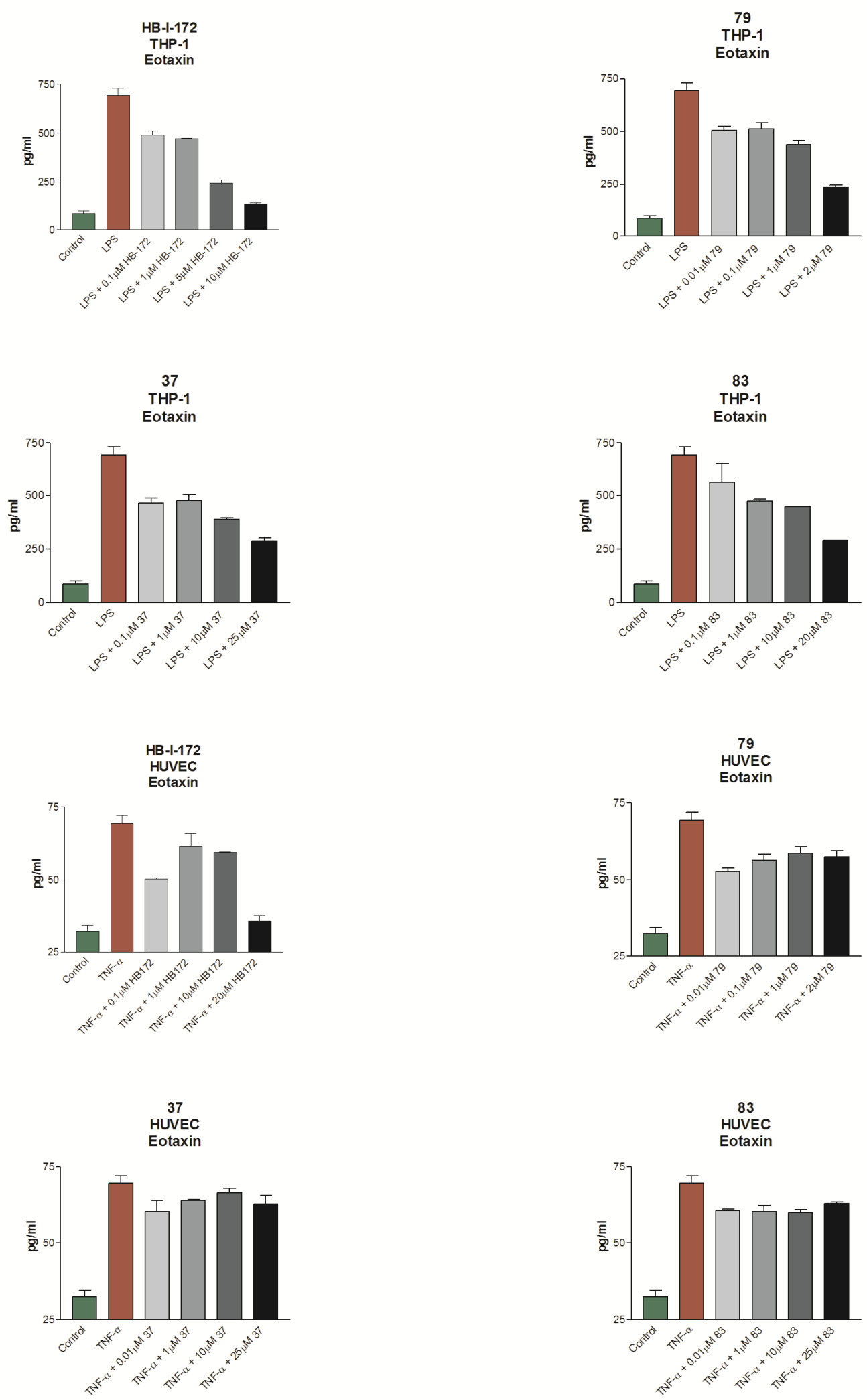

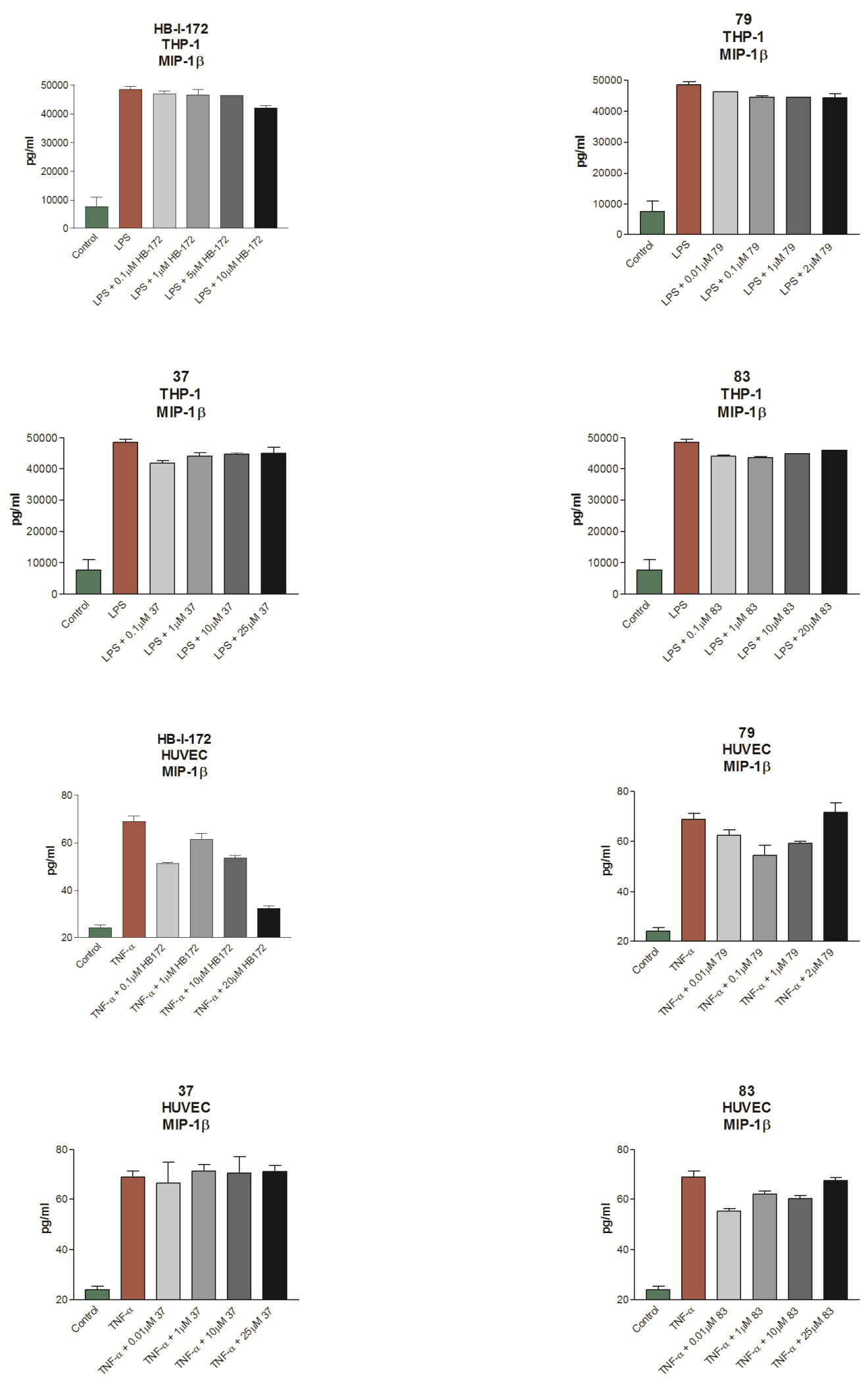

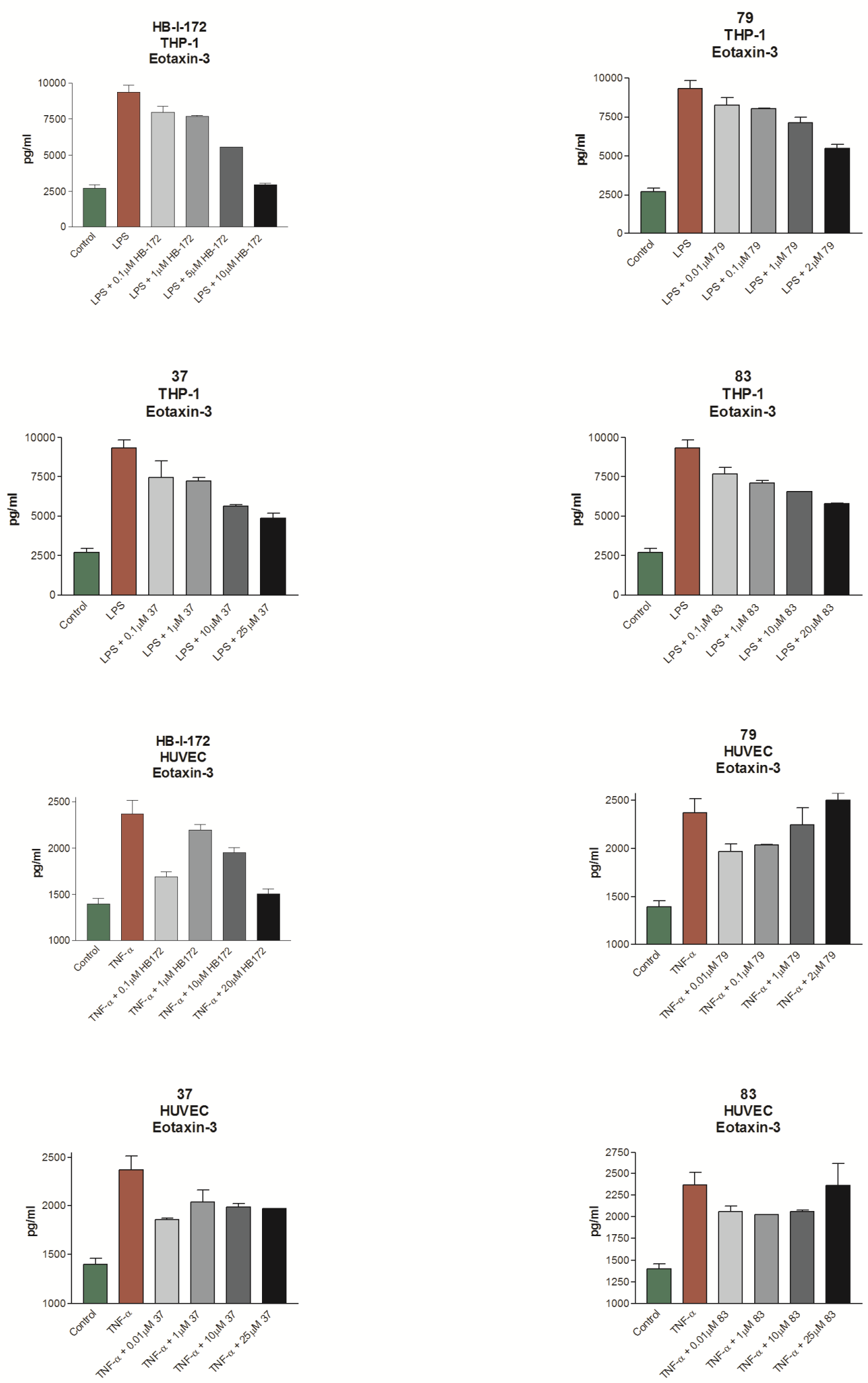

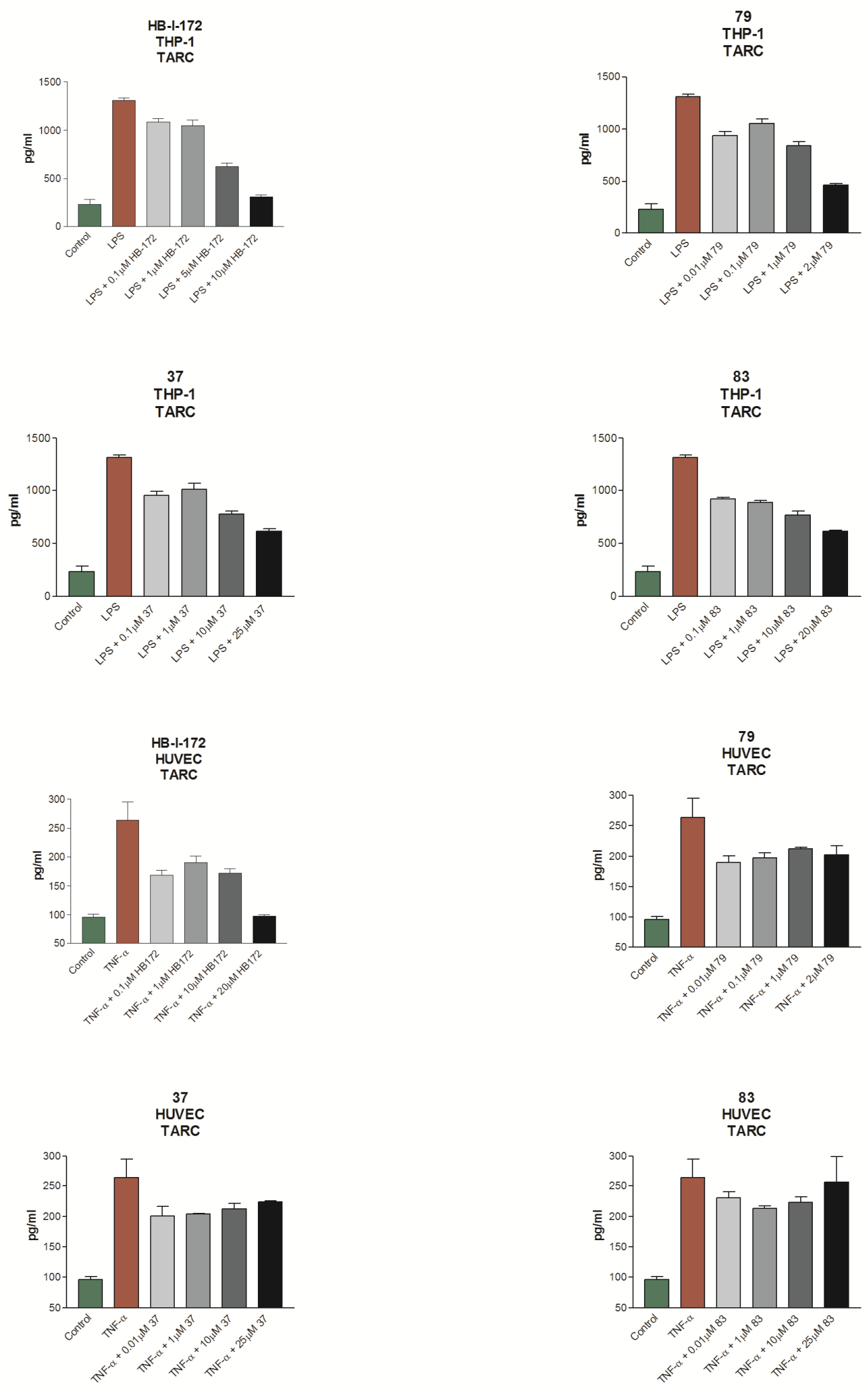

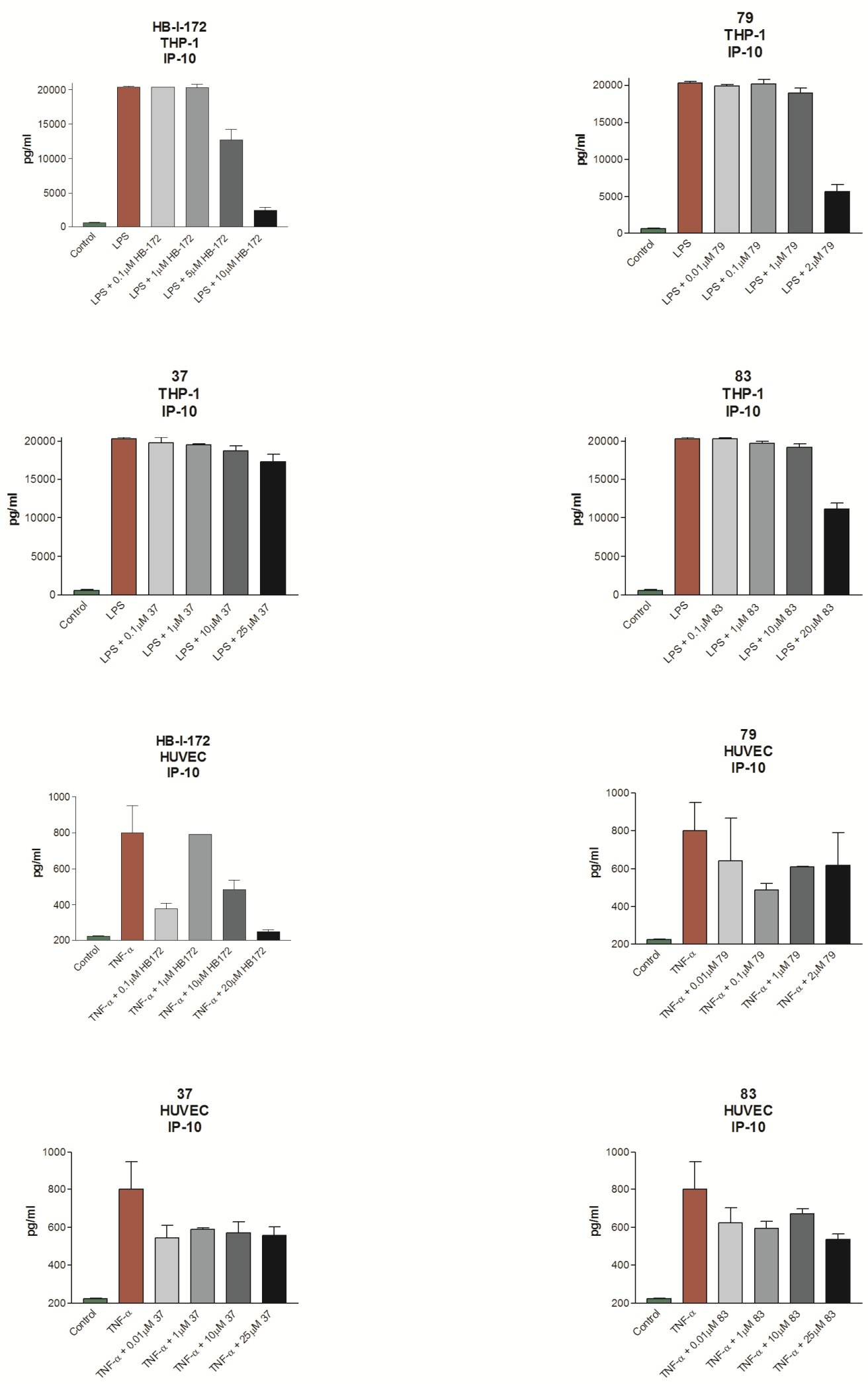

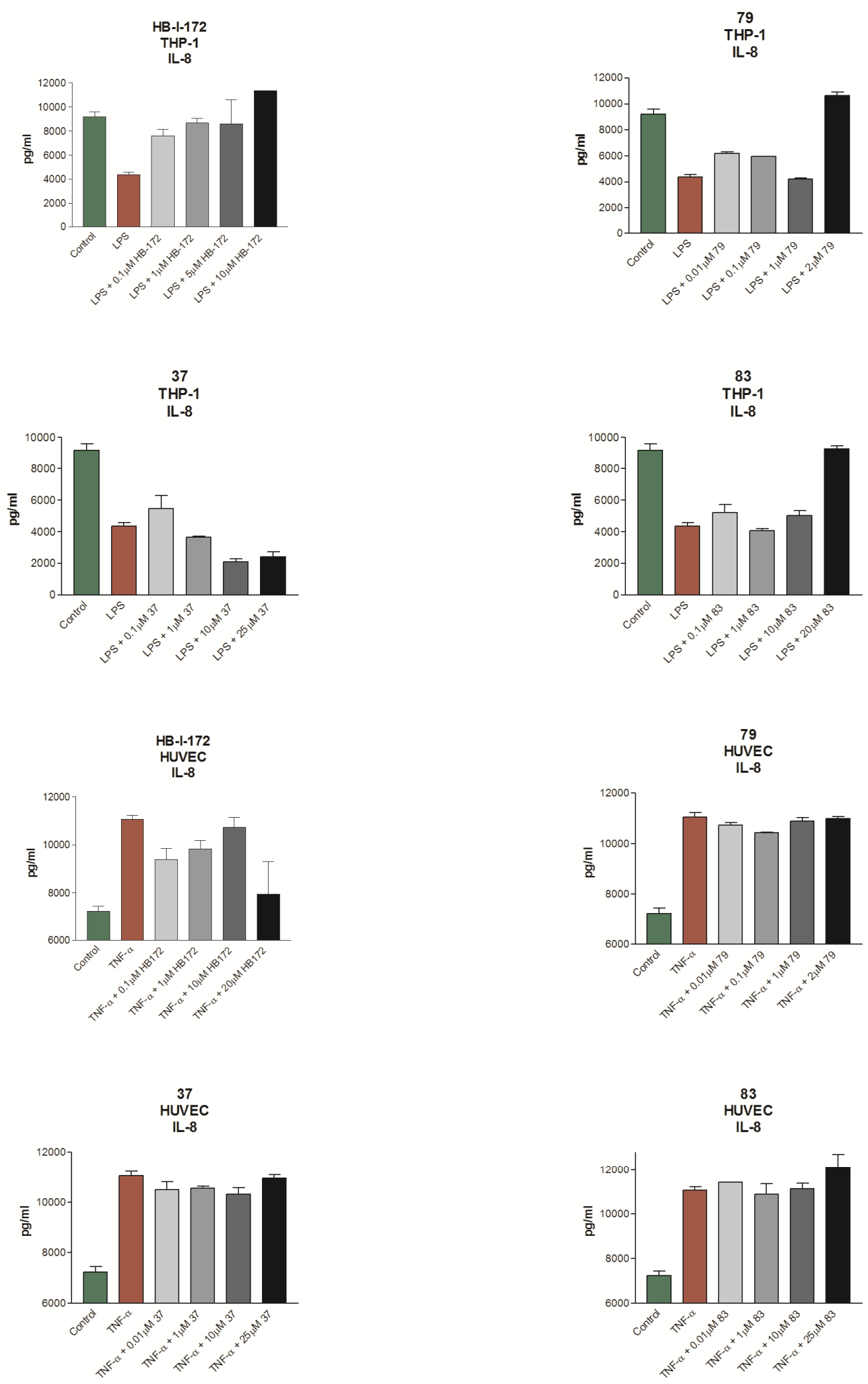

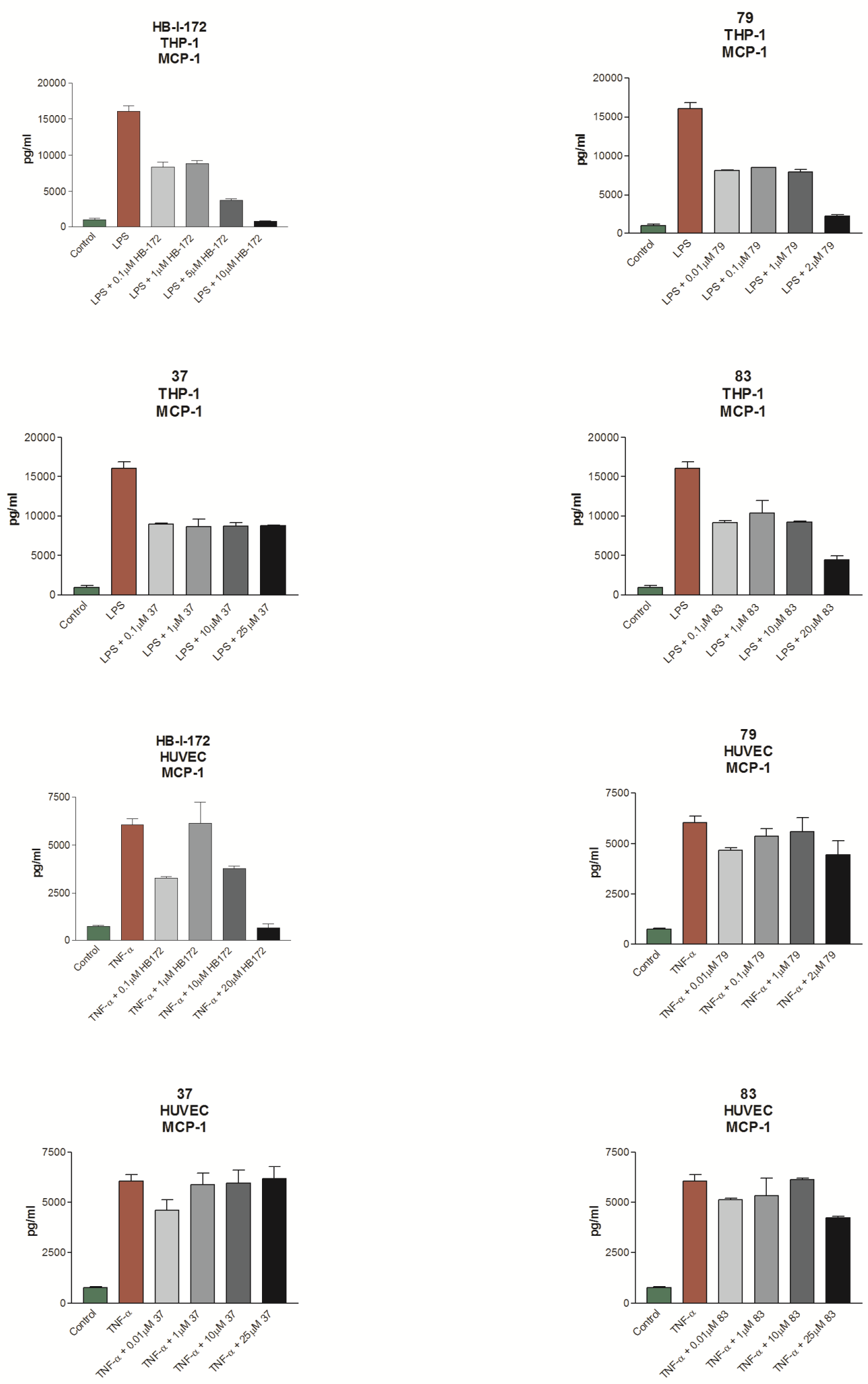

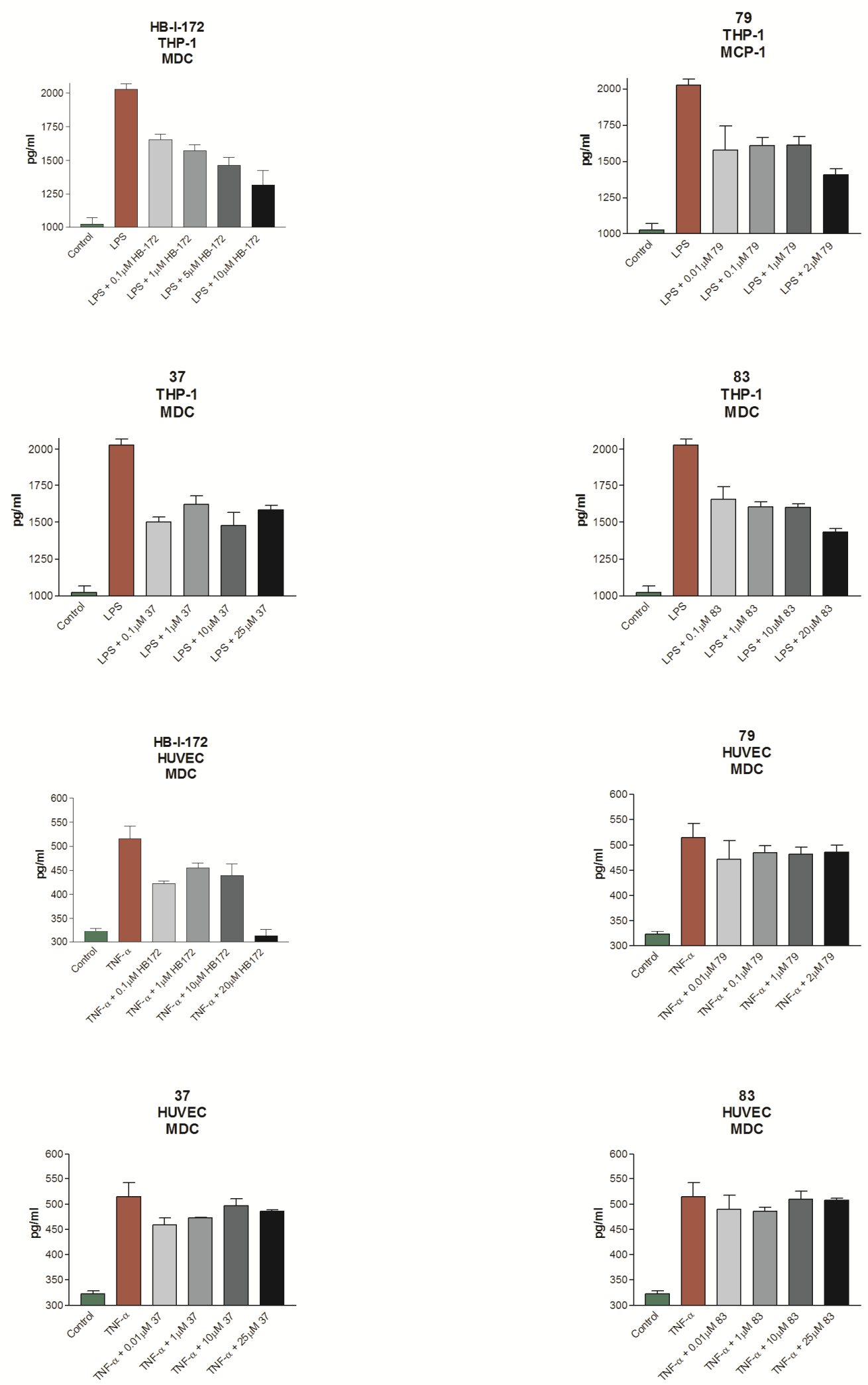

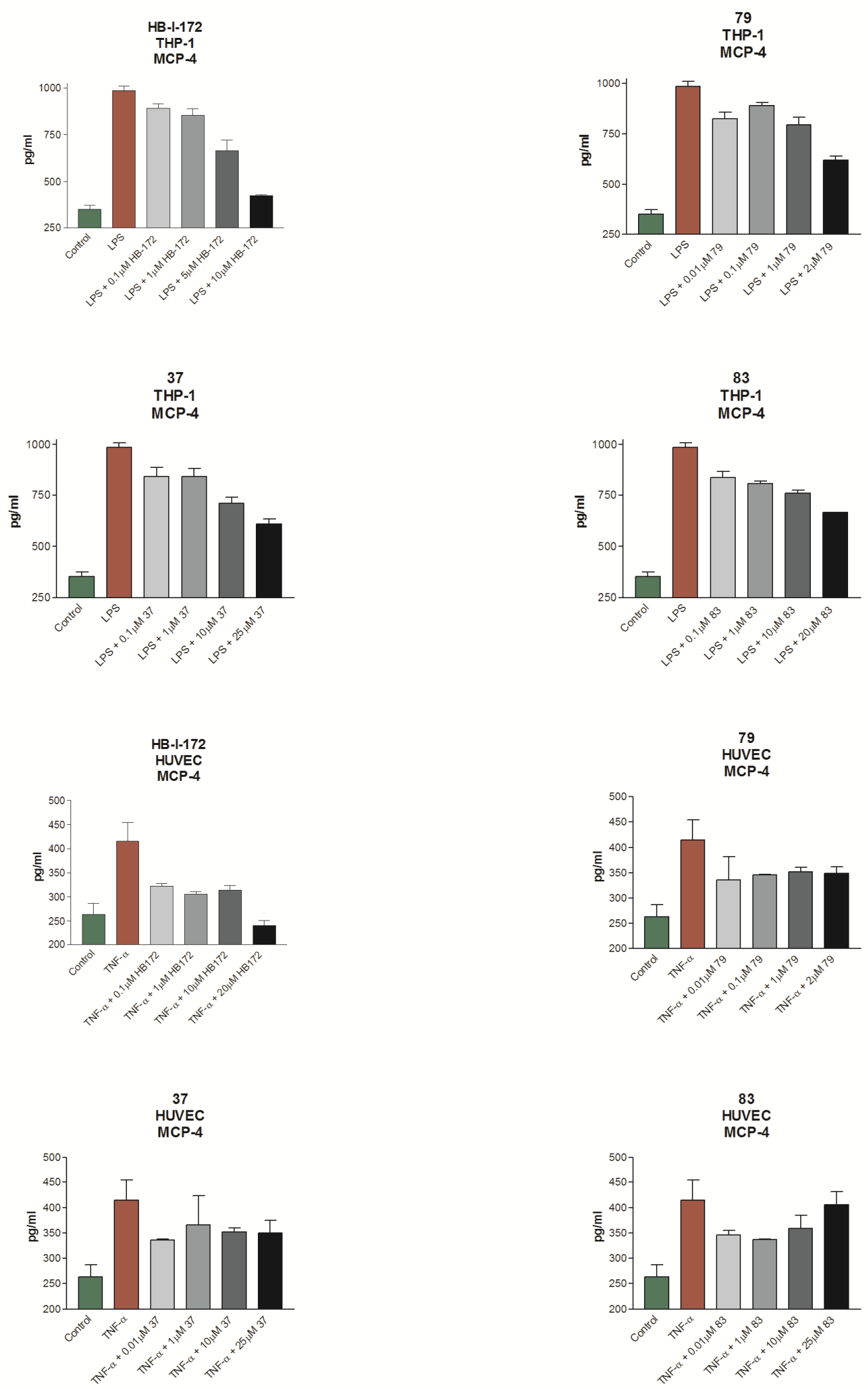
Appendix D: Cytokine and Chemokine Release Profiles of HMVEC-LBI, Monocyte Derived Macrophages, and SAEC 

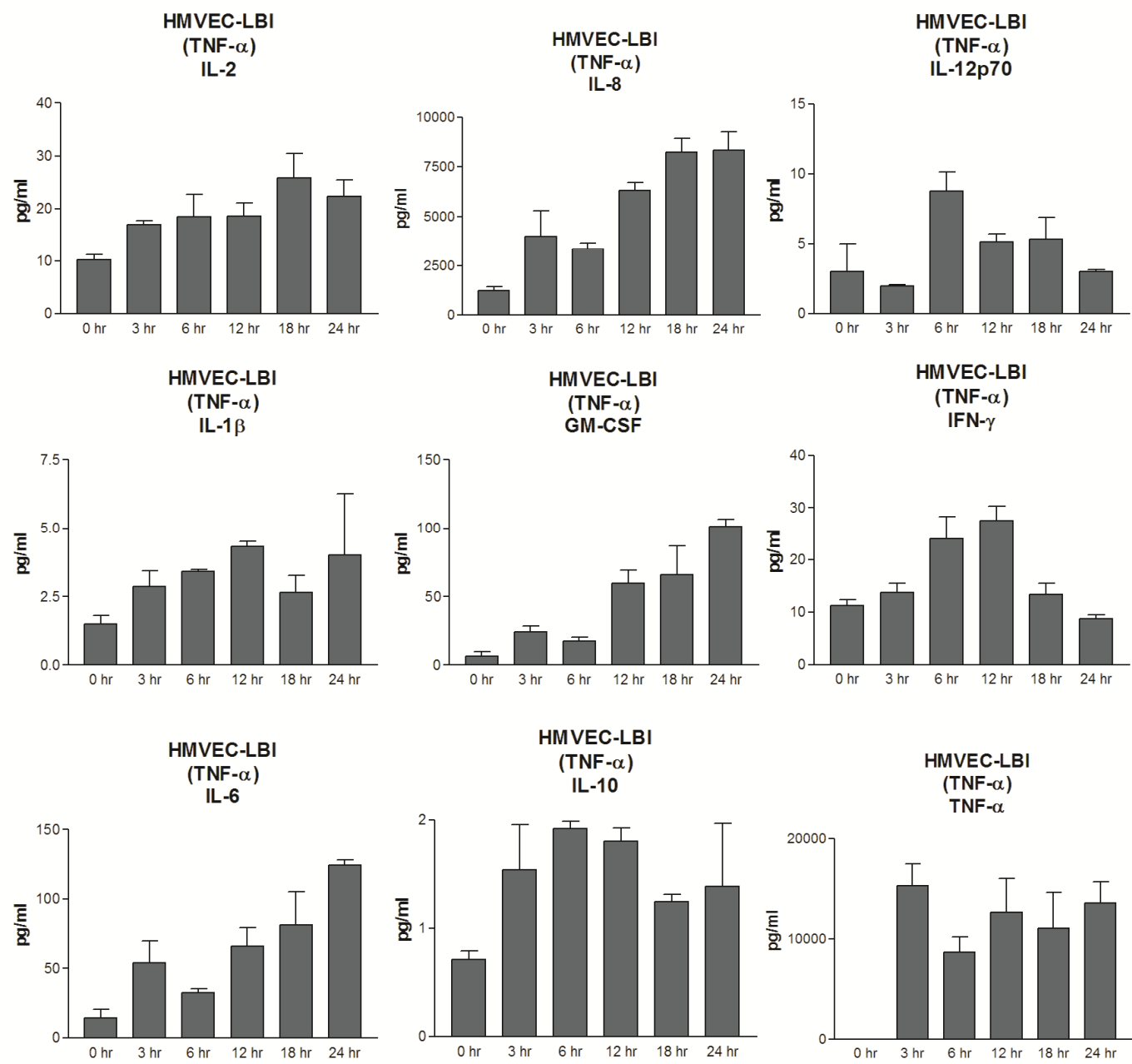

HMVEC-LBI
(TNF- $\alpha$ )

TNF- $\alpha$

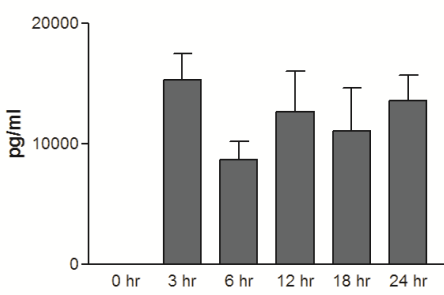



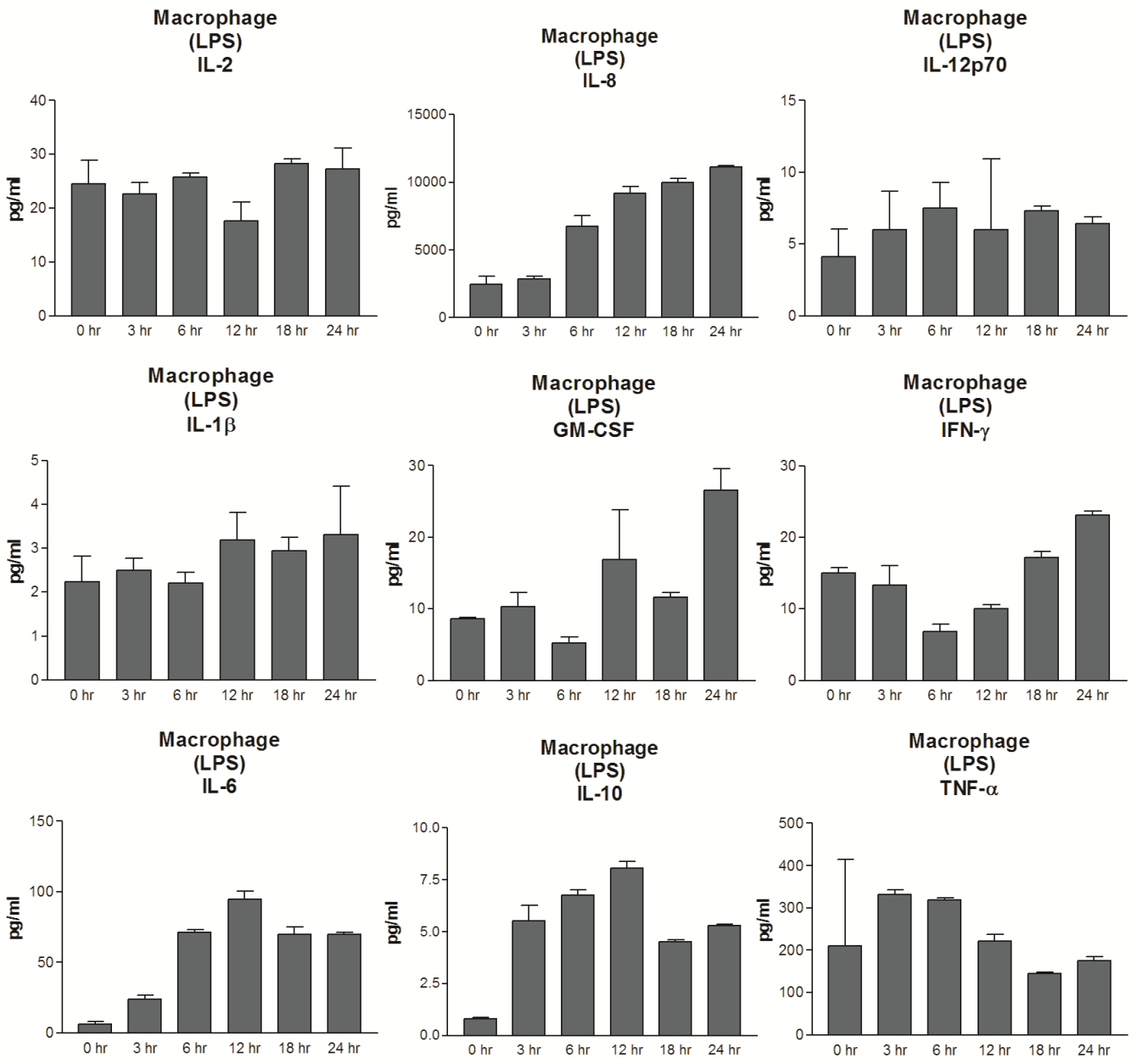

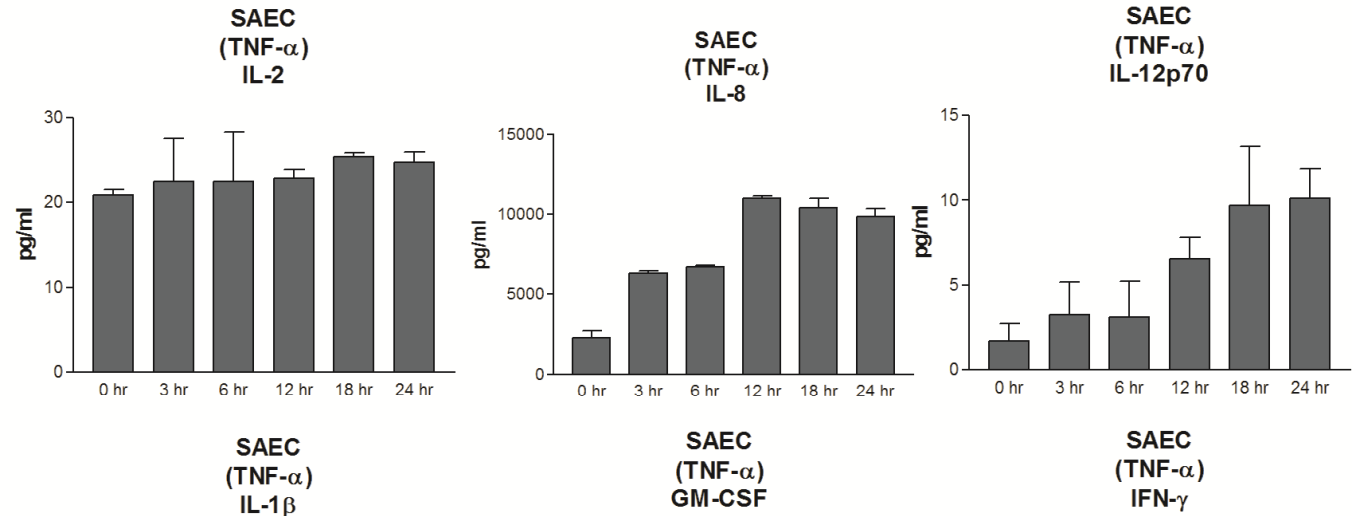

SAEC

(TNF- $\alpha$ )

SAEC

(TNF- $\alpha$ )
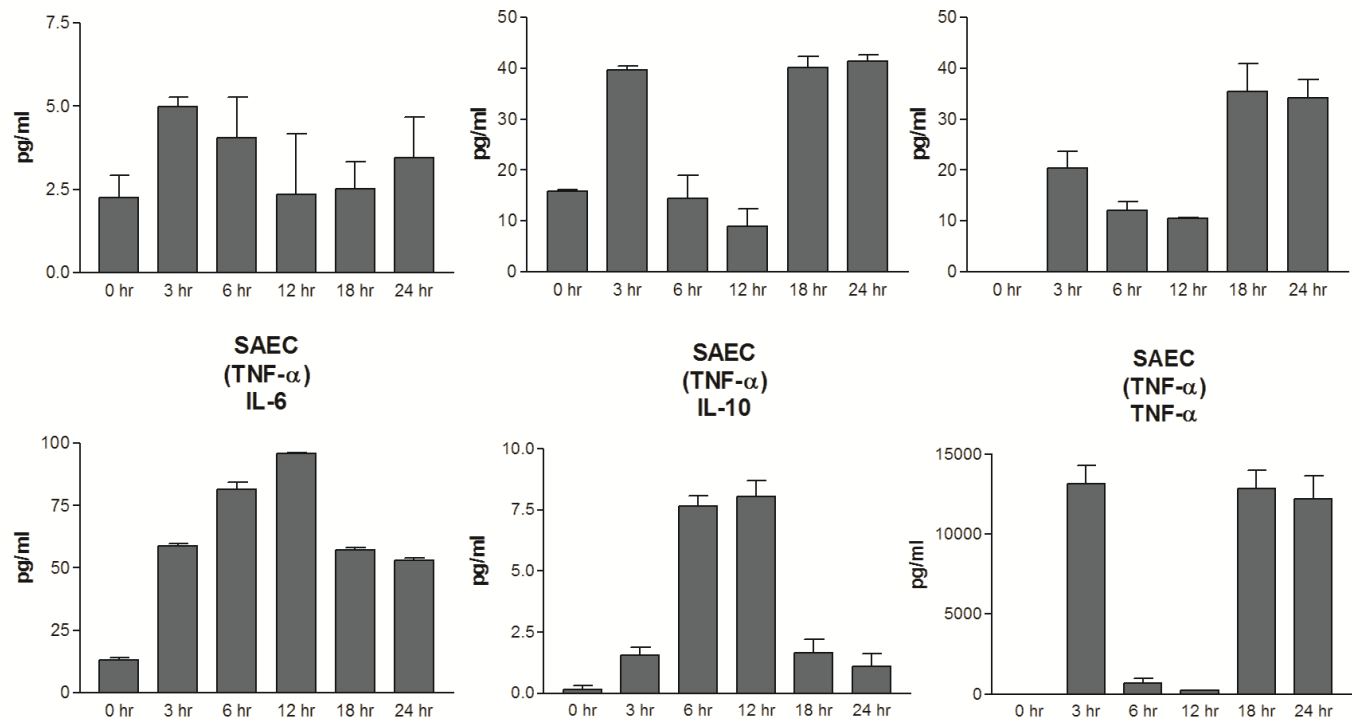

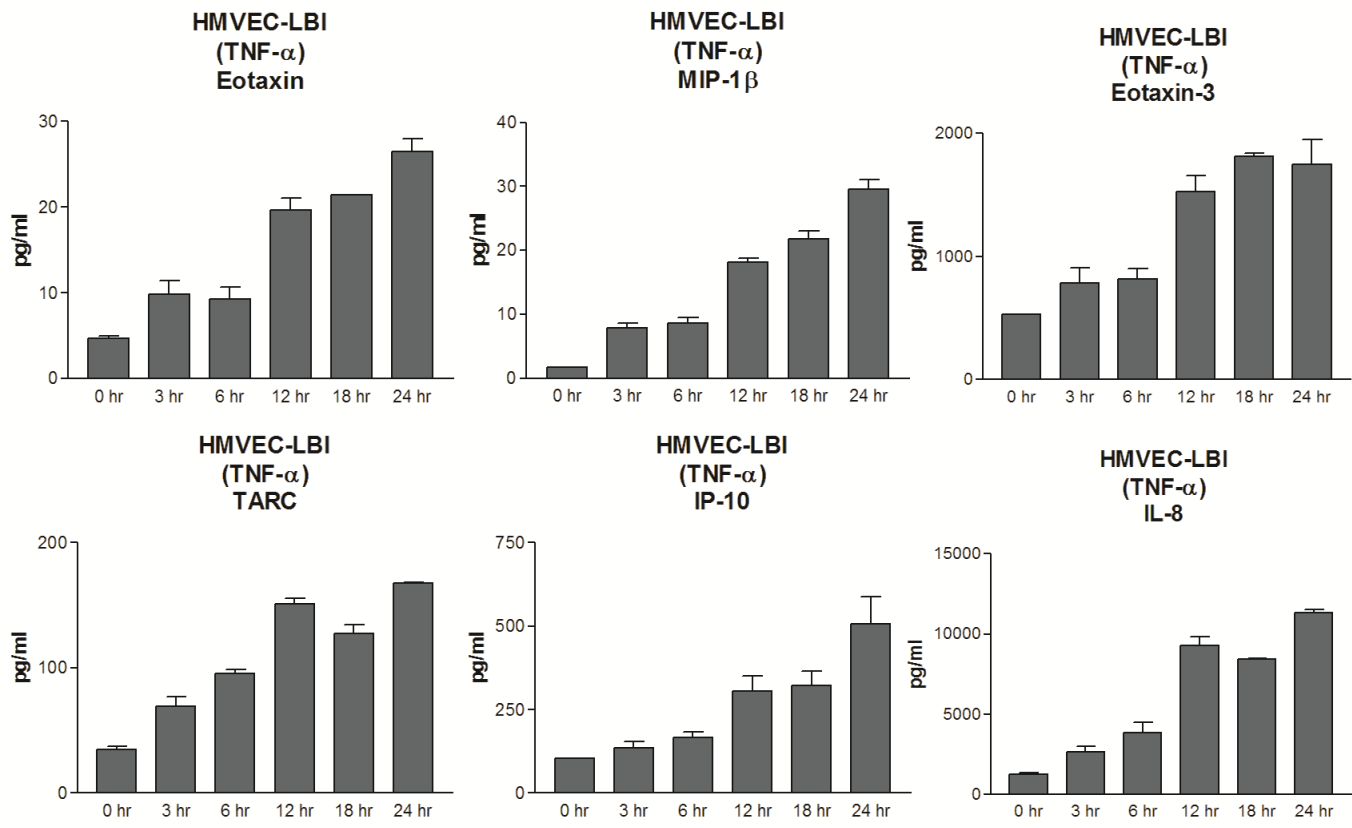

HMVEC-LB

(TNF- $\alpha$ )

MCP-1
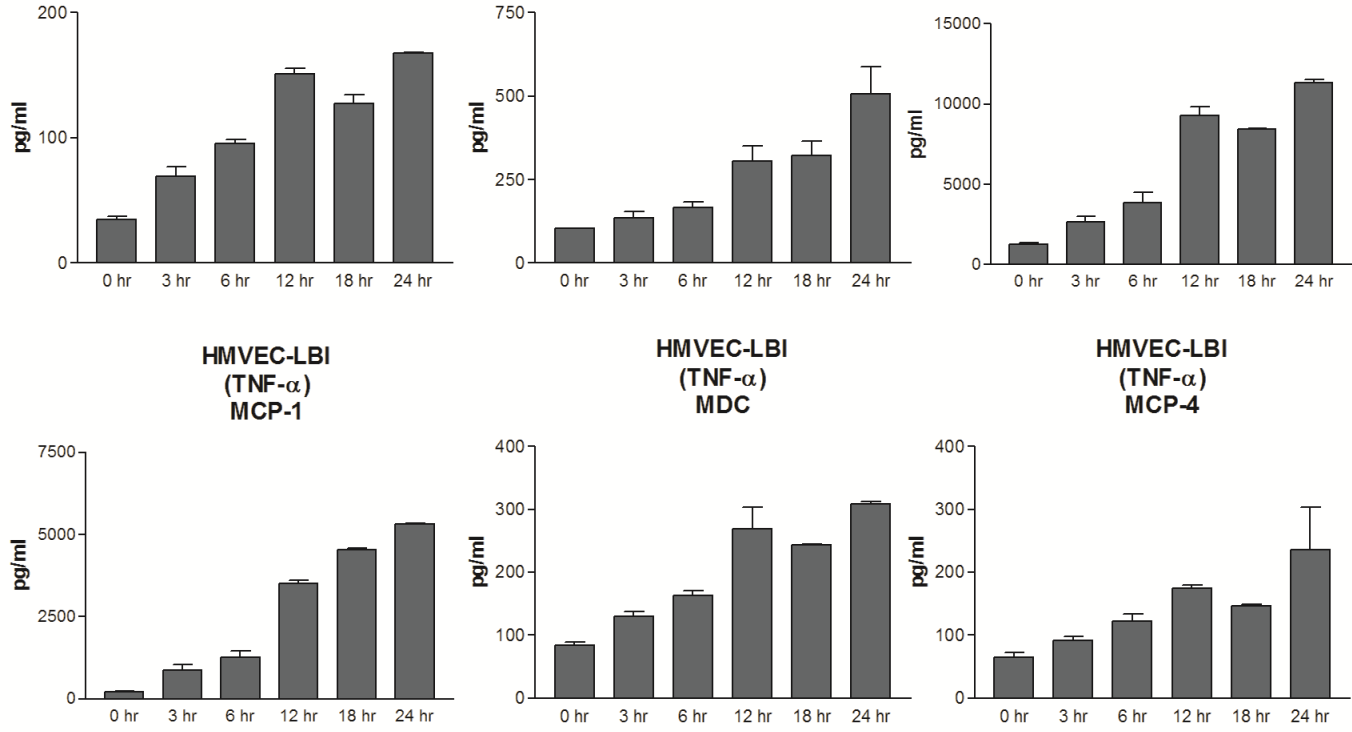

HMVEC-LBI

(TNF- $\alpha$ )

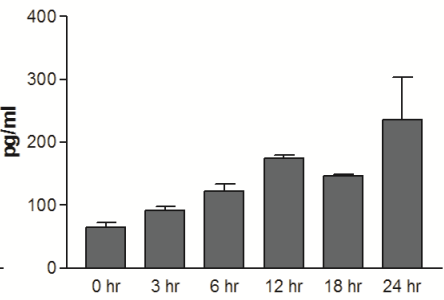



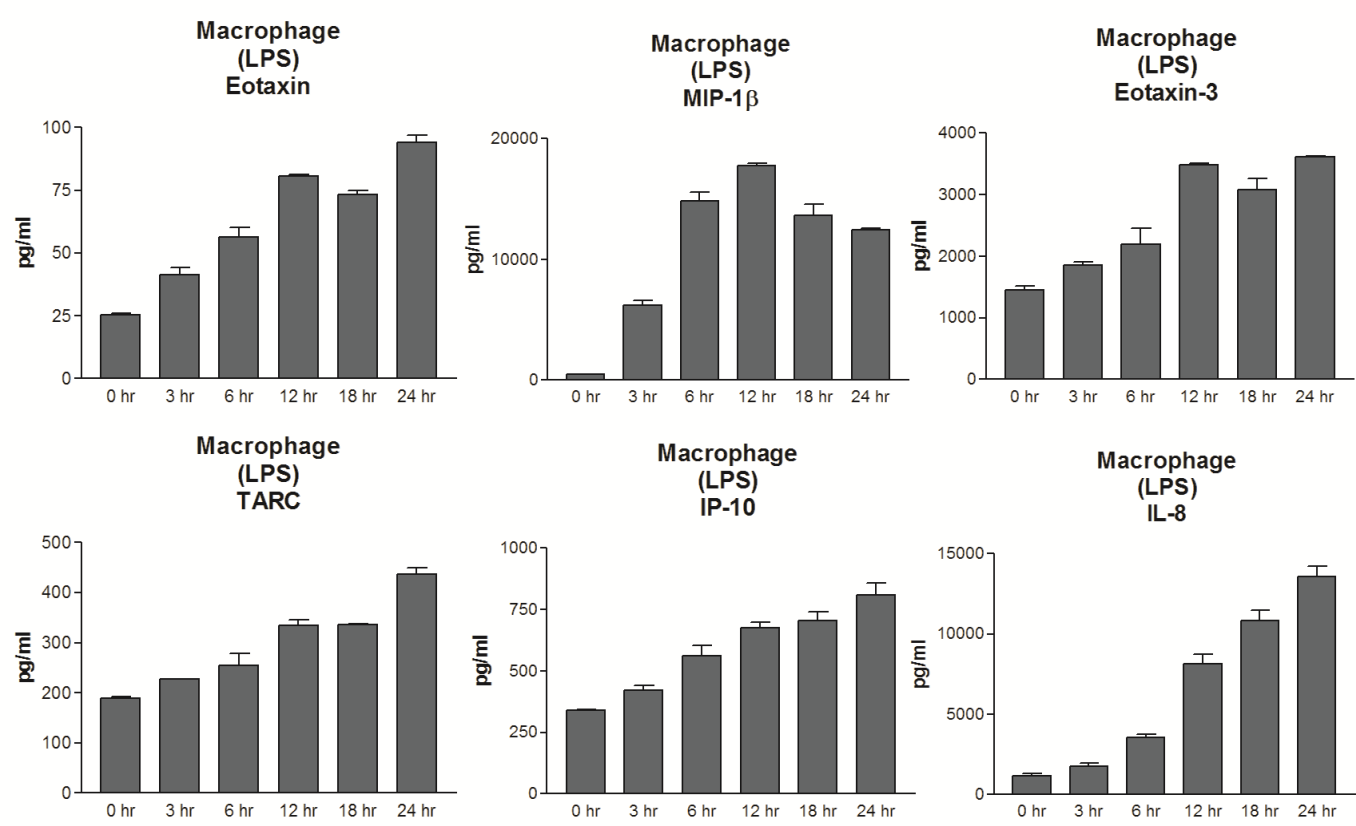

Macrophage

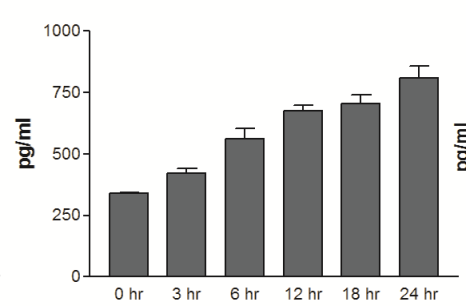

(LPS)
IL-8
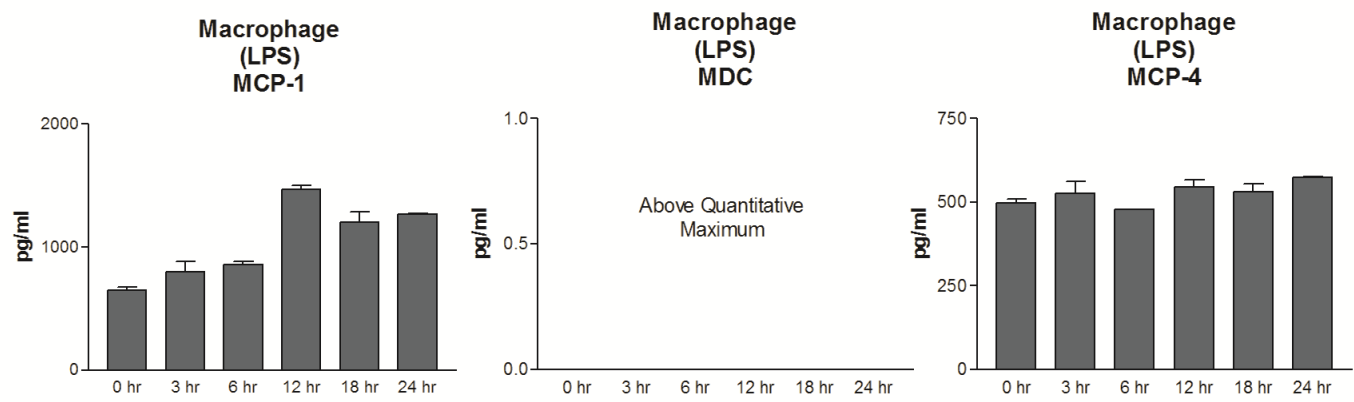

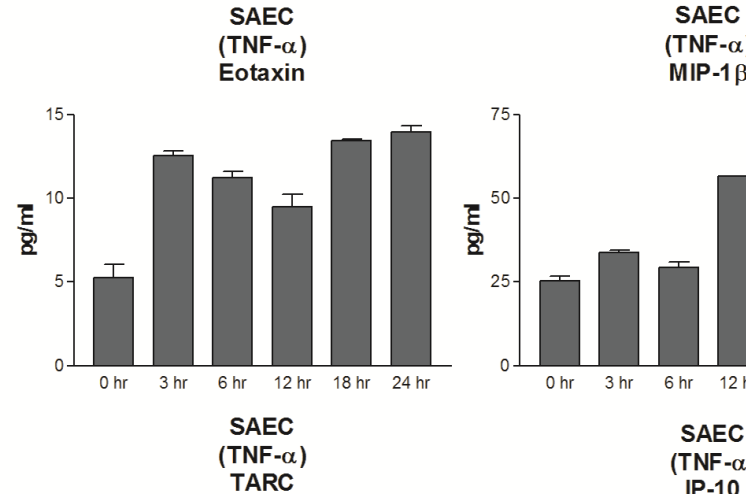

AEC
NF- $\alpha)$

TNF- $\alpha$ )
MIP-1 $\beta$

SAEC

(TNF- $\alpha$ )

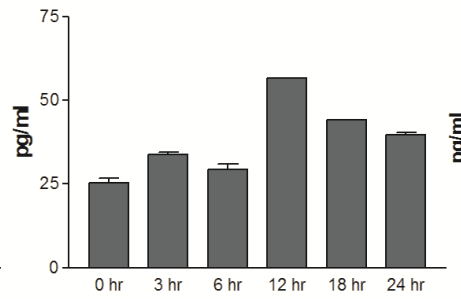

Eotaxin-3

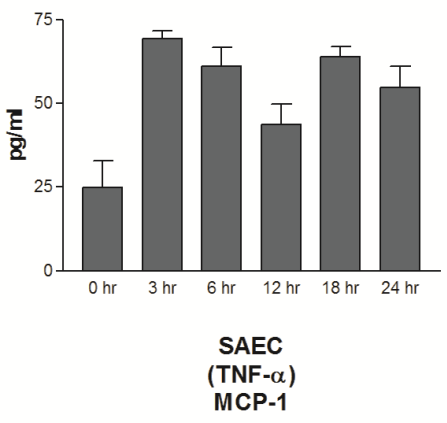

SAEC

(TNF- $\alpha$ )

(TNF- $\alpha$ )
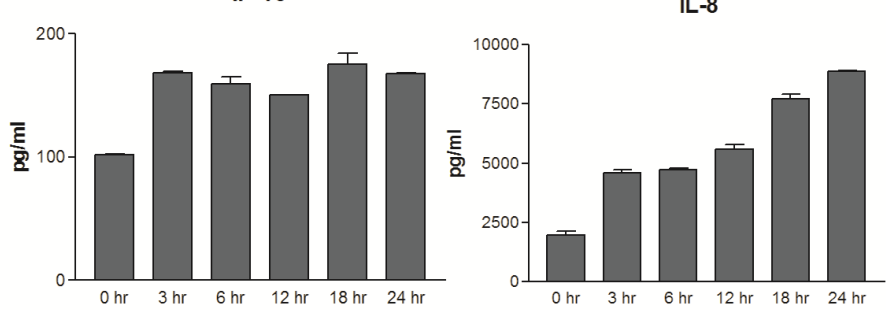

SAEC

(TNF- $\alpha$ )

SAEC

(TNF- $\alpha$ )
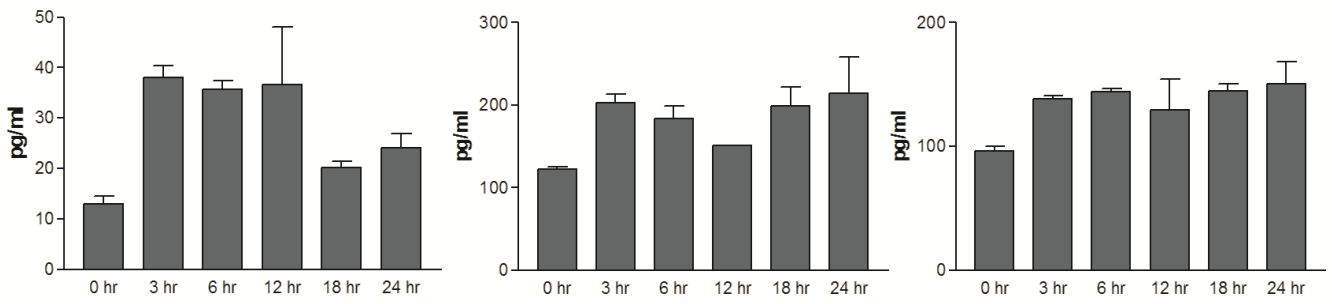
Appendix E: Effects of HB-I-172 and SMM-I-229 on the Cytokine and Chemokine Release of HMVEC-LBI, Monocyte Derived Macrophages, and SAEC 

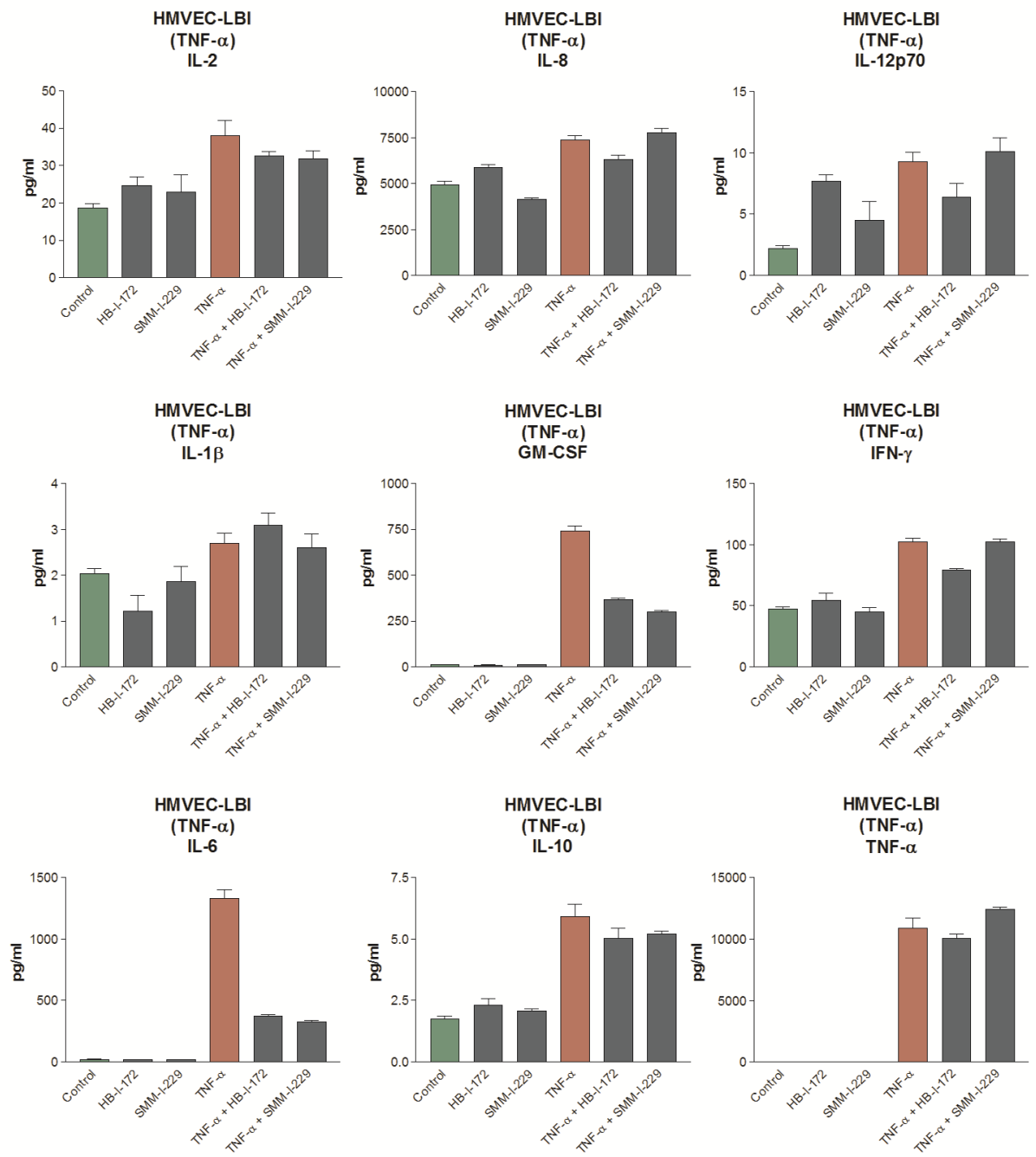

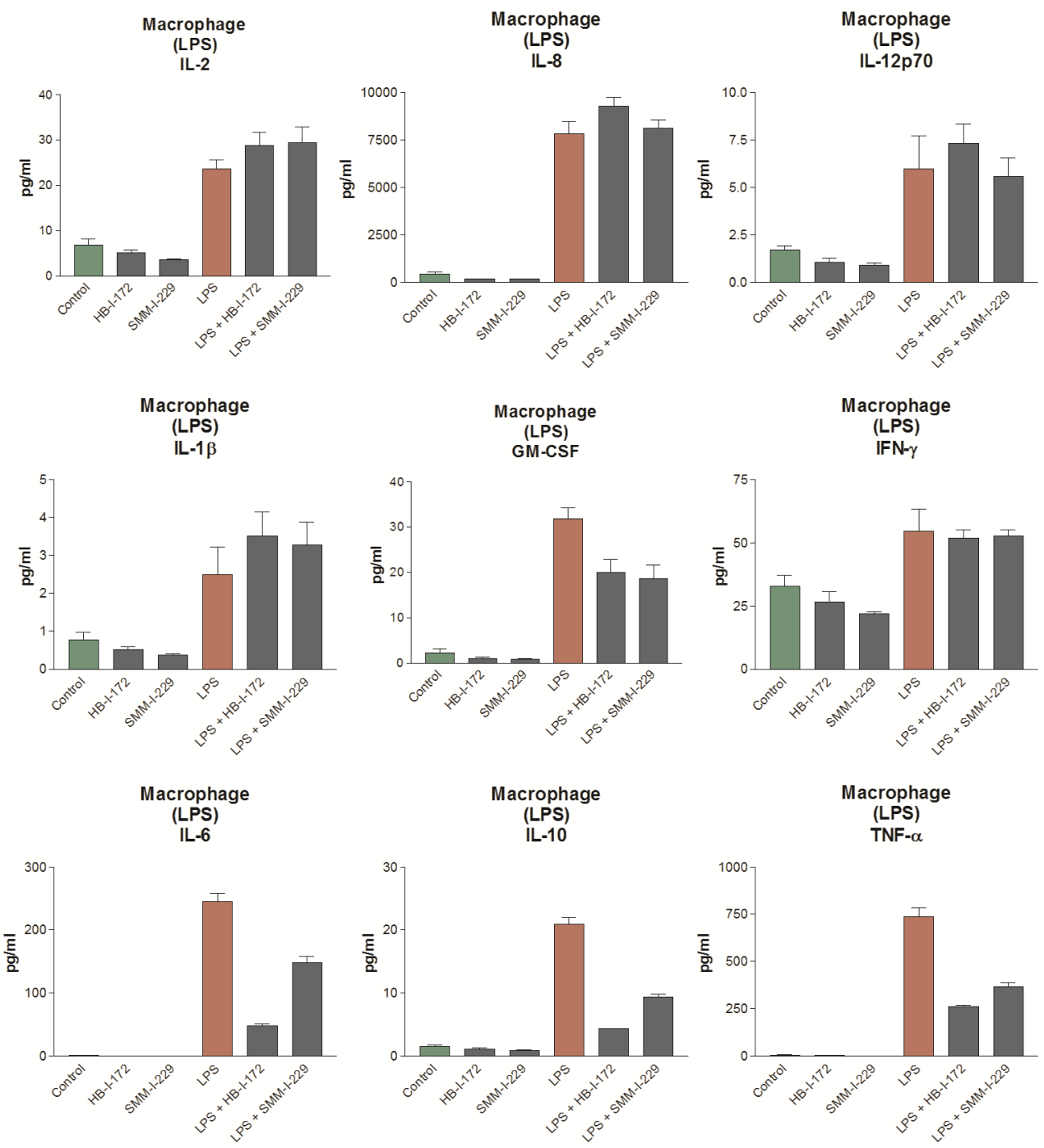

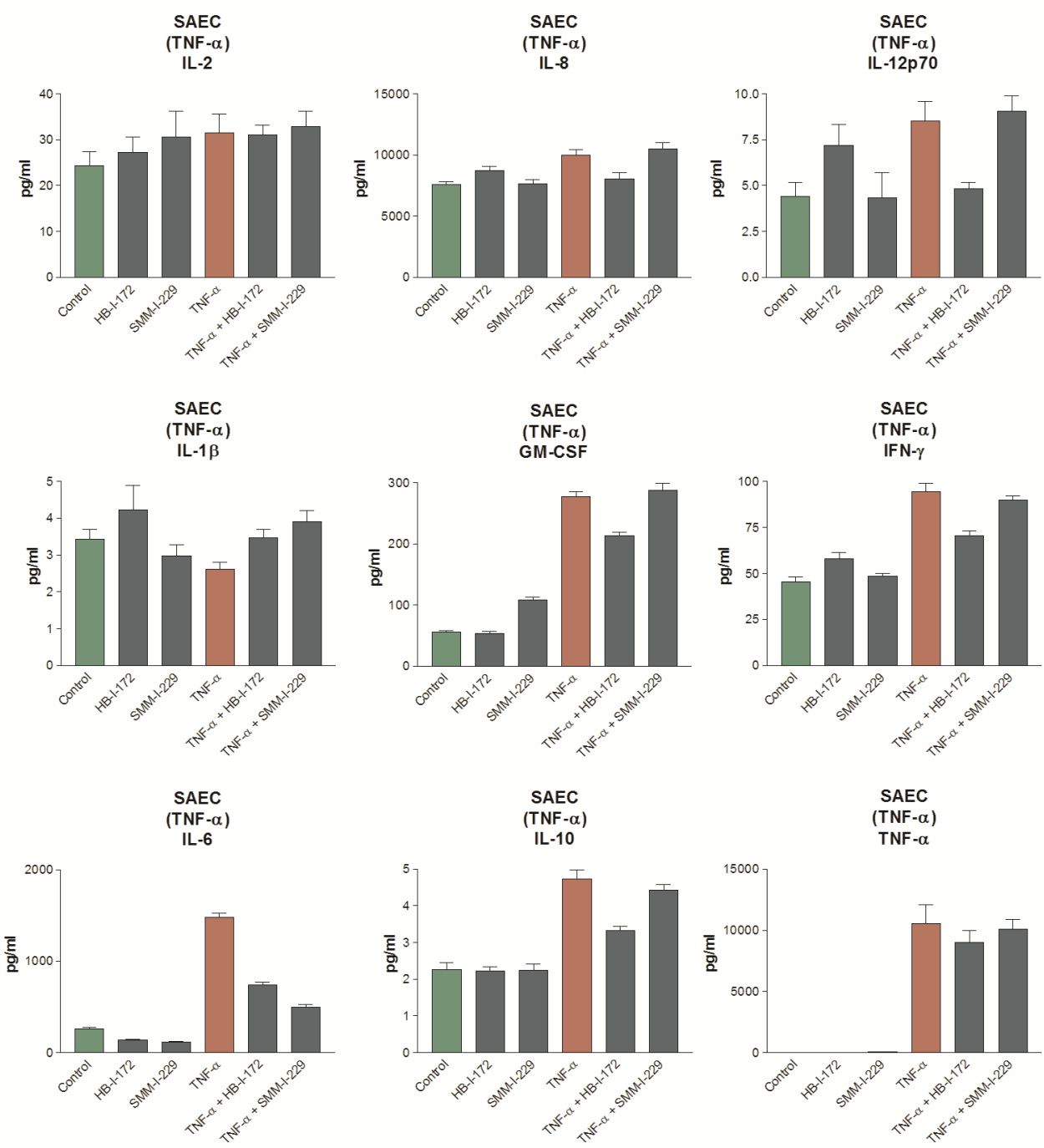

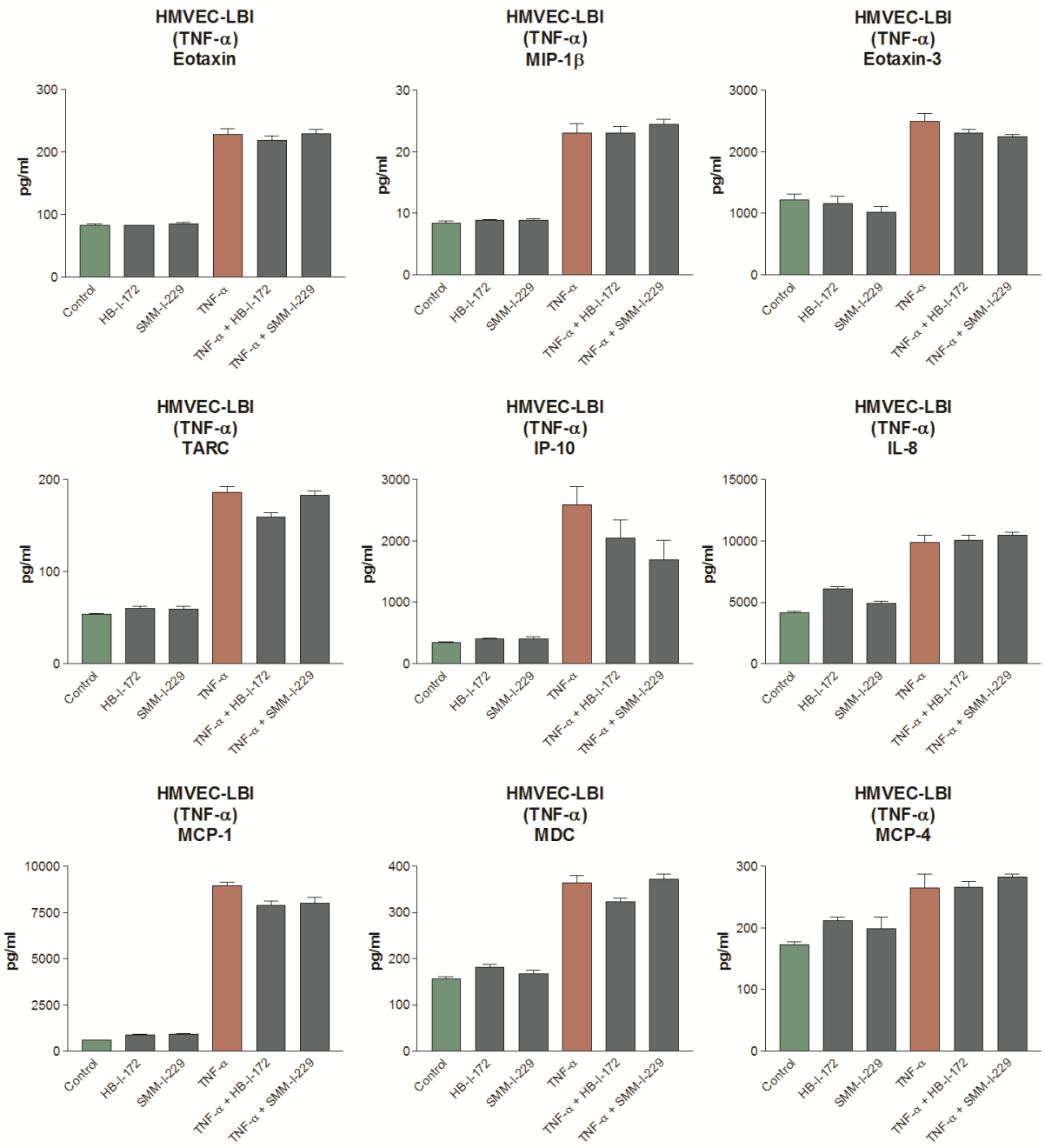

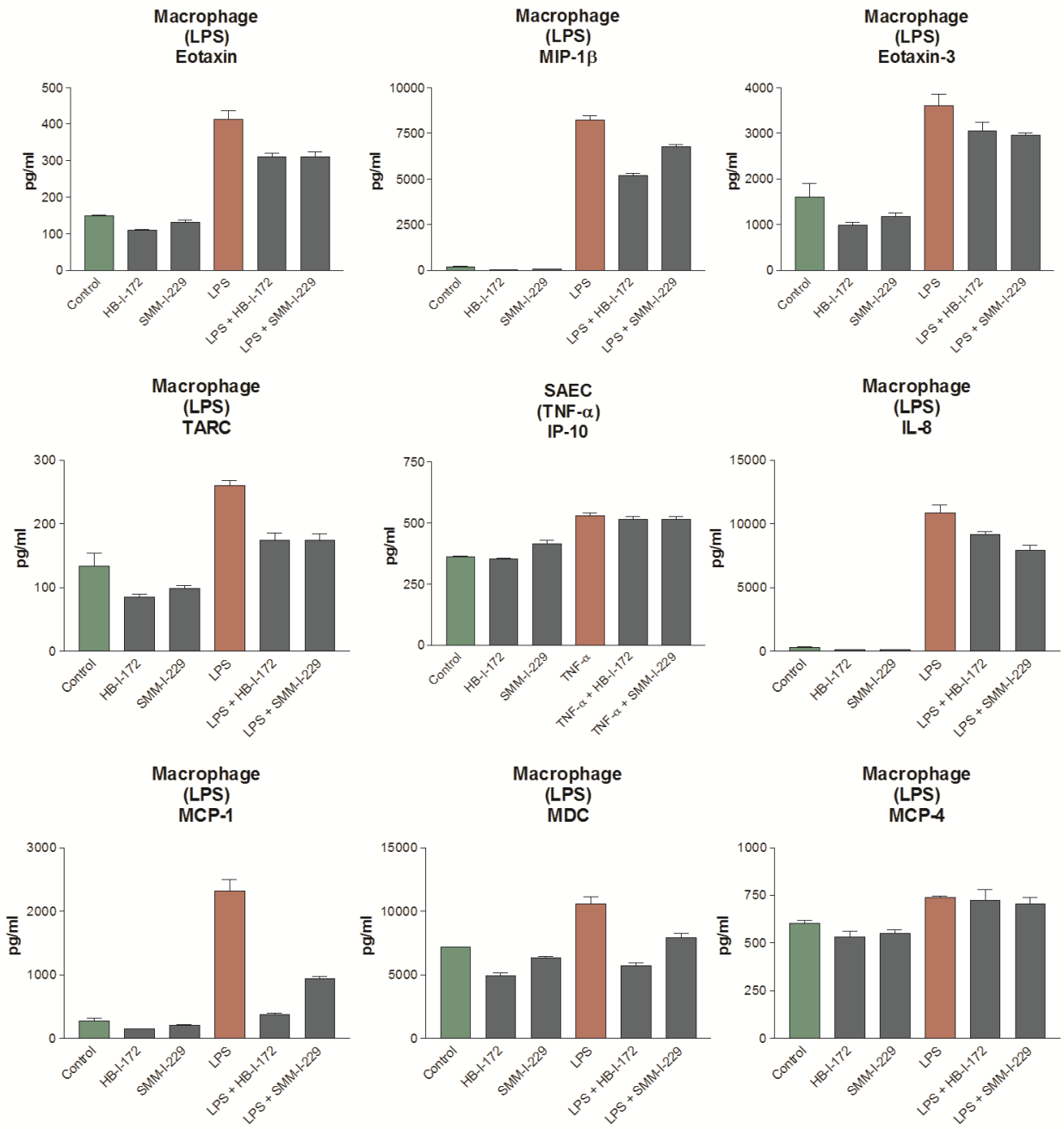

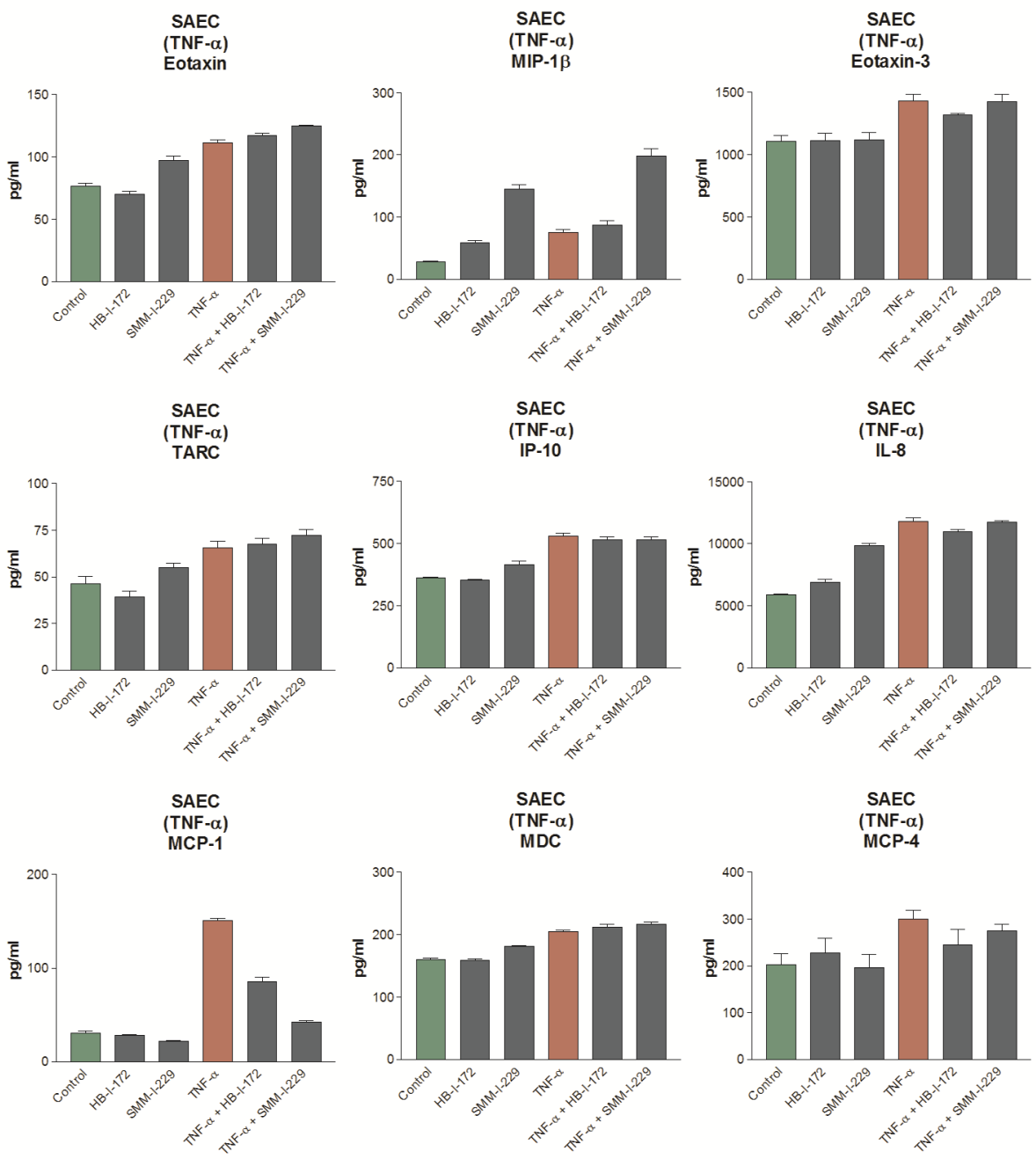
Appendix F: Cytokine and Chemokine Release Profiles of Primary Human Microglia 

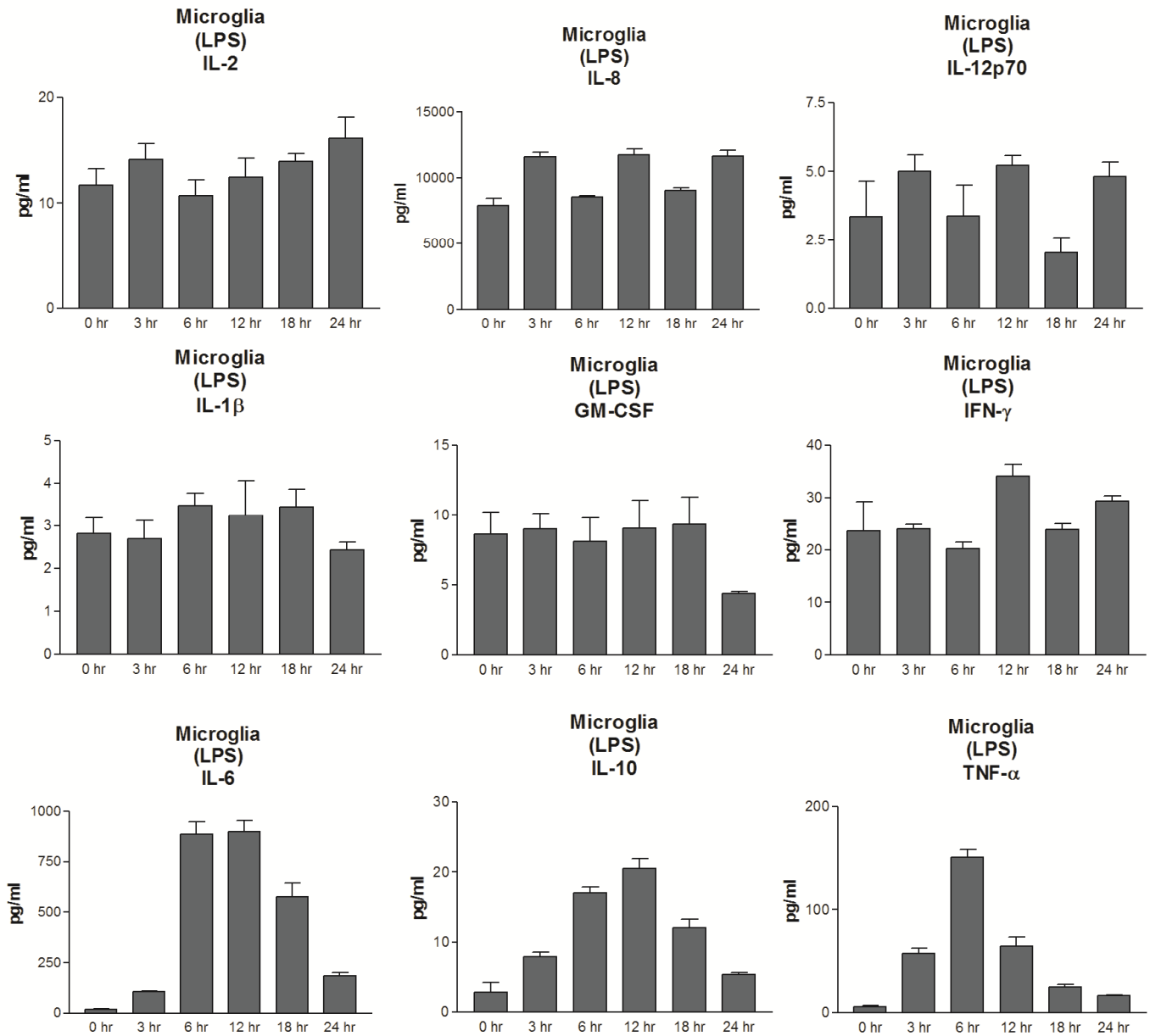

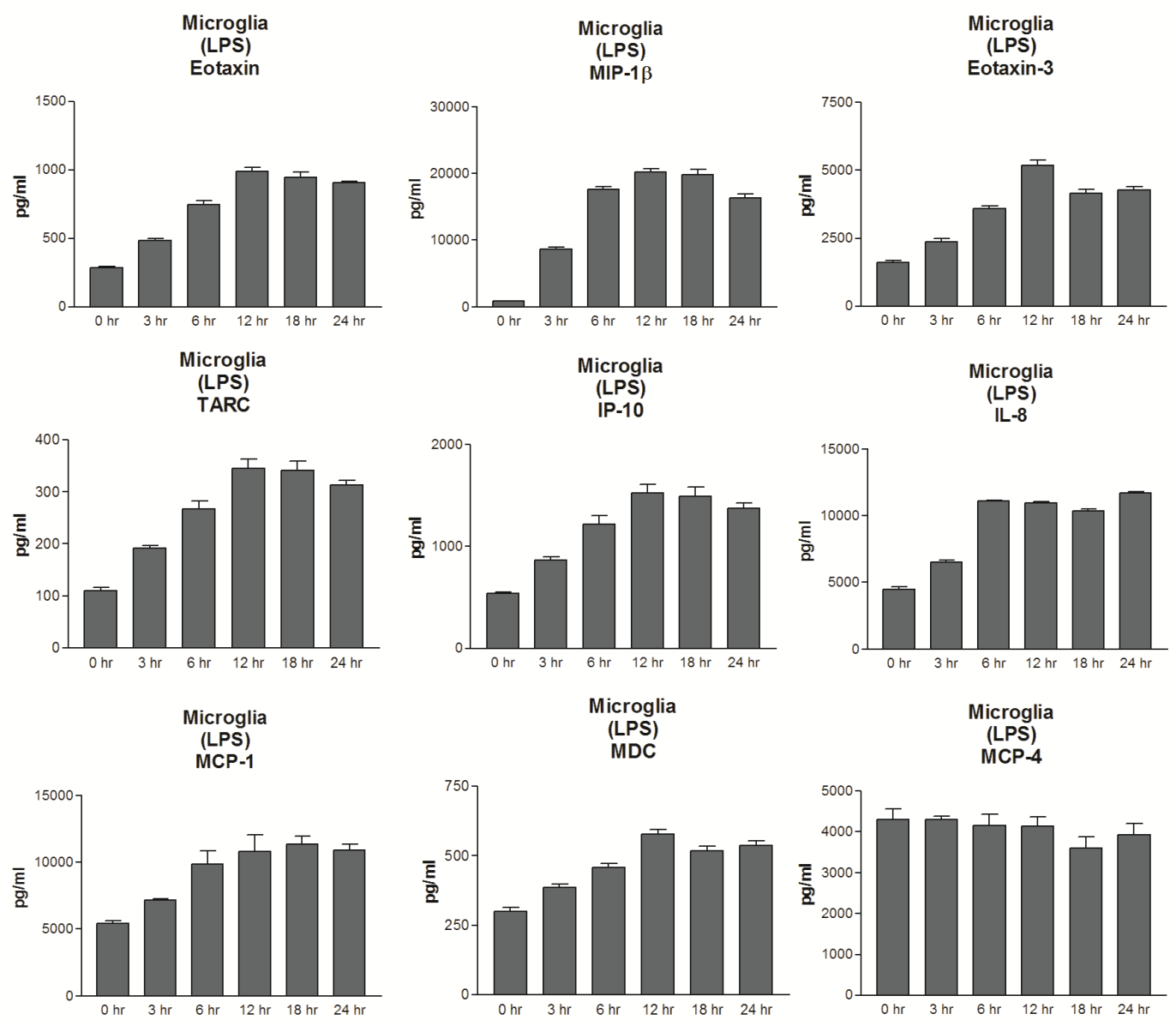
Appendix G: Effects of HB-I-172 and KM-233 on the Cytokine and Chemokine Release of Primary Human Microglia 

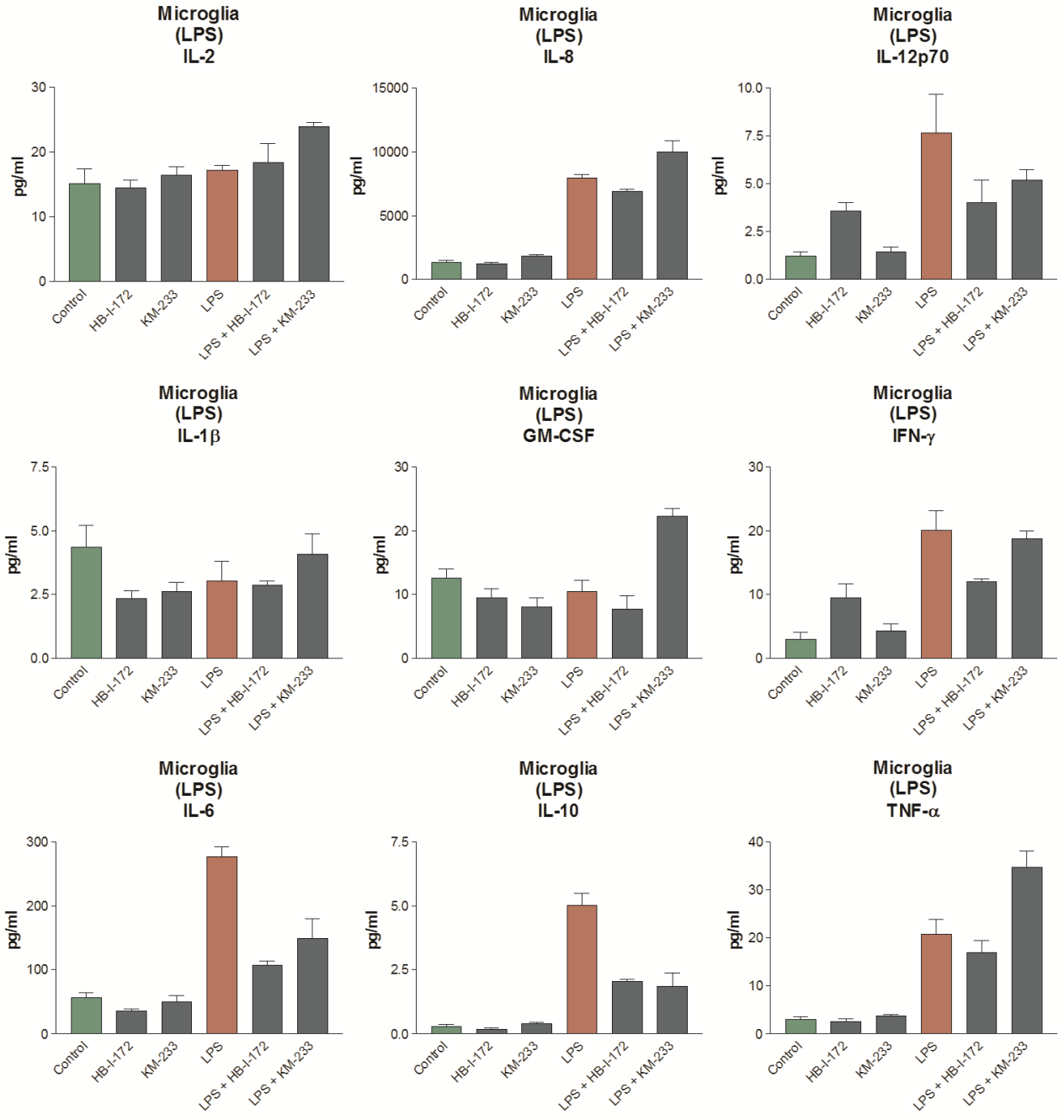

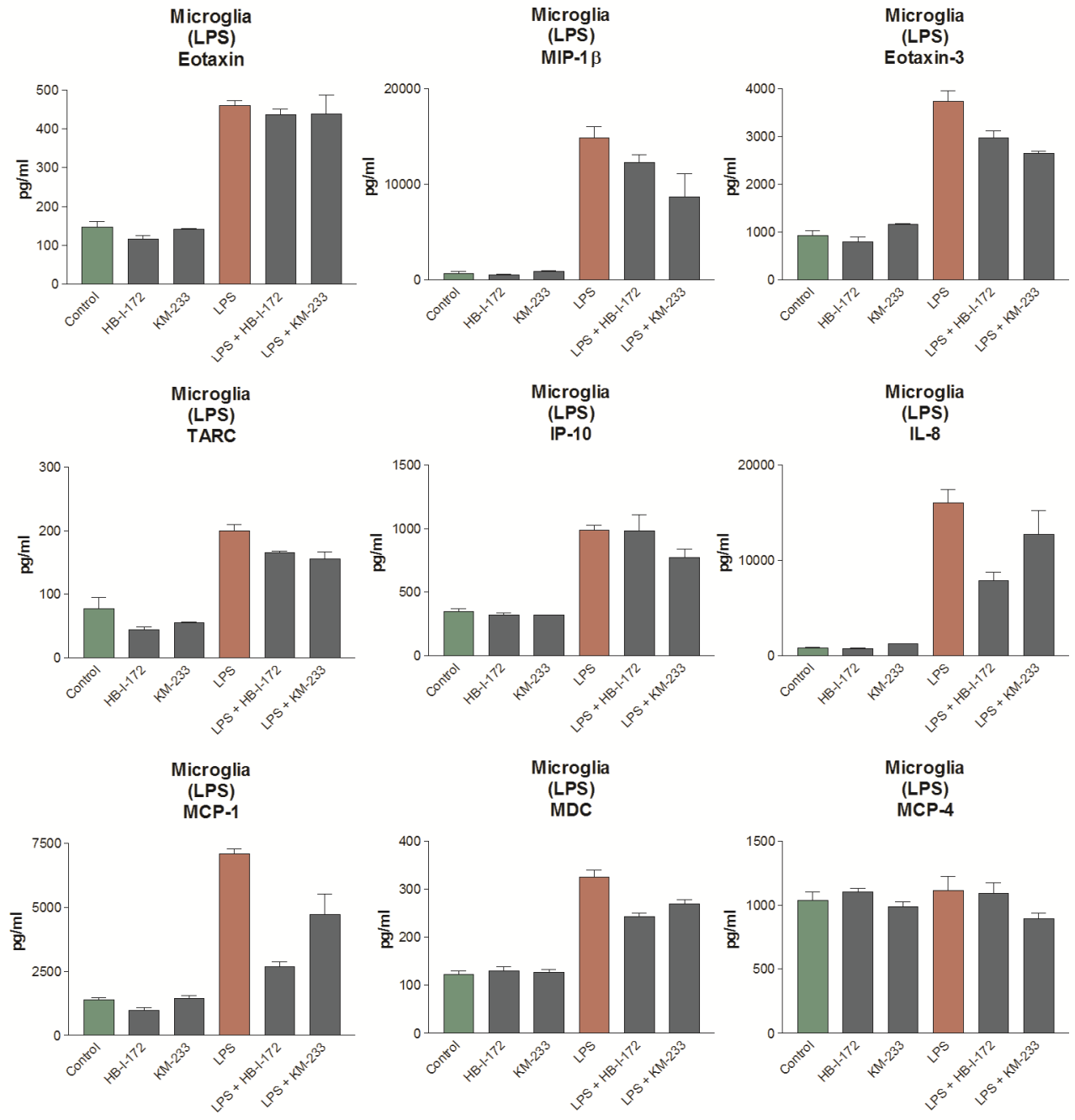

Microglia

IL-8
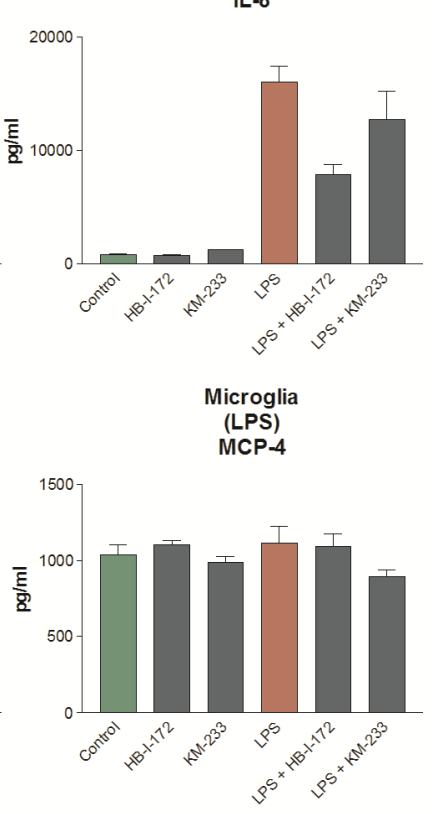
Appendix H: Comparison of the Effects of HB-I-172 to Dexamethasone on the Cytokine and Chemokine Release of THP-1, A549, and HMVEC-LBI 

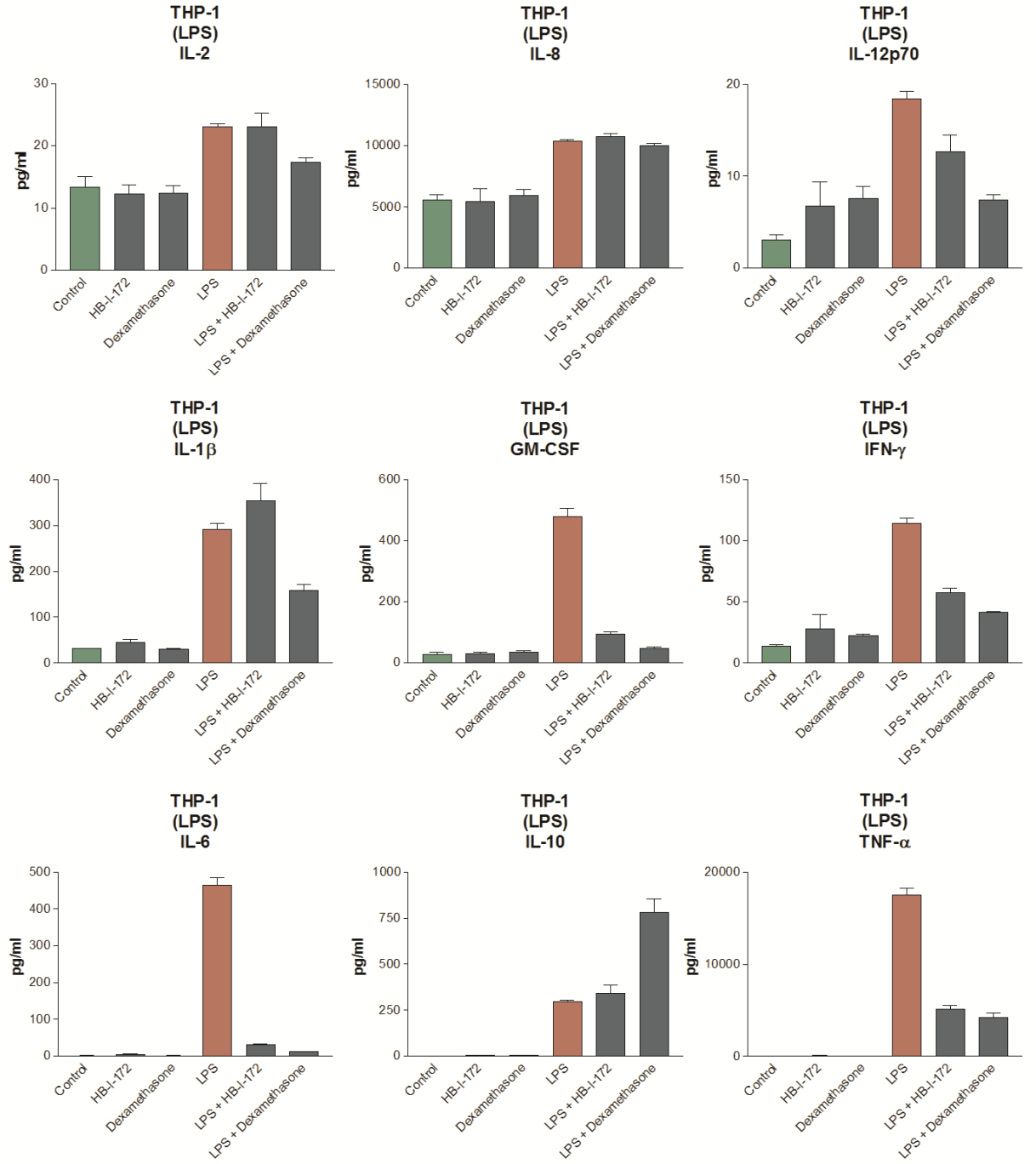

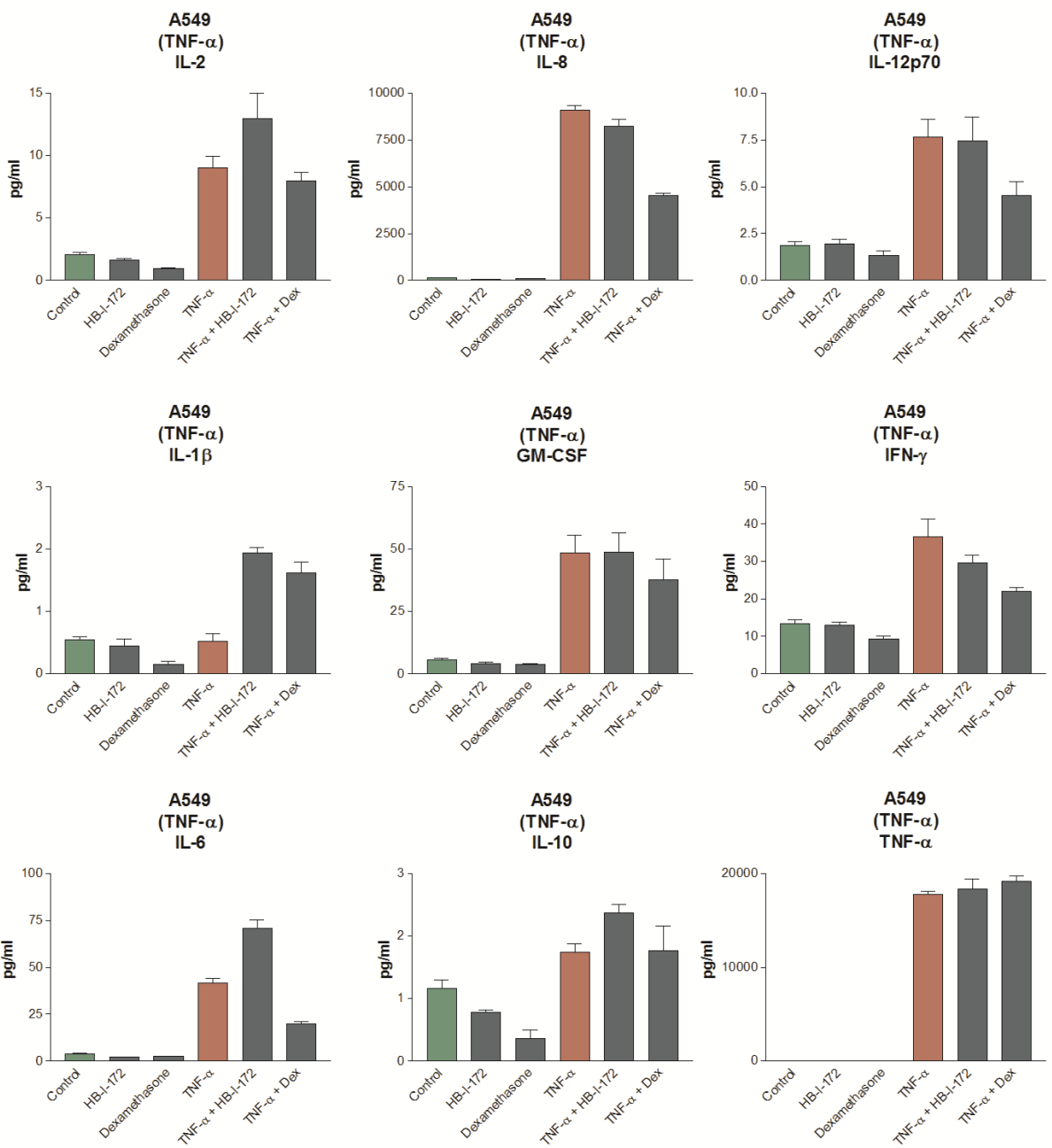

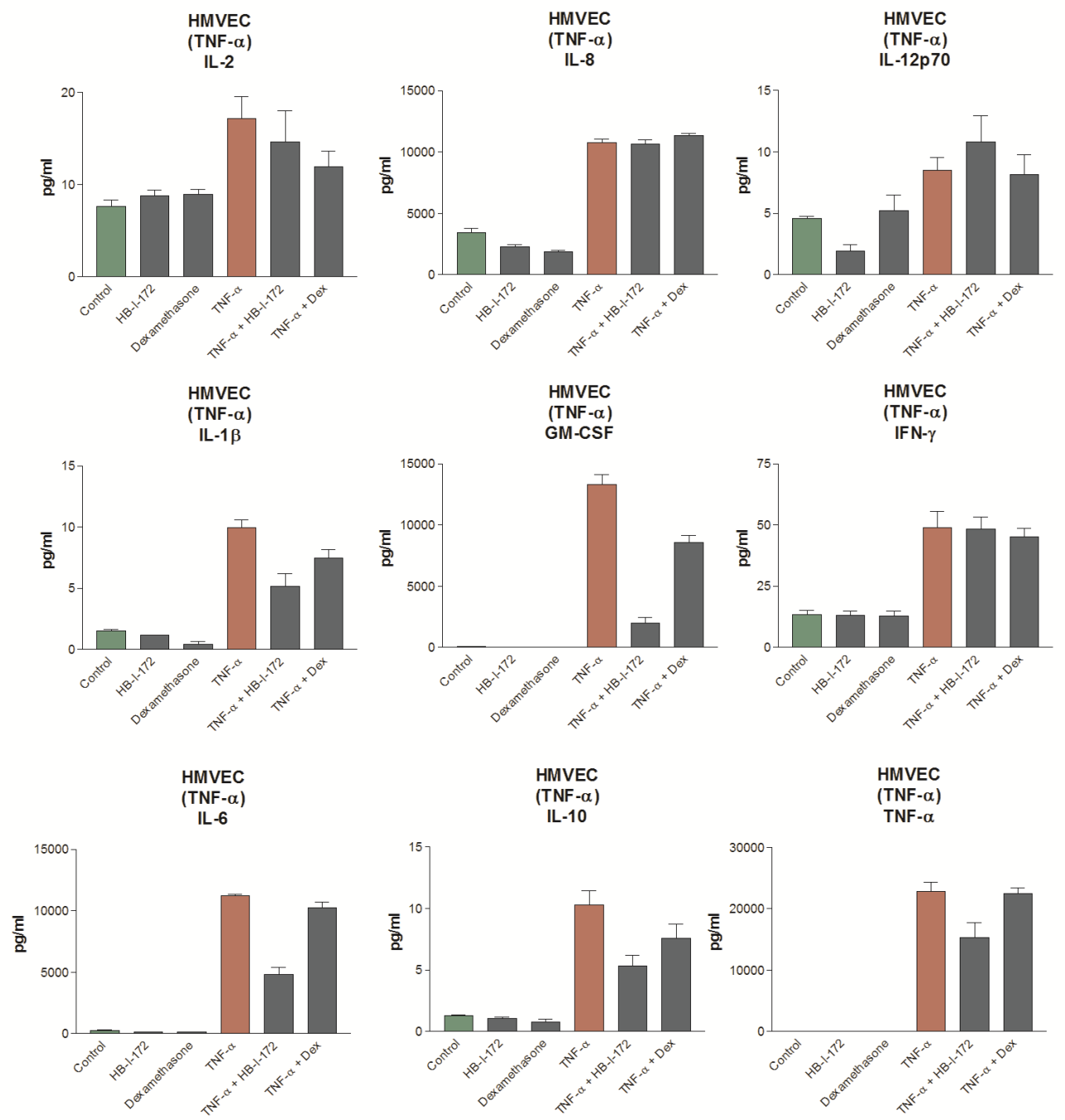

TNF- $\alpha$ )

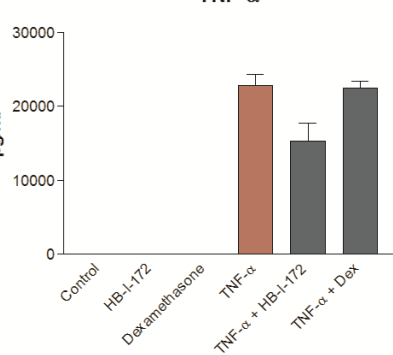



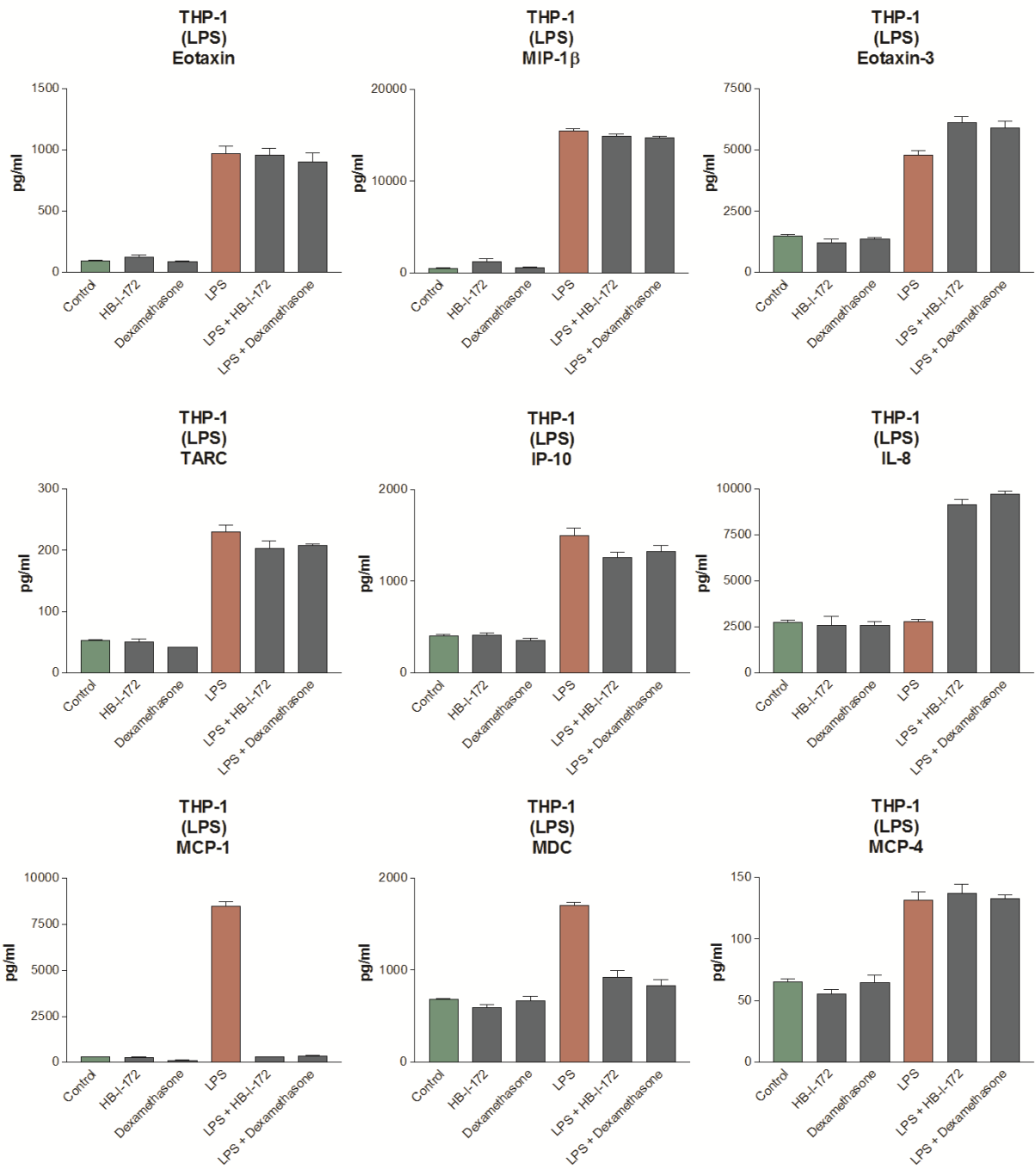

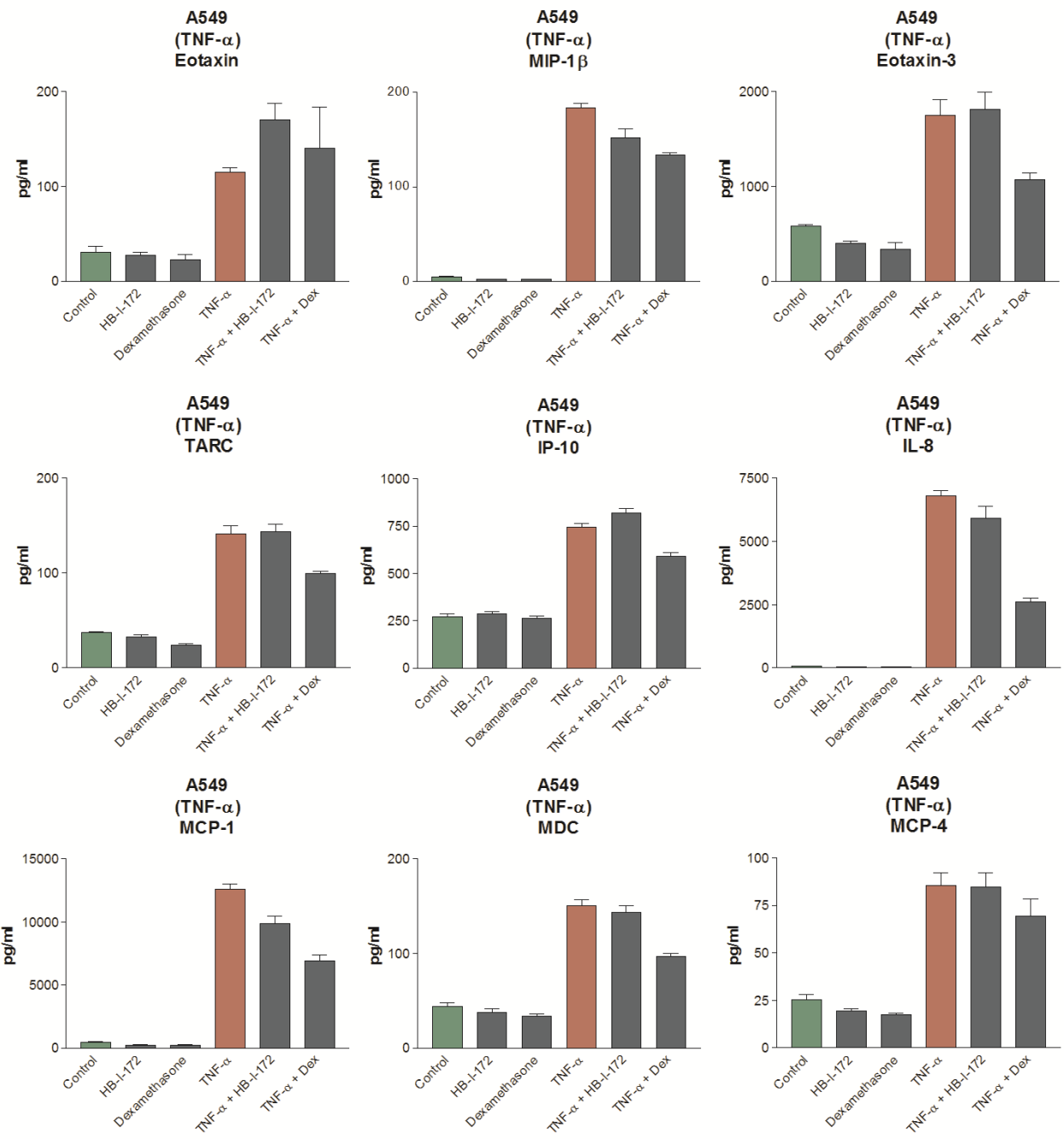

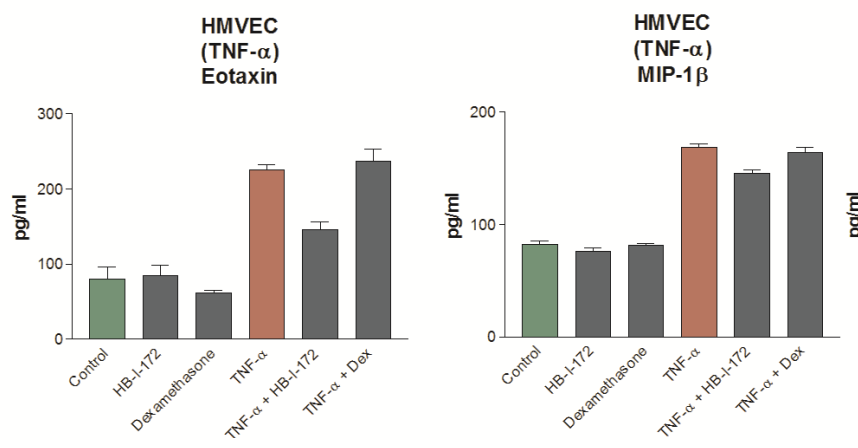

HMVEC

(TNF- $\alpha$ )

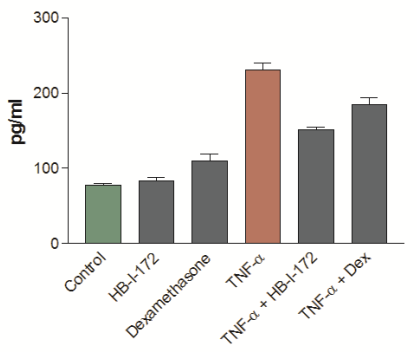

HMVEC

(TNF- $\alpha$ )

MCP-1

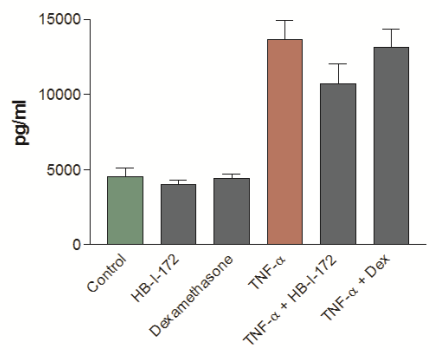

(TNF- $\alpha$ )
IP-10

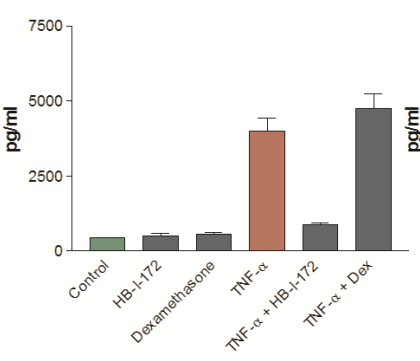

HMVEC

(TNF- $\alpha$ )

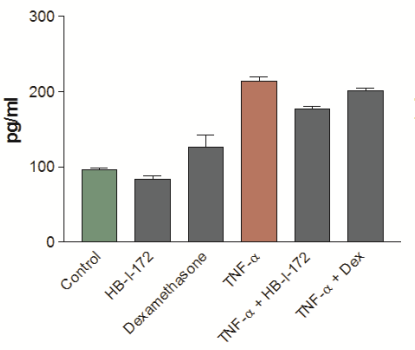

HMVEC

(TNF- $\alpha$ )

Eotaxin-3

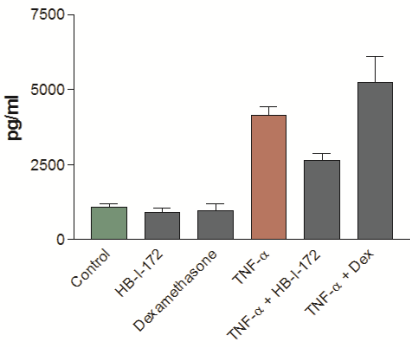

HMVEC

(TNF- $\alpha$ )

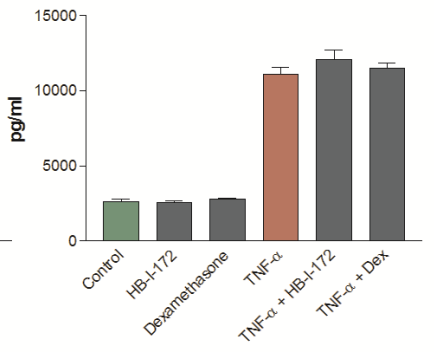

HMVEC

(TNF- $\alpha$ )

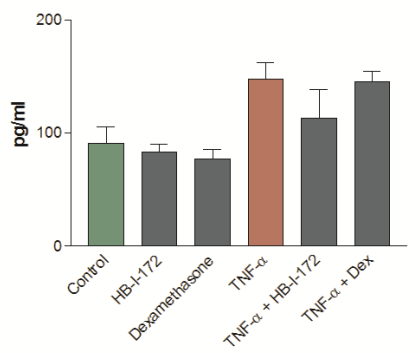




\section{Vita}

Steven Neal Gurley was born on September 12, 1972 in South Williamson, Kentucky. He received the degree Bachelor of Science in May, 1995 from the University of Tennessee at Martin majoring in Chemistry and Biology. He joined the College of Pharmacy and Graduate Health Sciences at the University of Tennessee Health Science Center in the summer of 2001 and earned the degree of PharmD in May, 2005, and the degree of Ph.D. in May, 2010. 